\author{
Universidade de São Paulo \\ Instituto de Astronomia, Geofísica e Ciências Astmosféricas \\ Departamento de Astronomia
}

\author{
Bruno Dias
}

\title{
Formation and evolution of globular clusters in the Galaxy and Magellanic Clouds
}

Formação e evolução de aglomerados globulares da Galáxia e Nuvens de Magalhães

São Paulo 

Bruno Dias

\section{Formation and evolution of globular clusters in the Galaxy and Magellanic Clouds}

Formação e evolução de aglomerados globulares da Galáxia e Nuvens de Magalhães

Thesis presented to Departamento de Astronomia of Instituto de Astronomia, Geofísica e Ciências Astmosféricas, Universidade de São Paulo, as partial requirement to get the title of $\mathrm{PhD}$ in Science.

Area: Astronomy

Advisor: Prof. Dr. Beatriz Barbuy

Updated version. Original can be found at the Institute.

Versão Corrigida. O original encontra-se disponível na Unidade.

São Paulo 

To my family. 



\section{Acknowledgements}

To my advisor Beatriz Barbuy for making me a professional astrophysicist;

To my internship advisor Ivo Saviane for receiving me at ESO/Santiago, and to Oscar Gonzalez for being my fellow mentor at ESO;

To the professor Silvia Rossi for helping me during the whole process;

To the professors Jorge Melendez and Walter Maciel for evaluating my PhD project;

To the astronomers Paula Coelho, Alan Alves Brito, Claudio Melo for many important discussions for my thesis and carreer;

To the astronomers Enrico Held, Ana Chies-Santos, Ricardo Schiavon, Leandro Kerber for inviting me as visitor astronomer in their institutes and for excellent discussions;

To the astronomers Sergio Ortolani and Dimitri Gadotti for teaching me in situ about NTT/ESO and FLAMES@VLT/ESO, respectively

To the professor Dante Minniti for accepting me as member of the VVV survey;

To the colleagues Marina Trevisan, Cesar Siqueira Mello, Marcelo Tucci for sharing the office, discussions and coffees with me;

To the colleagues Phillip, Eduardo, Beatriz, Thais for staying together for many years;

To the ESO colleagues Anna, Julien, Jos, Amy, Sergio, Matias, Florian, Joachim, Daniela, Paul, Marylou, Vera, Mirjam.

To CNPq by the financial support under project 142047/2010-4;

To ESO by the award of a studentship for one year in Santiago/Chile.

To my family Alvino, Nilda, Gabriel, Paula for supporting me all the time;

To my friends for making my life easier during the last four years.

This thesis was written in LTEX $_{\mathrm{E}} \mathrm{X}$ with the class IAGTESE for thesis and dissertations of IAG. 

"One's ideas must be as broad as Nature if they are to interpret Nature" Sherlock Holmes on "A Study in Scarlet". Sir Arthur Conan Doyle (1887)

"I suppose it is clear to everyone that astronomy at all events compels the soul to look upwards, and draws it from the things of this world to the other" Glaucon on the Republic, Book Seven. Plato (BC 427-BC 347) 



\section{Resumo}

Aglomerados globulares são traçadores da formação e evolução de suas galáxias. Cinemática, abundâncias químicas, idades e posições dos aglomerados permitem traçar interações entre Via Láctea e galáxias vizinhas e suas histórias de enriquecimento químico. Nesta tese analisamos espectros de média resolução de mais de 800 estrelas gigantes vermelhas em 51 aglomerados globulares Galácticos. É a primeira vez que $[\mathrm{Fe} / \mathrm{H}]$ and $[\mathrm{Mg} / \mathrm{Fe}]$ determinados de modo consistente são publicados para uma amostra desse porte, $\sim 1 / 3$ dos objetos catalogados. Nossas metalicidades são mais precisas que trabalhos anteriores similares. Uma quebra em $[\mathrm{Fe} / \mathrm{H}] \sim-1.0$ é encontrada no gráfico $[\mathrm{Fe} / \mathrm{H}]$ vs. $[\mathrm{Mg} / \mathrm{Fe}]$ para o bojo e halo, embora bojo parece ter uma quebra em $[\mathrm{Fe} / \mathrm{H}]$ maior, i.e, bojo tem formação mais eficiente que o halo. Comparando abundâncias com idade, a escala de tempo para SNIa ficar importante é 1Gano. [Fe/H] vs. idade corrobora diferentes eficiências de formação do bojo e halo, mas $[\mathrm{Mg} / \mathrm{Fe}]$ vs. idade não mostra isso. O halo foi formado em mini halos ou galáxias anãs, e dois aglomerados com dispersão em $[\mathrm{Fe} / \mathrm{H}]$ tiveram suas origens analisadas. M 22 parece ter sido formado na Via Láctea e NGC 5824 possivelmente foi originado em uma galáxia anã, embora os resultados são inconclusivos para NGC 5824. O bojo parece ter sido formado rapidamente e deve possuir o aglomerado mais velho. De fato, HP 1 tem um ramo horizontal mais azul que o esperado para sua metalicidade e vemos isso como um efeito da idade. Determinamos sua distância usando curvas de luz de RR Lyrae de maneira a restringir futuras determinações de idade via diagrama cor-magnitude. Finalmente, investigamos a interação entre Via Láctea e sua galáxia vizinha SMC. Encontramos aglomerados sendo removidos do corpo central da SMC, consistente com cenário de remoção por força de maré para a interação entre as galáxias, em vez de "ram pressure" que afeta só gás. 



\section{Abstract}

Globular clusters are tracers of the formation and evolution of their host galaxies. Kinematics, chemical abundances, age and position of the clusters allows tracing interactions between Milky Way and surrounding galaxies and outlines their chemical enrichment history. In this thesis we analyse mid-resolution spectra of about 800 red giant stars in 51 Galactic globular clusters. It is the first time that $[\mathrm{Fe} / \mathrm{H}]$ and $[\mathrm{Mg} / \mathrm{Fe}]$ derived in a consistent way are published for such a huge sample of globular clusters, almost $1 / 3$ of the total number of catalogued clusters. Our metallicities are showed to be more precise than previous works based on mid-resolution spectroscopy. A turnover at $[\mathrm{Fe} / \mathrm{H}] \sim-1.0$ is found in the plot $[\mathrm{Fe} / \mathrm{H}]$ vs. $[\mathrm{Mg} / \mathrm{Fe}]$ for bulge and halo, although bulge seems to have a more metal-rich turnover, i.e, bulge has more efficient formation than the halo. Comparing the abundances with age the timescale for SNIa to start to become important is $1 \mathrm{Gyr}$. $[\mathrm{Fe} / \mathrm{H}]$ vs. age corroborates the different star formation efficiency of bulge and halo while $[\mathrm{Mg} / \mathrm{Fe}]$ does not follow that. Halo was formed in mini halos or dwarf galaxies, and two multiple population clusters had their origin analysed to check it. M 22 seems to have been formed in the Milky Way while NGC 5824 possibly was originated in a dwarf galaxy, although our results are inconclusive for NGC 5824. The Galactic bulge seems to have been formed fast i.e., probably the oldest globular cluster is there. In fact HP 1 has a bluer horizontal branch than expected for its metallicity and we interpret that as an age effect. We determine its distance using light curves of variable stars in order to constrain future age determinations via colour-magnitude diagram. Finally, we investigate interaction between Milky Way

and its neighbour galaxy SMC. We find that some star clusters are being stripped out of the SMC main body, which is consistent with tidal stripping scenario for the interaction between the galaxies, instead of ram pressure that would only affect gas. 



\section{List of Figures}

1.1 Schematic view of the X-shaped bulge of the Milky Way. . . . . . . . . . 26

1.2 Magellanic Clouds and Magellanic Stream in terms of H I . . . . . . . . . 28

2.1 Sky maps of the six SMC clusters . . . . . . . . . . . . . . 40

2.2 Completeness curves for the six SMC clusters . . . . . . . . . . . . 41

$2.3 \mathrm{~V}$ and $(\mathrm{B}-\mathrm{V})$ photometric errors for the six SMC clusters. . . . . . . . . 42

$2.4 \mathrm{~V},(\mathrm{~B}-\mathrm{V})$ colour-magnitude diagrams for all clusters and control fields . . . 42

2.5 Radial density profiles fitted by King profiles for all clusters . . . . . . . . 44

2.6 Process of generation of a synthetic V, (B-V) CMD . . . . . . . 47

2.7 Best isochrone fittings for all clusters . . . . . . . . . . . . . . 49

2.8 Distribution of star clusters in the projected plane of the SMC . . . . . . 53

2.9 Age-metallicity relation for the $\mathrm{SMC} \ldots \ldots \ldots \ldots \ldots$

2.10 Age and $[\mathrm{Fe} / \mathrm{H}]$ as a function of the distance to the centre of SMC . . . . 55

3.1 Colour-Magnitude diagrams of the six Milky Way globular clusters . . . . . 62

3.2 Sky maps of the six Milky Way globular clusters . . . . . . . . . . . . . 63

3.3 Identificantion of most important atomic lines in each strong feature of $\mathrm{R} \sim 2000$ metal-poor and metal-rich stellar spectra . . . . . . . . . . 64

3.4 Heliocentric radial velocities for all clusters . . . . . . . . . . . . . . 65

3.5 Examples of spectral fitting for a metal-rich and a metal-poor star . . . . . 67

3.6 Parameter space of MILES library displayed in a HR diagram . . . . . . 68

3.7 Procedure to find best $\mathrm{T}_{\text {eff }}, \log (g)$ and $[\mathrm{Fe} / \mathrm{H}]$ for star HD122956 . . . . . 72

3.8 Comparisons of $\mathrm{T}_{\text {eff }}, \log (g),[\mathrm{Fe} / \mathrm{H}]$ from this work with literature for wellknown field stars 
3.9 Comparison of derived $\mathrm{T}_{\text {eff }}$ and $\log (g)$ with isochrones for member stars of

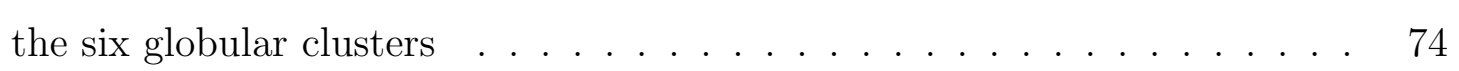

3.10 Comparison of derived $[\mathrm{Fe} / \mathrm{H}]$ with $\mathrm{CaT}$ results from literature for the stars from all clusters . . . . . . . . . . . . . . . . . . 75

3.11 Procedure for membership selection of stars in each globular cluster . . . . 77

3.12 HR diagram for Terzan 8 comparing Teff, $\log (g)$ from this work with high-

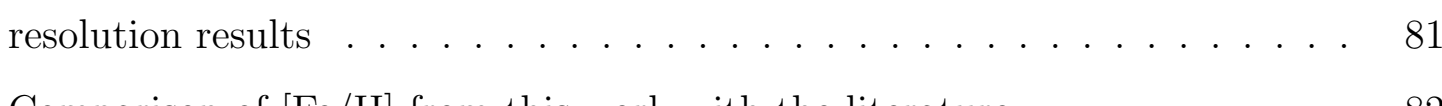

3.13 Comparison of $[\mathrm{Fe} / \mathrm{H}]$ from this work with the literature . . . . . . . 82

$3.14[\mathrm{Mg} / \mathrm{Fe}]$ vs. $[\mathrm{Fe} / \mathrm{H}]$ of Milky Way field stars overplotted by the average results for each cluster from this cluster.

4.1 Sky position of the 51 clusters over Milky Way image . . . . . . . . . 91

4.2 Reddening versus distance of the 51 globular clusters . . . . . . . . . . 92

4.3 Comparison of $[\mathrm{Fe} / \mathrm{H}]$ with results of Carretta's scale for 13 clusters in com-

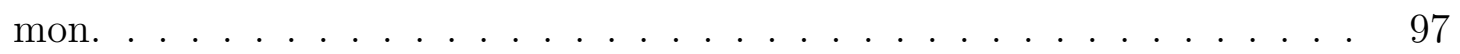

4.4 Comparison of calibrated $[\mathrm{Fe} / \mathrm{H}]$ with those from Carretta et al. (2009) for the 51 clusters . . . . . . . . . . . . . . . . . . . 101

4.5 Chemical evolution of the Milky Way in terms of $[\mathrm{Mg} / \mathrm{Fe}]$ vs. $[\mathrm{Fe} / \mathrm{H}] \ldots$. . 103

4.6 Age vs. $[\mathrm{Fe} / \mathrm{H}]$ and $[\mathrm{Mg} / \mathrm{Fe}]$ for 17 clusters . . . . . . . . . . . . 104

4.7 Metallicity radial gradients for Milky Way globular clusters . . . . . . . . 105

5.1 Magnitude vs. radius for GCs and UCDs . . . . . . . . . . . . . 109

5.2 Colour-Magnitude diagrams of two globular clusters with multiple stellar

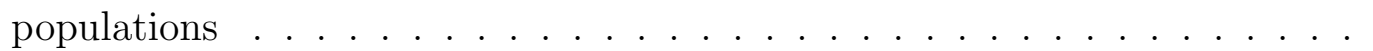

5.3 Sky map of two globular clusters with multiple stellar populations . . . . . 111

5.4 Comparison of derived $\mathrm{T}_{\text {eff }}$ and $\log (g)$ with isochrones for member stars of the six globular clusters

5.5 Procedure for membership selection of stars in M 22 and NGC 5824 . . . 113

$5.6[\mathrm{Fe} / \mathrm{H}]$ and $[\mathrm{Mg} / \mathrm{H}]$ distribution function of $\mathrm{M} 22 \ldots \ldots \ldots$

5.7 Comparison of sample stars from this work with Marino et al. (2011) for M 22

5.8 Chemical evolution history of $\mathrm{M} 22 \ldots \ldots \ldots \ldots \ldots \ldots$

5.9 Metallicity distribution function of NGC $5824 \ldots \ldots$. . . . . . . 118 
5.10 Chemical evolution history of NGC 5824

6.1 Colour-magnitude diagram of HP 1 with the six RR Lyrae candidates . . . 123

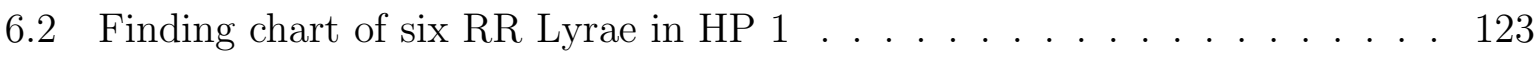

6.3 Light curves of five confirmed RR Lyrae in HP 1 . . . . . . . . . . . 124

6.4 Periodogram $\theta$ vs. period for the five confirmed RR Lyrae in HP 1 . . . . 125

6.5 Folded light curves for five RR Lyrae candidates in HP1, using the best periods found in Figure 6.4 . . . . . . . . . . . . . . . 126

6.6 Period-luminosity relation applied to calculate the distance . . . . . . . . 127

A.1 Likelihood from the CMD fits for different combinations of age, metallicity, reddening, and distance modulus for AM 3. . . . . . . . . . 158

A.2 Same as Fig. A.1 for HW 1. . . . . . . . . . . . . . . . . 158

A.3 Same as Fig. A.1 for HW 40. . . . . . . . . . . . . . 159

A.4 Same as Fig. A.1 for Lindsay 2. . . . . . . . . . . . . . . . . . 159

A.5 Same as Fig. A.1 for Lindsay 3. . . . . . . . . . . . . . . 159

C.1 Output two dimensional spectra of FORS2 pipeline using default parameters 210

C.2 Default list of skylines from FORS2 manual . . . . . . . . . . . . . 211

C.3 New list of skylines proposed in this work . . . . . . . . . . . . 212

C.4 Output two dimensional spectra of FORS2 pipeline using the new approach 213

C.5 Comparison between extracted spectrum from slit \#8 using default parameters with that using the new procedure . . . . . . . . . . 214

C.6 Same of Figure C.5, but for the slit \#6. . . . . . . . . . . . . . . . . . . . 215

C.7 Comparison between extracted spectra from slit \#8 with that from slit \#6 using default parameters and the new procedure . . . . . . . . . 216

D.1 Abstract of Borissova et al. (2011). . . . . . . . . . . . . . 217

D.2 Abstract of Saito et al. (2012) . . . . . . . . . . . . . . 218

D.3 Abstract of Saito et al. (2012) . . . . . . . . . . . . . . . 219

D.4 Abstract of Dias et al. (2014) . . . . . . . . . . . . . . 220

D.5 Abstract of Mauro et al. (2014) . . . . . . . . . . . . . . . . . 221

D.6 Abstract of Dias et al. (2014b), submitted to A\&A and accepted after revision.222 



\section{List of Tables}

2.1 Log of observations of the six SMC clusters . . . . . . . . . . . . 38

2.2 Colour coefficient and photometric zero point from standard stars calibration 39

2.3 Structural parameters derived from King profile fitting for the six SMC clusters 44

2.4 Stellar counts in the cluster and control field CMDs of the six clsuters . . . 46

2.5 Age, metallicity, distance and reddening derived for the SMC clusters . . . 49

3.1 FORS2 spectrograph details . . . . . . . . . . . . . . . . 60

3.2 Log of observations and parameters from literature for the six globular clusters 61

3.5 Final average parameters for each globular cluster and respective internal

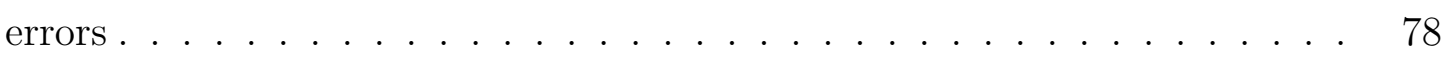

3.7 Atmospheric parameters for the three stars of M 71 in common with Cohen et al. (2001) . . . . . . . . . . . . . . . . . . .

3.8 Atmospheric parameters for the three stars of NGC 6558 in common with Barbuy et al. (2007)

3.9 Atmospheric parameters for the three stars of Terzan 8 in common with Carretta et al. (2014) . . . . . . . . . . . . . . . . 80

4.1 Log of observations of the 51 globular clusters . . . . . . . . . . . . 93

4.2 $[\mathrm{Fe} / \mathrm{H}]$ and radial velocities for the 13 globular clusters in common with Carretta et al. (2009) . . . . . . . . . . . . . . . . . . .

4.3 Final average parameters for the 51 clusters $[\mathrm{Fe} / \mathrm{H}],[\mathrm{Mg} / \mathrm{Fe}],[\alpha / \mathrm{Fe}]$ and $v_{\text {helio }} 99$

5.1 Final average parameters for M 22 and NGC 5824 and respective internal errors . . . . . . . . . . . . . . . . . . . . . 113 
B.1 Star by star coordinates, magnitude, colour, heliocentric radial velocity for the six globular clusters . . . . . . . . . . . . . . . . . 161

B.2 Atmospheric parameters of the 49 well known stars used to validate the

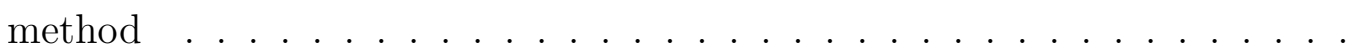

B.3 Atmospheric parameters for all 758 stars analysed in the six clusters: $\mathrm{T}_{e f f}$, $\log (g),[\mathrm{Fe} / \mathrm{H}],[\mathrm{Mg} / \mathrm{Fe}]$ and $[\alpha / \mathrm{Fe}] \ldots \ldots \ldots \ldots \ldots$ 


\section{Contents}

1. Introduction . . . . . . . . . . . . . . . . . . 23

1.1 Formation and evolution of galaxies . . . . . . . . . . . . 23

1.1.1 Milky Way bulge: monolithic collapse or secular evolution? . . . . . 24

1.1.2 Milky Way halo: minor mergers with surrounding galaxies . . . . . 26

1.1.3 Magellanic Clouds: tidal interactions in the system MW-SMC-LMC 28

1.2 Summary and goals of this thesis . . . . . . . . . . . . 31

1.2.1 Milky Way - Magellanic Clouds interactions . . . . . . . . . . 31

1.2.2 Formation and evolution of Galactic bulge and halo . . . . . . . . 32

2. Small Magellanic Cloud . . . . . . . . . . . . . . . . . . 35

2.1 Abstract of this chapter . . . . . . . . . . . . . . 35

2.2 Introduction to this chapter $\ldots \ldots \ldots \ldots \ldots \ldots \ldots$

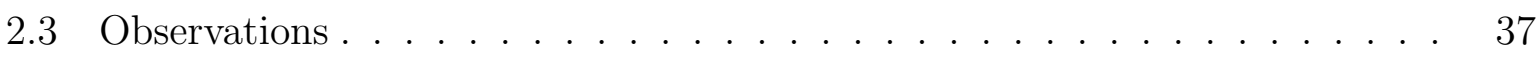

2.3.1 The SOAR/SOI data ........................... 37

2.3.2 Photometric errors and completeness curves . . . . . . . . . . 39

2.3.3 The radial density profiles . . . . . . . . . . . . . 43

2.3.4 The observed CMDs and cluster membership probability . . . . . 43

2.4 Statistical isochrone fitting . . . . . . . . . . . . . . 45

2.4 .1 CMD modelling . . . . . . . . . . . . . . . 46

2.4 .2 Likelihood statistics . . . . . . . . . . . . . . . . . . . 48

2.5 Results . . . . . . . . . . . . . . . . . . . . 48

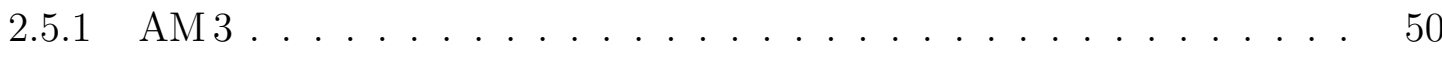

2.5.2 HW 1 and Lindsay $2 \ldots \ldots \ldots \ldots$ 


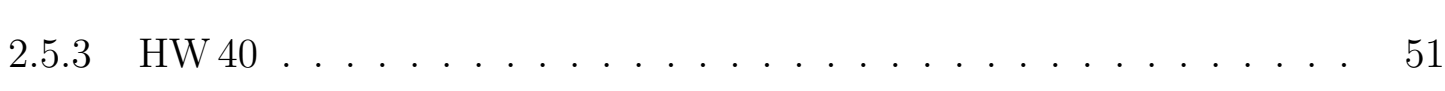

2.5 .4 Lindsay $3 \ldots \ldots \ldots \ldots \ldots$

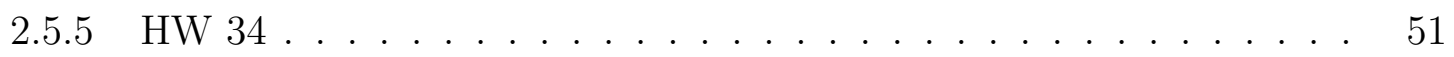

2.6 The age-metallicity relation and spatial distribution . . . . . . . 52

2.7 Summary of this chapter . . . . . . . . . . . . . . . . . . . 55

3. FORS2/VLT survey of Milky Way globular clusters. I. Method validation . . . . 57

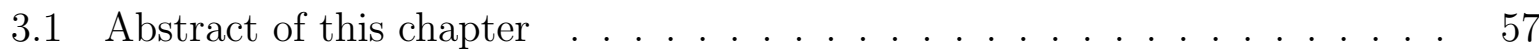

3.2 Introduction to this chapter $\ldots \ldots \ldots \ldots \ldots \ldots$

3.3 Observations and data reduction . . . . . . . . . . . . . . 59

3.4 Stellar parameters determination . . . . . . . . . . . . . . . 64

3.4 .1 Radial velocities . . . . . . . . . . . . . . . . . . . . 64

3.4 .2 Atmospheric parameters . . . . . . . . . . . . . . . 66

3.4.2.1 Stellar libraries . . . . . . . . . . . . . . . . . . 68

3.4.2.2 Average results and errors: validation with well-known stars 69

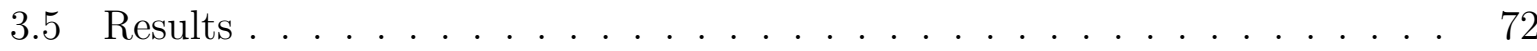

$3.5 .1 \mathrm{~T}_{\mathrm{eff}}, \log (g)$ against isochrones $\ldots \ldots \ldots \ldots \ldots \ldots \ldots$

3.5.2 Comparison with $[\mathrm{Fe} / \mathrm{H}]$ derived from $\mathrm{CaT} \ldots \ldots \ldots \ldots$

3.5 .3 Membership selection . . . . . . . . . . . . . . . . 76

3.5.4 Validation with high-resolution spectroscopy . . . . . . . . 78

$3.5 .4 .1 \quad$ M $71 \ldots \ldots \ldots \ldots \ldots \ldots \ldots \ldots$

3.5.4.2 NGC $6558 \ldots \ldots \ldots \ldots \ldots \ldots . \ldots \ldots \ldots$

$3.5 .4 .3 \quad \operatorname{Terzan} 8 \ldots \ldots \ldots \ldots \ldots$

3.6 Discussion . . . . . . . . . . . . . . . . . . . . . . . . . 81

3.6.1 Metal-rich clusters NGC 6528, NGC 6553 . . . . . . . . . . 82

3.6.2 Moderately metal-rich clusters M 71, NGC $6558 \ldots \ldots$. . . . 84

3.6.3 Metal-poor clusters NGC 6426, Terzan $8 \ldots \ldots \ldots \ldots \ldots$

3.7 Summary and conclusions . . . . . . . . . . . . . . . . . 86

4. FORS2/VLT survey of Milky Way globular clusters. II. Parameters for 51 clusters 89

4.1 Introduction to this chapter $\ldots \ldots \ldots \ldots \ldots$

4.2 Targets selection and observations . . . . . . . . . . . . 90

4.3 Method - summary from Chapter 3 . . . . . . . . . . . . . . . . . . . 94 
4.4 Calibration of results . . . . . . . . . . . . . 95

$4.4 .1[\mathrm{Fe} / \mathrm{H}]$ in Carretta scale . . . . . . . . . . . . 95

4.4 .2 Calibration of $[\mathrm{Mg} / \mathrm{Fe}]$ results $\ldots \ldots \ldots \ldots \ldots$

4.5 Discussion on abundances results . . . . . . . . . . . . . . . 98

4.5.1 Chemical evolution of the Milky Way . . . . . . . . . . 102

4.5 .2 Origin of globular clusters . . . . . . . . . . . 103

4.6 Summary and conclusions . . . . . . . . . . . . . . . . . 105

5. FORS2/VLT survey of Milky Way globular clusters. III. Clusters with multiple

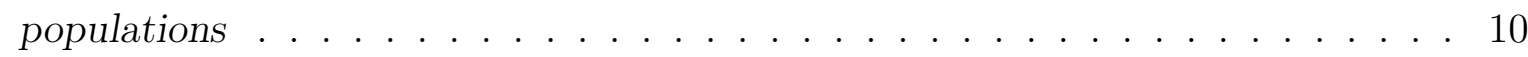

5.1 Introduction to this chapter $\ldots \ldots \ldots \ldots \ldots \ldots$

5.2 Observations and analysis $\ldots \ldots \ldots \ldots \ldots \ldots \ldots$

5.3 Results . . . . . . . . . . . . . . . . . . . . . . . 111

$5.3 .1 \mathrm{~T}_{\text {eff }}, \log (g)$ against isochrones $\ldots \ldots \ldots \ldots \ldots \ldots \ldots$

5.3 .2 Membership selection . . . . . . . . . . . . . . . . 112

$5.4[\mathrm{Fe} / \mathrm{H}]$ and $[\mathrm{Mg} / \mathrm{H}]$ intrinsic spread of M 22 and NGC $5824 \ldots \ldots \ldots$.

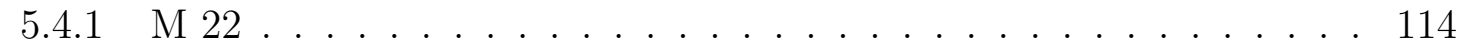

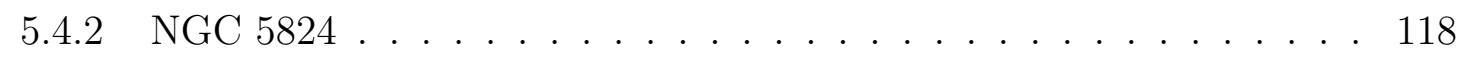

5.5 Summary and conclusions . . . . . . . . . . . . . . . 120

6. RR Lyrae variable stars in HP1 . . . . . . . . . . . . . . . . . . 121

6.1 Introduction to this chapter . . . . . . . . . . . . . . 121

6.2 Observations . . . . . . . . . . . . . . . . . . . . . . . 122

6.3 RR Lyrae candidates . . . . . . . . . . . . . . . 123

6.4 Period determination . . . . . . . . . . . . . . . . 124

6.5 Constraining the distance $\ldots \ldots \ldots \ldots \ldots \ldots$

6.6 Conclusions . . . . . . . . . . . . . . . . . . . . . . . 127

7. Conclusions and future work . . . . . . . . . . . . . . . . . 129

7.1 Summary . . . . . . . . . . . . . . . . . . 129

7.2 Perspectives . . . . . . . . . . . . . . . . . . . 132

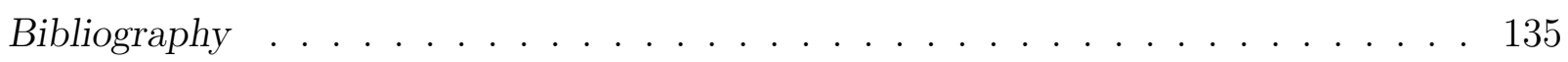


A. SMC clusters CMD statistical analysis . . . . . . . . . . . . . . 157

A.1 Likelihood results and degeneracy investigation . . . . . . . . . . 157

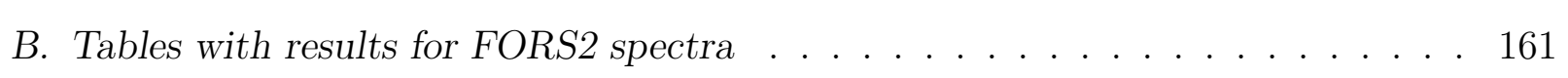

C. FORS2 pipeline - wavelength calibration for low resolution spectra of GRIS1400V 209

C.1 Default pipeline reduction ....................... 209

C.2 New list of sky lines for GRIS1400V . . . . . . . . . . . . . . 211

C.3 Comparisons bewteen the 2 procedures . . . . . . . . . . . . . 212

D. Papers in the period $2010-2014 \ldots \ldots \ldots \ldots \ldots$. . . . . . . . . . . . . . . . . . . 
Chapter 1

\section{Introduction}

\subsection{Formation and evolution of galaxies}

A major question to understand the large scale structure of the Universe presently observed is: how did galaxies form and evolve? There are basically two ways to study galaxy formation and evolution, one way is to observe galaxies in different redshifts, i.e, in different evolutionary phases and reconstruct a common history. Another possibility is to observe nearby galaxies in detail looking at different stellar populations covering ages from the beginning of the galaxy until today. In this second case, it is possible to recover the star formation history of the host galaxy and also detect traces of interactions with nearby galaxies. Such information can be compared with scenarios of galaxy formation and evolution and provide constrains that are only possible for close objects, such as metallicity of individual stars. Globular clusters are perfect targets for this purpose, because it is relatively simple to derive their ages and place them in a timeline of the evolution of their host galaxy. In this thesis we adopt the second approach and analyse globular clusters in the Milky Way to explore its formation and evolution. We also study some stellar clusters in the Small Magellanic Cloud since it is suffering gravitational influence of the Milky Way. We think that this set of objects covers almost all stages of formation and evolution of galaxies and can be used as templates for models. In the following sections we detail the cases of the Milky Way, Large and Small Magellanic Clouds focusing on the role of star clusters as tracers of galaxy formation and evolution. 


\subsubsection{Milky Way bulge: monolithic collapse or secular evolution?}

In the two last decades there were important discussions about the nature of galactic bulges. Wyse et al. (1997) pointed out the unclearness of the definitions of the nucleus of galaxies, "namely a centrally-concentrated stellar distribution with an amorphous smooth - appearance.". The classical scenario of monolithic collapse (Eggen et al., 1962) was proposed based on observation of 221 old dwarf stars in the Milky Way. The authors found that more metal-poor stars have highly eccentric orbits, and more metal-rich stars have nearly circular orbits, which was interpreted as the scenario where the galaxy started to collapse in a radial direction more than $10 \mathrm{Gyr}$ ago, and the first stars were formed in this process (more metal-poor population), whereas less than 1 Gyr later the gas found an equilibrium in circular orbits, starting the formation of the more metal-rich stellar population. Bulge and halo are the spheroidal galactic components formed first, and the disc is the flat component formed later. This scenario was extended to all galaxies, but the best way to prove it is observing spatially resolved stars in the different components of our own Galaxy.

Milky Way bulge and halo stars are expected to be old and metal-poor following the scenario of Eggen et al. (1962). Concerning the ages, Lee (1992) observed RR Lyrae in the bulge (typical of ol stellar populations), measured their metallicities and compare with horizontal branch models to conclude that there is an age gradient in the inner regions of the Milky Way, indicating that the oldest objects of the Galaxy are located in the bulge. Dékány et al. (2013) have shown that the bulge RR Lyrae form a spheroid. Stars in this region are more metal-rich than halo stars with $[\mathrm{Fe} / \mathrm{H}] \approx-1.0$, though. Following the logic of the monolithic collapse, it would be expected that the central region of the spheroidal component of the Milky Way (with a higher density of gas) would have formed stars in a more efficient way than the halo. However, bulge stellar content has a prolonged and bimodal (if not multimodal) metallicity distribution from $[\mathrm{Fe} / \mathrm{H}] \sim-1.0$ to $\sim+0.5$ (e.g. McWilliam and Rich, 1994; Sadler et al., 1996; Hill et al., 2011). This is not expected by simply collapsing a huge gas cloud about 14 Gyr ago. Moreover, Morrison (1993) debated about bulge and halo structures in terms of their density profiles. They showed that the power law density of the halo $\rho(r) \propto r^{-3.2}$ disagrees with the extension up to solar neighbourhood of the only surface brightness profile that fits the bulge (de Vaucouleurs and 
Pence, 1978). As a result, bulge and halo may have been formed by different processes, furthermore the bulge has a complex history with probably more than one scenario of formation. Recent models describe the collapsing scenario for the bulge formation as hierachical merging of subclumps (Noguchi, 1999; Aguerri et al., 2001).

Many spiral galaxies are barred, as already noted by Hubble (1926) in his original morphological classification of these "nebulae", as they were called in that epoch. Bars can transport gas into the inner regions of the galaxy and trigger star formation in a timescale of a few billion years. Many barred late-type galaxies have their central stellar populations formed only by the secular evolution driven by their bars, and these inner regions are called pseudobulges (see Kormendy and Kennicutt, 2004; Gadotti, 2009). The Milky Way is a barred spiral galaxy and its bulge can be considered as a combination of both a classical (merger driven) and a pseudobulge (bar driven). In fact, Hill et al. (2011) present a bimodal metallicity distribution of bulge red giant stars in the Baade's window $(1, b)=\left(1^{\circ},-4^{\circ}\right)$ - and discuss possible models to explain each peak. The best scenario that fitted both peaks is a combination of a closed-box model to explain the more metal-poor peak $([\mathrm{Fe} / \mathrm{H}] \approx-0.3)$ and an extension of the inner disc stellar population to describe the more metal-rich peak $([\mathrm{Fe} / \mathrm{H}] \approx+0.3)$.

Kinematics of a stellar population formed by a gravitational collapse would have a higher velocity dispersion, while a bar-driven population would be supported by rotation. Babusiaux et al. (2010) confirmed that based on [Fe/H], radial velocities and proper motion of red giant stars in the Baade's window compared with N-body simulations. Their findings are in agreement with Hill et al. (2011) as discussed above. Recently, it was found that the Milky Way bulge has an X-shape, and many works are devoted to decribe its kinematics and chemistry from observations and models, e.g. Gardner et al. (2014); Zoccali et al. (2014); Vásquez et al. (2013); Gonzalez et al. (2013); Saito et al. (2012). In particular, Saito et al. (2011) have mapped the X-shaped bulge using its stellar contents. Using their findings it is possible to sketch the position of the $\mathrm{X}$ whose plane is parallel to the Galactic bar (Figure 1.1).

As firstly described by Baade (1944), stellar populations in galaxies can be classified in two types: population I constituded by luminous OB stars and open clusters, and population II represented by short-period Cepheids and globular clusters. Early-type galaxies have predominantly population II, while late-type galaxies share both populations, as in 


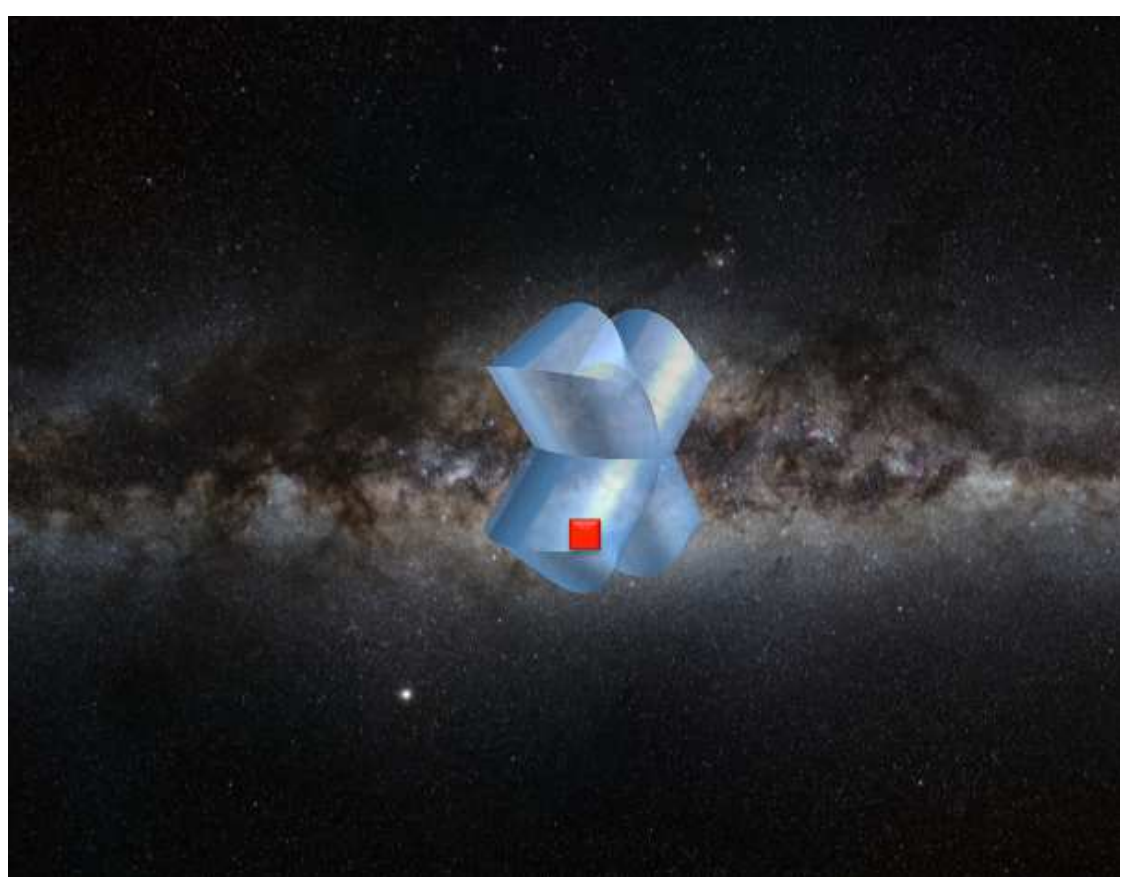

Figure 1.1: Schematic view of the X-shaped bulge of the Milky Way in transparent blue over an ESO/S. Brunier image. Approximate position of Baade's window is indicated in red.

the case of the Milky Way. In our Galaxy, globular clusters are located in the spheroidal components, bulge and halo, and hence they are relics to probe the nature of the bulge. The amount and density of gas to form a globular cluster which has typical mass of $\sim 10^{5} M_{\odot}$ is only found in the huge initial gravitational collapse that originated the Milky Way. In this case, globular clusters can be used as fossils of Galactic formation, and be compared with the more metal-poor population of the bulge. Barbuy et al. (1998) studied 15 bulge clusters and revised their metallicities, showing that the metallicity distribution is following the more metal-poor population of the bulge, as expected.

There is one interesting object located in the bulge that seems like a globular cluster, but it seems to be a pristine fragment of the bulge: Terzan 5. Ferraro et al. (2009) found two populations with different ages from photometry, Origlia et al. (2011) confirmed the two populations spectroscopically with $[\mathrm{Fe} / \mathrm{H}] \approx-0.3$ and +0.3 , which corresponds to the two peaks of the metallicity distribution of bulge stars. Origlia et al. (2013) found three stars with metallicity around $[\mathrm{Fe} / \mathrm{H}] \approx-0.79$, and Massari et al. (2014) found other three stars with metallicities around $[\mathrm{Fe} / \mathrm{H}] \approx+0.7$. They also analysed the alpha-elements for these stars and concluded that Terzan 5 is probably one of the subclumps that were merged to form the bulge. 


\subsubsection{Milky Way halo: minor mergers with surrounding galaxies}

Globular clusters (GCs) are tracers of the star formation history of their host galaxy, since most of the stars are formed in globular clusters, but only few of them keep this structure (Gieles et al., 2007). They also give constraints to galaxy formation scenarios, for instance the monolithic collapse (Eggen et al., 1962) predicts old and metal-poor globular clusters in the halo of the host galaxy. Disrupted dwarf galaxies could help forming the halo stellar populations, and transfer their globular clusters and/or nuclei to the Milky Way (Bekki and Freeman, 2003). This process could be the origin of multiple stellar populations found in few massive halo globular clusters. Therefore, globular clusters are useful to understand the chemical and dynamical evolution of the host galaxy. Another interesting feature of globular cluster systems in galaxies, and maybe one of the most important from the last 2 decades is their bimodal metallicity distribution, that reflects the chemical evolution of the galaxy, star formation history, and it constrains galaxy formation scenarios. There are 3 main scenarios to explain this bimodality: major disk-disk mergers (Zepf \& Ashman 1992), in situ formation through multiphase dissipational collapse (Forbes 1997), and dissipationless accretion (Cote et al. 1998, 2000, 2002).

In order to explore all the cases above, it is necessary to have large samples of spectroscopic and photometric data, with homogeneous determinations of ages, metallicities, some specific chemical abundances, and also the knowledge of the kinematics of the clusters. In particular $[\alpha / \mathrm{Fe}]$ ratios against $[\mathrm{Fe} / \mathrm{H}]$ are good tracers of star formation history, because it reflects the ratio and timing of supernovae type II and type Ia (e.g. Brodie \& Strader 2004). Therefore it is useful to study Milky Way and Local Group globular cluster systems from which one can get more detailed information, as well as in external galaxies. The average $[\mathrm{Fe} / \mathrm{H}]$, and $[\mathrm{Mg} / \mathrm{Fe}]$ for all stars within a same cluster can be placed in the Milky Way star formation history scenario and constrain the timescale of the supernovae II and supernovae Ia, which can be also compared to age determinations for these clusters.

Stars in distant galaxies (out of Local Group) are not spatially resolved by $8-10 \mathrm{~m}$ class telescopes, therefore only integrated spectra of globular clusters can be observed. In order to compare the Milky Way metallicity and $[\mathrm{Mg} / \mathrm{Fe}]$ to extragalactic globular cluster systems we use integrated light spectroscopy from extragalactic globular clusters (Trager et al. 2000, Thomas et al. 2003, Schiavon et al. 2005). This comparison is important in the 
sense that individual stars studies allow to proceed with membership selection, and exclude stars that can introduce bias in the $[\mathrm{Fe} / \mathrm{H}]$ and $[\mathrm{Mg} / \mathrm{Fe}]$ as derived by integrated spectra. Although elliptical galaxies present a different scenario of star formation, and different pattern of $[\mathrm{Mg} / \mathrm{Fe}]$ vs. $[\mathrm{Fe} / \mathrm{H}]$, the calibration described for Milky Way globular clusters are very useful to understand extragalactic globular clusters. Note that extragalactic clusters reach high metallicities, which are not covered by previous metallicity scales for Milky Way GCs, and that the scale of this work covers all metallicities between $[\mathrm{Fe} / \mathrm{H}]=$ -2.5 dex up to solar.

\subsubsection{Magellanic Clouds: tidal interactions in the system MW-SMC-LMC}

Evolution of galaxies depends on environmental effects, such as tidal stripping, that occurs when a given galaxy is subject to differential gravitational forces due to a companion and/or host galaxy. The gas of the galaxy is withdrawn and in a shorter timescale the stellar content is also removed from their original positions (Broeils and van Woerden, 1994). Another common process is the ram-pressure stripping (Gunn and Gott, 1972), where the gas of an interacting galaxy is lost because of the pressure of a hot gas in the intergalactic medium.

The Magellanic Stream (MS), discovered and named 40 years ago by Mathewson et al. (1974), is the trailing filament of neutral hydrogen of the Large and Small Magellanic Clouds (LMC and SMC) system. It is $\sim 200^{\circ}$ long and only $\sim 10^{\circ}$ wide, located close to the great circle that passes by the southern Galactic pole and crosses the Galactic plane at a longitude $l \sim 306^{\circ}$ (Nidever et al., 2010). Mathewson et al. (1974) argued that it is unlikely that the Magellanic Clouds had been closer than $40 \mathrm{kpc}$ from the Galactic centre, due to the smooth velocity gradient of the gas connecting the Clouds, forming a bridge between them, and of both galaxies with the Stream. They also found evidence of a leading component of the gas stream. Figure 1.2 shows the map of Mathewson et al. (1974) and the new one of Nidever et al. (2010), 36 years later.

H I Parkes All-Sky Survey (HIPASS) team has confirmed and characterized the so-called Leading Arm of the Magellanic system, endorsing the scenarios of interaction between Milky Way and Magellanic Clouds (Putman et al., 1998). They summarize the most probable explanations for the origin of these gas structures in two main scenarios: rampressure (Mathewson et al., 1987; Barnes, 1996; Sofue, 1994; Moore and Davis, 1994) or 


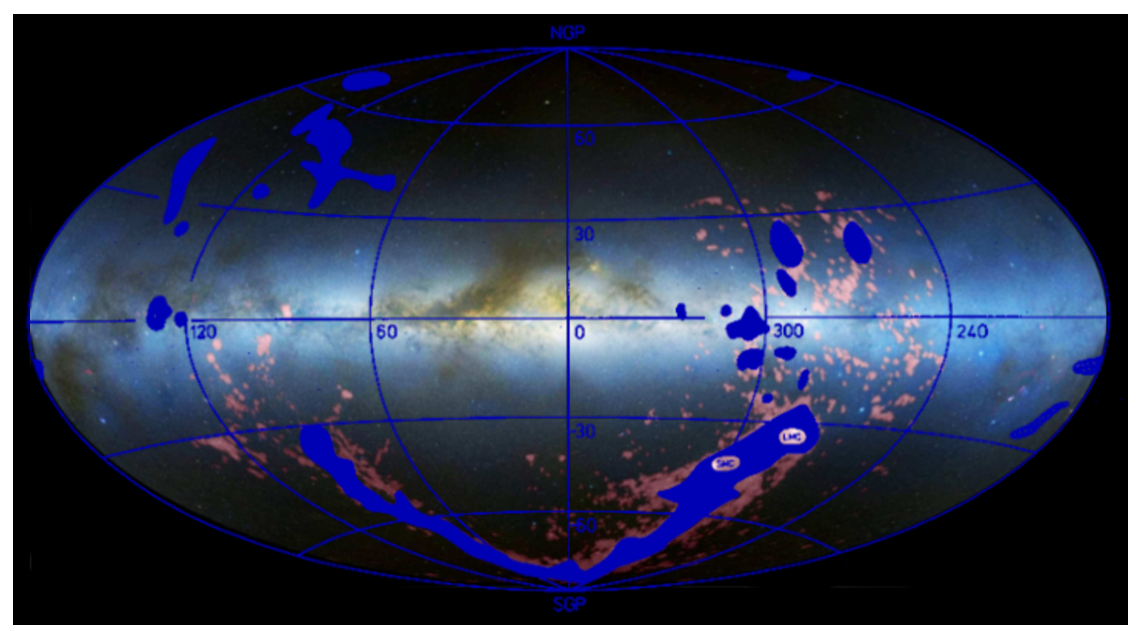

Figure 1.2: Aitoff projection of the Milky Way plane overplotted by the Magellanic System in terms of H I. Milky Way image with pink H I maps from Nidever et al. (2010) are overplotted with the blue H I maps and galactic coordinates grid from Mathewson et al. (1974).

tidal stripping (Murai and Fuijmoto, 1980; Gardiner and Noguchi, 1996; Gardiner et al., 1994; Lin et al., 1995; Davies and Wright, 1977; Lin and Lynden-Bell, 1977). The first one explains the gas content of the MS, but to explain the presence of both the Leading Arm and the MS the model has to degrade the quality of the velocity profile of the Stream (Sofue, 1994; Moore and Davis, 1994) . Ram-pressure is only valid for gas, not for stars. On the other hand, tidal stripping scenario predicts the presence of both Magellanic Stream (detected by Mathewson et al., 1974), a Leading Arm (confirmed by Putman et al., 1998) and the presence of a stellar counterpart.

On the one hand Nidever et al. (2008) traced back the velocity of the MS and LA, and showed that they originated in the LMC, not in the SMC or in the bridge. The authors suggest that a blowout of the LMC gas followed by a stretch of this expelled gas by the Milky Way potential can explain the formation of both gas structures, as an alternative scenario for the two most popular ones (ram-pressure and tidal stripping). On the other hand Diaz and Bekki (2012) realized N-body simulations to reproduce the morphology and kinematics of the MS, and proposed that LMC and SMC were separated galaxies until 2 Gyr ago. This first encounter and the following ones generated many tidal gas structures considering gravitational interactions with Milky Way: MS, LA, Bridge (between LMC and SMC) and a structure that they called Counter-bridge, which lies behind the SMC along the line-of-sight. The authors predict the presence of a stellar counterpart of the gas structures to support their scenario. Besla et al. (2013) also foresee stars in the Magellanic 
Stream.

Nidever et al. (2013) found a stellar component of the Magellanic Brigde coming from the SMC and called it the "SMC eastern stellar structure". The stars are about 1-2 Gyr old and the observations are qualitativelv consistent with Diaz and Bekki (2012) models. Noël et al. (2013) found intermediate-age stars in the Magellanic Bridge, close to the SMC, and detect much less stars with similar ages in the opposite direction, on the Western borders of SMC. This is evidence supporting the idea of the intermediate-age stars present in the Bridge had been originated in the SMC. Casetti-Dinescu et al. (2014) went further and detect star formation in the Leading Arm, triggered by the strong interactions of Milky Way and Magellanic system.

With the advent of many surveys targeting the stellar content of the Magellanic System, such as VMC (Cioni et al., 2011), MAGIC (Noël et al., 2013) and SMASH (Olsen et al., 2014), stellar counterparts of the gas structures have been detected for the Bridge and are being observed in Magellanic Stream and Leading Arm. Together with the difficulty to model a Leading Arm from ram-pressure effects, these evidences tend to rule out this scenario as the origin of LA, MS and other structures of the Magellanic System. Therefore, the most accepted framework for the complex structure of this system is tidal stripping, or at least a combination of this effect with others, such as gas blowout from the LMC.

As mentioned by Noël et al. (2013), field stars are telling something about the chemodynamical evolution of the Magellanic Clouds by comparisons of stars in the Clouds line-ofsight against stars stripped towards the Magellanic Brigde, for instance. What about the stellar clusters? It is not broadly accepted that the stellar populations from field star and stellar cluster should have the same star formation history (SFH). Holtzman et al. (1999) says that the SFH of LMC field stars is different from that derived from clusters. In fact, more than 25 years ago Jensen et al. (1988) proposed that the LMC cluster population has an age gap between $\sim 4$ and $\sim 10$ Gyr, and up to now no object was found with ages in this range (Balbinot et al., 2010). On the contrary, LMC field star populations show no dependency on the location in the galaxy, and none of those small age gaps coincide with the cluster age gap (Javiel et al., 2005). One possibility is that the 4-10 Gyr old clusters have been formed in the LMC and were tidally stripped from the galaxy. If this is true, where are these clusters? Were they destroyed? If they exist, they could be found in one of the tidal structures of the Magellanic System, or even in the SMC. 
Rafelski and Zaritsky (2005), Piatti et al. (2005) and Piatti (2012b) showed that for the SMC the SFH from field and cluster populations agree. These works have shown a recent burst of star formation, which could have been triggered by close encounters of LMC and SMC up to $\sim 2$ Gvr ago, as described by the dvnamical models of Diaz and Bekki (2012), being at variance with continuous star formation suggested by Da Costa and Hatzidimitriou (1998). However, there was also an older burst in the SMC at about 5-7 Gyr, equally described by Cignoni et al. (2013) that is not predicted in the dynamical model of Diaz and Bekki (2012). A major merger with mass ratio between 1:1 to 1:4 could have generated this peak in the SMC SFH 5-7 Gyr ago, as proposed by Tsujimoto and Bekki (2009). However this model predicts a dip in the age-metallicity relation (AMR) correlated with this major merger, which is not found by the observational results of Cignoni et al. (2013). The AMR for SMC star clusters presents a broad distribution of metallicities at a given age, as shown by Parisi et al. (2014) and it is not possible to see a dip in metallicity, but in average the metallicity increases monotonically with time, in agreement with the findings of Cignoni et al. (2013) for field stars. The scatter of metallicity values for clusters does not permit a choice for a unique chemical evolution model (Parisi et al., 2014). Is it possible that some of this variety of metallicity values, in particular for intermediate-age clusters, are due to accreted clusters from LMC? SMC has a more complex star formation and chemo-dynamical evolution histories, consequently, is that valid to analyse the galaxy as a whole, or is it worth analysing region by region of the Small Cloud? Bica et al. (2008) catalogued over 3700 stellar systems in the LMC, SMC and Magellanic Bridge, and some Bridge clusters are being observed to look for intermediate-age objects. Many works have been studying areas of the SMC in terms of ages, the next step towards a complete scenario of the evolution of this galaxy is deriving metallicities.

\subsection{Summary and goals of this thesis}

In this thesis we use intermediate-age and old star clusters in the Small Magellanic Cloud (SMC, Chapter 2) and globular clusters in the Milky Way as tracers of the chemical and dynamical evolution of these galaxies. It is relevant to study in detail clusters that can be spatially resolved of nearby galaxies in order to understand more distant galaxies and trace a general scenario for formation and evolution of galaxies. The Milky Way is studied 
in several chapters, initially presenting the method for chemical abundances derivation (Chapter 3), then releasing of a homogeneous metallicity and alpha-enhancement scale of Galactic globular clusters, and a catalgoue of member stars for about $1 / 3$ of the clusters in the Milky Way discussing their origin (Chapter 4). We discuss in particular, the origin of some halo clusters possibly captured from disrupted dwarf galaxies (Chapter 5), and one peculiar bulge cluster that could be the oldest object of our Galaxy (Chapter 6). Finally, in Chapter 7 we give a summary of the thesis and perspectives of works for the near future following the present results. An overview of each topic of the thesis is given below.

\subsubsection{Milky Way - Magellanic Clouds interactions}

Assuming that stellar clusters follow the streams of gas formed by tidal forces, they are pivotal objects to track down the time evolution of the disruption of the stellar content of the galaxy. In particular, using colour-magnitude diagrams of individual clusters, it is possible to derive age and metallicity with enough precision for radial gradients characterization of the population of clusters.

As shown in Chapter 2 we find radial gradients of ages and metallicities in the West Halo region of the SMC, based on star clusters, for the first time. This indicates the existence of gravitational effects stripping out the stellar content of the galaxy. As discussed in Section 1.1.3, this results exclude the possibility of ram pressure effects, which only affects gas. Instead, this disruption of star clusters in the West Halo could be explained by tidal stripping due to the combination of orbits of Small and Large Magellanic Clouds around the Milky Way.

\subsubsection{Formation and evolution of Galactic bulge and halo}

Efficiency of star formation can be indicated by the chemistry of stars, in particular in a plot of $[\mathrm{Fe} / \mathrm{H}]$ vs. $[\alpha / \mathrm{Fe}]$ because iron and $\alpha$-elements comes from supernovae type II (massive stars with rapid evolution), but supernovae type Ia provide only iron, not $\alpha$ elements. Therefore, when supernova type Ia becomes to be important the ratio $[\alpha / \mathrm{Fe}]$ decreases. The $[\mathrm{Fe} / \mathrm{H}]$ where $[\alpha / \mathrm{Fe}]$ starts to decrease $\left([\mathrm{Fe} / \mathrm{H}]_{\text {knee }}\right)$ reveals how efficiently a given stellar population was formed. Moreover, if these abundance values for globular clusters are compared with their ages this efficiency can be easily converted to a timescale of supernovae explosions, which is crucial information for galaxy formation models. This 
analysis can be done for the bulge and halo separately, as we perform in this thesis.

In Chapter 3 we define a method for derivation of chemical abundances $([\mathrm{Fe} / \mathrm{H}]$ and $[\alpha / \mathrm{Fe}])$ of red giant stars in globular clusters based on spectroscopy with resolution typical for extragalactic observations $(\mathrm{R} \sim 2,000)$. We validate the method of full spectrum fitting with well-known stars and apply to six globular clusters located in the bulge, disc and halo, covering metallicities from $[\mathrm{Fe} / \mathrm{H}]=-2.5$ to -0.1 . The results are in agreement with high-resolution spectroscopic analysis available in the literature. We apply the method to all 51 globular clusters of our sample and we present a homogeneous metallicity scale for Milky Way globular clusters in Chapter 4, as an update of Harris catalogue, with aditional information on $[\alpha / \mathrm{Fe}]$. We also present a catalogue of member stars for about $1 / 3$ of the system of Galactic globular clusters, selected from spectroscopy and photometry. It is the first time that $[\mathrm{Fe} / \mathrm{H}]$ and $[\mathrm{Mg} / \mathrm{Fe}]$ derived in a consistent way are published for such a huge sample of globular clusters. We find a $[\mathrm{Fe} / \mathrm{H}]_{\text {knee }} \sim-1.0$ for bulge and halo based on the clusters, although the bulge seems to be more efficient with a more metal-rich $[\mathrm{Fe} / \mathrm{H}]_{\text {knee }}$. Comparing the abundances and $[\mathrm{Fe} / \mathrm{H}]_{\text {knee }}$ with ages it implies in a timescale of $\sim 1$ Gyr for supernovae type Ia to start to become important in these components, which agrees with the value widely adopted in models for the Milky Way. Based on $[\mathrm{Fe} / \mathrm{H}]$ vs. age bulge was formed more rapid than halo, and surprisingly $[\mathrm{Mg} / \mathrm{Fe}]$ vs. age does not show this difference.

Halo globular clusters show a lower efficient star formation which is compatible with a scenario where its stellar content was formed in mini halos and/or dwarf galaxies. Some globular clusters may have been originated in the nucleus of dwarf galaxies, already captured by the Milky Way potential well and destroyed in the process. If this is true, their origin should be registered in their chemical contents, showing a complex star formation, continuous or with multiple bursts. There are six massive globular clusters with known internal spread in their $[\mathrm{Fe} / \mathrm{H}]$ distribution, such as $\omega$ Centauri, each one with a particular origin. We search for other clusters with spread in $[\mathrm{Fe} / \mathrm{H}]$ in our sample to check their origin.

Two of the 51 clusters in our survey host multiple populations in terms of [Fe/H], M 22 and NGC 5824, and possibly there are more among our observations to be investigated. In Chapter 5 we confirm the spread in $[\mathrm{Fe} / \mathrm{H}]$ and show that $\mathrm{M} 22$ has a star formation history similar to that of the Milky Way and it is unlikely that it had an origin in a dwarf 
galaxy, in agreement with Marino et al. (2011). NGC 5824 has a position and velocity consistent with the orbit of the Cetus Polar Stream which leads to investigations if the cluster and the stream share a same origin. possibly a disrupted dwarf galaxv Da Costa et al. (2014). With our data it is not possible to answer this question because the spread in $[\mathrm{Fe} / \mathrm{H}]$ has a inter-quartile range of only 0.10 dex which is the order of magnitude of the error bars of our measurements.

If bulge experienced a more efficient star formation and it hosts the centre of the Milky Way, it is possible that the oldest globular clusters are located there. In particular, there are some moderately metal-poor bulge clusters presenting a bluer horizontal branch (HB) than expected by their metallicity, as listed by Barbuy et al. (2009). The main parameter that causes a change in the HB morphology is the metallicity, known as the first parameter. The second most important parameter is age in most cases (Lee et al., 1994), although other parameters can play the role, such as He abundance, CNO abundance or AGB mass loss. Metal-poor clusters have a blue HB, and old clusters too, but these bulge clusters are moderately metal-poor with a blue HB, which indicates that they are very old, possibly the oldest objects of the Milky Way, witnesses of the initial collapse of the Galaxy.

The innermost Milky Way globular cluster is HP 1, for which we determine a metallicity of $[\mathrm{Fe} / \mathrm{H}]=-1.3 \pm 0.4$ in Chapter 4, has a HB index 1 of 0.75 , as determined by Mackey and van den Bergh (2005). Therefore this central cluster seems to have a bluer horizontal branch than expected. To constrain future age determination using colour-magnitude diagrams we determine its distance using light curves of RR Lyrae in Chapter 6. We find a distance of $8.0 \pm 0.6 \mathrm{kpc}$ consistent with the distance of the Galaxy centre. If this cluster is the oldest of our Galaxy it will confirm that the bulge had a rapid star formation at the beginning of the Milky Way.

\footnotetext{
${ }^{1}(\mathrm{~B}-\mathrm{R}) /(\mathrm{B}+\mathrm{V}+\mathrm{R})$, where $\mathrm{V}$ is the number of variable stars, $\mathrm{B}$ and $\mathrm{R}$ are the numbers of stars bluer and redder than the variables, respectively.
} 
Chapter 2

\section{Small Magellanic Cloud}

\subsection{Abstract of this chapter}

Stellar clusters in the Small Magellanic Cloud (SMC) are useful probes for studying the chemical and dynamical evolution of this neighbouring dwarf galaxy, enabling inspection of a large period covering over 10 Gyr. The main goals of this work are the derivation of age, metallicity, distance modulus, reddening, core radius and central density profiles for six sample clusters, in order to place them in the context of the Small Cloud evolution. The studied clusters are AM 3, HW 1, HW 34, HW 40, Lindsay 2, and Lindsay 3; HW 1, HW 34, and Lindsay 2 are studied for the first time. Optical Colour-Magnitude Diagrams (V, B-V CMDs) and radial density profiles were built from images obtained with the $4.1 \mathrm{~m}$ Southern Astrophysical Research (SOAR) telescope, reaching V 23. The determination of structural parameters were carried out by applying King profile fitting. The other parameters were derived in a self-consistent way by means of isochrone fitting, which uses likelihood statistics to identify the synthetic CMDs that best reproduce the observed ones. Membership probabilities were determined comparing the cluster and control field CMDs. Completeness and photometric uncertainties were obtained by performing artificial star tests. The results confirm that these clusters (except HW 34, identified as a field fluctuation) are intermediate-age clusters, with ages between 1.2 Gyr (Lindsay 3) and 5.0 Gyr (HW 1). In particular HW 1, Lindsay 2 and Lindsay 3 are located in a region that we called West Halo, where studies of ages and metallicity gradients are still lacking. Moreover Lindsay 2 was identified as a moderately metal-poor cluster with $[\mathrm{Fe} / \mathrm{H}]=-1.4 \pm 0.2 \mathrm{dex}$, lower than expected from the age-metallicity relation by Pagel \& Tautvaisiene (1998). We also found distances varying from $\sim 53 \mathrm{kpc}$ to $66 \mathrm{kpc}$, compatible with the large depth of 
the SMC.

\subsection{Introduction to this chapter}

Star clusters (SCs) are useful objects for studying the complex stellar content observed in nearby galaxies, as most of them may be modelled as simple stellar populations (SSP) of a fixed age and metallicity. The Magellanic Clouds (SMC for the Small Cloud, LMC for the Large Cloud, and MCs for both) form a rich system with over > 3700 stellar systems (Bica et al.,,2008), with combinations of age and metallicity that are not found in the Milky Way (Santos and Piatti, 2004). This information can be used to probe the dynamical and chemical evolution of these neighbouring and interacting dwarf irregular galaxies, and in particular the age-metallicity relation (AMR) of the Magellanic Clouds.

According to Holtzman et al. (1999), the age distribution based on clusters is probably distinct from the star formation history $(\mathrm{SFH})$ as inferred from field stars in the LMC. For the SMC instead, Rafelski and Zaritsky (2005) analysed a sample of 195 clusters, showing that the populations of field stars and star clusters are similar. In particular, Piatti et al. (2005) showed evidence of two peaks in the ages of SMC star clusters at 6.5 Gyr and 2.5 Gyr, and Piatti (2012b) indicates peaks of star formation for field stars at 2 Gyr and 7.5 Gyr. The more recent peak could be due to an encounter with the LMC. This leads to a model of bursts of star formation, in contrast with suggestions of continuous star formation (Da Costa and Hatzidimitriou, 1998). The large period of quiescent star formation in the MCs between $~ 3$ Gyr and 10 Gyr (Harris and Zaritskv, 2001, 2004) is indicated by the low number of populous SCs with these ages (Rich et al., 2000, 2001), and almost all of them are in the SMC (Mighell et al., 1998; Piatti et al., 2005, ??, 2007, a , b).

Cignoni et al. (2012) studied the SFH of two fields in the SMC, and compared their results to the SFH behaviour suggested by Harris and Zaritsky (2004). They concluded that stars older than 8.4 Gyr do not dominate the SMC stellar population, and the period between 2 Gyr and 8.4 Gyr agrees with Harris and Zaritsky (2004) in one region, but has a much higher star formation rate (SFR) in another region. Therefore, further studies of the SMC cluster and field AMR are needed.

Metallicity values for SMC star clusters, as given in the literature compilation by Parisi et al. (2009) for example, seem to be slightly underestimated when compared with the 
chemical evolution model predictions of Pagel and Tautvaisiene (1998), for ages in the range 3 Gyr to 10 Gyr. By adding more data points to this region of the AMR, Piatti (2011b) included a few metal-poor intermediate age star clusters (IACs). To further improve the age-metallicity relation of the SMC, it is important to identify other IACs, and the metalpoor ones are particularly interesting.

In spite of its great interest, the SMC cluster system has been studied less than that of the LMC. Literature data on SMC star clusters were reviewed by Dias et al. (2010). In their Table 6, ages and metallicities for 33 among the most well-studied SMC star clusters are reported, giving particular attention to old/intermediate-age ones. In addition, based on integrated spectra, the analysis by Dias et al. showed that the clusters HW 1 and Lindsay 3 (as well as NGC 152) can be added to the list of intermediate/old clusters. Since then, newly revealed intermediate-age star clusters (IACs) were studied by Piatti (2011a,$\underline{b})$, Piatti et al. (2011) and Piatti (2012a).

The compilation of ages and metallicities for SMC clusters by Dias et al. (2010) showed that the literature results have variations up to $7 \mathrm{Gyr}$ in age and 0.9 dex in metallicity, for a given cluster. In the present work, $\mathrm{B}$ and $\mathrm{V}$ photometry combined with self-consistent statistical tools are employed to determine ages and metallicities for AM 3, HW 1, HW 40, Lindsay 2 and Lindsay 3, in order to provide more precise constraints for the AMR of the SMC. For these clusters, there are the spectroscopic analysis by Dias et al. (2010) of HW 1 and Lindsay 3, and the Washington photometry analysis of AM 3, HW 40 and Lindsay 3 (Piatti, 2011a; Piatti et al., 2011). The target HW 34 is probably only a field fluctuation. For HW 1 and Lindsay 2 no previous CMD data were available in the literature. The confirmation of some of these clusters as intermediate or old age significantly improves the poor census in the age range corresponding to the age gap for the LMC clusters $(\sim 3-10$ Gyr).

In Sect. 2 the observations, data reduction, and photometry are described. In Sect. 3 the modelling of CMDs and statistical comparisons carried out to find the best fit of synthetic vs. observed CMDs are detailed. In Sect. 4 the results and a discussion of each cluster are given. In Sect. 5 comparisons with the literature, and the age-metallicity relation for the SMC are presented. Finally, a summary is given in Sect. 6 . 


\subsection{Observations}

\subsubsection{The SOAR/SOI data}

Using the SOAR Optical Imager (SOI) mounted on the 4.1m Southern Astrophysical Research (SOAR) Telescope, B and V images were obtained for SMC SCs, under projects SO2007B-013 and SO2008B-017. We chose filters B and V since they provide the best temperature resolution for $-0.2<\mathrm{B}-\mathrm{V}<1.4$, which corresponds to the colours of the present CMDs; V, I would be better in high extinction fields, which is not the case of our fields, and B, V is less affected by differential reddening than is V, I (Soldera, 2008). This imager has a field of view of $5.26^{\prime} \times 5.26^{\prime}$, and a pixel scale of $0.077^{\prime \prime} /$ pixel, which is converted to $0.154 "$ / pixel because of the $2 \times 2$ binned observations presented here. The seeing was $\sim 0.8$ arcsec, and magnitudes up to $\mathrm{V} \sim 23$ were detected. The log of observations is reported in Table 2.1 .

Table 2.1 - Log of observations. The CCDs were displaced by $\sim 20^{\prime}$ from the cluster centre to avoid the gap between the set of two E2V CCDs in SOI, as shown in Figure 2.1, The $(\alpha, \delta)$ coordinates are from Bica et al. (2008).

\begin{tabular}{|c|c|c|c|c|c|c|}
\hline Name(s) & $\begin{array}{c}\alpha(2000) \\
\text { h:m:s }\end{array}$ & $\begin{array}{c}\delta(2000) \\
\circ::^{\prime \prime}\end{array}$ & ilter & $\begin{array}{l}\text { Exp. } \\
\text { sec. }\end{array}$ & Airmass & $\begin{array}{c}\text { seeing } \\
"\end{array}$ \\
\hline \multicolumn{7}{|c|}{ 2007-09-07 } \\
\hline \multirow[t]{2}{*}{ AM 3} & $23: 48: 59$ & $-72: 56.7$ & B & 600 & 1.42 & 1.00 \\
\hline & & & $\mathrm{V}$ & 200 & 1.40 & 1.03 \\
\hline \multirow[t]{2}{*}{ Lindsay 2} & $00: 12: 55$ & $-73: 29.2$ & B & 600 & 1.38 & 1.10 \\
\hline & & & V & 200 & 1.38 & 1.03 \\
\hline \multicolumn{7}{|c|}{ 2008-09-21 } \\
\hline \multirow[t]{2}{*}{ HW 1} & $00: 18: 27$ & $-73: 23.7$ & B & 600 & 1.48 & 0.86 \\
\hline & & & V & 200 & 1.53 & 0.87 \\
\hline \multirow[t]{2}{*}{ Lindsay 3} & $00: 18: 25$ & $-74: 19.1$ & B & 600 & 1.42 & 1.13 \\
\hline & & & $\mathrm{V}$ & 200 & 1.44 & 0.86 \\
\hline \multirow[t]{2}{*}{ HW 34} & $00: 57: 52$ & $-73: 32.7$ & B & 600 & 1.38 & 0.95 \\
\hline & & & $\mathrm{V}$ & 200 & 1.39 & 0.82 \\
\hline \multirow[t]{2}{*}{ HW 40} & $01: 00: 25$ & $-71: 17.7$ & B & 600 & 1.34 & 0.89 \\
\hline & & & V & 200 & 1.33 & 0.91 \\
\hline
\end{tabular}

Reduction procedures were based on the SOAR/IRAF packages and the photometry 
procedures were based on the DAOPHOT/IRAF package (Stetson, 1987). Classical procedures of aperture and then PSF photometry were performed in the B and V bands. Since PSF fitting resulted in better quality photometry, all the analyses in this paper are based on this method.

The standard stars were chosen from Sharpee et al. (2002), having coordinates close to the SMC direction, in order to save time. Airmass corrections were applied assuming constant values of $0.22 \pm 0.03 \mathrm{mag} /$ airmass and $0.14 \pm 0.03 \mathrm{mag} /$ airmass for the $\mathrm{B}$ and $\mathrm{V}$ bands, respectively (as can be found at the CTIO website1 ). Only standard stars observed with a seeing lower than 1.0", and airmasses close to the cluster observations were considered. Moreover, stars with magnitude variations between an aperture radius of 5 and $8 \times$ FWHM larger than 0.010 mag were not considered in this fit. Then, the following calibration curves were fitted to the standard stars; the results are presented in Table 2.2. The coefficient of determination $r^{2}$ that indicates how well the data fits a line (close to 1 is best) and the low residual values show that both nights were photometric, which is confirmed by the CTIO monitoring 2

$$
M-m=\alpha \cdot(B-V)+\beta
$$

where $M$ corresponds to either $\mathrm{B}$ or $\mathrm{V}$, and $m$ corresponds to the respective instrumental magnitudes (given by $-2.5 \times \log$ (counts/exptime) already corrected by airmass effects.

Table 2.2 - Coefficients of Eq. 2.1 from the fits of the 2007 and 2008 standard stars.

\begin{tabular}{l|cc}
\hline Coef. & B & $\mathrm{V}$ \\
\hline$\alpha$ & $0.30 \pm 0.10$ & $0.30 \pm 0.09$ \\
$\beta$ (mag) & $27.02 \pm 0.04$ & $26.83 \pm 0.07$ \\
$\mathrm{r}^{2}$ & 0.83 & 0.44 \\
$\mid$ residuals $\mid(\mathrm{mag})$ & $<0.04$ & $<0.10$ \\
\hline
\end{tabular}

Figure 2.1 presents the sky map for each cluster, showing the stellar spatial distribution of the SOAR/SOI images, and the selected cluster and field areas. The adopted cluster $\left(R_{\text {clus }}\right)$ and field radii $\left(R_{\text {field }}\right)$ for all clusters are 30 arcsec and 90 arcsec, respectively,

\footnotetext{
${ }^{1}$ http://www.ctio.noao.edu/noao/content/13-m-photometric-standards

2 http://www.ctio.noao.edu/site/phot/sky_conditions.php
} 
covering solid angles $\left(\Omega_{\text {clus }}\right.$ and $\left.\Omega_{\text {field }}\right)$ of about $0.79 \operatorname{arcmin}^{2}$ and $20.6 \operatorname{arcmin}^{2}$. These sky maps enable us to visually identify the overdensity inside the cluster radii (except for HW 34) and to verify which are the more/less crowded lines of sight.
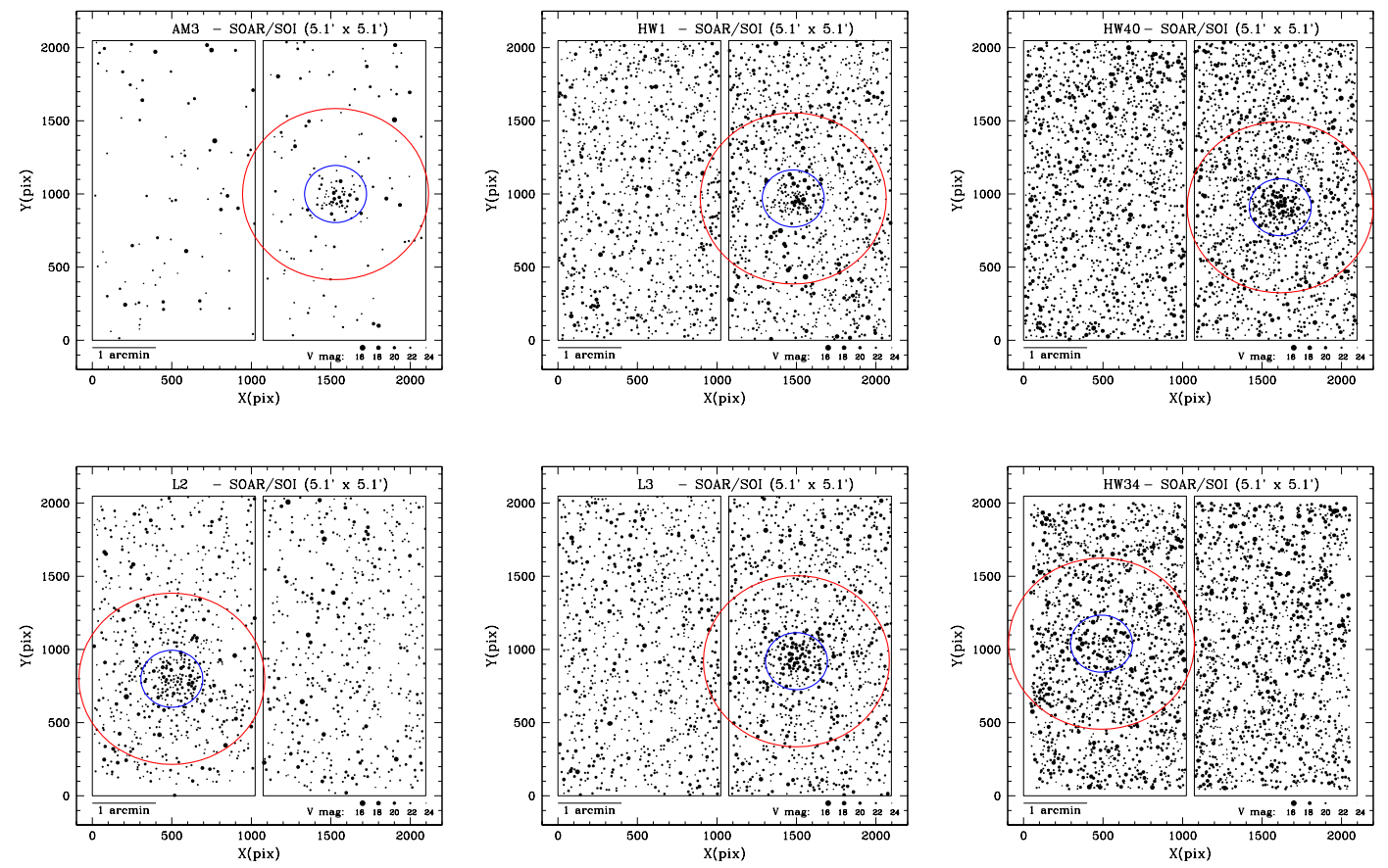

Figure 2.1: Sky maps showing the stellar distribution for all clusters, as imaged by the SOAR/SOI (1 pixel $=0.154$ arcsec $)$. The small circle $\left(R<R_{\text {clus }}=30\right.$ arcsec $)$ corresponds to the cluster sample, whereas the large circle limits the area for the control field sample $\left(R>R_{\text {field }}=90 \operatorname{arcsec}\right)$.

\subsubsection{Photometric errors and completeness curves}

To properly determine the completeness of the photometry and the photometric errors we performed artificial star tests (ASTs). These consist of adding stars (with known magnitudes and colours) in random positions to the reduced images, and then carrying out the photometry exactly as was done with the original data. The ratio of recovered to input stars is called completeness (see Figure 2.2).

The procedure was carried out for both $\mathrm{B}$ and $\mathrm{V}$ images assuming an average colour of $(\mathrm{B}-\mathrm{V})=0.5$ for all the stars. The most important curves are those from the $\mathrm{V}$ band, since a V magnitude limit is applied on Figure 2.4, and the curves are used to correct the radial density profiles (Fig. 2.5), which are better sampled by $\mathrm{V}$ images. In fact, $\mathrm{B}$ completeness curves were used only by internal checks: they differ by $<0.5 \mathrm{mag}$ from $\mathrm{V}$ curves with the same completeness values. We considered that this is a good approximation for the 

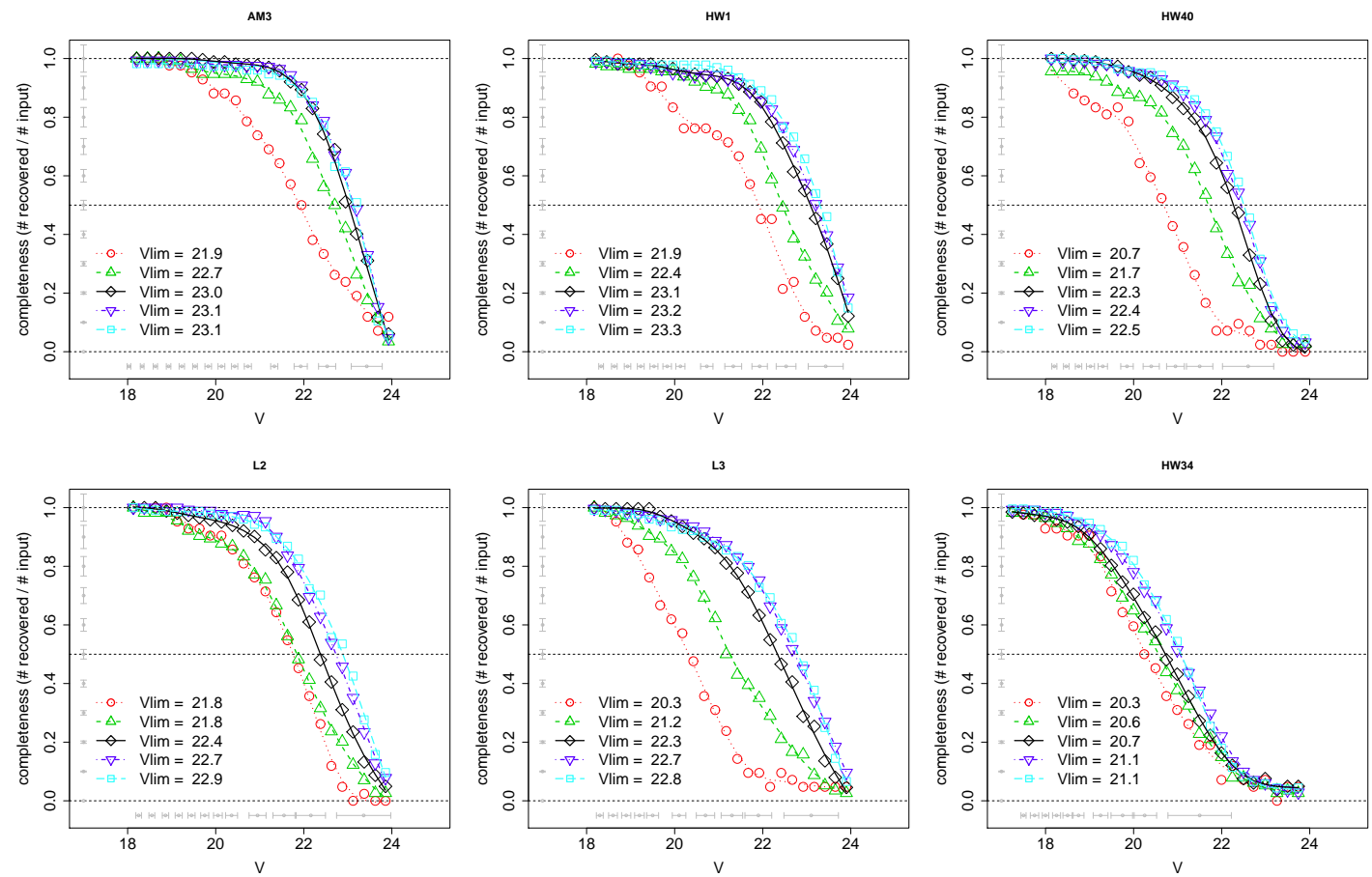

Figure 2.2: Completeness curves for different distances from the cluster centre, based on V-band photometry. These curves correspond to a ten-order smoothed spline fitted to the data. Different lines are the completeness curves for different annuli, with the respective data points: $0 \leq \mathrm{r}<10^{\prime \prime}$ (red dotted/circle); $10 \leq \mathrm{r}<20^{\prime \prime}$ (green dashed/triangle); $20 \leq \mathrm{r}<30^{\prime \prime}$ (black solid/rhombus); $30 \leq \mathrm{r}<40^{\prime \prime}$ (blue dash dotted/inverted triangle); $40 \leq \mathrm{r}<50^{\prime \prime}$ (cyan long dashed/square). The magnitude limit corresponding to $50 \%$ of completeness are indicated in the plots. Completeness uncertainties are indicated in grey vertically (assumed as Poissonian), and typical magnitude uncertainties are indicated in grey horizontally.

observed stars with colours ranging from $\sim 0.0<B-V<1.0$. Therefore, we used only $\mathrm{V}$ magnitude limits, as follows. The total range of $\mathrm{V}$ magnitudes covering all observed stars (from $\sim 17$ to $\sim 24 \mathrm{mag}$ ) were divided into 32 intervals. For each interval, two sets of 465 stars were simulated inside a radius of 50" from the cluster centre, with random magnitudes, separated by at least 3 " ( $\gtrsim 3.5 \times<$ FWHM $>$ ) to avoid introducing additional crowding (e.g. Rubele et al., 2011). The resulting completeness curves as a function of the V magnitude are presented in Figure 2.2 for diferent annuli around the cluster centre. The limiting magnitude is set where the completeness ratio is equal to $50 \%$ (see values in the figure). We adopted the $\mathrm{V}_{\text {limit }}$ values of the annulus $20^{\prime \prime}<r<R_{\text {clus }} \equiv 30^{\prime \prime}$, where $\mathrm{R}_{\text {clus }}$ is defined as the outer cluster radius used for the CMD analysis.

The correspondence between Figures 2.1 and 2.2 is evident. In particular, for the central regions (red solid curve), completeness for HW 34, HW 40, and Lindsay 3 are similar, as expected from the similar central densities of the stars. Instead, for external annuli, 
HW 40 and Lindsay 3 are more complete (2 magnitudes more), while HW 34 presents roughly similar completeness to the centre. This indicates a crowded and homogeneous field in the HW 34 direction.

The observational photometric errors for $\mathrm{V}$ and $(\mathrm{B}-\mathrm{V})$ as given by DAOPHOT are presented in Figure 2.3. The photometric uncertainties, i.e., the standard deviations of the differences between input and output magnitudes and colours derived from the ASTs, are presented together with the CMDs (Section 2.3.4).
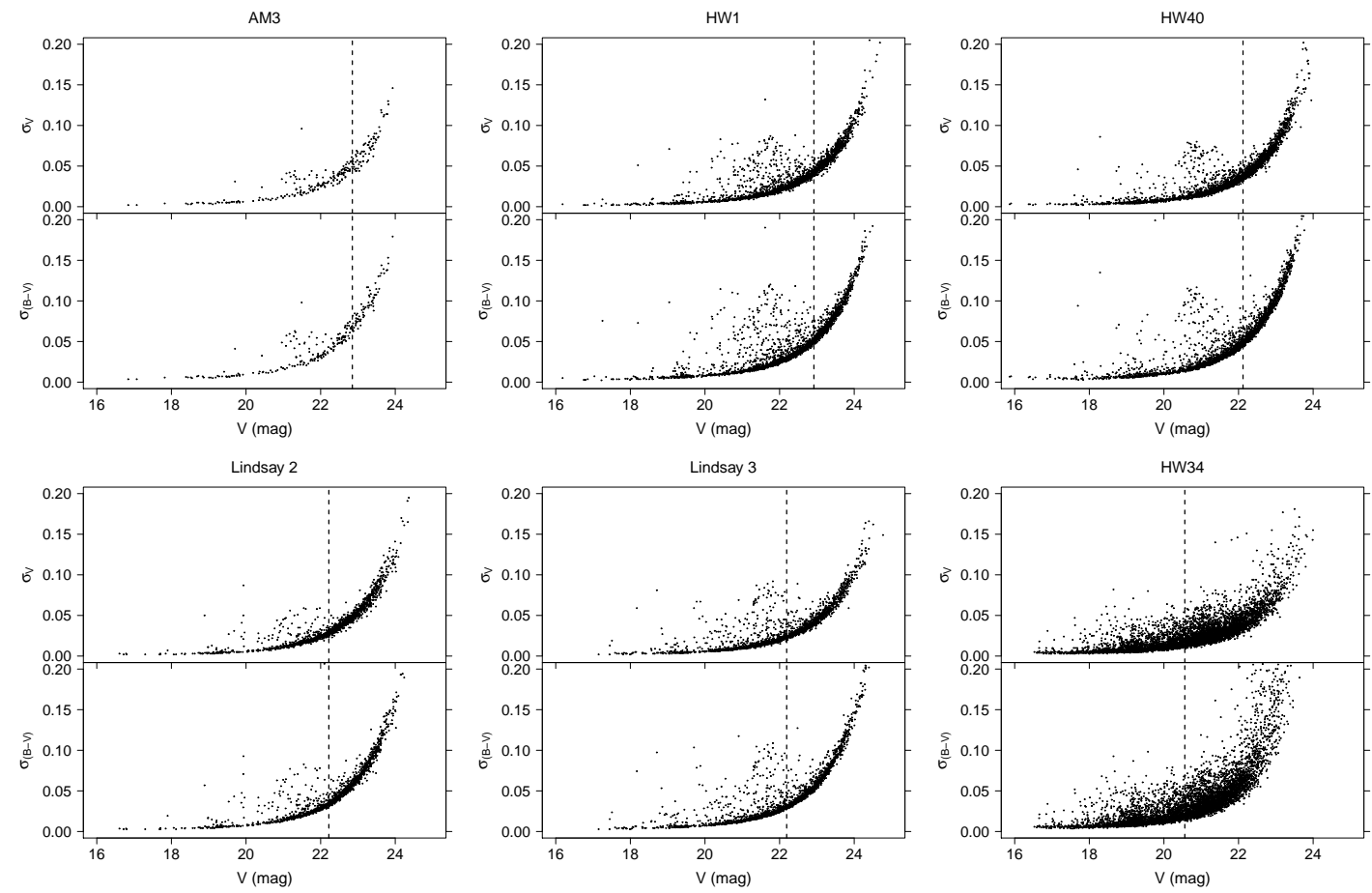

Figure 2.3: V and (B-V) photometric errors given by DAOPHOT outputs. Vertical dashed lines correspond to magnitude limits in a radius of 30", as shown in Figure 2.2.

\subsubsection{The radial density profiles}

The radial density profiles for all clusters are shown in Fig. 2.5. Only stars brighter than the magnitude limit presented in Fig. 2.4 were considered. Two profiles are presented: a) the lower density, which is observed, and b) the higher density corresponding to those corrected for completeness. Each star has a value for the completeness that comes from an interpolation in radius and magnitude, based on the curves presented in Figure 2.2. The error bars are the propagation of the Poissonic errors on the star counts $(\sqrt{N} /$ area), corrected by the incompleteness of the sample. Each profile is fitted with the empirical 

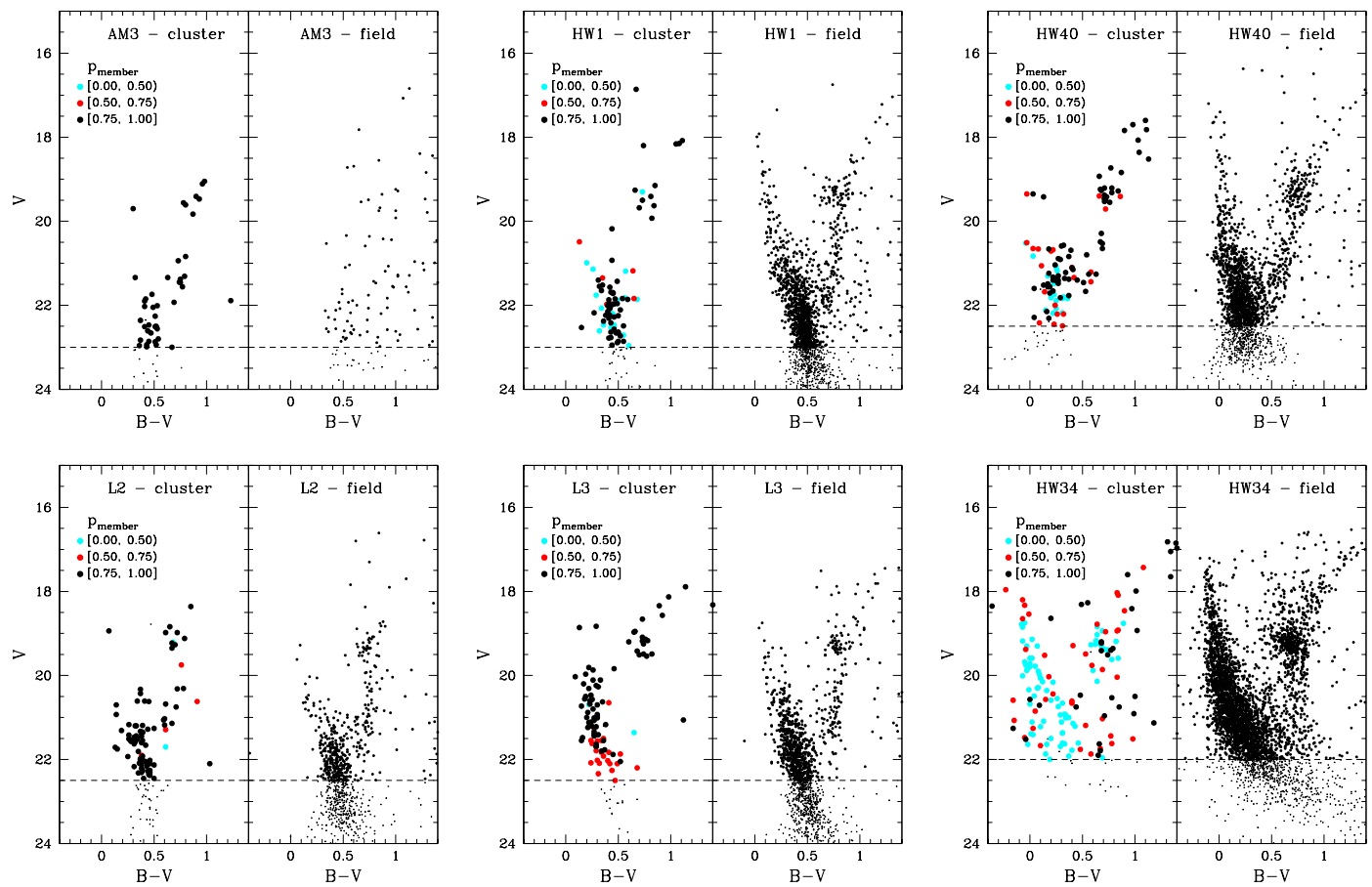

Figure 2.4: V, (B-V) colour-magnitude diagrams for all clusters. Left panels: cluster stars $\left(\mathrm{R}<\mathrm{R}_{\text {clus }}\right)$. Right panels: control field stars $\left(\mathrm{R}>\mathrm{R}_{\text {field }}\right)$. The colours depend on the membership probabilities ( $\left.\mathrm{p}_{\text {member }}\right)$ for each star in the cluster direction $\left(\mathrm{R}<\mathrm{R}_{\text {clus }}\right)$. The horizontal dashed lines correspond to the magnitude limits, derived from completeness curves around $R_{\text {clus }}$.

density law of King (1962), expressed by equation 2.2 below, using a nonlinear least-squares routine

$$
n=n_{0}\left\{\frac{1}{\left[1+\left(r / r_{c}\right)^{2}\right]^{1 / 2}}-\frac{1}{\left[1+\left(r_{t} / r_{c}\right)^{2}\right]^{1 / 2}}\right\}^{2}+n_{\text {field }},
$$

where $n_{0}$ is the central density of the cluster, $n_{\text {field }}$ is the density of field stars, $r_{c}$ is the core radius, and $r_{t}$ is the tidal radius. From these parameters one can quantify the concentration parameter $c$, defined as $c \equiv \log \left(r_{t} / r_{c}\right)$. For each cluster, the value of $n_{\text {field }}$ was assumed to the average density of stars outside $R_{\text {field }} \equiv 90^{\prime \prime}$, and it was kept constant in the fits. The final fits are presented in Fig. 2.5, whereas all recovered structural parameters are shown in Table 2.3.

The comparison of the panels in Fig. 2.5 and the parameters in Table 2.3 reveal, as would be predicted from a visual analysis of the sky maps, that the objects present quite different stellar concentrations and field stellar densities. In all cases the core radius $r_{c}<R_{\text {cluster }}$ and the tidal radius $r_{t}<R_{\text {field }}$, which confirm that the adopted values of $R_{\text {cluster }}$ and $R_{\text {field }}$ are reasonable. All clusters present roughly the same level of concentration, and 
the central density contrast with respect to the field is about 10 to 80 times the field density. We note that the original profile of Lindsay 2 and both profiles of HW 34 did not converge. The first is due to incompleteness, and the second does not have enough density contrast in the centre to be fitted by a King profile, which can be interpreted as evidence of a non-physical system.
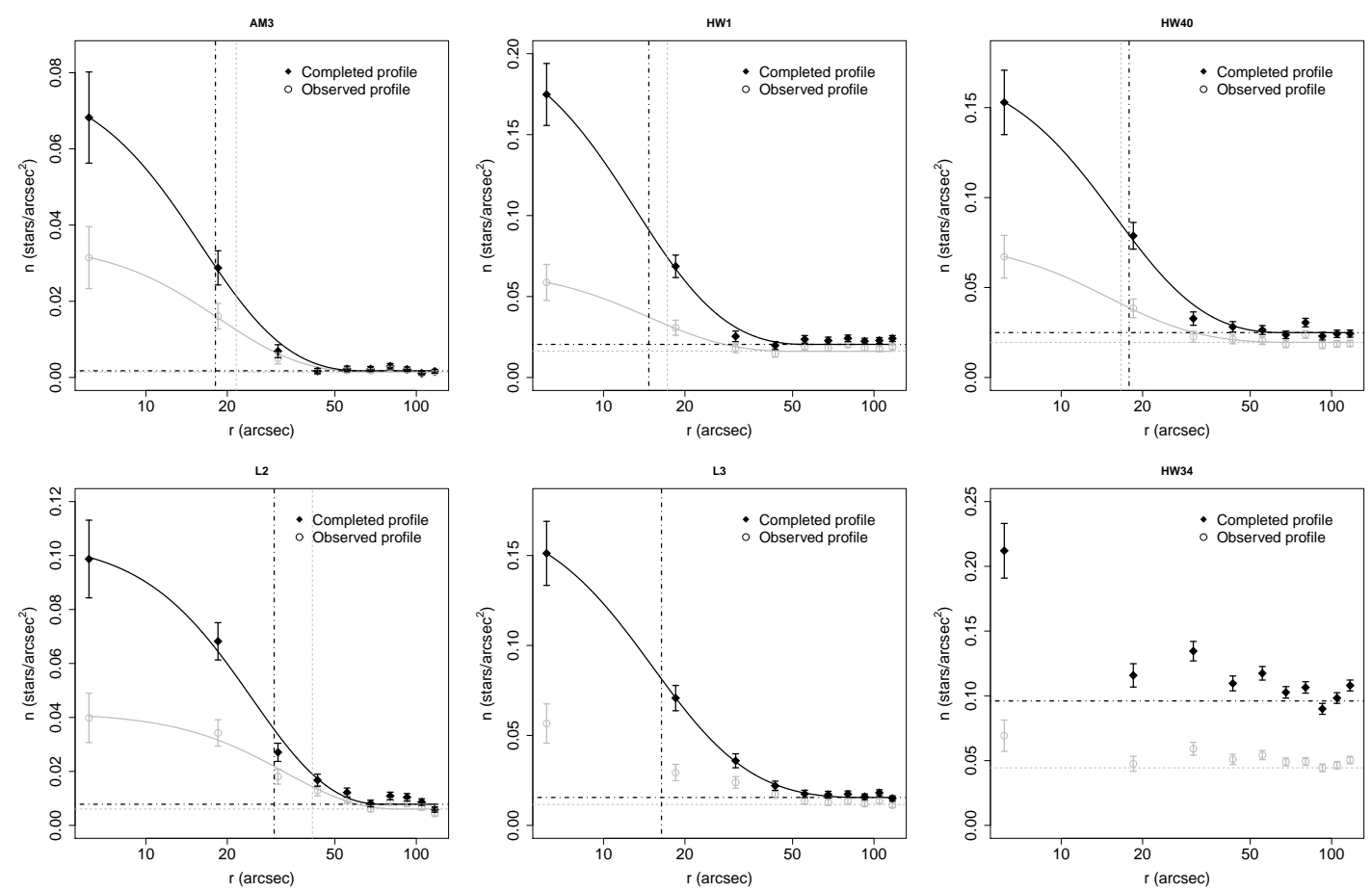

Figure 2.5: Radial numerical density profiles fitted by King profiles. The empty grey circles and filled black diamonds are the observed profiles, original and corrected by completeness (Figure 2.2), respectively. Correspondent lines are King profiles fitted to the data points. The horizontal and vertical dashed grey lines are $n_{\text {field }}$ and $\mathrm{r}_{c}$, respectively, for the original profile, the dash dotted black lines for the corrected profile. HW34 does not present a high enough density contrast to be fitted by a King profile.

\subsubsection{The observed CMDs and cluster membership probability}

Figure 2.4 shows the $\mathrm{V} \times \mathrm{B}-\mathrm{V}$ CMDs for the present sample. For each object, the cluster $\left(R<R_{\text {clus }}\right)$ and field $\left(R>R_{\text {field }}\right)$ CMDs are presented, with stars from $\mathrm{V} \sim 17$ up to $\mathrm{V} \sim 23$. It is possible to identify main sequence (MS) stars as well as red giant stars belonging to the subgiant branch (SGB), red giant branch (RGB), and to the red clump (RC). The comparisons with the field CMDs confirm these stellar identifications. We note that the field CMDs are much richer in stars than the cluster CMDs, as a natural consequence of the ratio between their covered areas $\left(\frac{\Omega_{\text {field }}}{\Omega_{\text {clus }}} \sim 26\right)$. 
Table 2.3 - Structural parameters derived from King profile fitting, as described in Section 2.3.3. The overdensity column indicates how much bigger the central density is with respect to the field density. Notes: a) cases where fit did not converge, and we attribute the density of the innermost region of the profile as $n_{0}$ to estimate a lower limit of the overdensity.

\begin{tabular}{|c|c|c|c|c|c|c|}
\hline Cluster & $\begin{array}{c}n_{\text {field }} \\
\left(\text { stars } /{ }^{\prime \prime 2}\right)\end{array}$ & $\begin{array}{c}n_{0} \\
\left(\text { stars } /{ }^{\prime \prime 2}\right)\end{array}$ & $\begin{array}{l}r_{c} \\
(")\end{array}$ & $\begin{array}{l}r_{t} \\
(")\end{array}$ & $\begin{array}{c}c \\
\log \left(r_{t} / r_{c}\right)\end{array}$ & $\begin{array}{c}\text { overdensity } \\
\left(n_{0}-n_{\text {field }}\right) /\left(n_{\text {field }}\right)\end{array}$ \\
\hline AM 3 - original & 0.0014 & $0.072 \pm 0.009$ & $21.6 \pm 1.4$ & $65 \pm 6$ & $0.48 \pm 0.05$ & 50 \\
\hline AM 3 - completed & 0.0018 & $0.150 \pm 0.015$ & $18.1 \pm 1.1$ & $62 \pm 6$ & $0.54 \pm 0.05$ & 82 \\
\hline Lindsay 2 - original & 0.0061 & $0.11 \pm 0.05$ & $41 \pm 8$ & $85 \pm 17$ & $0.31 \pm 0.12$ & 17 \\
\hline Lindsay 2 - completed & 0.0078 & $0.25 \pm 0.05$ & $29.8 \pm 3.2$ & $74 \pm 9$ & $0.39 \pm 0.07$ & 31 \\
\hline HW 1 - original & 0.0162 & $0.114 \pm 0.009$ & $17.2 \pm 1.3$ & $49.2 \pm 3.0$ & $0.46 \pm 0.04$ & 6 \\
\hline HW 1 - completed & 0.0204 & $0.349 \pm 0.021$ & $14.7 \pm 0.8$ & $55.3 \pm 3.7$ & $0.58 \pm 0.04$ & 16 \\
\hline Lindsay 3 - original $^{a}$ & 0.0116 & $0.057 \pm 0.011$ & - & - & - & 3.9 \\
\hline Lindsay 3 - completed & 0.0155 & $0.238 \pm 0.010$ & $16.4 \pm 0.5$ & $89 \pm 8$ & $0.73 \pm 0.04$ & 14 \\
\hline HW 40 - original & 0.0195 & $0.096 \pm 0.019$ & $16.6 \pm 2.3$ & $69 \pm 17$ & $0.62 \pm 0.12$ & 4 \\
\hline HW 40 - completed & 0.0249 & $0.28 \pm 0.04$ & $17.8 \pm 1.6$ & $65 \pm 9$ & $0.56 \pm 0.7$ & 10 \\
\hline HW 34 - original ${ }^{a}$ & 0.0444 & $0.069 \pm 0.012$ & - & - & - & 0.5 \\
\hline HW $34-$ completed $^{a}$ & 0.0960 & $0.212 \pm 0.021$ & - & - & - & 1.2 \\
\hline
\end{tabular}

Since the stellar samples in the direction of the clusters are contaminated by SMC field stars, a procedure to determine the cluster membership probability ( $\left.p_{\text {member }}\right)$ was applied. In summary, the adopted procedure follows the one from Kerber and Santiago (2005) and Alves et al. (2012), where $p_{\text {member }}$ for each cluster star is determined by comparing the density of stars inside the CMD in the cluster direction $\left(R<R_{\text {clus }}\right)$, with a CMD representative of the local SMC field $\left(R>R_{\text {field }}\right)$ (see Fig. 2.4). The cluster and field CMDs are divided into a grid of small boxes in $\mathrm{V}$ magnitude and $\mathrm{B}-\mathrm{V}$ colour, centred on each cluster star. So, for the $\mathrm{i}^{\text {th }}$ cluster star, the number of cluster $\left(N_{\text {clus, }}\right)$ and field stars $\left(N_{\text {field,i }}\right)$ contained in a box in magnitude and colour (with a 3-sigma size in photometric errors) are computed, taking the inverse of the completeness into account. Finally, the cluster membership probability for the $\mathrm{i}^{\text {th }}$ cluster star is given by

$$
p_{\text {member }, \mathrm{i}}=1-\frac{N_{\text {field }, \mathrm{i}}}{N_{\text {clus }, \mathrm{i}}} \times \frac{\Omega_{\text {clus }}}{\Omega_{\text {field }}} .
$$

The results of these determinations are shown in Fig. 2.4 in a colour scale. The analysis of this figure reveals that most probable cluster members $\left(p_{\text {member }}>75 \%\right)$ are red giants or stars in the upper MS. In particular, almost all stars in the AM 3 cluster sample can be considered physical cluster members. On the other hand, the MS stars in the HW 34 cluster direction have a higher probability of being field stars rather than cluster stars since they present $p_{\text {member }}<50 \%$. This strongly reinforces the argument that this stellar 
concentration is not a physical system, but only a field stellar fluctuation (see also Sect. 2.3.3). Table 2.4 summarizes the star counts in the sample CMDs.

Table 2.4 - Stellar counts in the sample CMDs. Columns correspond to: the cluster's name, the number of stars without the completeness corrections and with them for the cluster $\left(\mathrm{N}_{\text {clus }}^{(\text {obs })}\right.$ and $\mathrm{N}_{\text {clus }}^{\text {(comp) }}$ ) and field direction ( $\mathrm{N}_{\text {field }}^{(\mathrm{obs})}$ and $\mathrm{N}_{\text {field }}^{(\text {comp })}$ ), as well as the expected number of field stars in the completed cluster sample, with pmember $<0.5$ (contamination $C$ ). The last column is the number of cluster stars in terms of the field standard deviation (assumed as Poissonian), given by the expression $N_{\sigma}=\left(N_{\text {clus }}^{(\text {comp })}-C\right) / \sqrt{N_{\text {field }}^{(\text {comp })}}$.

\begin{tabular}{lcccccc}
\hline Target & $\mathrm{N}_{\text {clus }}^{(\text {obs }}$ & $\mathrm{N}_{\text {clus }}^{(\text {comp }}$ & $\mathrm{N}_{\text {field }}^{(\text {obs })}$ & $\mathrm{N}_{\text {field }}^{(\text {comp })}$ & $C$ & $\mathrm{~N}_{\sigma}$ \\
\hline AM 3 & 56 & 172 & 135 & 209 & 8 & 11 \\
Lindsay 2 & 127 & 490 & 847 & 2434 & 93 & 8.1 \\
HW 1 & 112 & 370 & 1659 & 3686 & 141 & 3.8 \\
Lindsay 3 & 116 & 342 & 1412 & 3818 & 145 & 3.2 \\
HW 40 & 133 & 393 & 1906 & 3790 & 143 & 4.1 \\
HW 34 & 171 & 562 & 4014 & 13594 & 515 & 0.4
\end{tabular}

\subsection{Statistical isochrone fitting}

The isochrone fitting used in this work follows a numerical and statistical approach that combines CMD modelling and an objective criterion of comparing synthetic CMDs with the observed ones. It was initially developed to analyse CMDs of rich LMC clusters, observed with the Hubble Space Telescope (HST) (Kerber et al.,, 2002), and recently it was applied to determining physical parameters of Galactic open clusters imaged by 2MASS (Alves et al., 2012). The central idea behind this method is to statistically determine the synthetic CMDs that best reproduce the observed CMD, recovering as a consequence the physical parameters for the stellar cluster. In the present work the statistics adopted to select the best models is the likelihood, in particular following a Bayesian approach. This approach has been successfully applied to analvsing CMDs of stellar clusters (Naylor and Jeffries, 2006; Hernandez and Valls-Gabaud, 2008; Monteiro et al., 2010) as well as CMDs of composite stellar populations (Hernandez et al., 2000, 1999; Vergely et al., 2002).

The generation of synthetic CMDs, as well as the likelihood statistics, are detailed in the next subsections. 


\subsubsection{CMD modelling}

The synthetic CMDs are generated considering that they are SSPs, characterized by stars with the same age $(\tau)$ and metallicity $(Z)$. The basic steps to generate a specific synthetic CMD are the following (see Fig. 2.6):

(i) adoption of an evolutionary stellar model. The new Padova-Trieste models (PARSEC isochrones, Bressan et al., 2012) were adopted in this work;

(ii) selection of the age and metallicity of the SSP, which is equivalent to selecting an isochrone from the library;

(iii) application of the distance modulus $(m-M)_{0}$ and reddening $E(B-V)$ values to shift the isochrone from the absolute magnitudes to the observed ones;

(iv) generation of $10^{5}$ synthetic stars belonging to stellar systems (single and binary stars) following an IMF and a fraction of unresolved binaries $\left(f_{\text {bin }}\right)$. A Salpeter IMF $(d N / d m \propto$ $\left.m^{-2.35}\right)$ and $f_{\text {bin }}=30 \%$ were adopted, with a minimum mass ratio $\left(q_{\min }=m_{2} / m_{1}\right)$ of 0.7 and a constant mass-ratio distribution $(d N / d q=$ const. $)$;

(v) introduction of the observational photometric errors in magnitude and colour.

To determine the cluster parameters, a wide and regular grid of models was build for each cluster, composed typically of $\sim 9000$ combinations in $\log (\tau / \mathrm{yr}), Z,(m-M)_{0}$ and $E(B-V)$ centred on the parameters found by a visual isochrone fit. To take into account the line-of-sight depth and the reddening values found for the SMC stellar clusters (Glatt et al., 2008; Crowl et al., 2001), the grids of models span distance modulii and reddening values in the range $18.50<(m-M)_{0}<19.20$ and $0.00<E(B-V) 0.20$, in steps of 0.05 and 0.01 , respectively. For $\log (\tau / \mathrm{yr})$ the step is 0.05 , and for $Z$ all values provided by the isochrones are considered: $0.0001,0.0004,0.001,0.002,0.004,0.006,0.008,0.010,0.012$ and 0.014 .

\subsubsection{Likelihood statistics}

The first step in computing the likelihood for a synthetic CMD is to establish the probabilities for each observed star cluster that it belong to that choice of SSP $\left(p_{\mathrm{CMD}}\right)$. In general, this is done using analytical expressions (e.g. Hernandez and Valls-Gabaud, 2008; Monteiro et al., 2010), assuming that the observed CMD positions would represent stars scattered from the isochrones following Gaussian distributions for the photometric 
errors. However, in the present work these probabilities are computed assuming that they are proportional to the density of points generated by the synthetic CMD in each of the respective CMD positions $(N[V,(B-V)])$, which naturally incorporates the effect of unresolved binaries and photometric uncertainties. This density map on the CMD is commonly called a Hess diagram, even though its application is not directly related with the likelihood statistics. Therefore, the likelihood statistics in this numerical approach is given by the product of these probabilities over all the $N_{\text {clus }}$ observed cluster stars as stated by the expression

$$
\mathrm{L} \propto \prod_{\mathrm{i}=1}^{\mathrm{N}_{\text {clus }}} \mathrm{p}_{\mathrm{CMD}, \mathrm{i}} \times \mathrm{p}_{\text {member }, \mathrm{i}} \propto \prod_{\mathrm{i}=1}^{\mathrm{N}_{\text {clus }}} \mathrm{N}\left[\mathrm{V}_{\mathrm{i}},(\mathrm{B}-\mathrm{V})_{\mathrm{i}}\right] \times \mathrm{p}_{\text {member }, \mathrm{i}},
$$

where the $i$ index corresponds to the $\mathrm{i}^{\text {th }}$ observed cluster star. It can thus be stated that the best model is the one that provides the synthetic CMD/Hess diagram that maximizes the above expression $\left(\mathrm{L}_{\max }\right)$. The final parameters for each cluster, as well as corresponding

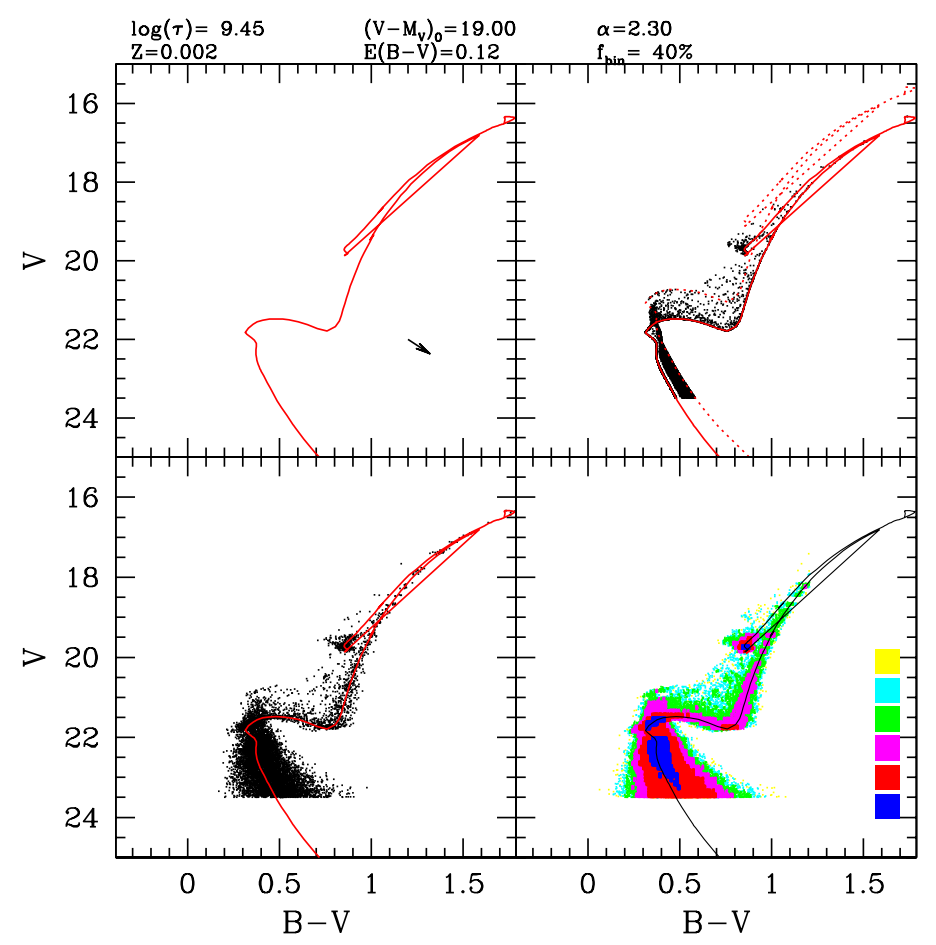

Figure 2.6: The generation of synthetic V vs. B-V CMDs. Panel a: the adopted isochrone shifted by distance modulus and reddening. Panel $b$ : the distribution of stars in accordance to the IMF and the fraction of binaries; Panel $c$ : the introduction of photometric errors in magnitude and colour. Panel $d$ : colours following the density of points in the CMD in a logarithmic scale. The density of points is related to $p_{\mathrm{CMD}, \mathrm{i}}$ (see Sect. \#2.4.2 ). The reddening vector and the values for all physical parameters are shown in the figure. 
uncertainties, are determined by computing the average and standard deviation over the physical parameters of the set of models that have likelihood values at the 1-sigma level from $\mathrm{L}_{\max }$ (see Appendix A for details).

\subsection{Results}

The results of the isochrone fittings for the sample clusters are presented in Figure 2.7 and Table 2.5. The panels in Fig. 2.7 give isochrones corresponding to the bestfit parameters, together with another two isochrones indicating 1- $\sigma$ uncertainties in age and metallicity: one younger and more metal-rich, and the other older and more metalpoor. The three isochrones cover most of the data points, as expected, allowing the reader to visually inspect the quality of the fits. For the $[\mathrm{Fe} / \mathrm{H}]$ derivation we adopted a solar metallicity Z=0.0152 (Caffau et al., 2011) in order to be compatible with the value assumed in the PARSEC isochrones (Bressan et al., 2012). These results are discussed cluster by cluster bellow.

Table 2.5 - Physical parameters determined in this work. Columns refer to: cluster name, logarithm of the age, age, metallicity $\mathrm{Z}$ and $[\mathrm{Fe} / \mathrm{H}]$ (assuming $\mathrm{Z}_{\odot}=0.0152$, Caffau et al., 2011), distance modulus, distance, reddening, and semi-major axis corresponding to the distance of the cluster to the centre of SMC (as explained in Section 2.6).

\begin{tabular}{lcccccccc}
\hline Name & $\log (\tau / y r)$ & Age $(\mathrm{Gyr})$ & $\mathrm{Z}$ & {$[\mathrm{Fe} / \mathrm{H}]$} & $(\mathrm{m}-\mathrm{M})_{0}$ & $\mathrm{~d}(\mathrm{kpc})$ & $\mathrm{E}(\mathrm{B}-\mathrm{V})$ & $a\left(^{\circ}\right)$ \\
\hline AM 3 & $9.69 \pm 0.15$ & $4.9_{-1.5}^{+2.1}$ & $0.0022 \pm 0.0016$ & $-0.8_{-0.6}^{+0.2}$ & $18.99 \pm 0.16$ & $63.1_{-1.7}^{+1.8}$ & $0.08 \pm 0.05$ & 7.3 \\
Lindsay 2 & $9.60 \pm 0.09$ & $4.0_{-0.7}^{+0.9}$ & $0.0007 \pm 0.0004$ & $-1.4_{-0.2}^{+0.2}$ & $18.68 \pm 0.14$ & $54.4_{-1.5}^{+1.5}$ & $0.09 \pm 0.04$ & 3.9 \\
HW 1 & $9.70 \pm 0.12$ & $5.0_{-1.2}^{+1.5}$ & $0.0013 \pm 0.0007$ & $-1.1_{-0.3}^{+0.2}$ & $18.84 \pm 0.16$ & $58.7_{-1.6}^{+1.6}$ & $0.07 \pm 0.04$ & 3.4 \\
Lindsay 3 & $9.07 \pm 0.11$ & $1.2_{-0.3}^{+0.3}$ & $0.0057 \pm 0.0020$ & $-0.4_{-0.2}^{+0.1}$ & $18.64 \pm 0.14$ & $53.4_{-1.5}^{+1.5}$ & $0.07 \pm 0.04$ & 2.9 \\
HW 40 & $9.41 \pm 0.06$ & $2.5_{-0.3}^{+0.4}$ & $0.0018 \pm 0.0005$ & $-0.9_{-0.2}^{+0.1}$ & $19.08 \pm 0.14$ & $65.6_{-1.8}^{+1.8}$ & $0.03 \pm 0.03$ & 2.0 \\
\hline
\end{tabular}

\subsubsection{AM3}

Although this is the cluster with the smallest number of stars in our sample, it has the advantage of being located in the SMC field with the lowest stellar density. The results in the present work indicate an age of $4.9_{-1.5}^{+2.1}$ Gyr. The recovered metallicity, $[\mathrm{Fe} / \mathrm{H}]=-0.8_{-06}^{+0.2}$, is consistent with the value predicted by the SMC chemical evolution model from Pagel and Tautvaisiene (1998) for this age. Piatti (2011a) also determined 

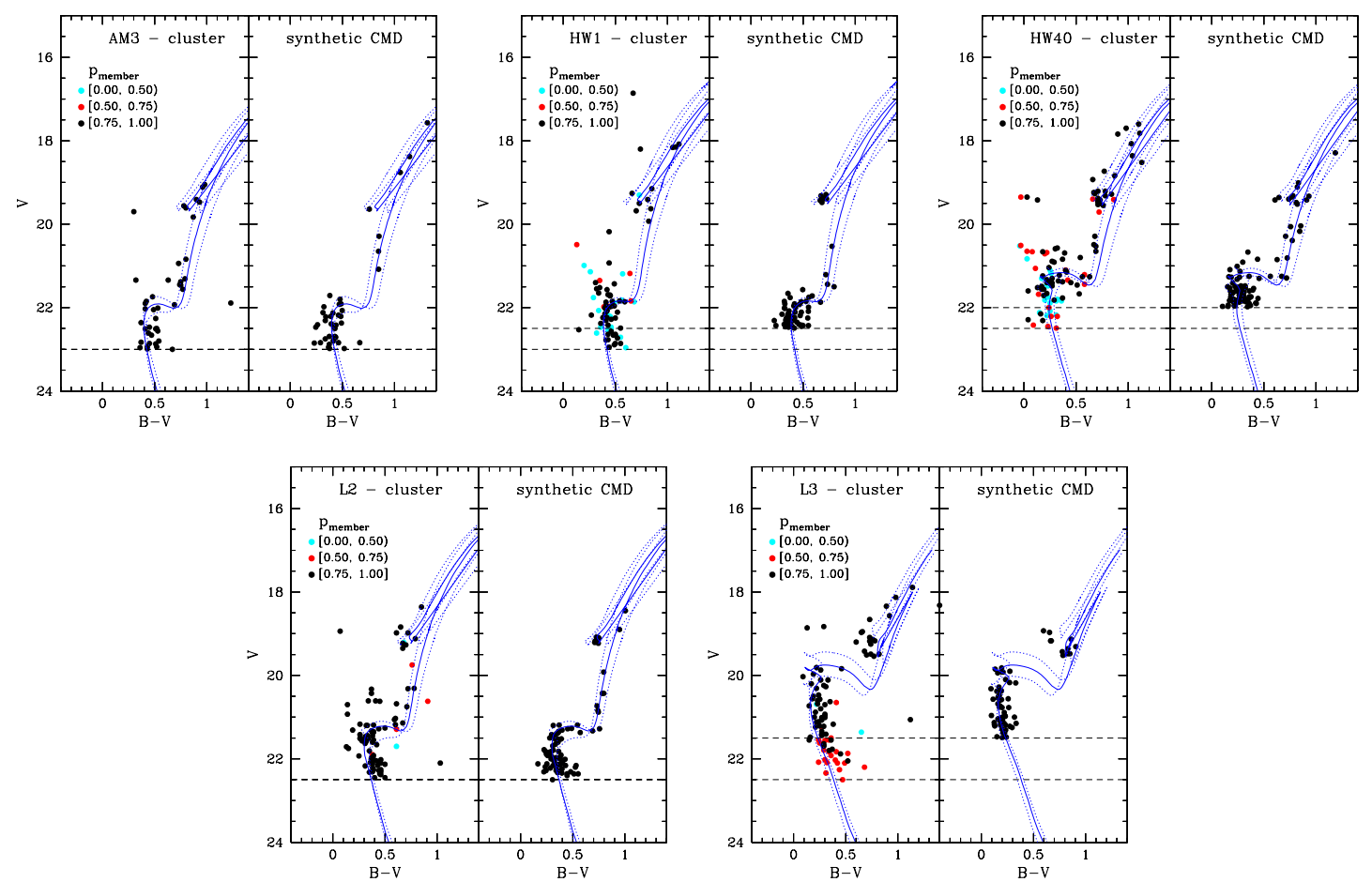

Figure 2.7: Best isochrone fittings for all clusters. Left panels: cluster stars with colour symbols in accordance to the membership probabilities ( $p_{\text {member }}$ ). Right panels: synthetic CMDs generated with the parameters found for the best solution. The number of points is equal to the observed CMDs within the same magnitude limits. The horizontal dashed black lines correspond to the magnitude limits used to compute the likelihood (brighter mag) and pmember (fainter mag). The solid and dashed blue lines correspond to the isochrones with the parameters found in Table2.5 and take into account the uncertainties on ages and metallicities.

physical parameters for this cluster, based on the analysis of a CMD built with Washington photometry. Taking the uncertainties into account, our results are in good agreement with the ones from Piatti et al., although they derived slightly older ages (6.0 $\pm 1.0 \mathrm{Gyr})$ and more metal poor values $([\mathrm{Fe} / \mathrm{H}]=-1.25 \pm 0.25)$. Our results are also close to the parameters derived by Da Costa (1999) of $[\mathrm{Fe} / \mathrm{H}]=-1.27$ and age of $5.5 \pm 0.5$ Gyr.

The differences in age can be explained by the distance modulus. While we fitted a $(\mathrm{m}-\mathrm{M})_{0}=19.00$ for this cluster, Piatti (2011a) assumed an average value of 18.90. With a distance modulus $\sim 0.1$ lower, the isochrone should be $\sim 0.6$ Gyr older to fit the data.

This western SMC cluster was considered a galaxy member by Da Costa (1999) because it is close to the projected plan of the SMC, and its red clump magnitude (distance indicator) is comparable to other SMC clusters. It is located in the West Halo region, but it is not as old and metal poor as one could expected in the case of gradients in age and $[\mathrm{Fe} / \mathrm{H}]$ for this group (see Sect. 2.6). Although AM 3 is close to the SMC main body in the 
projected plan, its distance is higher than the other West Halo clusters (HW 1, Lindsay 2, and Lindsay 3). Therefore its age, metallicity, and distance do not follow exactly the general characteristics of the West Halo, which indicates a more complex history, possibly a capture by the potential well of the SMC.

\subsubsection{HW 1 and Lindsay 2}

The CMD analysis for these clusters which have never been published before, identifies these objects as intermediate-age metal-poor SMC clusters, with similar metallicities $([\mathrm{Fe} / \mathrm{H}] \sim-1.1$ and -1.4 , respectively) and ages (4.98 and $4.0 \mathrm{Gyr})$. In particular, the age and metallicity values for HW 1 confirm the previous results from the integrated spectral analysis performed by Dias et al. (2010). We note that these two clusters are located close to each other, in the West Halo region (Fig. 2.8), at angular distances from the SMC centre of $\mathrm{a}=3.4^{\circ}$ to $3.9^{\circ}$, and distances from the Milky Way of 58.7 and $54.4 \mathrm{kpc}$, respectively. Their ages correspond to the epoch of a probable recent tidal interaction between the Magellanic Clouds, therefore they could be remnants of this period.

\subsubsection{HW 40}

The CMD analysis for this cluster indicates that it is an intermediate-age SMC cluster with $2.5_{-0.3}^{+0.4}$ Gyr. The presently derived metallicity $\left([\mathrm{Fe} / \mathrm{H}]=-0.9_{-0.2}^{+0.1}\right)$ is in agreement with the results from Piatti (2011a) based on photometry $([\mathrm{Fe} / \mathrm{H}]=-1.10 \pm 0.25)$, and by Parisi et al. (2013, private comm.; $[\mathrm{Fe} / \mathrm{H}]=-0.78 \pm 0.05 \mathrm{dex})$ using CaT spectra. The age determined by Piatti et al. is significantly older (5.4 \pm 1.0 Gyr) than our result. More accurate CMDs are needed for this cluster to constrain its age.

\subsubsection{Lindsay 3}

The age $(1.2 \pm 0.3 \mathrm{Gyr})$ and metallicity $\left.-0.4_{-0.2}^{+0.1}\right)$ for this cluster is in good agreement with the results from Piatti et al. (2011): $1.25 \pm 0.20 \mathrm{Gyr}$ and $[\mathrm{Fe} / \mathrm{H}]=-0.65 \pm 0.20$ dex. A remarkable result from our isochrone fitting is the small distance modulus for this cluster $(18.64 \pm 0.14)$, suggesting that it is one of the closest SMC clusters. We note that here too Piatti et al. (2011) assumed a distance modulus of 18.90 and also a reddening of 0.04 instead of our fitted value of 0.07 . The combination of these two values tends to compensate the effects on age and metallicity. Lindsay 3 is younger than the bulk of the 
surrounding field stellar population in the West Halo region (HW 1 and Lindsay 2 are $\sim 3$ Gyr older, for instance). If the Magellanic Clouds suffered interactions at about 4 Gyr ago, this could be a resulting cluster in an older field, or else it is possible that there is an age gradient in the West Halo. More data on the star clusters of this region are needed to confirm these conclusions (see Sect. 2.6).

\subsubsection{HW 34}

No star cluster was identified, as made evident by the following:

- Sky maps given in Fig. 2.1 and radial density profiles in Fig. 2.5 show no significant overdensity in the region centred on HW 34 coordinates;

- the photometric data are of comparable quality to the other clusters (see Figs. 2.2 and 2.3), so a cluster would be recovered if present;

- Almost all stars in the direction of HW 34 coordinates were given the probability of being field stars, as shown in Fig. 2.4 and Table 2.4;

- Based on the very few RGB stars with $\mathrm{p}_{\text {member }}>0.5$, and no clear main sequence turnoff, if there was a cluster there, it would be older than $\sim 5$ Gyr, which is not expected for the central younger regions of the SMC.

\subsection{The age-metallicity relation and spatial distribution}

Figure 2.8 shows the 2D positions of the five clusters in the sky plane, where clusters from the catalog of Bica et al. (2008) were overplotted. Ellipses are indicated to find the clusters' distances from the SMC centre, following the procedure of Piatti et al. (2005). We adopt the coordinates of the SMC centre from Crowl et al. $(2001),(\alpha, \delta)=\left(0^{\mathrm{h}} 52^{\mathrm{m}} 45^{\mathrm{s}}\right.$, $-72^{\circ} 49^{\prime} 43^{\prime \prime}$ ), and a minor to major axis ratio of $b / a=1 / 2$. Thus, the indicator of distance to the SMC centre would be the semi-major axis $a$, which is more appropriate for a ellipsoidal galaxy like the SMC (see Figure 2.8). We consider an inclination of $45^{\circ}$ for the major axis of all the ellipses in the projected plane of the figure. The distances $a$ for all the clusters can be found in Table 2.5.

Most of the sample clusters are located outside the region sampled by Harris and Zaritsky (2004), but inspired by the different SFR that they found in different regions of 
the SMC, we located our targets in different groups for a $>2^{\circ}$ (see Fig. 2.8). These regions were simply divided into the sky plane based on the HI structures, as described by Diaz and Bekki (2012), assuming that these structures have stellar/star cluster counterparts. They are: Bridge, Counter-Bridge, and a third region not related with the HI structures that we have called West Halo. They are indicated by different colours and symbols in Figs. 2.8, 2.9, and 2.10.

The 3D structure of the SMC was shown to be complex (Subramanian and Subramaniam, 2012), with a spread in depth of about $14 \mathrm{kpc}$, and an inclination along the main axis with the north-eastern portion towards the observer. The five clusters studied in this work follow this dispersion, in particular for West Halo clusters, where we found distances between $\sim 54$ and $63 \mathrm{kpc}$, revealing that more statistics on this region are needed in order to better describe the 3D structure of the SMC.

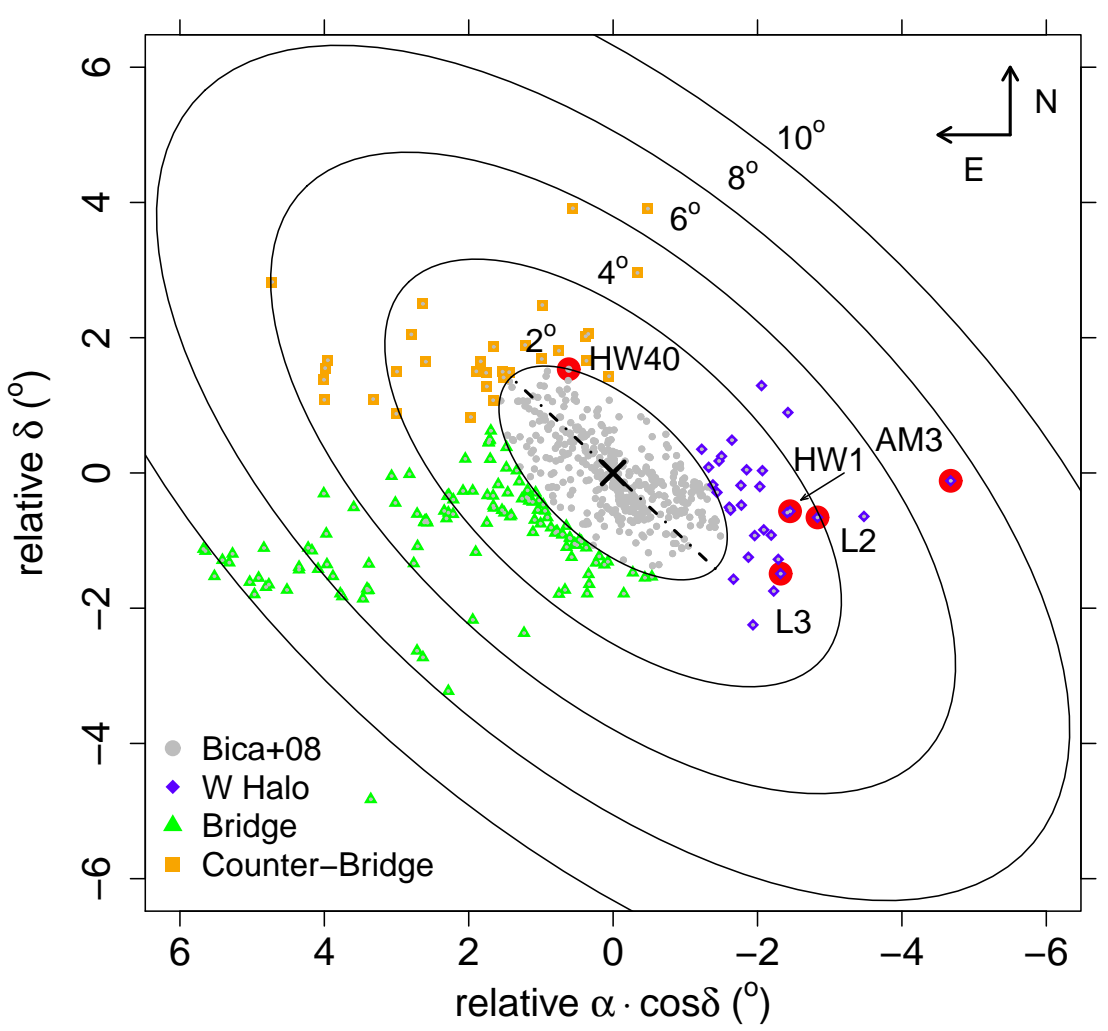

Figure 2.8: On sky distribution of the SMC star clusters, including the catalog clusters (Bica et al., 2008), overplotted with the five sample clusters. The ellipses are used to illustrate distances from the SMC centre (Table 2.5). Different colours indicate subgroups, as indicated in the panel (see text for details).

Figure 2.9 shows the age-metallicity relation (AMR) of the SMC, by plotting data for well-studied star clusters (Dias et al., 2010; Piatti et al., 2011; Piatti, 2011a, b), overplotted 
by the model of Pagel and Tautvaisiene (1998). The results for the sample clusters fit well the Pagel and Tautvaisiene (1998) model, and agree with a dispersion in metallicity for the $\mathrm{SMC}$ cluster system in the metallicity and age ranges of $-1.5<[\mathrm{Fe} / \mathrm{H}]<-1.0$, and $5<$ age $<10$ Gyr, as indicated by Piatti (2011b), in their Fig. 3. The field stars also agree with this statement, as can be seen in Fig. 8 of Piatti (2012b). In this case, the model of Pagel and Tautvaisiene (1998) would represent an average of the chemical enrichment history of the SMC. Spectroscopic results by Parisi et al. (2009) also show a spread in [Fe/H] among intermediate-age clusters; however, their values are in the range $-1.2<[\mathrm{Fe} / \mathrm{H}]<-0.7$ dex. Therefore, both photometric and spectroscopic metallicities show a spread of $\sim 0.5$ dex in metallicity, with limits defined by different metallicity scales.

Another important product of this work concerns AM 3, Lindsay 2, and HW 1, the oldest clusters of the sample, with 4.9, 4.0, and $4.98 \mathrm{Gyr}$, respectively. They are close to the end of the quiescent star formation as pointed out by Harris and Zaritsky (2004), and can be among the first products of the reactivation of star formation in the MC system $\sim$ 3-4 Gyr ago.

The cluster distribution in the $2 \mathrm{D}$ projected plane of the sky is also useful for investigating gradients of age and metallicity. This is presented in Figure 2.10, where the marked labels correspond to those of Figure 2.9. The five clusters presented in this work are located outside the $2^{\circ}$ ellipse. The small sample combined with the error bars do not permit us to establish a strong gradient. Nevertheless, it is possible to identify that the most internal regions of the SMC, below $a=1-2^{\circ}$ might indicate a gradient in age and metallicity, whereas the outer regions have a spread in these parameters. Otherwise, these trends seem to be consistent with those of the SMC field population (Piatti, 2012b).

Even so, if one looks at different regions of the projected SMC, they reveal different superimposed gradients (which would generate the overall dispersion with no gradients), but the dispersion is too high and more data are needed for this to be confirmed. Lindsay 2, Lindsay 3, and HW 1, which are located in the West Halo region, tend to show a possible age gradient in this region, as indicated by the blue diamonds in the figure. 


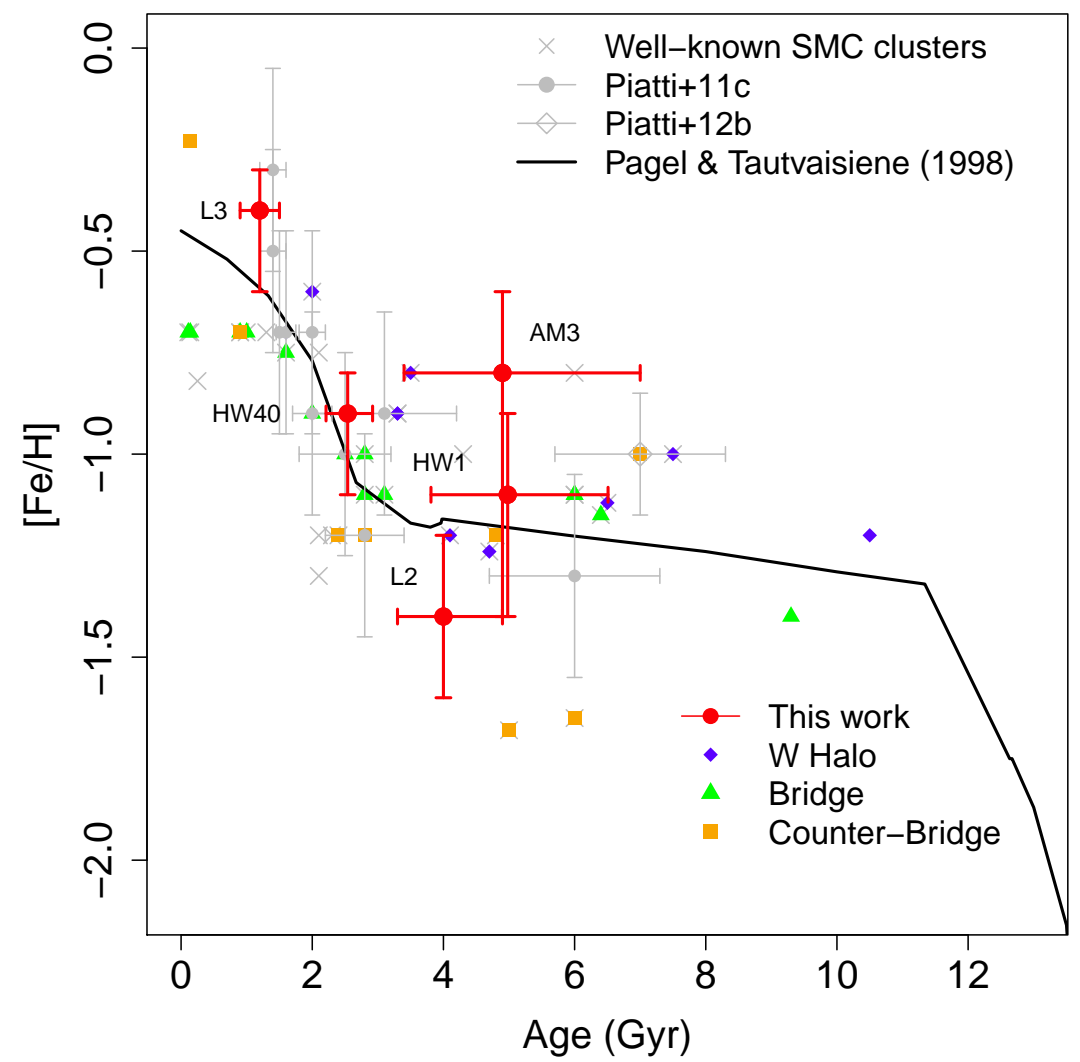

Figure 2.9: Age-metallicity relation for the SMC, including the new additions from the present work. The well-known clusters are listed in Table 6 of Dias et al. (2010), and other clusters from literature. The model of Pagel and Tautvaisiene (1998) is overplotted.

\subsection{Summary of this chapter}

We studied intermediate-age stellar clusters in the SMC. The parameters were derived by statistical fitting of the observed CMDs with a grid of synthetic CMDs based on the PARSEC isochrones. Six targets were observed in the visible filters B and V, and we derived ages for five of them (AM 3, HW 1, HW 40, Lindsay 2, and Lindsay 3) in the age range of 1.2 Gyr to 5.0 Gyr, whereas HW 34 was identified as a field fluctuation. In particular, Lindsay 2 and HW 1 were studied for the first time in this work, and for the other clusters we derived self-consistent parameters compatible with the literature, and in principle with more precision.

The five clusters essentially follow the chemical enrichment historv modelled by Pagel and Tautvaisiene (1998). Figure 2.9 shows a strong spread in metallicity for clusters with ages of 2-5 Gyr, also found by Piatti (2011b). Piatti (2012b) finds a metallicity spread of $\sim 0.4$ dex at ages of 2-4 Gyr, and Parisi et al. (2010) finds a dispersion of $\sim 0.32$ dex around 


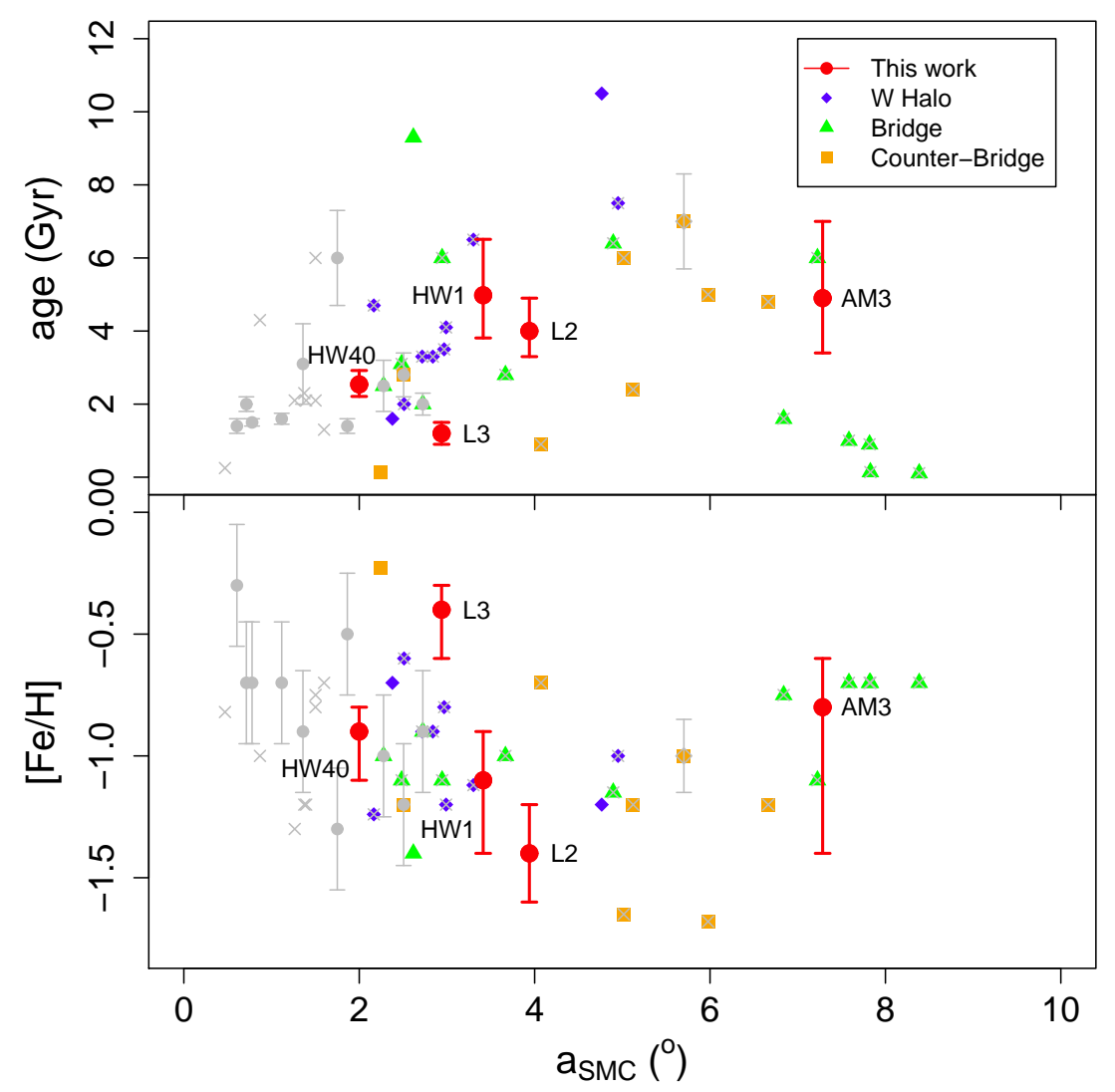

Figure 2.10: Age and $[\mathrm{Fe} / \mathrm{H}]$ as a function of the distance indicator $a$. The dots are the same as described in Figure 2.9.

the mean value of $[\mathrm{Fe} / \mathrm{H}]=-1.0$ from 15 fields.

This suggests that, after an initial period of rapid enrichment, that brought the metallicity up to around $[\mathrm{Fe} / \mathrm{H}] \approx-1.2$, the $\mathrm{SMC}$ chemical enrichment is very inhomogeneous, since there is a spread in metallicity from $\sim 11$ to $\sim 3$ Gyr, with a trend for slow enrichment. Finally an intense burst occurred at around 2 Gyr ago, when most of the star clusters started to form.

Piatti et al. (2011) suggested the occurrence of two recent bursts at $\sim 2$ Gyr and $\sim$ 6 Gyr ago (preceded by a quiescent period lasting $\sim 3$ Gyr), with roughly no metallicity variations. Our clusters improve the statistics for the two more recent bursts identifed by Piatti et al. (2011), in particular HW 1 and Lindsay 2, which are located in the West Halo region, at almost the same distance.

A gradient of age and metallicity can be found in the inner part of the SMC $\left(\mathrm{a}<2^{\circ}\right)$, but in the outer regions the high dispersion in ages and metallicities prevents the identification of any trends. More data on age, metallicity/abundances, distance, and kinematics of star 
clusters are needed in order to prove different star formation rates and chemical evolution histories in the regions due to tidal forces caused by the interactions in the SMC-LMCMilky Way system. 
Chapter 3

\section{FORS2/VLT survey of Milky Way globular clusters I. Method validation}

\subsection{Abstract of this chapter}

We have observed almost 1/3 of the globular clusters in the Milky Way, targeting distant and/or highly reddened objects, besides a few reference clusters. A large sample of red giant stars was observed with FORS2@VLT/ESO at R 2000. The method for derivation of stellar parameters is presented with application to six reference clusters. We aim at deriving the stellar parameters effective temperature, gravity, metallicity and alpha-element enhancement, as well as radial velocity, for membership confirmation of individual stars in each cluster. We analyse the spectra collected for the reference globular clusters NGC 6528 $([\mathrm{Fe} / \mathrm{H}] \sim-0.1)$, NGC $6553([\mathrm{Fe} / \mathrm{H}] \sim-0.2)$, M 71 ([Fe/H] -0.8), NGC $6558([\mathrm{Fe} / \mathrm{H}] \sim-1.0)$, NGC $6426([\mathrm{Fe} / \mathrm{H}] \sim-2.1)$ and Terzan $8([\mathrm{Fe} / \mathrm{H}] \sim-2.2)$. They cover the full range of globular cluster metallicities, and are located in the bulge, disc and halo. Full spectrum fitting techniques are applied, by comparing each target spectrum with a stellar library in the optical region at 4560-5860 А. We employed the library of observed spectra MILES, and the synthetic library by Coelho et al. (2005). Validation of the method is achieved through recovery of the known atmospheric parameters for 49 well-studied stars that cover a wide range in the parameter space. We identified 4 member stars in NGC 6528, 13 in NGC 6553, 10 in M 71, 5 in NGC 6558, 5 in NGC 6426 and 12 in Terzan 8. Radial velocities, effective temperatures, gravities, metallicities and alpha-element enhancements were derived. We derived $\left\langle\mathrm{v}_{\text {helio }}\right\rangle=-242 \pm 11 \mathrm{~km} / \mathrm{s},[\mathrm{Fe} / \mathrm{H}]=-2.39 \pm 0.04,[\mathrm{Mg} / \mathrm{Fe}]=$ $0.38 \pm 0.06$ for NGC 6426 from spectroscopy for the first time. The method proved to be reliable for red giant stars observed with resolution $\mathrm{R} \sim 2000$, yielding results compatible 
with high-resolution spectroscopy. The derived $\alpha$-element abundances show $[\alpha / \mathrm{Fe}]$ vs. $[\mathrm{Fe} / \mathrm{H}]$ consistent with that of field stars at the same metallicities.

\subsection{Introduction to this chapter}

The derivation of stellar metallicities and abundances are best defined when based on high spectral resolution and high signal-to-noise (S/N) data. Cavrel (1988) showed that higher resolution carries more information than higher S/N. Such kind of data require however substantial telescope time. For this reason, very large samples of stellar spectra have been gathered in recent years, or are planned to be collected in the near future, with multi-object low and medium-resolution instruments. A few examples are the Sloan Digital Sky Survey (SDSS, York et al., 2000), at a resolution R 1800, the Radial Velocity Experiment survey (RAVE, Steinmetz et al., 2006) of R 7500 in the CaT region, and other large ongoing surveys such as LAMOST at the Guoshoujing telescope (GSJT, Wu et al., 2011) of $\mathrm{R} \sim 2000$, and future ones such as GAIA (Perryman et al., 2001). Large data sets of low/medium-resolution spectra are reachable for extragalactic stars, such as presented in Kirby et al. (2009). A few recent surveys are able to use medium/high-resolution spectra focused on specific targets such as provided by the APOGEE (R 22,500, Mészáros et al., 2013), GAIA-ESO using the FLAMES-GIRAFFE spectrograph $(\mathrm{R} \sim 22,000)$ at the Very Large Telescope (VLT, Gilmore et al.., 2012) and HERMES (R 28.000 or 45.000. Wyliede Boer and Freeman, 2010) at the AAT. More complete reviews of available, ongoing and future surveys, as well as automated methods for stellar parameter derivation can be found in Allende Prieto et al. (2008), Lee et al. (2008), Koleva et al. (2009), Mészáros et al. (2013), and Wu et al. (2011), among others.

In most analyses of medium to low-resolution spectra, the least squares $\left(\chi^{2}\right.$ minimization), or "euclidian distance", also called minimum distance method, such as Université de Lyon Spectroscopic Analysis Software (ULySS, Koleva et al.,, 2009), and the k-means clustering described in Sánchez Almeida and Allende Prieto (2013), are employed.

In the present work we analyse spectra in the optical, in the range 4560-5860 $\AA$, obtained at the FORS2/VLT at a resolution $\mathrm{R} \sim 2,000$, carrying out full spectrum fitting. This spectral region, in particular from $\mathrm{H}_{\beta}$ to $\mathrm{Na}$ I lines, is sensitive to metallicity and temperature, to gravity due to $\mathrm{MgH}$ molecular bands (as part of the $\mathrm{Mg}_{2}$ index), and it 
includes the Lick indices Fe5270, Fe5335 and $\mathrm{Mg}_{2}$, that are usual Fe and $\mathrm{Mg}$ abundance indicators (Katz et al., 2011; Cayrel et al., 1991; Faber et al., 1985; Worthey et al., 1994).

The same sample was observed in the near-infrared (CaT), as presented in Saviane et al. (2012), Da Costa et al. (2009) and Vasquez et al. (in prep), where two among the triplet Ca II lines were used to derive velocities and metallicities. A comparison of their results with the present ones show good consistency, as will be discussed in the present paper.

In this work we study six reference globular clusters, spanning essentially the full range of metallicities of globulars: the metal-poor halo clusters NGC 6426 and Terzan $8([\mathrm{Fe} / \mathrm{H}] \sim-$ 2.1 and -2.2 , respectively), the moderately metal-poor NGC $6558([\mathrm{Fe} / \mathrm{H}] \sim-1.0)$ in the bulge, the template "disc" metal-rich cluster M 71 (NGC 6838, [Fe/H] -0.7), and the metal-rich bulge clusters NGC 6528 and NGC 6553 ([Fe/H] - 0.1 and -0.2 , respectively).

These reference clusters are analysed with the intent of testing and improving the method, and verifying the metallicity range of applicability of each library of template spectra. In all cases, member stars and surrounding field stars are analysed. For some of these clusters previous high-resolution spectroscopic and photometric data of a few member stars are available.

The minimum distance method was adopted by Cayrel et al. (1991), by measuring residuals in each of the stellar parameters effective temperature, gravity, and metallicity; the method required the input of reference parameters. In the present work, we adopt the code ETOILE (Katz et al., 2011) that uses the minimum distance method, where the reliability and coverage of $\mathrm{T}_{\text {eff }}, \log (g),[\mathrm{Fe} / \mathrm{H}],[\alpha / \mathrm{Fe}]$ of the template stars are important to find well-founded parameters for the target stars. We adopted two different libraries of spectra, the MILES1 library of low-resolution spectra $(\mathrm{R} \sim 2,000)$ and the grid of synthetic spectra by Coelho et al. (2005)2.

In Sect. 2 the observations are described. In Sect. 3 the method of stellar parameter derivation is detailed. In Sect. 4 the method is applied to six cluster as a validation of the procedures. In Sect. 5 the results are discussed, and in Sect. 6 a summary is given.

\footnotetext{
${ }^{1}$ http://miles.iac.es/

${ }^{2}$ http://www.mpa-garching.mpg.de/PUBLICATIONS/DATA/SYNTHSTELLIB/synthetic_stellar_spectra.html
} 


\subsection{Observations and data reduction}

We observed respectively 17, 17, 12, 17, 10 and 13 red giant stars of the globular clusters NGC 6528, NGC 6553, M 71, NGC 6558, NGC 6426, Terzan 8, and surrounding fields, using FORS2@VLT/ESO, under projects 077.D-0775(A) and 089.D-0493(B). Table 3.1 summarizes the setup of the observations. Pre-images were taken using filters JohnsonCousins $\mathrm{V}$ and $\mathrm{I}$ in order to select only stars in the red giant branch (RGB) brighter than the Red Clump (RC) level. Zero points in colours and magnitudes were fitted to match isochrones with parameters from Table 3.2 (see Colour-Magnitude Diagrams, CMDs, in Figure 3.11). We selected stars covering the whole interval in colour of the RGB, and when possible trying to avoid Asymptotic Giant Branch (AGB) stars. These stars are spatially distributed as shown in Figure 3.2, partly due to the slitlet configuration. Cluster parameters and log of observations are given in Table 3.2. The list of individual stars, their coordinates and VI magnitudes from the present FORS2 observations are given in Table B.1.

Table 3.1 - Telescope and spectrograph

\begin{tabular}{l|c}
\hline \multicolumn{2}{c}{ Observing information } \\
\hline \hline Telescope & Antu/UT1-VLT@ESO \\
Instrument & FORS2 \\
Grism & $1400 \mathrm{~V}$ \\
FoV & $6.8^{\prime} \times 6.8^{\prime}$ \\
Pixel scale & $0.25^{\prime \prime} / \mathrm{pixel}$ \\
Slit width & $0.53 \mathrm{~mm}$ \\
Spec. resolution & 2,000 \\
\hline
\end{tabular}


Table 3.2 - Log of observations and clusters parameters from literature. Main reference is Harris (1996, 2010 edition), when not indicated explicitly.

\begin{tabular}{|c|c|c|c|c|c|c|}
\hline Parameter & NGC 6528 & NGC 6553 & M 71 & NGC 6558 & NGC 6426 & Terzan 8 \\
\hline Date of obs. & 29.05 .2006 & 29.05 .2006 & 29.05 .2006 & 29.05 .2006 & 13.07.2012 & 12.07.2012 \\
\hline $\mathrm{UT}$ & $08: 36: 22$ & $08: 57: 50$ & $09: 14: 32$ & $06: 55: 32$ & $02: 31: 12$ & $07: 47: 29.346$ \\
\hline$\tau$ & $149.4 \mathrm{~s}$ & $79.4 \mathrm{~s}$ & $17.2 \mathrm{~s}$ & $148.3 \mathrm{~s}$ & $500.0 \mathrm{~s}$ & $360 \mathrm{~s}$ \\
\hline RA & $18^{h} 04^{\prime} 49.64^{\prime \prime}$ & $18^{h} 09^{\prime} 17.60^{\prime \prime}$ & $19^{h} 53^{\prime} 46.49^{\prime \prime}$ & $18^{h} 10^{\prime} 17.60^{\prime \prime}$ & $17^{h} 44^{\prime} 54.65^{\prime \prime}$ & $19^{h} 41^{\prime} 44.41^{\prime \prime}$ \\
\hline $\mathrm{DEC}$ & $-30^{\circ} 03^{\prime} 22.6^{\prime \prime}$ & $-25^{\circ} 54^{\prime} 31.3 \prime \prime$ & $+18^{\circ} 46^{\prime} 45.1^{\prime \prime}$ & $-31^{\circ} 45^{\prime} 50.0^{\prime \prime}$ & $+03^{\circ} 10^{\prime} 12.5^{\prime \prime}$ & $-33^{\circ} 59^{\prime} 58.1^{\prime \prime}$ \\
\hline age & $13 \mathrm{Gyr}^{(1)}$ & $13 \mathrm{Gyr}^{(1)}$ & $11.00 \pm 0.38 \mathrm{Gyr}^{(2)}$ & $14 \mathrm{Gyr}^{(3)}$ & $13.0 \pm 1.5 \mathrm{Gyr}^{(4)}$ & $13.00 \pm 0.38 \mathrm{Gyr}^{(2)}$ \\
\hline$[\mathrm{Fe} / \mathrm{H}]$ & -0.11 dex & -0.18 dex & -0.78 dex & $-0.97 \pm 0.15 \mathrm{dex}^{(3)}$ & -2.15 dex & -2.16 dex \\
\hline$[\mathrm{Mg} / \mathrm{Fe}]^{a}$ or $[\alpha / \mathrm{Fe}]^{b}$ & $0.24^{(b, 5)}$ & $0.26 \mathrm{dex}^{(b, 6)}$ & $0.19 \pm 0.04^{(a, 7)}, 0.40^{(b, 5)}$ & $0.24^{(a, 3)}$ & $0.4^{(b, 4)}$ & $0.47 \pm 0.09(a, 8)$ \\
\hline $\mathrm{E}(\mathrm{B}-\mathrm{V})$ & 0.54 & 0.63 & 0.25 & 0.44 & 0.36 & 0.12 \\
\hline$(\mathrm{m}-\mathrm{M})_{\mathrm{V}}$ & 16.17 & 15.83 & 13.80 & 15.70 & 17.68 & 17.47 \\
\hline $\mathrm{R}_{\text {Sun }}$ & $7.9 \mathrm{kpc}$ & $6.0 \mathrm{kpc}$ & $4.0 \mathrm{kpc}$ & $7.4 \mathrm{kpc}$ & $20.6 \mathrm{kpc}$ & $26.3 \mathrm{kpc}$ \\
\hline $\mathrm{R}_{\mathrm{GC}}$ & $0.6 \mathrm{kpc}$ & $2.2 \mathrm{kpc}$ & $6.7 \mathrm{kpc}$ & $1.0 \mathrm{kpc}$ & $14.4 \mathrm{kpc}$ & $19.4 \mathrm{kpc}$ \\
\hline$<$ v $_{\text {helio }}>$ & $206.6 \pm 1.4 \mathrm{~km} / \mathrm{s}$ & $-3.2 \pm 1.5 \mathrm{~km} / \mathrm{s}$ & $-22.8 \pm 0.2 \mathrm{~km} / \mathrm{s}$ & $-197.3 \pm 4 \mathrm{~km} / \mathrm{s}^{(3)}$ & $-162.0 \mathrm{~km} / \mathrm{s}$ & $130.0 \mathrm{~km} / \mathrm{s}$ \\
\hline $\mathrm{r}_{\text {tidal }}$ & $4.11^{\prime}$ & $7.66^{\prime}$ & $8.90^{\prime}$ & $9.49^{\prime}$ & 13.03 & 3.98 \\
\hline $\mathrm{r}_{\text {half-light }}$ & 0.38 & $1.03^{\prime}$ & $1.67^{\prime}$ & 2.15 & 0.92 & 0.95 \\
\hline
\end{tabular}

1 Zoccali et al. (2001) ${ }^{2}$ VandenBerg et al. (2013) ${ }^{3}$ Barbuy et al. (2007) ${ }^{4}$ Dotter et al. (2011) ${ }^{5}$ Carretta et al. (2010) 9 Cohen et al. (1999) ${ }^{7}$ Meléndez and Cohen (2009) \& Carretta et al. (2014) 

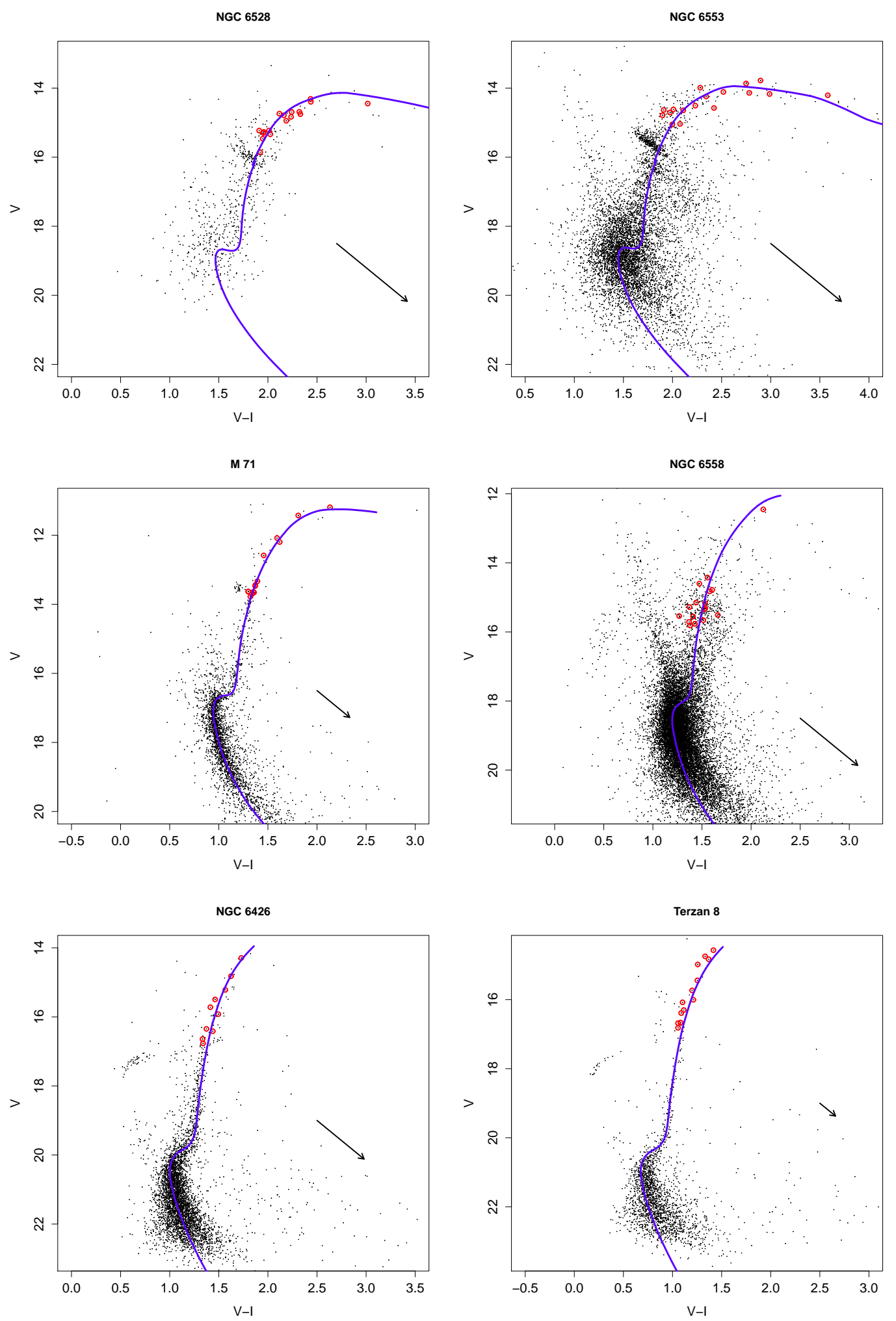

Figure 3.1: Colour-Magnitude diagrams of all clusters analysed in the present work. Left panels are metalrich clusters, middle panels are intermediate metallicity clusters and right panels correspond to the more metal-poor ones. All stars within $2 \times \mathrm{r}_{\text {half-light }}$ are plotted, without any cleaning procedure. Dartmouth isochrones with literature parameters (Table 3.2) are overplotted. Selected RGB stars for spectroscopic observations are in red. Reddening vectors are shown in each CMD based on E(B-V) listed in Table 3.2 

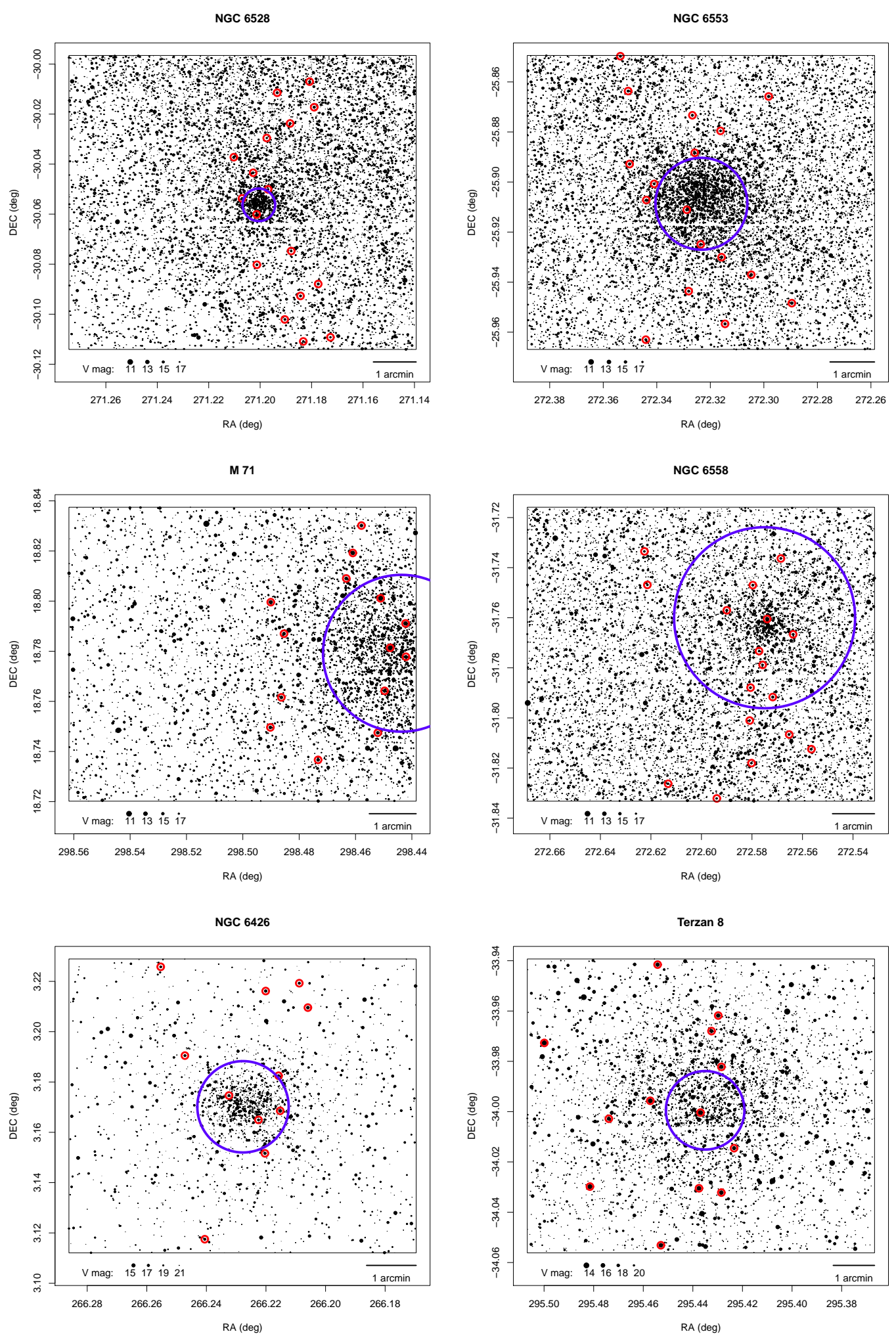

Figure 3.2: Sky map of all clusters analysed in the present work. Panels are displayed as in Figure 3.1 Only brightest stars are shown, and the size of the dots are scaled with the stars magnitudes as indicated in each plot. Selected RGB stars for spectroscopic observations are in red. The blue circle corresponds to the half-light radius of each cluster from Table 3.2 
The spectra were taken using the grism $1400 \mathrm{~V}$, centred at $5200 \AA$, covering the range 4560 - $5860 \AA$, with a resolution of $\mathrm{R} \sim 2000$. Figure 3.3 illustrates the spectra of a metalpoor and a metal-rich red giant star, where many of the strongest lines are indicated.
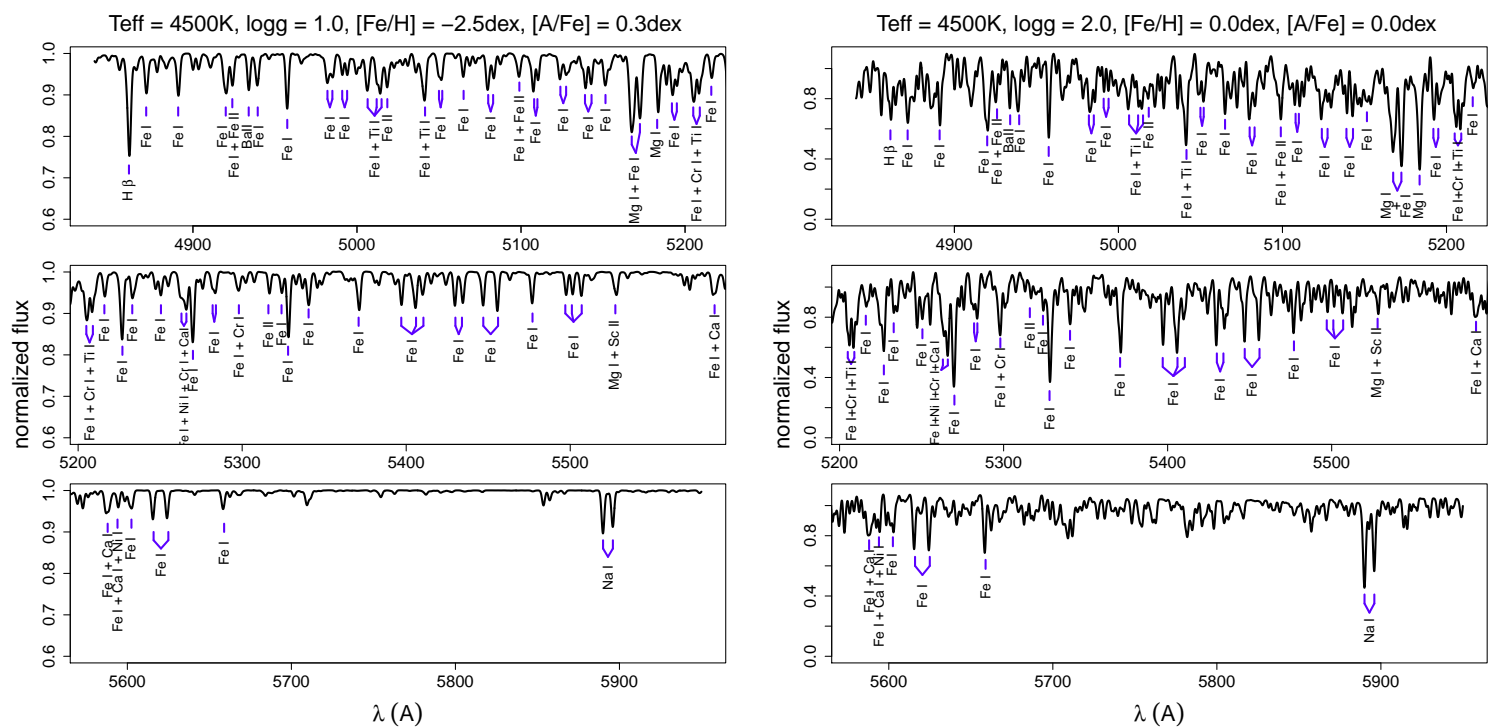

Figure 3.3: Identificantion of most important atomic lines in each strong feature of spectra with FORS2 resolution $(\mathrm{R} \sim 2000)$. The most important molecular band in this region is $\mathrm{MgH}$ around $\lambda=5165 \AA$, on top of the Mg I triplet lines. Left panel shows a metal-poor red giant star from Coelho et al. (2005) library, and right panel shows a metal-rich one. The left panels were zoomed in on y-axis direction for better visualization.

The spectra were reduced using esorex/FORS2 pipeline 3 with default parameters for bias and flatfield correction, spectra extraction, and wavelength calibration. The only modification relative to default parameters, has been the introduction of a list of skylines, since the default list had only one line. The wavelength calibration proved to be satisfactory with such line list (see Appendix $\mathrm{C}$ for further details). A last step in the reduction procedure was a manual removal of cosmic rays.

\subsection{Stellar parameters determination}

\subsubsection{Radial velocities}

Radial velocities were measured using the ETOILE code through cross correlation with a template spectrum from the chosen library. Tests were done in order to check the results, by measuring radial velocities using fxcor@IRAF (cross correlation), and rvidlines@IRAF

\footnotetext{
${ }^{3}$ http://www.eso.org/sci/software/pipelines/
} 


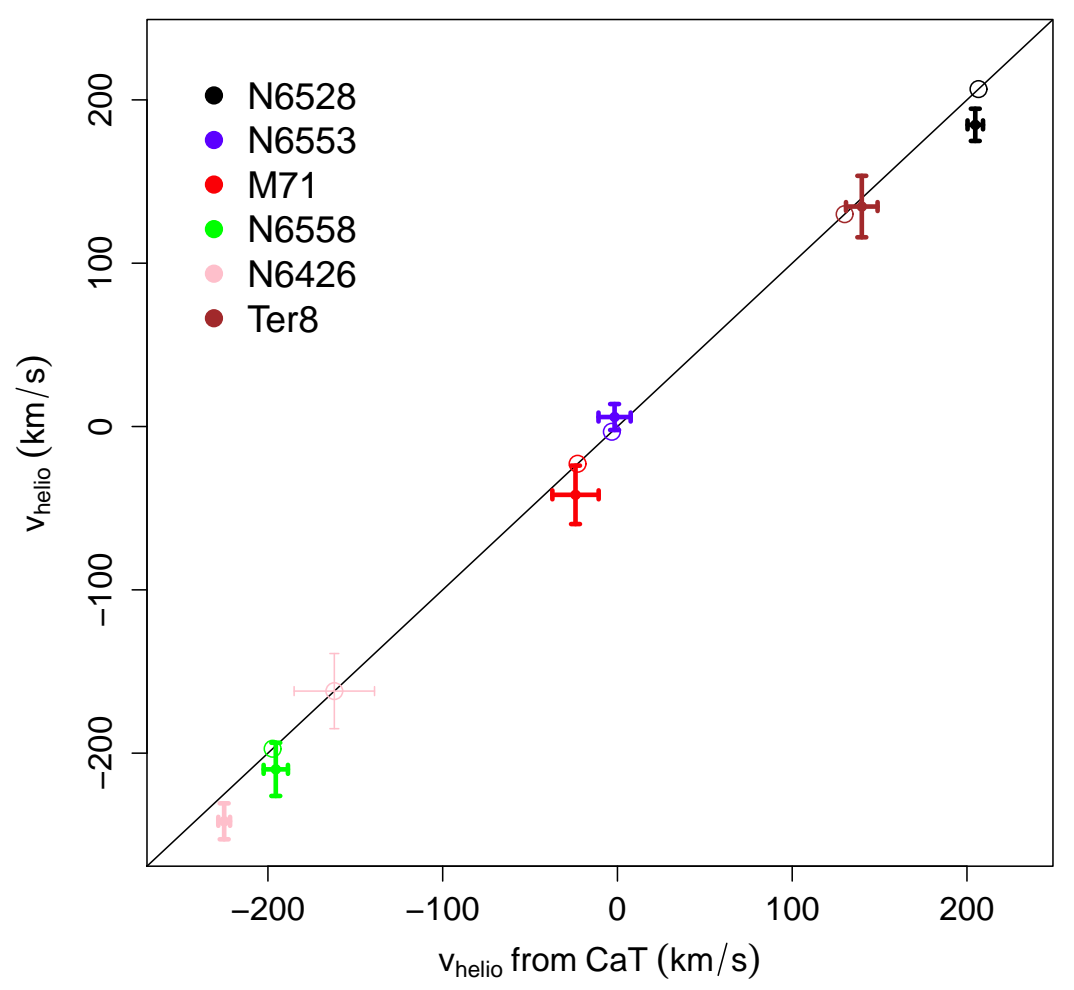

Figure 3.4: Average of heliocentric radial velocities of member stars (see details in Section 3.5.3 and values in Table B.1) for each globular cluster. Our results are plotted against those from CaT spectroscopy (Saviane et al., 2012) showing good agreement. Error bars are the standard deviation of the average. One-to-one line is plotted for visual guidance, where radial velocities from Harris (1996, 2010 edition) are overplotted as empty circles.

(using wavelength of MgI triplet lines as a reference). The derived velocities are consistent, therefore we used ETOILE also to determine radial velocities. Heliocentric radial velocities for each star can be found in Table B.1, where the last column refers to the values measured from the CaII triplet (CaT) lines in the near infrared by Saviane et al. (2012) for member stars for NGC 6528, NGC 6553, M 71 and NGC 6558, and by Vasquez et al. (2014 in prep.) for NGC 6426 and Terzan 8. There is good agreement between the present radial velocity values and those from the $\mathrm{CaT}$ line region. A few exceptions are cool stars presenting strong TiO molecular bands, e.g. \#8 in NGC 6558. Radial velocities of stars \#2, \#10 of M 71 are also discrepant between CaT and our determinations. Average values for member stars in each cluster are presented in Figure 3.4, where our results are compared to CaT results (Saviane et al., 2012 and Vasquez et al. (priv. communication)), and with Harris (1996, 2010 edition) catalogue. Error bars from the literature are smaller than the empty 


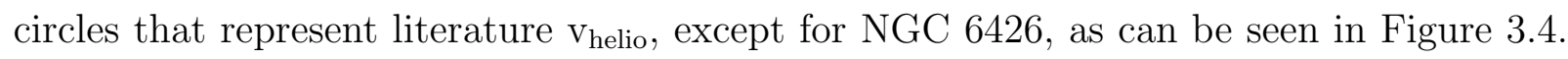
Our results are in good agreement with both references. In particular, the radial velocity measured for NGC 6426 is in agreement between this work and CaT results based on individual member stars, but it is only compatible with the literature value within $3 \sigma$. The explanation is that the only work that measured radial velocities for this cluster was based on integrated light from photographic plates (Hesser et al., 1986). Therefore, the present radial velocity derivation for NGC 6426 is more reliable.

\subsubsection{Atmospheric parameters}

Full spectrum fitting with minimum distance method is employed, using the ETOILE code described in Katz et al. (2011) and Katz (2001). We apply the calculations to the wavelength region 4600-5600 $\AA$, similarly to the procedure described in Katz et al. (2011).

Automated derivation of the atmospheric parameters $\left(\mathrm{T}_{e f f}, \log (g),[\mathrm{Fe} / \mathrm{H}],[\alpha / \mathrm{Fe}]\right)$ of a stellar spectrum is carried out by comparing the target spectrum with each library spectrum, thus covering a large range of atmospheric parameters. In each comparison, ETOILE fits a polynomial to both spectra, which compensates for continuum differences between the two spectra. The distance between the two spectra is calculated through weighted least-squares. The estimation of the parameters is done by taking an average of the most similar librarv spectra. This is called the similarity method introduced by Katz et al. (1998). For a more detailed explanation see Katz (2001).

Before running the code, two important steps are needed: to convolve all the library spectra to the same resolution of the target spectra, and to correct for radial velocities $v_{r}$. Convolution calculations were performed for the library spectra using the task GAUSS in IRAF. The code ETOILE measures the radial velocities by comparison with template spectra from the library, a reliable way to measure $v_{r}$ in each observed spectrum and correct them.

Figure 3.5 shows two examples of spectral fitting, one for a metal-poor (Terzan8_11) and another for a metal-rich (NGC6528_11) star. The template stars that best fit these cluster stars among the available spectra from MILES library are BD+060648 and HD161074, respectively. The residuals shown at the bottom of these figures indicate that the metalpoor target spectrum is similar to the template spectrum within $2 \%$, except for a few strong features. The residuals for the metal-rich star shows a similarity between target 

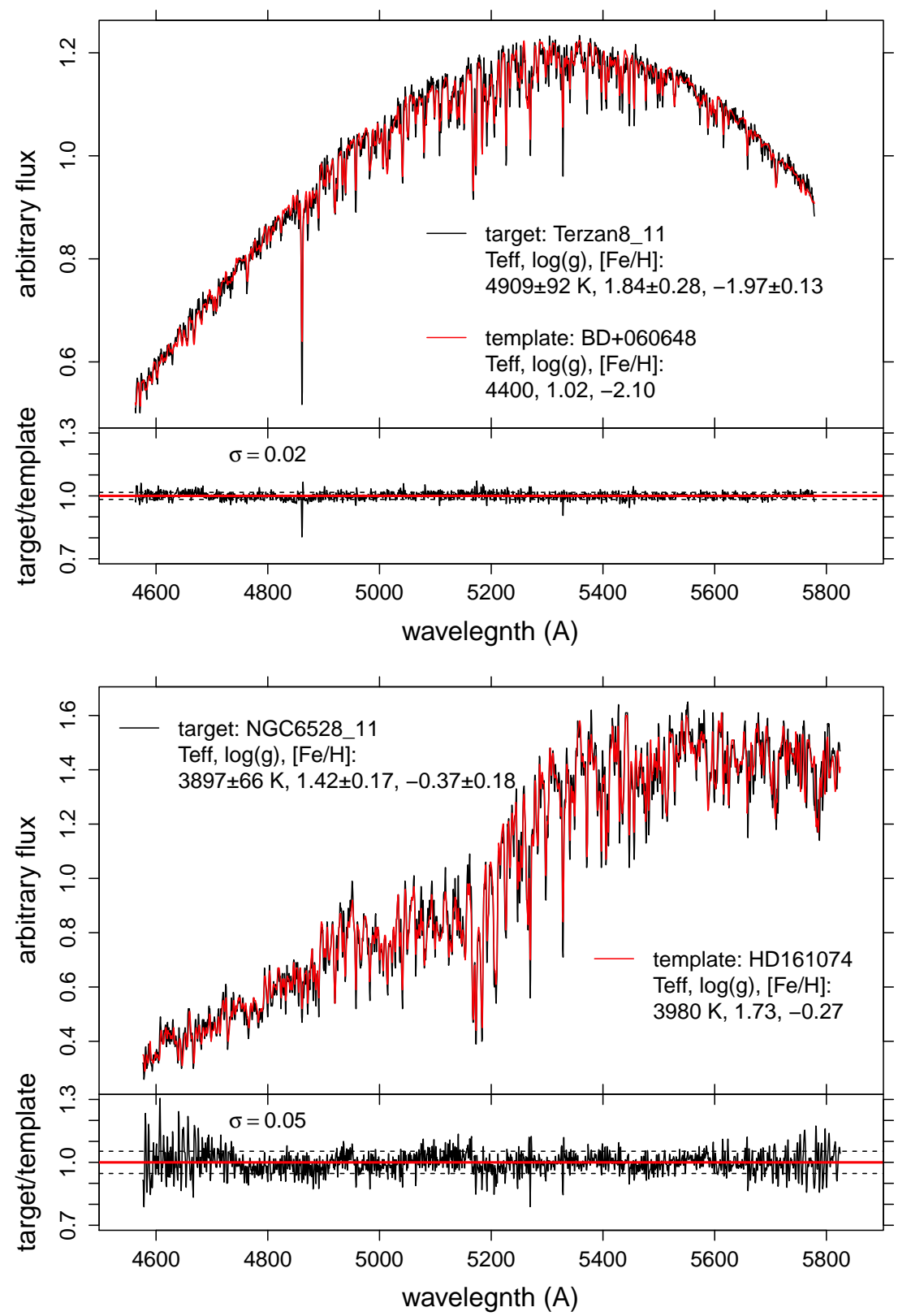

Figure 3.5: Examples of spectral fitting carried out with ETOILE for a metal-poor (Terzan8_11) and a metal-rich (NGC6528_11) star displayed in the upper and bottom panels, respectively. For each star, its spectrum (black line) is overplotted by the MILES spectrum (red line) that best fits it, BD+060648 and HD161074, respectively. Below each stellar spectrum the residuals of each fit are presented. The match between the spectra is done following the procedures explained in Section 3.4.2. The fit appears very satisfactory for the whole wavelength interval.

and template spectra of 5\%, except for the boundaries $\lambda \gtrsim 5700 \AA$ and $\lesssim 4700 \AA$, and for a few strong features. These differences between the spectra are reflected in the atmospheric parameters, and they are compensated by taking the average of parameters of the most similar spectra. For Terzan8_11, there are 8 MILES spectra close enough which were averaged, and for NGC6528_11, 21 MILES spectra were considered. Details on the criterion 
to select the number of template spectra are discussed in Section 3.4 .2 .2 ,

\subsubsection{Stellar libraries}

The core of the atmospheric parameters derivation in this work is the choice of a stellar library. There are two classes of stellar libraries: based on observed or synthetic spectra. The real spectra are more reliable, but the drawback is that they have abundances typical of nearby stellar populations. The synthetic libraries have no noise, and a large and uniform coverage of the atmospheric parameters space, however there are still limitations on the completeness of atomic and molecular line lists, plus uncertainties on oscillator strengths, and assumptions on atmospheric models, such as 1-D and local thermodynamical equilibrium. For these reasons, it is useful to use both observational and synthetic libraries. In the present work, we use two libraries, one observed and one synthetic, as described below:

The MILES library (Sánchez-Blázquez et al., 2006) has 985 stellar spectra with resolution $\mathrm{R} \sim 2,200$, and mean signal-to-noise ratio of 150 per pixel for field and open cluster stars, and 50 for globular cluster stars. Atmospheric parameters coverage is (Cenarro et al., 2007; Milone et al., 2011):

$$
\begin{aligned}
352.5 \mathrm{~nm} & <\lambda<750 \mathrm{~nm} \\
2,747 \mathrm{~K} & <T_{\text {eff }}<36,000 \mathrm{~K} \\
-0.20 & <\log (g)<5.50 \\
-2.86 & <[\mathrm{Fe} / \mathrm{H}]<+1.65
\end{aligned}
$$

$-0.54<[\mathrm{Mg} / \mathrm{Fe}]<+0.74$
The COELHO library (Coelho et al., 2005$)$ has 6367 synthetic stellar spectra 4 with wavelength steps of $0.02 \AA$ (resolution $\mathrm{R}=525,000$ ). Atmospheric parameters coverage is:

\footnotetext{
${ }^{4}$ Interpolation on the original library was carried out to produce spectra with $[\alpha / \mathrm{Fe}]=0.1,0.2,0.3$ dex from the provided 0.0 and 0.4 dex spectra.
} 


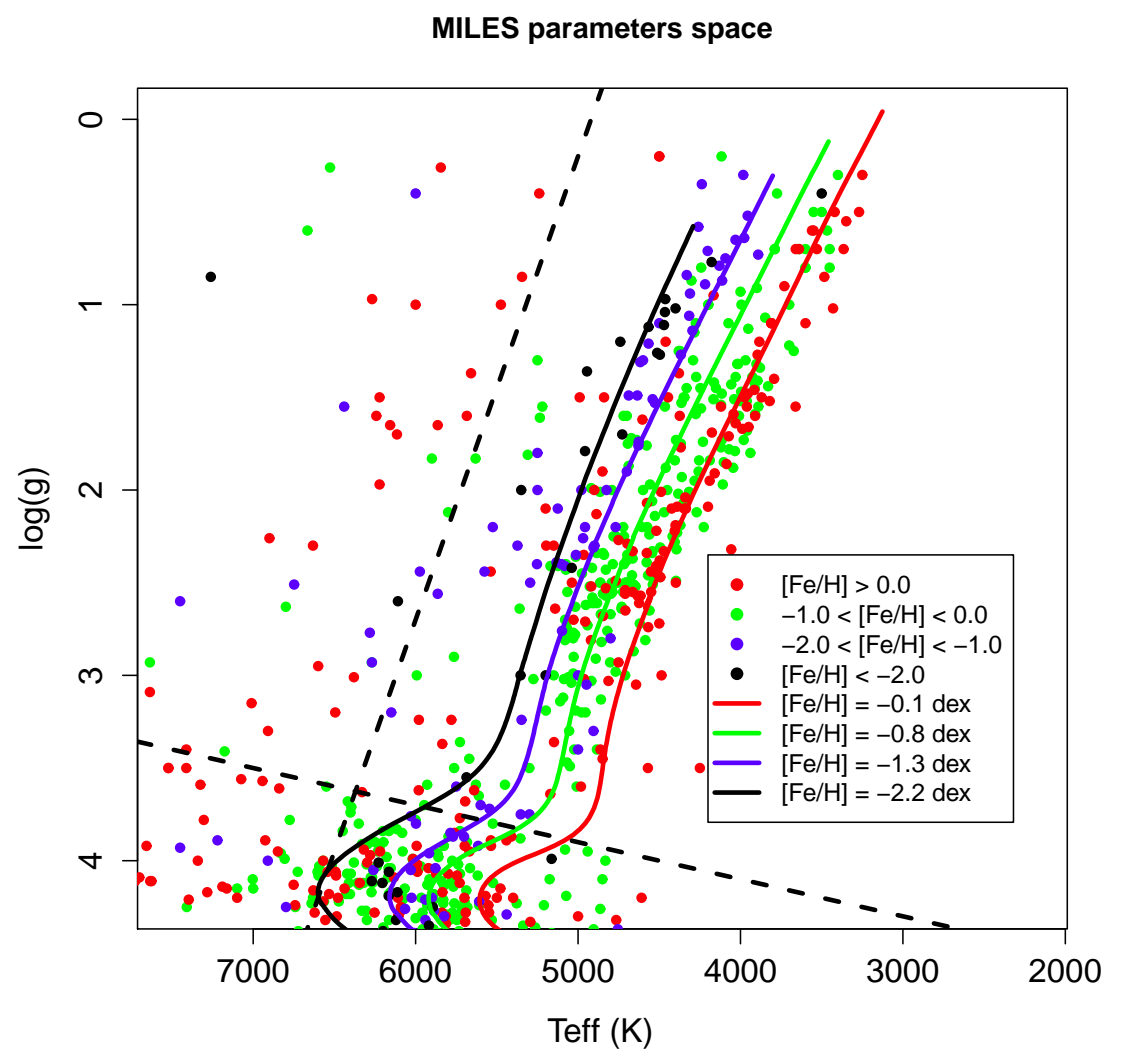

Figure 3.6: HR diagram showing the parameter space available from the MILES library. Dartmouth isochrones (Dotter et al., 2008) are overplotted for metallicities close to those of the six analysed globular clusters: $[\mathrm{Fe} / \mathrm{H}]=-0.1,-0.8,-1.3,-2.2$ dex, with $[\alpha / \mathrm{Fe}]=0.2,0.4,0.4,0.4,0.4 \mathrm{dex}$, respectively, and ages $=13$ Gyr for all cases. Colours of the dots indicate the metallicity range closer to the isochrones. Dashed black lines are the adopted limit to select RGB stars from MILES used as reference for the fits.

$$
\begin{aligned}
300 \mathrm{~nm} & <\lambda<1800 \mathrm{~nm} \\
3,500 \mathrm{~K} & <T_{\text {eff }}<7,000 \mathrm{~K} \\
0.0 & <\log (g)<5.0 \\
-2.5 & <[\mathrm{Fe} / \mathrm{H}]<+0.5 \\
0.0 & <[\alpha / \mathrm{Fe}]<+0.4
\end{aligned}
$$

where $\alpha$-elements considered in this library are: $\mathrm{O}, \mathrm{Mg}, \mathrm{Si}, \mathrm{S}, \mathrm{Ca}$ and $\mathrm{Ti}$.

Once all cluster stars are located in the red giant branch, as shown in Figure 3.1, we selected only stars in this region in the parameters space of the libraries (see Figure 3.6) to avoid non-physical results. 


\subsubsection{Average results and errors: validation with well-known stars}

We define different criteria for MILES and COELHO libraries for taking the average of stellar parameters from reference spectra, as mentioned in Section 3.4.2. For MILES the average results are based on different numbers of templates depending on the sampling as shown in Figure 3.6. For the synthetic library COELHO the sampling is homogeneous, therefore a constant number of templates is adopted. We found that 10 templates for COELHO cover satisfactorily the variations in the four stellar parameters $\left(\mathrm{T}_{\mathrm{eff}}, \log (g)\right.$, $[\mathrm{Fe} / \mathrm{H}]$ and $[\alpha / \mathrm{Fe}])$. The COELHO library was built by varying all alpha-elements $(\mathrm{O}, \mathrm{Mg}$, $\mathrm{Si}, \mathrm{S}, \mathrm{Ca}, \mathrm{Ti})$ together, therefore $[\alpha / \mathrm{Fe}]$ is an average of the effect from enhancement of these element abundances. In this spectral region $\mathrm{Mg}$ lines are the dominant source of line strengthening.

The criterion to define average results from the MILES library is more complex, as follows. The code provides a list of the closest reference spectra from the library, ranked by the similarity parameter ( $S$, see Katz et al., 2011). The final parameters $\mathrm{T}_{\text {eff }}, \log (g)$, $[\mathrm{Fe} / \mathrm{H}]$ and $[\mathrm{Mg} / \mathrm{Fe}]$ are the average of the parameters of first $\mathrm{N}$ reference stars from the ETOILE output, where $\mathrm{N}$ depends on the sampling of the library for each combination of parameters. The average is weighted by $1 / S^{2}$ as shown in the equation below for $\mathrm{T}_{\text {eff }}$ (the same is valid for the other three parameters):

$$
\mathrm{T}_{\text {eff }}(N)=\frac{\sum_{i=1}^{N} \mathrm{~T}_{\text {eff }, \mathrm{i}} \times \frac{1}{S_{i}^{2}}}{\sum_{i=1}^{N} \frac{1}{S_{i}^{2}}}
$$

The errors are defined as the average of the squared residuals, weighted by $1 / S^{2}$, as shown in the equation below for $\mathrm{T}_{\text {eff }}$ (the same is valid for the other three parameters). For $\mathrm{N}=1$, we adopted the same error of $\mathrm{N}=2$.

$$
\sigma_{\mathrm{T}_{\mathrm{eff}}(N)}=\sqrt{\frac{\sum_{i=1}^{N}\left(\mathrm{~T}_{\mathrm{eff}, \mathrm{i}}-\mathrm{T}_{\mathrm{eff}}\right)^{2} \times \frac{1}{S_{i}^{2}}}{\sum_{i=1}^{N} \frac{1}{S_{i}^{2}}}}
$$

To estimate the number of reference stars to be averaged in each case, we proceeded with some tests using 59 spectra of 49 well-known stars, listed in Table B.2. These stars were selected among red giant stars (same $\log (g)$ and $\mathrm{T}_{\text {eff }}$ intervals defined in Figure 
3.6) presented in the ELODIE library 5 (Prugniel et al., 2007). Reference atmospheric parameters were averaged from the PASTEL catalogue (Soubiran et al., 2010), and the quality filter was determined by a threshold in the standard deviation: $\sigma_{T_{\text {eff }}}<200 \mathrm{~K}$, $\sigma_{\log (\mathrm{g})}<0.5, \sigma_{[\mathrm{Fe} / \mathrm{H}]}<0.2$. We calculated the average parameters and respective errors for different $\mathrm{N}$ and compared the results with the average values of $\mathrm{T}_{\text {eff }}, \log (g),[\mathrm{Fe} / \mathrm{H}]$ from the PASTEL catalogue (Soubiran et al., 2010). We minimize the equation below to find the best $\mathrm{N}$ that will give the final parameters and respective errors. This equation considers the distance between the average for a given $\mathrm{N}$ and literature average; in this way all the three parameters are minimized at the same time. Milone et al. (2011) have measured $[\mathrm{Mg} / \mathrm{Fe}]$ for MILES spectra, therefore it is possible to take averages for this parameter as a function of $\mathrm{N}$, and use $[\mathrm{Mg} / \mathrm{Fe}]$ for the best $\mathrm{N}$ as an estimation of the $\alpha$-enrichment for each star.

$$
\operatorname{RR}_{\text {tot }}(N)=\sqrt{\mathrm{RR}_{\mathrm{N}}\left(\mathrm{T}_{\text {eff }}\right)^{2}+\mathrm{RR}_{\mathrm{N}}(\log (g))^{2}+\mathrm{RR}_{\mathrm{N}}([\mathrm{Fe} / \mathrm{H}])^{2}}
$$

where $R_{N}\left(T_{\text {eff }}\right)$ is given by the equation below (the same is valid for the other three parameters):

$$
\mathrm{RR}_{\mathrm{N}}\left(\mathrm{T}_{\text {eff }}\right)=\frac{\mathrm{T}_{\text {eff }}(N)-\mathrm{T}_{\text {eff }}^{(l i t)}(N)}{\mathrm{T}_{\text {eff }}^{(l i t)}(N)}
$$

Figure 3.7illustrates the finding procedure of $\mathrm{N}$ for the case of star HD122956, showing that ETOILE could recover all the four parameters accurately. The resulting parameters, $\mathrm{RR}_{\text {tot }}, \mathrm{N}$, and literature values are presented in Table B.2. Different stars need different number $\mathrm{N}$ of templates to find the best result. Moreover the ratio $S(\mathrm{~N}) / S(1)$ for the best $\mathrm{N}$ is roughly constant for all ETOILE template spectra, with an average value of $1.1 \pm 0.1$. The best number $\mathrm{N}$ and the respective ratio $S(\mathrm{~N}) / S(1)$ are related to the library sampling, for example, for a given star with best $\mathrm{N}=1$ it means that there is only one reference star with $S(\mathrm{~N}) / S(1) \lesssim 1.1$, and there are two possible explanations: either the target star matches perfectly some reference star, or the library has no other reference spectra similar enough to that star to be considered. In the cases with $\mathrm{N}=15$, for instance, the library has 15 reference spectra very similar $(S(\mathrm{~N}) / S(1) \lesssim 1.1)$ to the target spectra, and their parameters must be averaged in order to get the parameters for the target star.

\footnotetext{
${ }^{5}$ http://www.obs.u-bordeaux1.fr/m2a/soubiran/elodie_library.html
} 


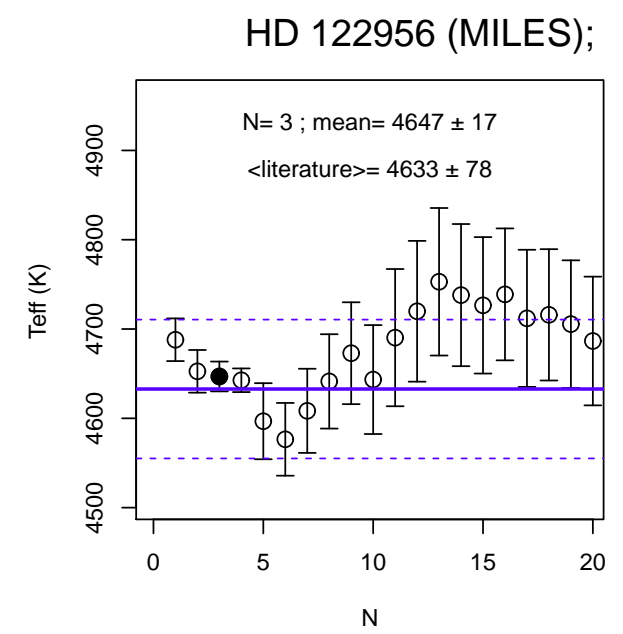

RRtot $=0.02 ; \mathrm{N}=3$
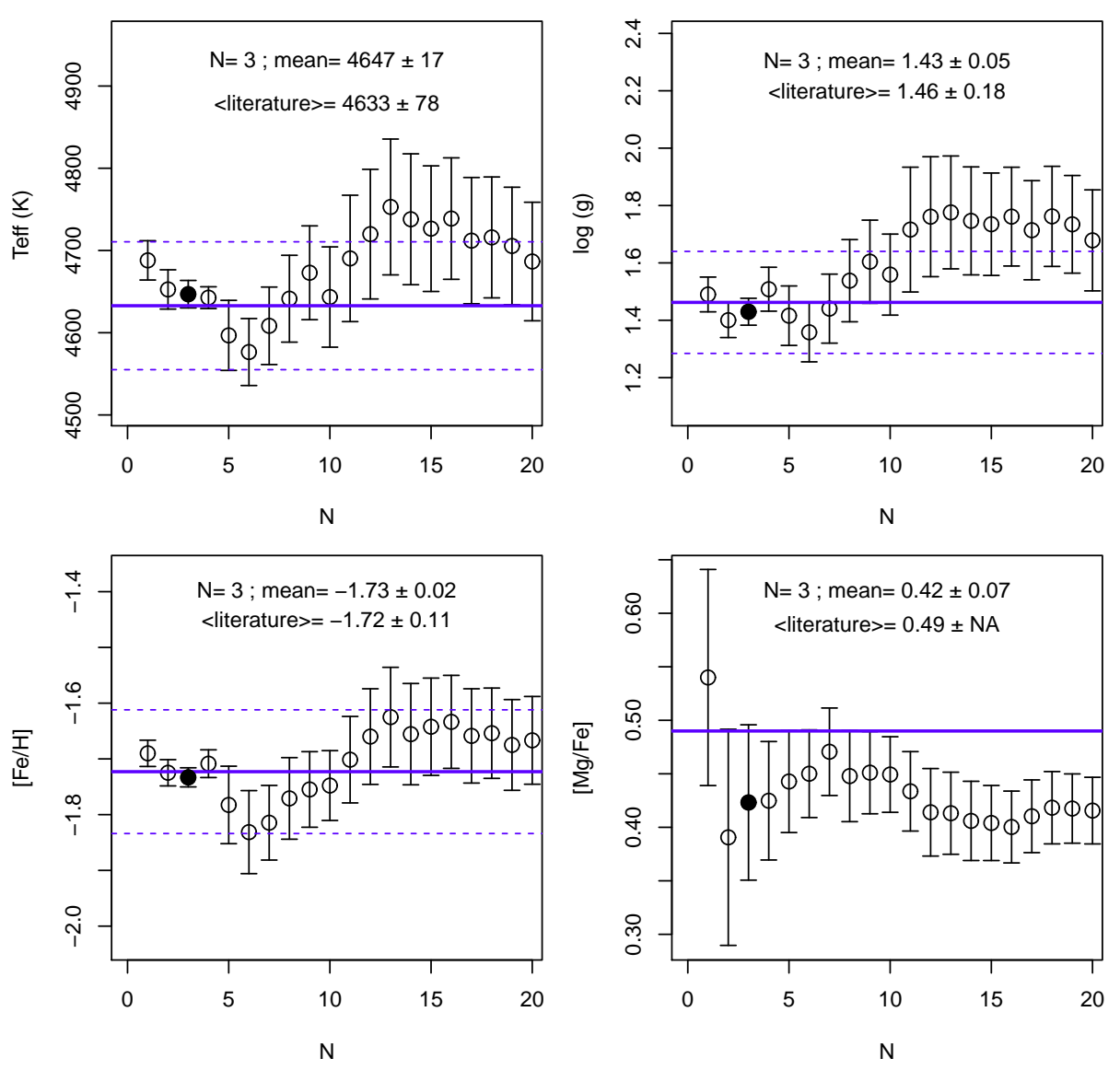

Figure 3.7: Finding procedure of $\mathrm{N}$ for the case of star HD122956, based on the minimization of the residuals of $\mathrm{T}_{\text {eff }}, \log (g)$ and $[\mathrm{Fe} / \mathrm{H}]$ (equation 3.3 ). Circles represent the averages of the parameters of $\mathrm{N}$ best reference stars. Filled black circle indicates the closest values to the references. Blue solid lines and blue dashed lines are average from PASTEL catalogue and standard deviation (Table B.2). For this star, Fulbright (2000) published $[\mathrm{Mg} / \mathrm{Fe}]$ and we compare also with the averages as a function of $\mathrm{N}$. All the four parameters for the best $\mathrm{N}=3$ are compatible with literature.

All results are plotted in Figure 3.8 showing the good agreement of ETOILE results and PASTEL catalogue average for $\mathrm{T}_{\text {eff }}, \log (g),[\mathrm{Fe} / \mathrm{H}]$ in the whole range for RGB stars analysed in this work. The behaviour of the derived values of $[\mathrm{Mg} / \mathrm{Fe}]$ vs. $[\mathrm{Fe} / \mathrm{H}]$ has a similar behaviour to field stars (see e.g. Figure 6 of Alves-Brito et al., 2010).

After these tests we can consider that ETOILE code together with the MILES library works well for low-resolution spectra of red giant stars in the optical region. Additionally we define the criterion to consider a reference spectrum similar enough to be considered in the average of the parameters as $S(\mathrm{~N}) / S(1) \leq 1.1$. 

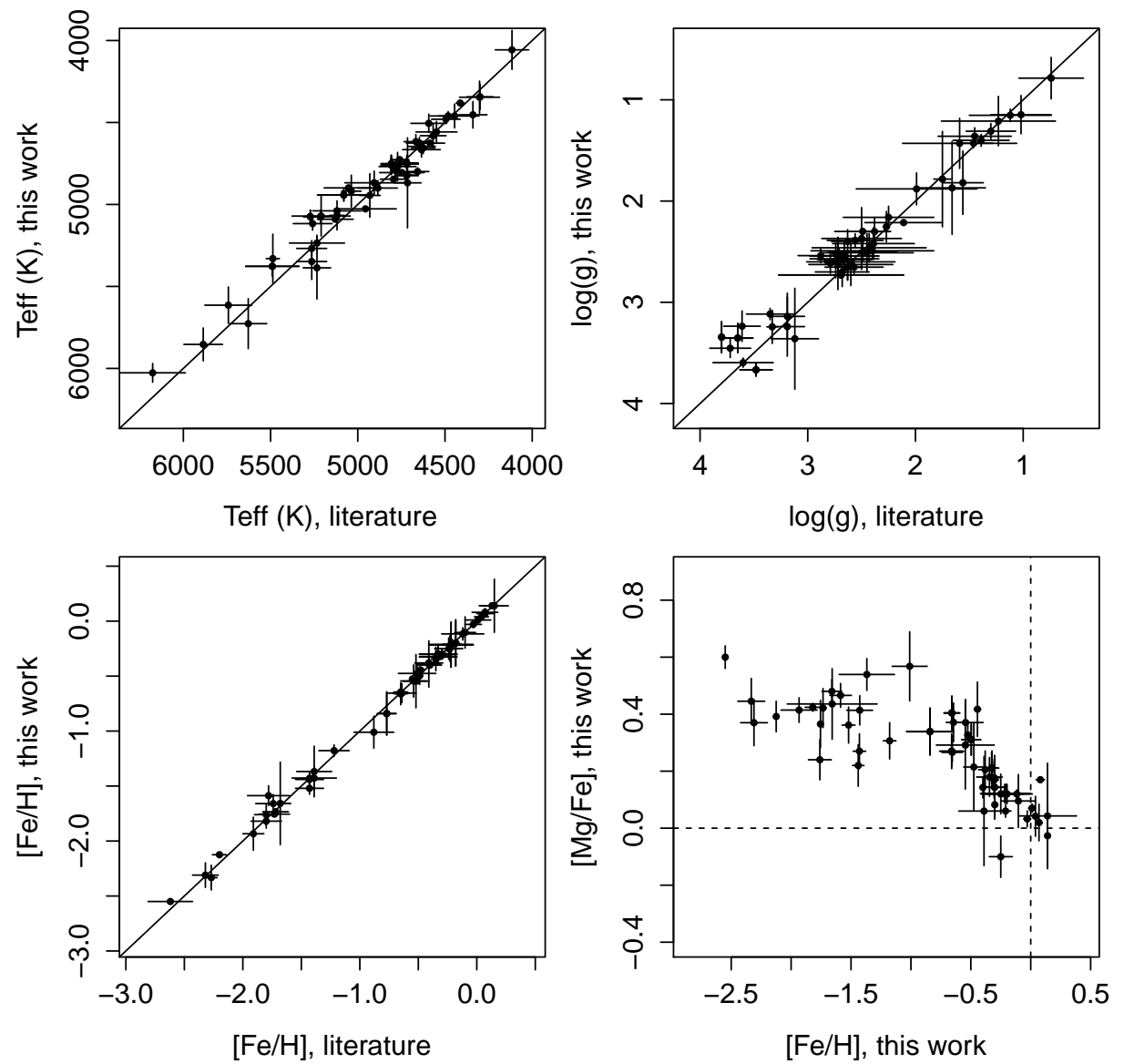

Figure 3.8: Comparison of the parameters determined in this work with PASTEL catalogue average values for the well-known stars presented in Table B.2. These plots endorse the usage of ETOILE code for atmospheric parameters determination for red giant stars in the optical spectral region. Only stars with $\sigma_{T_{\text {eff }}}<200 \mathrm{~K}, \sigma_{\log (\mathrm{g})}<0.5, \sigma_{[\mathrm{Fe} / \mathrm{H}]}<0.2$ from PASTEL catalogue were selected as good quality candidates for validation of the method.

\subsection{Results}

The derived $\mathrm{T}_{\text {eff }}, \log (g),[\mathrm{Fe} / \mathrm{H}],[\mathrm{Mg} / \mathrm{Fe}]$ and $[\alpha / \mathrm{Fe}]$ are presented in Table B.3. In order to discuss these results, we proceed as follows: in Sect. 3.5.1 we plot $\mathrm{T}_{\text {eff }}$ and $\log (g)$ for stars in each cluster together with isochrones of age and metallicity given in Table 3.2 . Section 3.5 .2 compares $[\mathrm{Fe} / \mathrm{H}]$ with CaT results from Saviane et al. (2012) and Vasquez et al. (in prep.). Subsequently all checked parameters are used to select member stars for each cluster (Section 3.5.3). Finally, all parameters for member stars are compared individually with high-resolution analysis, when available in the literature. M 71 has three stars in common with Cohen et al. (2001), NGC 6558 has three stars in common with Barbuy et al. (2007) and Terzan 8 has four stars in common with Carretta et al. (2014), as 
described in Sections 3.5.4.1, 3.5.4.2 and 3.5.4.3, respectively. For NGC 6528, NGC 6553 and NGC 6426 we did not find any star in common with high-resolution spectroscopic studies.

\subsection{1 $T_{\text {eff }}, \log (g)$ against isochrones}

In high-resolution spectroscopy studies, usually $\mathrm{T}_{\text {eff }}$ is estimated from photometry and $\log (g)$ from theoretical equation 6 . These parameters are employed as initial guesses to derive $[\mathrm{Fe} / \mathrm{H}]$, which is applied to redetermine $\mathrm{T}_{\text {eff }}$ and $\log (g)$ iteratively, until reaching a convergence of the three parameters. In this work we fit all the three parameters at the same time (Section 3.4.2), and a check on these parameters is carried out as explained below.

Figure 3.9 displays the results of all stars in the six clusters in a Hertzprung-Russell diagram form. Left, middle and right panels show the results using MILES library, COELHO library and the average of both results, respectively. Black dots represent member stars of each cluster, and grey dots are not members, based on the selection described in the next Section 3.5.3. Dartmouth isochrones (Dotter et al., 2008) with age, $[\mathrm{Fe} / \mathrm{H}]$ and $[\alpha / \mathrm{Fe}]$ from Table 3.2 are overplotted in the diagrams of Figure 3.9 in blue. Cyan lines have the same age and $[\mathrm{Fe} / \mathrm{H}]$ as the respective blue lines, but with the extreme values of $[\alpha / \mathrm{Fe}]=-0.2$ and +0.8 , available from the models.

The results on $\mathrm{T}_{\text {eff }}$ and $\log (g)$ from MILES and COELHO are in good agreement with the isochrones. We also computed an average of the results weighted by their uncertainties that are displayed in the right panels of Figure 3.9. We adopted as the final results in this work the weighted average of MILES and COELHO results.

\subsubsection{Comparison with $[\mathrm{Fe} / \mathrm{H}]$ derived from $\mathrm{CaT}$}

All selected stars from photometrv were observed both in the near-infrared (CaT. Saviane et al., 2012 and Vasquez et al. in prep) and in the optical spectral region. Metallicities from CaT were calibrated by Saviane et al. (2012), to be in an homogeneous scale. The optical region studied here is suitable to provide robust values of $[\mathrm{Fe} / \mathrm{H}]$ for each cluster, to be compared with the CaT value, and to converge ultimately to the average $[\mathrm{Fe} / \mathrm{H}]$ for each cluster.

\footnotetext{
${ }^{6} \log (g)=4.44+4 \log \frac{\mathrm{T}}{\mathrm{T}_{\odot}}+0.4\left(\mathrm{M}_{\mathrm{bol}}-4.75\right)+\log \frac{M}{M_{\odot}}$, see for example Barbuv et al. (2009)
} 

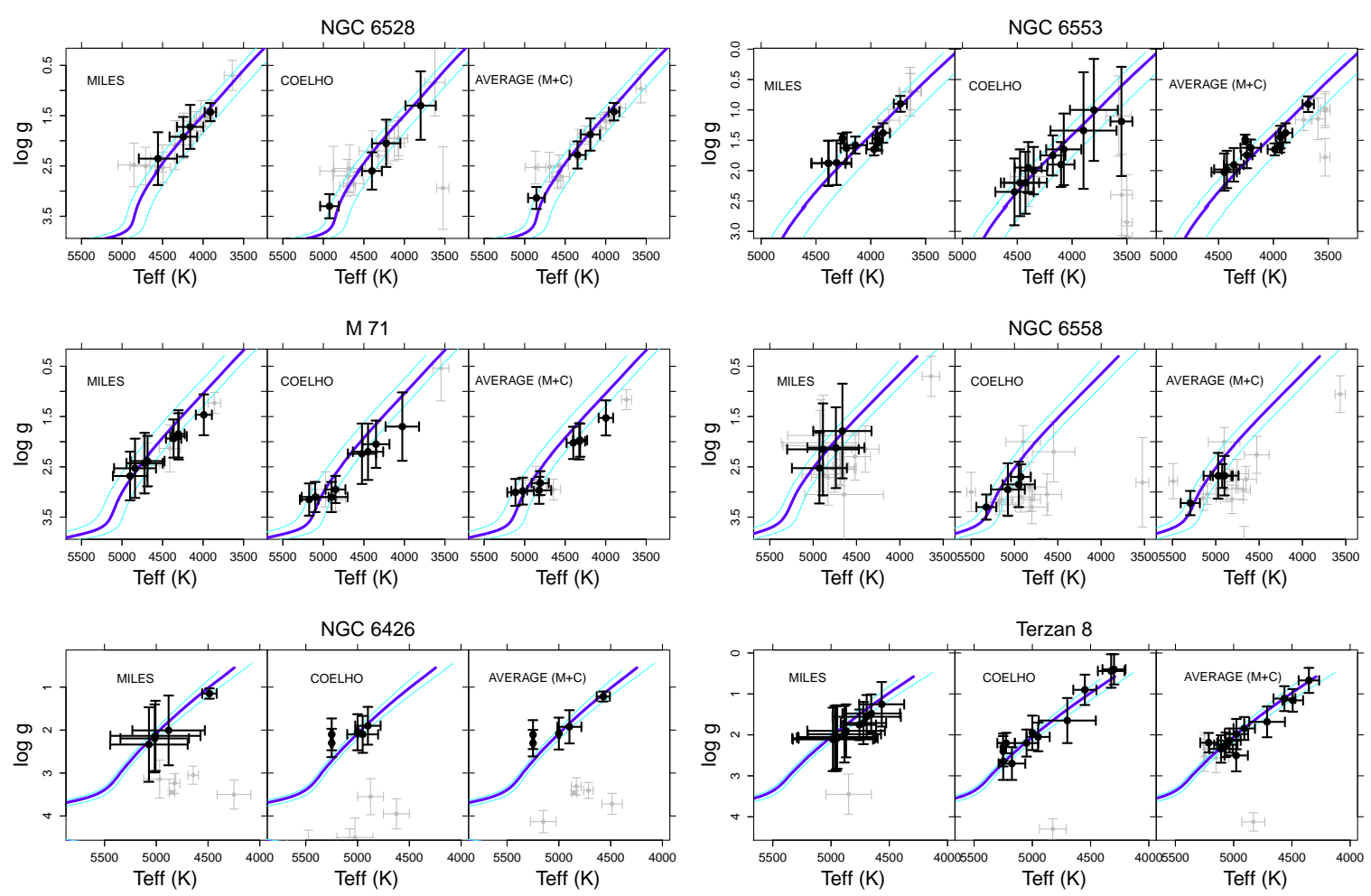

Figure 3.9: Comparison of $\mathrm{T}_{\mathrm{eff}}$ and $\log (q)$ of stars in each cluster with Dartmouth isochrones (Dotter et al., 2008). For each cluster we show the results based on MILES and COELHO libraries and a third panel with the weighted average of the results from both libraries. The parameters of age, $[\mathrm{Fe} / \mathrm{H}]$ and $[\alpha / \mathrm{Fe}]$ for the blue thick isochrones were taken from Table 3.2. Cyan thin isochrones have same age and $[\mathrm{Fe} / \mathrm{H}]$ as blue ones, but with the limits $[\alpha / \mathrm{Fe}]=-0.2$ dex and +0.8 dex. Black dots represent member stars of each cluster, and grey dots are not members.

Figure 3.10 shows the comparisons of the metallicity values presented in Table B.3 with those from CaT analysis. Upper left panel shows that $[\mathrm{Fe} / \mathrm{H}]$ using MILES library is in good agreement with CaT results for the three most metal-rich clusters NGC 6528, NGC 6553 and M 71. This is in agreement with the sampling of metal-rich stars for all combinations of $\mathrm{T}_{\text {eff }}$ and $\log (g)$ as displayed in Figure 3.6 in red and green. For NGC 6558 with $[\mathrm{Fe} / \mathrm{H}] \sim-1.0$ the dispersion on the parameters is larger which is explained by the smaller number of stars available in the library with such metallicity. MILES is based on the solar neighbourhood showing therefore only a few stars with $[\mathrm{Fe} / \mathrm{H}] \sim-1.0$. For the metal-poor clusters NGC 6426 and Terzan 8 the library sampling is even more sparse, as becomes evident in Figure 3.6. In this case, the average of parameters from the library takes into account some more metal-rich reference stars which results in higher values of [Fe/H] for NGC 6426 and Terzan 8 stars.

Metallicities using COELHO library are compared with CaT results in the upper right 

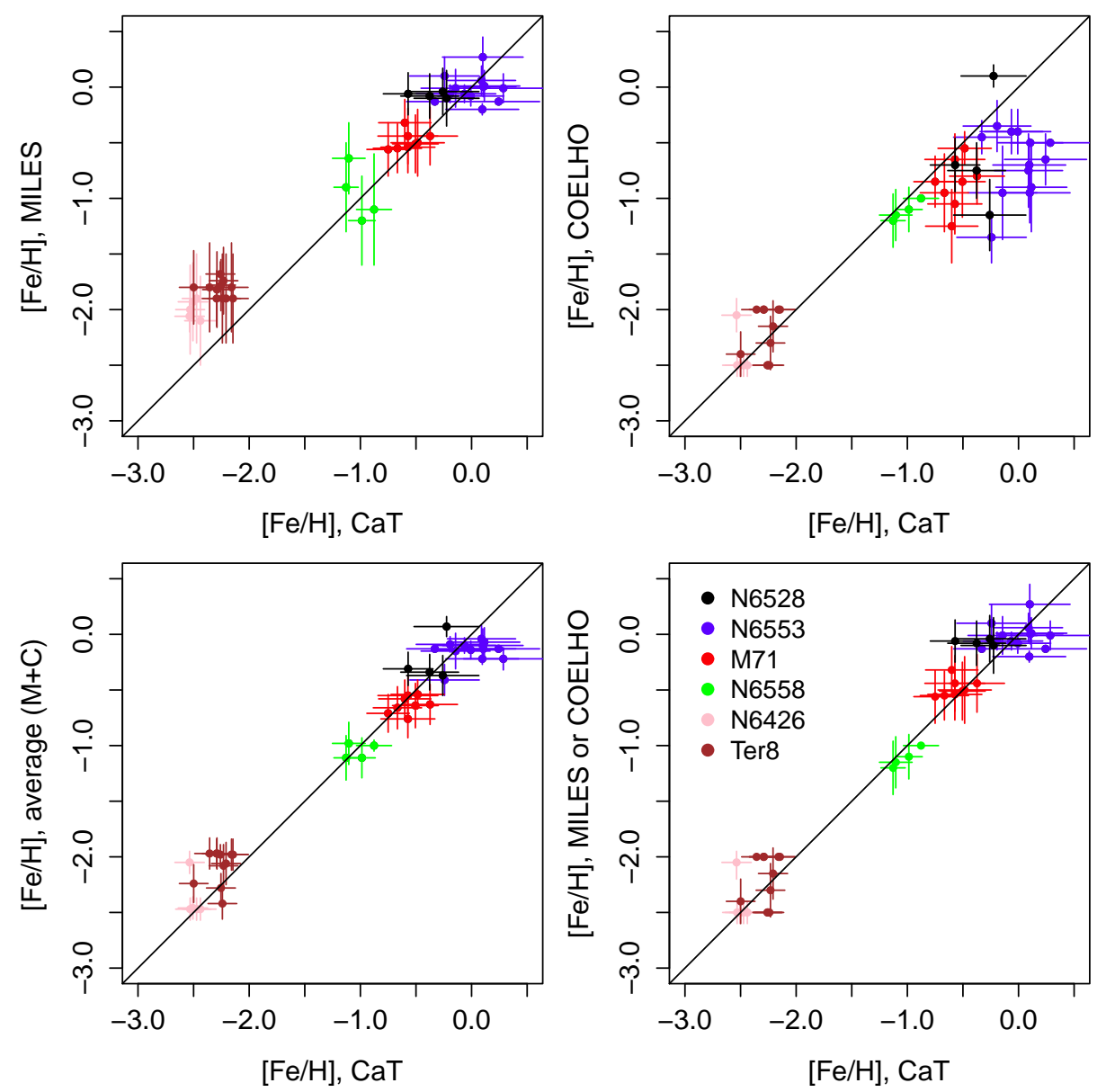

Figure 3.10: Comparison of $[\mathrm{Fe} / \mathrm{H}]$ from this work (Table B.3) with those from equivalent widths of $\mathrm{Ca}$ II triplet for the same stars with the same instrument by Saviane et al. (2012) and Vasquez et al. (in prep). Upper panels compare CaT metallicities with those obtained with MILES and COELHO libraries. Bottom panels are two types of combination of the results: average of each star on the left, and assuming MILES results for more metal-rich and COELHO results for more metal-poor stars, on the right.

panel of Figure 3.10. The synthetic spectra reproduce less well the metal-rich stars, given the missing opacity due to millions of very weak lines, not taken into account in the calculations; this blanketing effect lowers the continuum in real stars, and the measurable lines are shallower than in the present synthetic spectra calculations by Coelho et al. Because of this effect, stars of NGC 6528, NGC 6553 and M 71 are more metal-poor than CaT results. On the other hand, COELHO library is suitable to reproduce the stars of the three more metal-poor clusters of this sample, NGC 6558, NGC 6426 and Terzan 8.

To summarize, for the three more metal-rich clusters, MILES results are better, and for the other three, COELHO results are preferable. The bottom right panel of Figure 3.10 shows the concatenation of this conclusion, i.e., it displays MILES results for NGC 6528, 
NGC 6553 and M 71, and COELHO results for NGC 6558, NGC 6426 and Terzan 8. An alternative combination of results from MILES and COELHO is to take the average of the results weighted by their uncertainties. This average combines the best of both libraries and gives a good correlation with CaT results, as shown in the bottom left panel of Figure 3.10. Both criteria to combine MILES and COELHO (two bottom panels) are in good agreement with $\mathrm{CaT}$ results, and we adopted $[\mathrm{Fe} / \mathrm{H}]$ from the average results represented in the bottom left panel.

\subsubsection{Membership selection}

In Figure 3.11 we show four panels for each globular cluster. In upper left panels radial velocities against metallicities are displayed. Blue solid lines are parameters from Table 3.2 and red dashed lines are the average of parameters for cluster members only. For all clusters there is a clear concentration of stars around literature values, and these stars were selected as cluster members. Upper right panels show Teff vs. $\log (g)$ compared with

Dartmouth isochrones (Dotter et al., 2008) as done in Sect. 3.5.1. Blue solid lines are isochrones with parameters from Table 3.2, and red dashed lines are based on the average (Table 3.5) of the parameters for member stars (Table B.3). All member stars are close to the isochrones while non-member stars are out of these lines, confirming the membership selection. Bottom left panels shows $[\mathrm{Fe} / \mathrm{H}]$ against distance to the cluster centre, and no trends were found for any sample cluster. Bottom right panels shows no correlation between $\mathrm{T}_{\text {eff }}$ and $[\mathrm{Fe} / \mathrm{H}]$ for any of the six clusters analysed in this work.

In conclusion all Teff, $\log (g)$ and $[\mathrm{Fe} / \mathrm{H}]$ values are found to sit in well defined sequences following the isochrones. Bottom-right panels show no correlation between $[\mathrm{Fe} / \mathrm{H}]$ and Teff, suggesting that the $[\mathrm{Fe} / \mathrm{H}] /$ Teff degeneracy does not affect our fitting procedure. Further evidence of this is provided by the comparisons of our results with $[\mathrm{Fe} / \mathrm{H}]$ measures from high-resolution analysis available in the literature, presented in Sections 3.4.2.2, 3.5.4.1, 3.5 .4 .2 and 3.5.4.3.

\subsubsection{Validation with high-resolution spectroscopy}

We found stars in common with literature high-resolution spectroscopy for three clusters: M 71, NGC 6558 and Terzan 8. In Sect. 3.5.3 we were able to identify ten member stars of M 71, five member stars of NGC 6558, and twelve member stars in Terzan 8, these 


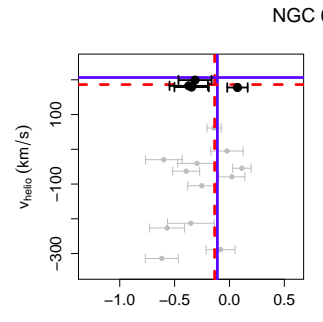

NGC 6528
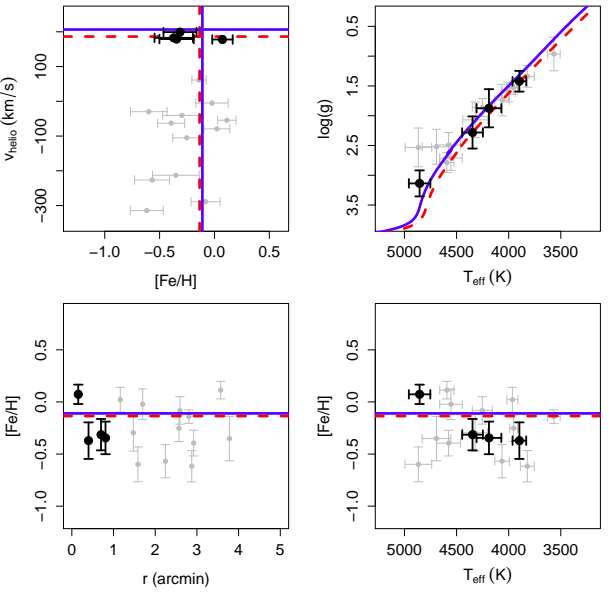

M 71
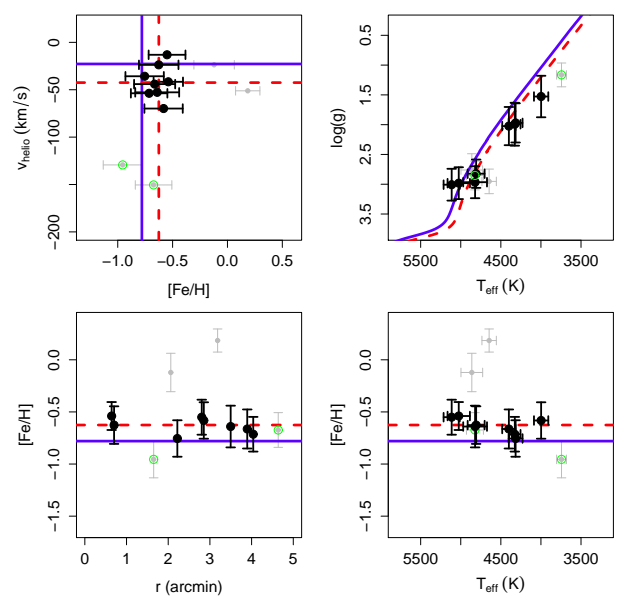

NGC 6426
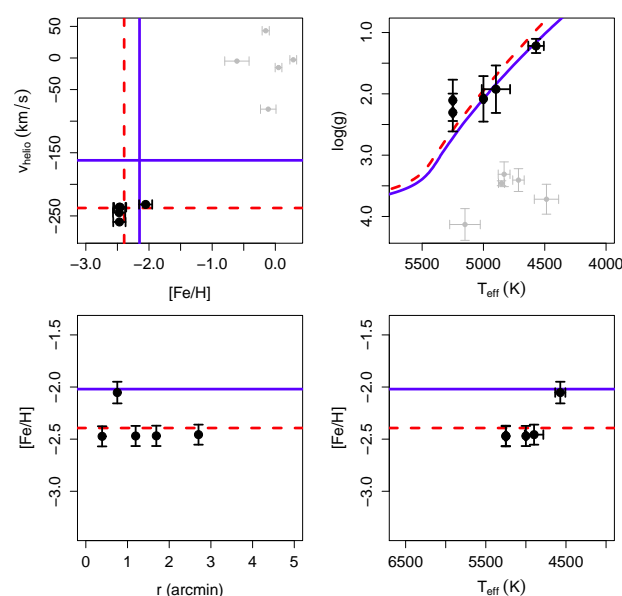
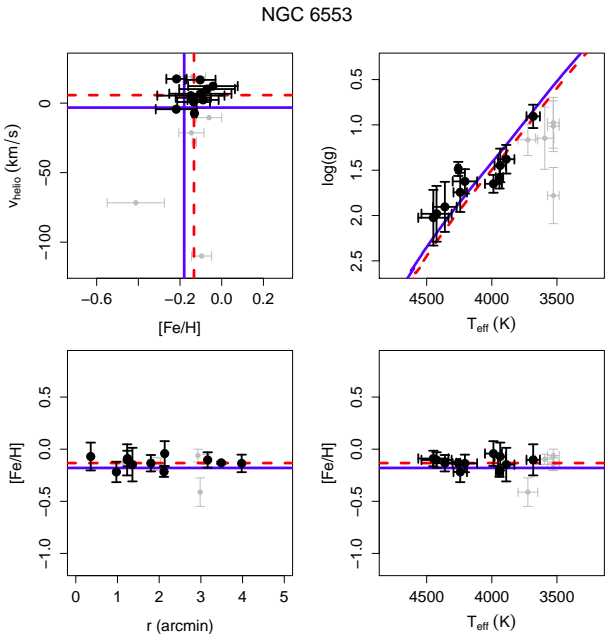

NGC 6558
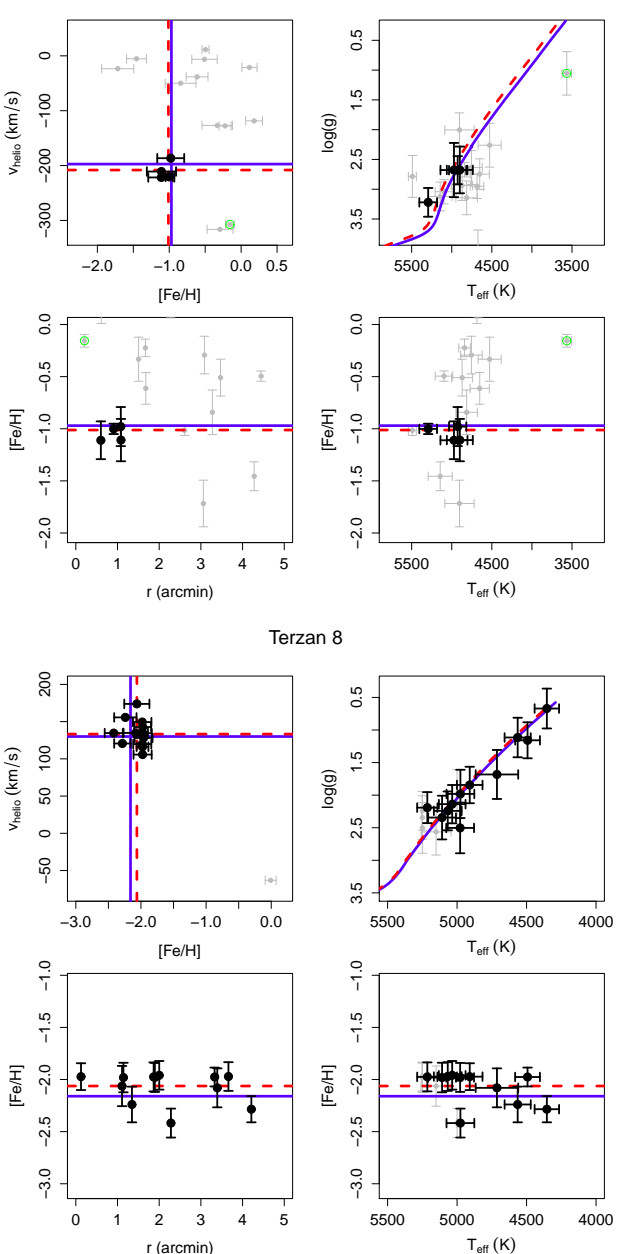

Figure 3.11: Step-by-step of selection of member stars for each cluster (four panels per cluster). Black dots are selected member stars, grey dots are non-members and green circles shows stars considered as cluster member by Saviane et al. (2012) but non-members in the present work. Blue solid lines are drawn based on values of Table 3.2 which were also applied to the ischrones from Dotter et al. (2008). Red dashed lines refer to the weighted average of the member stars parameters. 
Table 3.5 - Final average parameters for member stars in each globular cluster, and respective internal errors.

\begin{tabular}{l|cccccc}
\hline \hline Cluster & $\langle$ vhelio $>(\mathrm{km} / \mathrm{s})$ & $<[\mathrm{Fe} / \mathrm{H}]\rangle^{(a)}$ & $<[\mathrm{Fe} / \mathrm{H}]\rangle^{(b)}$ & $<[\mathrm{Fe} / \mathrm{H}]\rangle^{(a v g)}$ & $<[\mathrm{Mg} / \mathrm{Fe}]>^{(a)}$ & $<[\alpha / \mathrm{Fe}]\rangle^{(b)}$ \\
\hline NGC 6528 & $185 \pm 10$ & $-0.07 \pm 0.10$ & $-0.18 \pm 0.08$ & $-0.13 \pm 0.05$ & $0.05 \pm 0.09$ & $0.26 \pm 0.05$ \\
NGC 6553 & $6 \pm 8$ & $-0.125 \pm 0.009$ & $-0.55 \pm 0.07$ & $-0.133 \pm 0.017$ & $0.107 \pm 0.009$ & $0.302 \pm 0.025$ \\
M 71 & $-42 \pm 18$ & $-0.48 \pm 0.08$ & $-0.77 \pm 0.08$ & $-0.63 \pm 0.15$ & $0.25 \pm 0.07$ & $0.293 \pm 0.032$ \\
NGC 6558 & $-210 \pm 16$ & $-0.88 \pm 0.20$ & $-1.02 \pm 0.05$ & $-1.012 \pm 0.013$ & $0.26 \pm 0.06$ & $0.23 \pm 0.06$ \\
NGC 6426 & $-242 \pm 11$ & $-2.03 \pm 0.11$ & $-2.46 \pm 0.05$ & $-2.39 \pm 0.11$ & $0.38 \pm 0.06$ & $0.24 \pm 0.05$ \\
Terzan 8 & $135 \pm 19$ & $-1.76 \pm 0.07$ & $-2.18 \pm 0.05$ & $-2.06 \pm 0.17$ & $0.41 \pm 0.04$ & $0.21 \pm 0.04$ \\
\hline
\end{tabular}

${ }^{a}$ MILES library ${ }^{b}$ COELHO library ${ }^{a v g}$ Average of MILES and COELHO results

being the same selected by Saviane et al. (2012), and Vasquez et al. (in preparation). The derived stellar parameters are reported in Table B.3 for member and non-member stars.

\subsubsection{M 71}

Cohen et al. (2001) observed 25 member red giant stars of M 71 using HIRES@Keck $(\mathrm{R} \sim 34,000)$, and derived their $\mathrm{T}_{\text {eff }}$ and $\log (q)$. In two subsequent papers, they derived [Fe/H] (Ramírez et al., 2001) and [Mg/Fe] (Ramírez and Cohen, 2002) for them. We have three stars in common that are presented in Table 3.7. Temperature and gravity values are compatible within 0.5 to $2-\sigma,[\mathrm{Fe} / \mathrm{H}]$ and $[\mathrm{Mg} / \mathrm{Fe}]$ are compatible within 0.1 to $1.5-\sigma$.

Table 3.7 - Final atmospheric parameters for the three stars of M 71 in common with Cohen et al. (2001), and also their determinations for the respective parameters.

\begin{tabular}{l|l|l|l|l}
\hline \hline Star & $\begin{array}{l}\mathrm{T}_{\text {eff }}(\mathrm{K}) \\
\mathrm{T}_{\text {eff-C01 }(\mathrm{K})}\end{array}$ & $\begin{array}{l}\log (g) \\
\log (g)-\mathrm{C} 01\end{array}$ & $\begin{array}{l}{[\mathrm{Fe} / \mathrm{H}]} \\
{[\mathrm{Fe} / \mathrm{H}]-\mathrm{C} 01}\end{array}$ & $\begin{array}{l}{[\mathrm{Mg} / \mathrm{Fe}]} \\
{[\mathrm{Mg} / \mathrm{Fe}]-\mathrm{C} 01}\end{array}$ \\
\hline M71_7 & $3997 \pm 89$ & $1.53 \pm 0.35$ & $-0.58 \pm 0.17$ & $0.15 \pm 0.18$ \\
$1-45$ & 3950 & 0.9 & $-0.60 \pm 0.03$ & $0.43 \pm 0.09$ \\
M71_9 & $4316 \pm 87$ & $1.97 \pm 0.33$ & $-0.76 \pm 0.17$ & $0.27 \pm 0.21$ \\
1-64 & 4200 & 1.35 & $-0.61 \pm 0.03$ & $0.43 \pm 0.09$ \\
M71_13 & $4808 \pm 106$ & $2.82 \pm 0.24$ & $-0.63 \pm 0.18$ & $0.23 \pm 0.20$ \\
G53476_4543 & 4900 & 2.65 & $-0.61 \pm 0.03$ & $0.36 \pm 0.06$ \\
\hline
\end{tabular}

\subsubsection{NGC 6558}

Barbuy et al. (2007) observed six RGB stars using the high-resolution ( R 22,000) spectrograph FLAMES+GIRAFFE@VLT/ESO, and derived $\mathrm{T}_{\text {eff }}, \log (g),[\mathrm{Fe} / \mathrm{H}]$, and $[\mathrm{Mg} / \mathrm{Fe}]$ 
for each of them. We have three stars in common with their sample: \#6, \#8, \#9, corresponding to their identification as B11, F42, F97, respectively (see Table 3.8). For stars \#6 and \#9, full spectrum fitting recovers all parameters within 1- $\sigma$. Star \#8 is a more complicated case because it is a very cool star $\left(\mathrm{T}_{\text {eff }}<4000 \mathrm{~K}\right)$ and molecular bands of TiO are important. They change a lot the continuum which is not fitted perfectly. In fact, the derived parameters for this star led us to select it as non-member. Although temperature agrees with that from Barbuy et al. (2007), the gravity is much lower than their results.

These results show that full spectrum fitting method is reliable, consistent among all libraries, and present reasonable errors for RGB stars hotter then $\sim 4000$ K. Stars cooler than that must be analysed with a better suited reference library, cointaining a sufficient number of cool stars at all metallicities.

Table 3.8 - Final atmospheric parameters for the three stars of NGC 6558 in common with Barbuy et al. (2007), and also their determinations for the respective parameters.

\begin{tabular}{l|l|l|l|l}
\hline \hline Star & $\begin{array}{l}\mathrm{T}_{\text {eff }}(\mathrm{K}) \\
\mathrm{T}_{\text {eff-B07 }(\mathrm{K})}\end{array}$ & $\begin{array}{l}\log (g) \\
\log (g)-\mathrm{B} 07\end{array}$ & $\begin{array}{l}{[\mathrm{Fe} / \mathrm{H}]} \\
{[\mathrm{Fe} / \mathrm{H}]-\mathrm{B} 07}\end{array}$ & $\begin{array}{l}{[\mathrm{Mg} / \mathrm{Fe}]} \\
{[\mathrm{Mg} / \mathrm{Fe}]-\mathrm{B} 07}\end{array}$ \\
\hline $6558 \_6$ & $4899 \pm 162$ & $2.68 \pm 0.39$ & $-1.11 \pm 0.20$ & $0.22 \pm 0.07$ \\
B11 & 4650 & 2.2 & -1.04 & 0.20 \\
$6558 \_8$ & $3565 \pm 59$ & $1.05 \pm 0.36$ & $-0.16 \pm 0.06$ & $0.23 \pm 0.00$ \\
F42 & 3800 & 0.5 & -1.01 & 0.30 \\
$6558 \_9$ & $4972 \pm 168$ & $2.68 \pm 0.46$ & $-1.11 \pm 0.18$ & $0.41 \pm 0.16$ \\
F97 & 4820 & 2.3 & -0.97 & 0.23 \\
\hline
\end{tabular}

\subsubsection{Terzan 8}

Carretta et al. (2014) observed six stars with UVES@VLT/ESO (R 45,000) and 14 with GIRAFFE@VLT/ESO (R 22,500 - 24,200), with among them four stars in common with our FORS2@VLT/ESO sample. Their parameters for these stars are presented in Table 3.9. For temperature and gravity the compatibility is within 1 to $3-\sigma$, except for $\mathrm{T}_{\text {eff }}$ of star Ter8_8 which is in the limit of $3.9-\sigma$ of distance. For $[\mathrm{Fe} / \mathrm{H}]$ all stars have compatible values with Carretta et al. (2014) within 1- $\sigma$, except for star Ter8_1 which is in the limit of $3.9-\sigma$ of distance. $[\mathrm{Mg} / \mathrm{Fe}]$ is compatible within $1-\sigma$.

Differently from M 71 and NGC 6558, the comparison between our results and Carretta et al. (2014) for Terzan 8 give all three parameters $\mathrm{T}_{\text {eff }}, \log (g)$ and $[\mathrm{Fe} / \mathrm{H}]$ systematically larger. For this reason we inspected the $\mathrm{T}_{\text {eff- }} \log (g)$ diagram of both sets of data, compared 
Table 3.9 - Final atmospheric parameters for the four stars of Terzan 8 in common with Carretta et al. (2014), together with their determinations for the respective parameters.

\begin{tabular}{l|l|l|l|l}
\hline \hline Star & $\begin{array}{l}\mathrm{T}_{\text {eff }}(\mathrm{K}) \\
\mathrm{T}_{\text {eff-C14 }(\mathrm{K})}\end{array}$ & $\begin{array}{l}\log (g) \\
\log (g)-\mathrm{C} 14\end{array}$ & $\begin{array}{l}{[\mathrm{Fe} / \mathrm{H}]} \\
{[\mathrm{Fe} / \mathrm{H}]-\mathrm{C} 14}\end{array}$ & $\begin{array}{l}{[\mathrm{Mg} / \mathrm{Fe}]} \\
{[\mathrm{Mg} / \mathrm{Fe}]-C 14}\end{array}$ \\
\hline Ter8_1 & $5067 \pm 314$ & $2.24 \pm 0.30$ & $-1.97 \pm 0.14$ & $0.40 \pm 0.13$ \\
2913 & 4628 & 1.49 & $-2.52 \pm 0.07$ & 0.58 \\
Ter8_4 & $4354 \pm 88$ & $0.67 \pm 0.31$ & $-2.28 \pm 0.13$ & $0.40 \pm 0.14$ \\
2357 & 4188 & 0.66 & $-2.29 \pm 0.10$ & $0.48 \pm 0.14$ \\
Ter8_8 & $5151 \pm 108$ & $2.56 \pm 0.36$ & $-2.06 \pm 0.19$ & $0.40 \pm 0.18$ \\
2124 & 4730 & 1.67 & $-2.28 \pm 0.26$ & 0.56 \\
Ter8_9 & $4564 \pm 94$ & $1.12 \pm 0.30$ & $-2.24 \pm 0.17$ & $0.42 \pm 0.13$ \\
1658 & 4264 & 0.80 & $-2.40 \pm 0.07$ & $0.51 \pm 0.02$ \\
\hline
\end{tabular}

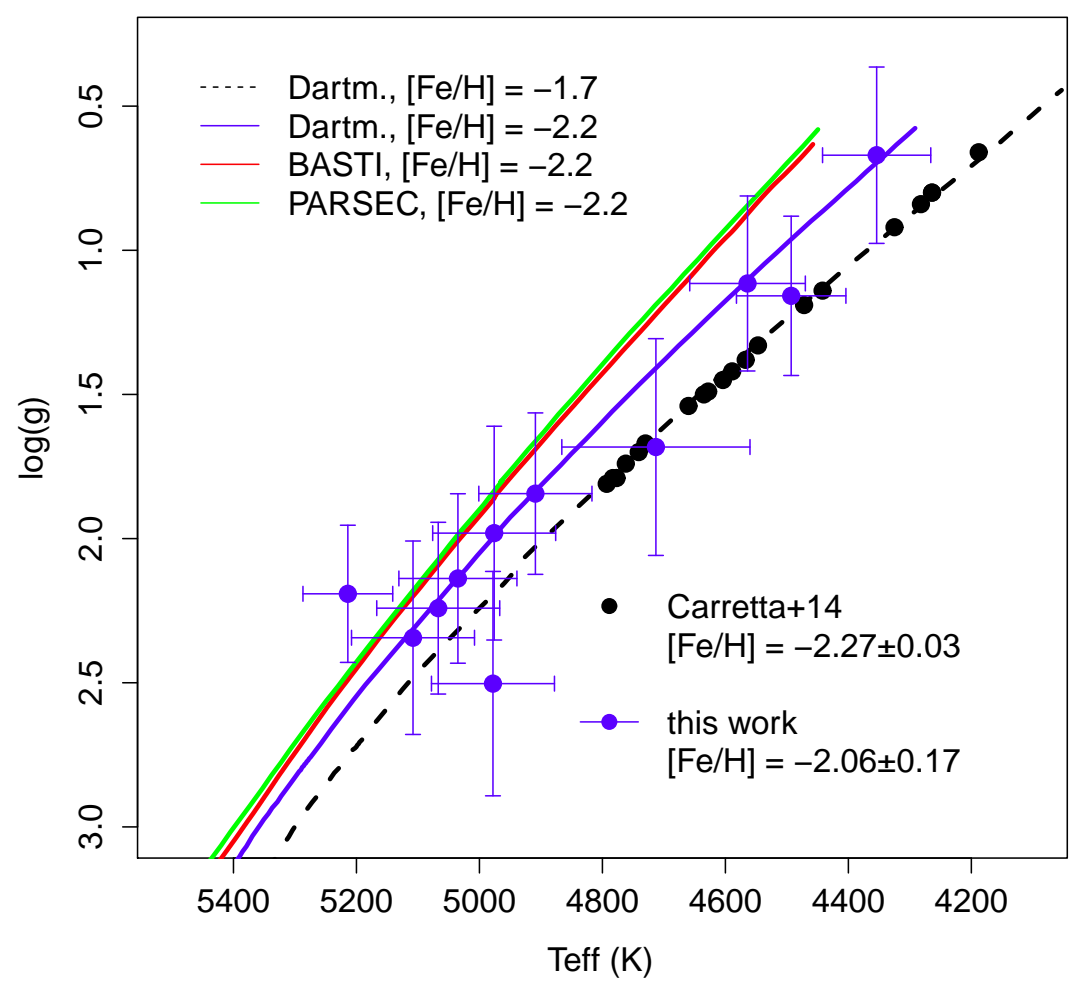

Figure 3.12: Diagram $\log (g)$ vs. Teff for Terzan 8 showing present (blue points with error bars) and Carretta et al. (2014, black filled circles) results, overplotted with Dartmouth, PARSEC and BASTI isochrones for ages $=13 \mathrm{Gyr}$, and metallicities indicated on the Figure.

with the Dartmouth (Dotter et al., 2008), PARSEC (Bressan et al., 2012) and BASTI (Pietrinferni et al., 2004) isochrones, as shown in Figure 3.12. The Carretta et al. (2014) results are compatible with an isochrone of $[\mathrm{Fe} / \mathrm{H}]=-1.7,[\alpha / \mathrm{Fe}]=+0.4$ and $13 \mathrm{Gyr}$, whereas the present results fit better with an isochrone $[\mathrm{Fe} / \mathrm{H}]=-2.2,[\alpha / \mathrm{Fe}]=+0.4$ and 13 Gyr. 
Except for star Terzan8_4 showing very similar gravities, for the other stars the difference could come from a different mass/distance/bolometric correction and is probably within the gravity uncertainty. Over-ionization at low temperature atmospheres makes it hard to get a reliable gravity from Fe I/Fe II lines.

\subsection{Discussion}

Results for individual stars in each cluster (Table B.3. $)$ and average results (Table 3.5) are discussed below and compared with literature results. Figure 3.13 displays the comparison of our $[\mathrm{Fe} / \mathrm{H}]$ results for each cluster with reference values, showing good agreement for the whole range of metallicities from $[\mathrm{Fe} / \mathrm{H}]=-2.5$ to solar. Figure 3.14 gives the comparison of the average results of $[\mathrm{Fe} / \mathrm{H}]$ and $[\mathrm{Mg} / \mathrm{Fe}]$ with abundances for field stars of the different Galactic components: bulge, thin/thick disc and inner/outer halo. We discuss case by case below.

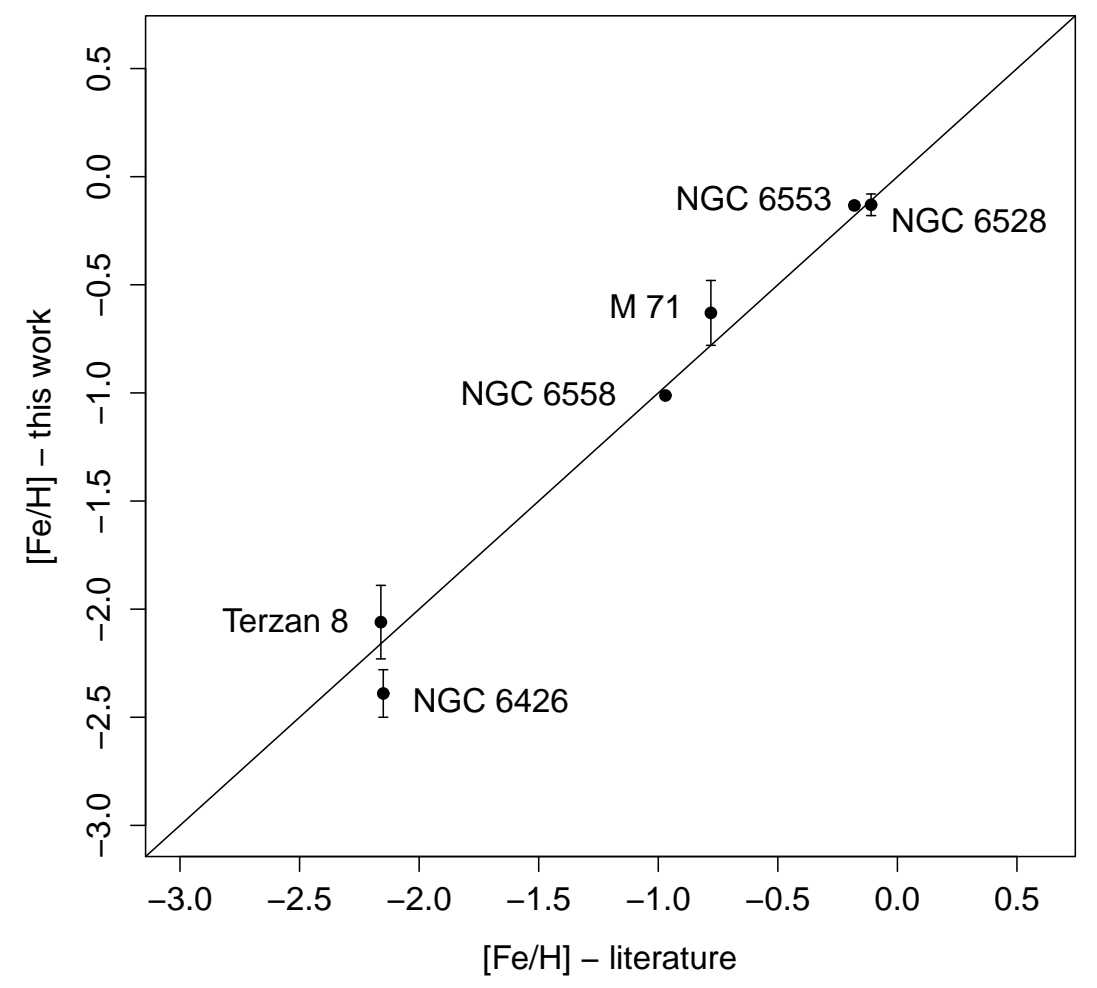

Figure 3.13: Comparison of $[\mathrm{Fe} / \mathrm{H}]$ from this work (Table 3.5) with those from literature, as revised in Table 3.2. Error bars are the weighted average of the $[\mathrm{Fe} / \mathrm{H}]$ of member stars in each cluster, as presented in Table B.3. For NGC 6553 and NGC 6558 the error bars are not visible because they are too small (see Table 3.5. 


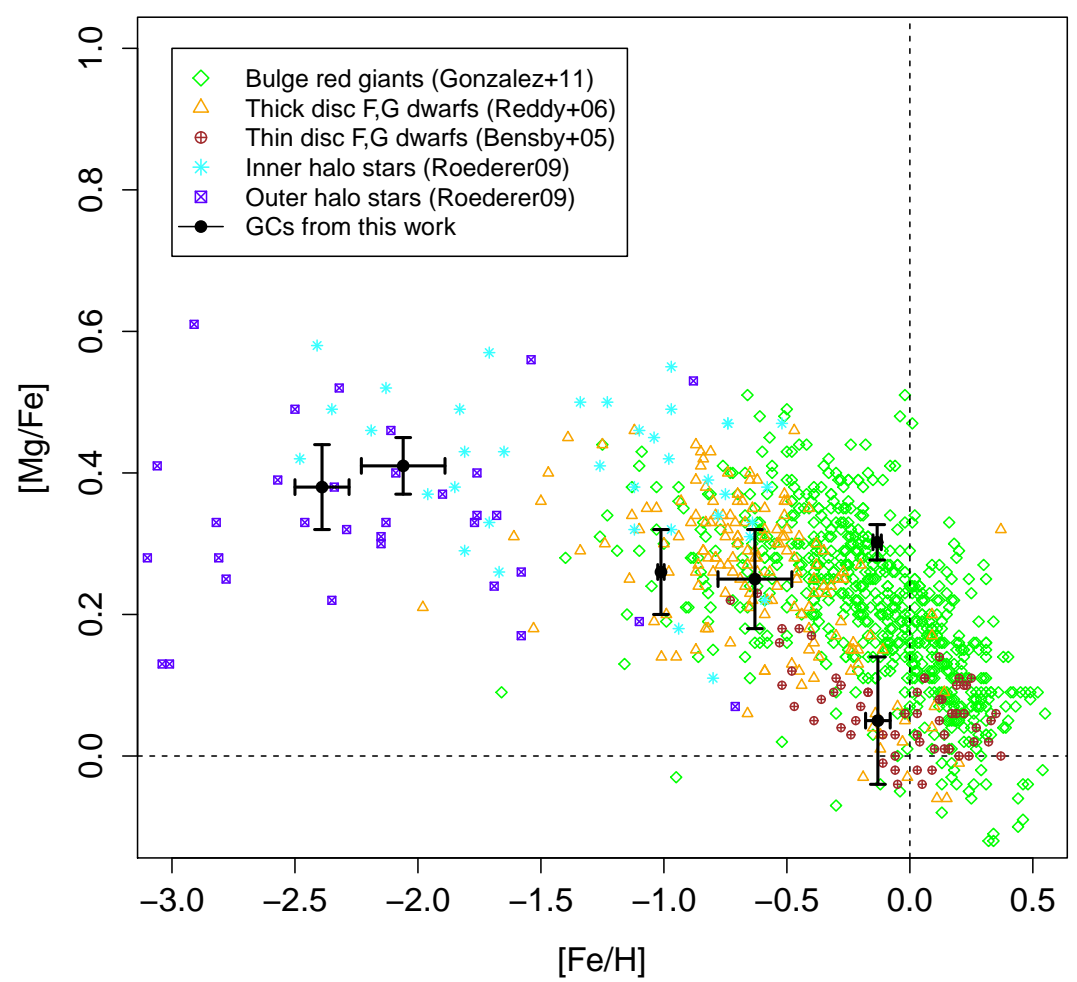

Figure 3.14: Chemical evolution of the Milky Way based on field stars from bulge (Gonzalez et al., 2011), thin disc (Bensby et al., 2005), thick disc (Reddy et al., 2006), inner and outer halo (Roederer, 2009). Results from this work for globular clusters are overplotted. For NGC 6553 the point corresponds to $[\alpha / \mathrm{Fe}]$ instead of $[\mathrm{Mg} / \mathrm{Fe}]$, as discussed in Section 3.6 .2

\subsubsection{Metal-rich clusters NGC 6528, NGC 6553}

NGC 6528 and NGC 6553 are known as "twin clusters" (Ortolani et al., 1995) with almost identical CMDs, as shown in Figure 3.1. As given in Table 3.2 they have reddening $\mathrm{E}(\mathrm{B}-\mathrm{V})=0.54 \mathrm{mag}$ and $0.63 \mathrm{mag},[\mathrm{Fe} / \mathrm{H}]=-0.11$ and $-0.18,[\mathrm{Mg} / \mathrm{Fe}]=0.24$ and 0.26 , respectively. They are located in the Milky Way bulge, distant $0.6 \mathrm{kpc}$ and $2.2 \mathrm{kpc}$ from the Galactic centre, and in opposite "southern legs" of the X-shaped bulge (see Figure 3 of Saito et al., 2011). Figure 3.9 shows that ETOILE recovers parameters for member stars coherent with a simple stellar population represented by the isochrones. Although all the results are compatible between the libraries, error bars for MILES results are lower than from COELHO results. This synthetic library, on the other hand, gives better alphaenhancement values compatible with average values from high-resolution studies $([\alpha / \mathrm{Fe}]=$ $0.26 \pm 0.05$ and $0.302 \pm 0.025$, respectively, from Table 3.5). In this respect, MILES cannot 
give an alpha-enhancement, since their metal-rich stars are basically solar neighbourhood stars, that have no alpha-enhancement for metal-rich stars (see Figure 2 in Milone et al., 2011). The results from MILES are $[\mathrm{Mg} / \mathrm{Fe}]=0.05 \pm 0.09$ and $0.107 \pm 0.009$, respectively. This is a particularity of the bulge. where stars are metal-rich and old. However. Zoccali et al. (2004) finds $[\mathrm{Mg} / \mathrm{Fe}]=+0.07$ from high-resolution spectroscopy of three stars of NGC 6528, which is compatible with MILES and not with COELHO. For N6553, Cohen et al. (1999) finds $[\mathrm{Mg} / \mathrm{Fe}]=+0.41$ also from high-resolution spectroscopy of five stars, which is closer to COELHO results.

Figure 3.11 compares the final results (average of MILES and COELHO results) with isochrones with literature parameters (same as Figure 3.9) and an additional isochrone considering the parameters derived from this work (Table 3.5). The isochrones consider $[\alpha / \mathrm{Fe}]$ from COELHO results as discussed above. We derived $[\mathrm{Fe} / \mathrm{H}]=-0.13 \pm 0.06$ and $-0.133 \pm 0.009$ for NGC 6528 and NGC 6553, respectively, in agreement with high spectral resolution analyses of Carretta et al. (2001) and Alves-Brito et al. (2006).

\subsubsection{Moderately metal-rich clusters M 71, NGC 6558}

According to Harris (1996, 2010 edition), M 71 (or NGC 6838) is located $6.7 \mathrm{kpc}$ from the Galactic centre, with a perpendicular distance to the Galactic plane of only $0.3 \mathrm{kpc}$ towards the South Galactic Pole, which means that this globular cluster is located in the Milky Way disc. Because of that its reddening is high, although not as high as for most bulge clusters, with $\mathrm{E}(\mathrm{B}-\mathrm{V})=0.25$. NGC 6558 is located only $1.0 \mathrm{kpc}$ from the Galactic centre, in particular between the two "southern legs" of the X-shaped bulge (see Figure 3 of Saito et al., 2011). It has a reddening of $\mathrm{E}(\mathrm{B}-\mathrm{V})=0.44$. Although they are located in different components of the Milky Way, these clusters share similar metallicities, $[\mathrm{Fe} / \mathrm{H}] \sim-0.8$, and $[\mathrm{Fe} / \mathrm{H}] \sim-1.0$, respectively.

Figure 3.1 shows a red horizontal branch (HB) for M 71 and a blue HB for NGC 6558, and this difference is not due to metallicity (Lee et al., 1994). It is true that a few other parameters can change the HB morphology at a fixed metallicity, as discussed by Catelan et al. (2001), for instance, but in this case the age is probably playing the major role. Literature ages are 11 Gyr (VandenBerg et al., 2013) and 14 Gyr old (Barbuy et al., 2007), respectively. M 71 is younger and moderately metal-rich, therefore a red horizontal branch is expected. In particular, we derived a slightly higher metallicity for this cluster 
in comparison with the literature, $[\mathrm{Fe} / \mathrm{H}]=-0.63 \pm 0.06$. NGC 6558 has a high metallicity for such a blue horizontal branch, and Barbuy et al. (2007) argue that if this is interpreted as pure factor of age, this cluster should be one of the oldest objects in the Milky Way. We derived $[\mathrm{Fe} / \mathrm{H}]=-1.01 \pm 0.04$, compatible with their findings $([\mathrm{Fe} / \mathrm{H}]=-0.97 \pm 0.15)$, and more metal-rich than Harris (1996. 2010 edition) value of $[\mathrm{Fe} / \mathrm{H}]=-1.32$. Saviane et al. (2012) also found $[\mathrm{Fe} / \mathrm{H}]=-1.03+/-0.14$ for NGC 6558 from their Ca II triplet spectroscopy, with error being dominated by the external calibration uncertainty.

Comparing the error bars of $\mathrm{T}_{\text {eff }}$ and $\log (g)$ in Figure 3.9 between the two libraries, for M 71 they are of the same order, but for NGC 6558 MILES results present larger error bars. The main reason for this is that MILES library is based on solar neighbouhood stars, and only a few of them have metallicities $[\mathrm{Fe} / \mathrm{H}] \approx-1.0$ (see Figure 2 of SánchezBlázquez et al., 2006). Synthetic libraries such as that of Coelho et al. (2005) have spectra for any combination of atmospheric parameters evenly distributed in the parameter space. Therefore COELHO library is more suitable for the analysis of these moderately metallicity clusters.

For this range of metallicity and for expected values of $[\mathrm{Mg} / \mathrm{Fe}]$ from the literature (0.19 and 0.24, respectively), MILES and COELHO results are compatible, as shown in Table [3.5, $[\mathrm{Mg} / \mathrm{Fe}]=0.25 \pm 0.07$ and $0.26 \pm 0.06,[\alpha / \mathrm{Fe}]=0.293 \pm 0.032$ and $0.23 \pm 0.06$, respectively. Ramírez and Cohen (2002) give an average $[\mathrm{Mg} / \mathrm{Fe}]=+0.37$ from 24 stars observed with high-resolution, which is compatible with our findings. The number of stars in MILES with these metallicities is low, but it is sampled enough for determinations of $[\mathrm{Fe} / \mathrm{H}]$ and $[\mathrm{Mg} / \mathrm{Fe}]$ for Milky Way stars. COELHO spectra have $[\alpha / \mathrm{Fe}]$ varying from 0.0 to 0.4 , which is also enough for this kind of objects.

\subsubsection{Metal-poor clusters NGC 6426, Terzan 8}

NGC 6426 and Terzan 8 present similar CMDs with a same literature age and metallicity of 13 Gyr (Dotter et al., 2011; VandenBerg et al., 2013) and [Fe/H] = -2.15 (Harris, 1996, 2010 edition). NGC 6426 is located in the northern halo of the Milky Way, $14.4 \mathrm{kpc}$ from the Galactic centre and $5.8 \mathrm{kpc}$ above the Galactic plane. Its height is much larger than the height scale of the thick disc $(0.75 \mathrm{kpc}$, de Jong et al., 2010), but it has a non-negligible reddening of $\mathrm{E}(\mathrm{B}-\mathrm{V})=0.36$, at a galactic latitude of $\mathrm{b}=16.23^{\circ}$. The best CMD available for this cluster was observed with the ACS imager onboard the Hubble 
Space Telescope by Dotter et al. (2011), and they derived an age of 13.0 \pm 1.5 Gyr from isochrone fitting. Our pre-image photometry based on observations with the Very Large Telescope/ESO produced a rather well-defined CMD for this cluster which is compatible with a 13 Gyr isochrone (see Figure 3.1).

Terzan 8 is located in the southern halo of the Milky Way, $19.4 \mathrm{kpc}$ from the Galactic centre and $10.9 \mathrm{kpc}$ below the Galactic plane. It has the lowest reddening of all clusters analysed in this work, $\mathrm{E}(\mathrm{B}-\mathrm{V})=0.12$. This is one of the four Milky Way globular clusters believed to be captured from the Sagittarius dwarf galaxy (Ibata et al., 1994), the other three being M 54, Terzan 7 and Arp 2 (Da Costa and Armandroff, 1995). Carretta et al. (2014) did not find a strong evidence for Na-O anticorrelation, typical for globular clusters which may indicate that these clusters may have simple stellar populations.

Atmospheric parameters derived in this work for both clusters are in good agreement with literature values, when compared to the isochrones in Figure 3.9. As for the moderately metal-rich clusters, MILES results present larger error bars than COELHO results for $\mathrm{T}_{\text {eff }}$ and $\log (g)$, and the reason is the same as mentioned above, i.e., the sampling of MILES library is poorer for this metallicity range (see Figure 2 of Sánchez-Blázquez et al., 2006). Derived metallicities for these clusters are $[\mathrm{Fe} / \mathrm{H}]=-2.39 \pm 0.04$ and $-2.06 \pm 0.04$, respectively. For NGC 6426 our determination is 0.24 more metal-poor than given in Harris's catalogue $([\mathrm{Fe} / \mathrm{H}]=-2.15)$. However the only derivation of the metallicity for this cluster was done by Zinn and West (1984) based on integrated light ([Fe/H] $=-2.20 \pm 0.17)$ which is compatible with our findings. The value from Harris catalogue was obtained by applying the Carretta et al. (2009) metallicity scale. We present for the first time a direct measurement of metallicity of individual red giant stars finding a more metal-poor value than previously attributed to this cluster. Terzan 8 is compatible with the Harris (2010 edition) catalogue of $[\mathrm{Fe} / \mathrm{H}]=-2.16$. There are three papers with metallicities for this cluster: Mottini et al. (2008) and Carretta et al. (2014), based on high-resolution spectra of individual star and average metallicity $[\mathrm{Fe} / \mathrm{H}]=-2.35 \pm 0.04$ and $-2.27 \pm 0.08$, respectively. The third paper is Da Costa and Armandroff (1995) who derived $[\mathrm{Fe} / \mathrm{H}]=-1.99 \pm 0.08$ based on CaII triplet spectroscopy. Our result is compatible with the two more metal-rich results.

For alpha-enhancement in this metallicity range, MILES spectra goes up to $[\mathrm{Mg} / \mathrm{Fe}]$ $=0.74$, while COELHO is limited to the models of $[\alpha / \mathrm{Fe}]=0.4$. The results based on 
COELHO $([\alpha / \mathrm{Fe}]=0.24 \pm 0.05$ and $0.21 \pm 0.04$, respectively $)$ are less enhanced than MILES $([\mathrm{Mg} / \mathrm{Fe}]=0.38 \pm 0.06$ and $0.41 \pm 0.04$, respectively $)$, the latter being closer to literature abundance ratios.

\subsection{Summary and conclusions}

We present a method of full spectrum fitting, based on the ETOILE code, to derive $\mathrm{V}_{\text {helio }}, \mathrm{T}_{\text {eff }}, \log (g),[\mathrm{Fe} / \mathrm{H}],[\mathrm{Mg} / \mathrm{Fe}]$ and $[\alpha / \mathrm{Fe}]$ for red giant stars in Milky Way globular clusters. The observations were carried out with FORS2@VLT/ESO with resolution $\mathrm{R} \sim 2000$.

We validated the method using well known red giant stars covering the parameter space of $4000 \mathrm{~K}<\mathrm{T}_{\text {eff }}<6000 \mathrm{~K}, 0.0<\log (g)<4.0,-2.5<[\mathrm{Fe} / \mathrm{H}]<+0.3$ and $-0.2<$ $[\mathrm{Mg} / \mathrm{Fe}]<+0.6$. The spectra of these reference stars are from the ELODIE library and the parameters from the PASTEL catalogue. The parameters were recovered for the whole range of parameters.

We selected six clusters from our large sample of observed clusters in order to establish the methodology. We chose two metal-rich (NGC 6528, NGC 6553), two moderately metalrich (M 71, NGC 6558) and two metal-poor (NGC 6426 and Terzan 8) clusters. NGC 6528, NGC 6553 and NGC 6558 are located in the bulge, M 71 in the disc, NGC 6426 and Terzan 8 in the halo. For all clusters the effective temperatures and gravities are well determined using the MILES and Coelho et al. (2005) libraries of spectra. Metallicities and alphaelement enhancement are also derived with the caveats that for alpha-enhanced bulge clusters with $[\mathrm{Fe} / \mathrm{H}]>-0.5$, MILES is not suitable, since it has only solar neighbourhood stars, therefore in this case we use COELHO results because synthetic libraries have all combinations of parameters. For $[\mathrm{Fe} / \mathrm{H}] \approx-1.0$ MILES has few stars due to a lack of such stars in the solar vicinity. For metal-poor clusters, with high $[\alpha / \mathrm{Fe}]$, MILES may be more indicated than COELHO because the latter is limited to $0<[\alpha / \mathrm{Fe}]<0$.4dex, if such high Mg enhancements are confirmed.

The present results are in agreement with literature parameters available for five of the six template clusters. NGC 6426 is analysed for the first time using spectroscopy of individual stars. Therefore we provide a more precise radial velocity of $-242 \pm 11 \mathrm{~km} / \mathrm{s}$, a metallicity $[\mathrm{Fe} / \mathrm{H}]=-2.39 \pm 0.04$, and $[\mathrm{Mg} / \mathrm{Fe}]=0.38 \pm 0.06$. The comparison of our results 
of $[\mathrm{Fe} / \mathrm{H}]$ and $[\mathrm{Mg} / \mathrm{Fe}]$ to those from field stars from all Galactic components shows that the globular clusters follows the same chemical enrichment pattern as the field stars.

In conclusion, full spectrum fitting technique using ETOILE code together with MILES and COELHO libraries appears to be suitable to derive chemical abundances for Milky Way globular clusters, from low/medium-resolution spectra. Depending on the stellar population studied, the choice of library with parameter space covering the expected values for the clusters is a crucial ingredient, observed library being better for more metal-rich stars and synthetic library being preferable for the more metal-poor ones. This method will be applied to the other Milky Way globular clusters from this survey. It is also promising for extragalactic stars, that can be more easily observed with similar resolutions of $\mathrm{R} \sim 2000$, for studies of galaxy formation and evolution. 
Chapter 4

\section{FORS2/VLT survey of Milky Way globular clusters II. Parameters for 51 clusters}

\subsection{Introduction to this chapter}

The system of Galactic globular clusters (GGC) is of paramount importance to reconstruct the early evolution of the Milky Way: if their ages were known with sufficient precision, then correlations of their kinematics and chemical abundances with time would shed light on the dynamical and chemical evolution of the protogalactic halo and bulge. The present study allows a first order derivation of metallicity and alpha-enhancement from low-resolution spectra, together with radial velocities, and it provides a reliable target list for follow-up with high resolution spectroscopy. This is particularly important for the bulge clusters.

With the advent of multifiber spectrographs at 8m-class telescopes, high-resolution spectra of sufficient $\mathrm{S} / \mathrm{N}$ can now be obtained for the giant stars of clusters out to (m$\mathrm{M})_{V} \approx 19$, i.e. for $>80 \%$ of the GGCs. Nevertheless, the largest homogeneous samples of metallicities are still based on low-resolution spectroscopy (and calibrated versus highresolution results). Even so, more than 50\% of GGCs do not have any spectroscopic estimation of their $[\mathrm{Fe} / \mathrm{H}]$. Our survey improves this statistics targetting mostly distant and highly reddened clusters, which are poorly studied in the literature. We also observed some well-known clusters for calibration purposes, in a total of 51 objects, which is $\sim 1 / 3$ of the total number of catalogued Galactic globular clusters (see Saviane et al., 2012 for a review of $[\mathrm{Fe} / \mathrm{H}]$ values available in the literature).

Homogeneous determinations of $[\mathrm{Fe} / \mathrm{H}]$ and $[\alpha / \mathrm{Fe}]$ for a large set of globular clusters are useful to analyse the chemical evolution of the Milky Way for its different components 
(bulge, disc, inner and outer halo) and compare with field stars. The combination of these abundances with distance to the Galactic centre and ages leads to discussions about the origin of the globular clusters and it constrains models of Galaxy formation and evolution. Usually, $[\mathrm{Fe} / \mathrm{H}]$ for all globular clusters are averaged from different sources, using different methods and spectral resolution, and put them in a same scale. This procedure is useful to draw an overall picture of the metallicity distribution of our Galaxy, but it is not exactly an homogeneous set of $[\mathrm{Fe} / \mathrm{H}]$ and may contain some biases. In the present work we present for the first time a large set of $[\mathrm{Fe} / \mathrm{H}]$ and $[\mathrm{Mg} / \mathrm{Fe}]$ for the globular cluster population of the Milky Way, observed with the same setup, and analysed in a homogeneous way, finally calibrated using high-resolution results. We discuss how these results can help to understand the formation and evolution of the Milky Way. Similar observations and analysis may be done using this method to study extragalactic globular clusters, such as Magellanic Clouds, dwarf galaxies and more distant galaxies with the emergence of 40m-class telescopes, such as E-ELT.

\subsection{Targets selection and observations}

The targets were selected among the faintest globular clusters cataloged by (Harris, 1996, 2010 edition) which are more distant and/or highly reddened, many of them poorly studied. Some well-known brighter objects were also observed for comparison with high-resolution spectroscopic studies available in the literature. The sample clusters were divided into four groups defined by their stellar populations, following the criteria discussed by Carretta et al. (2010). Bulge clusters are all objects within $3.5 \mathrm{kpc}$ from the Galactic centre, outer halo clusters have $R_{\mathrm{GC}} \geq 15.0 \mathrm{kpc}$, the other objects are classified as disc or inner halo depending on their kynematics and vertical distance in relation to the Galactic plane (see Carretta et al., 2010 for further details). In Table 4.1 we show the population that each globular cluster belongs to, and their sky position are displayed over an all-sky image in Figure 4.1 with letters indicating the respective populations.

Figure 4.2 shows reddening versus distance for all clusters in the bulge, disc, inner and outer halo. Bulge clusters are located in a similar distance from the Sun $(\sim 8 \mathrm{kpc})$ and are spread over a wide range of reddening values between $\sim 0.2$ and 1.5 depending on the direction. The closest disc clusters to the Sun have distances of $\sim 2.3 \mathrm{kpc}$ and the farthest 


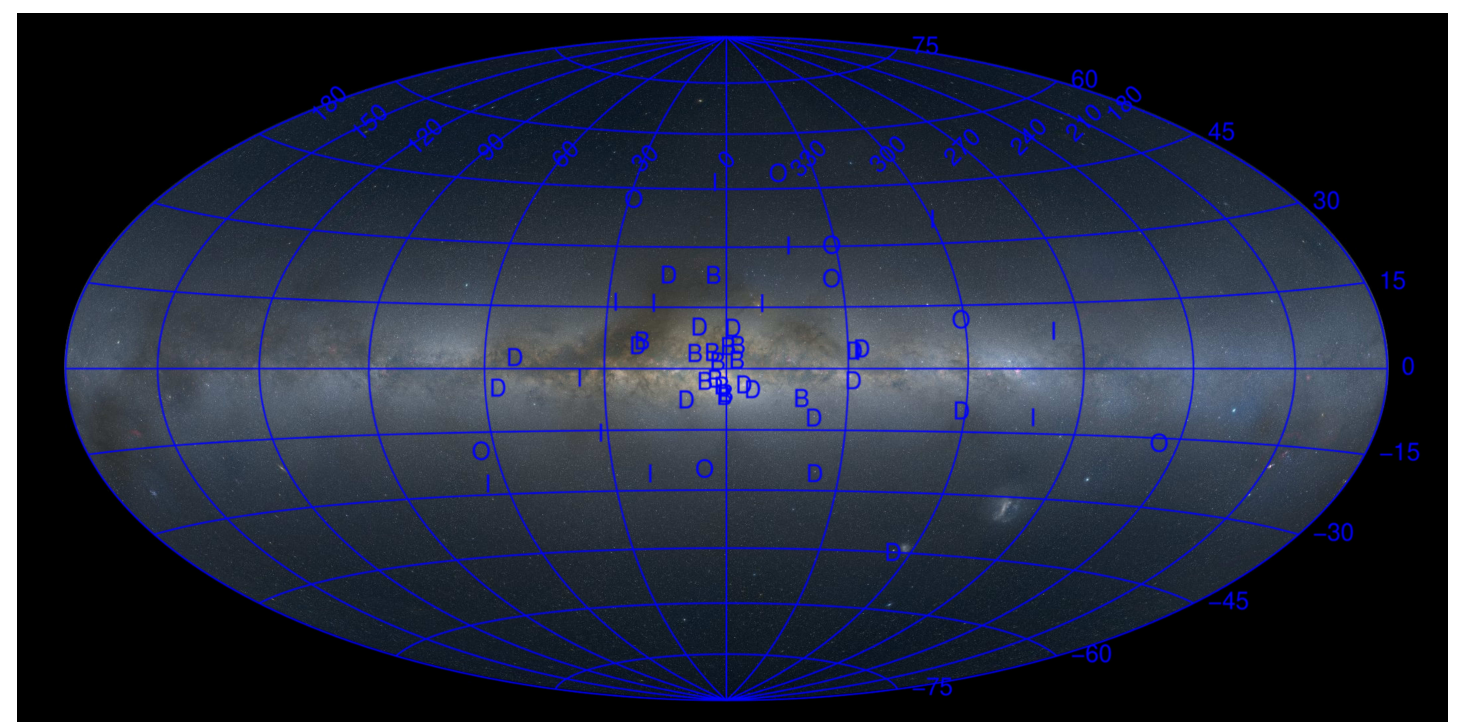

Figure 4.1: Sky position of the 51 clusters over Milky Way image (Mellinger, 2009) in terms of Galactic coordinates in a Aitoff projection. In the position of each cluster, a letter indicates its population, namely (B)ulge, (D)isc, (I)nner halo and (O)uter halo, as classified in Table 4.1.

are $\sim 19 \mathrm{kpc}$ from here. Because of that distribution and the low latitudes of these clusters, reddening values varies from $\sim 0.04$ to $\sim 1$.7. Inner halo has similar intervals of $\mathrm{E}(\mathrm{B}-\mathrm{V})$ and distance as disc clusters. Outer halo clusters have low reddening, $\mathrm{E}(\mathrm{B}-\mathrm{V})<0.2$ and are faraway $(11<\mathrm{d} / \mathrm{kpc}<77)$.

We defined $\mathrm{V}_{\mathrm{HB}}=17.5$ as a threshold for observations with high-resolution spectrographs in $8 \mathrm{~m}$-class telescopes. FLAMES/VLT user manual 1 indicates that observations of stars with $\mathrm{V}=17.5$ with one hour of exposure will result a $\mathrm{S} / \mathrm{N} \sim 30$ using GIRAFFE fibers and $\mathrm{S} / \mathrm{N} \sim 10$ using UVES fibers. Stars fainter than that would require too much telescope time to get useful spectra and would forbid observations. We selected red giant stars usually brighter than the horizontal branch level (see Figure ??) in each cluster, therefore the classification of globular clusters with $\mathrm{V}_{\mathrm{HB}} \gtrless 17.5$ indicates which clusters could be observed with high-resolution spectroscopy. In Figure 4.2 we call attention to the fact that half of the sample (25 of 51) are not bright enough and hence our observations of lowresolution spectra are perfect to cover the whole range of parameters of all populations of globular clusters in the Galaxy even for the very distant and highly reddened clusters.

Around 10 - 20 red giant stars were selected from photometry in each cluster as described in Chapter 3, for a total of 819 stars. We obtained FORS2@VLT/ESO spectra for them, and 61 spectra (7\%) were not considered in the analysis due to very low signal-

\footnotetext{
${ }^{1}$ FLAMES User Manual VLT-MAN-ESO-13700-2994, Issue 92, 06/03/2013, Table 1
} 


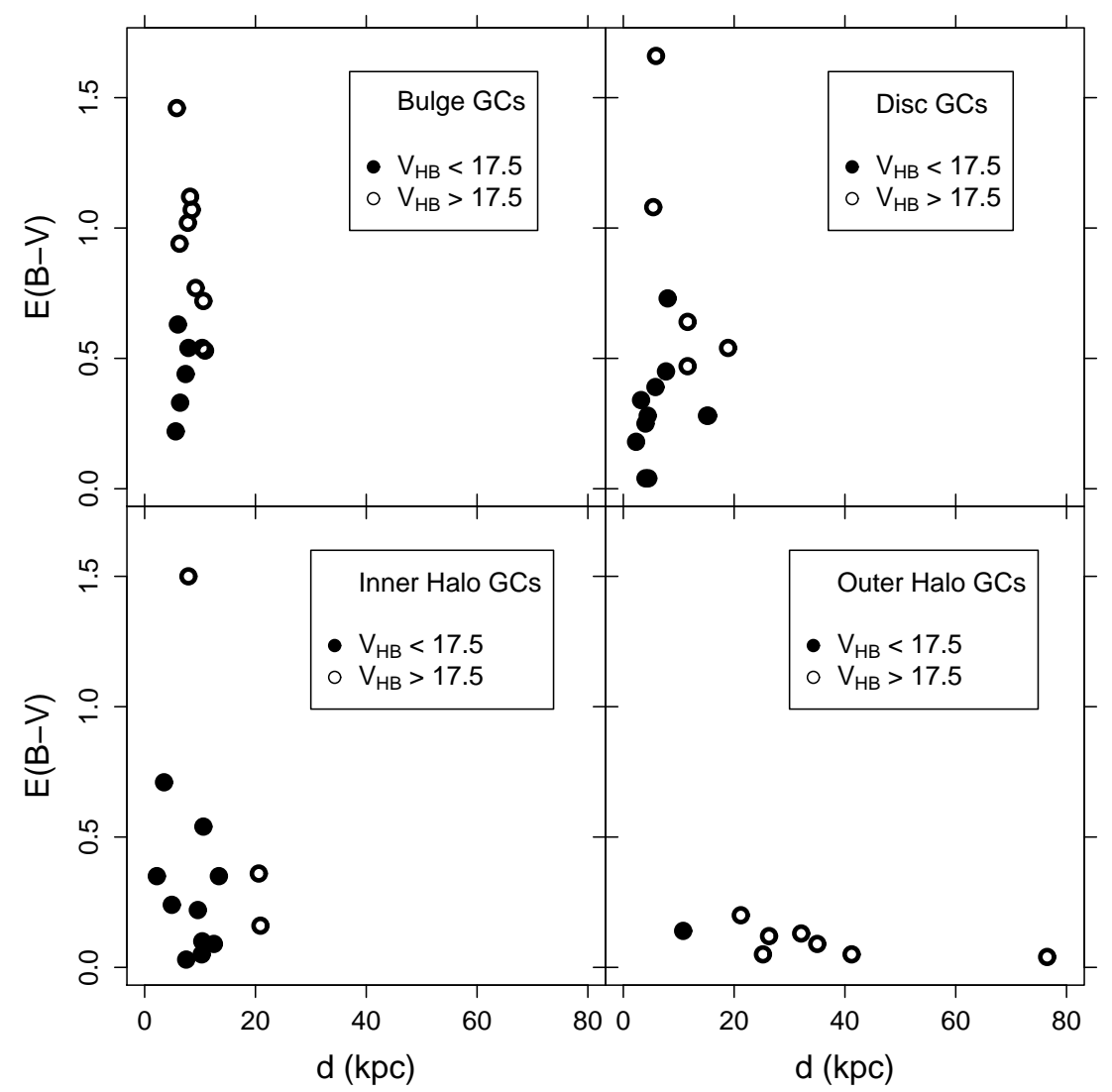

Figure 4.2: Reddening versus distance of the 51 Milky Way globular clusters for each population: bulge, disc, inner halo, outer halo. Empty circles represent clusters with horizontal branch level fainter than V $=17.5$, filled circles are clusters with $\mathrm{V}_{\mathrm{HB}}$ brigther than 17.5.

to-noise ratio or data reduction problems. From the remaining 758 useful spectra, 464 $(61 \%)$ are of confirmed members of the 51 clusters, and the other 294 spectra (39\%) are of non-members or at least they do not have results good enough to be classified as members. The spectra were observed in the visible region (grism 1400V, 456 - $586 \mathrm{~nm}$ ) with resolution of $\Delta \lambda=2.5 \AA$ and typical $\mathrm{S} / \mathrm{N} \sim 30$ - 100. The data was colected from 2001 to 2012 under projects ID 68.B-0482(A, 2001), ID 69.D-0455(A, 2002), ID 71.D-0219(A, 2003), ID 077.D-0775(A, 2006) and ID 089.D-0493(B, 2012). Table 4.1 lists the selected clusters, their coordinates, observing date and exposure time. Coordinates of the 758 analysed stars and their magnitudes are displayed in Table B.1. The spectra were reduced using FORS2 pipeline inside the EsoRex software 2 following the procedure described in Section 3.3 and Appendix C.

\footnotetext{
${ }^{2}$ https://www.eso.org/sci/software/cpl/esorex.html
} 
Table 4.1 - Log of observations of the 51 globular clusters using FORS2/VLT with grism 1400V.a) 2001 observations, ID 68.B-0482(A); b) 2002 observations, ID 69.D-0455(A); c) 2003 observations, ID 71.D0219(A); d) 2006 observations, ID 077.D-0775(A); e) 2012 observations, ID 089.D-0493(B). Classification of each cluster as (B)ulge, (D)isc, inner (IH) or outer halo $(\mathrm{OH})$, as well as, open cluster (DOpen) and dwarf galaxy-related cluster (OHdSph) follow the criteria defined by Carretta et al. (2010). In the last column we show the numbers of analysed stars that belong to each cluster $(\mathrm{C})$ and those that we classified as field stars $(\mathrm{F})$.

\begin{tabular}{|c|c|c|c|c|c|c|c|c|}
\hline Cluster & $\begin{array}{l}\text { Other } \\
\text { names }\end{array}$ & $\begin{array}{l}\alpha(\mathrm{J} 2000) \\
\mathrm{h} \mathrm{m} \mathrm{s}\end{array}$ & $\begin{array}{l}\delta(\mathrm{J} 2000) \\
\circ,,\end{array}$ & $\begin{array}{c}\text { obs. date } \\
\text { dd.mm.yyyy }\end{array}$ & $\begin{array}{c}\text { UT } \\
\mathrm{h}: \mathrm{m}: \mathrm{s}\end{array}$ & $\begin{array}{r}\tau \\
(\mathrm{s})\end{array}$ & Pop. & $\begin{array}{c}\text { \# stars } \\
\mathrm{C} / \mathrm{F}\end{array}$ \\
\hline NGC $104^{a}$ & 47 Tuc & 002405.67 & -720452.6 & 22.10 .2001 & $07: 16: 53$ & 120.0 & $\mathrm{D}$ & $15 / 1$ \\
\hline NGC $2298^{a}$ & & 064859.41 & -360019.1 & 23.10 .2001 & $06: 25: 42$ & 120.0 & $\mathrm{OH}$ & $5 / 0$ \\
\hline NGC $2808^{d}$ & & 091203.10 & -645148.6 & 29.05 .2006 & 00:06:07 & 45.0 & $\mathrm{IH}$ & $14 / 4$ \\
\hline NGC $3201^{d}$ & & 101736.82 & -462444.9 & 28.05 .2006 & $22: 52: 56$ & 20.8 & $\mathrm{IH}$ & $13 / 2$ \\
\hline $\mathrm{NGC} 4372^{c}$ & & 122545.40 & -723932.4 & 25.05 .2003 & 01:34:41 & 300.0 & $\mathrm{D}$ & $8 / 2$ \\
\hline Rup $106^{d}$ & & 123840.2 & -510901 & 28.05 .2006 & $23: 15: 01$ & 758.6 & $\mathrm{OH}$ & $8 / 7$ \\
\hline NGC $4590^{b}$ & M 68 & 123927.98 & -264438.6 & 07.05 .2002 & 03:38:10 & 60.0 & $\mathrm{IH}$ & $7 / 3$ \\
\hline NGC $5634^{e}$ & & 142937.23 & $-05 \quad 5835.1$ & 26.06.2012 & 00:12:03 & 240.0 & $\mathrm{OH}$ & $8 / 1$ \\
\hline NGC $5694^{e}$ & & 143936.29 & -263220.2 & 25.06 .2012 & $23: 27: 18$ & 540.0 & $\mathrm{OH}$ & $8 / 3$ \\
\hline NGC $5824^{d}$ & & 150358.63 & -330405.6 & 29.05 .2006 & $00: 26: 28$ & 553.3 & $\mathrm{OH}$ & $15 / 3$ \\
\hline NGC $5897^{b}$ & & 151724.50 & -210037.0 & 07.05 .2002 & $03: 53: 29$ & 60.0 & $\mathrm{IH}$ & $8 / 0$ \\
\hline NGC $5904^{c}$ & M 5 & 151833.22 & +020451.7 & 04.05 .2003 & 06:00:38 & 300.0 & $\mathrm{IH}$ & $9 / 0$ \\
\hline NGC $5927^{b}$ & & 152800.69 & -504022.9 & 07.05 .2002 & $04: 14: 29$ & 300.0 & $\mathrm{D}$ & $6 / 0$ \\
\hline NGC $5946^{e}$ & & 153528.52 & -503934.8 & 23.06 .2012 & 02:52:00 & 180.0 & $\mathrm{IH}$ & $5 / 10$ \\
\hline $\mathrm{BH} 176^{e}$ & & 153907.45 & -500309.8 & 22.05 .2012 & 03:06:04 & 600.0 & DOpen & $11 / 4$ \\
\hline Lynga $7^{d}$ & BH 184 & 161103.65 & -551904.0 & 29.05 .2006 & 01:17:18 & 451.6 & $\mathrm{D}$ & $3 / 10$ \\
\hline Pal $14^{e}$ & AvdB & 161100.6 & +145728 & 15.06 .2012 & $02: 49: 24$ & 1140.0 & $\mathrm{OH}$ & $6 / 1$ \\
\hline $\mathrm{NGC} 6121^{d}$ & M 4 & 162335.22 & -263132.7 & 29.05 .2006 & 02:53:01 & 5.8 & $\mathrm{IH}$ & $8 / 6$ \\
\hline NGC $6171^{b}$ & M 107 & 163231.86 & -130313.6 & 07.05 .2002 & $04: 39: 29$ & 60.0 & B & $1 / 4$ \\
\hline NGC $6254^{d}$ & M 10 & 165709.05 & -040601.1 & 29.05 .2006 & 03:11:21 & 54.5 & $\mathrm{D}$ & $13 / 2$ \\
\hline NGC $6284^{e}$ & & 170428.51 & -244553.5 & 22.07 .2012 & 03:17:03 & 180.0 & $\mathrm{D}$ & $7 / 10$ \\
\hline NGC $6316^{e}$ & & 171637.30 & -280824.4 & 22.07 .2012 & $03: 29: 22$ & 180.0 & B & $7 / 9$ \\
\hline NGC $6356^{d}$ & & 172334.93 & -174846.9 & 29.05 .2006 & $04: 40: 47$ & 167.9 & $\mathrm{D}$ & $13 / 5$ \\
\hline NGC $6355^{e}$ & & 172358.59 & -262112.3 & 11.09 .2012 & $23: 19: 52$ & 240.0 & B & $6 / 10$ \\
\hline NGC $6352^{e}$ & & 172529.11 & -482519.8 & 22.05 .2012 & 05:33:04 & 60.0 & B & $12 / 2$ \\
\hline NGC $6366^{e}$ & & 172744.24 & -050447.5 & 15.06 .2012 & $04: 53: 54$ & 60.0 & $\mathrm{IH}$ & $14 / 3$ \\
\hline $\mathrm{HP} 1^{d}$ & BH 229 & 173105.2 & -295854 & 30.05 .2006 & 05:19:08 & 1037.5 & B & $8 / 19$ \\
\hline NGC $6401^{e}$ & & 173836.60 & -235434.2 & 14.07 .2012 & $05: 36: 29$ & 300.0 & B & $6 / 12$ \\
\hline NGC $6397^{c}$ & & 174042.09 & -534027.6 & 06.05 .2003 & 03:54:19 & 300.0 & $\mathrm{D}$ & $18 / 3$ \\
\hline NGC $6397^{d}$ & & $"$ & $"$ & 29.05 .2006 & $05: 24: 27$ & 7.7 & $"$ & $"$ \\
\hline $\mathrm{Pal} 6^{e}$ & & 174342.2 & -261321 & 12.09 .2012 & 01:01:28 & 780.0 & $\mathrm{~B}$ & $4 / 13$ \\
\hline NGC $6426^{e}$ & & 174454.65 & +031012.5 & 13.07 .2012 & $02: 31: 12$ & 500.0 & $\mathrm{IH}$ & $5 / 5$ \\
\hline NGC $6440^{d}$ & & 174852.70 & -202136.9 & 20.05 .2006 & $05: 38: 49$ & 649.2 & B & $7 / 9$ \\
\hline NGC $6441^{d}$ & & 175013.06 & -370305.2 & 29.05 .2006 & 06:27:01 & 227.2 & $\mathrm{D}$ & $8 / 10$ \\
\hline NGC $6453^{e}$ & & 175051.70 & -343557.0 & 12.09 .2012 & 00:50:14 & 300.0 & $\mathrm{D}$ & $3 / 13$ \\
\hline Djorg $2^{e}$ & ESO456-SC38 & 180149.1 & -274933 & 14.07 .2012 & $05: 52: 59$ & 180.0 & B & $4 / 11$ \\
\hline
\end{tabular}


Table 4.1 - continued.

\begin{tabular}{|c|c|c|c|c|c|c|c|c|}
\hline Cluster & $\begin{array}{l}\text { Other } \\
\text { names }\end{array}$ & $\begin{array}{l}\alpha(\mathrm{J} 2000) \\
\mathrm{h} \mathrm{m} \mathrm{s}\end{array}$ & $\begin{array}{l}\delta(\mathrm{J} 2000) \\
\circ, ",\end{array}$ & $\begin{array}{c}\text { obs. date } \\
\text { dd.mm.yyyy }\end{array}$ & $\begin{array}{c}\text { UT } \\
\text { h:m:s }\end{array}$ & $\begin{array}{r}\tau \\
(\mathrm{s})\end{array}$ & Pop. & $\begin{array}{c}\text { \# stars } \\
\mathrm{C} / \mathrm{F}\end{array}$ \\
\hline NGC $6528^{d}$ & & $"$ & $"$ & 29.05 .2006 & $08: 36: 22$ & 149.4 & B & $4 / 13$ \\
\hline NGC $6539^{e}$ & & 180449.68 & -073509.1 & 12.09 .2012 & 00:13:01 & 360.0 & B & $7 / 8$ \\
\hline $\mathrm{NGC} 6553^{d}$ & & 180917.60 & -255431.3 & 29.05 .2006 & $08: 57: 50$ & 79.4 & B & $11 / 6$ \\
\hline NGC $6558^{d}$ & & 181017.60 & -314550.0 & 29.05 .2006 & $06: 55: 32$ & 148.3 & B & $4 / 13$ \\
\hline IC $1276^{d}$ & Pal 7 & 181044.20 & $-07 \quad 12 \quad 27.4$ & 29.05 .2006 & $07: 17: 06$ & 229.8 & $\mathrm{D}$ & $12 / 5$ \\
\hline NGC $6569^{d}$ & & 181338.80 & -314936.8 & 29.05 .2006 & 07:43:05 & 210.4 & B & $7 / 11$ \\
\hline NGC $6656^{d}$ & M 22 & 183623.94 & $-2354 \quad 17.1$ & 29.05 .2006 & $08: 22: 25$ & 36.1 & $\mathrm{D}$ & $44 / 9$ \\
\hline$"$ & $"$ & $"$ & $"$ & $"$ & $09: 57: 32$ & 36.1 & $"$ & $"$ \\
\hline$"$ & $"$ & $"$ & $"$ & $"$ & 08:08:05 & 36.1 & $"$ & $"$ \\
\hline NGC $6749^{e}$ & & 190515.3 & +015403 & 27.05 .2012 & 05:04:40 & 810.0 & $\mathrm{IH}$ & $4 / 13$ \\
\hline NGC $6752^{a}$ & & 191052.11 & -595904.4 & 25.05 .2003 & $06: 55: 40$ & 300.0 & $\mathrm{D}$ & $5 / 1$ \\
\hline Pal $10^{e}$ & & 191802.1 & +183418 & 16.06 .2012 & $05: 38: 57$ & 900.0 & $\mathrm{D}$ & $9 / 14$ \\
\hline Terzan $8^{e}$ & & 194144.41 & -335958.1 & 12.07 .2012 & $07: 37: 44$ & 360.0 & OHdSph & $12 / 1$ \\
\hline$"$ & & $"$ & $"$ & 13.07 .2012 & 08:10:51 & 360.0 & $"$ & $"$ \\
\hline$"$ & & $"$ & $"$ & 14.07 .2012 & $05: 18: 10$ & 360.0 & $"$ & $"$ \\
\hline Pal $11^{e}$ & & 194514.4 & -080026 & 13.06 .2012 & $07: 32: 42$ & 180.0 & $\mathrm{IH}$ & $10 / 2$ \\
\hline$"$ & & $"$ & $"$ & $"$ & $07: 50: 37$ & 300.0 & $"$ & $"$ \\
\hline NGC $6838^{d}$ & M 71 & 195346.49 & +184645.1 & 29.05 .2006 & 09:14:32 & 17.2 & $\mathrm{D}$ & $8 / 4$ \\
\hline NGC $6864^{e}$ & M 75 & 200604.69 & -215516.2 & 27.07 .2012 & $05: 35: 32$ & 240.0 & $\mathrm{IH}$ & $10 / 2$ \\
\hline NGC $7006^{d}$ & & 210129.38 & +161114.4 & 30.05 .2006 & 09:08:54 & 1200.0 & $\mathrm{OH}$ & $5 / 9$ \\
\hline NGC $7078^{d}$ & M 15 & 212958.33 & +121001.2 & 29.05 .2006 & 09:30:56 & 47.7 & $\mathrm{IH}$ & $15 / 0$ \\
\hline
\end{tabular}

\subsection{Method - summary from Chapter 3}

Atmospheric parameters $\left(\mathrm{T}_{\text {eff }}, \log (q),[\mathrm{Fe} / \mathrm{H}],[\mathrm{Mg} / \mathrm{Fe}],[\alpha / \mathrm{Fe}]\right)$ were derived using full spectrum fitting with code ETOILE (Katz et al., 2011 and Katz, 2001). As reference library we chose one empirical (MILES, Sánchez-Blázquez et al., 2006) and one synthetic (COELHO, Coelho et al., 2005) to complement each other. We adopted the average results from MILES and COELHO as the final $\mathrm{T}_{\text {eff }}, \log (g),[\mathrm{Fe} / \mathrm{H}]$, and the others are the results without taking the average, i.e., $[\mathrm{Mg} / \mathrm{Fe}]$ from MILES results and $[\alpha / \mathrm{Fe}]$ from COELHO results. Before run the comparison of a given target spectrum with the entire library of reference spectra two important steps are needed: convolute all the library spectra to the same resolution of the target spectrum, and correct of radial velocities, measured also with ETOILE code. For detailed discussion of this method, arguments for each choice and validation with known stars and high-resolution analysis, see Chapter 3 . 


\subsection{Calibration of results}

\subsection{1 $[\mathrm{Fe} / \mathrm{H}]$ in Carretta scale}

Carretta et al. (2009) published a new metallicity scale for Milky Way globular clusters based on their observations of 19 clusters with UVES (Carretta et al., 2009) and GIRAFFE (Carretta et al., 2009) @ VLT/ESO. In our survey we have 13 objects in common with their sample, and our $[\mathrm{Fe} / \mathrm{H}]$ results are compared with theirs in Figure 4.3, where the names of the clusters are indicated. Black line is the one-to-one relation, the red line is the linear fit to the dots without considering the error bars, and blue dashed line is the second-order polynomial fit. The transformation of our results into Carretta's UVES results is given by one of the two equations below:

$$
[\mathrm{Fe} / \mathrm{H}]_{\mathrm{C} 09}=-0.12( \pm 0.05)+0.953( \pm 0.032)[\mathrm{Fe} / \mathrm{H}]_{\mathrm{D} 14}
$$

$$
[\mathrm{Fe} / \mathrm{H}]_{\mathrm{C} 09}=-0.13( \pm 0.11)+0.94( \pm 0.18)[\mathrm{Fe} / \mathrm{H}]_{\mathrm{D} 14}-0.00( \pm 0.06)[\mathrm{Fe} / \mathrm{H}]_{\mathrm{D} 14}^{2}
$$

From Figure 4.3 it is possible to see that second-order polynomial function coincides with the linear function, which is confirmed by the null second-order coefficient of Equation 4.2 and the similarity of the other coefficients between Equations 4.1 and 4.2 . The angular coefficient of the linear function is compatible with 1.0, which means that our measurements have no important trends with respect to Carretta's scale. Although the linear coefficient of Equation 4.1 is compatible with 0.0, our metallicities are slightly higher than those of Carretta et al. (2009), in particular for the more metal-rich clusters. In Table 4.2 we present our $[\mathrm{Fe} / \mathrm{H}]$ results and also those from Carretta et al. (2009).

Table 4.2 - Average $[\mathrm{Fe} / \mathrm{H}],[\mathrm{Mg} / \mathrm{H}]$ and radial velocities for the 13 globular clusters in common with Carretta et al. (2009) and number of selected member stars averaged in each cluster as specified in Table B.3. $[\mathrm{Fe} / \mathrm{H}]$ and $[\mathrm{Mg} / \mathrm{H}]$ from Carretta et al. (2009) are also presented for comparison.

\begin{tabular}{lc|ccc|c}
\hline \hline Cluster & Other & $\begin{array}{c}{[\mathrm{Fe} / \mathrm{H}]} \\
(\mathrm{MILES})\end{array}$ & $\begin{array}{c}{[\mathrm{Fe} / \mathrm{H}]} \\
(\mathrm{COELHO})\end{array}$ & $\begin{array}{c}{[\mathrm{Fe} / \mathrm{H}]} \\
\text { (average) }\end{array}$ & $\begin{array}{c}{[\mathrm{Fe} / \mathrm{H}]} \\
(\mathrm{Car09})\end{array}$ \\
\hline NGC 104 & \multirow{2}{*}{47 Tuc } & $-0.46 \pm 0.06$ & $-0.95 \pm 0.06$ & $-0.71 \pm 0.25$ & $-0.77 \pm 0.05$ \\
NGC 2808 & & $-0.67 \pm 0.09$ & $-1.21 \pm 0.06$ & $-1.06 \pm 0.22$ & $-1.15 \pm 0.07$ \\
\hline
\end{tabular}


Table 4.2 - continued.

\begin{tabular}{ll|ccc|c}
\hline \hline Cluster & Other & {$[\mathrm{Fe} / \mathrm{H}]$} & $\begin{array}{c}{[\mathrm{Fe} / \mathrm{H}]} \\
(\mathrm{MILES})\end{array}$ & $\begin{array}{c}{[\mathrm{Fe} / \mathrm{H}]} \\
\text { (C)erage) }\end{array}$ & $\begin{array}{c}{[\mathrm{Fe} / \mathrm{H}]} \\
(\mathrm{Car} 09)\end{array}$ \\
\hline names & & $-1.52 \pm 0.08$ & $-1.51 \pm 0.04$ & $-1.5126 \pm 0.0011$ & $-1.51 \pm 0.06$ \\
NGC 4590 & M 68 & $-1.86 \pm 0.15$ & $-2.23 \pm 0.05$ & $-2.20 \pm 0.06$ & $-2.26 \pm 0.05$ \\
NGC 5904 & M 5 & $-1.02 \pm 0.13$ & $-1.30 \pm 0.06$ & $-1.25 \pm 0.08$ & $-1.34 \pm 0.05$ \\
NGC 6121 & M 4 & $-0.80 \pm 0.13$ & $-1.05 \pm 0.06$ & $-1.01 \pm 0.07$ & $-1.17 \pm 0.05$ \\
NGC 6171 & M 107 & $-0.55 \pm 0.27$ & $-1.00 \pm 0.10$ & $-0.95 \pm 0.09$ & $-1.03 \pm 0.04$ \\
NGC 6254 & M 10 & $-1.59 \pm 0.08$ & $-1.55 \pm 0.04$ & $-1.558 \pm 0.015$ & $-1.57 \pm 0.06$ \\
NGC 6397 & & $-1.75 \pm 0.06$ & $-2.154 \pm 0.031$ & $-2.07 \pm 0.13$ & $-1.99 \pm 0.04$ \\
NGC 6441 & & $-0.18 \pm 0.09$ & $-0.71 \pm 0.10$ & $-0.41 \pm 0.26$ & $-0.43 \pm 0.06$ \\
NGC 6752 & & $-1.49 \pm 0.13$ & $-1.59 \pm 0.08$ & $-1.57 \pm 0.04$ & $-1.55 \pm 0.05$ \\
NGC 6838 & M 71 & $-0.44 \pm 0.08$ & $-0.87 \pm 0.08$ & $-0.66 \pm 0.22$ & $-0.83 \pm 0.06$ \\
NGC 7078 & M 15 & $-2.114 \pm 0.020$ & $-2.487 \pm 0.030$ & $-2.23 \pm 0.16$ & $-2.32 \pm 0.06$ \\
\hline
\end{tabular}

\subsubsection{Calibration of $[\mathrm{Mg} / \mathrm{Fe}]$ results}

We derived $[\mathrm{Mg} / \mathrm{Fe}]$ using MILES library (see Table B.3) which does not have metalrich stars with higher alpha-elements enhancement, typical in the Milky Way bulge. In previous subsection we showed that for the more metal-rich clusters we derived higher metallicities than those from Carretta et al. (2009). This can be explained as follows: for metal-rich alpha-enhanced target stars the features depth are fitted not by stars with higher $[\mathrm{Mg} / \mathrm{Fe}]$ but it is compensated by a higher $[\mathrm{Fe} / \mathrm{H}]$. Therefore, if we scale our $[\mathrm{Fe} / \mathrm{H}]$ as done above, we should correct the $[\mathrm{Mg} / \mathrm{Fe}]$ in terms of this difference.

Salaris et al. (1993) showed how $[\mathrm{Fe} / \mathrm{H}]$ and $[\alpha / \mathrm{Fe}]$ are combined to get the total metallicity $[\mathrm{M} / \mathrm{H}]$, expressed in the equation below.

$$
[\mathrm{M} / \mathrm{H}]=[\mathrm{Fe} / \mathrm{H}]+\log (0.638 f+0.362)
$$

where $\log (f)=[\alpha / \mathrm{Fe}]$

To find the difference in $[\mathrm{Mg} / \mathrm{Fe}]$ equivalent to the difference found in $[\mathrm{Fe} / \mathrm{H}]$ for a given spectrum of total metallicity $[\mathrm{M} / \mathrm{H}]$ we have to subtract Equation 4.3 for one case and the other as follows: 


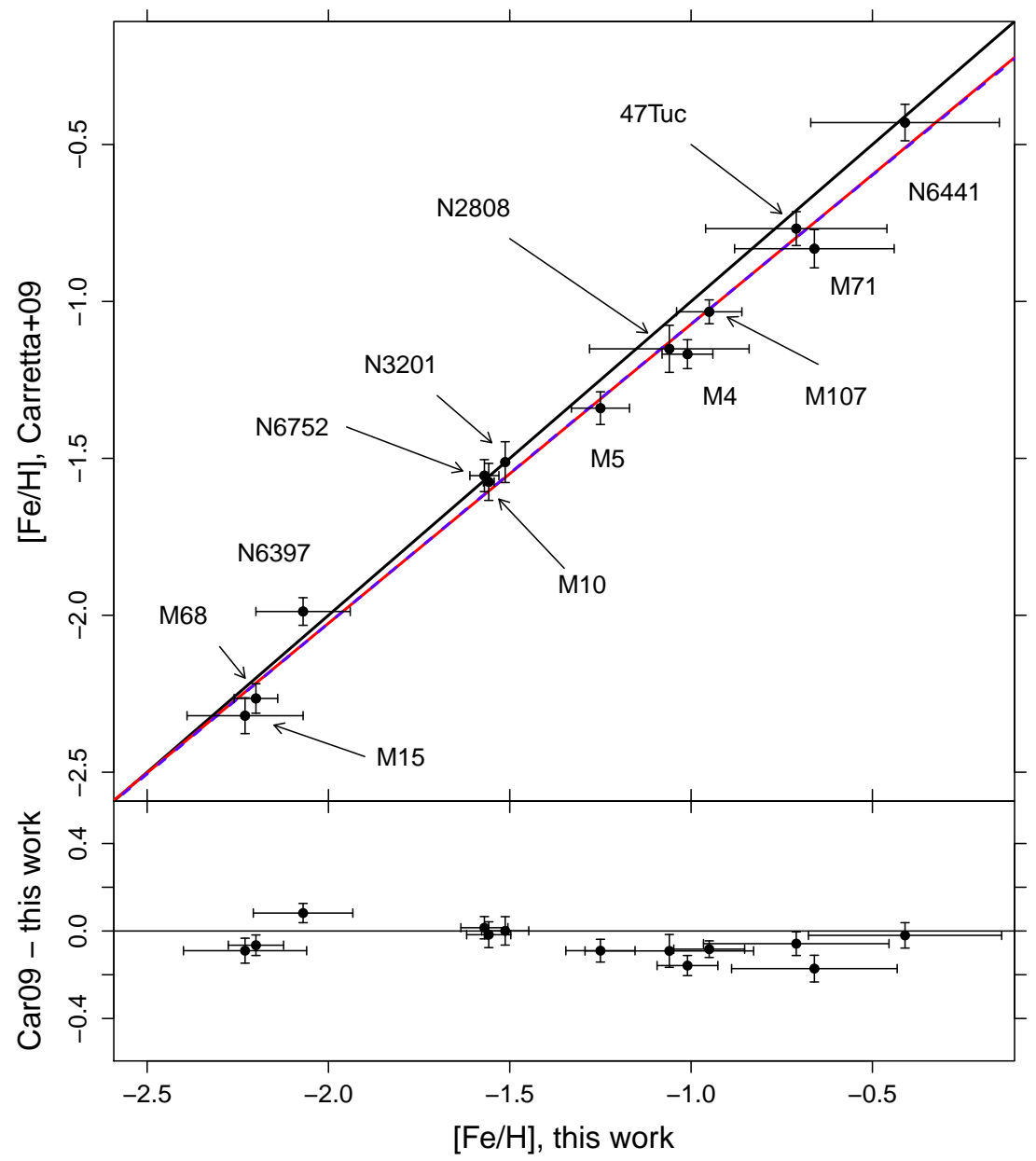

Figure 4.3: Comparison of $[\mathrm{Fe} / \mathrm{H}]$ with results of Carretta's scale for 13 clusters in common. Black line is the one-to-one relation and the red line is the linear fit to the dots without considering the error bars, and blue dashed line is the second-order polynomial fit.

$$
\begin{gathered}
{[\mathrm{M} / \mathrm{H}]_{\mathrm{C} 09}=[\mathrm{Fe} / \mathrm{H}]_{\mathrm{C} 09}+\log \left(0.638 f_{\mathrm{C} 09}+0.362\right)} \\
- \\
{[\mathrm{M} / \mathrm{H}]_{\mathrm{D} 14}=[\mathrm{Fe} / \mathrm{H}]_{\mathrm{D} 14}+\log \left(0.638 f_{\mathrm{D} 14}+0.362\right)}
\end{gathered}
$$

$$
\Delta[\mathrm{M} / \mathrm{H}]=\Delta[\mathrm{Fe} / \mathrm{H}]+\log \left[\left(0.638 f_{\mathrm{C} 09}+0.362\right) /\left(0.638 f_{\mathrm{D} 14}+0.362\right)\right]
$$

Manipulating this subtraction and assuming that $\Delta[\mathrm{M} / \mathrm{H}]=0$, and that the differential behaviour of $[\mathrm{Mg} / \mathrm{Fe}]$ is the same of $[\alpha / \mathrm{Fe}]$, it is possible to isolate $f_{\mathrm{C} 09}$ :

$$
f_{\mathrm{C} 09}=\left\{10^{\left[\log \left(0.638 f_{\mathrm{D} 14}+0.362\right)-\Delta[\mathrm{Fe} / H]\right]}-0.362\right\} / 0.638
$$


where for $\Delta[\mathrm{Fe} / \mathrm{H}]=0$, obviously $f_{\mathrm{C} 09}=f_{\mathrm{D} 14}$.

To find the final $[\mathrm{Mg} / \mathrm{Fe}]$ compatible with the calibrated $[\mathrm{Fe} / \mathrm{H}]$ found in Section 4.4.1]we determine the difference $\Delta[\mathrm{Fe} / \mathrm{H}]$ between the uncalibrated $[\mathrm{Fe} / \mathrm{H}]_{\mathrm{D} 14}$ and the calibrated $[\mathrm{Fe} / \mathrm{H}]_{\mathrm{C} 09}$. From Equation 4.3 we calculate $f_{\mathrm{D} 14}=10^{[\mathrm{Mg} / \mathrm{Fe}]_{\mathrm{D} 14}}$ using the uncalibrated $[\mathrm{Mg} / \mathrm{Fe}]_{\mathrm{D} 14}$. Lastly, we apply Equation 4.5 to find the calibrated $[\mathrm{Mg} / \mathrm{Fe}]_{\mathrm{C} 09}=\log \left(f_{\mathrm{C} 09}\right)$.

\subsection{Discussion on abundances results}

Final parameters $\left([\mathrm{Fe} / \mathrm{H}],[\mathrm{Mg} / \mathrm{Fe}],[\alpha / \mathrm{Fe}]\right.$ and $\left.v_{\text {helio }}\right)$ for the 51 clusters are presented in Table 4.3. The average of $[\mathrm{Fe} / \mathrm{H}]$ from individual stars using MILES and COELHO are in the Table, as well as the combined average of MILES and COELHO, that we assume as our results. The final values of $[\mathrm{Fe} / \mathrm{H}]$ are those calibrated as described in Section 4.4.1, Average $[\mathrm{Mg} / \mathrm{Fe}]$ and $[\alpha / \mathrm{Fe}]$ of individual stars were calculated with results in Table B.3. The final values of $[\mathrm{Mg} / \mathrm{Fe}]$ are those calibrated following the procedures of Section 4.4.2. It is the first time that $[\mathrm{Fe} / \mathrm{H}]$ and $[\mathrm{Mg} / \mathrm{Fe}]$ derived in a consistent way are published for such a huge sample of globular clusters (51 objects), almost 1/3 of the total number of catalogued clusters (157 as compiled by Harris, 1996, 2010 edition).

In Figure 4.4 we compare our calibrated results of $[\mathrm{Fe} / \mathrm{H}]$ with those from Carretta et al. (2009) for the 51 Milky Way globular clusters of our FORS2 survey (see Table 4.3). In the main panel a one-to-one line is plotted for reference showing a good agreement of both sets of $[\mathrm{Fe} / \mathrm{H}]$, with no trends and a dispersion of $\sigma=0.16 \mathrm{dex}$, as supported by the residuals in the bottom panel. The 13 clusters used to scale our [Fe/H] to Carretta's scale in Section 4.4 .1 are highlighted as red triangles to show that the application of Equation 4.1 scales their metallicities with low dispersion in comparison with the other clusters $(\sigma=0.07 \mathrm{dex})$. There are six clusters in our sample that were not scaled by Carretta et al. (2009), therefore we plotted them with $[\mathrm{Fe} / \mathrm{H}]$ from our work only in the main plot in blue. The names of the clusters are indicated in the Figure and our work publish for the first time homogeneous metallicities for these objects already scaled to Carretta's scale. 
Table 4.3 - Final parameters for the 51 clusters $[\mathrm{Fe} / \mathrm{H}],[\mathrm{Mg} / \mathrm{Fe}],[\alpha / \mathrm{Fe}]$ and $v_{\text {helio. }}$ Values labelled with (MILES), (COELHO) and $v_{\text {helio }}$ are averages of individual stars from Tables B.1 and B.3. Car09 are the metallicities of Carretta et al. (2009). [Fe/H] (calibrated) is determined in Section 4.4.1 and [Mg/Fe] (calibrated) in Section 4.4 .2 .

\begin{tabular}{|c|c|c|c|c|c|c|c|c|c|c|}
\hline Cluster & $\begin{array}{l}\text { Other } \\
\text { names }\end{array}$ & $\begin{array}{c}v_{\text {helio }} \\
(\mathrm{km} / \mathrm{s})\end{array}$ & $\begin{array}{c}{[\mathrm{Fe} / \mathrm{H}]} \\
(\mathrm{MILES})\end{array}$ & $\begin{array}{c}{[\mathrm{Fe} / \mathrm{H}]} \\
(\mathrm{COELHO})\end{array}$ & $\begin{array}{c}{[\mathrm{Fe} / \mathrm{H}]} \\
\text { (average) }\end{array}$ & $\begin{array}{c}{[\mathrm{Fe} / \mathrm{H}]} \\
\text { (calibrated) }\end{array}$ & $\begin{array}{c}{[\mathrm{Fe} / \mathrm{H}]} \\
(\mathrm{Car} 09)\end{array}$ & $\begin{array}{c}{[\mathrm{Mg} / \mathrm{Fe}]} \\
(\mathrm{MILES})\end{array}$ & $\begin{array}{c}{[\mathrm{Mg} / \mathrm{Fe}]} \\
\text { (calibrated) }\end{array}$ & $\begin{array}{c}{[\alpha / \mathrm{Fe}]} \\
(\mathrm{COELHO})\end{array}$ \\
\hline NGC 104 & 47 Tuc & $-48 \pm 10$ & $-0.46 \pm 0.06$ & $-0.95 \pm 0.06$ & $-0.71 \pm 0.25$ & $-0.78 \pm 0.24$ & $-0.76 \pm 0.02(1)$ & $0.26 \pm 0.05$ & $0.37 \pm 0.05$ & $0.264 \pm 0.027$ \\
\hline NGC 2298 & & $134 \pm 14$ & $-1.68 \pm 0.16$ & $-1.98 \pm 0.05$ & $-1.95 \pm 0.05$ & $-1.98 \pm 0.09$ & $-1.96 \pm 0.04(1)$ & $0.44 \pm 0.06$ & $0.47 \pm 0.06$ & $0.19 \pm 0.06$ \\
\hline NGC 2808 & & $96 \pm 25$ & $-0.67 \pm 0.09$ & $-1.21 \pm 0.06$ & $-1.06 \pm 0.22$ & $-1.13 \pm 0.22$ & $-1.18 \pm 0.04(1)$ & $0.22 \pm 0.04$ & $0.31 \pm 0.04$ & $0.240 \pm 0.031$ \\
\hline NGC 3201 & & $472 \pm 19$ & $-1.52 \pm 0.08$ & $-1.51 \pm 0.04$ & $-1.5126 \pm 0.0011$ & $-1.56 \pm 0.07$ & $-1.51 \pm 0.02(1)$ & $0.430 \pm 0.040$ & $0.49 \pm 0.04$ & $0.219 \pm 0.035$ \\
\hline NGC 4372 & & $63 \pm 10$ & $-1.83 \pm 0.12$ & $-2.34 \pm 0.07$ & $-2.22 \pm 0.18$ & $-2.24 \pm 0.19$ & $-2.19 \pm 0.08$ (1) & $0.40 \pm 0.04$ & $0.42 \pm 0.04$ & $0.21 \pm 0.04$ \\
\hline Rup 106 & & $-47 \pm 12$ & $-1.60 \pm 0.12$ & $-1.54 \pm 0.05$ & $-1.545 \pm 0.013$ & $-1.59 \pm 0.07$ & $-1.78 \pm 0.08$ & $0.45 \pm 0.05$ & $0.51 \pm 0.05$ & $0.12 \pm 0.035$ \\
\hline NGC 4590 & M 68 & $-92 \pm 25$ & $-1.86 \pm 0.15$ & $-2.23 \pm 0.05$ & $-2.20 \pm 0.06$ & $-2.22 \pm 0.10$ & $-2.27 \pm 0.04(1)$ & $0.39 \pm 0.05$ & $0.41 \pm 0.05$ & $0.19 \pm 0.05$ \\
\hline NGC 5634 & & $-29 \pm 42$ & $-1.60 \pm 0.10$ & $-1.82 \pm 0.07$ & $-1.75 \pm 0.09$ & $-1.79 \pm 0.11$ & $-1.93 \pm 0.09(2)$ & $0.43 \pm 0.05$ & $0.47 \pm 0.05$ & $0.20 \pm 0.04$ \\
\hline NGC 5694 & & $-150 \pm 9$ & $-1.76 \pm 0.12$ & $-2.00 \pm 0.04$ & $-1.98 \pm 0.04$ & $-2.01 \pm 0.09$ & $-2.02 \pm 0.07(1)$ & $0.41 \pm 0.05$ & $0.44 \pm 0.05$ & $0.17 \pm 0.04$ \\
\hline NGC 5824 & & $-35 \pm 12$ & $-1.95 \pm 0.05$ & $-2.011 \pm 0.031$ & $-1.995 \pm 0.024$ & $-2.02 \pm 0.08$ & $-1.94 \pm 0.14(1)$ & $0.436 \pm 0.030$ & $0.467 \pm 0.030$ & $0.240 \pm 0.029$ \\
\hline NGC 5897 & & $88 \pm 13$ & $-1.63 \pm 0.12$ & $-2.02 \pm 0.04$ & $-1.97 \pm 0.08$ & $-2.00 \pm 0.11$ & $-1.90 \pm 0.06(1)$ & $0.43 \pm 0.05$ & $0.46 \pm 0.05$ & $0.23 \pm 0.04$ \\
\hline NGC 5904 & M 5 & $46 \pm 7$ & $-1.02 \pm 0.13$ & $-1.30 \pm 0.06$ & $-1.25 \pm 0.08$ & $-1.31 \pm 0.10$ & $-1.33 \pm 0.02(1)$ & $0.35 \pm 0.05$ & $0.42 \pm 0.05$ & $0.24 \pm 0.04$ \\
\hline NGC 5927 & & $-99 \pm 11$ & $-0.165 \pm 0.026$ & $-0.82 \pm 0.10$ & $-0.21 \pm 0.08$ & $-0.32 \pm 0.09$ & $-0.29 \pm 0.07(1)$ & $0.09 \pm 0.04$ & $0.24 \pm 0.04$ & $0.30 \pm 0.04$ \\
\hline NGC 5946 & & $134 \pm 29$ & $-1.50 \pm 0.17$ & $-1.55 \pm 0.07$ & $-1.544 \pm 0.015$ & $-1.59 \pm 0.07$ & $-1.29 \pm 0.14(1)$ & $0.42 \pm 0.07$ & $0.48 \pm 0.07$ & $0.22 \pm 0.05$ \\
\hline BH 176 & & $-6 \pm 14$ & $-0.08 \pm 0.04$ & $-0.07 \pm 0.06$ & $-0.078 \pm 0.007$ & $-0.19 \pm 0.05$ & - & $0.10 \pm 0.05$ & $0.26 \pm 0.05$ & $0.20 \pm 0.029$ \\
\hline Lynga 7 & BH 184 & $-13 \pm 28$ & $-0.41 \pm 0.13$ & $-0.87 \pm 0.15$ & $-0.61 \pm 0.23$ & $-0.70 \pm 0.23$ & - & $0.21 \pm 0.11$ & $0.33 \pm 0.11$ & $0.27 \pm 0.06$ \\
\hline Pal 14 & AvdB & $45 \pm 9$ & $-1.07 \pm 0.18$ & $-1.27 \pm 0.10$ & $-1.22 \pm 0.07$ & $-1.28 \pm 0.09$ & $-1.63 \pm 0.08$ & $0.32 \pm 0.06$ & $0.40 \pm 0.06$ & $0.24 \pm 0.04$ \\
\hline NGC 6121 & M 4 & $23 \pm 35$ & $-0.80 \pm 0.13$ & $-1.05 \pm 0.06$ & $-1.01 \pm 0.07$ & $-1.08 \pm 0.09$ & $-1.18 \pm 0.02(1)$ & $0.35 \pm 0.06$ & $0.44 \pm 0.06$ & $0.27 \pm 0.04$ \\
\hline NGC 6171 & M 107 & $-122 \pm 0$ & $-0.55 \pm 0.27$ & $-1.00 \pm 0.10$ & $-0.95 \pm 0.09$ & $-1.02 \pm 0.10$ & $-1.03 \pm 0.02(1)$ & $0.28 \pm 0.21$ & $0.37 \pm 0.21$ & $0.20 \pm 0.14$ \\
\hline NGC 6254 & M 10 & $43 \pm 34$ & $-1.59 \pm 0.08$ & $-1.55 \pm 0.04$ & $-1.558 \pm 0.015$ & $-1.60 \pm 0.07$ & $-1.57 \pm 0.02(1)$ & $0.436 \pm 0.034$ & $0.492 \pm 0.034$ & $0.214 \pm 0.035$ \\
\hline NGC 6284 & & $35 \pm 21$ & $-0.84 \pm 0.15$ & $-1.12 \pm 0.07$ & $-1.07 \pm 0.09$ & $-1.14 \pm 0.10$ & $-1.31 \pm 0.09(2)$ & $0.33 \pm 0.06$ & $0.42 \pm 0.06$ & $0.27 \pm 0.04$ \\
\hline NGC 6316 & & $81 \pm 40$ & $-0.28 \pm 0.08$ & $-0.84 \pm 0.10$ & $-0.50 \pm 0.27$ & $-0.60 \pm 0.26$ & $-0.36 \pm 0.14(1)$ & $0.11 \pm 0.06$ & $0.24 \pm 0.06$ & $0.303 \pm 0.031$ \\
\hline NGC 6356 & & $36 \pm 41$ & $-0.30 \pm 0.06$ & $-0.91 \pm 0.07$ & $-0.55 \pm 0.30$ & $-0.64 \pm 0.29$ & $-0.35 \pm 0.14(1)$ & $0.12 \pm 0.04$ & $0.25 \pm 0.04$ & $0.303 \pm 0.024$ \\
\hline NGC 6355 & & $-215 \pm 7$ & $-1.38 \pm 0.09$ & $-1.54 \pm 0.08$ & $-1.46 \pm 0.08$ & $-1.51 \pm 0.10$ & $-1.33 \pm 0.14(1)$ & $0.264 \pm 0.010$ & $0.330 \pm 0.010$ & $0.27 \pm 0.05$ \\
\hline
\end{tabular}


Table 4.3 - continued.

\begin{tabular}{|c|c|c|c|c|c|c|c|c|c|c|}
\hline Cluster & $\begin{array}{l}\text { Other } \\
\text { names }\end{array}$ & $\begin{array}{c}v_{\text {helio }} \\
(\mathrm{km} / \mathrm{s})\end{array}$ & $\begin{array}{c}{[\mathrm{Fe} / \mathrm{H}]} \\
(\mathrm{MILES})\end{array}$ & $\begin{array}{c}{[\mathrm{Fe} / \mathrm{H}]} \\
(\mathrm{COELHO})\end{array}$ & $\begin{array}{c}{[\mathrm{Fe} / \mathrm{H}]} \\
\text { (average) }\end{array}$ & $\begin{array}{c}{[\mathrm{Fe} / \mathrm{H}]} \\
\text { (calibrated) }\end{array}$ & $\begin{array}{c}{[\mathrm{Fe} / \mathrm{H}]} \\
(\mathrm{Car} 09)\end{array}$ & $\begin{array}{l}{[\mathrm{Mg} / \mathrm{Fe}]} \\
(\mathrm{MILES})\end{array}$ & $\begin{array}{c}{[\mathrm{Mg} / \mathrm{Fe}]} \\
\text { (calibrated) }\end{array}$ & $\begin{array}{c}{[\alpha / \mathrm{Fe}]} \\
(\mathrm{COELHO})\end{array}$ \\
\hline NGC 6352 & & $-138 \pm 36$ & $-0.41 \pm 0.06$ & $-0.76 \pm 0.07$ & $-0.54 \pm 0.17$ & $-0.63 \pm 0.17$ & $-0.62 \pm 0.05(1)$ & $0.15 \pm 0.05$ & $0.28 \pm 0.05$ & $0.304 \pm 0.025$ \\
\hline NGC 6366 & & $-137 \pm 54$ & $-0.41 \pm 0.07$ & $-0.81 \pm 0.07$ & $-0.61 \pm 0.20$ & $-0.70 \pm 0.20$ & $-0.59 \pm 0.08$ & $0.22 \pm 0.05$ & $0.34 \pm 0.05$ & $0.303 \pm 0.023$ \\
\hline HP 1 & BH 229 & $54 \pm 5$ & $-0.70 \pm 0.11$ & $-1.49 \pm 0.09$ & $-1.2 \pm 0.4$ & $-1.26 \pm 0.39$ & $-1.57 \pm 0.09$ & $0.33 \pm 0.07$ & $0.41 \pm 0.07$ & $0.28 \pm 0.04$ \\
\hline NGC 6401 & & $-120 \pm 17$ & $-0.59 \pm 0.14$ & $-1.34 \pm 0.09$ & $-1.12 \pm 0.31$ & $-1.19 \pm 0.30$ & $-1.01 \pm 0.14$ & $0.32 \pm 0.08$ & $0.40 \pm 0.08$ & $0.269 \pm 0.041$ \\
\hline NGC 6397 & & $-27 \pm 55$ & $-1.75 \pm 0.06$ & $-2.154 \pm 0.031$ & $-2.07 \pm 0.13$ & $-2.09 \pm 0.15$ & $-1.99 \pm 0.02(1)$ & $0.402 \pm 0.030$ & $0.430 \pm 0.030$ & $0.229 \pm 0.028$ \\
\hline Pal 6 & & $177 \pm 5$ & $-0.27 \pm 0.14$ & $-1.66 \pm 0.17$ & $-0.8 \pm 0.7$ & $-0.88 \pm 0.67$ & $-1.06 \pm 0.09$ & $0.14 \pm 0.10$ & $0.25 \pm 0.10$ & $0.28 \pm 0.05$ \\
\hline NGC 6426 & & $-242 \pm 11$ & $-2.03 \pm 0.11$ & $-2.46 \pm 0.05$ & $-2.39 \pm 0.11$ & $-2.40 \pm 0.14$ & - & $0.38 \pm 0.06$ & $0.39 \pm 0.06$ & $0.24 \pm 0.05$ \\
\hline NGC 6440 & & $-59 \pm 26$ & $-0.03 \pm 0.06$ & $-0.80 \pm 0.10$ & $-0.24 \pm 0.30$ & $-0.35 \pm 0.29$ & $-0.20 \pm 0.14$ & $0.11 \pm 0.04$ & $0.26 \pm 0.04$ & $0.314 \pm 0.031$ \\
\hline NGC 6441 & & $-6 \pm 32$ & $-0.18 \pm 0.09$ & $-0.71 \pm 0.10$ & $-0.41 \pm 0.26$ & $-0.51 \pm 0.25$ & $-0.44 \pm 0.07$ & $0.11 \pm 0.06$ & $0.25 \pm 0.06$ & $0.26 \pm 0.04$ \\
\hline NGC 6453 & & $-153 \pm 11$ & $-1.45 \pm 0.18$ & $-1.57 \pm 0.10$ & $-1.54 \pm 0.04$ & $-1.59 \pm 0.08$ & $-1.48 \pm 0.14$ & $0.42 \pm 0.09$ & $0.48 \pm 0.09$ & $0.16 \pm 0.06$ \\
\hline Djorg 2 & ESO456SC38 & $-150 \pm 28$ & $-0.50 \pm 0.12$ & $-1.19 \pm 0.14$ & $-0.79 \pm 0.33$ & $-0.87 \pm 0.32$ & - & $0.28 \pm 0.10$ & $0.38 \pm 0.10$ & $0.27 \pm 0.05$ \\
\hline NGC 6528 & & $185 \pm 10$ & $-0.07 \pm 0.10$ & $-0.18 \pm 0.08$ & $-0.13 \pm 0.05$ & $-0.24 \pm 0.07$ & $+0.07 \pm 0.08$ & $0.05 \pm 0.09$ & $0.21 \pm 0.09$ & $0.26 \pm 0.05$ \\
\hline NGC 6539 & & $30 \pm 18$ & $-0.23 \pm 0.09$ & $-0.89 \pm 0.09$ & $-0.55 \pm 0.33$ & $-0.64 \pm 0.32$ & $-0.53 \pm 0.14$ & $0.16 \pm 0.07$ & $0.29 \pm 0.07$ & $0.302 \pm 0.028$ \\
\hline NGC 6553 & & $6 \pm 8$ & $-0.125 \pm 0.009$ & $-0.55 \pm 0.07$ & $-0.133 \pm 0.017$ & $-0.25 \pm 0.05$ & $-0.16 \pm 0.06(1)$ & $0.107 \pm 0.009$ & $0.263 \pm 0.009$ & $0.302 \pm 0.025$ \\
\hline NGC 6558 & & $-210 \pm 16$ & $-0.88 \pm 0.20$ & $-1.02 \pm 0.05$ & $-1.012 \pm 0.013$ & $-1.08 \pm 0.06$ & $-1.37 \pm 0.14$ & $0.26 \pm 0.06$ & $0.35 \pm 0.06$ & $0.23 \pm 0.06$ \\
\hline IC 1276 & Pal 7 & $155 \pm 15$ & $-0.13 \pm 0.06$ & $-1.11 \pm 0.07$ & $-0.56 \pm 0.49$ & $-0.65 \pm 0.47$ & $-0.65 \pm 0.09(2)$ & $0.09 \pm 0.05$ & $0.22 \pm 0.05$ & $0.304 \pm 0.026$ \\
\hline NGC 6569 & & $-51 \pm 9$ & $-0.53 \pm 0.09$ & $-0.85 \pm 0.11$ & $-0.66 \pm 0.15$ & $-0.75 \pm 0.15$ & $-0.72 \pm 0.14(1)$ & $0.30 \pm 0.07$ & $0.41 \pm 0.07$ & $0.29 \pm 0.032$ \\
\hline NGC 6656 & M 22 & $-152 \pm 25$ & $-1.77 \pm 0.05$ & $-1.945 \pm 0.020$ & $-1.92 \pm 0.05$ & $-1.95 \pm 0.09$ & $-1.70 \pm 0.08$ & $0.501 \pm 0.008$ & $0.536 \pm 0.008$ & $0.225 \pm 0.018$ \\
\hline NGC 6749 & & $-66 \pm 8$ & $-0.64 \pm 0.15$ & $-2.14 \pm 0.11$ & $-1.59 \pm 0.70$ & $-1.63 \pm 0.67$ & $-1.62 \pm 0.09(2)$ & $0.34 \pm 0.10$ & $0.40 \pm 0.10$ & $0.17 \pm 0.06$ \\
\hline NGC 6752 & & $-28 \pm 7$ & $-1.49 \pm 0.13$ & $-1.59 \pm 0.08$ & $-1.57 \pm 0.04$ & $-1.62 \pm 0.08$ & $-1.55 \pm 0.01$ & $0.47 \pm 0.06$ & $0.52 \pm 0.06$ & $0.22 \pm 0.05$ \\
\hline Pal 10 & & $-38 \pm 17$ & $-0.080 \pm 0.039$ & $-0.53 \pm 0.05$ & $-0.24 \pm 0.20$ & $-0.35 \pm 0.20$ & - & $0.118 \pm 0.010$ & $0.266 \pm 0.010$ & $0.273 \pm 0.034$ \\
\hline Terzan 8 & & $135 \pm 19$ & $-1.76 \pm 0.07$ & $-2.18 \pm 0.05$ & $-2.06 \pm 0.17$ & $-2.08 \pm 0.18$ & - & $0.41 \pm 0.04$ & $0.44 \pm 0.04$ & $0.21 \pm 0.04$ \\
\hline Pal 11 & & $-81 \pm 15$ & $-0.22 \pm 0.05$ & $-0.62 \pm 0.08$ & $-0.35 \pm 0.17$ & $-0.45 \pm 0.17$ & $-0.45 \pm 0.08$ & $0.12 \pm 0.05$ & $0.26 \pm 0.05$ & $0.303 \pm 0.030$ \\
\hline NGC 6838 & M 71 & $-42 \pm 18$ & $-0.48 \pm 0.08$ & $-0.77 \pm 0.08$ & $-0.63 \pm 0.15$ & $-0.72 \pm 0.15$ & $-0.82 \pm 0.02(1)$ & $0.25 \pm 0.07$ & $0.37 \pm 0.07$ & $0.293 \pm 0.032$ \\
\hline NGC 6864 & M 75 & $-190 \pm 20$ & $-0.75 \pm 0.10$ & $-1.09 \pm 0.06$ & $-1.00 \pm 0.13$ & $-1.07 \pm 0.14$ & $-1.29 \pm 0.14$ & $0.35 \pm 0.05$ & $0.44 \pm 0.05$ & $0.222 \pm 0.035$ \\
\hline NGC 7006 & & $-391 \pm 24$ & $-1.54 \pm 0.19$ & $-1.74 \pm 0.11$ & $-1.69 \pm 0.07$ & $-1.73 \pm 0.10$ & $-1.46 \pm 0.06$ & $0.42 \pm 0.07$ & $0.47 \pm 0.07$ & $0.25 \pm 0.05$ \\
\hline NGC 7078 & M 15 & $-159 \pm 40$ & $-2.114 \pm 0.020$ & $-2.487 \pm 0.030$ & $-2.23 \pm 0.16$ & $-2.24 \pm 0.18$ & $-2.33 \pm 0.02(1)$ & $0.410 \pm 0.034$ & $0.428 \pm 0.034$ & $0.237 \pm 0.030$ \\
\hline
\end{tabular}




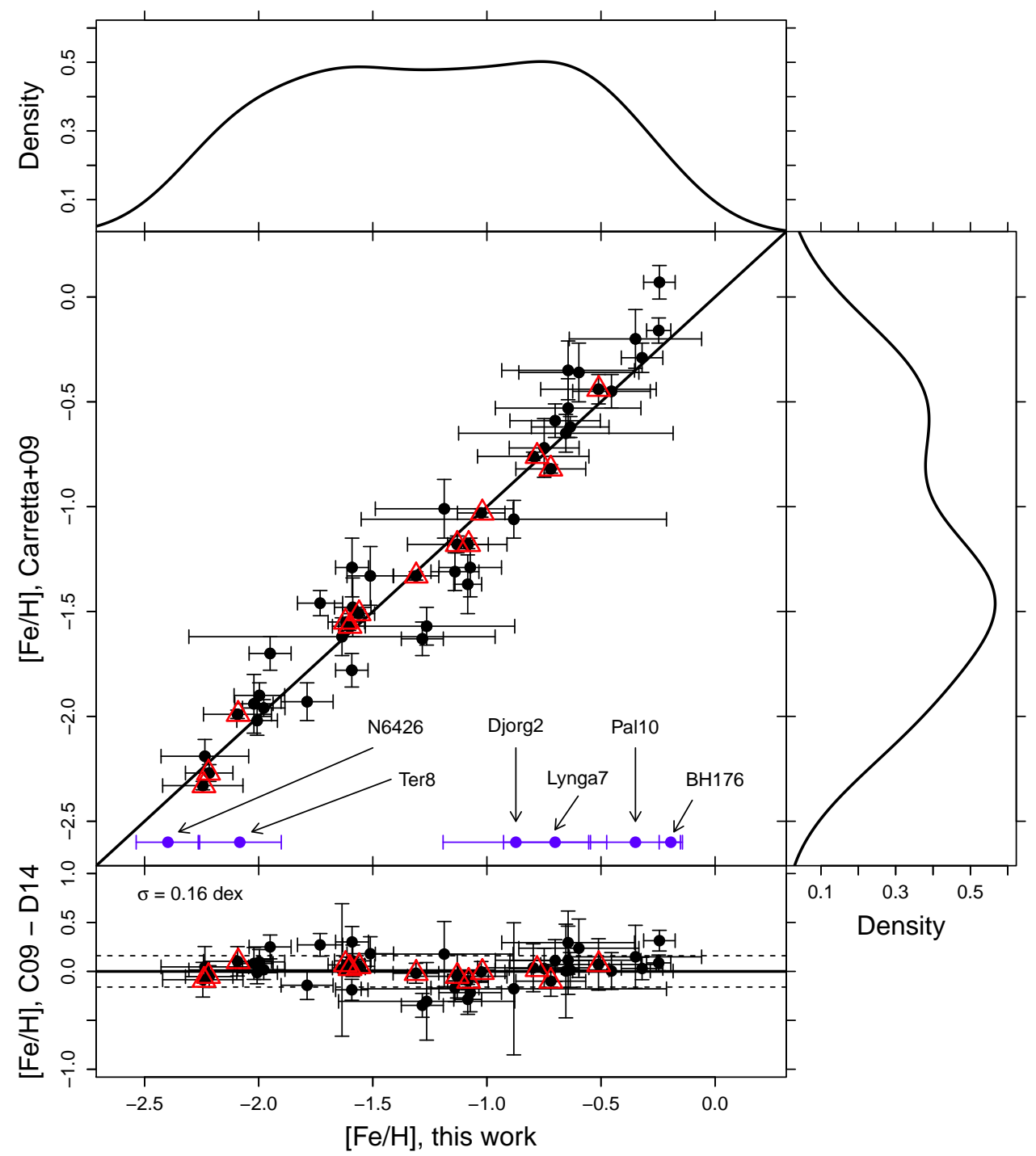

Figure 4.4: Comparison of calibrated $[\mathrm{Fe} / \mathrm{H}]$ from this work (D14) with those from (Carretta et al., 2009, C09) for the 51 clusters of our survey using values of Table 4.3. Main panel: red triangles emphasize the 13 clusters used for calibration in Section 4.4.1. Blue points are the six clusters not calibrated bv Carretta et al. (2009) and analysed in this work. One-to-one line is plotted for reference. Bottom panel: residuals of the comparison, with standard deviation equal to 0.16 dex. Top and right panels: smoothed histogram of metallicities from this work and from Carretta, respectively, only for the 45 clusters in common.

Another subproduct of this comparison is the metallicity distribution of the Milky Way for the 45 clusters in common with Carretta et al. (2009). This group of clusters represents $\sim 30 \%$ of the total number of catalogued objects in our Galaxy by (Harris, 1996, 2010 edition), which is significant to draw the known bimodal metallicity distribution of the Milky Way globular cluster population. In the right panel of Figure 4.4 we show the metallicity distribution based on Carretta et al. (2009) values, while in the upper panel our results are displayed. In both cases is possible to identify two peaks, although this 
is clearer using Carretta's results since they are based on high-resolution spectroscopic analysis and their uncertainties are lower than ours.

\subsubsection{Chemical evolution of the Milky Way}

As mentioned in Chapter 1 the ratio $[\alpha / \mathrm{Fe}]$ plotted against $[\mathrm{Fe} / \mathrm{H}]$ is an evidence of how efficient the star formation in the Galaxy was. Supernovae type II (SNII) explode quickly after the formation of the progenitor massive star and release $\alpha$-elements and iron to the interstellar medium. Later, supernovae type Ia (SNIa) explode liberating iron, which decrease the ratio $[\alpha / \mathrm{Fe}]$. Magnesium is one of the $\alpha$-elements and represents well these processes. Increasing values of $[\mathrm{Fe} / \mathrm{H}]$ represents many generations of stars enriched by the material expelled by SNII, lower values representing the first stars and higher values standing for more recent objects.

In Figure 4.5 we show our (final calibrated) results of $[\mathrm{Fe} / \mathrm{H}]$ and $[\mathrm{Mg} / \mathrm{Fe}]$ for the 51 clusters (see Table 4.3), splitted according to their populations: bulge, disc, inner halo, outer halo (see Table 4.1). As a reference we plot in the respective panels of Figure 4.5 also field stars from Gonzalez et al. (2011) for bulge, Bensby et al. (2005) for thin disc, Reddy et al. (2006) for thick disc, Roederer (2009) for inner and outer halo. Clearly globular clusters abundances trends follow field stars values, and the range of metallicities covered by each population is equivalent also to the respective field stars population. We note that bulge and disc stars reach high metallicities, above solar, and the cluster populations are mostly more metal-poor than our Sun. These plots are indicatives that globular clusters and field stars populations were formed in the same processes in all Galactic components or, at least, they were formed in processes with similar star formation rate, given by the drop of $[\mathrm{Mg} / \mathrm{Fe}]$ at about $[\mathrm{Fe} / \mathrm{H}] \sim-1.0$.

From Figure 4.5 we conclude that SNII were able to enrich stellar generations up to $[\mathrm{Fe} / \mathrm{H}] \sim-1.0$ before SNIa start to become important, which typically happens $\sim 1 \mathrm{Gyr}$ after the formation of the progenitor stars. Matteucci and Recchi (2001) discussed in detail the relationship of the timescale of SNIa events and the distribution of $[\alpha / \mathrm{Fe}]$ vs. $[\mathrm{Fe} / \mathrm{H}]$. They concluded that the timescale of 1 Gyr for the knee shown in Figure 4.5 is specific for solar neighbourhood stars, and it is not a general rule. However the four Galactic components seem to share the same $[\mathrm{Fe} / \mathrm{H}]_{\text {knee }} \sim-1.0$ (see Figure 4.5), and we assume that the SNIa timescale is approximately the same for all components. 


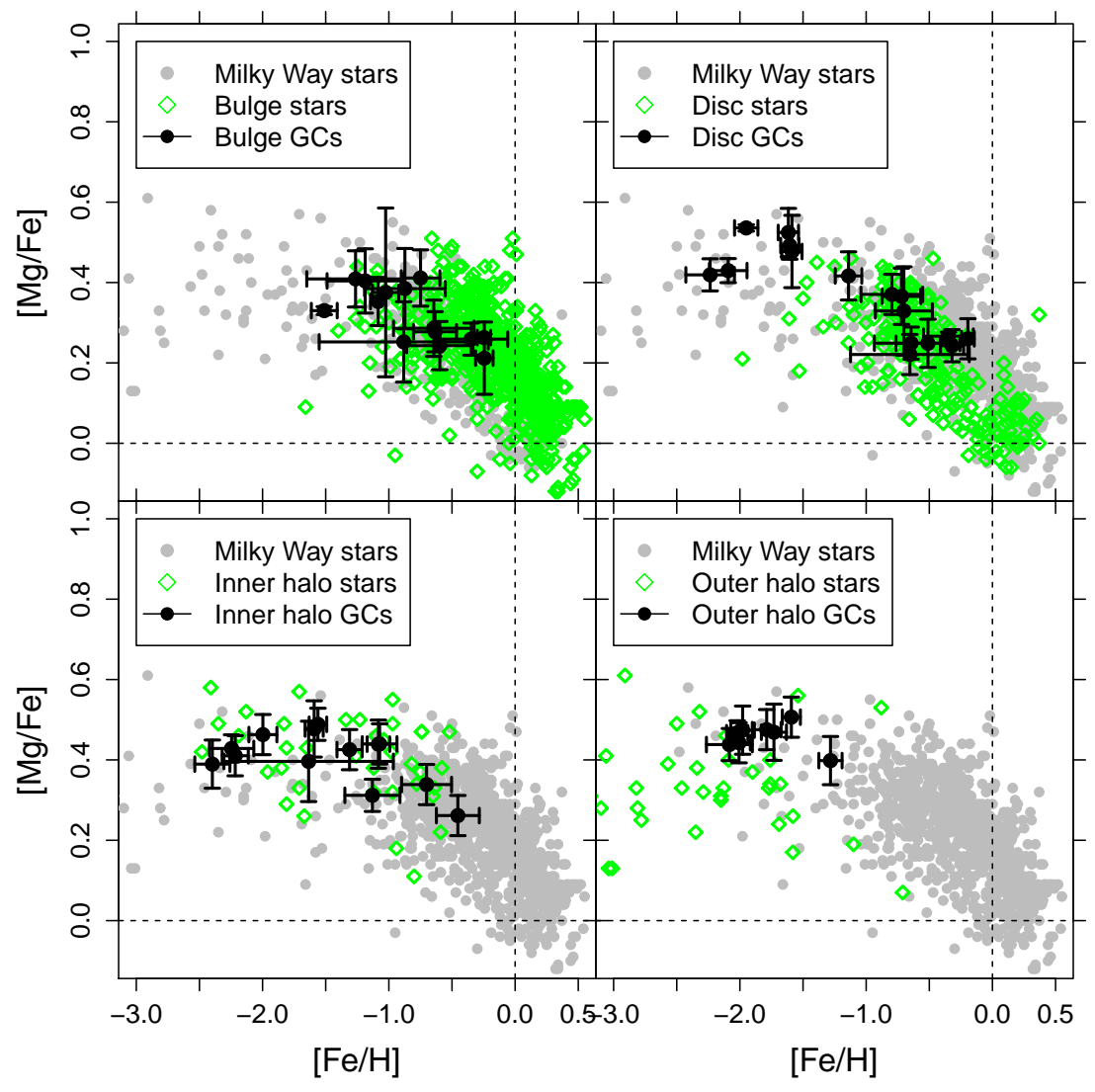

Figure 4.5: Chemical evolution of the Milky Way in terms of $[\mathrm{Mg} / \mathrm{Fe}]$ vs. $[\mathrm{Fe} / \mathrm{H}]$ for 51 globular clusters from this work. Field stars are from Gonzalez et al. (2011) for bulge, Bensby et al. (2005) for thin disc, Reddy et al. (2006) for thick disc. Roederer (2009) for inner and outer halo.

VandenBerg et al. (2013) have derived the ages of 55 Milky Way globular clusters in a same scale, of which 17 are in common with our sample. In Figure 4.6 we compare ages with $[\mathrm{Fe} / \mathrm{H}]$ and $[\mathrm{Mg} / \mathrm{Fe}]$ for the common clusters in the left and right panels, respectively. In the left panel we can see that the first clusters were formed between 12-13 Gyr ago, and the next generations were enriched up to $[\mathrm{Fe} / \mathrm{H}] \sim-1.0$ about 1 Gyr later. The right panel shows a similar behaviour than that of Figure 4.5, with a knee at about 12 Gyr ago. The oldest objects are $\sim 1$ Gyr older than that. These plots indicate that the timescale of 1 Gyr for SNIa to become important is a reasonable value for Milky Way globular clusters populations.

\subsubsection{Origin of globular clusters}

There is further discussions about Figure 4.6 concerning the formation/evolution scenario of the clusters. VandenBerg et al. (2013) noted that for $[\mathrm{Fe} / \mathrm{H}]>-1.7$ there is a visual impression of a split between halo (red) and bulge/disc (blue) clusters with 

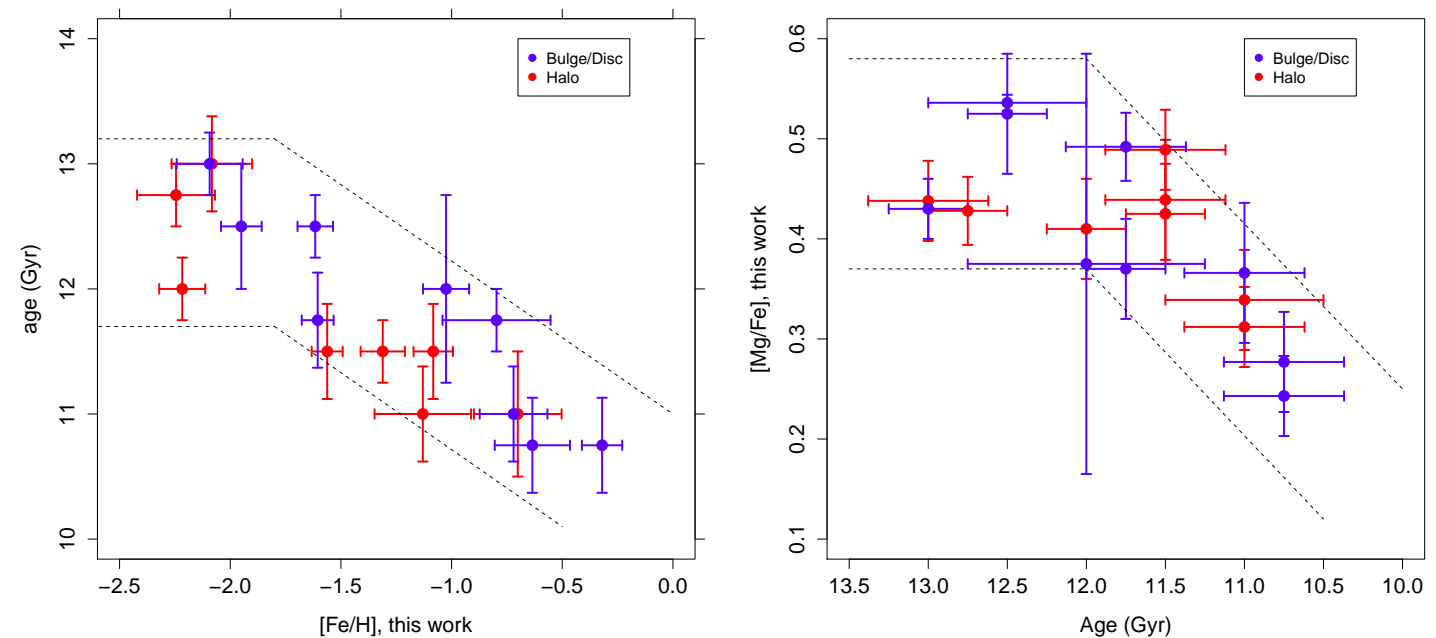

Figure 4.6: Age vs. $[\mathrm{Fe} / \mathrm{H}]$ and $[\mathrm{Mg} / \mathrm{Fe}]$ for 17 clusters

$\Delta[\mathrm{Fe} / \mathrm{H}] \sim 0.6$ dex for all ages (left panel of the Figure). Leaman et al. (2013) argued that this effect is real and sperates a population of halo clusters from those with disc kinematics. Clusters lying in the more metal-rich branch would have been formed in situ while the other objects would have their origin in low-mass dwarf galaxies that were later accreted to the Milky Way.

If the scenario proposed by Leaman et al. (2013) is true it would be reflected also in $[\mathrm{Mg} / \mathrm{Fe}]$ ratio. Dwarf galaxies have less efficient star formation (Kirby et al., 2010), and they do not reach higher metallicities before SNIa start to become important. Therefore, if halo clusters were formed in low-efficient star formation environments as dwarf galaxies we would observe metal-poor clusters with lower $[\mathrm{Mg} / \mathrm{Fe}]$ ratios, which is not the case (see bottom panels of Figure 4.5).

Figure 4.7 shows the radial gradient of $[\mathrm{Fe} / \mathrm{H}]$ for halo and bulge/disc clusters. More distant halo clusters are more metal-poor than the closest one to the Galactic centre. This is in agreement with a scenario of monolithic collapse of Eggen et al. (1962). For bulge/disc clusters there is also a metallicity gradient with increasing $[\mathrm{Fe} / \mathrm{H}]$ towards the centre of the Milky Way. Combining these results with the discussions before we can say that halo clusters may have been formed in building blocks during the initial collapse of the Milky Way, which explains their kinematics. In this scenario they experienced the same efficiency of star formation as the whole Milky Way, which is corroborated by the chemical patterns discuted above. Bulge/disc globular clusters were formed in situ, which 
explain their kinematics, and is also compatible with their abundances patterns.

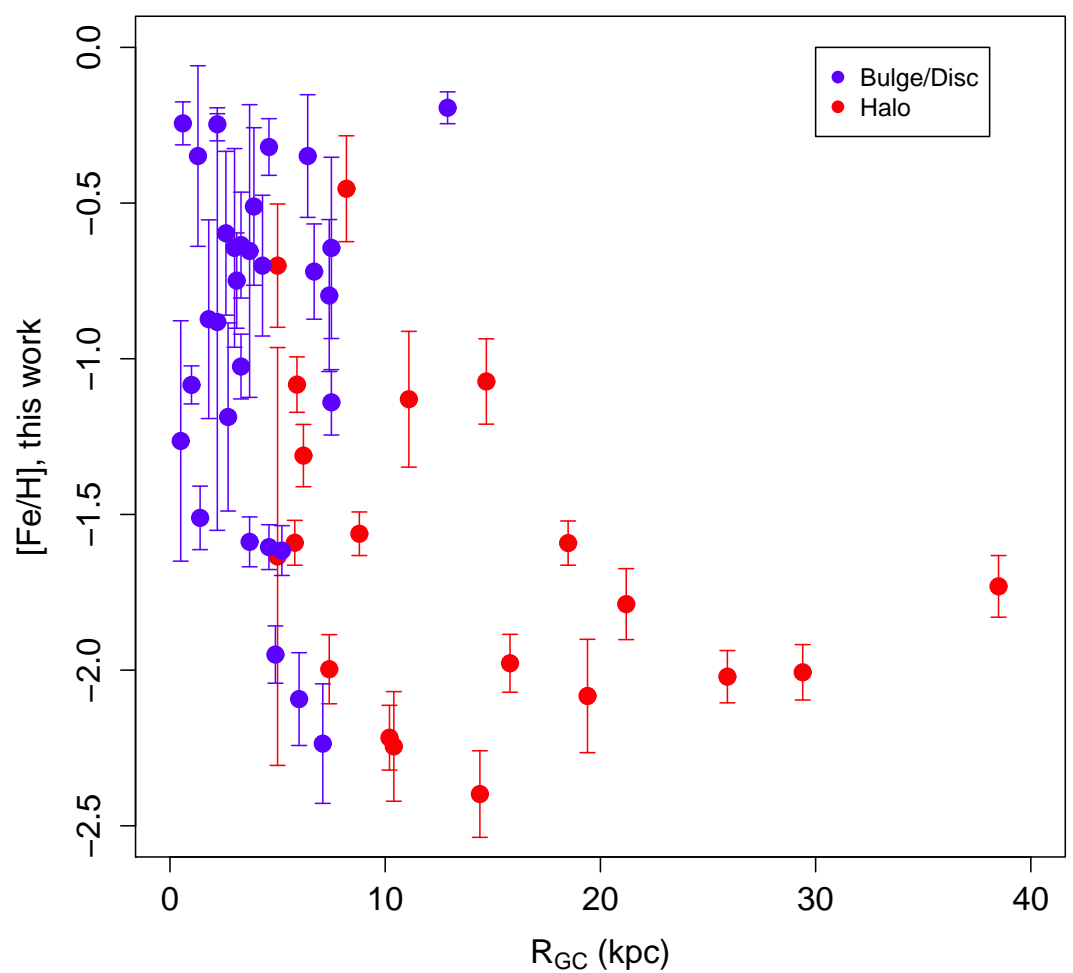

Figure 4.7: Metallicity radial gradients for the 51 Milky Way globular clusters of our survey. Pal 14 is the only object that is out of the plot area, with $\mathrm{R}_{\mathrm{GC}}=71.6 \mathrm{kpc}$ and $[\mathrm{Fe} / \mathrm{H}]=-1.28 \pm 0.09$. We prefer to show a shorter interval in $\mathrm{R}_{\mathrm{GC}}$ in order to get a better visualization of the gradients.

\subsection{Summary and conclusions}

In this Chapter we present all parameters derived for the whole survey of 51 Galactic globular clusters, based on the method described and vastly discussed in Chapter 3. We observed 819 red giant stars, analysed 758 useful spectra and classified 464 as members of the 51 clusters, and 294 as non-members. To proceed with membership selection we derived radial velocities for all 758 spectra. $\mathrm{T}_{\text {eff }}, \log (g),[\mathrm{Fe} / \mathrm{H}]$ were derived using MILES and COELHO libraries and the results from both libraries were averaged to get the final results from our method. We have 13 clusters in common with observations of Carretta et al. (2009), that we used to calibrate our average metallicities per cluster to Carretta's scale. $[\mathrm{Mg} / \mathrm{Fe}]$ were derived using only MILES library and $[\alpha / \mathrm{Fe}]$ using only COELHO library. Once COELHO library provides a more limited interval of $[\alpha / \mathrm{Fe}]$ than MILES for 
$[\mathrm{Mg} / \mathrm{Fe}]$, we adopted $[\mathrm{Mg} / \mathrm{Fe}]$ as our proxy for studying the chemical evolution of the Milky Way. We calibrated $[\mathrm{Mg} / \mathrm{Fe}]$ to a scale compatible with that found for $[\mathrm{Fe} / \mathrm{H}]$ considering that metal-rich and alpha-enhanced stars had their abundances not perfectly recovered by our method due to a lack of reference stars with these characteristics.

The comparison of our final calibrated $[\mathrm{Fe} / \mathrm{H}]$ with those from Carretta's scale are in good agreement with a total dispersion of $\sigma=0.16$ dex, and a lower dispersion $(\sigma=0.07$ dex) considering only the 13 clusters used for calibration. We recover the known bimodal metallicity distribution of the globular cluster populations. Chemical evolution history of the Milky Way showed to be similar between field stars and globular clusters for the four Galactic components: bulge, disc, inner and outer halo. This shows that the whole Milky Way have experienced star formation and galactic evolution with similar efficiency of chemical enrichment. We found that the typical timescale of 1 Gyr for SNIa to start to become important is reasonable for all components of the Milky Way, based on the agemetallicity relation based on globular clusters. Finally we discussed the origin of the halo and bulge/disc clusters that present different kinematics which seems to indicate different formation scenarios for the two groups. For the halo clusters, there is a clear metallicity radial gradient with relation to the Galactic centre, which corroborates the scenario of a initial collapse of the Galaxy, where building blocks clumps were the environment of formation of the halo clusters. Bulge/disc group of clusters would have been formed in situ which would explain their kinematics compatible with disc stars. A radial metallicity gradient is found for this group also which endorse this formation scenario. 
Chapter 5

\section{FORS2/VLT survey of Milky Way globular clusters III. Clusters with multiple populations}

\subsection{Introduction to this chapter}

The definition of globular clusters as templates of simple stellar population systems has changed in the last decade (see Gratton et al., 2012, and references therein). The advent of high-precision photometric and spectroscopic data have shown instrinsic abundance variations of light elements, such as $\mathrm{He}, \mathrm{C}, \mathrm{N}, \mathrm{O}, \mathrm{Na}, \mathrm{Al}, \mathrm{Mg}, \mathrm{Si}$ and $\mathrm{F}$. In particular, the Na-O anticorrelation was considered a characteristic of many globular clusters, from where it is possible to classify a first generation (primordial) and a second generation (intermediate and extreme) components. The presence of this anticorrelation is one more characteristic that differentiate globular and open clusters (Carretta et al., 2010, 2009).

The Milky Way has some peculiar globular clusters presenting also a variation in iron abundance. The classical case of $\omega$ Centauri (NGC 5139) has stars with $[\mathrm{Fe} / \mathrm{H}]$ in a range of about 1.5 dex (e.g. Marino et al.,, 2011, and references therein). Another case is Terzan 5, discussed in Section 1.1.1, that shows at least two populations with metallicity peaks at $[\mathrm{Fe} / \mathrm{H}] \approx-0.3$ and +0.3 (e.g. Ferraro et al., 2009) and it is probably not a globular cluster. The nucleus of the Sagittarius dwarf galaxy, the globular cluster M 54 (NGC 6715) has $\sigma_{[\mathrm{Fe} / \mathrm{H}]} \approx 0.19 \operatorname{dex}$ (Bellazzini et al., 2008; Carretta et al., 2010; Saviane et al., 2012). The massive cluster M 22 (NGC 6656) has been studied for peculiarities in $\mathrm{CN}$ and $\mathrm{CH}$ band strengths, e.g. Hesser and Harris (1979). The cluster had its iron abundance dispersion of $\sigma_{[\mathrm{Fe} / \mathrm{H}]} \approx 0.15$ dex found out simultaneously bv Marino et al. (2009) and bv our group (Da Costa et al., 2009). Alves-Brito et al. (2012) found a range of $\Delta[\mathrm{Fe} / \mathrm{H}]$ of 0.4 dex from nine red giant stars using high-resolution spectra. There are other more subtle cases: NGC 2419 
with $\sigma_{[\mathrm{Ca} / \mathrm{H}]} \approx 0.2$ dex as an indication of $\sigma_{[\mathrm{Ca} / \mathrm{H}]}($ Cohen et al., 2010); NGC 1851, with a range in $[\mathrm{Fe} / \mathrm{H}]$ of only 0.08 dex (Carretta et al., 2010, see also Villanova et al., 2010); the recently characterized by our group NGC 5824 with a total $[\mathrm{Fe} / \mathrm{H}]$ range of 0.3 dex (Saviane et al., 2012; Da Costa et al., 2014).

It seems natural to classify these massive $\left(\mathrm{M}_{V} \lesssim-7 \mathrm{mag}\right.$, see Figure [5.1) multiple population globular clusters with intrinsic $[\mathrm{Fe} / \mathrm{H}]$ spread in a same subgroup of clusters. In order to characterize this group as a population sharing similar origins, many papers have been devoted to deeply examine high quality spectroscopy and photometry for these objects. The following possible scenarios for these unusual clusters are proposed $\omega$ Cen is probably the remnant nucleus of a disrupted dwarf galaxy (Lee et al., 1999), M 54 is located in the nucleus of Sagittarius dwarf galaxy and could be an early snapshot of a $\omega$ Cen-type cluster (Da Costa and Armandroff, 1995; Carretta et al., 2010). The dispersion in $[\mathrm{Fe} / \mathrm{H}]$ of M 22 is similar to that of $\omega$ Cen, and Da Costa et al. (2009) suggested that M 22 could share the same origin as $\omega$ Cen. NGC 1851 is surrounded by a considerable stellar halo that may be a vestige of a dwarf galaxy in which the cluster was once embedded (Olszewski et al., 2009). Carretta et al. (2010) discuss the possibility of NGC 1851 being the result of a merger of two clusters. Lee et al. (2013) indicate NGC 2419 as having an origin in the nucleus of a disrupted dwarf galaxy. Newberg et al. (2009) recently reported the discovery of the Cetus Polar Stream and argued that the cluster NGC 5824 is around $3^{\circ}$ from the fitted orbit of the stream. This cluster shares the same distance of the stream, has compatible radial velocity and metallicity. Therefore, NGC 5824 was possibly located in the nucleus of a dwarf galaxy that was captured and destroyed by the potential well of the Milky Way and originated the Cetus Polar Stream (Da Costa et al., 2014).

In a bigger picture, finding evidence of disrupting satellites in the Galactic halo has great significance for the current $\Lambda \mathrm{CDM}$ galaxy formation paradigm. In this scenario, the Galaxy is built-up through the merger and accretion of lower mass systems, predominantly at early epochs. Indeed, while the spatial and kinematic signatures of this process have been erased in the inner parts of the Galaxy, in its halo where dynamical times are long, several stellar streams have been identified in the past (e.g. Maiewski et al., 2003: Duffau et al., 2006; Newberg et al., 2010). There is however still an order of magnitude discrepancy between the large number of predicted MW satellites and the observed ones. Any addition of a new remnant is therefore important to confirm the theory. Drinkwater et al. (2000) 


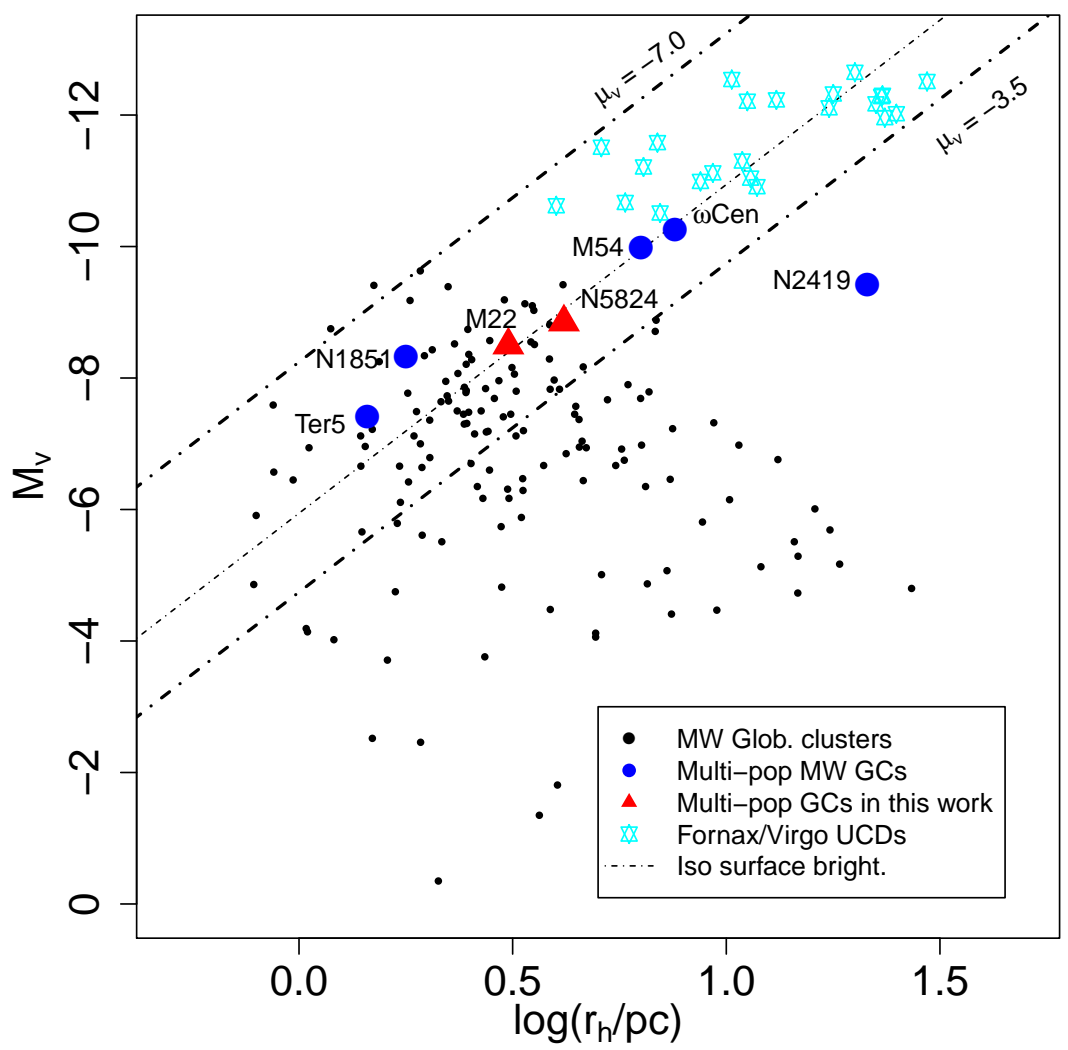

Figure 5.1: Integrated magnitude $\mathrm{M}_{V}$ and half-light radius for Milky Way globular clusters (black dots) and ultra compact dwarfs (UCDs) from Fornax and Virgo clusters (cyan stars) from Evstigneeva et al. (2008). The two globular clusters discussed in this Chapter are indicated as red triangles.

reported the existence of ultra compact old objects in the centre of the Fornax cluster of galaxies. They are more massive (brighter, see Figure 5.1) and distributed over larger distances to the centre of NGC 1399 (the central galaxy of the cluster) in comparison with its globular cluster system. They propose that they were either tidally stripped from the nucleus of disrupted dwarf galaxies, as the multiple population globular clusters described above, or they are a new type of galaxy (for a detailed discussion about the so-called ultra compact dwarf (UCD) galaxies see Mieske et al., 2002, 2008, and references therein). In the working hypothesis that Galactic GC with metallicity spreads signpost halo substructures, our FORS2/VLT survey of Milky Way globular clusters has the potential of finding still unknown examples of these peculiar clusters.

Saviane et al. (2012) revised the available $[\mathrm{Fe} / \mathrm{H}]$ for Milky Way globular clusters from spectroscopy of individual stars, and found that for only half of the objects it would be possible to detect intrinsic metallicity dispersion. Up to now, only seven such peculiar clusters have been found, which means a frequency of about 1/10. In our FORS2 survey 
we observed 51 clusters, of which $\sim 40$ are poorly studied, therefore we expect to find about four clusters with metallicity spread in our sample. In this Chapter we present the results for M 22 and NGC 5824 based on optical spectra.

\subsection{Observations and analysis}

The selection of targets was carried out as described in Section 3.3. The CMDs of M 22 and NGC 5824 are presented in Figure 5.2 where the selected targets are shown in red. Dartmouth isochrones with parameters from (Harris, 1996, 2010 edition) catalogue are overplotted. Sky maps of the two clusters are displayed in Figure 5.3 showing that there was no bias in the position of the selected targets.
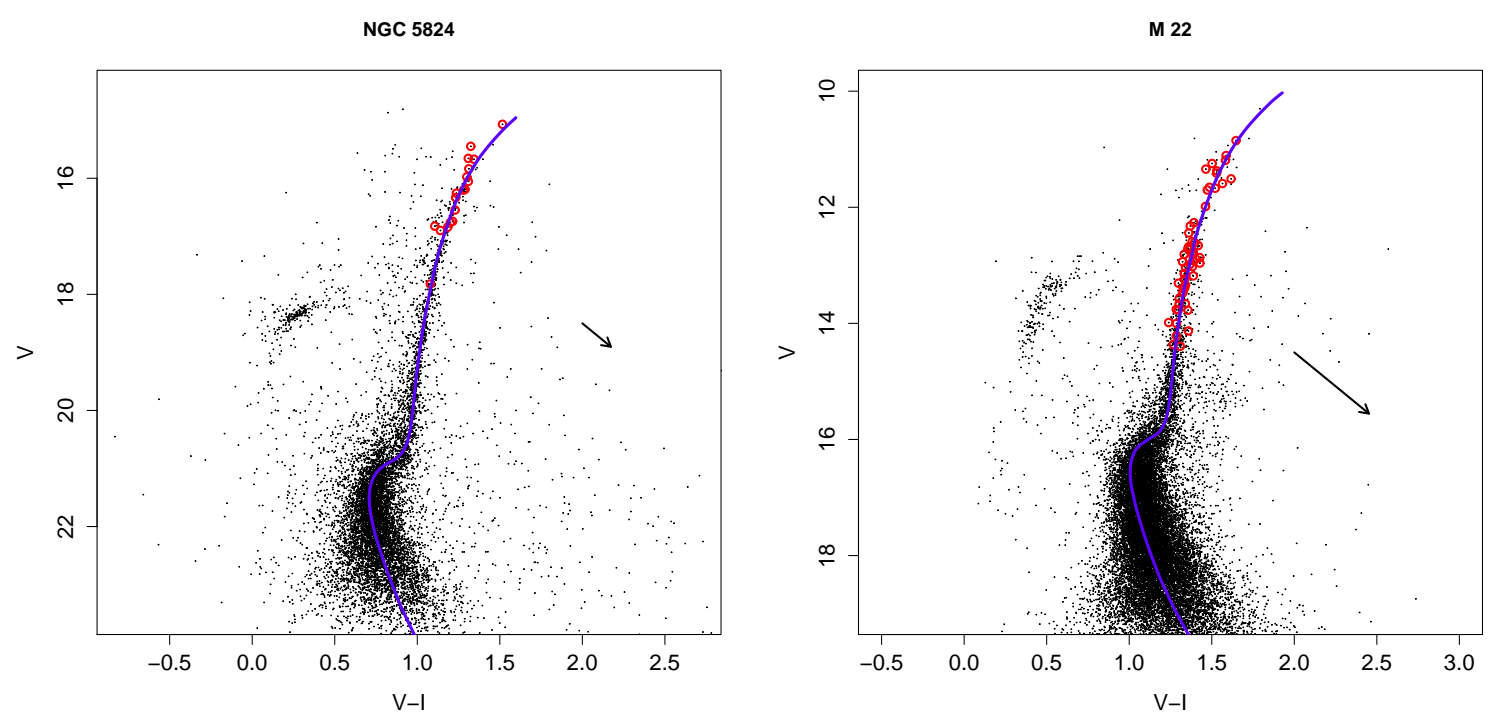

Figure 5.2: Colour-Magnitude diagrams of M 22 and NGC 5824. All stars within $2 \times \mathrm{r}_{\text {half-light }}$ are plotted, without any cleaning procedure. Dartmouth isochrones with literature parameters (Harris, 1996, 2010 edition catalogue) are overplotted. Selected RGB stars for spectroscopic observations are in red. Reddening vectors are shown in each CMD.

Radial velocities were measured by cross correlation and atmospheric parameters were determined following the full spectrum fitting techniques with ETOILE code plus MILES and COELHO stellar libraries in the same way as discussed and validated in Section 3.4. Results for all stars in these two clusters are presented in Tables B.1 (star ID, coordinates, magnitudes, colours, heliocentric radial velocities form this work and from CaT analysis, membership) and $\mathrm{B} .3\left(\mathrm{~T}_{\text {eff }}, \log (g),[\mathrm{Fe} / \mathrm{H}],[\mathrm{Mg} / \mathrm{Fe}]\right.$ and $\left.[\alpha / \mathrm{Fe}]\right)$. 

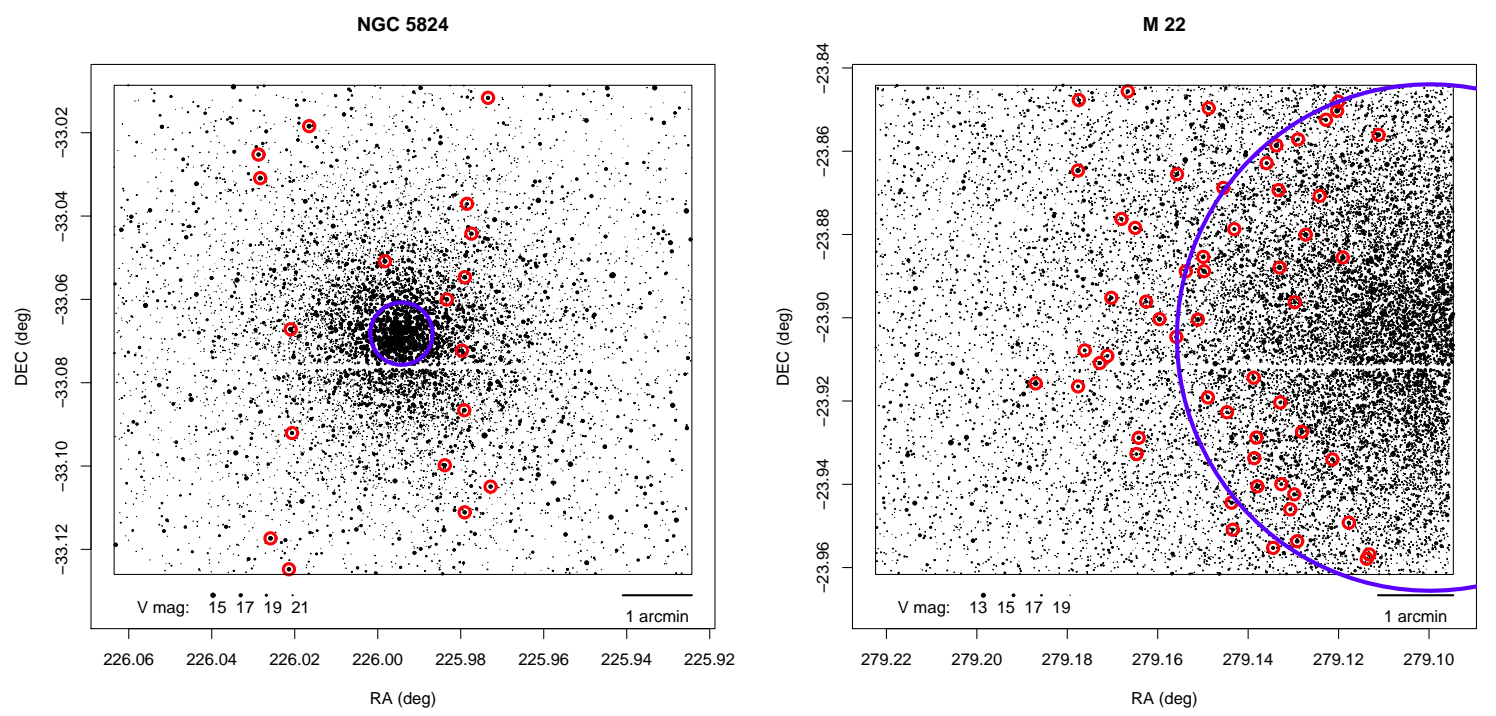

Figure 5.3: Sky map of M 22 and NGC 5824. The size of dots are scaled with the stellar magnitudes as indicated in each plot. Selected RGB stars for spectroscopic observations are in red. The blue circle corresponds to the half-light radius of each cluster from (Harris, 1996, 2010 edition) catalogue.

\subsection{Results}

\subsection{1 $T_{\text {eff }}, \log (g)$ against isochrones}

Figure 5.4 displays the results of all stars in the two clusters in a Hertzprung-Russell diagram form. Left, middle and right panels show the results using MILES library, COELHO library and the average of both results weighted by their uncertainties, respectively. Black dots represent member stars of each cluster, and grey dots are non-members, based on the selection described in the next Section 5.3.2. Dartmouth isochrones (Dotter et al., $2008)$ with age, $[\mathrm{Fe} / \mathrm{H}]$ and $[\alpha / \mathrm{Fe}]$ from (Harris, 1996, 2010 edition) catalogue are overplotted in the diagrams of Figure 5.4 in blue. Cyan lines have the same age and $[\mathrm{Fe} / \mathrm{H}]$ as the respective blue lines, but with the extreme values of $[\alpha / \mathrm{Fe}]=-0.2$ and +0.8 , available from the models.

The results on $\mathrm{T}_{\text {eff }}$ and $\log (g)$ from MILES and COELHO are in good agreement with the isochrones, as well as the average of both reuslts. Uncertainties of results based on MILES are larger than those of COELHO results because of the poor sampling of MILES for metal-poor stars, as discussed in Section 3.6.3. We adopted as the final results in this work the weighted average of MILES and COELHO results. 

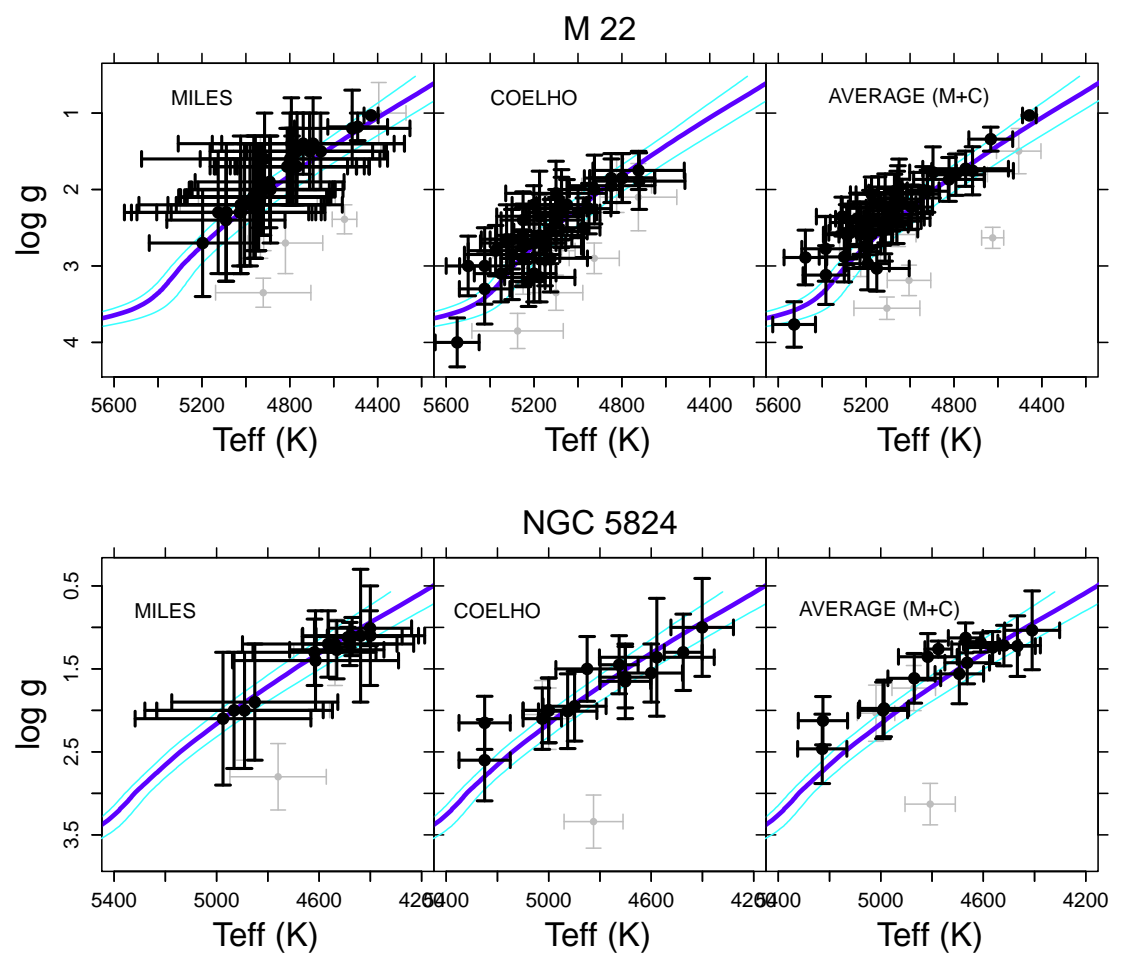

Figure 5.4: Comparison of $\mathrm{T}_{\text {eff }}$ and $\log (a)$ of stars in each cluster with Dartmouth isochrones (Dotter et al., 2008). For each cluster we show the results based on MILES and COELHO libraries and a third panel with the weighted average of the results from both libraries. The parameters of age, $[\mathrm{Fe} / \mathrm{H}]$ and $[\alpha / \mathrm{Fe}]$ for the blue thick isochrones were taken from Table 3.2. Cyan thin isochrones have same age and $[\mathrm{Fe} / \mathrm{H}]$ as blue ones, but with the limits $[\alpha / \mathrm{Fe}]=-0.2$ and +0.8 . Black dots represent member stars of each cluster, and grey dots are not members.

\subsubsection{Membership selection}

In Figure 5.5 we show four panels for each globular cluster, the same as in Figure 3.11. Black dots represent member stars of each cluster, and grey dots are non-members, green circles are classified as non-members but Saviane et al. (2012) considered them as members from their CaT analysis, cyan diamonds are member stars that were considered as nonmembers by Saviane et al. (2012). In upper left panels radial velocities against metallicities are displayed. Blue solid lines are parameters from (Harris, 1996, 2010 edition) and red dashed lines are the average of parameters for cluster members only. For all clusters there is a clear concentration of stars around literature values, and these stars were selected as cluster members. Upper right panels show $\mathrm{T}_{\text {eff }}$ vs. $\log (g)$ compared with Dartmouth isochrones (Dotter et al., 2008). Blue solid lines are isochrones with parameters from (Harris, 1996, 2010 edition), and red dashed lines are based on the average (Table 5.1) of the parameters for member stars (Table B.3). All member stars are close to the isochrones while 
non-member stars are outside these lines, confirming the membership selection. Bottom left panels show $[\mathrm{Fe} / \mathrm{H}]$ against distance to the cluster centre, and no trends were found for any sample cluster. Bottom right panels show no correlation between $\mathrm{T}_{\text {eff }}$ and $[\mathrm{Fe} / \mathrm{H}]$ for any of the six clusters analysed in this work.

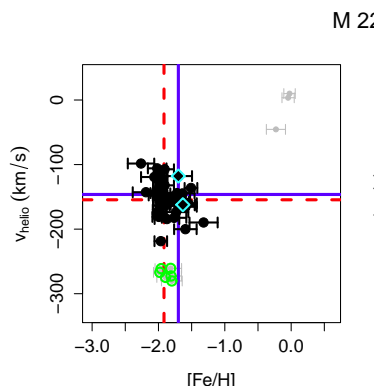

M 22
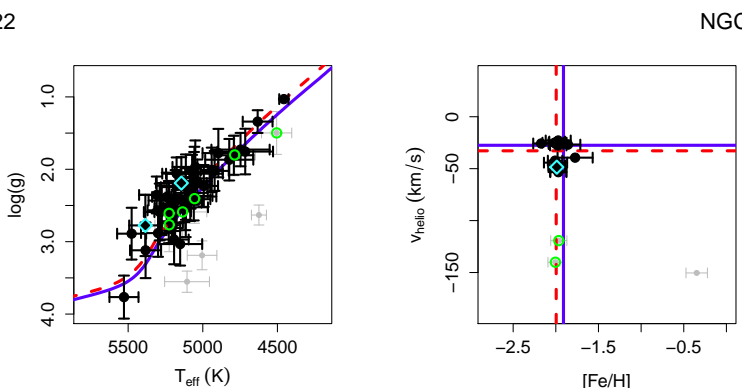

NGC 5824
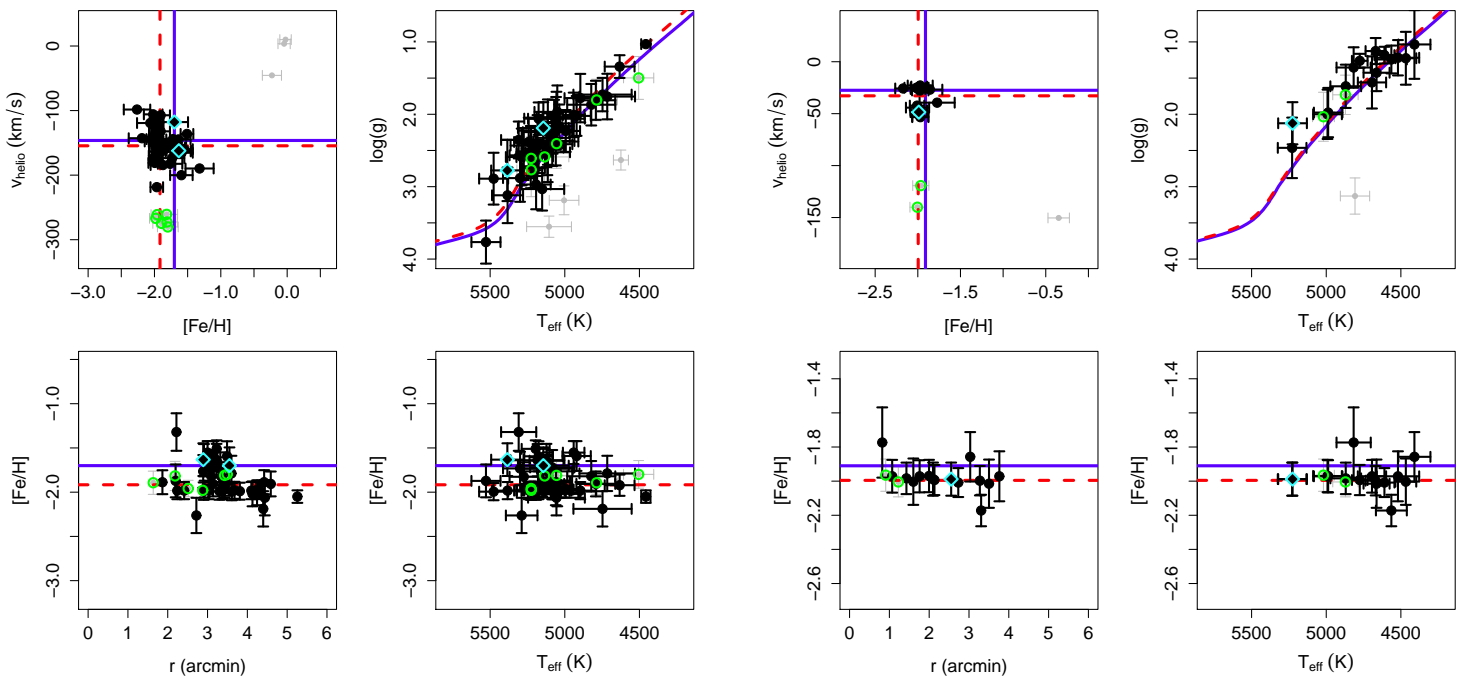

Figure 5.5: Same as Figure 3.11 for the clusters M 22 and NGC 5824.

Table 5.1 - Final average parameters for M 22 and NGC 5824 and respective internal errors.

\begin{tabular}{l|cccccc}
\hline \hline Cluster & $\langle$ vhelio $>(\mathrm{km} / \mathrm{s})$ & $<[\mathrm{Fe} / \mathrm{H}]>^{(a)}$ & $<[\mathrm{Fe} / \mathrm{H}]>^{(b)}$ & $<[\mathrm{Fe} / \mathrm{H}]>^{(a v g)}$ & $<[\mathrm{Mg} / \mathrm{Fe}]>^{(a)}$ & $<[\alpha / \mathrm{Fe}]>^{(b)}$ \\
\hline M 22 & $-152 \pm 25$ & $-1.77 \pm 0.05$ & $-1.945 \pm 0.020$ & $-1.92 \pm 0.05$ & $0.501 \pm 0.008$ & $0.225 \pm 0.018$ \\
NGC 5824 & $-35 \pm 12$ & $-1.95 \pm 0.05$ & $-2.011 \pm 0.031$ & $-1.995 \pm 0.024$ & $0.436 \pm 0.030$ & $0.240 \pm 0.029$
\end{tabular}

${ }^{a}$ MILES library ${ }^{b}$ COELHO library ${ }^{a v g}$ Average of MILES and COELHO results

In conclusion all $\mathrm{T}_{\text {eff }}, \log (g)$ and $[\mathrm{Fe} / \mathrm{H}]$ values are found to sit in well defined sequences following the isochrones. Bottom-right panels show no correlation between $[\mathrm{Fe} / \mathrm{H}]$ and $\mathrm{T}_{\text {eff }}$, suggesting that the $[\mathrm{Fe} / \mathrm{H}] / \mathrm{T}_{\text {eff }}$ degeneracy does not affect our fitting procedure.

\section{$5.4[\mathrm{Fe} / \mathrm{H}]$ and $[\mathrm{Mg} / \mathrm{H}]$ intrinsic spread of $M 22$ and $\mathrm{NGC} 5824$}

Once the member stars are selected and their atmospheric parameters are determined, it is possible to analyse in detail their metal content seeking for multiple populations. We discuss each cluster in the following subsections. 


\section{$5.4 .1 \quad M 22$}

From the 53 observed stars we selected 44 as M 22 members, giving an average radial velocity of $-152 \pm 25 \mathrm{~km} / \mathrm{s}$. compatible with $-150 \pm 2 \mathrm{~km} / \mathrm{s}$ from 51 red giant stars of Da Costa et al. (2009) observed in the CaT with $\mathrm{R} \sim 2,000$ (from our group) and $-146 \pm 2 \mathrm{~km} / \mathrm{s}$ derived by Marino et al. (2009) using high-resolution ( $\mathrm{R} \sim 20,000$ - 45,000) spectra in the optical region of 17 red giant stars. The metallicity distribution function (MDF) using our results is presented in Figure [5.6, as the smoothed histogram in black. From this work we present also the distribution of $[\mathrm{Mg} / \mathrm{H}]$ in the right panel of the same figure. Dashed red and solid blue lines in Figure 5.6 are gaussians with $\sigma$ equal to the average errors of individual stars abundances, and centre at the main peak of the distributions and at the average abundances, respectively. The comparison of the red gaussians with the data (black lines) shows a lack of metal-poor (and low $[\mathrm{Mg} / \mathrm{H}]$ ) stars and an excess of metal-rich (and high $[\mathrm{Mg} / \mathrm{H}]$ ) stars. This comparison shows that the dispersion in $[\mathrm{Fe} / \mathrm{H}]$ and $[\mathrm{Mg} / \mathrm{H}]$ are not due to errors in the abundance derivations for each star, but it is a real dispersion related to the formation of the cluster. The blue gaussians compared to the black observed ones reveals that the shape of the abundance distributions are not uniform around the average values. This could not be a bias in the target selection because we chose stars spread over the cluster area in the sky and along the red giant branch with no preferences, as shown in Figures 5.2 and 5.3. Therefore the two populations are real, and we can split them as population $\mathrm{A}$ with $[\mathrm{Fe} / \mathrm{H}]<-1.8$ and population $\mathrm{B}$ with $[\mathrm{Fe} / \mathrm{H}]>-1.8$.

The main peak of $[\mathrm{Fe} / \mathrm{H}]$ at $\sim-1.9$ and the metal-rich tail of the MDF up to $\sim-1.3$ coincides with the findings of Da Costa et al. (2009). We derived an average $[\mathrm{Fe} / \mathrm{H}]=$ $-1.92 \pm 0.05$ and $[\mathrm{Mg} / \mathrm{Fe}]=0.501 \pm 0.008$, and Marino et al. $(2009,2011)$ derived $[\mathrm{Fe} / \mathrm{H}]=$ $-1.76 \pm 0.02$ and $[\mathrm{Mg} / \mathrm{Fe}]=0.39 \pm 0.03$. We derived a higher value of $[\mathrm{Mg} / \mathrm{Fe}]$ because we have few stars with $[\mathrm{Mg} / \mathrm{Fe}] \sim 0.5$ with lower error bars that are biasing the average. The mean and standard deviation of our results give $[\mathrm{Mg} / \mathrm{Fe}]=0.43 \pm 0.04$ which is compatible with Marino et al. (2009). The difference in [Fe/H] from our sample and that of Marino's papers are explained by the fact that we observed more stars from population $\mathrm{A}([\mathrm{Fe} / \mathrm{H}]$ $<-1.8)$ and they observed more stars from population $\mathrm{B}([\mathrm{Fe} / \mathrm{H}]>-1.8)$, as shown in Figure 5.7 and discussed below.

Marino et al. (2011) and us have selected red giant stars spread over the cluster with no 

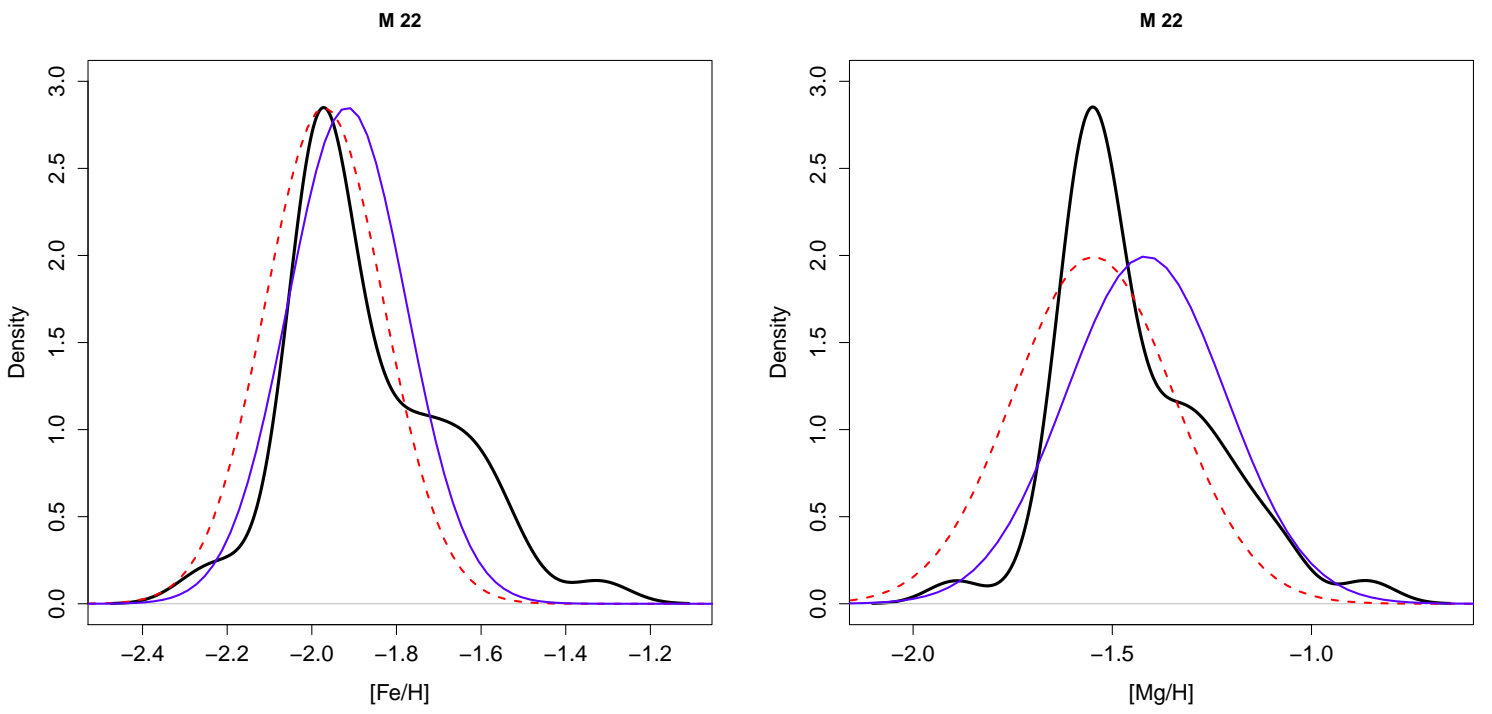

Figure 5.6: Left panel: $[\mathrm{Fe} / \mathrm{H}]$ distribution function of $\mathrm{M} 22$ showing a spread in $[\mathrm{Fe} / \mathrm{H}]$ (solid black line). Dashed red line is a normal distribution centred in the main peak with $\sigma$ equal to the mean uncertainty of individual stars. Blue solid line is the same, but with the peak displaced to the $<[\mathrm{Fe} / \mathrm{H}]>$ from all cluster stars. Right panel: the same, but for $[\mathrm{Mg} / \mathrm{H}]$.

biases for any region for both populations. The stars of both populations lay out along the RGB with no preferences of any region, although we observed stars systematically fainter than Marino et al. (2011). We have three stars in common with their sample, our M22a_3, M22b_7, M22b_10 correspoding to their I-86, I-27, I-53, respectively. We derived [Fe/H] $=-1.92 \pm 0.12,-1.32 \pm 0.21,-1.79 \pm 0.20$, and they derived $[\mathrm{Fe} / \mathrm{H}]=-1.82 \pm 0.04,-1.73 \pm 0.04$, $-1.74 \pm 0.05$, respectively. All stars have compatible $[\mathrm{Fe} / \mathrm{H}]$ and no systematic difference was detected. Among our fainter RGB stars there are 30 classified as population A (metal-poor) and 14 as population B (metal-rich). The brighter stars observed by Marino et al. (2011) are separated in 14 from population A and 21 from population B. Once we observed more metal-poor stars, our metallicity average is lower than that of Marino et al. (2011) who observed more metal-rich stars. Considering the three stars in common, and combining both samples, the total number of stars is 43 from population A and 33 from population B. As a result, the ratio of populations is $(\mathrm{popA}) /(\mathrm{popB}) \approx 1.3$, i.e, metal-poor population in M 22 represent $\sim 57 \%$ of all stars, and metal-rich population, $\sim 43 \%$. Despite that, this ratio is not uniform with magnitude (a proxy for the stellar mass), as displayed in the right panel of Figure 5.7, where smoothed histograms of the distributions of populations A and B (summed samples of this work with Marino's) are shown in blue and red, respectively. These historgrams shows a predominancy of population $\mathrm{A}$ in the fainter part of the RGB 
and population B in the brighter part. In fact, we observed fainter stars and Marino et al. (2011) observed brighter ones, as well as Alves-Brito et al. (2012).

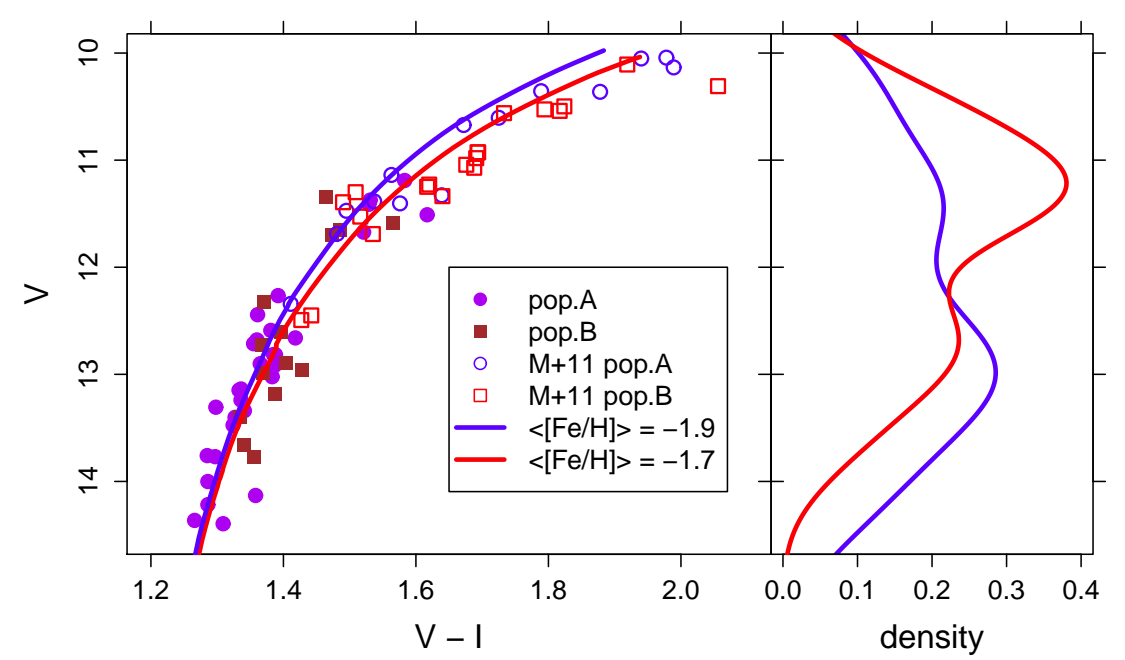

Figure 5.7: Comparison of sample stars from this work with Marino et al. (2011) for M 22. Purple and blue circles are population A stars $([\mathrm{Fe} / \mathrm{H}]<-1.8)$ from this work and from Marino et al. (2011), respectively. Brown and red squares are population B stars $([\mathrm{Fe} / \mathrm{H}]>-1.8)$ from this work and from Marino et al. (2011), respectively. In the main panel, blue and red lines are Dartmouth $14 \mathrm{Gyr}$ old isochrones with $[\mathrm{Fe} / \mathrm{H}]=$ -1.9 and -1.7 , respectively. In the right panel, smoothed histograms of the distributions of populations A and B (summed samples of this work with Marino's) are shown in blue and red, respectively.

The metal-poor population A and metal-rich population B are related to a first and second generation in the cluster formation process. Carretta et al. (2009) have shown that $47-49 \%$ of the stars in globular clusters are from first generation. Other authors argued that $\sim 50 \%$ is an upper limit for the amount of first generation stars in a clusters (Vesperini et al., 2010, and references therein). If it is true that second generation stars are predominant in all clusters, more observations should be done to complete Figure 5.7. However, Carretta et al. (2009) observed only brighter RGB stars, and the histograms in Figure 5.7 show that for stars with $\mathrm{M}_{V}<12 \mathrm{mag}$, population A (first generation, metalpoor) represents $45 \%$ of the total, which is in agreement with their results. Vesperini et al. (2010) proceeded hydrodynamical simulations to find that $4-9 \%$ of the field halo second generation stars were originated in globular clusters and then stripped to the halo. This process could be responsible for the non-uniform sample of first and second generation stars in the RGB of M 22.

The shapes of the observed distributions of $[\mathrm{Fe} / \mathrm{H}]$ and $[\mathrm{Mg} / \mathrm{H}]$ seem to be similar, displaced by $\sim 0.5$ dex, which is coherent with the derived average $[\mathrm{Mg} / \mathrm{Fe}]$. If this displacement is approximately constant from Figure [5.6, the variance in $[\mathrm{Mg} / \mathrm{Fe}]$ should be 


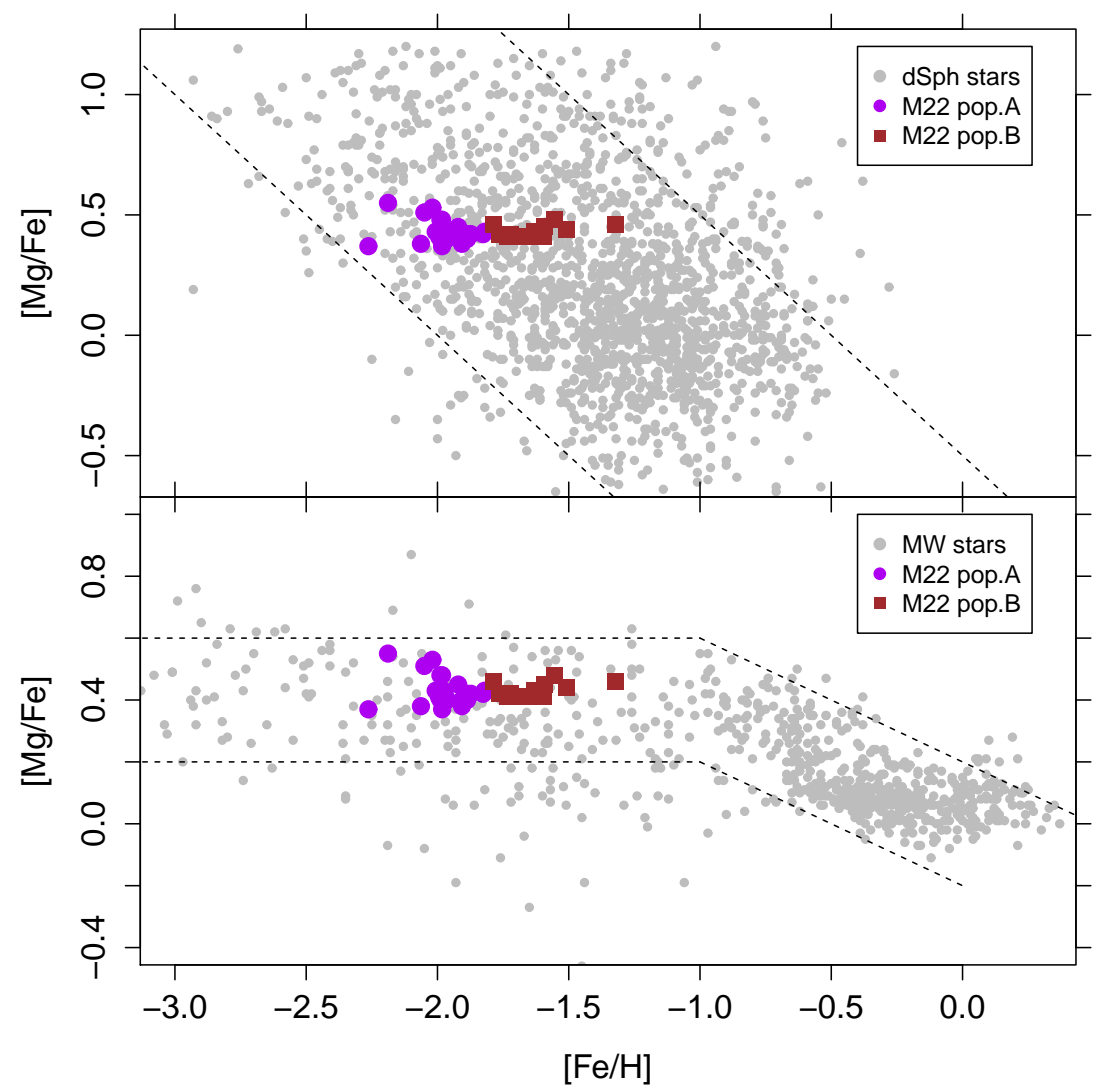

Figure 5.8: Chemical evolution history of M 22. Purple filled circles are M 22 stars from population A $([\mathrm{Fe} / \mathrm{H}]<-1.8)$, brown filled squares are M 22 stars from population B $([\mathrm{Fe} / \mathrm{H}]>-1.8)$. Grey filled circles are from dwarf spheroidal galaxies (compilation of Kirby et al., 2010) in the upper panel, and from Milky Way stars (compilation of Venn et al., 2004). Dashed lines are guidelines for the trends observed in dSph and MW stars.

negligible. In fact, Figure 5.8 shows clearly a dispersion in $[\mathrm{Fe} / \mathrm{H}]$ and no dispersion in $[\mathrm{Mg} / \mathrm{Fe}]$. In the upper panel of Figure 5.8 we compare M 22 stars with stars from dwarf spheroidal galaxies (Kirby et al., 2010), and in the lower panel we compare the same M 22 stars with Milky Way stars (Venn et al., 2004). The trends in this plot reveal the efficiency of star formation in a galaxy, i.e., if the efficiency is high, many type II supernovae enrich the interstellar medium with $\mathrm{Mg}$ and Fe up to high metallicities before type Ia supernovae start to explode providing $\mathrm{Fe}$ and no $\mathrm{Mg}$ (which starts to decrease the $[\mathrm{Mg} / \mathrm{Fe}]$ ratio). For the Milky Way this break occurs at about $[\mathrm{Fe} / \mathrm{H}] \sim-1.0$ (bottom panel of Figure 5.8), and for dwarf galaxies this knee is located at lower metallicities, as shown in the upper panel of Figure 5.8, M 22 stars have $-2.0<[\mathrm{Fe} / \mathrm{H}]<-1.5$, approximately, and for that range of metallicities, SNIa already started to explode. $[\mathrm{Mg} / \mathrm{Fe}]$ is constant for both populations A and B. It means that the more metal-rich population B should have been enriched by 
SNII, and M 22 possibly was formed in a galaxy with a higher star formation rate, such as the Milky Way. This evidence that M 22 possibly was not originated in a nucleus of a disrupted dwarf galaxy as $\omega$ Cen or M 54 is in agreement with the findings and conclusions of Marino et al. (2011) who analysed in detail chemical abundances of 35 M 22 red giant stars using high-resolution spectroscopy. They proposed that M 22 is the result of a merger between two clusters and discuss the possibility of this to happen in the Milky Way halo or in a dwarf galaxy.

\subsubsection{NGC 5824}

We have selected 15 member stars from the 17 observed red giant stars of NGC 5824 from which we derived an average radial velocity of $-35 \pm 12 \mathrm{~km} / \mathrm{s}$. This is consistent with Da Costa et al. (2014) who derived $-28.9 \pm 1.0 \mathrm{~km} / \mathrm{s}$ based on $\mathrm{R} 2,000$ spectra in the CaT region for 147 red giant stars of the cluster. The $\mathrm{MDF}$ and $[\mathrm{Mg} / \mathrm{H}]$ distribution of NGC 5824 using our results are presented in Figure 5.9, in the same way as done for M 22 in the previous section. We derived a mean $[\mathrm{Fe} / \mathrm{H}]=-1.995 \pm 0.024$, and Da Costa et al. (2014) found a trimodal distribution in $[\mathrm{Fe} / \mathrm{H}]$ with peaks at about $-2.09,-2.01$ and -1.91 with a metal-rich tail up to $\sim-1.8$ and metal-poor stars down to $\sim-2.2$. Their three peaks are within our main peak that is not resolved in this work because of the lower number of stars observed. Besides, the spread in $[\mathrm{Fe} / \mathrm{H}]$ is much lower than that of $\mathrm{M} 22$, and our metallicity errors for each star would explain such small dispersion, as can be seen in Figure 5.9.

The $[\mathrm{Mg} / \mathrm{H}]$ distribution is similar to the MDF, as shown in Figure 5.9, which indicates that $[\mathrm{Mg} / \mathrm{Fe}]$ should be constant for all observed stars in NGC 5824. In fact, in Figure 5.10 we show that all stars are concentrated and it is difficult to find any dispersion from this data only. The selected stars from Da Costa et al. (2014) could be observed in the optical region in order to derive $[\mathrm{Mg} / \mathrm{Fe}]$ and discuss in more detail the chemical evolution of this cluster. 

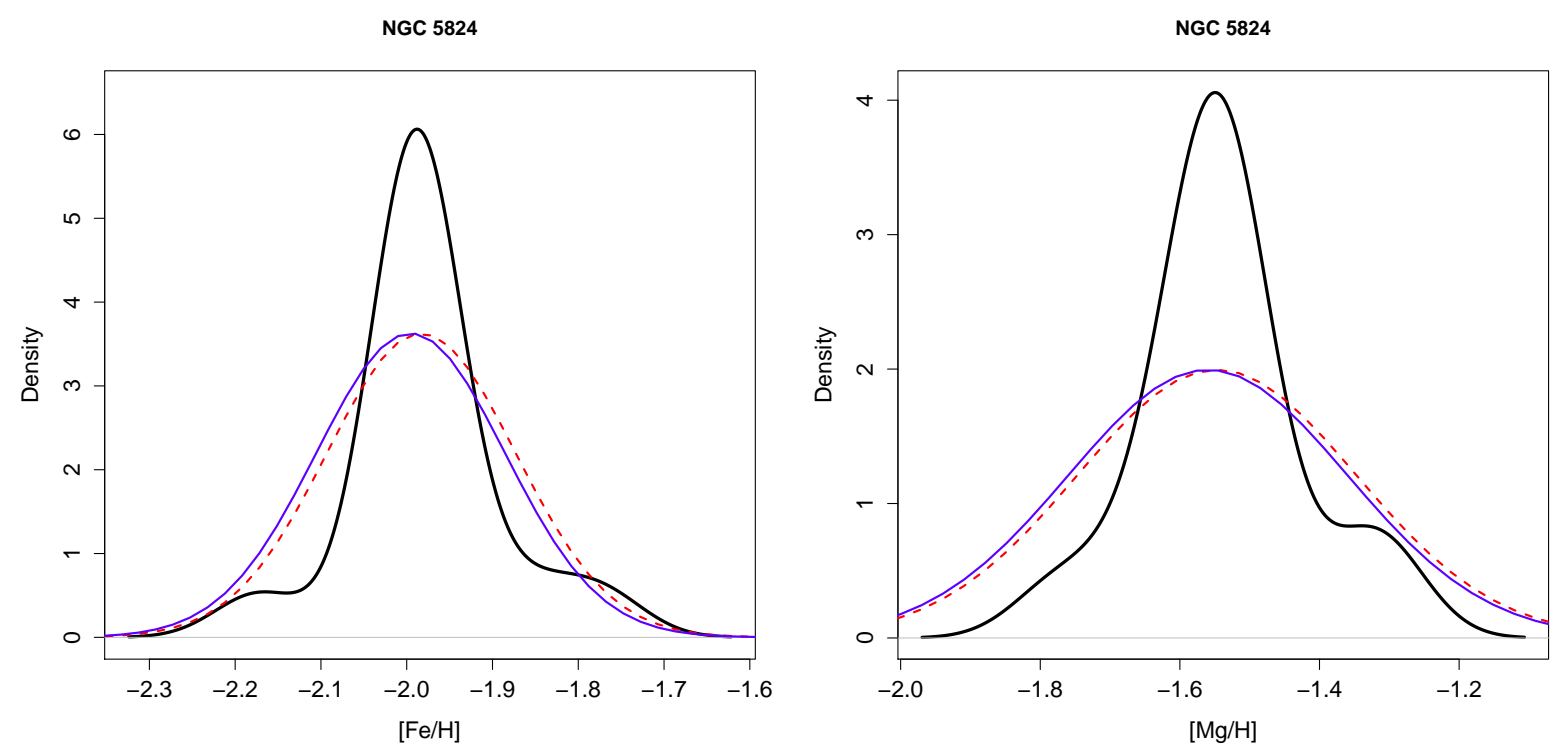

Figure 5.9: Same as Figure 5.6 for NCG 5824.

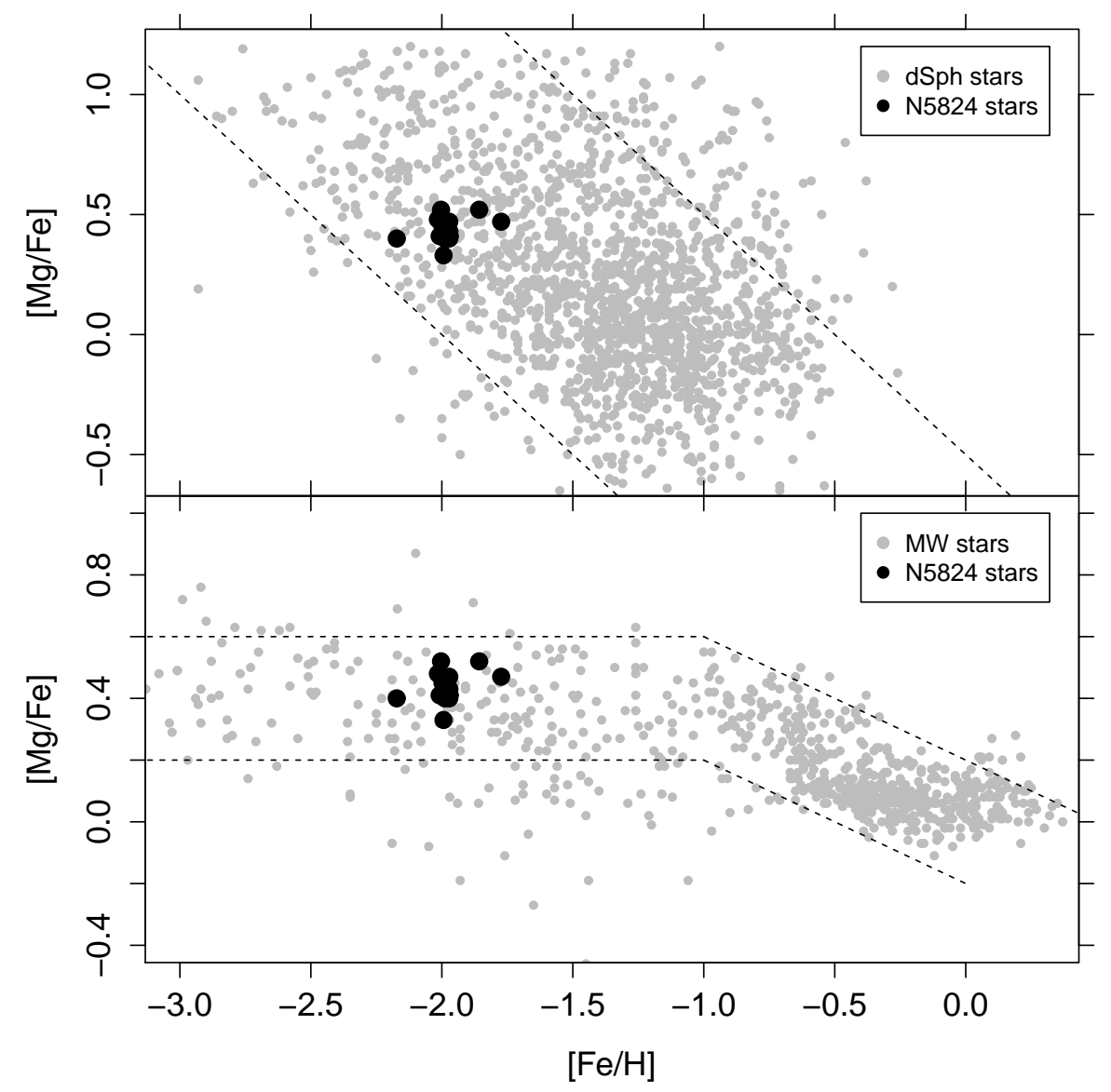

Figure 5.10: Same as Figure 5.8 for NGC 5824. 


\subsection{Summary and conclusions}

From the 44 selected red giant stars, we confirmed and characterized the two populations of $\mathrm{M} 22$ with metallicity peaks at $[\mathrm{Fe} / \mathrm{H}] \approx-1.9$ and -1.6 , in a total range of $-2.3<$ $[\mathrm{Fe} / \mathrm{H}]<-1.3$. Also for $[\mathrm{Mg} / \mathrm{H}]$ we found a similar distribution with peaks at $[\mathrm{Mg} / \mathrm{H}] \approx$ -1.5 and -1.3 , in a total range of $-1.9<[\mathrm{Fe} / \mathrm{H}]<-0.9$. From these results, $\mathrm{M} 22$ has an average $[\mathrm{Mg} / \mathrm{Fe}]=0.43 \pm 0.04$ with negligible star-to-star variations, i.e, both populations have the same $[\mathrm{Mg} / \mathrm{Fe}]$ ratio. This means that the second generation stars were enriched by core-collapse supernovae and not by type Ia supernovae, as one could expect if M 22 would had been formed in a dwarf galaxy. We conclude that if M 22 had an extragalactic origin, it should be in a galaxy with efficient star formation. It is unlikely the Milky Way halo would have had enough density to produce 1.0 dex range of metallicity within a globular cluster. Further chemo-dynamical observations and models must performed to explain if such cluster can be formed in another environment but a dwarf galaxy with high star formation rate.

For NGC 5824 we found a symmetrical metallicity distribution function that peaks at $[\mathrm{Fe} / \mathrm{H}] \sim-2.0$ and covers $-2.2<[\mathrm{Fe} / \mathrm{H}]<-1.8$. This distribution is based on 15 selected member red giant stars and it is close to the distribution found by Da Costa et al. (2014). Once they selected 147 members, they could resolve the main peak in the MDF presented in this work into three peaks of metallicity. The MDF found here is also compatible with $[\mathrm{Fe} / \mathrm{H}]$ errors of each star. $[\mathrm{Mg} / \mathrm{H}]$ has a similar distribution with main peak at $[\mathrm{Mg} / \mathrm{H}]$ $\sim-1.5$ and ranging $-1.8<[\mathrm{Mg} / \mathrm{H}]<-1.3$. This is consistent with an average $[\mathrm{Mg} / \mathrm{Fe}]$ $=0.436 \pm 0.030$. Although compatible with chemical enrichment of dwarf galaxies, if this cluster was formed in a dSph, its $[\mathrm{Mg} / \mathrm{Fe}]$ ratio could be higher. The adopted stellar libraries MILES and COELHO does not have stars with alpha-enhancement as high as $[\mathrm{Mg} / \mathrm{Fe}] \sim 1.0$, and further spectroscopic analysis must be executed in order to stablish the origin of NGC 5824 . 
Chapter 6

\section{RR Lyrae variable stars in HP1}

\subsection{Introduction to this chapter}

If bulge experienced a more efficient star formation and it hosts the centre of the Milky Way, it is possible that the oldest globular clusters are located there. In particular, there are some moderately metal-poor bulge clusters presenting a bluer horizontal branch (HB) than expected by their metallicity, as listed by Barbuy et al. (2009). The main parameter that causes a change in the HB morphology is the metallicity, known as the first parameter. The second most important parameter is age in most cases (Lee et al., 1994), although other parameters can play the role, such as He abundance, CNO abundance or AGB mass loss. Metal-poor clusters have a blue HB, and old clusters too, but these bulge clusters are moderately metal-poor with a blue HB, which indicates that they are very old, possibly the oldest objects of the Milky Way, witnesses of the initial collapse of the Galaxy.

The innermost Milky Way globular cluster is HP 1, for which we determine a metallicity of $[\mathrm{Fe} / \mathrm{H}]=-1.3 \pm 0.4$ in Chapter 4, has a HB index ${ }^{1}$ of 0.75 , as determined bv Mackey and van den Bergh (2005). Therefore this central cluster seems to have a bluer horizontal branch than expected. If this cluster is the oldest of our Galaxy it will confirm that the bulge had a rapid star formation at the beginning of the Milky Way. To better constrain the age determination via colour-magnitude diagram, it is important to have a precise estimation of distance from a different source. Moreover, the distance itself is important to define better the position in relation to the Galactic centre and correlate with bulge formation and evolution models. Ortolani et al. (2011) published a cleaned CMD for this

\footnotetext{
${ }^{1}(\mathrm{~B}-\mathrm{R}) /(\mathrm{B}+\mathrm{V}+\mathrm{R})$, where $\mathrm{V}$ is the number of variable stars, $\mathrm{B}$ and $\mathrm{R}$ are the numbers of stars bluer and redder than the variables, respectively.
} 
bulge cluster, using proper motion techniques to remove field stars contamination and indicated six RR Lyrae candidates. We aim at measuring light curves of the six RR Lyrae candidates, determine their periods and the distance of the cluster, using V,I photometry from EFOSC2@NTT.

\subsection{Observations}

The observations were performed with EFOSC2@NTT/ESO-La Silla in May 14, 2013, under project 091.D-0711(A), PI. Sergio Ortolani. Once typical periods of RR Lyrae are of a few hours, 18 images were taken in a range of six hours, trying to keep an almost regular time step. Filter Gunn-i was used for these images. PSF photometry using DAOPhot@IRAF was performed, and relative magnitudes were obtained in order to construct the light curves. In Table 6.1 the log of observations is given.

\subsection{RR Lyrae candidates}

In Figure 6.1 we show the V vs. V-I Colour-Magnitude Diagram (CMD) of HP 1. The original data was collected with the NTT (Ortolani et al., 1997), and later it was corrected for proper motion using the JHK VLT/MAD data (Ortolani et al., 2011). Six candidate stars are located in the RR Lyrae gap region. Photometry of star \#2 was not good, and this star is not considered in the analysis.

\subsection{Period determination}

One of the most used techniques to determine the period of a light-curve is Phase Dispersion Minimization (PDM), which is available within IRAF. The best period for each curve was found using PDM, as shown in Figures 6.3 and 6.4. The folded curves using the best period found are plotted in Figure 6.5.

\subsection{Constraining the distance}

From the period-luminosity relationship by Catelan et al. (2004) combined with the distance modulus formula it is possible to derive the distance of the RR Lyrae stars, as follows: 
Table 6.1 - Log of observations

\begin{tabular}{|c|c|c|c|c|}
\hline obs. date & exp. time & filter & seeing & airmass \\
\hline \multicolumn{5}{|c|}{ block \# 1} \\
\hline 2013-05-14T04:17:51.712 & 30.0005 & i-705 & 0.92 & 1.181 \\
\hline 2013-05-14T04:19:44.535 & 30.0005 & i- 705 & 0.92 & 1.176 \\
\hline 2013-05-14T04:22:45.035 & 180.0005 & i- 705 & 0.92 & 1.168 \\
\hline 2013-05-14T04:27:07.647 & 180.0006 & i- 705 & 0.84 & 1.157 \\
\hline 2013-05-14Т04:31:35.321 & 180.0005 & $\mathrm{i}-705$ & 0.84 & 1.146 \\
\hline 2013-05-14T04:35:58.353 & 167.0005 & i- 705 & 0.81 & 1.136 \\
\hline \multicolumn{5}{|c|}{$\begin{array}{ll}\text { block \# } 2\end{array}$} \\
\hline 2013-05-14T05:31:10.934 & 30.0005 & i-705 & 0.70 & 1.042 \\
\hline 2013-05-14T05:33:09.520 & 180.0006 & i- 705 & 0.70 & 1.040 \\
\hline \multicolumn{5}{|c|}{ block \# 3} \\
\hline 2013-05-14T07:24:18.491 & 30.0005 & i-705 & 0.61 & 1.011 \\
\hline 2013-05-14T07:26:16.036 & 180.0005 & i- 705 & 0.61 & 1.012 \\
\hline \multicolumn{5}{|c|}{ block \# 4} \\
\hline 2013-05-14T08:10:24.651 & 30.0005 & i-705 & 0.53 & 1.053 \\
\hline 2013-05-14T08:12:22.536 & 180.0005 & i-705 & 0.53 & 1.056 \\
\hline \multicolumn{5}{|c|}{ block \# 5} \\
\hline 2013-05-14T08:55:37.961 & 30.0005 & i-705 & 0.82 & 1.133 \\
\hline 2013-05-14T08:57:35.486 & 180.0005 & i- 705 & 0.82 & 1.138 \\
\hline \multicolumn{5}{|c|}{ block \# 6} \\
\hline 2013-05-14T09:26:43.353 & 30.0005 & i-705 & 0.63 & 1.217 \\
\hline 2013-05-14T09:28:41.128 & 180.0006 & i- 705 & 0.72 & 1.223 \\
\hline \multicolumn{5}{|c|}{ block \# 7} \\
\hline 2013-05-14T10:10:59.721 & 30.0005 & i-705 & 0.69 & 1.394 \\
\hline 2013-05-14T10:12:57.406 & 180.0005 & i- 705 & 0.69 & 1.404 \\
\hline
\end{tabular}

$$
\begin{aligned}
M_{V} & =a+b \cdot \log P, \text { where } \\
a & =\sum_{i=o}^{3} a_{i} \cdot(L)^{i} \\
b & =\sum_{i=o}^{3} b_{i} \cdot(L)^{i}
\end{aligned}
$$

The parameter $L=(B-R) /(B+V+R)$ characterizes the ratio of $(\mathrm{B})$ lue, (R)ed, and (V)ariable stars, and has the value 0.75 for HP1 (Mackey and van den Bergh, 2005). The coefficients $a_{i}$ and $b_{i}$ were fitted by Catelan et al. (2004) for four different values of metalicity Z: $0.0060,0.0020,0.0010,0.0005$. Distance can be derived from the difference between apparent and absolute magnitude, which results in the equation below:

$$
d(p c)=10^{\left(m_{V}-M_{V}+5\right) / 5}
$$




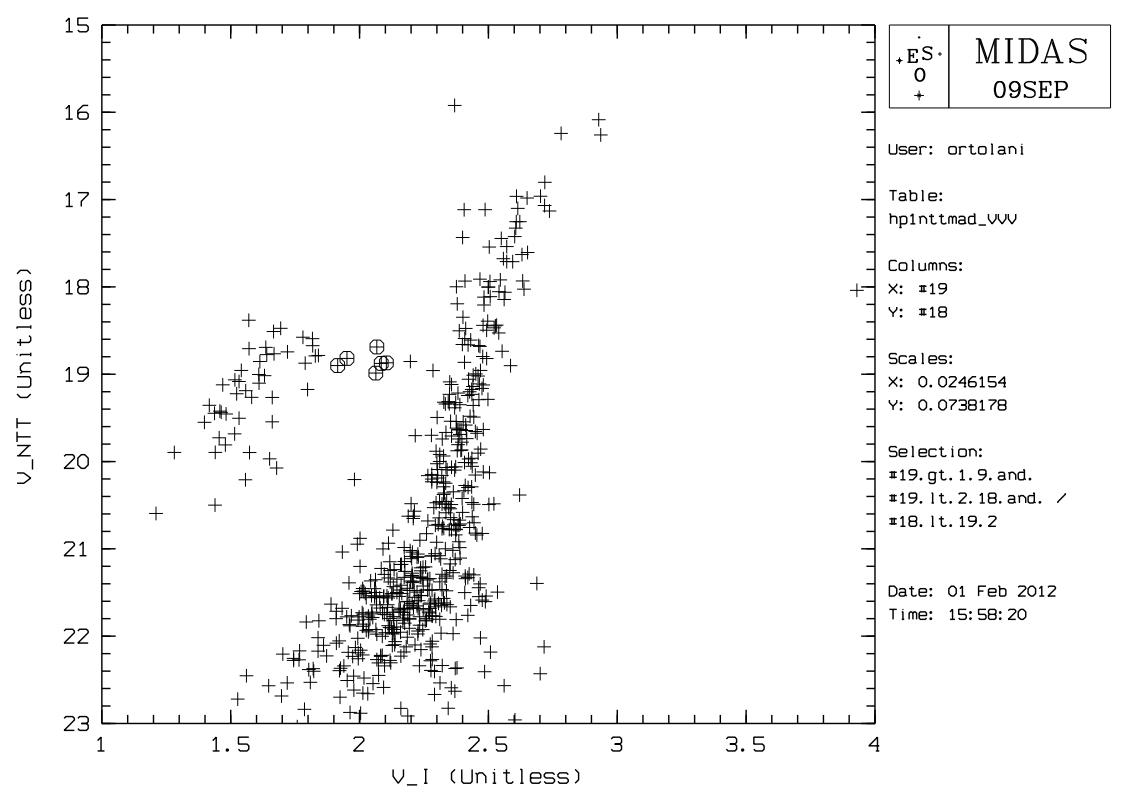

Figure 6.1: Colour magnitude diagram showing six RR Lyrae candidates.

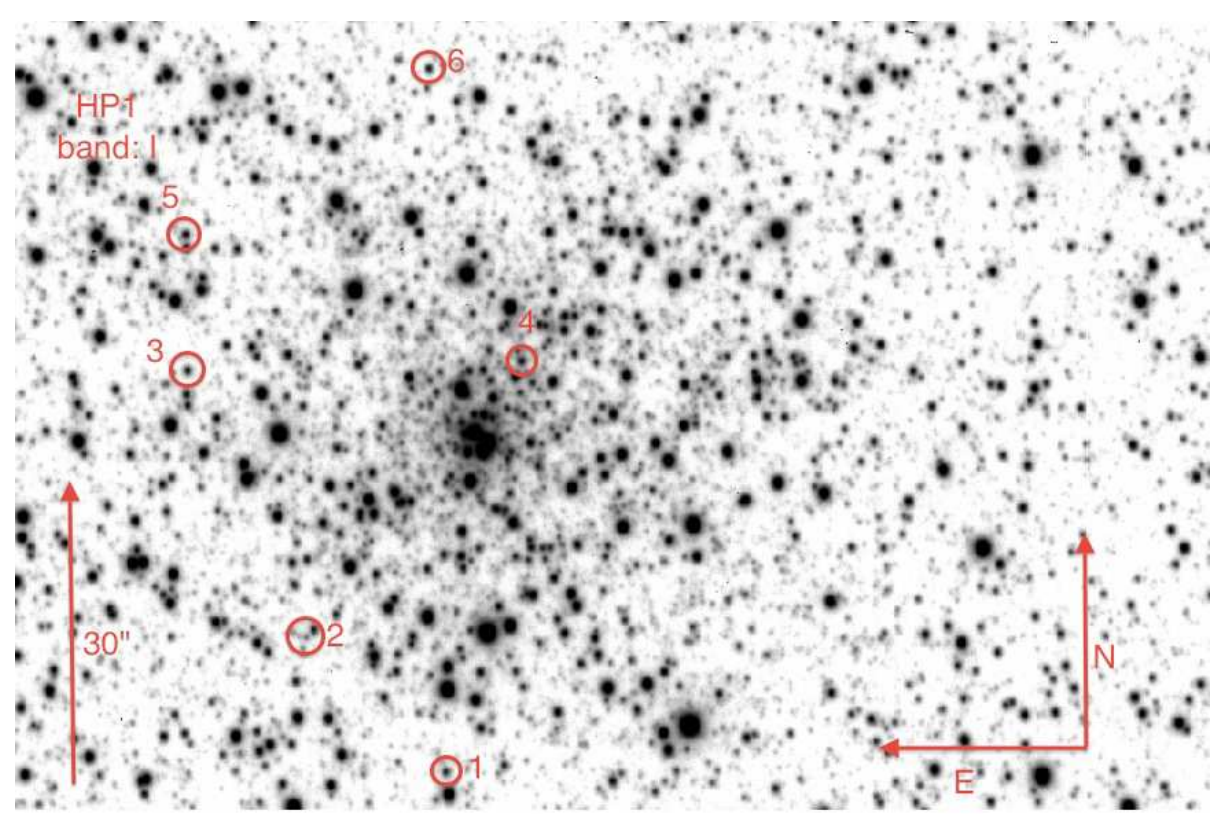

Figure 6.2: HP 1: Finding chart of the six RR Lyrae candidates. Central extraction of the NTT I image.

Combination of Equations 6.1 and 6.2 give the direct relationship between period and distance. Figure 6.6 shows the parameter space for distance as a function of period for different magnitudes and metallicities.

Assuming a cluster metallicity of $[\mathrm{Fe} / \mathrm{H}]=-1.26 \pm 0.39$ from Chapter 4 and the solar metallicity to be $\mathrm{Z}=0.0153$ (Caffau et al., 2011), we get $\mathrm{Z}=0.0008$ for the present $\mathrm{RR}$ Lyrae. A reddening $\mathrm{E}(\mathrm{B}-\mathrm{V})=1.12$ (Harris, 1996, 2010 edition) is adopted, that together 


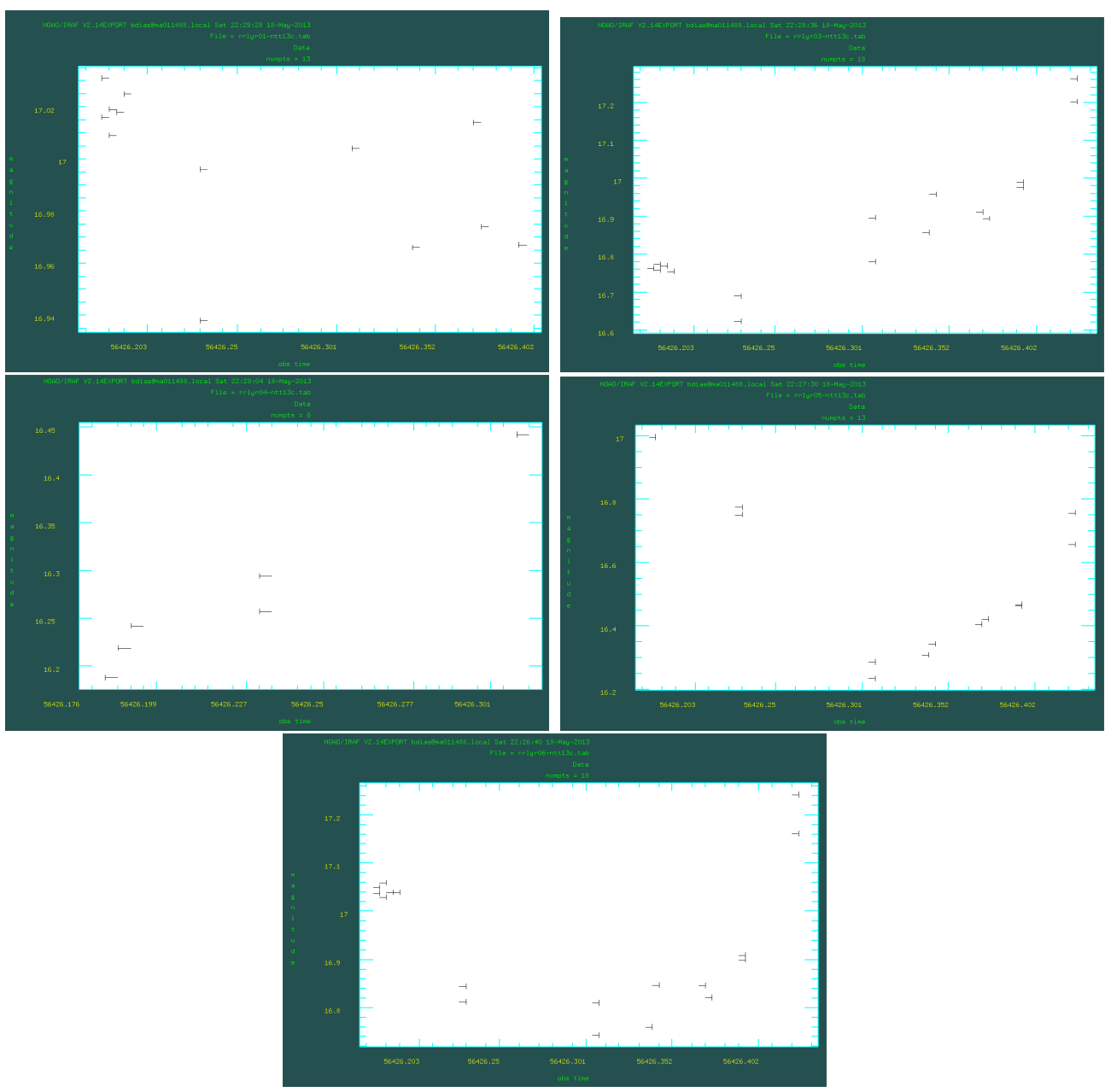

Figure 6.3: Light curves of five RR Lyrae candidates in HP1.

with $R_{V}=3.1$ gives $A_{V}=3.472$. $\mathrm{V}$ magnitudes corrected by this value are presented in Table 6.2, together with values for periods, and resulting distances using the fits for $\mathrm{Z}=0.0010$ and $\mathrm{Z}=0.0020$ by Catelan et al. (2004).

Table 6.2 - Distance determination. V magnitude is already corrected by extinction of $A_{V}=3.1 \times$ $\mathrm{E}(\mathrm{B}-\mathrm{V})=3.472$, where $\mathrm{E}(\mathrm{B}-\mathrm{V})=1.12$ is from (Harris, 1996, 2010 edition).

\begin{tabular}{lccccc}
\hline ID & period (days) & $\mathrm{m}_{\mathrm{V}}$ & $\Delta m_{i}$ & $\operatorname{dist} \mathrm{A}(\mathrm{kpc})$ & $\operatorname{distB}(\mathrm{kpc})$ \\
\hline 1 & 0.129 & 15.429 & 0.093 & 7.1 & 6.9 \\
2 & - & 15.347 & - & - & - \\
3 & 0.406 & 15.404 & 0.640 & 8.8 & 8.5 \\
4 & 0.102 & 15.398 & 0.254 & 6.6 & 6.5 \\
5 & 0.270 & 15.216 & 0.760 & 7.5 & 7.2 \\
6 & 0.243 & 15.515 & 0.499 & 8.4 & 8.1 \\
\hline
\end{tabular}

Star \#1 has no magnitude variations, star \#2 is so faint that the photometry did not 


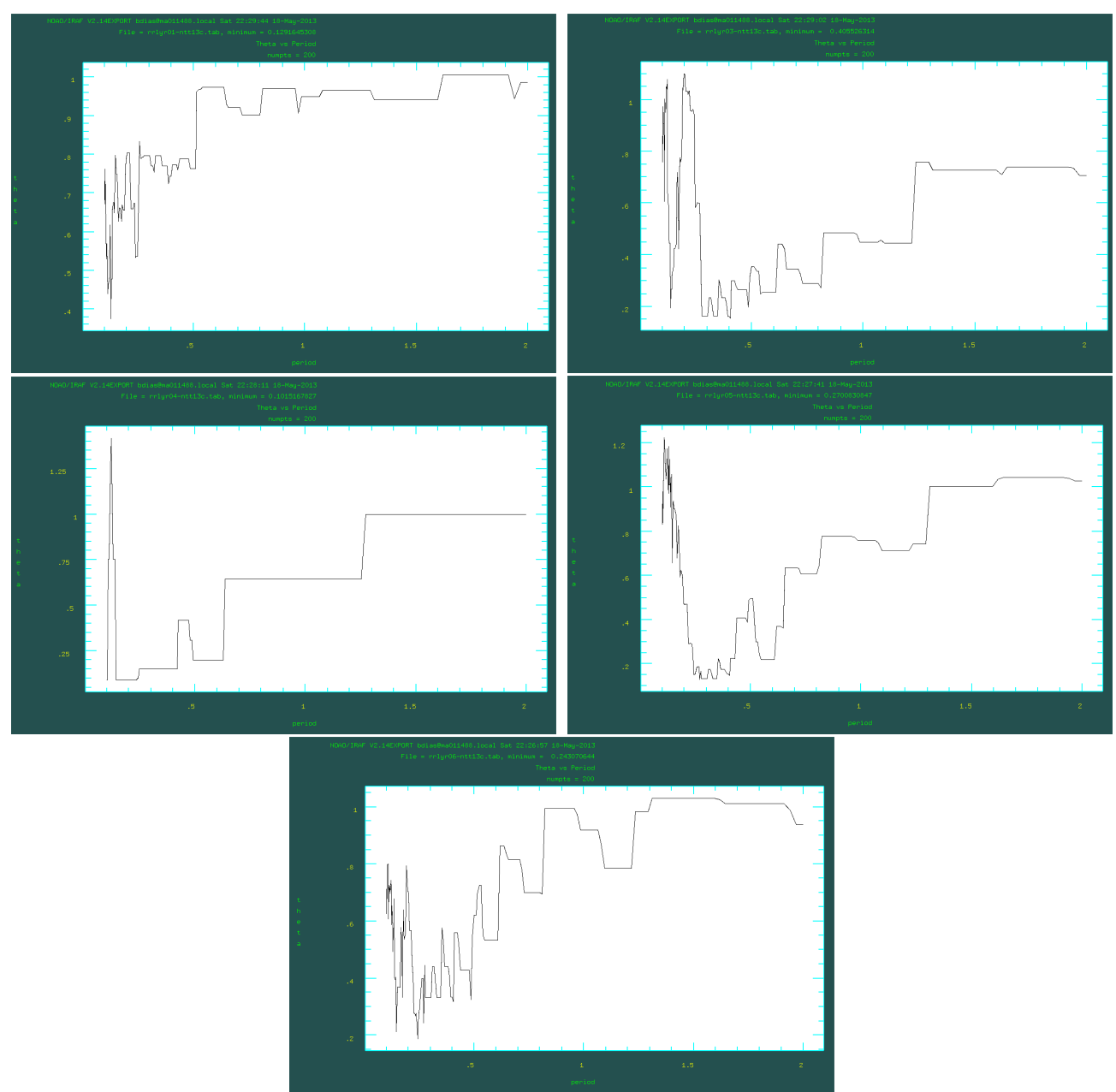

Figure 6.4: Theta vs. period for five RR Lyrae candidates in HP1.

recover its magnitudes, star \#4 is undersampled. The other three stars $(\# 3, \# 5, \# 6)$ present magnitude variations above 0.5 mag in Gunn-i, and well sampled light-curves in the phase range. The periods and amplitudes reported in Table 6.2 imply, from the Bailey diagram, that the five RR Lyrae stars are of type c (e.g. Bono et al., 1997). An average distance of HP1 based on the 3 confirmed RR Lyrae is then $8.0 \pm 0.6 \mathrm{kpc}$.

\subsection{Conclusions}

We present for the first time 3 confirmed RR Lyrae stars for the inner Galactic bulge globular cluster. Combining their periods with magnitude, metallicity, horizontal branch parameter, we could apply a period-luminosity relation and derive the distance of $8.0 \pm$ $0.6 \mathrm{kpc}$ for HP1. This is consistent with the distance of the Galactic center to the Sun, as well as with distance of this cluster in the catalog of (Harris, 1996, 2010 edition), which 


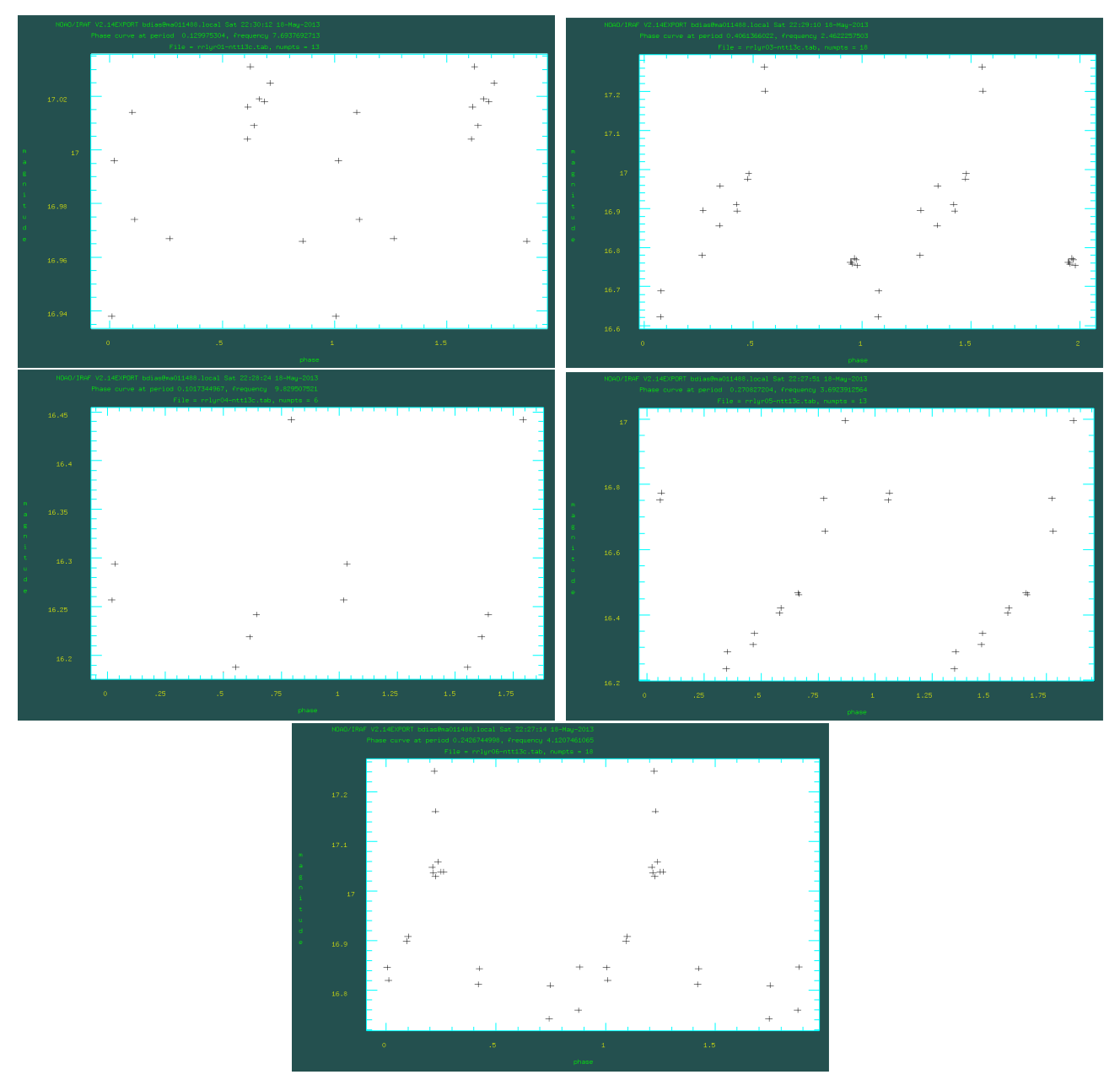

Figure 6.5: Folded light curves for five RR Lyrae candidates in HP1, using the best periods found in Figure 6.4.

is $8.2 \mathrm{kpc}$. A comparison with VVV survey data is under way, in order to conclude this project. 


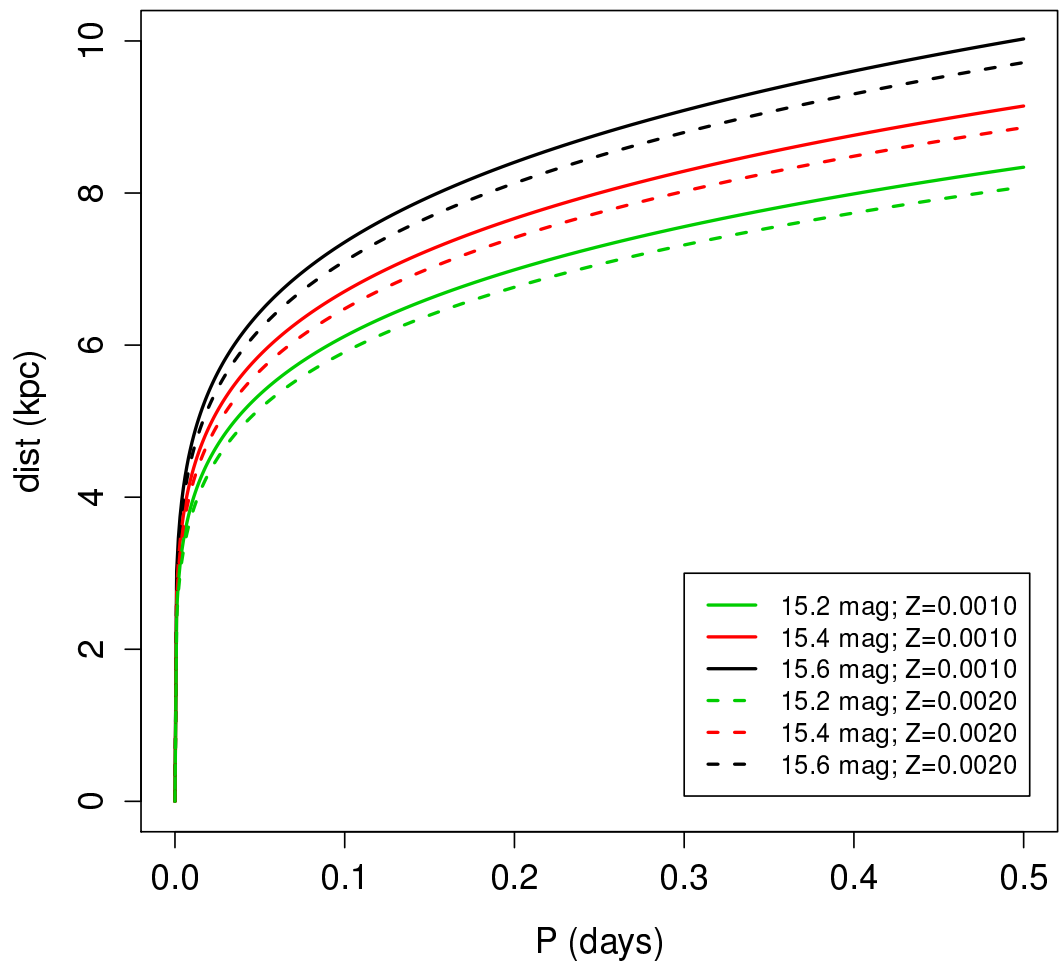

Figure 6.6: Theoretical curves showing Equations 6.1 and 6.2. 
Chapter

\section{Conclusions and future work}

\subsection{Summary}

In this thesis we use intermediate-age and old star clusters in the Small Magellanic Cloud and globular clusters in the Milky Way as tracers of the chemical and dynamical evolution of these galaxies. Since these clusters are spatially resolved they can be studied in detail and the system Milky Way - SMC - LMC becomes a good laboratory for studying galaxy interactions, giving hints on how distant galaxies evolve.

In Chapter 2 we derive ages and metallicities for five star clusters in the SMC and we defined a particular group of clusters called West Halo. Four clusters are in this region and present a gradient in age and metallicity, which indicates that the scenario of tidal stripping in the SMC is true, instead of ram pressure which only affects gas content of the galaxy. Other clusters in this region have ages and metallicities published in the literature and also corroborates the gradients.

The FORS2/VLT survey of Milky Way globular clusters is presented in Chapters 3 and 4. In the first one, we detail the method for derivation of parameters $\left(v_{\text {helio }}, \mathrm{T}_{\text {eff }}\right.$, $\log (g),[\mathrm{Fe} / \mathrm{H}],[\mathrm{Mg} / \mathrm{Fe}]$ and $[\alpha / \mathrm{Fe}])$ from mid-resolution stellar spectra $(\mathrm{R} \sim 2000, \lambda \lambda 456$ - $586 \mathrm{~nm}$ ) based on full spectrum fitting code ETOILE with reference stellar libraries the observed MILES and the synthetic COELHO. We validate the method recovering the parameters of 49 well-known stars in the whole parameter space for our FORS2 targets using spectra from ELODIE library and parameters from PASTEL catalogue. Six clusters were used as template with metallicities varying from -2.5 to solar, and the resulting $[\mathrm{Fe} / \mathrm{H}]$ are in agreement with high-resolution spectroscopic determinations and also with CaT metallicities for the same stars. 
In the following Chapter 4, the whole sample of about 800 red giant stars distributed over the 51 Galactic globular clusters is analysed. We present a new metallicity scale for $\sim 1 / 3$ of the total number of catalogued clusters, as well as alpha-element enhancement indicator $[\mathrm{Mg} / \mathrm{Fe}]$ for all clusters with homogeneous observations and analysis, recovering the bimodal metallicity distribution for globular clusters. We fit a linear function with Carretta et al. (2009) metallicity scale based on 13 clusters in common with their observations, and the dispersion of the comparison is only $\sigma=0.07$ dex. Carretta et al. (2009) compiled metallicities from literature and put them in their scale, however comparing these values with our derivations we have a higher dispersion of $\sigma=0.16$ dex. We conclude that if all 51 clusters were analysed with high-resolution spectroscopy, the comparison with our results would keep the dispersion of $\sigma=0.07 \mathrm{dex}$, therefore our metallicities are more precise and self-consistent than previous works based on low-resolution spectroscopy. Six clusters from our sample were not calibrated by Carretta et al. (2009), and we present a complete analysis for them: NGC 6426, Terzan 8, Djorg 2, Lynga 7, Pal 10 and BH 176. It is the first time that $[\mathrm{Fe} / \mathrm{H}]$ and $[\mathrm{Mg} / \mathrm{Fe}]$ derived in a consistent way are published for such a huge sample of globular clusters.

The behaviour of $[\mathrm{Mg} / \mathrm{Fe}]$ vs. $[\mathrm{Fe} / \mathrm{H}]$ of the 51 clusters are similar to their field stars counterparts in the bulge, disc, inner and outer halo. $[\mathrm{Mg} / \mathrm{Fe}]$ starts to decrease at $[\mathrm{Fe} / \mathrm{H}]_{\text {knee }} \sim-1.0$ for all components, although we do not exclude the possibility of the bulge having $[\mathrm{Fe} / \mathrm{H}]_{\text {knee }}$ at a higher metallicity showing that it had a more efficient star formation. Comparing our results with ages for the clusters, the timescale for SNIa to become important is about 1 Gyr for all components in the Milky Way which corroborates the value adopted vastly in Galactic models. [Fe/H] vs. age exhibit a difference between bulge and halo clusters that is interpreted as a more efficient star formation in the bulge than in the halo. This should be reflect also in $[\mathrm{Mg} / \mathrm{Fe}]$ vs. age but this comparison does not allow a clear separation between bulge and halo clusters. A metallicity gradient for the halo and a different one for the bulge/disc populations are found and reflects different origins of these clusters. Halo clusters would have been assembled in the building blocks of the primordial Galaxy, while bulge/disc clusters would have been formed in situ. This is consistent with a more rapid star formation in the bulge than in the halo.

In the context of understanding the origin of halo globular clusters and discuss if the environment of less efficient star formation was a dwarf galaxy or a mini halo, we study 
two interesting massive clusters from our FORS2 survey in Chapter 5, namely M 22 and NGC 5824. These clusters present a dispersion in $[\mathrm{Fe} / \mathrm{H}]$ and in $[\mathrm{Mg} / \mathrm{H}]$ which indicates a different formation scenario for them, possibly related to a disrupted dwarf galaxy captured by the Milky Way potential well. M 22 has peaks at $[\mathrm{Fe} / \mathrm{H}] \approx-1.9$ and -1.6 , and $[\mathrm{Mg} / \mathrm{H}] \approx-$ 1.5 and -1.3 . $[\mathrm{Fe} / \mathrm{H}]$ and $[\mathrm{Mg} / \mathrm{H}]$ range 1.0 dex and average uncertainty of individual stars is $\sigma_{[\mathrm{Fe} / \mathrm{H}]}=0.14$ dex and $\sigma_{[\mathrm{Mg} / \mathrm{H}]}=0.20$ dex. This cluster seems to follow an internal chemical evolution closer to that of the Milky Way, instead of dwarf galaxies, which indicates that the formation scenario of M 22 is more complex than previously thought, possibly formed by the merger of two other clusters as proposed by Marino et al. (2011). For NGC 5824 there is only one peak at $[\mathrm{Fe} / \mathrm{H}] \sim-2.0$ and $[\mathrm{Mg} / \mathrm{H}] \sim-1.5$. The ranges are 0.4 dex and 0.5 dex, respectively, with average uncertainty of individual stars of $\sigma_{[\mathrm{Fe} / \mathrm{H}]}=0.11 \mathrm{dex}$ and $\sigma_{[\mathrm{Mg} / \mathrm{H}]}=0.20$ dex. In fact, Da Costa et al. (2014) showed that a inter-quartile range for the $[\mathrm{Fe} / \mathrm{H}]$ in this clusters is only 0.10 dex, which is of the order of the uncertainties in the present work. Consequently, a cluster with a large spread in $[\mathrm{Fe} / \mathrm{H}]$ such as $\mathrm{M} 22$ probably was not originated in a dwarf galaxy, and a cluster that seems to share a similar origin with Cetus Polar Stream, possibly a disrupted dwarf galaxy has a very small spread in $[\mathrm{Fe} / \mathrm{H}]$ such as NGC 5824. This reinforces that each cluster with spread in $[\mathrm{Fe} / \mathrm{H}]$ seems to have a different origin and must be analysed independently of the others.

Barbuy et al. (2009) listed some moderately metal-poor bulge clusters presenting a blue horizontal branch (HB). This horizontal branch morphology can be due to very metalpoor (main parameter) or very old stars (second parameter), as modelled by Lee et al. (1994), among other weaker effects (other options for second parameter effect, such as He abundance, CNO abundance, AGB mass lost etc). Once these clusters are not too metalpoor, they could be the oldest objects in the Milky Way, which would be in agreement with a classical component of the bulge, as discussed in Section 1.1.1. We analyse in detail the innermost Galactic globular cluster, HP 1 and determine $[\mathrm{Fe} / \mathrm{H}]=-1.3 \pm 0.4$. Mackey and van den Bergh (2005) published its HB index equal to +0.75 . Therefore this central cluster seems to have a bluer horizontal branch than expected. To constrain future age determination using colour-magnitude diagrams we determine its distance using light curves of RR Lyrae in Chapter 6. We find a distance of $8.0 \pm 0.6 \mathrm{kpc}$ consistent with the distance of the Galaxy centre. If this cluster is the oldest of our Galaxy it will confirm that the bulge had a rapid star formation at the beginning of the Milky Way. 


\subsection{Perspectives}

The SMC West Halo clusters that do not have ages and metallicities measured were observed by us and the data are under analysis to get a general picture of the West Halo of the SMC and to constrain models of tidal interaction between the Magellanic Clouds and our Galaxy. The list of newly observed clusters is: B 1, B 2, B 4, B 6, BS 1, HW 5, HW 6, Kron 8, Kron 11, Lindsay 14 and a calibration cluster NGC 152.

For the FORS2/VLT survey of globular clusters further discussion should be adress to explain why $[\mathrm{Fe} / \mathrm{H}]$ vs. age and $[\mathrm{Fe} / \mathrm{H}]$ vs. $[\mathrm{Mg} / \mathrm{Fe}]$ indicate different efficiency of star formation for bulge and halo populations, but looking at $[\mathrm{Mg} / \mathrm{Fe}]$ vs. age both population seems to share similar histories. Only few clusters were in common with large samples of homogeneous age determinations, that being the case we intend to use the pre-images observed to select our targets to produce calibrated colour-magnitude diagrams to derive ages for all the 51 clusters and trace a complete scenario for these objects.

NGC 5824 follows the orbit of the Cetus Polar Stream and it was possibly related to a disrupted dwarf galaxy in the past. Detailed chemistry of this cluster have to be analysed in order to better characterize its origin as the nucleus of a disrupted dwarf galaxy or not.

For the innermost bulge globular cluster HP 1, more images were observed at SOI/SOAR in 2013 and also at EFOSC2/NTT in 2014, and they should be analysed soon to get better sampled light curves and improve the precision of distance determination. An independent analysis of its distance have to be done using $\mathrm{VVV} / \mathrm{ESO}^{1}$ photometry in $\mathrm{K}_{S}$ band to make well sampled light curves using a different photometric filter and determine the cluster distance, that should not be so different of our findings here. Having its distance and metallicity defined using independent methods, the next step it to observe HP 1 with the Hubble Space Telescope to make a colour-magnitude diagram reaching the main sequence turnoff and measure its age and prove if this is (among) the oldest object in the Milky Way.

In conclusion, the techniques developed in this thesis to use full spectrum fitting to derive $[\mathrm{Fe} / \mathrm{H}]$ and $[\alpha / \mathrm{Fe}]$ can be applied to cluster systems in other galaxies and even for entire dwarf galaxies. One first target would be the system of stellar clusters of the SMC. From our works on photometry of SMC clusters, we have ages in a homogeneous scale,

\footnotetext{
${ }^{1}$ http://mwm.astro.puc.cl/mw/index.php/Main_Page
} 
and we also have selected RGB stars to be observed with spectroscopy. We can study a scenario of efficiency of star formation in the SMC in terms of $[\alpha / \mathrm{Fe}]$ vs. $[\mathrm{Fe} / \mathrm{H}]$. With the upcoming giant 30-40m class telescopes (E-ELT, GMT, TMT) we will be able to observe individual stars up to several magnitudes fainter than now. As we need stellar libraries for reference, and observed libraries are based on solar neighbourhood with a specific chemical evolution history and a set of solar abundances, this analysis will need synthetic spectra as reference. The observation of individual stars in extragalactic globular clusters will encourage the development of synthetic stellar libraries, in particular with different patterns of chemical abundances and alpha-enhancement elements. 


\section{Bibliography}

Aguerri J. A. L., Balcells M., Peletier R. F., Growth of galactic bulges by mergers. I. Dense satellites, A\&A, 2001, vol. 367, p. 428

Allende Prieto C., Sivarani T., Beers T. C., Lee Y. S., Koesterke L., Shetrone M., Sneden C., Lambert D. L., Wilhelm R., Rockosi C. M., Lai D. K., Yanny B., Ivans I. I., Johnson J. A., Aoki W., Bailer-Jones C. A. L., Re Fiorentin P., The SEGUE Stellar Parameter Pipeline. III. Comparison with High-Resolution Spectroscopy of SDSS/SEGUE Field Stars, AJ, 2008, vol. 136, p. 2070

Alves V. M., Pavani D. B., Kerber L. O., Bica E., On open cluster physical parameters from 2MASS data, New A, 2012, vol. 17, p. 488

Alves-Brito A., Barbuy B., Zoccali M., Minniti D., Ortolani S., Hill V., Renzini A., Pasquini L., Bica E., Rich R. M., Meléndez J., Momany Y., VLT-UVES abundance analysis of four giants in NGC 6553, A\&A, 2006, vol. 460, p. 269

Alves-Brito A., Meléndez J., Asplund M., Ramírez I., Yong D., Chemical similarities between Galactic bulge and local thick disk red giants: O, Na, Mg, Al, $\mathrm{Si}, \mathrm{Ca}$, and Ti, A\&A, 2010, vol. 513, p. A35

Alves-Brito A., Yong D., Meléndez J., Vásquez S., Karakas A. I., CNO and F abundances in the globular cluster M 22 (NGC 6656), A\&A, 2012, vol. 540, p. A3

Baade W., The Resolution of Messier 32, NGC 205, and the Central Region of the Andromeda Nebula., ApJ, 1944, vol. 100, p. 137

Babusiaux C., Gómez A., Hill V., Royer F., Zoccali M., Arenou F., Fux R., Lecureur A., Schultheis M., Barbuy B., Minniti D., Ortolani S., Insights on the Milky Way bulge 
formation from the correlations between kinematics and metallicity, A\&A, 2010, vol. 519, p. A77

Balbinot E., Santiago B. X., Kerber L. O., Barbuy B., Dias B. M. S., Probing the Large Magellanic Cloud age gap at intermediate cluster masses, MNRAS, 2010, vol. 404, p. 1625

Barbuy B., Bica E., Ortolani S., Globular clusters within 5(deg) of the Galactic center, A\&A, 1998, vol. 333, p. 117

Barbuy B., Zoccali M., Ortolani S., Hill V., Minniti D., Bica E., Renzini A., Gómez A., VLT-FLAMES analysis of 8 giants in the bulge metal-poor globular cluster NGC 6522: oldest cluster in the Galaxy?. Analysis of 8 giants in NGC 6522, A\&A, 2009, vol. 507, p. 405

Barbuy B., Zoccali M., Ortolani S., Minniti D., Hill V., Renzini A., Bica E., Gómez A., NGC 6558: A Blue Horizontal Branch Moderately Metal-Poor Globular Cluster in the Bulge, AJ, 2007, vol. 134, p. 1613

Barnes J. E., Memory in Mergers Major and Minor. In Formation of the Galactic Halo...Inside and Out , vol. 92 of Astronomical Society of the Pacific Conference Series, 1996, p. 415

Bekki K., Freeman K. C., Formation of $\omega$ Centauri from an ancient nucleated dwarf galaxy in the young Galactic disc, MNRAS, 2003, vol. 346, p. L11

Bellazzini M., Ibata R. A., Chapman S. C., Mackey A. D., Monaco L., Irwin M. J., Martin N. F., Lewis G. F., Dalessandro E., The Nucleus of the Sagittarius Dsph Galaxy and M54: a Window on the Process of Galaxy Nucleation, AJ, 2008, vol. 136, p. 1147

Bensby T., Feltzing S., Lundström I., Ilyin I., $\alpha-$, r-, and s-process element trends in the Galactic thin and thick disks, A\&A, 2005, vol. 433, p. 185

Besla G., Hernquist L., Loeb A., The origin of the microlensing events observed towards the LMC and the stellar counterpart of the Magellanic stream, MNRAS, 2013, vol. 428, p. 2342 
Bica E., Bonatto C., Dutra C. M., Santos J. F. C., A general catalogue of extended objects in the Magellanic System, MNRAS, 2008, vol. 389, p. 678

Bono G., Caputo F., Castellani V., Marconi M., Nonlinear investigation of the pulsational properties of RR Lyrae variables, A\&AS, 1997, vol. 121, p. 327

Borissova J., Bonatto C., Kurtev R., Clarke J. R. A., Peñaloza F., Sale S. E. e. a., New Galactic star clusters discovered in the VVV survey, A\&A, 2011, vol. 532, p. A131

Bressan A., Marigo P., Girardi L., Salasnich B., Dal Cero C., Rubele S., Nanni A., PARSEC: stellar tracks and isochrones with the PAdova and TRieste Stellar Evolution Code, MNRAS, 2012, vol. 427, p. 127

Broeils A. H., van Woerden H., A search for spiral galaxies with extended HI disks., A\&AS, 1994, vol. 107, p. 129

Caffau E., Ludwig H.-G., Steffen M., Freytag B., Bonifacio P., Solar Chemical Abundances Determined with a CO5BOLD 3D Model Atmosphere, Sol. Phys., 2011, vol. 268, p. 255

Carretta E., Bragaglia A., Gratton R., D’Orazi V., Lucatello S., Intrinsic iron spread and a new metallicity scale for globular clusters, A\&A, 2009, vol. 508, p. 695

Carretta E., Bragaglia A., Gratton R., Lucatello S., Na-O anticorrelation and HB. VIII. Proton-capture elements and metallicities in 17 globular clusters from UVES spectra, A\&A, 2009, vol. 505, p. 139

Carretta E., Bragaglia A., Gratton R. G., D'Orazi V., Lucatello S., Sollima A., Terzan 8: a Sagittarius-flavoured globular cluster, A\&A, 2014, vol. 561, p. A87

Carretta E., Bragaglia A., Gratton R. G., Lucatello S., Bellazzini M., Catanzaro G., Leone F., Momany Y., Piotto G., D’Orazi V., M54 + Sagittarius = $\omega$ Centauri, ApJ, 2010, vol. 714 , p. L7

Carretta E., Bragaglia A., Gratton R. G., Lucatello S., Catanzaro G., Leone F., Bellazzini M., Claudi R., D’Orazi V., Momany Y., Ortolani S., Pancino E., Piotto G., Recio-Blanco A., Sabbi E., Na-O anticorrelation and HB. VII. The chemical composition of first and second-generation stars in 15 globular clusters from GIRAFFE spectra, A\&A, 2009, vol. 505 , p. 117 
Carretta E., Bragaglia A., Gratton R. G., Recio-Blanco A., Lucatello S., D'Orazi V., Cassisi S., Properties of stellar generations in globular clusters and relations with global parameters, A\&A, 2010, vol. 516, p. A55

Carretta E., Cohen J. G., Gratton R. G., Behr B. B., An Abundance Analysis for Four Red Horizontal-Branch Stars in the Extremely Metal-Rich Globular Cluster NGC 6528, AJ, 2001, vol. 122, p. 1469

Carretta E., Gratton R. G., Lucatello S., Bragaglia A., Catanzaro G., Leone F., Momany Y., D’Orazi V., Cassisi S., D'Antona F., Ortolani S., Abundances for a Large Sample of Red Giants in NGC 1851: Hints for a Merger of Two Clusters?, ApJ, 2010, vol. 722, p. L1

Casetti-Dinescu D. I., Moni Bidin C., Girard T. M., Méndez R. A., Vieira K., Korchagin V. I., van Altena W. F., Recent Star Formation in the Leading Arm of the Magellanic Stream, ApJ, 2014, vol. 784, p. L37

Catelan M., Ferraro F. R., Rood R. T., Horizontal-Branch Models and the SecondParameter Effect. IV. The Case of M3 and Palomar 3, ApJ, 2001, vol. 560, p. 970

Catelan M., Pritzl B. J., Smith H. A., The RR Lyrae Period-Luminosity Relation. I. Theoretical Calibration, ApJS, 2004, vol. 154, p. 633

Cayrel R., Data Analysis. In The Impact of Very High S/N Spectroscopy on Stellar Physics , vol. 132 of IAU Symposium, 1988, p. 345

Cayrel R., Perrin M.-N., Barbuy B., Buser R., A grid of synthetic spectra for the determination of effective temperature, gravity and metallicity of F, G, and K stars. I Description of the method. II - Application to 41 stellar spectra taken in the Basel field of SA 141, A\&A, 1991, vol. 247, p. 108

Cenarro A. J., Peletier R. F., Sánchez-Blázquez P., Selam S. O., Toloba E., Cardiel N., Falcón-Barroso J., Gorgas J., Jiménez-Vicente J., Vazdekis A., Medium-resolution Isaac Newton Telescope library of empirical spectra - II. The stellar atmospheric parameters, MNRAS, 2007, vol. 374, p. 664 
Cignoni M., Cole A. A., Tosi M., Gallagher J. S., Sabbi E., Anderson J., Grebel E. K., Nota A., Star Formation History in Two Fields of the Small Magellanic Cloud Bar, ApJ, 2012, vol. 754 , p. 130

Cignoni M., Cole A. A., Tosi M., Gallagher J. S., Sabbi E., Anderson J., Grebel E. K., Nota A., Mean Age Gradient and Asymmetry in the Star Formation History of the Small Magellanic Cloud, ApJ, 2013, vol. 775, p. 83

Cioni M.-R. L., Clementini G., Girardi L., Guandalini R., Gullieuszik M., Miszalski B., Moretti M.-I., Ripepi V., Rubele S., Bagheri G. e. a., The VMC survey. I. Strategy and first data, A\&A, 2011, vol. 527, p. A116

Coelho P., Barbuy B., Meléndez J., Schiavon R. P., Castilho B. V., A library of high resolution synthetic stellar spectra from $300 \mathrm{~nm}$ to $1.8 \mu \mathrm{m}$ with solar and $\alpha$-enhanced composition, A\&A, 2005, vol. 443, p. 735

Cohen J. G., Behr B. B., Briley M. M., Abundances in Stars from the Red Giant Branch Tip to Near the Main-Sequence Turnoff in M71. I. Sample Selection, Observing Strategy, and Stellar Parameters, AJ, 2001, vol. 122, p. 1420

Cohen J. G., Gratton R. G., Behr B. B., Carretta E., An Abundance Analysis for Five Red Horizontal-Branch Stars in the Extremely Metal-rich Globular Cluster NGC 6553, ApJ, 1999, vol. 523, p. 739

Cohen J. G., Kirby E. N., Simon J. D., Geha M., NGC 2419-Another Remnant of Accretion by the Milky Way, ApJ, 2010, vol. 725, p. 288

Crowl H. H., Sarajedini A., Piatti A. E., Geisler D., Bica E., Clariá J. J., Santos Jr. J. F. C., The Line-of-Sight Depth of Populous Clusters in the Small Magellanic Cloud, AJ, 2001, vol. 122 , p. 220

Da Costa G. S., AM-3: An Intermediate-Age Star Cluster in the Extreme Outskirts of the SMC. In New Views of the Magellanic Clouds, vol. 190 of IAU Symposium, 1999, p. 446

Da Costa G. S., Armandroff T. E., Abundances and Kinematics of the Globular Cluster Systems of the Galaxy and of the Sagittarius Dwarf, AJ, 1995, vol. 109, p. 2533 
Da Costa G. S., Hatzidimitriou D., Ca II Triplet Spectroscopy of Giants in Small Magellanic Cloud Star Clusters: Abundances, Velocities, and the Age-Metallicity Relation, AJ, 1998, vol. 115, p. 1934

Da Costa G. S., Held E. V., Saviane I., NGC 5824: a luminous outer halo globular cluster with an intrinsic abundance spread, MNRAS, 2014, vol. 438, p. 3507

Da Costa G. S., Held E. V., Saviane I., Gullieuszik M., M22: An [Fe/H] Abundance Range Revealed, ApJ, 2009, vol. 705, p. 1481

Davies R. D., Wright A. E., A tidal origin for the Magellanic Stream, MNRAS, 1977, vol. 180 , p. 71

de Jong J. T. A., Yanny B., Rix H.-W., Dolphin A. E., Martin N. F., Beers T. C., Mapping the Stellar Structure of the Milky Way Thick Disk and Halo Using SEGUE Photometry, ApJ, 2010, vol. 714, p. 663

de Vaucouleurs G., Pence W. D., An outsider's view of the Galaxy - Photometric parameters, scale lengths, and absolute magnitudes of the spheroidal and disk components of our Galaxy, AJ, 1978, vol. 83, p. 1163

Dékány I., Minniti D., Catelan M., Zoccali M., Saito R. K., Hempel M., Gonzalez O. A., VVV Survey Near-infrared Photometry of Known Bulge RR Lyrae Stars: The Distance to the Galactic Center and Absence of a Barred Distribution of the Metal-poor Population, ApJ, 2013, vol. 776, p. L19

Dias B., Coelho P., Barbuy B., Kerber L., Idiart T., Age and metallicity of star clusters in the Small Magellanic Cloud from integrated spectroscopy, A\&A, 2010, vol. 520, p. A85

Dias B., Kerber L. O., Barbuy B., Santiago B., Ortolani S., Balbinot E., Self-consistent physical parameters for five intermediate-age SMC stellar clusters from CMD modelling, A\&A, 2014, vol. 561, p. A106

Diaz J. D., Bekki K., The Tidal Origin of the Magellanic Stream and the Possibility of a Stellar Counterpart, ApJ, 2012, vol. 750, p. 36

Dotter A., Chaboyer B., Jevremović D., Kostov V., Baron E., Ferguson J. W., The Dartmouth Stellar Evolution Database, ApJS, 2008, vol. 178, p. 89 
Dotter A., Sarajedini A., Anderson J., Globular Clusters in the Outer Galactic Halo: New Hubble Space Telescope/Advanced Camera for Surveys Imaging of Six Globular Clusters and the Galactic Globular Cluster Age-metallicity Relation, ApJ, 2011, vol. 738, p. 74

Drinkwater M. J., Jones J. B., Gregg M. D., Phillipps S., Compact stellar systems in the Fornax Cluster: Super-massive star clusters or extremely compact dwarf galaxies?, PASA, 2000, vol. 17, p. 227

Duffau S., Zinn R., Vivas A. K., Carraro G., Méndez R. A., Winnick R., Gallart C., Spectroscopy of QUEST RR Lyrae Variables: The New Virgo Stellar Stream, ApJ, 2006, vol. 636, p. L97

Eggen O. J., Lynden-Bell D., Sandage A. R., Evidence from the motions of old stars that the Galaxy collapsed., ApJ, 1962, vol. 136, p. 748

Evstigneeva E. A., Drinkwater M. J., Peng C. Y., Hilker M., De Propris R., Jones J. B., Phillipps S., Gregg M. D., Karick A. M., Structural Properties of Ultra-Compact Dwarf Galaxies in the Fornax and Virgo Clusters, AJ, 2008, vol. 136, p. 461

Faber S. M., Friel E. D., Burstein D., Gaskell C. M., Old stellar populations. II - an analysis of K-giant spectra, ApJS, 1985, vol. 57, p. 711

Ferraro F. R., Dalessandro E., Mucciarelli A., Beccari G., Rich R. M., Origlia L., Lanzoni B., Rood R. T., Valenti E., Bellazzini M., Ransom S. M., Cocozza G., The cluster Terzan 5 as a remnant of a primordial building block of the Galactic bulge, Nature, 2009, vol. 462, p. 483

Fulbright J. P., Abundances and Kinematics of Field Halo and Disk Stars. I. Observational Data and Abundance Analysis, AJ, 2000, vol. 120, p. 1841

Gadotti D. A., Structural properties of pseudo-bulges, classical bulges and elliptical galaxies: a Sloan Digital Sky Survey perspective, MNRAS, 2009, vol. 393, p. 1531

Gardiner L. T., Noguchi M., N-body simulations of the Small Magellanic Cloud and the Magellanic Stream, MNRAS, 1996, vol. 278, p. 191 
Gardiner L. T., Sawa T., Fujimoto M., Numerical Simulations of the Magellanic System Part One - Orbits of the Magellanic Clouds and the Global Gas Distribution, MNRAS, 1994, vol. 266, p. 567

Gardner E., Debattista V. P., Robin A. C., Vásquez S., Zoccali M., N-body simulation insights into the X-shaped bulge of the Milky Way: kinematics and distance to the Galactic Centre, MNRAS, 2014, vol. 438, p. 3275

Gieles M., Lamers H. J. G. L. M., Portegies Zwart S. F., On the Interpretation of the Age Distribution of Star Clusters in the Small Magellanic Cloud, ApJ, 2007, vol. 668, p. 268

Gilmore G., Randich S., Asplund M., Binney J., Bonifacio P., Drew J., Feltzing S., Ferguson A., The Gaia-ESO Public Spectroscopic Survey, The Messenger, 2012, vol. 147, p. 25

Glatt K., Grebel E. K., Sabbi E., Gallagher J. S., Nota A., Sirianni M., Clementini G., Tosi M., Harbeck D., Koch A., Kayser A., Da Costa G., Age Determination of Six Intermediate-Age Small Magellanic Cloud Star Clusters with Hst/acs, AJ, 2008, vol. 136, p. 1703

Gonzalez O. A., Rejkuba M., Zoccali M., Hill V., Battaglia G., Babusiaux C., Minniti D., Barbuy B., Alves-Brito A., Renzini A., Gomez A., Ortolani S., Alpha element abundances and gradients in the Milky Way bulge from FLAMES-GIRAFFE spectra of $650 \mathrm{~K}$ giants, A\&A, 2011, vol. 530, p. A54

Gonzalez O. A., Rejkuba M., Zoccali M., Valent E., Minniti D., Tobar R., Reddening and metallicity maps of the Milky Way bulge from VVV and 2MASS. III. The first global photometric metallicity map of the Galactic bulge, A\&A, 2013, vol. 552, p. A110

Gratton R. G., Carretta E., Bragaglia A., Multiple populations in globular clusters. Lessons learned from the Milky Way globular clusters, A\&A Rev., 2012, vol. 20, p. 50

Gunn J. E., Gott III J. R., On the Infall of Matter Into Clusters of Galaxies and Some Effects on Their Evolution, ApJ, 1972, vol. 176, p. 1

Harris J., Zaritsky D., A Method for Determining the Star Formation History of a Mixed Stellar Population, ApJS, 2001, vol. 136, p. 25 
Harris J., Zaritsky D., The Star Formation History of the Small Magellanic Cloud, AJ, 2004, vol. 127, p. 1531

Harris W. E., A Catalog of Parameters for Globular Clusters in the Milky Way, AJ, 1996, vol. 112 , p. 1487

Hernandez X., Gilmore G., Valls-Gabaud D., Non-parametric star formation histories for four dwarf spheroidal galaxies of the Local Group, MNRAS, 2000, vol. 317, p. 831

Hernandez X., Valls-Gabaud D., A robust statistical estimation of the basic parameters of single stellar populations - I. Method, MNRAS, 2008, vol. 383, p. 1603

Hernandez X., Valls-Gabaud D., Gilmore G., Deriving star formation histories: inverting Hertzsprung-Russell diagrams through a variational calculus maximum likelihood method, MNRAS, 1999, vol. 304, p. 705

Hesser J. E., Harris G. L. H., Further spectroscopic evidence bearing on the M22-Omega Centauri comparison, ApJ, 1979, vol. 234, p. 513

Hesser J. E., Shawl S. J., Meyer J. E., Systematic reinvestigation of the radial velocities of the galactic globular clusters - Image-tube results, PASP, 1986, vol. 98, p. 403

Hill V., Lecureur A., Gómez A., Zoccali M., Schultheis M., Babusiaux C., Royer F., Barbuy B., Arenou F., Minniti D., Ortolani S., The metallicity distribution of bulge clump giants in Baade's window, A\&A, 2011, vol. 534, p. A80

Holtzman J. A., Gallagher III J. S., Cole A. A., Mould J. R., Grillmair C. J., Ballester G. E., Burrows C. J., Clarke J. T., Crisp D., Evans R. W., Griffiths R. E., Hester J. J., Hoessel J. G., Scowen P. A., Stapelfeldt K. R., Trauger J. T., Watson A. M., Observations and Implications of the Star Formation History of the Large Magellanic Cloud, AJ, 1999, vol. 118, p. 2262

Hubble E. P., Extragalactic nebulae., ApJ, 1926, vol. 64, p. 321

Ibata R. A., Gilmore G., Irwin M. J., A dwarf satellite galaxy in Sagittarius, Nature, 1994, vol. 370 , p. 194

Javiel S. C., Santiago B. X., Kerber L. O., Constraints on the star formation history of the Large Magellanic Cloud, A\&A, 2005, vol. 431, p. 73 
Jensen J., Mould J., Reid N., The continuity of cluster formation in the Large Magellanic Cloud, ApJS, 1988, vol. 67, p. 77

Katz D., Automatic determination of stellar atmospheric parameters, Journal of Astronomical Data, 2001, vol. 7, p. 8

Katz D., Soubiran C., Cayrel R., Adda M., Cautain R., On-line determination of stellar atmospheric parameters T_eff, log g, [Fe/H] from ELODIE echelle spectra. I. The method, A\&A, 1998, vol. 338, p. 151

Katz D., Soubiran C., Cayrel R., Barbuy B., Friel E., Bienaymé O., Perrin M.-N., Probing the Galactic thick disc vertical properties and interfaces, A\&A, 2011, vol. 525, p. A90+

Kerber L. O., Santiago B. X., Physical parameters of rich LMC clusters from modeling of deep HST colour-magnitude diagrams, A\&A, 2005, vol. 435, p. 77

Kerber L. O., Santiago B. X., Castro R., Valls-Gabaud D., Analysis of colour-magnitude diagrams of rich LMC clusters: NGC 1831, A\&A, 2002, vol. 390, p. 121

King I., The structure of star clusters. I. an empirical density law, AJ, 1962, vol. 67, p. 471

Kirby E. N., Guhathakurta P., Bolte M., Sneden C., Geha M. C., Multi-element Abundance Measurements from Medium-resolution Spectra. I. The Sculptor Dwarf Spheroidal Galaxy, ApJ, 2009, vol. 705, p. 328

Kirby E. N., Guhathakurta P., Simon J. D., Geha M. C., Rockosi C. M., Sneden C., Cohen J. G., Sohn S. T., Majewski S. R., Siegel M., Multi-element Abundance Measurements from Medium-resolution Spectra. II. Catalog of Stars in Milky Way Dwarf Satellite Galaxies, ApJS, 2010, vol. 191, p. 352

Koleva M., Prugniel P., Bouchard A., Wu Y., ULySS: a full spectrum fitting package, A\&A, 2009, vol. 501, p. 1269

Kormendy J., Kennicutt Jr. R. C., Secular Evolution and the Formation of Pseudobulges in Disk Galaxies, ARA\&A, 2004, vol. 42, p. 603 
Leaman R., VandenBerg D. A., Mendel J. T., The bifurcated age-metallicity relation of Milky Way globular clusters and its implications for the accretion history of the galaxy, MNRAS, 2013, vol. 436, p. 122

Lee Y. S., Beers T. C., Sivarani T., Allende Prieto C., Koesterke L., Wilhelm R., Re Fiorentin P., Bailer-Jones C. A. L., Norris J. E., Rockosi C. M., Yanny B., Newberg H. J., Covey K. R., Zhang H.-T., Luo A.-L., The SEGUE Stellar Parameter Pipeline. I. Description and Comparison of Individual Methods, AJ, 2008, vol. 136, p. 2022

Lee Y.-W., Evidence for an old Galactic bulge from RR Lyrae stars in Baade's window - Implications for the formation of the Galaxy and the age of the universe, AJ, 1992, vol. 104, p. 1780

Lee Y.-W., Demarque P., Zinn R., The horizontal-branch stars in globular clusters. 2: The second parameter phenomenon, ApJ, 1994, vol. 423, p. 248

Lee Y.-W., Han S.-I., Joo S.-J., Jang S., Na C., Okamoto S., Arimoto N., Lim D., Kim H.-S., Yoon S.-J., Two Distinct Red Giant Branch Populations in the Globular Cluster NGC 2419 as Tracers of a Merger Event in the Milky Way*, ApJ, 2013, vol. 778, p. L13

Lee Y.-W., Joo J.-M., Sohn Y.-J., Rey S.-C., Lee H.-C., Walker A. R., Multiple stellar populations in the globular cluster $\omega$ Centauri as tracers of a merger event, Nature, 1999, vol. 402 , p. 55

Lin D. N. C., Jones B. F., Klemola A. R., The motion of the Magellanic clouds, origin of the Magellanic Stream, and the mass of the Milky Way, ApJ, 1995, vol. 439, p. 652

Lin D. N. C., Lynden-Bell D., Simulation of the Magellanic Stream to estimate the total mass of the Milky Way, MNRAS, 1977, vol. 181, p. 59

Mackey A. D., van den Bergh S., The properties of Galactic globular cluster subsystems, MNRAS, 2005, vol. 360, p. 631

Majewski S. R., Skrutskie M. F., Weinberg M. D., Ostheimer J. C., A Two Micron All Sky Survey View of the Sagittarius Dwarf Galaxy. I. Morphology of the Sagittarius Core and Tidal Arms, ApJ, 2003, vol. 599, p. 1082 
Marino A. F., Milone A. P., Piotto G., Villanova S., Bedin L. R., Bellini A., Renzini A., A double stellar generation in the globular cluster NGC 6656 (M 22). Two stellar groups with different iron and s-process element abundances, A\&A, 2009, vol. 505, p. 1099

Marino A. F., Milone A. P., Piotto G., Villanova S., Gratton R., D’Antona F., Anderson J., Bedin L. R., Bellini A., Cassisi S., Geisler D., Renzini A., Zoccali M., Sodium-Oxygen Anticorrelation and Neutron-capture Elements in Omega Centauri Stellar Populations, ApJ, 2011, vol. 731, p. 64

Marino A. F., Sneden C., Kraft R. P., Wallerstein G., Norris J. E., da Costa G., Milone A. P., Ivans I. I., Gonzalez G., Fulbright J. P., Hilker M., Piotto G., Zoccali M., Stetson P. B., The two metallicity groups of the globular cluster M 22: a chemical perspective, A\&A, 2011, vol. 532, p. A8

Massari D., Ferraro F. R., Mucciarelli A., Origlia L., Dalessandro E., Lanzoni B., Terzan 5: a pristine fragment of the Galactic Bulge?, ArXiv e-prints, 2014

Mathewson D. S., Cleary M. N., Murray J. D., The Magellanic stream, ApJ, 1974, vol. 190, p. 291

Mathewson D. S., Wayte S. R., Ford V. L., Ruan K., The 'high velocity cloud' origin of the Magellanic system, Proceedings of the Astronomical Society of Australia, 1987, vol. 7, p. 19

Matteucci F., Recchi S., On the Typical Timescale for the Chemical Enrichment from Type Ia Supernovae in Galaxies, ApJ, 2001, vol. 558, p. 351

Mauro F., Moni Bidin C., Geisler D., Saviane I., Da Costa G. S., Gormaz-Matamala A. C., Vasquez S., Chené A.-N., Cohen R., Dias B., Deriving metallicities from calcium triplet spectroscopy in combination with near-infrared photometry, A\&A, 2014, vol. 563, p. A76

McWilliam A., Rich R. M., The first detailed abundance analysis of Galactic bulge K giants in Baade's window, ApJS, 1994, vol. 91, p. 749

Meléndez J., Cohen J. G., The Rise of the AGB in the Galactic Halo: Mg Isotopic Ratios and High Precision Elemental Abundances in M71 Giants, ApJ, 2009, vol. 699, p. 2017 
Mellinger A., A Color All-Sky Panorama Image of the Milky Way, PASP, 2009, vol. 121, p. 1180

Mészáros S., Holtzman J., García Pérez A. E., Allende Prieto C., Schiavon R. P., Basu S. e. a., Calibrations of Atmospheric Parameters Obtained from the First Year of SDSS-III APOGEE Observations, AJ, 2013, vol. 146, p. 133

Mieske S., Hilker M., Infante L., Ultra compact objects in the Fornax cluster of galaxies: Globular clusters or dwarf galaxies?, A\&A, 2002, vol. 383, p. 823

Mieske S., Hilker M., Jordán A., Infante L., Kissler-Patig M., Rejkuba M., Richtler T., Côté P., Baumgardt H., West M. J., Ferrarese L., Peng E. W., The nature of UCDs: Internal dynamics from an expanded sample and homogeneous database, A\&A, 2008, vol. 487 , p. 921

Mighell K. J., Sarajedini A., French R. S., WFPC2 Observations of Star Clusters in the Magellanic Clouds. II. The Oldest Star Clusters in the Small Magellanic Cloud, AJ, 1998, vol. 116, p. 2395

Milone A. D. C., Sansom A. E., Sánchez-Blázquez P., Element abundances in the stars of the MILES spectral library: the Mg/Fe ratio, MNRAS, 2011, vol. 414, p. 1227

Monteiro H., Dias W. S., Caetano T. C., Fitting isochrones to open cluster photometric data. A new global optimization tool, A\&A, 2010, vol. 516, p. A2

Moore B., Davis M., The Origin of the Magellanic Stream, MNRAS, 1994, vol. 270, p. 209

Morrison H. L., The local density of halo giants, AJ, 1993, vol. 106, p. 578

Mottini M., Wallerstein G., McWilliam A., ARP 2 and Terzan 8: a Detailed Chemical Analysis, AJ, 2008, vol. 136, p. 614

Murai T., Fujimoto M., The Magellanic Stream and the Galaxy with a Massive Halo, PASJ, 1980, vol. 32, p. 581

Naylor T., Jeffries R. D., A maximum-likelihood method for fitting colour-magnitude diagrams, MNRAS, 2006, vol. 373, p. 1251 
Newberg H. J., Willett B. A., Yanny B., Xu Y., The Orbit of the Orphan Stream, ApJ, 2010, vol. 711, p. 32

Newberg H. J., Yanny B., Willett B. A., Discovery of a New, Polar-Orbiting Debris Stream in the Milky Way Stellar Halo, ApJ, 2009, vol. 700, p. L61

Nidever D. L., Majewski S. R., Burton W. B., The Origin of the Magellanic Stream and Its Leading Arm, ApJ, 2008, vol. 679, p. 432

Nidever D. L., Majewski S. R., Butler Burton W., Nigra L., The 200deg Long Magellanic Stream System, ApJ, 2010, vol. 723, p. 1618

Nidever D. L., Monachesi A., Bell E. F., Majewski S. R., Muñoz R. R., Beaton R. L., A Tidally Stripped Stellar Component of the Magellanic Bridge, ApJ, 2013, vol. 779, p. 145

Noël N. E. D., Conn B. C., Carrera R., Read J. I., Rix H.-W., Dolphin A., The MAGellanic Inter-Cloud Project (MAGIC). I. Evidence for Intermediate-age Stellar Populations in between the Magellanic Clouds, ApJ, 2013, vol. 768, p. 109

Noguchi M., Early Evolution of Disk Galaxies: Formation of Bulges in Clumpy Young Galactic Disks, ApJ, 1999, vol. 514, p. 77

Olsen K. A., Nidever D. L., Gruendl R. A., Blum R. D., Walker A. R., Saha A., Olszewski E. W., Munoz R., Kunder A. M., Kaleida C. C., Conn B. e. a., SMASH: The Survey of the MAgellanic Stellar History. In American Astronomical Society Meeting Abstracts , vol. 223 of American Astronomical Society Meeting Abstracts, 2014, p. 254.44

Olszewski E. W., Saha A., Knezek P., Subramaniam A., de Boer T., Seitzer P., A 500 Parsec Halo Surrounding the Galactic Globular NGC 1851, AJ, 2009, vol. 138, p. 1570

Origlia L., Massari D., Rich R. M., Mucciarelli A., Ferraro F. R., Dalessandro E., Lanzoni B., The Terzan 5 Puzzle: Discovery of a Third, Metal-poor Component, ApJ, 2013, vol. 779 , p. L5

Origlia L., Rich R. M., Ferraro F. R., Lanzoni B., Bellazzini M., Dalessandro E., Mucciarelli A., Valenti E., Beccari G., Spectroscopy Unveils the Complex Nature of Terzan 5, ApJ, 2011, vol. 726, p. L20 
Ortolani S., Barbuy B., Momany Y., Saviane I., Bica E., Jilkova L., Salerno G. M., Jungwiert B., A Fossil Bulge Globular Cluster Revealed by very Large Telescope Multiconjugate Adaptive Optics, ApJ, 2011, vol. 737, p. 31

Ortolani S., Bica E., Barbuy B., HP 1: a blue horizontal branch globular cluster in the bulge, MNRAS, 1997, vol. 284, p. 692

Ortolani S., Renzini A., Gilmozzi R., Marconi G., Barbuy B., Bica E., Rich R. M., Nearcoeval formation of the Galactic bulge and halo inferred from globular cluster ages, Nature, 1995, vol. 377, p. 701

Pagel B. E. J., Tautvaisiene G., Chemical evolution of the Magellanic Clouds: analytical models, MNRAS, 1998, vol. 299, p. 535

Parisi M. C., Geisler D., Carraro G., Clariá J. J., Costa E., Grocholski A. J., Sarajedini A., Leiton R., Piatti A. E., Age Determination of 15 Old to Intermediate-age Small Magellanic Cloud Star Clusters, AJ, 2014, vol. 147, p. 71

Parisi M. C., Geisler D., Grocholski A. J., Clariá J. J., Sarajedini A., Ca II Triplet Spectroscopy of Small Magellanic Cloud Red Giants. II. Abundances for a Sample of Field Stars, AJ, 2010, vol. 139, p. 1168

Parisi M. C., Grocholski A. J., Geisler D., Sarajedini A., Clariá J. J., Ca II Triplet Spectroscopy of Small Magellanic Cloud Red Giants. I. Abundances and Velocities for a Sample of Clusters, AJ, 2009, vol. 138, p. 517

Perryman M. A. C., de Boer K. S., Gilmore G., Høg E., Lattanzi M. G., Lindegren L., Luri X., Mignard F., Pace O., de Zeeuw P. T., GAIA: Composition, formation and evolution of the Galaxy, A\&A, 2001, vol. 369, p. 339

Piatti A. E., New candidate intermediate-age star clusters in the Small Magellanic Cloud, MNRAS, 2011a, vol. 416, p. L89

Piatti A. E., Towards a comprehensive picture of the star cluster age-metallicity relationship in the Small Magellanic Cloud, MNRAS, 2011b, vol. 418, p. L69

Piatti A. E., Identification of a New Relatively Old Star Cluster in the Small Magellanic Cloud, ApJ, 2012a, vol. 756, p. L32 
Piatti A. E., The star field age-metallicity relationship of the Small Magellanic Cloud, MNRAS, 2012b, vol. 422, p. 1109

Piatti A. E., Clariá J. J., Bica E., Geisler D., Ahumada A. V., Girardi L., Washington photometry of 14 intermediate-age to old star clusters in the Small Magellanic Cloud, MNRAS, 2011, vol. 417, p. 1559

Piatti A. E., Santos Jr. J. F. C., Clariá J. J., Bica E., Ahumada A. V., Parisi M. C., Integrated spectral analysis of 18 concentrated star clusters in the Small Magellanic Cloud, A\&A, 2005, vol. 440, p. 111

Piatti A. E., Sarajedini A., Geisler D., Clark D., Seguel J., Young star clusters immersed in intermediate-age fields in the Small Magellanic Cloud, MNRAS, 2007, vol. 377, p. 300

Piatti A. E., Sarajedini A., Geisler D., Gallart C., Wischnjewsky M., Five young star clusters in the outer region of the Small Magellanic Cloud, MNRAS, 2007a, vol. 382, p. 1203

Piatti A. E., Sarajedini A., Geisler D., Gallart C., Wischnjewsky M., Two newly identified, relatively old star clusters in the Small Magellanic Cloud, MNRAS, 2007b, vol. 381, p. L84

Piatti A. E., Sarajedini A., Geisler D., Seguel J., Clark D., Tracing the formation history of intermediate-age star clusters in the Small Magellanic Cloud, MNRAS, 2005, vol. 358, p. 1215

Pietrinferni A., Cassisi S., Salaris M., Castelli F., A Large Stellar Evolution Database for Population Synthesis Studies. I. Scaled Solar Models and Isochrones, ApJ, 2004, vol. 612, p. 168

Prugniel P., Soubiran C., Koleva M., Le Borgne D., New release of the ELODIE library: Version 3.1, ArXiv Astrophysics e-prints, 2007

Putman M. E., Gibson B. K., Staveley-Smith L., Banks G., Barnes D. G., Bhatal R., Disney M. J. e. a., Tidal disruption of the Magellanic Clouds by the Milky Way, Nature, 1998, vol. 394, p. 752 
Rafelski M., Zaritsky D., The Star Clusters of the Small Magellanic Cloud: Age Distribution, AJ, 2005, vol. 129, p. 2701

Ramírez S. V., Cohen J. G., Abundances in Stars from the Red Giant Branch Tip to Near the Main-Sequence Turnoff in M71. III. Abundance Ratios, AJ, 2002, vol. 123, p. 3277

Ramírez S. V., Cohen J. G., Buss J., Briley M. M., Abundances in Stars from the Red Giant Branch Tip to Near the Main-Sequence Turnoff in M71. II. Iron Abundance, AJ, 2001, vol. 122, p. 1429

Reddy B. E., Lambert D. L., Allende Prieto C., Elemental abundance survey of the Galactic thick disc, MNRAS, 2006, vol. 367, p. 1329

Rich R. M., Shara M., Fall S. M., Zurek D., Two Groups of Nearly Coeval Star Clusters in the Small Magellanic Cloud, AJ, 2000, vol. 119, p. 197

Rich R. M., Shara M. M., Zurek D., New Photometry for the Intermediate-Age Large Magellanic Cloud Globular Cluster NGC 2121 and the Nature of the LMC Age Gap, AJ, 2001, vol. 122, p. 842

Roederer I. U., Chemical Inhomogeneities in the Milky Way Stellar Halo, AJ, 2009, vol. 137, p. 272

Rubele S., Girardi L., Kozhurina-Platais V., Goudfrooij P., Kerber L., The star formation history of the Large Magellanic Cloud star cluster NGC 1751, MNRAS, 2011, vol. 414, p. 2204

Sadler E. M., Rich R. M., Terndrup D. M., K Giants in Baade's Window. II. The Abundance Distribution, AJ, 1996, vol. 112, p. 171

Saito R. K., Hempel M., Minniti D., Lucas P. W., Rejkuba M., Toledo I., Gonzalez O. A., Alonso-García J. e. a., VVV DR1: The first data release of the Milky Way bulge and southern plane from the near-infrared ESO public survey VISTA variables in the Vía Láctea, A\&A, 2012, vol. 537, p. A107

Saito R. K., Minniti D., Dias B., Hempel M., Rejkuba M., Alonso-García J., Barbuy B., Catelan M., Emerson J. P., Gonzalez O. A., Lucas P. W., Zoccali M., Milky Way 
demographics with the VVV survey. I. The 84-million star colour-magnitude diagram of the Galactic bulge, A\&A, 2012, vol. 544, p. A147

Saito R. K., Zoccali M., McWilliam A., Minniti D., Gonzalez O. A., Hill V., Mapping the X-shaped Milky Way Bulge, AJ, 2011, vol. 142, p. 76

Salaris M., Chieffi A., Straniero O., The alpha-enhanced isochrones and their impact on the FITS to the Galactic globular cluster system, ApJ, 1993, vol. 414, p. 580

Sánchez Almeida J., Allende Prieto C., Automated Unsupervised Classification of the Sloan Digital Sky Survey Stellar Spectra using k-means Clustering, ApJ, 2013, vol. 763, p. 50

Sánchez-Blázquez P., Peletier R. F., Jiménez-Vicente J., Cardiel N., Cenarro A. J., FalcónBarroso J., Gorgas J., Selam S., Vazdekis A., Medium-resolution Isaac Newton Telescope library of empirical spectra, MNRAS, 2006, vol. 371, p. 703

Santos Jr. J. F. C., Piatti A. E., Ages and metallicities of star clusters: New calibrations and diagnostic diagrams from visible integrated spectra, A\&A, 2004, vol. 428, p. 79

Saviane I., da Costa G. S., Held E. V., Sommariva V., Gullieuszik M., Barbuy B., Ortolani S., Homogeneous metallicities and radial velocities for Galactic globular clusters. First CaT metallicities for twenty clusters, A\&A, 2012, vol. 540, p. A27

Saviane I., Held E. V., Da Costa G. S., Sommariva V., Gullieuszik M., Barbuy B., Ortolani S., New Surprises in Old Stellar Clusters, The Messenger, 2012, vol. 149, p. 23

Sharpee B., Stark M., Pritzl B., Smith H., Silbermann N., Wilhelm R., Walker A., BV Photometry of Variable Stars in the Northeast Arm of the Small Magellanic Cloud, AJ, 2002, vol. 123 , p. 3216

Sofue Y., Fate of the Magellanic Stream, PASJ, 1994, vol. 46, p. 431

Soldera L., L'effetto dell'arrossamento interstellare sulla misura delle temperature stellari (The effect of the interstellar reddening on the stellar temperature measurements), University of Padova, Department of Astronomy, 2008, Ph.D. Thesis

Soubiran C., Le Campion J.-F., Cayrel de Strobel G., Caillo A., The PASTEL catalogue of stellar parameters, A\&A, 2010, vol. 515, p. A111 
Steinmetz M., Zwitter T., Siebert A., Watson F. G., Freeman K. C., Munari U., Campbell R., Williams M. e. a., The Radial Velocity Experiment (RAVE): First Data Release, AJ, 2006, vol. 132, p. 1645

Stetson P. B., DAOPHOT - A computer program for crowded-field stellar photometry, PASP, 1987, vol. 99, p. 191

Subramanian S., Subramaniam A., The Three-dimensional Structure of the Small Magellanic Cloud, ApJ, 2012, vol. 744, p. 128

Tsujimoto T., Bekki K., Chemical Signature of a Major Merger in the Early Formation of Small Magellanic Cloud, ArXiv e-prints, 2009

VandenBerg D. A., Brogaard K., Leaman R., Casagrande L., The Ages of 55 Globular Clusters as Determined Using an Improved \Delta V^HB_TO Method along with ColorMagnitude Diagram Constraints, and Their Implications for Broader Issues, ApJ, 2013, vol. 775 , p. 134

Vásquez S., Zoccali M., Hill V., Renzini A., González O. A., Gardner E., Debattista V. P., Robin A. C., Rejkuba M., Baffico M., Monelli M., Motta V., Minniti D., 3D kinematics through the X-shaped Milky Way bulge, A\&A, 2013, vol. 555, p. A91

Venn K. A., Irwin M., Shetrone M. D., Tout C. A., Hill V., Tolstoy E., Stellar Chemical Signatures and Hierarchical Galaxy Formation, AJ, 2004, vol. 128, p. 1177

Vergely J.-L., Köppen J., Egret D., Bienaymé O., An inverse method to interpret colourmagnitude diagrams, A\&A, 2002, vol. 390, p. 917

Vesperini E., McMillan S. L. W., D'Antona F., D'Ercole A., The Fraction of Globular Cluster Second-generation Stars in the Galactic Halo, ApJ, 2010, vol. 718, p. L112

Villanova S., Geisler D., Piotto G., Detailed Abundances of Red Giants in the Globular Cluster NGC 1851: C+N+O and the Origin of Multiple Populations, ApJ, 2010, vol. 722, p. L18

Worthey G., Faber S. M., Gonzalez J. J., Burstein D., Old stellar populations. 5: Absorption feature indices for the complete LICK/IDS sample of stars, ApJS, 1994, vol. 94, p. 687 
Wu Y., Luo A.-L., Li H.-N., Shi J.-R., Prugniel P., Liang Y.-C., Zhao Y.-H., Zhang J.N., Bai Z.-R., Wei P., Dong W.-X., Zhang H.-T., Chen J.-J., Automatic determination of stellar atmospheric parameters and construction of stellar spectral templates of the Guoshoujing Telescope (LAMOST), Research in Astronomy and Astrophysics, 2011, vol. 11, p. 924

Wylie-de Boer E., Freeman K., HERMES - An instrument of the future. In IAU Symposium , vol. 262 of IAU Symposium, 2010, p. 448

Wyse R. F. G., Gilmore G., Franx M., Galactic Bulges, ARA\&A, 1997, vol. 35, p. 637

York D. G., Adelman J., Anderson Jr. J. E., Anderson S. F., Annis J., Bahcall N. A., Bakken J. A., SDSS Collaboration The Sloan Digital Sky Survey: Technical Summary, AJ, 2000, vol. 120, p. 1579

Zinn R., West M. J., The globular cluster system of the galaxy. III - Measurements of radial velocity and metallicity for 60 clusters and a compilation of metallicities for 121 clusters, ApJS, 1984, vol. 55, p. 45

Zoccali M., Barbuy B., Hill V., Ortolani S., Renzini A., Bica E., Momany Y., Pasquini L., Minniti D., Rich R. M., The metal content of the bulge globular cluster NGC 6528, A\&A, 2004, vol. 423, p. 507

Zoccali M., Gonzalez O. A., Vasquez S., Hill V., Rejkuba M., Valenti E., Renzini A., RojasArriagada A., Martinez-Valpuesta I., Babusiaux C., Brown T., Minniti D., McWilliam A., The GIRAFFE Inner Bulge Survey (GIBS). I. Survey description and a kinematical map of the Milky Way bulge, A\&A, 2014, vol. 562, p. A66

Zoccali M., Renzini A., Ortolani S., Bica E., Barbuy B., The Proper Motion of the Globular Cluster NGC 6553 and of Bulge Stars with the Hubble Space Telescope, AJ, 2001, vol. 121 , p. 2638 
Appendix 

Appendix A

\section{SMC clusters CMD statistical analysis}

\section{A.1 Likelihood results and degeneracy investigation}

We analysed the likelihood maps to investigate the degeneracy in age, metallicity, reddening, and distance modulus. The likelihood values were obtained from a comparison of a given synthetic CMD with the observed one (see Section 3 for details).

To establish the standard deviation of the likelihood statistics, we proceeded as follows. A synthetic best model CMD was created with the same numbers of stars as the observed one. We call this a false observation. We then compared this CMD with another one with the same parameters but with many more stars (e.g. $10^{5}$ ). From this model vs. model comparison, a likelihood value is obtained, as previously done for data vs. model comparions. Then simulating 300 false observations, and comparing them with the star rich synthetic CMD, we derived the standard deviation $\sigma$. This tells how much the likelihood can vary due only to stochastic effects, i.e., due to the statistical fluctuations on the position of stars in a CMD with a number of points compatible with that of the observed CMDs.

The likelihood maps of these comparisons are displayed in Figures A.1 to A.5, in the space of parameters of $[\mathrm{Fe} / \mathrm{H}]$ vs. $\log ($ age $), \log ($ age $)$ vs. $(\mathrm{m}-\mathrm{M})$, and $\mathrm{E}(\mathrm{B}-\mathrm{V})$ vs. (m-M), centred in the parameters of the best-fit. The colour scale is red, green, cyan or yellow, corresponding to a difference from the best fit solution up to 1-sigma, between 1- and 2-sigma, between 2- and 3-sigma, and larger than 3-sigma of the best fit, respectively. The central panel in each figure gives the value obtained for the two fixed parameters, since in each ma two parameters are varied and the two others are fixed.

These maps clearly reveal the expected anti-correlation beteween age and metallicity, distance modulus and age, and between distance modulus and reddening. These degene- 
racies are directly reflected in the physical parameter uncertainties since they correspond to the standard deviation in each parameter for the set of models with likelihood values up to 1-sigma (red points) from the best-fit solution.
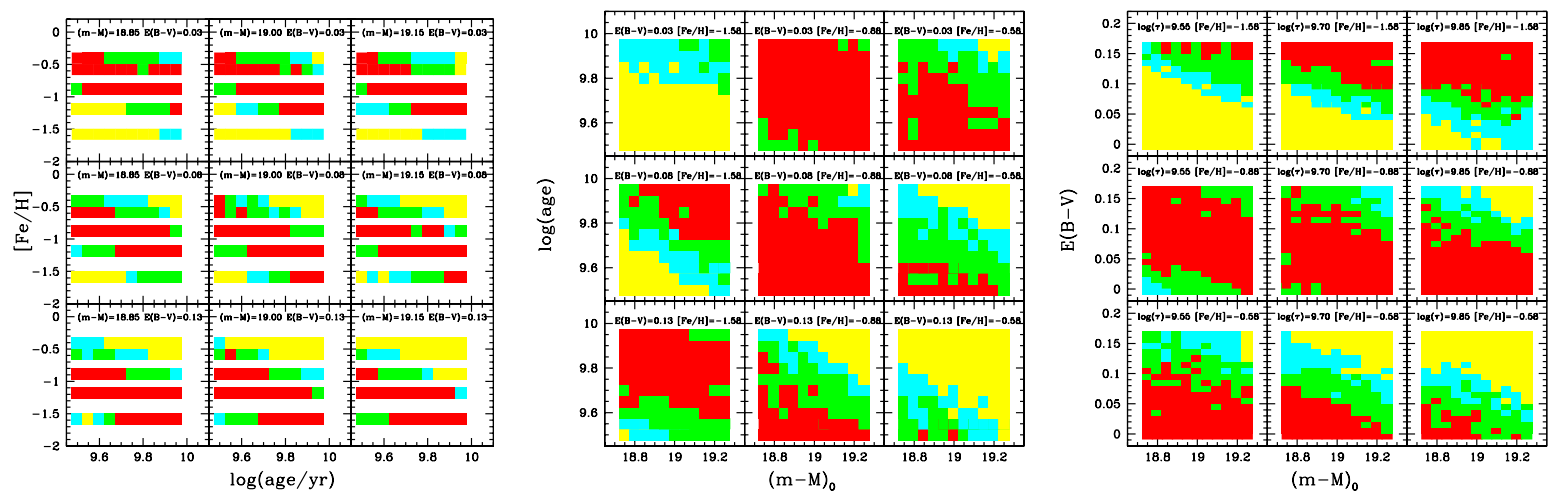

Figure A.1: Likelihood from the fits for different combinations of age, metallicity, reddening, and distance modulus for AM 3. Upper panel: metallicity vs. age; middle panel: age vs. distance modulus; bottom panel: reddening vs. distance modulus. The colour scale is red, green, cyan or yellow, corresponding to a difference from the best-fit solution up to 1-sigma, between 1- and 2-sigma, between 2- and 3-sigma, and larger than 3-sigma of the best fit, respectively.
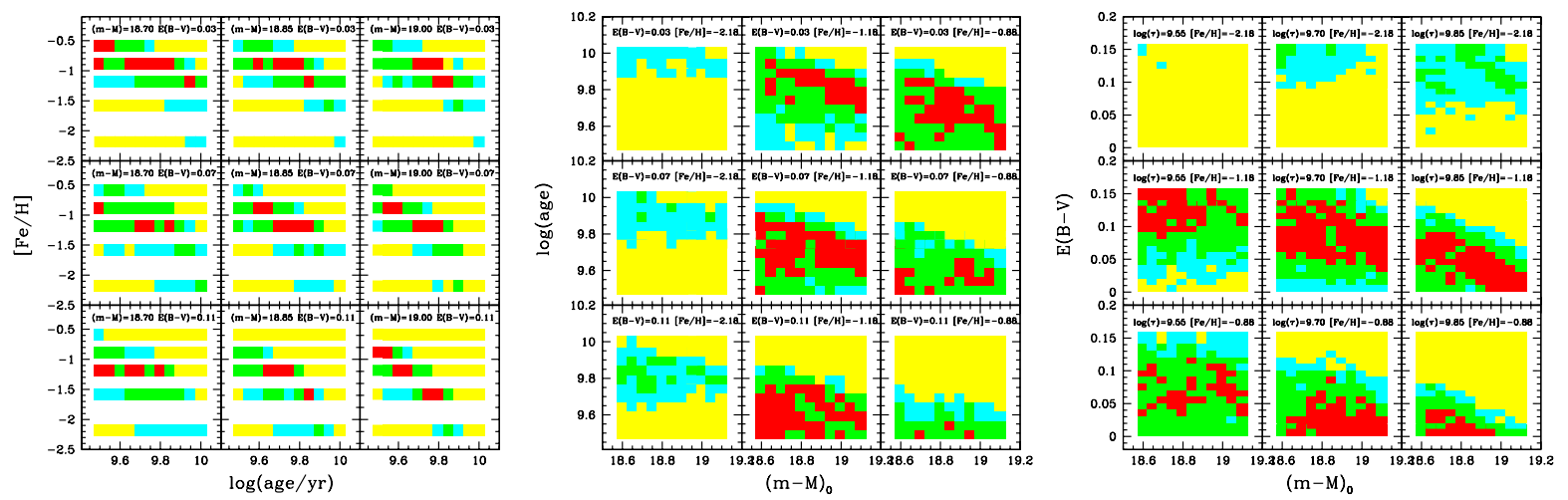

Figure A.2: Same as Fig. A.1 for HW 1. 

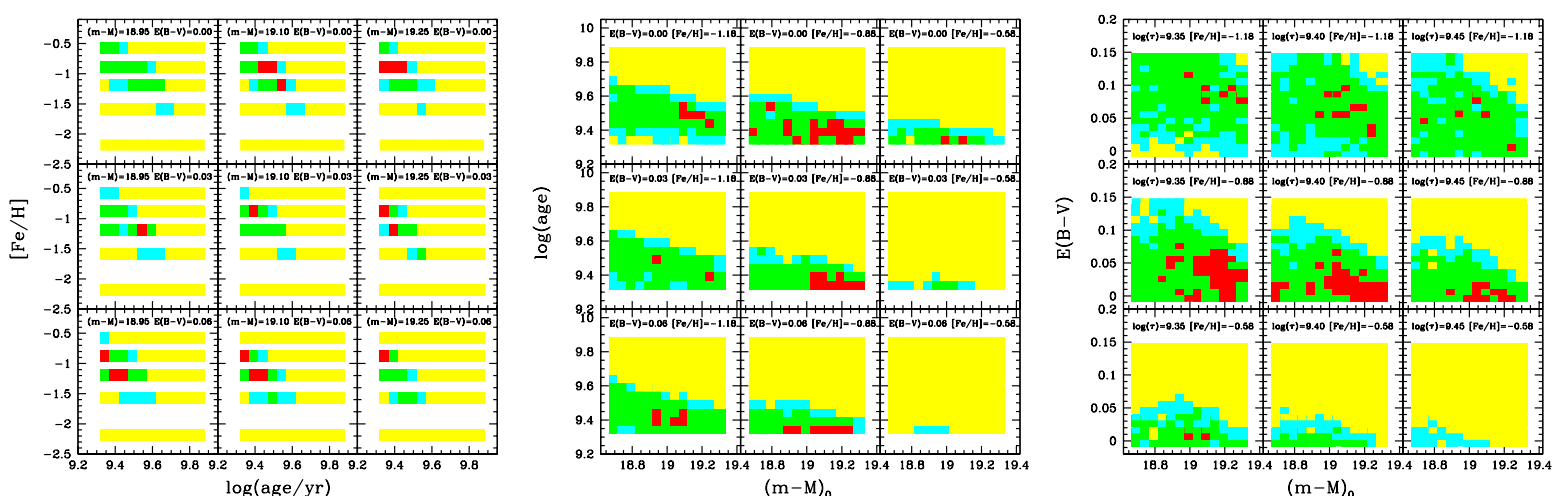

Figure A.3: Same as Fig. A.1 for HW 40.
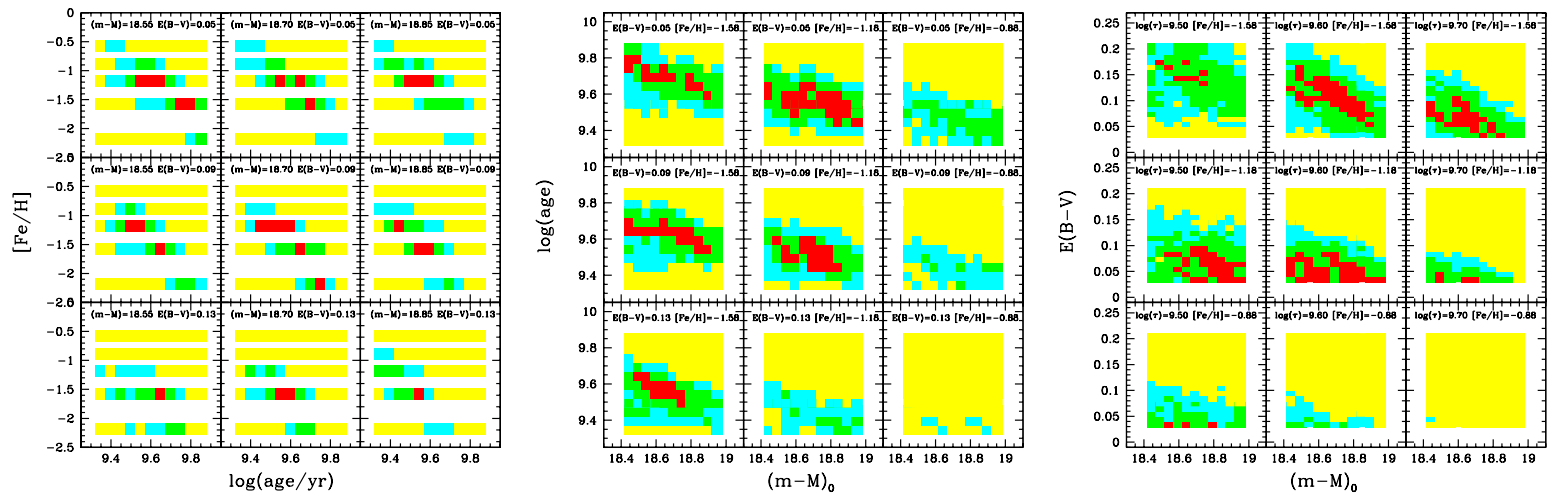

Figure A.4: Same as Fig. A.1 for Lindsay 2.
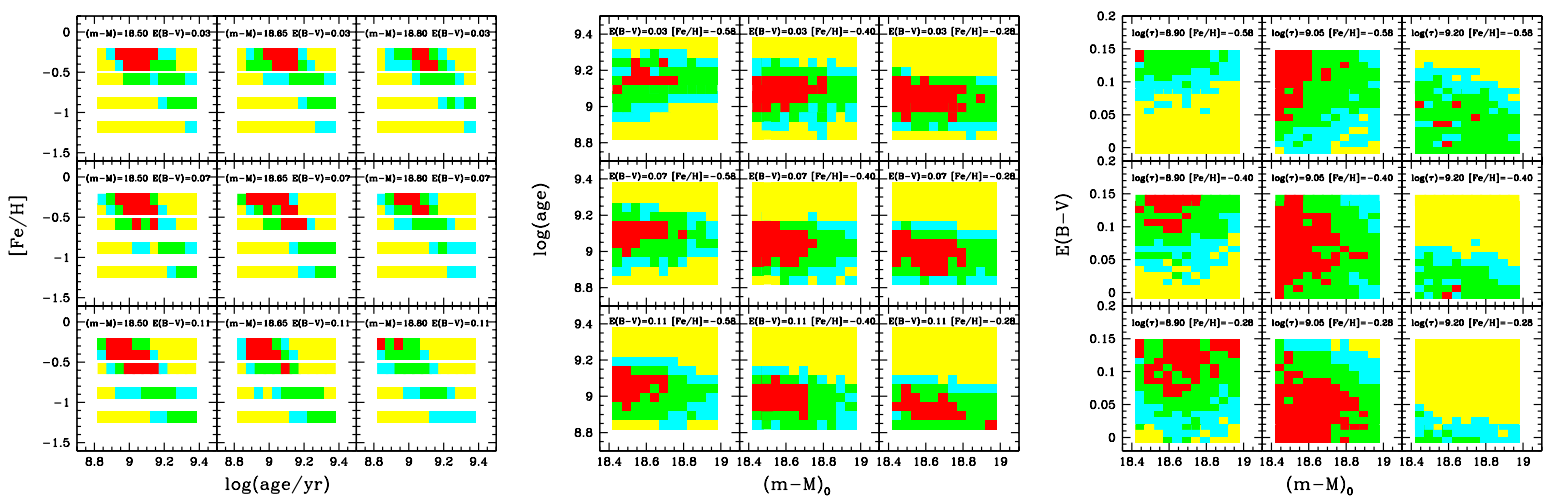

Figure A.5: Same as Fig. A.1 for Lindsay 3. 
Appendix B

\section{Tables with results for FORS2 spectra}

Table B.1 - Star by star coordinates, magnitude, colour, heliocentric radial velocity. Velocities from CaT were taken from from Saviane et al. (2012) for NGC 6528, NGC 6553, M 71 and NGC 6558, and from Vasquez et al. (2014, in prep.) for NGC 6426 and Terzan 8.

\begin{tabular}{|c|c|c|c|c|c|c|c|c|}
\hline Star ID & $\begin{array}{l}\text { RA (J2000) } \\
(\mathrm{deg})\end{array}$ & $\begin{array}{l}\text { DEC (J2000) } \\
(\mathrm{deg})\end{array}$ & $\begin{array}{c}\mathrm{V} \\
(\mathrm{mag})\end{array}$ & $\begin{array}{c}\text { V-I } \\
(\mathrm{mag})\end{array}$ & $\begin{array}{l}\mathrm{B}-\mathrm{V} \\
(\mathrm{mag})\end{array}$ & $\begin{array}{r}\mathrm{V}_{\text {helio }} \\
(\mathrm{km} / \mathrm{s})\end{array}$ & $\begin{array}{r}\text { Vhelio-CaT } \\
(\mathrm{km} / \mathrm{s})\end{array}$ & members \\
\hline NGC6528_2 & 271.1807715771910 & -30.0070234836130 & 15.987 & 1.982 & - & -51.55 & - & \\
\hline NGC6528_3 & 271.1933667526377 & -30.0114575457940 & 15.647 & 2.836 & - & 61.28 & - & \\
\hline NGC6528_4 & 271.1789935185810 & -30.0172537974630 & 15.596 & 2.257 & - & -314.24 & - & \\
\hline NGC6528_5 & 271.1883528713980 & -30.0237445060760 & 15.939 & 1.937 & - & -226.44 & - & \\
\hline NGC6528_6 & 271.1974416677920 & -30.0295599638440 & 17.060 & 1.741 & - & -4.93 & - & \\
\hline NGC6528_7 & 271.2102328036860 & -30.0372195159180 & 15.887 & 2.140 & - & -79.24 & - & \\
\hline NGC6528_8 & 271.2027175539120 & -30.0434602130820 & 16.428 & 1.831 & - & 179.09 & 200 & M \\
\hline NGC6528_9 & 271.1969387171677 & -30.0500739849720 & 16.501 & 1.774 & - & 199.18 & 208 & M \\
\hline NGC6528_10 & 271.2070341687930 & -30.0537176170110 & 16.145 & 2.007 & - & 177.87 & 209 & M \\
\hline NGC6528_11 & 271.2013524700317 & -30.0600904185180 & 15.511 & 2.255 & - & 182.67 & 202 & M \\
\hline NGC6528_13 & 271.1879324598030 & -30.0747465095010 & 16.429 & 1.731 & - & -29.84 & - & \\
\hline NGC6528_14 & 271.2012738946260 & -30.0802412070160 & 16.032 & 2.056 & - & -40.56 & - & \\
\hline NGC6528_15 & 271.1774205888063 & -30.0879237445260 & 15.892 & 2.062 & - & -288.86 & - & \\
\hline NGC6528_16 & 271.1843122189650 & -30.0926974725130 & 15.954 & 2.150 & - & -104.91 & - & \\
\hline NGC6528_17 & 271.1904270294383 & -30.1021087576640 & 16.537 & 1.844 & - & -62.96 & - & \\
\hline NGC6528_18 & 271.1726747862950 & -30.1092237523470 & 16.666 & 1.767 & - & -212.87 & - & \\
\hline NGC6528_19 & 271.1832358189100 & -30.1108940656500 & 16.468 & 1.778 & - & -54.47 & - & \\
\hline NGC6553_1 & 272.3536106669400 & -25.8497112721910 & 15.816 & 2.109 & - & 3.90 & -8 & M \\
\hline NGC6553_3 & 272.3507085422689 & -25.8636412811220 & 15.832 & 2.010 & - & 16.71 & 19 & M \\
\hline NGC6553_4 & 272.2982167426244 & -25.8658039783470 & 15.310 & 2.618 & - & -71.71 & -39 & M \\
\hline NGC6553_5 & 272.3267021788947 & -25.8733214097360 & 15.775 & 2.522 & - & 12.23 & -12 & M \\
\hline NGC6553_6 & 272.3161974470756 & -25.8795540059450 & 16.237 & 2.177 & - & 0.81 & -6 & M \\
\hline NGC6553_7 & 272.3258216397713 & -25.8883122417460 & 15.338 & 2.881 & - & 6.76 & 3 & M \\
\hline NGC6553_8 & 272.3501542761960 & -25.8928074210270 & 15.370 & 3.088 & - & 18.98 & - & \\
\hline NGC6553_9 & 272.3409674217040 & -25.9007568498840 & 16.253 & 2.097 & - & -27.72 & -32 & M \\
\hline NGC6553_10 & 272.3439213760840 & -25.9071903257530 & 15.985 & 1.998 & - & 2.34 & 2 & M \\
\hline NGC6553_11 & 272.3287891424960 & -25.9110912319810 & 15.441 & 2.443 & - & 10.12 & -3 & M \\
\hline
\end{tabular}


Table B.1 - continued.

\begin{tabular}{|c|c|c|c|c|c|c|c|c|}
\hline Star ID & $\begin{array}{l}\text { RA (J2000) } \\
(\mathrm{deg})\end{array}$ & $\begin{array}{l}\text { DEC (J2000) } \\
(\mathrm{deg})\end{array}$ & $\begin{array}{c}\mathrm{V} \\
(\mathrm{mag})\end{array}$ & $\begin{array}{c}\text { V-I } \\
(\mathrm{mag})\end{array}$ & $\begin{array}{c}\text { B-V } \\
(\mathrm{mag})\end{array}$ & $\begin{array}{r}\mathrm{v}_{\text {helio }} \\
(\mathrm{km} / \mathrm{s})\end{array}$ & $\begin{array}{r}\text { Vhelio-CaT } \\
(\mathrm{km} / \mathrm{s})\end{array}$ & members \\
\hline NGC6553_13 & 272.3235733284760 & -25.9249134402580 & 15.906 & 2.075 & - & -4.38 & -12 & M \\
\hline NGC6553_14 & 272.3157993633750 & -25.9300477560180 & 15.187 & 2.382 & - & 5.40 & -5 & M \\
\hline NGC6553_15 & 272.3047106358403 & -25.9370597367310 & 14.980 & 2.996 & - & -21.36 & - & \\
\hline NGC6553_16 & 272.3280756666470 & -25.9436286781210 & 15.708 & 2.332 & - & 17.42 & 8 & M \\
\hline NGC6553_17 & 272.2895515368990 & -25.9484055025060 & 15.065 & 2.849 & - & -110.00 & - & \\
\hline NGC6553_18 & 272.3144982937293 & -25.9566926017970 & 15.407 & 3.681 & - & -10.44 & - & \\
\hline NGC6553_19 & 272.3440933085433 & -25.9630097530170 & 15.843 & 2.209 & - & -7.38 & -4 & M \\
\hline M71_2 & 298.4578984488130 & 18.8301452486084 & 14.606 & 1.269 & - & -150.46 & -34 & M \\
\hline M71_4 & 298.4609554670447 & 18.8192204176758 & 13.030 & 1.503 & - & -53.75 & -41 & M \\
\hline M71_5 & 298.4632902665389 & 18.8090499016740 & 14.391 & 1.282 & - & -52.74 & -26 & M \\
\hline M71_6 & 298.4900822754500 & 18.7995866372160 & 13.534 & 1.367 & - & -44.07 & -37 & M \\
\hline M71_7 & 298.4510869475747 & 18.8011235588184 & 12.376 & 1.719 & - & -69.84 & -34 & M \\
\hline M71_8 & 298.4854008510480 & 18.7869940682184 & 14.421 & 1.280 & - & -51.13 & - & \\
\hline M71_9 & 298.4422411559920 & 18.7910962573810 & 13.146 & 1.530 & - & -35.78 & -24 & M \\
\hline M71_10 & 298.4476878071520 & 18.7813713381542 & 12.140 & 2.042 & - & -129.33 & -26 & M \\
\hline M71_13 & 298.4496765965810 & 18.7641970806387 & 14.281 & 1.303 & - & -23.72 & -5 & M \\
\hline M71_14 & 298.4902210311380 & 18.7496150618418 & 14.582 & 1.210 & - & -13.11 & -17 & M \\
\hline M71_15 & 298.4521026881480 & 18.7474809643955 & 14.580 & 1.209 & - & -41.62 & -8 & M \\
\hline M71_16 & 298.4732927595957 & 18.7366583486676 & 14.727 & 1.231 & - & -23.67 & - & \\
\hline NGC6558_3 & 272.6225545017980 & -31.7335169754310 & 16.862 & 1.434 & - & -6.32 & - & \\
\hline NGC6558_4 & 272.5685475821600 & -31.7363376028500 & 16.541 & 1.441 & - & -38.29 & - & \\
\hline NGC6558_5 & 272.6214120279947 & -31.7468917846890 & 16.349 & 1.360 & - & -23.46 & - & \\
\hline NGC6558_6 & 272.5796695743913 & -31.7469946267620 & 15.982 & 1.524 & - & -210.85 & -196 & M \\
\hline NGC6558_7 & 272.5899712149563 & -31.7570504471050 & 15.803 & 1.393 & - & -186.59 & -187 & M \\
\hline NGC6558_8 & 272.5739816190383 & -31.7605125893880 & 13.651 & 2.044 & - & -307.26 & -210 & M \\
\hline NGC6558_9 & 272.5637020636180 & -31.7666306345590 & 16.026 & 1.499 & - & -221.66 & -204 & M \\
\hline NGC6558_10 & 272.5771880085779 & -31.7733227718450 & 16.710 & 1.580 & - & -21.13 & - & \\
\hline NGC6558_11 & 272.5757815235553 & -31.7788392433250 & 16.753 & 1.329 & - & -220.56 & -195 & M \\
\hline NGC6558_12 & 272.5805489015007 & -31.7879129654280 & 16.521 & 1.452 & - & -126.79 & - & \\
\hline NGC6558_13 & 272.5717825299750 & -31.7916739864870 & 15.626 & 1.477 & - & -127.74 & - & \\
\hline NGC6558_14 & 272.5809044425367 & -31.8010529051890 & 16.740 & 1.188 & - & -118.59 & - & \\
\hline NGC6558_15 & 272.5652668675210 & -31.8066271885810 & 16.480 & 1.293 & - & 105.58 & - & \\
\hline NGC6558_16 & 272.5564861827613 & -31.8124812867920 & 16.366 & 1.450 & - & -316.32 & - & \\
\hline NGC6558_17 & 272.5801632324050 & -31.8180552937480 & 16.964 & 1.348 & - & -49.93 & - & \\
\hline NGC6558_18 & 272.6131914789087 & -31.8262981571080 & 16.911 & 1.288 & - & 11.27 & - & \\
\hline NGC6558_19 & 272.5939188278727 & -31.8321512784620 & 17.013 & 1.299 & - & -5.38 & - & \\
\hline NGC6426_1 & 266.2553357204670 & 3.2257821720617 & 18.018 & 1.359 & - & -80.97 & -49.927 & \\
\hline NGC6426_2 & 266.2088188480099 & 3.2192772570129 & 17.597 & 1.394 & - & -2.85 & 11.325 & \\
\hline NGC6426_3 & 266.2200960712349 & 3.2161573577027 & 16.468 & 1.586 & - & -15.18 & 12.662 & \\
\hline NGC6426_4 & 266.2059346355020 & 3.2095717676480 & 16.072 & 1.644 & - & -236.29 & -230.019 & M \\
\hline NGC6426_7 & 266.2472044411759 & 3.1904905620518 & 17.666 & 1.459 & - & -259.55 & -225.413 & M \\
\hline NGC6426_9 & 266.2324246578110 & 3.1746245634344 & 17.170 & 1.515 & - & -244.78 & -222.660 & M \\
\hline NGC6426_10 & 266.2152297889280 & 3.1685396261324 & 15.544 & 1.749 & - & -231.87 & -221.297 & M \\
\hline
\end{tabular}


Table B.1 - continued.

\begin{tabular}{|c|c|c|c|c|c|c|c|c|}
\hline Star ID & $\begin{array}{l}\text { RA (J2000) } \\
(\mathrm{deg})\end{array}$ & $\begin{array}{l}\text { DEC (J2000) } \\
(\mathrm{deg})\end{array}$ & $\begin{array}{c}\mathrm{V} \\
(\mathrm{mag})\end{array}$ & $\begin{array}{c}\mathrm{V}-\mathrm{I} \\
(\mathrm{mag})\end{array}$ & $\begin{array}{c}\text { B-V } \\
\text { (mag) }\end{array}$ & $\begin{array}{r}\mathrm{V}_{\text {helio }} \\
(\mathrm{km} / \mathrm{s})\end{array}$ & $\begin{array}{r}\text { Vhelio-CaT } \\
(\mathrm{km} / \mathrm{s})\end{array}$ & members \\
\hline NGC6426_11 & 266.2225277929239 & 3.1649406775723 & 17.892 & 1.354 & - & -4.92 & 12.270 & \\
\hline NGC6426_13 & 266.2203523684530 & 3.1516328071727 & 16.745 & 1.482 & - & -236.15 & -225.730 & M \\
\hline NGC6426_18 & 266.2405437043161 & 3.1175053813749 & 16.970 & 1.434 & - & 43.08 & 72.570 & \\
\hline Terzan8_1 & 295.4542164758450 & -33.9415478435970 & 16.706 & 1.232 & - & 134.45 & 138.8238 & M \\
\hline Terzan8_4 & 295.4999075670249 & -33.9726804796670 & 15.268 & 1.436 & - & 120.63 & 137.4998 & M \\
\hline Terzan8_5 & 295.4297741922069 & -33.9618073863440 & 17.002 & 1.135 & - & 134.61 & 152.6001 & M \\
\hline Terzan8_6 & 295.4324851623650 & -33.9679803480730 & 17.517 & 1.076 & - & 119.81 & 138.3819 & M \\
\hline Terzan8_8 & 295.4284871648290 & -33.9821965410250 & 17.089 & 1.108 & - & 173.81 & 150.6620 & M \\
\hline Terzan8_9 & 295.4571652028810 & -33.9956864225960 & 15.447 & 1.352 & - & 155.67 & 139.3673 & M \\
\hline Terzan8_10 & 295.4738836006140 & -34.0028288269560 & 17.381 & 1.076 & - & -63.09 & -25.6758 & \\
\hline Terzan8_11 & 295.4369091804230 & -34.0003943267700 & 15.682 & 1.277 & - & 142.74 & 150.6086 & M \\
\hline Terzan8_13 & 295.4233824419659 & -34.0145360437400 & 17.364 & 1.101 & - & 149.42 & 144.2031 & M \\
\hline Terzan8_14 & 295.4815071780459 & -34.0298070702590 & 15.530 & 1.388 & - & 116.26 & 131.9558 & M \\
\hline Terzan8_15 & 295.4375324314209 & -34.0304794278020 & 16.433 & 1.219 & - & 105.77 & 121.5507 & $\mathrm{M}$ \\
\hline Terzan8_16 & 295.4285209120099 & -34.0321898355090 & 16.778 & 1.122 & - & 128.12 & 140.9906 & M \\
\hline Terzan8_18 & 295.4529047094940 & -34.0531331777060 & 16.143 & 1.273 & - & 134.81 & 131.1500 & M \\
\hline HP1_8 & 262.779492341903 & -29.98247389275 & 16.11 & 2.853 & - & 51.39 & 42 & M \\
\hline HP1_10 & 262.7747217681897 & -29.98806695748 & 17.089 & 2.611 & - & 57.23 & 33 & M \\
\hline HP1_11 & 262.774686991011 & -29.976234361979 & 17.023 & 2.582 & - & 51.05 & 49 & M \\
\hline HP1_12 & 262.7843264886397 & -29.986110981196 & 17.436 & 2.461 & - & 60.54 & 46 & M \\
\hline HP1_13 & 262.77288295732 & -29.97799391478 & 16.314 & 2.729 & - & 50.66 & - & M \\
\hline HP1_14 & 262.775818435468 & -29.973607362265 & 17.981 & 2.346 & - & 61.72 & - & \\
\hline HP1_15 & 262.7658913221563 & -29.970602559014 & 18.155 & 2.408 & - & 43.66 & - & \\
\hline HP1_19 & 262.7710596683369 & -29.961417147298 & 18.707 & 2.373 & - & 23.73 & - & \\
\hline HP1_20 & 262.773834846011 & -29.959064708418 & 18.298 & 2.48 & - & -79.38 & - & \\
\hline HP1_21 & 262.764621912009 & -29.95728051885 & 18.698 & 2.436 & - & 62.94 & - & \\
\hline HP1_22 & 262.767341454344 & -29.990314369868 & 17.48 & 2.575 & - & 46.12 & - & M \\
\hline HP1_23 & 262.7795572868710 & -29.993509304553 & 16.625 & 2.757 & - & 55.28 & 41 & M \\
\hline HP1_24 & 262.7845610691 & -29.992512837865 & 17.688 & 2.467 & - & 56.36 & - & M \\
\hline HP1_31 & 262.7669832075777 & -30.015181033202 & 18.99 & 2.419 & - & -127.76 & - & \\
\hline HP1_32 & 262.7748099931330 & -30.010978851507 & 16.993 & 2.679 & - & -33.81 & - & \\
\hline HP1_33 & 262.757315085811 & -30.009544004121 & 18.927 & 2.372 & - & -34.45 & 55 & \\
\hline HP1_34 & 262.781520651355 & -30.013632577769 & 18.249 & 2.5 & - & 93.67 & - & \\
\hline HP1_35 & 262.783949679813 & -30.017159403528 & 18.318 & 2.495 & - & 71.42 & - & \\
\hline HP1_36 & 262.763821357723 & -30.019233977044 & 17.942 & 2.4 & - & -86.25 & 23 & \\
\hline HP1_37 & 262.7988439553969 & -30.026125010639 & 18.517 & 2.493 & - & 15.55 & - & \\
\hline HP1_38 & 262.804671214119 & -30.030509267249 & 18.292 & 2.477 & - & -208.68 & - & \\
\hline HP1_40 & 262.7792611041633 & -30.035669352705 & 18.775 & 2.470 & - & -109.28 & - & \\
\hline HP1_41 & 262.755386489532 & -30.039545559857 & 18.547 & 2.301 & - & -85.63 & - & \\
\hline HP1_42 & 262.7626463939883 & -30.054216574809 & 18.140 & 2.330 & - & -59.15 & - & \\
\hline HP1_43 & 262.7845333499400 & -30.055806701214 & 17.987 & 2.423 & - & 67.24 & - & \\
\hline HP1_44 & 262.7813860411687 & -30.06779264653 & 18.21 & 2.453 & - & -5.16 & - & \\
\hline HP1_45 & 262.77203229412 & -30.069404519862 & 17.79 & 2.448 & - & 60.36 & - & \\
\hline
\end{tabular}


Table B.1 - continued.

\begin{tabular}{|c|c|c|c|c|c|c|c|c|}
\hline Star ID & $\begin{array}{l}\text { RA (J2000) } \\
(\mathrm{deg})\end{array}$ & $\begin{array}{l}\text { DEC (J2000) } \\
(\mathrm{deg})\end{array}$ & $\begin{array}{c}\mathrm{V} \\
(\mathrm{mag})\end{array}$ & $\begin{array}{c}\mathrm{V}-\mathrm{I} \\
(\mathrm{mag})\end{array}$ & $\begin{array}{c}\text { B-V } \\
\text { (mag) }\end{array}$ & $\begin{array}{r}\mathrm{V}_{\text {helio }} \\
(\mathrm{km} / \mathrm{s})\end{array}$ & $\begin{array}{r}\text { Vhelio-CaT } \\
(\mathrm{km} / \mathrm{s})\end{array}$ & members \\
\hline Djorg2_1 & 270.417078411407 & -27.770336770561 & 16.105 & 2.446 & - & -31.19 & -45.945 & \\
\hline Djorg2_3 & 270.484188183519 & -27.780979087074 & 17.191 & 2.166 & - & 56.59 & 28.664 & \\
\hline Djorg2_4 & 270.438318422190 & -27.788128353318 & 16.569 & 2.217 & - & -197.17 & -152.971 & \\
\hline Djorg2_5 & 270.447758172525 & -27.794258449012 & 16.688 & 2.221 & - & 108.52 & 117.805 & \\
\hline Djorg2_6 & 270.428848388335 & -27.802121606205 & 17.102 & 2.235 & - & -27.09 & 16.752 & \\
\hline Djorg2_7 & 270.463779615994 & -27.806532752427 & 16.879 & 2.186 & - & -111.15 & -105.065 & M \\
\hline Djorg2_8 & 270.473027706742 & -27.811879575782 & 17.271 & 2.226 & - & -53.58 & -33.666 & \\
\hline Djorg2_9 & 270.449730151511 & -27.818858148134 & 16.404 & 2.363 & - & -170.86 & -171.734 & M \\
\hline Djorg2_10 & 270.452491503247 & -27.825727319070 & 15.707 & 2.336 & - & -146.38 & -152.576 & $\mathrm{M}$ \\
\hline Djorg2_11 & 270.452744340045 & -27.829940713150 & 16.082 & 2.273 & - & -127.83 & -114.725 & \\
\hline Djorg2_13 & 270.486191567274 & -27.844114862668 & 17.103 & 2.191 & - & 12.25 & 25.8682 & \\
\hline Djorg2_14 & 270.460387721211 & -27.849287982906 & 17.275 & 2.190 & - & -0.23 & 18.0543 & \\
\hline Djorg2_15 & 270.444232735907 & -27.856516596341 & 16.995 & 2.294 & - & -170.02 & -155.4192 & M \\
\hline Djorg2_16 & 270.483310147638 & -27.862658567924 & 16.964 & 2.302 & - & 74.48 & 91.3211 & \\
\hline Djorg2_17 & 270.451485414403 & -27.867894607897 & 17.254 & 2.268 & - & 10.35 & 26.5939 & \\
\hline M22a_1 & 279.1666250722699 & -23.8456280526570 & 14.067 & 1.387 & - & 9.93 & - & \\
\hline M22a_2 & 279.1203368876777 & -23.8502790889930 & 12.051 & 1.607 & - & -280.25 & -175 & \\
\hline M22a_3 & 279.1337615252070 & -23.8586133574210 & 12.390 & 1.543 & - & -150.98 & -157 & M \\
\hline M22a_4 & 279.1776372616150 & -23.8646361547940 & 12.612 & 1.489 & - & -144.13 & -159 & M \\
\hline M22a_5 & 279.1454915186590 & -23.8688173810320 & 13.807 & 1.356 & - & -200.07 & -152 & M \\
\hline M22a_6 & 279.1649925293353 & -23.8784140984930 & 14.101 & 1.325 & - & -154.39 & -163 & M \\
\hline M22a_7 & 279.1190633242593 & -23.8855144892020 & 12.446 & 1.462 & - & -274.77 & -173 & \\
\hline M22a_8 & 279.1497676262920 & -23.8888179678540 & 12.902 & 1.433 & - & -165.38 & -170 & M \\
\hline M22a_9 & 279.1296876926750 & -23.8962744818240 & 14.602 & 1.287 & - & -162.76 & -162 & M \\
\hline M22a_10 & 279.1511268265590 & -23.9004626206730 & 12.872 & 1.481 & - & -151.26 & -148 & M \\
\hline M22a_11 & 279.1761564462446 & -23.9078387986190 & 14.959 & 1.245 & - & -107.27 & -144 & M \\
\hline M22a_13 & 279.1329002956070 & -23.9203534699920 & 15.331 & 1.318 & - & -184.37 & -144 & $\mathrm{M}$ \\
\hline M22a_14 & 279.1381784590147 & -23.9287792077520 & 13.860 & 1.378 & - & -98.39 & -159 & M \\
\hline M22a_15 & 279.1213787575467 & -23.9340248401570 & 13.966 & 1.347 & - & -260.77 & -151 & \\
\hline M22a_16 & 279.1297951311230 & -23.9424120237370 & 14.094 & 1.364 & - & -182.46 & -162 & M \\
\hline M22a_17 & 279.1177440796620 & -23.9492310681210 & 14.242 & 1.301 & - & -266.90 & -136 & \\
\hline M22a_18 & 279.1344761999463 & -23.9552663000650 & 14.348 & 1.293 & - & -154.45 & -165 & M \\
\hline M22a_19 & 279.1137715179620 & -23.9578959925670 & 14.507 & 1.258 & - & -169.73 & -135 & M \\
\hline M22b_2 & 279.1487212876030 & -23.8496758353150 & 13.879 & 1.320 & - & -150.36 & -160 & M \\
\hline M22b_3 & 279.1111623406380 & -23.8560708266330 & 12.856 & 1.446 & - & -159.81 & -148 & M \\
\hline M22b_4 & 279.1557209203100 & -23.8655332874450 & 13.790 & 1.341 & - & -136.49 & -140 & M \\
\hline M22b_5 & 279.1332777302069 & -23.8693388862050 & 13.643 & 1.321 & - & -218.64 & -152 & $\mathrm{M}$ \\
\hline M22b_6 & 279.1680264045470 & -23.8762713491130 & 14.540 & 1.301 & - & -119.28 & -148 & M \\
\hline M22b_7 & 279.1272594035789 & -23.8800383502430 & 12.543 & 1.424 & - & -189.70 & -143 & M \\
\hline M22b_8 & 279.1330535403420 & -23.8879255853180 & 12.573 & 1.491 & - & -175.18 & -162 & M \\
\hline M22b_9 & 279.1625562094060 & -23.8961356996120 & 15.186 & 1.199 & - & 3.52 & - & \\
\hline M22b_10 & 279.1596042399243 & -23.9003368086050 & 12.791 & 1.525 & - & -149.92 & -147 & $\mathrm{M}$ \\
\hline M22b_11 & 279.1728016834153 & -23.9108669779370 & 14.337 & 1.296 & - & -142.83 & -148 & M \\
\hline
\end{tabular}


Table B.1 - continued.

\begin{tabular}{|c|c|c|c|c|c|c|c|c|}
\hline Star ID & $\begin{array}{l}\text { RA (J2000) } \\
(\operatorname{deg})\end{array}$ & $\begin{array}{l}\text { DEC (J2000) } \\
(\mathrm{deg})\end{array}$ & $\begin{array}{c}\mathrm{V} \\
(\mathrm{mag})\end{array}$ & $\begin{array}{c}\mathrm{V}-\mathrm{I} \\
(\mathrm{mag})\end{array}$ & $\begin{array}{c}\text { B-V } \\
(\mathrm{mag})\end{array}$ & $\begin{array}{r}\text { Vhelio } \\
(\mathrm{km} / \mathrm{s})\end{array}$ & $\begin{array}{r}\text { Vhelio-CaT } \\
(\mathrm{km} / \mathrm{s})\end{array}$ & members \\
\hline M22b_12 & 279.1387917919590 & -23.9144019494200 & 14.015 & 1.345 & - & -138.58 & -139 & $\mathrm{M}$ \\
\hline M22b_13 & 279.1446715959634 & -23.9226894359160 & 15.595 & 1.269 & - & -144.68 & -138 & M \\
\hline M22b_15 & 279.1647014983650 & -23.9327436018760 & 15.201 & 1.246 & - & -147.84 & -156 & M \\
\hline M22b_16 & 279.1379540852260 & -23.9404817327490 & 12.710 & 1.577 & - & -132.18 & -146 & M \\
\hline M22b_17 & 279.1436723242483 & -23.9444067248200 & 14.382 & 1.348 & - & -117.82 & - & M \\
\hline M22b_18 & 279.1433891813503 & -23.9509077647760 & 14.440 & 1.296 & - & -148.89 & -166 & M \\
\hline M22b_19 & 279.1132162240080 & -23.9568469309490 & 13.522 & 1.330 & - & -136.20 & -136 & M \\
\hline M22c_1 & 279.1200663503463 & -23.8480911555530 & 15.564 & 1.226 & - & -181.06 & -166 & M \\
\hline M22c_2 & 279.1227957715860 & -23.8524548018580 & 14.138 & 1.283 & - & -272.56 & -158 & \\
\hline M22c_3 & 279.1289637909800 & -23.8571515884500 & 14.969 & 1.257 & - & -158.88 & -126 & M \\
\hline M22c_4 & 279.1359228263940 & -23.8628693264100 & 14.595 & 1.295 & - & -173.62 & -149 & M \\
\hline M22c_5 & 279.1241844719839 & -23.8707070342580 & 14.011 & 1.293 & - & -261.18 & -148 & \\
\hline M22c_6 & 279.1430691671390 & -23.8787067306810 & 14.675 & 1.284 & - & -163.65 & -148 & M \\
\hline M22c_7 & 279.1499272095340 & -23.8853796867550 & 13.924 & 1.328 & - & -163.13 & -140 & M \\
\hline M22c_8 & 279.1537927426139 & -23.8888545995990 & 13.914 & 1.315 & - & -127.20 & -124 & M \\
\hline M22c_9 & 279.1702654177500 & -23.8952114942570 & 14.116 & 1.347 & - & -142.68 & -149 & M \\
\hline M22c_10 & 279.1558910801040 & -23.9046055967410 & 14.017 & 1.348 & - & -151.16 & -146 & M \\
\hline M22c_11 & 279.1711564217420 & -23.9091704786310 & 15.417 & 1.246 & - & -118.45 & -136 & M \\
\hline M22c_12 & 279.1776201241823 & -23.9164834140020 & 14.867 & 1.262 & - & -45.50 & - & \\
\hline M22c_13 & 279.1489093190013 & -23.9191389245750 & 14.183 & 1.329 & - & -144.23 & -133 & $\mathrm{M}$ \\
\hline M22c_14 & 279.1642129850940 & -23.9288310549590 & 14.222 & 1.343 & - & -105.87 & -158 & M \\
\hline M22c_15 & 279.1386592958460 & -23.9337122259620 & 14.161 & 1.388 & - & -144.71 & -138 & M \\
\hline M22c_16 & 279.1326771879260 & -23.9399533005910 & 14.973 & 1.316 & - & -162.20 & - & M \\
\hline M22c_17 & 279.1306698772860 & -23.9460284799480 & 14.862 & 1.300 & - & -157.68 & -132 & M \\
\hline M22c_18 & 279.1291621975290 & -23.9536741591000 & 13.465 & 1.352 & - & -177.36 & -150 & M \\
\hline NGC5824_1 & 225.9734215705400 & -33.0116093771520 & 17.225 & 1.056 & - & -150.40 & - & \\
\hline NGC5824_2 & 226.0165356446810 & -33.0184212781050 & 16.237 & 1.262 & - & -26.26 & -3 & $\mathrm{M}$ \\
\hline NGC5824_3 & 226.0287325923800 & -33.0252307354730 & 16.592 & 1.239 & - & -25.75 & -14 & M \\
\hline NGC5824_4 & 226.0283231480360 & -33.0308974205760 & 15.471 & 1.466 & - & -26.86 & -12 & M \\
\hline NGC5824_5 & 225.9784884294890 & -33.0370597656750 & 18.222 & 1.029 & - & -46.76 & -11 & M \\
\hline NGC5824_6 & 225.9774276480950 & -33.0442239249250 & 17.164 & 1.150 & - & -52.68 & -15 & M \\
\hline NGC5824_7 & 225.9982707978390 & -33.0508289721770 & 16.058 & 1.259 & - & -27.67 & -11 & M \\
\hline NGC5824_8 & 225.9790189358050 & -33.0546714164150 & 16.733 & 1.183 & - & -140.08 & -29 & \\
\hline NGC5824_9 & 225.9833503351730 & -33.0600078905660 & 16.072 & 1.293 & - & -39.41 & -8 & M \\
\hline NGC5824_10 & 226.0209718810750 & -33.0671784622460 & 15.850 & 1.274 & - & -44.20 & -27 & M \\
\hline NGC5824_11 & 225.9797147609669 & -33.0722923238210 & 17.244 & 1.134 & - & -119.30 & -2 & \\
\hline NGC5824_13 & 225.9791626677340 & -33.0866087907630 & 16.657 & 1.187 & - & -23.92 & 12 & M \\
\hline NGC5824_14 & 226.0206452881650 & -33.0920727071730 & 17.300 & 1.090 & - & -24.94 & -4 & $\mathrm{M}$ \\
\hline NGC5824_15 & 225.9838476458860 & -33.0998016563950 & 16.947 & 1.179 & - & -53.36 & -21 & M \\
\hline NGC5824_16 & 225.9728026992450 & -33.1049387810300 & 17.142 & 1.164 & - & -48.56 & - & $\mathrm{M}$ \\
\hline NGC5824_17 & 225.9790519921090 & -33.1111104009490 & 16.454 & 1.259 & - & -42.29 & -8 & $\mathrm{M}$ \\
\hline NGC5824_18 & 226.0258405833140 & -33.1173468021370 & 16.604 & 1.230 & - & -25.02 & -13 & $\mathrm{M}$ \\
\hline NGC5824_19 & 226.0214064677600 & -33.1247697457440 & 16.376 & 1.250 & - & -22.89 & -10 & M \\
\hline
\end{tabular}


Table B.1 - continued.

\begin{tabular}{|c|c|c|c|c|c|c|c|c|}
\hline Star ID & $\begin{array}{l}\text { RA (J2000) } \\
\text { (deg) }\end{array}$ & $\begin{array}{l}\text { DEC (J2000) } \\
(\mathrm{deg})\end{array}$ & $\begin{array}{c}\mathrm{V} \\
(\mathrm{mag})\end{array}$ & $\begin{array}{c}\mathrm{V}-\mathrm{I} \\
(\mathrm{mag})\end{array}$ & $\begin{array}{c}\text { B-V } \\
(\mathrm{mag})\end{array}$ & $\begin{array}{r}\mathrm{v}_{\text {helio }} \\
(\mathrm{km} / \mathrm{s})\end{array}$ & $\begin{array}{r}\text { Vhelio-CaT } \\
(\mathrm{km} / \mathrm{s})\end{array}$ & members \\
\hline 47Tuc_502 & 6.39660800 & -72.03976000 & 11.40 & - & 2.66 & -72.61 & - & \\
\hline 47Tuc_509 & 6.32615400 & -72.08429000 & 12.09 & - & 1.47 & -35.21 & - & M \\
\hline 47Tuc_514 & 6.41895400 & -72.09905000 & 13.74 & - & 1.04 & -46.80 & - & M \\
\hline 47Tuc_517 & 6.42278700 & -72.10688000 & 13.44 & - & 1.16 & -54.61 & - & M \\
\hline 47Tuc_519 & 6.41735800 & -72.10936000 & 12.69 & - & 1.28 & -56.79 & - & M \\
\hline 47Tuc_525 & 6.36514200 & -72.02726000 & 12.84 & - & 1.27 & -44.54 & - & M \\
\hline 47Tuc_533 & 6.33341200 & -72.08765000 & 12.09 & - & 1.40 & -34.56 & - & M \\
\hline 47Tuc_534 & 6.31307100 & -72.03169000 & 14.05 & - & 0.83 & -50.90 & - & M \\
\hline 47Tuc_535 & 6.34090400 & -72.09150000 & 13.79 & - & 1.06 & -49.11 & - & M \\
\hline 47Tuc_539 & 6.36690000 & -72.05583000 & 14.68 & - & 0.90 & -32.26 & - & M \\
\hline 47Tuc_551 & 6.31267500 & -72.07024000 & 12.34 & - & 1.40 & -40.29 & - & M \\
\hline 47Tuc_553 & 6.39460000 & -72.07391000 & 14.15 & - & 0.84 & -46.89 & - & M \\
\hline 47Tuc_554 & 6.36475000 & -72.01599000 & 12.06 & - & 1.57 & -44.67 & - & M \\
\hline 47Tuc_559 & 6.40361700 & -72.01102000 & 13.98 & - & 1.05 & -68.65 & - & M \\
\hline 47Tuc_571 & 6.37052500 & -72.04336000 & 12.59 & - & 1.26 & -58.65 & - & M \\
\hline 47Tuc_581 & 6.40670800 & -72.02340000 & 11.82 & - & 1.66 & -56.46 & - & M \\
\hline NGC2808_1 & 138.0417278196900 & -64.8092533942590 & 15.171 & 1.278 & - & 102.73 & 87 & M \\
\hline NGC2808_2 & 137.9510644096820 & -64.8162521848410 & 15.678 & 1.227 & - & -38.59 & - & \\
\hline NGC2808_3 & 137.9891312000780 & -64.8207082107530 & 15.031 & 1.351 & - & 40.09 & 78 & M \\
\hline NGC2808_4 & 138.0316478749950 & -64.8267569570500 & 14.994 & 1.319 & - & 93.52 & 84 & M \\
\hline NGC2808_5 & 138.0084116317160 & -64.8341757324620 & 15.523 & 1.260 & - & 141.37 & 109 & M \\
\hline NGC2808_6 & 138.0903663713400 & -64.8427676002660 & 15.501 & 1.210 & - & 124.16 & 123 & M \\
\hline NGC2808_7 & 138.0705536048417 & -64.8471283547480 & 15.139 & 1.181 & - & 103.97 & 97 & M \\
\hline NGC2808_8 & 138.0760951541040 & -64.8526824542090 & 14.492 & 1.345 & - & 109.32 & 109 & M \\
\hline NGC2808_9 & 137.9401382663190 & -64.8584820105990 & 15.217 & 1.246 & - & -26.92 & 72 & \\
\hline NGC2808_10 & 138.0699371148180 & -64.8674965399320 & 13.513 & 1.800 & - & 94.61 & 69 & M \\
\hline NGC2808_11 & 137.9445503739920 & -64.8706333755900 & 14.246 & 1.370 & - & -26.70 & 88 & \\
\hline NGC2808_13 & 138.0748040594420 & -64.8849411022800 & 15.047 & 1.318 & - & 92.14 & 82 & M \\
\hline NGC2808_14 & 138.0088058861450 & -64.8908680622540 & 14.969 & 1.292 & - & 118.06 & 118 & M \\
\hline NGC2808_15 & 138.0277500441930 & -64.8968860105230 & 14.848 & 1.242 & - & 100.49 & 99 & M \\
\hline NGC2808_16 & 137.9794418613510 & -64.9039743986850 & 14.122 & 1.520 & - & 81.67 & 93 & M \\
\hline NGC2808_17 & 138.0253961697260 & -64.9118795070550 & 14.728 & 1.381 & - & 70.94 & 56 & M \\
\hline NGC2808_18 & 138.0462439846450 & -64.9176314464940 & 14.622 & 1.381 & - & 253.18 & - & \\
\hline NGC2808_19 & 137.9999025756700 & -64.9209206227550 & 15.689 & 1.219 & - & 68.92 & 99 & M \\
\hline NGC3201_1 & 154.4022188951080 & -46.3576365537710 & 14.905 & 1.172 & - & 391.27 & 522 & \\
\hline NGC3201_2 & 154.4238541394780 & -46.3651754747810 & 14.220 & 1.226 & - & 493.33 & 504 & M \\
\hline NGC3201_5 & 154.4123378691450 & -46.3821823854600 & 14.707 & 1.227 & - & 454.30 & 500 & M \\
\hline NGC3201_6 & 154.4113544203970 & -46.3901722205060 & 13.324 & 1.391 & - & 398.72 & 501 & \\
\hline NGC3201_7 & 154.4319366864960 & -46.3948909223600 & 14.684 & 1.221 & - & 470.72 & 487 & M \\
\hline NGC3201_8 & 154.4374161137010 & -46.4015263953670 & 12.803 & 1.465 & - & 466.05 & 487 & M \\
\hline NGC3201_9 & 154.4390300101110 & -46.4073415606250 & 13.969 & 1.286 & - & 461.17 & 484 & M \\
\hline NGC3201_10 & 154.4547170051770 & -46.4160504916910 & 13.901 & 1.313 & - & 504.96 & 486 & M \\
\hline NGC3201_11 & 154.4542427127600 & -46.4209906922740 & 14.449 & 1.294 & - & 510.49 & 475 & M \\
\hline
\end{tabular}


Table B.1 - continued.

\begin{tabular}{|c|c|c|c|c|c|c|c|c|}
\hline Star ID & $\begin{array}{l}\text { RA (J2000) } \\
(\mathrm{deg})\end{array}$ & $\begin{array}{l}\text { DEC (J2000) } \\
(\mathrm{deg})\end{array}$ & $\begin{array}{c}\mathrm{V} \\
(\mathrm{mag})\end{array}$ & $\begin{array}{c}\mathrm{V}-\mathrm{I} \\
(\mathrm{mag})\end{array}$ & $\begin{array}{c}\text { B-V } \\
\text { (mag) }\end{array}$ & $\begin{array}{r}\mathrm{V}_{\text {helio }} \\
(\mathrm{km} / \mathrm{s})\end{array}$ & $\begin{array}{r}\text { Vhelio-CaT } \\
(\mathrm{km} / \mathrm{s})\end{array}$ & members \\
\hline NGC3201_13 & 154.4761499745440 & -46.4350765100180 & 14.982 & 1.199 & - & 458.56 & 473 & M \\
\hline NGC3201_14 & 154.4567663989070 & -46.4403081429750 & 14.296 & 1.274 & - & 457.51 & 471 & M \\
\hline NGC3201_15 & 154.4221121767170 & -46.4465748973930 & 15.009 & 1.219 & - & 456.96 & 470 & $\mathrm{M}$ \\
\hline NGC3201_16 & 154.4274413445690 & -46.4540124607780 & 12.575 & 1.533 & - & 460.37 & 468 & M \\
\hline NGC3201_17 & 154.4703687642530 & -46.4583470341790 & 14.681 & 1.254 & - & 472.20 & 472 & M \\
\hline NGC3201_18 & 154.4485808118120 & -46.4630495798020 & 14.893 & 1.221 & - & 473.53 & 472 & M \\
\hline M68_49 & 189.81640000 & -26.76985000 & 14.62 & - & 0.62 & -117.70 & - & M \\
\hline M68_30 & 189.82090000 & -26.76078000 & 14.15 & - & 0.87 & -98.51 & - & M \\
\hline M68_74 & 189.82600000 & -26.77787000 & 14.59 & - & 0.84 & -131.91 & - & M \\
\hline M68_II47 & 189.83980000 & -26.80485000 & 15.03 & - & 0.85 & -82.49 & - & M \\
\hline M68_119 & 189.86280000 & -26.79759000 & 13.62 & - & 0.95 & -76.73 & - & M \\
\hline M68_144 & 189.87850000 & -26.79799000 & 12.80 & - & 1.29 & -425.94 & - & \\
\hline M68_185 & 189.89810000 & -26.77817000 & 17.82 & - & 0.75 & -18.11 & - & \\
\hline M68_239 & 189.90680000 & -26.75432000 & 14.19 & - & 0.87 & -76.57 & - & M \\
\hline M68_II72 & 189.91760000 & -26.79374000 & 15.03 & - & 0.85 & -63.83 & - & M \\
\hline M68_II79 & 189.94230000 & -26.76432000 & 14.39 & - & 0.84 & 38.06 & - & \\
\hline M5_II45 & 229.69710000 & 2.12638300 & 14.75 & - & 0.82 & 54.52 & - & M \\
\hline M5_II50 & 229.68660000 & 2.12951400 & 13.92 & - & 0.96 & 46.08 & - & M \\
\hline M5_II51 & 229.67630000 & 2.13015300 & 14.05 & - & 0.96 & 40.96 & - & M \\
\hline M5_II80 & 229.66510000 & 2.12146900 & 14.31 & - & 0.91 & 35.73 & - & M \\
\hline M5_I2 & 229.62980000 & 2.13470800 & 13.87 & - & 1.02 & 53.85 & - & M \\
\hline M5_I50 & 229.62030000 & 2.12908100 & 13.91 & - & 0.97 & 38.16 & - & M \\
\hline M5_I61 & 229.60180000 & 2.11524400 & 13.37 & - & 1.17 & 52.20 & - & M \\
\hline M5_I68 & 229.59390000 & 2.11570600 & 12.37 & - & 1.52 & 44.10 & - & M \\
\hline M5_I71 & 229.58890000 & 2.12383300 & 13.01 & - & 1.29 & 52.36 & - & M \\
\hline M4_1 & 245.9297297869130 & -26.4687300856480 & 13.567 & 1.351 & - & -23.23 & 55 & M \\
\hline M4_3 & 245.9083172618420 & -26.4781130777430 & 13.532 & 1.355 & - & -20.06 & 29 & M \\
\hline M4_4 & 245.9595428044260 & -26.4906387516420 & 13.432 & 1.304 & - & 73.45 & 64 & M \\
\hline M4_5 & 245.9285006629270 & -26.4910400283700 & 12.684 & 1.444 & - & 35.11 & 72 & M \\
\hline M4_6 & 245.9337478207980 & -26.5017457785090 & 11.809 & 1.540 & - & 59.27 & 96 & \\
\hline M4_8 & 245.9108397606630 & -26.5105926710210 & 12.961 & 1.402 & - & -59.65 & 58 & \\
\hline M4_9 & 245.9784456545850 & -26.5223373845770 & 13.145 & 1.338 & - & 136.54 & 53 & \\
\hline M4_10 & 245.9208888506110 & -26.5250639791230 & 12.787 & 1.448 & - & 8.85 & 69 & M \\
\hline M4_11 & 245.9301389427700 & -26.5316608572910 & 12.843 & 1.450 & - & 46.20 & 87 & M \\
\hline M4_13 & 245.9023430347580 & -26.5415733433870 & 13.566 & 1.371 & - & 54.24 & 94 & M \\
\hline M4_16 & 245.9743179217110 & -26.5645714434050 & 12.927 & 1.416 & - & 138.22 & 47 & \\
\hline M4_17 & 245.9732209310530 & -26.5708402941870 & 12.565 & 1.477 & - & 9.44 & 83 & M \\
\hline M4_18 & 245.9739292754490 & -26.5785037699360 & 11.802 & 1.470 & - & 112.24 & 127 & \\
\hline M4_19 & 245.9142992077440 & -26.5813663445440 & 13.593 & 1.291 & - & 62.37 & - & \\
\hline M107_F & 248.08860000 & -13.04748000 & 13.39 & - & 1.70 & -28.32 & - & \\
\hline M107_62 & 248.13850000 & -13.07172000 & 13.97 & - & 1.62 & -8.89 & - & \\
\hline M107_100 & 248.16340000 & -13.07844000 & 14.21 & - & 1.40 & -24.12 & - & \\
\hline
\end{tabular}


Table B.1 - continued.

\begin{tabular}{|c|c|c|c|c|c|c|c|c|}
\hline Star ID & $\begin{array}{l}\text { RA (J2000) } \\
(\mathrm{deg})\end{array}$ & $\begin{array}{l}\text { DEC (J2000) } \\
(\mathrm{deg})\end{array}$ & $\begin{array}{c}\mathrm{V} \\
(\mathrm{mag})\end{array}$ & $\begin{array}{c}\mathrm{V}-\mathrm{I} \\
(\mathrm{mag})\end{array}$ & $\begin{array}{c}\mathrm{B}-\mathrm{V} \\
(\mathrm{mag})\end{array}$ & $\begin{array}{r}\mathrm{v}_{\text {helio }} \\
(\mathrm{km} / \mathrm{s})\end{array}$ & $\begin{array}{r}\text { Vhelio-CaT } \\
(\mathrm{km} / \mathrm{s})\end{array}$ & members \\
\hline M107_I & 248.17360000 & -13.07616000 & 13.89 & - & 1.46 & -40.50 & - & \\
\hline M107_C & 248.18470000 & -13.06669000 & 11.79 & - & 1.17 & -121.78 & - & $\mathrm{M}^{(a)}$ \\
\hline M10_1 & 254.3171837281469 & -4.0411518459603 & 15.107 & 1.191 & - & 15.63 & 84 & M \\
\hline M10_2 & 254.3228668862143 & -4.0453713154607 & 13.949 & 1.262 & - & 11.74 & 71 & $\mathrm{M}$ \\
\hline M10_3 & 254.3398910591860 & -4.0509869791799 & 15.993 & 1.144 & - & 76.32 & 99 & M \\
\hline M10_4 & 254.2952316719980 & -4.0588352280029 & 14.742 & 1.153 & - & 66.80 & 86 & M \\
\hline M10_5 & 254.3056444118800 & -4.0644976762931 & 14.382 & 1.273 & - & 30.46 & 75 & M \\
\hline M10_6 & 254.3248227850040 & -4.0726104654236 & 13.725 & 1.297 & - & 12.85 & 87 & M \\
\hline M10_7 & 254.3138792124880 & -4.0797717909184 & 13.642 & 1.262 & - & 58.66 & 96 & M \\
\hline M10_8 & 254.3036166247190 & -4.0847652166142 & 15.089 & 1.143 & - & -37.99 & 86 & M \\
\hline M10_9 & 254.3092280291750 & -4.0925118847391 & 12.966 & 1.375 & - & 49.06 & 78 & M \\
\hline M10_10 & 254.3115913558880 & -4.0981498933977 & 13.542 & 1.358 & - & 67.55 & 85 & M \\
\hline M10_11 & 254.3160940089350 & -4.1026238369323 & 15.508 & 1.180 & - & 64.64 & 86 & M \\
\hline M10_13 & 254.3216643958510 & -4.1168053117431 & 15.606 & 1.191 & - & 80.99 & 94 & M \\
\hline M10_15 & 254.2960863303000 & -4.1269278482101 & 15.744 & 1.170 & - & 62.41 & 85 & M \\
\hline M10_16 & 254.3131486307350 & -4.1351867073021 & 14.650 & 1.233 & - & 45.67 & - & \\
\hline M10_17 & 254.3023740571860 & -4.1416695758784 & 15.863 & 1.163 & - & -39.54 & - & \\
\hline NGC6397_1 & 265.2212301649530 & -53.6299970684040 & 13.564 & 1.038 & - & -73.51 & 3 & $\mathrm{M}$ \\
\hline NGC6397_2 & 265.2574707418480 & -53.6511313057610 & 12.450 & 1.029 & - & -1.25 & - & \\
\hline NGC6397_4 & 265.1746135514710 & -53.6385099535620 & 12.826 & 1.098 & - & -90.63 & 12 & M \\
\hline NGC6397_5 & 265.1806579482867 & -53.6473420123450 & 13.687 & 1.049 & - & 9.55 & 37 & M \\
\hline NGC6397_6 & 265.1701016417030 & -53.6471000179280 & 13.935 & 1.022 & - & -95.51 & -7 & M \\
\hline NGC6397_7 & 265.1621423202240 & -53.6566342448740 & 13.106 & 1.080 & - & -79.15 & 25 & M \\
\hline NGC6397_8 & 265.1469821778820 & -53.6579510091340 & 13.243 & 1.073 & - & 0.10 & 16 & M \\
\hline NGC6397_9 & 265.2003518222717 & -53.6803241141170 & 12.609 & 1.101 & - & 64.56 & 5 & M \\
\hline NGC6397_10 & 265.1404006970820 & -53.6701161565620 & 13.074 & 1.056 & - & -88.88 & 6 & M \\
\hline NGC6397_11 & 265.1366858414970 & -53.6749607073660 & 12.082 & 1.137 & - & -108.30 & 10 & M \\
\hline NGC6397_13 & 265.1413447194420 & -53.6887671662460 & 12.196 & 1.182 & - & -29.43 & 25 & M \\
\hline NGC6397_14 & 265.1330615276170 & -53.6977610910050 & 12.888 & 1.122 & - & -29.37 & 17 & M \\
\hline NGC6397_14b & 265.13320000 & -53.69827000 & 12.78 & - & 0.89 & 35.51 & - & M \\
\hline NGC6397_15 & 265.1274256674430 & -53.7026382997840 & 13.728 & 1.052 & - & -32.80 & 0 & M \\
\hline NGC6397_16 & 265.1279607171810 & -53.7067037325510 & 12.217 & 1.169 & - & -26.86 & 2 & M \\
\hline NGC6397_16b & 265.12800000 & -53.70719000 & 12.07 & - & 0.93 & 24.78 & - & M \\
\hline NGC6397_19 & 265.0925299293380 & -53.7185477496650 & 13.519 & 1.026 & - & -54.15 & 12 & M \\
\hline NGC6397_319 & 265.09110000 & -53.71521000 & 12.95 & - & 1.02 & 28.31 & - & \\
\hline NGC6397_344 & 265.08000000 & -53.67509000 & 13.50 & - & 1.78 & 84.62 & - & \\
\hline NGC6397_361 & 265.11960000 & -53.64236000 & 11.67 & - & 1.08 & 40.74 & - & M \\
\hline NGC6397_365 & 265.11770000 & -53.62390000 & 13.08 & - & 0.88 & 46.89 & - & M \\
\hline NGC6441_1 & 267.5515879937640 & -36.9959965728170 & 17.300 & 1.553 & - & -179.54 & - & \\
\hline NGC6441_2 & 267.5674913837453 & -37.0010138178080 & 17.215 & 1.651 & - & -22.34 & - & \\
\hline NGC6441_3 & 267.5281735461197 & -37.0057433249170 & 17.327 & 1.516 & - & -62.90 & - & M \\
\hline NGC6441_4 & 267.5486118676010 & -37.0125519130730 & 16.858 & 1.549 & - & 8.19 & 14 & M \\
\hline NGC6441_5 & 267.5612985558160 & -37.0174913542750 & 17.425 & 1.507 & - & 5.78 & - & M \\
\hline
\end{tabular}


Table B.1 - continued.

\begin{tabular}{|c|c|c|c|c|c|c|c|c|}
\hline Star ID & $\begin{array}{l}\text { RA (J2000) } \\
(\mathrm{deg})\end{array}$ & $\begin{array}{l}\text { DEC (J2000) } \\
(\mathrm{deg})\end{array}$ & $\begin{array}{c}\mathrm{V} \\
(\mathrm{mag})\end{array}$ & $\begin{array}{c}\text { V-I } \\
(\mathrm{mag})\end{array}$ & $\begin{array}{c}\text { B-V } \\
\text { (mag) }\end{array}$ & $\begin{array}{r}\mathrm{V}_{\text {helio }} \\
(\mathrm{km} / \mathrm{s})\end{array}$ & $\begin{array}{r}\text { Vhelio-CaT } \\
(\mathrm{km} / \mathrm{s})\end{array}$ & members \\
\hline NGC6441_6 & 267.5617704376787 & -37.0263698501170 & 16.052 & 2.164 & - & 14.04 & 9 & M \\
\hline NGC6441_7 & 267.5192293599370 & -37.0314760337140 & 17.504 & 1.564 & - & -0.10 & 18 & \\
\hline NGC6441_8 & 267.5672891635543 & -37.0398682583090 & 17.086 & 1.696 & - & 29.56 & - & M \\
\hline NGC6441_9 & 267.5704202800800 & -37.0465647228760 & 17.211 & 1.742 & - & 18.87 & 37 & M \\
\hline NGC6441_10 & 267.5262345252269 & -37.0520198353200 & 17.432 & 1.625 & - & -22.21 & 25 & M \\
\hline NGC6441_11 & 267.5786097381969 & -37.0574957675210 & 17.039 & 1.639 & - & -188.15 & 6 & \\
\hline NGC6441_13 & 267.5335816428650 & -37.0708266216330 & 17.378 & 1.471 & - & -37.60 & - & M \\
\hline NGC6441_14 & 267.5445841061490 & -37.0766569572610 & 16.444 & 1.685 & - & -149.36 & - & \\
\hline NGC6441_15 & 267.5297434362500 & -37.0830384774400 & 16.789 & 1.757 & - & -148.33 & - & \\
\hline NGC6441_16 & 267.5704974086177 & -37.0899429393470 & 17.197 & 1.694 & - & -282.15 & - & \\
\hline NGC6441_17 & 267.5395804575260 & -37.0966969885660 & 17.214 & 1.695 & - & -22.99 & - & \\
\hline NGC6441_18 & 267.5270547394850 & -37.1012335037780 & 17.293 & 1.712 & - & -31.15 & - & \\
\hline NGC6441_19 & 267.5818116124880 & -37.1091236311610 & 17.515 & 1.729 & - & -15.20 & - & \\
\hline NGC6752_2 & 287.68260 & -60.024740 & 12.490 & - & 0.900 & -25.14 & - & M \\
\hline NGC6752_4 & 287.70260 & -60.031540 & 13.700 & - & 0.850 & -21.71 & - & M \\
\hline NGC6752_10 & 287.74220 & -60.029200 & 12.700 & - & 0.790 & -36.69 & - & M \\
\hline NGC6752_11 & 287.75580 & -60.034130 & 12.240 & - & 0.640 & -35.20 & - & M \\
\hline NGC6752_12 & 287.76250 & -60.029760 & 11.200 & - & 1.370 & -21.40 & - & M \\
\hline NGC6752_16 & 287.78380 & -60.030550 & 11.470 & - & 1.560 & 10.17 & - & \\
\hline M15_2 & 322.5214189696987 & 12.2227087625862 & 14.398 & 1.105 & - & -223.86 & -138 & M \\
\hline M15_3 & 322.5332798994560 & 12.2154610609082 & 14.313 & 1.135 & - & -143.96 & -145 & M \\
\hline M15_4 & 322.4994012317070 & 12.2084721190462 & 15.452 & 0.952 & - & -128.98 & -122 & M \\
\hline M15_5 & 322.5611079173707 & 12.2033365017587 & 15.648 & 0.971 & - & -136.13 & -130 & M \\
\hline M15_6 & 322.5020644576907 & 12.1940252155229 & 14.413 & 1.084 & - & -199.05 & -98 & M \\
\hline M15_7 & 322.5092035836513 & 12.1897419163095 & 12.809 & 1.396 & - & -212.69 & -102 & M \\
\hline M15_8 & 322.5178193978010 & 12.1826621976324 & 13.751 & 1.199 & - & -120.47 & -108 & M \\
\hline M15_9 & 322.5190490608820 & 12.1761618053724 & 14.037 & 1.147 & - & -115.34 & -101 & M \\
\hline M15_10 & 322.5235211413769 & 12.1714178109786 & 14.309 & 1.140 & - & -110.11 & -95 & M \\
\hline M15_12 & 322.5271887719680 & 12.1566131585421 & 15.432 & 1.044 & - & -189.77 & -102 & M \\
\hline M15_13 & 322.5164140411927 & 12.1498342128064 & 15.044 & 1.073 & - & -137.21 & -108 & M \\
\hline M15_14 & 322.5370046077370 & 12.1474231509706 & 14.844 & 1.069 & - & -113.98 & -124 & M \\
\hline M15_15 & 322.5309994712117 & 12.1375868809030 & 14.037 & 1.150 & - & -161.10 & -115 & M \\
\hline M15_17 & 322.5013766439880 & 12.1272243863982 & 13.792 & 1.180 & - & -196.66 & -78 & M \\
\hline M15_18 & 322.5230161717470 & 12.1185801689600 & 15.581 & 1.035 & - & -197.80 & -101 & M \\
\hline NGC2298_11 & 102.24680 & -36.013800 & 14.210 & - & 1.200 & 122.33 & - & M \\
\hline NGC2298_14 & 102.22770 & -35.987410 & 14.350 & - & 1.220 & 130.55 & - & M \\
\hline NGC2298_16 & 102.23500 & -36.004570 & 14.770 & - & 0.950 & 118.82 & - & M \\
\hline NGC2298_17 & 102.24570 & -36.021050 & 14.870 & - & 1.090 & 145.59 & - & M \\
\hline NGC2298_18 & 102.23460 & -36.012570 & 14.960 & - & 1.000 & 152.26 & - & M \\
\hline NGC4372_20 & 186.29310 & -72.647110 & 12.880 & - & 1.570 & 49.17 & - & M \\
\hline NGC4372_14 & 186.32230 & -72.632710 & 14.290 & - & 1.290 & 54.13 & - & M \\
\hline NGC4372_13 & 186.33780 & -72.626460 & 12.720 & - & 1.730 & 51.30 & - & M \\
\hline
\end{tabular}


Table B.1 - continued.

\begin{tabular}{|c|c|c|c|c|c|c|c|c|}
\hline Star ID & $\begin{array}{l}\text { RA (J2000) } \\
(\mathrm{deg})\end{array}$ & $\begin{array}{l}\text { DEC (J2000) } \\
(\mathrm{deg})\end{array}$ & $\begin{array}{c}\mathrm{V} \\
(\mathrm{mag})\end{array}$ & $\begin{array}{c}\text { V-I } \\
(\mathrm{mag})\end{array}$ & $\begin{array}{c}\text { B-V } \\
\text { (mag) }\end{array}$ & $\begin{array}{r}\mathrm{v}_{\text {helio }} \\
(\mathrm{km} / \mathrm{s})\end{array}$ & $\begin{array}{r}\text { Vhelio-CaT } \\
(\mathrm{km} / \mathrm{s})\end{array}$ & members \\
\hline NGC4372_95 & 186.46560 & -72.621520 & 14.480 & - & 1.300 & 69.04 & - & M \\
\hline NGC4372_91 & 186.48730 & -72.626130 & 14.450 & - & 1.330 & 69.51 & - & M \\
\hline NGC4372_89 & 186.50650 & -72.626710 & 14.490 & - & 1.290 & 61.49 & - & M \\
\hline NGC4372_85 & 186.55180 & -72.629520 & 14.930 & - & 1.030 & 17.25 & - & \\
\hline NGC4372_74 & 186.57040 & -72.657320 & 14.170 & - & 1.400 & 74.77 & - & M \\
\hline NGC4372_76 & 186.59190 & -72.649200 & 14.180 & - & 1.300 & 73.37 & - & M \\
\hline NGC4372_77 & 186.62360 & -72.648570 & 14.190 & - & 1.280 & 14.36 & - & \\
\hline Rup106_2 & 189.6768998880010 & -51.1056842484210 & 17.181 & 1.242 & - & 50.22 & - & \\
\hline Rup106_3 & 189.6932711237220 & -51.1128486896620 & 17.794 & 1.234 & - & -42.80 & -36 & M \\
\hline Rup106_4 & 189.6538310511420 & -51.1187718174390 & 15.628 & 1.510 & - & -148.79 & -54 & \\
\hline Rup106_5 & 189.6729033037630 & -51.1245846157830 & 18.594 & 1.185 & - & 72.46 & - & \\
\hline Rup106_6 & 189.7040715724640 & -51.1288122990960 & 16.676 & 1.343 & - & -58.38 & -49 & M \\
\hline Rup106_7 & 189.6986910763680 & -51.1380491648540 & 16.959 & 1.240 & - & -7.12 & - & \\
\hline Rup106_8 & 189.7272422891820 & -51.1424906917940 & 17.286 & 1.262 & - & 143.16 & - & \\
\hline Rup106_9 & 189.7134282848469 & -51.1486117989790 & 17.660 & 1.209 & - & -47.56 & -43 & M \\
\hline Rup106_10 & 189.6808605474090 & -51.1557282835810 & 17.756 & 1.200 & - & -40.06 & -34 & M \\
\hline Rup106_11 & 189.6755230715590 & -51.1623524677760 & 15.478 & 1.462 & - & -40.72 & -34 & M \\
\hline Rup106_12 & 189.6802353325200 & -51.1691042971530 & 17.053 & 1.255 & - & -37.67 & -28 & M \\
\hline Rup106_14 & 189.6397943947549 & -51.1828891117730 & 18.284 & 1.209 & - & -72.74 & -49 & M \\
\hline Rup106_16 & 189.6408966297330 & -51.1933813852430 & 18.518 & 1.172 & - & -67.91 & - & \\
\hline Rup106_17 & 189.6988243841690 & -51.2013261022120 & 17.279 & 1.286 & - & -37.35 & -23 & M \\
\hline Rup106_18 & 189.6706147758830 & -51.2082040394740 & 18.597 & 1.155 & - & 240.83 & - & \\
\hline NGC5634_6 & 217.4079843945940 & -5.9527883388807 & 17.124 & 1.044 & - & 40.71 & -7 & M \\
\hline NGC5634_7 & 217.3907924909180 & -5.9560295168940 & 17.267 & 0.961 & - & -33.63 & -45 & M \\
\hline NGC5634_8 & 217.3992275868640 & -5.9641932074133 & 16.922 & 0.968 & - & -89.80 & -44 & M \\
\hline NGC5634_9 & 217.3908871017030 & -5.9705719834359 & 17.456 & 1.006 & - & -76.17 & -32 & M \\
\hline NGC5634_11 & 217.4131014284530 & -5.9818178621402 & 15.728 & 1.163 & - & -5.86 & 7 & M \\
\hline NGC5634_13 & 217.3869685734450 & -5.9956708745018 & 17.634 & 0.856 & - & -26.60 & -20 & M \\
\hline NGC5634_14 & 217.4142199604380 & -6.0030100771986 & 16.571 & 1.102 & - & -41.82 & -34 & M \\
\hline NGC5634_15 & 217.3894998618240 & -6.0062776413795 & 15.316 & 1.321 & - & -3.16 & 9 & M \\
\hline NGC5634_17 & 217.3708007790040 & -6.0220435949273 & 17.283 & 0.957 & - & -47.96 & -31 & \\
\hline NGC5694_3 & 219.9348810287710 & -26.4946618047940 & 18.371 & 0.953 & - & -83.00 & -71 & \\
\hline NGC5694_5 & 219.8973138738160 & -26.5043633462540 & 16.864 & 1.120 & - & -168.02 & -144 & M \\
\hline NGC5694_6 & 219.9052304389680 & -26.5119464734560 & 18.410 & 0.982 & - & -153.94 & -140 & M \\
\hline NGC5694_7 & 219.9144178528140 & -26.5205518431080 & 18.058 & 1.012 & - & -143.70 & -129 & M \\
\hline NGC5694_8 & 219.8897697484850 & -26.5262032004710 & 18.374 & 0.999 & - & -142.23 & -119 & M \\
\hline NGC5694_9 & 219.8966177062100 & -26.5303560476950 & 16.666 & 1.196 & - & -157.48 & -136 & M \\
\hline NGC5694_10 & 219.9158491830350 & -26.5368552509350 & 18.443 & 0.972 & - & -177.81 & -159 & \\
\hline NGC5694_11 & 219.8819010796280 & -26.5433086004050 & 18.233 & 1.002 & - & -142.20 & -127 & M \\
\hline NGC5694_13 & 219.9051136155080 & -26.5567467822200 & 17.746 & 1.055 & - & -36.37 & -23 & \\
\hline NGC5694_15 & 219.8873222857810 & -26.5705548936230 & 17.915 & 1.042 & - & -149.53 & -137 & M \\
\hline NGC5694_17 & 219.8940840133800 & -26.5805245126430 & 17.326 & 1.070 & - & -147.01 & -127 & M \\
\hline
\end{tabular}


Table B.1 - continued.

\begin{tabular}{|c|c|c|c|c|c|c|c|c|}
\hline Star ID & $\begin{array}{l}\text { RA (J2000) } \\
(\operatorname{deg})\end{array}$ & $\begin{array}{l}\text { DEC (J2000) } \\
(\mathrm{deg})\end{array}$ & $\begin{array}{c}\mathrm{V} \\
(\mathrm{mag})\end{array}$ & $\begin{array}{c}\text { V-I } \\
(\mathrm{mag})\end{array}$ & $\begin{array}{l}\text { B-V } \\
(\mathrm{mag})\end{array}$ & $\begin{array}{r}\mathrm{v}_{\text {helio }} \\
(\mathrm{km} / \mathrm{s})\end{array}$ & $\begin{array}{r}\text { Vhelio-CaT } \\
(\mathrm{km} / \mathrm{s})\end{array}$ & members \\
\hline NGC5897_138 & 229.32940 & -21.024200 & 14.350 & - & 1.180 & 62.03 & - & M \\
\hline NGC5897_142 & 229.34370 & -21.041560 & 14.580 & - & 1.090 & 94.50 & - & M \\
\hline NGC5897_160 & 229.38830 & -21.039470 & 13.430 & - & 1.430 & 94.29 & - & M \\
\hline NGC5897_255 & 229.37750 & -21.034920 & 13.400 & - & 1.800 & 96.24 & - & M \\
\hline NGC5897_302 & 229.40740 & -21.006760 & 14.100 & - & 1.280 & 96.30 & - & M \\
\hline NGC5897_306 & 229.40020 & -21.041690 & 14.320 & - & 1.180 & 95.76 & - & M \\
\hline NGC5897_316 & 229.36370 & -21.006770 & 13.860 & - & 1.340 & 70.66 & - & M \\
\hline NGC5897_318 & 229.35660 & -21.039150 & 14.580 & - & 1.140 & 91.61 & - & M \\
\hline NGC5927_37 & 231.97970 & -50.662650 & 15.770 & - & 1.590 & -99.77 & - & M \\
\hline NGC5927_65 & 231.97280 & -50.665090 & 14.640 & - & 1.920 & -97.59 & - & M \\
\hline NGC5927_133 & 232.00010 & -50.657180 & 14.750 & - & 1.970 & -116.35 & - & M \\
\hline NGC5927_372 & 232.00540 & -50.696110 & 14.660 & - & 2.110 & -97.90 & - & M \\
\hline NGC5927_335 & 232.01040 & -50.689630 & 14.440 & - & 1.940 & -100.23 & - & M \\
\hline NGC5927_190 & 232.02400 & -50.663760 & 14.290 & - & 2.020 & -82.28 & - & M \\
\hline NGC5946_1 & 233.8046378431610 & -50.6330999838030 & 16.736 & 1.764 & - & -27.44 & -63 & \\
\hline NGC5946_2 & 233.8192784968850 & -50.6347502685450 & 17.017 & 1.610 & - & -84.94 & -105 & \\
\hline NGC5946_3 & 233.7968271675320 & -50.6664482079140 & 16.499 & 1.780 & - & -5.24 & -38 & \\
\hline NGC5946_4 & 233.8349967731580 & -50.6435873111980 & 16.315 & 1.728 & - & 71.73 & 85 & \\
\hline NGC5946_5 & 233.8351619708550 & -50.6556161766250 & 16.229 & 1.809 & - & 115.64 & 112 & M \\
\hline NGC5946_6 & 233.8533263570141 & -50.6441955351030 & 16.865 & 1.641 & - & 131.54 & 96 & \\
\hline NGC5946_7 & 233.8447146767090 & -50.6705180815020 & 15.955 & 1.793 & - & 106.48 & 96 & M \\
\hline NGC5946_8 & 233.8595939690180 & -50.6634568192010 & 15.780 & 1.866 & - & 162.46 & 116 & M \\
\hline NGC5946_9 & 233.8666866915190 & -50.6714124930590 & 16.745 & 1.745 & - & 118.45 & 100 & M \\
\hline NGC5946_10 & 233.8951206530490 & -50.6490984493930 & 17.119 & 1.698 & - & 139.52 & 130 & \\
\hline NGC5946_11 & 233.8903132732620 & -50.6670769475470 & 17.160 & 1.669 & - & 143.52 & 129 & \\
\hline NGC5946_14 & 233.9077804562540 & -50.6840449718950 & 16.261 & 1.756 & - & 109.52 & 172 & \\
\hline NGC5946_15 & 233.9313592396560 & -50.6687249102040 & 16.748 & 1.706 & - & 168.90 & 116 & M \\
\hline NGC5946_16 & 233.9304730779910 & -50.6845904460230 & 16.989 & 1.722 & - & 111.27 & -81 & \\
\hline NGC5946_19 & 233.9375579411780 & -50.7135206532660 & 16.543 & 1.643 & - & -83.91 & - & \\
\hline BH176_2 & 234.8322480854411 & -49.9847554870280 & 19.377 & 1.720 & - & 17.00 & 27 & M \\
\hline BH176_3 & 234.8258020595950 & -49.9905808846840 & 18.826 & 1.709 & - & 55.13 & 36 & \\
\hline BH176_4 & 234.7353061460010 & -49.9945984823580 & 19.073 & 1.718 & - & -15.41 & -12 & \\
\hline BH176_6 & 234.7674651957070 & -50.0097045530970 & 18.249 & 2.006 & - & -2.51 & -4 & M \\
\hline BH176_7 & 234.7792297073740 & -50.0131437707750 & 18.411 & 1.959 & - & -37.48 & 5 & M \\
\hline BH176_8 & 234.7325900671540 & -50.0193875514340 & 17.766 & 2.294 & - & 10.42 & 7 & \\
\hline BH176_9 & 234.7931783392740 & -50.0273857906780 & 18.985 & 1.721 & - & -17.84 & 5 & M \\
\hline BH176_10 & 234.8025091323060 & -50.0330453671780 & 17.976 & 2.058 & - & -5.52 & -5 & M \\
\hline BH176_11 & 234.8017269343900 & -50.0385856964930 & 18.066 & 2.113 & - & -1.22 & -2 & M \\
\hline BH176_13 & 234.7825982516510 & -50.0528938288310 & 17.408 & 2.755 & - & -3.58 & -5 & M \\
\hline BH176_14 & 234.7519927464920 & -50.0596122899570 & 18.101 & 1.909 & - & -6.45 & -6 & M \\
\hline BH176_15 & 234.7656274407960 & -50.0638176373480 & 18.775 & 1.796 & - & 7.40 & -2 & M \\
\hline BH176_16 & 234.8019923942540 & -50.0708836705370 & 18.617 & 1.810 & - & -14.69 & -0 & M \\
\hline BH176_17 & 234.7518063534750 & -50.0797145124840 & 18.144 & 2.010 & - & -25.68 & -16 & \\
\hline
\end{tabular}


Table B.1 - continued.

\begin{tabular}{|c|c|c|c|c|c|c|c|c|}
\hline Star ID & $\begin{array}{l}\text { RA (J2000) } \\
(\mathrm{deg})\end{array}$ & $\begin{array}{l}\text { DEC (J2000) } \\
(\mathrm{deg})\end{array}$ & $\begin{array}{c}\mathrm{V} \\
(\mathrm{mag})\end{array}$ & $\begin{array}{c}\text { V-I } \\
\text { (mag) }\end{array}$ & $\begin{array}{c}\text { B-V } \\
\text { (mag) }\end{array}$ & $\begin{array}{l}\text { Vhelio } \\
(\mathrm{km} / \mathrm{s})\end{array}$ & $\begin{array}{r}\text { Vhelio-CaT } \\
(\mathrm{km} / \mathrm{s})\end{array}$ & members \\
\hline BH176_18 & 234.7603405866310 & -50.0830708858910 & 17.951 & 2.030 & - & 0.20 & -75 & M \\
\hline Lynga7_1 & 242.7143087690700 & -55.2905208683670 & 16.706 & 1.988 & - & -112.43 & - & \\
\hline Lynga7_2 & 242.7653623624860 & -55.2979759401890 & 16.114 & 2.078 & - & 41.69 & 6 & \\
\hline Lynga7_3 & 242.7793235806400 & -55.3040472206520 & 16.402 & 1.973 & - & 12.75 & 23 & \\
\hline Lynga7_4 & 242.7493485490450 & -55.3104250625750 & 16.724 & 1.959 & - & -33.95 & 21 & M \\
\hline Lynga7_5 & 242.7686462362690 & -55.3153817813770 & 15.380 & 3.168 & - & 9.13 & 18 & \\
\hline Lynga7_6 & 242.7471523509420 & -55.3235157955460 & 17.174 & 1.806 & - & -56.46 & - & \\
\hline Lynga7_8 & 242.7497743196010 & -55.3351275639070 & 16.150 & 2.018 & - & -23.86 & 17 & $\mathrm{M}$ \\
\hline Lynga7_9 & 242.7942370919550 & -55.3409352713680 & 16.268 & 2.038 & - & -35.79 & - & \\
\hline Lynga7_10 & 242.7572950981700 & -55.3488247445700 & 17.110 & 1.999 & - & 18.90 & 27 & $\mathrm{M}$ \\
\hline Lynga7_11 & 242.7749423241250 & -55.3527911736560 & 16.418 & 2.102 & - & 16.30 & 34 & \\
\hline Lynga7_14 & 242.7817767699260 & -55.3749439667090 & 17.164 & 1.856 & - & 61.49 & - & \\
\hline Lynga7_15 & 242.7553826491850 & -55.3774876150520 & 15.606 & 2.554 & - & 8.66 & 27 & \\
\hline Lynga7_16 & 242.6969892779080 & -55.3836099651490 & 17.110 & 2.015 & - & -84.30 & - & \\
\hline Pal14_3 & 242.7289540671210 & 14.9768305596526 & 18.302 & 1.126 & - & 50.59 & 71 & M \\
\hline Pal14_4 & 242.7252997753470 & 14.9515772785923 & 18.755 & 1.117 & - & 46.46 & 66 & $\mathrm{M}$ \\
\hline Pal14_5 & 242.7372606969950 & 14.9656721115292 & 18.870 & 1.069 & - & 53.74 & 72 & $\mathrm{M}$ \\
\hline Pal14_7 & 242.7447676166120 & 14.9468796368414 & 17.749 & 1.351 & - & 48.97 & 75 & $\mathrm{M}$ \\
\hline Pal14_8 & 242.7560206091760 & 14.9665979935925 & 19.117 & 1.105 & - & 28.09 & 75 & $\mathrm{M}$ \\
\hline Pal14_10 & 242.7747630175660 & 14.9785733163268 & 19.312 & 1.079 & - & 20.98 & 58 & \\
\hline Pal14_11 & 242.7743710727580 & 14.9624234573138 & 17.367 & 1.421 & - & 43.94 & 81 & M \\
\hline NGC6284_1 & 256.1787421768040 & -24.7239654554000 & 15.863 & 1.460 & - & -128.96 & -56 & \\
\hline NGC6284_2 & 256.1815532872069 & -24.7319915884480 & 14.690 & 1.729 & - & -278.63 & -219 & \\
\hline NGC6284_3 & 256.1570456950431 & -24.7294863031900 & 16.947 & 1.205 & - & -0.67 & 10 & \\
\hline NGC6284_4 & 256.1338305379220 & -24.7250439225030 & 16.404 & 1.352 & - & -141.74 & -100 & \\
\hline NGC6284_5 & 256.1489134049400 & -24.7375092679340 & 16.038 & 1.393 & - & -171.37 & -194 & \\
\hline NGC6284_6 & 256.1745929082130 & -24.7556796415000 & 15.369 & 1.549 & - & -66.23 & -81 & \\
\hline NGC6284_7 & 256.1425619205140 & -24.7501496060640 & 16.571 & 1.441 & - & 40.74 & 22 & M \\
\hline NGC6284_8 & 256.1319097101249 & -24.7527760959490 & 15.690 & 1.497 & - & -24.10 & -19 & \\
\hline NGC6284_9 & 256.1289997288189 & -24.7592355039020 & 16.069 & 1.398 & - & 64.38 & 8 & M \\
\hline NGC6284_10 & 256.1127878908740 & -24.7569225247240 & 16.179 & 1.433 & - & 27.53 & 4 & M \\
\hline NGC6284_11 & 256.1418949500970 & -24.7775725446190 & 16.132 & 1.406 & - & -82.35 & -51 & \\
\hline NGC6284_13 & 256.1190234932520 & -24.7797550947810 & 16.274 & 1.426 & - & 52.78 & 23 & $\mathrm{M}$ \\
\hline NGC6284_14 & 256.1076461193801 & -24.7833849093920 & 16.543 & 1.392 & - & -1.56 & 33 & M \\
\hline NGC6284_15 & 256.1271039938770 & -24.7977459030990 & 16.966 & 1.379 & - & 28.95 & 22 & $\mathrm{M}$ \\
\hline NGC6284_16 & 256.1125261800070 & -24.8003947883760 & 15.310 & 1.581 & - & -40.52 & -94 & \\
\hline NGC6284_17 & 256.1021024388910 & -24.8009876097890 & 16.329 & 1.367 & - & -14.32 & -2 & \\
\hline NGC6284_18 & 256.1176796144840 & -24.8149306835980 & 16.278 & 1.388 & - & 29.26 & 32 & M \\
\hline NGC6316_1 & 259.0963084956139 & -28.0873501900400 & 16.646 & 1.978 & - & -81.46 & -49 & \\
\hline NGC6316_2 & 259.1166970225880 & -28.0861498907930 & 16.941 & 1.906 & - & -30.22 & -15 & \\
\hline NGC6316_3 & 259.1160790626469 & -28.0949073119040 & 16.716 & 1.733 & - & -55.20 & -32 & \\
\hline NGC6316_4 & 259.1377284457139 & -28.0971532857540 & 17.074 & 1.802 & - & -45.83 & -42 & \\
\hline
\end{tabular}


Table B.1 - continued.

\begin{tabular}{|c|c|c|c|c|c|c|c|c|}
\hline Star ID & $\begin{array}{l}\text { RA (J2000) } \\
(\mathrm{deg})\end{array}$ & $\begin{array}{l}\text { DEC (J2000) } \\
(\mathrm{deg})\end{array}$ & $\begin{array}{c}\mathrm{V} \\
(\mathrm{mag})\end{array}$ & $\begin{array}{c}\text { V-I } \\
\text { (mag) }\end{array}$ & $\begin{array}{l}\mathrm{B}-\mathrm{V} \\
(\mathrm{mag})\end{array}$ & $\begin{array}{r}\mathrm{V}_{\text {helio }} \\
(\mathrm{km} / \mathrm{s})\end{array}$ & $\begin{array}{r}\text { Vhelio-CaT } \\
(\mathrm{km} / \mathrm{s})\end{array}$ & members \\
\hline NGC6316_5 & 259.1312272086139 & -28.1036357862990 & 17.090 & 1.842 & - & -125.06 & 16 & \\
\hline NGC6316_6 & 259.1273755069050 & -28.1099285456990 & 16.835 & 1.726 & - & -85.98 & -74 & \\
\hline NGC6316_7 & 259.1477506136241 & -28.1154189764410 & 16.385 & 1.861 & - & 36.38 & 77 & M \\
\hline NGC6316_8 & 259.1311023859710 & -28.1247550044380 & 16.508 & 1.768 & - & -180.17 & -169 & \\
\hline NGC6316_9 & 259.1623377367640 & -28.1225520700910 & 15.828 & 2.199 & - & 154.08 & 97 & M \\
\hline NGC6316_10 & 259.1721802632810 & -28.1249274390170 & 16.684 & 1.876 & - & 87.13 & - & M \\
\hline NGC6316_11 & 259.1725801971090 & -28.1318873124630 & 16.462 & 1.839 & - & 100.53 & 96 & M \\
\hline NGC6316_14 & 259.1616428829790 & -28.1535857831530 & 16.856 & 1.828 & - & 86.61 & -78 & M \\
\hline NGC6316_15 & 259.1651348929910 & -28.1621833867960 & 16.452 & 2.006 & - & -80.73 & 42 & \\
\hline NGC6316_17 & 259.1734767954070 & -28.1732067264740 & 16.590 & 1.767 & - & 37.62 & 83 & M \\
\hline NGC6316_18 & 259.1483852088890 & -28.1861490142080 & 16.893 & 1.735 & - & 68.00 & -11 & M \\
\hline NGC6316_19 & 259.1654467639340 & -28.1876061552910 & 16.431 & 1.960 & - & -10.30 & - & \\
\hline NGC6356_1 & 260.9235074077297 & -17.7546041249480 & 16.660 & 1.491 & - & 5.87 & 45 & M \\
\hline NGC6356_2 & 260.9558057842270 & -17.7710305110450 & 16.723 & 1.422 & - & 0.40 & - & \\
\hline NGC6356_3 & 260.9558705466089 & -17.7784978788010 & 17.082 & 1.385 & - & 212.79 & - & \\
\hline NGC6356_4 & 260.8954703282600 & -17.7645922141030 & 15.741 & 1.953 & - & -232.62 & - & \\
\hline NGC6356_5 & 260.9239185919519 & -17.7846685306150 & 16.873 & 1.632 & - & 39.08 & 50 & M \\
\hline NGC6356_6 & 260.9169091259810 & -17.7881376496330 & 16.676 & 1.450 & - & -8.54 & - & M \\
\hline NGC6356_7 & 260.9279656805810 & -17.7966787950700 & 16.561 & 1.616 & - & 42.36 & 53 & M \\
\hline NGC6356_8 & 260.8932599793060 & -17.7917801507460 & 15.779 & 1.819 & - & -54.42 & 64 & M \\
\hline NGC6356_9 & 260.9156319574237 & -17.8087940477470 & 16.795 & 1.542 & - & 71.72 & 82 & M \\
\hline NGC6356_10 & 260.9214777632967 & -17.8143867084250 & 16.546 & 1.464 & - & 51.41 & 64 & M \\
\hline NGC6356_11 & 260.9114420443020 & -17.8179999655190 & 15.950 & 1.570 & - & 21.22 & - & \\
\hline NGC6356_13 & 260.9115145337869 & -17.8309059820990 & 15.934 & 1.643 & - & 93.58 & 88 & M \\
\hline NGC6356_14 & 260.9222838412350 & -17.8419564642120 & 16.165 & 1.640 & - & 70.53 & 75 & M \\
\hline NGC6356_15 & 260.9314184453367 & -17.8502978968420 & 15.256 & 1.917 & - & 3.79 & - & \\
\hline NGC6356_16 & 260.8948341251553 & -17.8448271016070 & 16.721 & 1.428 & - & 3.64 & 60 & M \\
\hline NGC6356_17 & 260.8988194150897 & -17.8524578021880 & 15.408 & 2.129 & - & 52.95 & - & M \\
\hline NGC6356_18 & 260.9039357772895 & -17.8638101621960 & 16.709 & 1.595 & - & 79.51 & 90 & M \\
\hline NGC6356_19 & 260.8706044317470 & -17.8568782386050 & 16.175 & 1.539 & - & 26.48 & 75 & M \\
\hline NGC6355_1 & 261.0139708308290 & -26.2986443619180 & 16.602 & 2.021 & - & -20.92 & -60 & \\
\hline NGC6355_2 & 260.9876263216710 & -26.3032970647830 & 17.364 & 1.956 & - & -42.22 & -50 & \\
\hline NGC6355_3 & 260.9645602791170 & -26.3095794146230 & 16.960 & 2.022 & - & 365.88 & 367 & \\
\hline NGC6355_4 & 261.0034840024430 & -26.3133409142560 & 17.475 & 1.871 & - & -127.08 & -124 & \\
\hline NGC6355_5 & 260.9765675424109 & -26.3222867639420 & 16.549 & 2.118 & - & -205.83 & -215 & \\
\hline NGC6355_6 & 260.9776929200140 & -26.3281193397190 & 17.150 & 2.021 & - & -50.62 & -53 & \\
\hline NGC6355_7 & 260.9909410424039 & -26.3357769969130 & 17.402 & 1.946 & - & -224.20 & -207 & M \\
\hline NGC6355_8 & 260.9811462177399 & -26.3404718116360 & 15.739 & 2.195 & - & -211.17 & -219 & M \\
\hline NGC6355_9 & 260.9819204193250 & -26.3455751719490 & 15.639 & 2.156 & - & -218.51 & -200 & M \\
\hline NGC6355_10 & 260.9994267993690 & -26.3538344802030 & 16.595 & 1.940 & - & -212.99 & -205 & M \\
\hline NGC6355_11 & 261.0034555787130 & -26.3596188513650 & 15.896 & 2.068 & - & -204.05 & -207 & M \\
\hline NGC6355_13 & 260.9972671624630 & -26.3741315224720 & 16.880 & 2.072 & - & -216.96 & -214 & M \\
\hline NGC6355_14 & 260.9929749853910 & -26.3766231832010 & 16.584 & 1.938 & - & -84.52 & -84 & \\
\hline
\end{tabular}


Table B.1 - continued.

\begin{tabular}{|c|c|c|c|c|c|c|c|c|}
\hline Star ID & $\begin{array}{l}\text { RA (J2000) } \\
(\mathrm{deg})\end{array}$ & $\begin{array}{l}\text { DEC (J2000) } \\
(\mathrm{deg})\end{array}$ & $\begin{array}{c}\mathrm{V} \\
(\mathrm{mag})\end{array}$ & $\begin{array}{c}\mathrm{V}-\mathrm{I} \\
(\mathrm{mag})\end{array}$ & $\begin{array}{c}\mathrm{B}-\mathrm{V} \\
(\mathrm{mag})\end{array}$ & $\begin{array}{r}\mathrm{v}_{\text {helio }} \\
(\mathrm{km} / \mathrm{s})\end{array}$ & $\begin{array}{r}\text { Vhelio-CaT } \\
(\mathrm{km} / \mathrm{s})\end{array}$ & members \\
\hline NGC6355_15 & 260.9830679562149 & -26.3874489320860 & 17.542 & 1.900 & - & -189.60 & -242 & \\
\hline NGC6355_17 & 260.9655114255959 & -26.3960577501580 & 15.973 & 2.185 & - & 102.94 & 94 & \\
\hline NGC6355_18 & 261.0137987038789 & -26.4056110757630 & 16.916 & 2.081 & - & 18.74 & 49 & \\
\hline NGC6352_1 & 261.4410166794020 & -48.3642026590000 & 14.948 & 1.301 & - & -121.65 & -124 & M \\
\hline NGC6352_2 & 261.4129538199270 & -48.3685108494390 & 15.079 & 1.310 & - & -7.12 & -37 & \\
\hline NGC6352_5 & 261.4355040079679 & -48.3897451899930 & 14.423 & 1.456 & - & -141.24 & -151 & M \\
\hline NGC6352_6 & 261.3453211212850 & -48.3877354011100 & 15.348 & 1.326 & - & -178.77 & -114 & M \\
\hline NGC6352_8 & 261.3562017080330 & -48.3991912951620 & 14.073 & 1.545 & - & -137.52 & -122 & M \\
\hline NGC6352_9 & 261.3785247710999 & -48.4085480198530 & 15.134 & 1.308 & - & -134.08 & -134 & M \\
\hline NGC6352_10 & 261.3407913170790 & -48.4109981946450 & 14.556 & 1.515 & - & -223.85 & -118 & M \\
\hline NGC6352_11 & 261.3749274397111 & -48.4222219762690 & 13.422 & 1.870 & - & -99.30 & -135 & M \\
\hline NGC6352_13 & 261.3708585654150 & -48.4354209604850 & 15.231 & 1.291 & - & -135.80 & -128 & M \\
\hline NGC6352_14 & 261.3334026622210 & -48.4350178054250 & 14.050 & 1.464 & - & -118.67 & -139 & \\
\hline NGC6352_15 & 261.3505553097240 & -48.4453305951550 & 15.146 & 1.323 & - & -98.24 & -134 & M \\
\hline NGC6352_16 & 261.3608831766571 & -48.4516101506590 & 14.494 & 1.402 & - & -113.99 & -130 & M \\
\hline NGC6352_17 & 261.3675556709620 & -48.4601120214010 & 13.750 & 1.617 & - & -161.47 & -113 & M \\
\hline NGC6352_18 & 261.4110421627360 & -48.4703858199440 & 14.523 & 1.458 & - & -108.31 & -113 & M \\
\hline NGC6366_1 & 261.9095291704430 & -5.0195910671494 & 16.479 & 1.895 & - & -170.57 & -138 & $\mathrm{M}$ \\
\hline NGC6366_2 & 261.9045681025580 & -5.0268560605880 & 15.875 & 1.820 & - & -174.56 & -108 & M \\
\hline NGC6366_3 & 261.9461497402918 & -5.0310026920141 & 16.626 & 1.888 & - & -143.24 & -133 & M \\
\hline NGC6366_4 & 261.9098940585019 & -5.0401070162215 & 16.271 & 1.893 & - & -191.77 & -113 & M \\
\hline NGC6366_5 & 261.9353344668059 & -5.0439214509264 & 14.786 & 2.005 & - & -121.28 & -126 & M \\
\hline NGC6366_6 & 261.9489990876769 & -5.0494799166147 & 15.874 & 1.814 & - & -119.21 & -117 & \\
\hline NGC6366_7 & 261.9582332068019 & -5.0596384523628 & 16.707 & 1.857 & - & -177.89 & -117 & $\mathrm{M}$ \\
\hline NGC6366_8 & 261.9226967769650 & -5.0629107784532 & 16.136 & 1.862 & - & -225.87 & -119 & M \\
\hline NGC6366_9 & 261.9194012789320 & -5.0716868933516 & 15.189 & 2.020 & - & -138.71 & -122 & M \\
\hline NGC6366_10 & 261.9612595505589 & -5.0771209262480 & 16.262 & 1.893 & - & -33.21 & -106 & M \\
\hline NGC6366_11 & 261.9221402785569 & -5.0835030019369 & 15.692 & 1.979 & - & -70.78 & -117 & M \\
\hline NGC6366_13 & 261.9387570683969 & -5.0951106568610 & 16.757 & 1.857 & - & -176.64 & -108 & M \\
\hline NGC6366_14 & 261.8998623184320 & -5.0999764100051 & 15.716 & 1.874 & - & -73.28 & -108 & \\
\hline NGC6366_15 & 261.9312457422168 & -5.1084049060473 & 14.424 & 2.005 & - & -121.52 & -126 & M \\
\hline NGC6366_16 & 261.9290313916749 & -5.1158275368716 & 16.134 & 1.858 & - & -96.74 & -121 & M \\
\hline NGC6366_17 & 261.9595558876100 & -5.1193281721352 & 15.535 & 1.859 & - & -36.46 & 39 & \\
\hline NGC6366_18 & 261.9501356527969 & -5.1277765435729 & 14.991 & 1.942 & - & -69.04 & -120 & M \\
\hline NGC6401_1 & 264.6740793857970 & -23.8556633971030 & 17.321 & 1.995 & - & -41.36 & -47 & \\
\hline NGC6401_2 & 264.6396680991930 & -23.8610421303060 & 16.599 & 2.058 & - & -31.56 & -38 & \\
\hline NGC6401_3 & 264.6568766516490 & -23.8647642881960 & 16.924 & 2.072 & - & -92.44 & -103 & M \\
\hline NGC6401_4 & 264.6900204428919 & -23.8740817309480 & 15.314 & 2.331 & - & -1.69 & 26 & \\
\hline NGC6401_5 & 264.6308167036780 & -23.8784535100230 & 17.142 & 2.193 & - & -11.11 & 3 & \\
\hline NGC6401_6 & 264.6856894020570 & -23.8866262403780 & 16.785 & 2.065 & - & -42.67 & -38 & \\
\hline NGC6401_7 & 264.6465304337050 & -23.8920274646890 & 17.166 & 2.039 & - & -120.97 & -96 & $\mathrm{M}$ \\
\hline NGC6401_8 & 264.6493729355540 & -23.8963685172360 & 16.706 & 2.175 & - & -144.76 & -137 & M \\
\hline NGC6401_9 & 264.6606431183770 & -23.9041214598170 & 16.114 & 2.081 & - & -125.41 & -111 & M \\
\hline
\end{tabular}


Table B.1 - continued.

\begin{tabular}{|c|c|c|c|c|c|c|c|c|}
\hline Star ID & $\begin{array}{l}\text { RA (J2000) } \\
(\mathrm{deg})\end{array}$ & $\begin{array}{l}\text { DEC (J2000) } \\
(\mathrm{deg})\end{array}$ & $\begin{array}{c}\mathrm{V} \\
(\mathrm{mag})\end{array}$ & $\begin{array}{c}\text { V-I } \\
(\mathrm{mag})\end{array}$ & $\begin{array}{l}\mathrm{B}-\mathrm{V} \\
(\mathrm{mag})\end{array}$ & $\begin{array}{r}\mathrm{V}_{\text {helio }} \\
(\mathrm{km} / \mathrm{s})\end{array}$ & $\begin{array}{r}\text { Vhelio-CaT } \\
(\mathrm{km} / \mathrm{s})\end{array}$ & members \\
\hline NGC6401_10 & 264.6672999336120 & -23.9085421356730 & 16.484 & 2.243 & - & -184.47 & -169 & \\
\hline NGC6401_11 & 264.6484872492470 & -23.9158401886910 & 16.911 & 2.215 & - & -106.33 & -63 & \\
\hline NGC6401_12 & 264.6638409242120 & -23.9232830795760 & 16.478 & 2.036 & - & -137.77 & -129 & \\
\hline NGC6401_13 & 264.6507855666670 & -23.9279831483290 & 17.374 & 2.054 & - & -113.77 & -110 & M \\
\hline NGC6401_14 & 264.6583349865899 & -23.9362185412110 & 17.236 & 2.139 & - & -120.74 & -121 & M \\
\hline NGC6401_15 & 264.6719233586430 & -23.9406429153550 & 16.807 & 2.202 & - & 38.81 & 63 & \\
\hline NGC6401_16 & 264.6476908074171 & -23.9471300975680 & 17.281 & 2.191 & - & -133.97 & -130 & \\
\hline NGC6401_17 & 264.6466533919830 & -23.9540215315450 & 17.528 & 2.167 & - & -106.95 & -103 & \\
\hline NGC6401_18 & 264.6693516511750 & -23.9612225098970 & 16.655 & 2.251 & - & 104.17 & 81 & \\
\hline Pal6_1 & 265.9138498528130 & -26.1649109273710 & 18.265 & 3.165 & - & -35.47 & -22 & \\
\hline Pal6_2 & 265.9034035887530 & -26.1709532492550 & 19.121 & 3.075 & - & 76.42 & 73 & \\
\hline Pal6_3 & 265.9530750251889 & -26.1800411651570 & 18.050 & 3.207 & - & 12.61 & -48 & \\
\hline Pal6_4 & 265.9495279366710 & -26.1869191371730 & 18.520 & 3.064 & - & -70.25 & -55 & \\
\hline Pal6_5 & 265.9369039009599 & -26.1886178964360 & 19.231 & 2.877 & - & -69.80 & -72 & \\
\hline Pal6_6 & 265.9227793854060 & -26.1978613619770 & 19.082 & 3.084 & - & 10.35 & 8 & \\
\hline Pal6_7 & 265.9224889666719 & -26.2047843344080 & 18.918 & 3.050 & - & 35.18 & 53 & \\
\hline Pal6_8 & 265.9065700629099 & -26.2094174719570 & 19.257 & 2.892 & - & 96.92 & 107 & \\
\hline Pal6_9 & 265.9415791651939 & -26.2176098802120 & 17.270 & 3.371 & - & 173.50 & 178 & M \\
\hline Pal6_10 & 265.9367765058209 & -26.2221073256090 & 18.846 & 3.052 & - & -90.74 & -82 & \\
\hline Pal6_11 & 265.9257829144390 & -26.2286324458830 & 18.153 & 3.114 & - & 182.53 & 186 & M \\
\hline Pal6_13 & 265.9337087726818 & -26.2412589944980 & 18.368 & 3.020 & - & 173.24 & 178 & M \\
\hline Pal6_14 & 265.9369008632410 & -26.2485857092230 & 17.903 & 3.162 & - & 180.24 & 174 & M \\
\hline Pal6_15 & 265.9447640007471 & -26.2521501635350 & 18.045 & 3.192 & - & -72.40 & -56 & \\
\hline Pal6_16 & 265.9375657316269 & -26.2585990959490 & 18.240 & 3.029 & - & 128.42 & 135 & \\
\hline Pal6_17 & 265.9316835487689 & -26.2652504769740 & 18.543 & 3.047 & - & 134.19 & 137 & \\
\hline Pal6_18 & 265.9419895113390 & -26.2712003357810 & 18.774 & 3.094 & - & 149.91 & 148 & \\
\hline NGC6440_1 & 267.1860577172569 & -20.3029171413500 & 17.359 & 2.790 & - & 73.91 & - & \\
\hline NGC6440_3 & 267.2094912561130 & -20.3149347371460 & 17.941 & 2.487 & - & 25.88 & - & \\
\hline NGC6440_4 & 267.2101077506937 & -20.3219764394440 & 18.251 & 2.406 & - & -160.93 & - & \\
\hline NGC6440_5 & 267.2035072858610 & -20.3291722920080 & 16.990 & 2.895 & - & 32.72 & - & \\
\hline NGC6440_6 & 267.2171388334789 & -20.3351667481490 & 18.048 & 2.572 & - & -31.95 & - & M \\
\hline NGC6440_7 & 267.2251585557580 & -20.3402378225310 & 18.500 & 2.397 & - & -23.02 & - & M \\
\hline NGC6440_8 & 267.2344986043180 & -20.3485639174310 & 17.291 & 2.986 & - & -93.13 & -97 & M \\
\hline NGC6440_10 & 267.2320462698550 & -20.3589215988520 & 17.623 & 2.702 & - & -86.97 & -84 & M \\
\hline NGC6440_11 & 267.2061834512457 & -20.3660566970390 & 17.028 & 2.752 & - & 9.90 & -78 & \\
\hline NGC6440_13 & 267.2269927367090 & -20.3783455444010 & 18.202 & 2.486 & - & -52.54 & -57 & M \\
\hline NGC6440_14 & 267.2193743591089 & -20.3852127997750 & 16.403 & 3.100 & - & -65.02 & -73 & M \\
\hline NGC6440_15 & 267.2241545171047 & -20.3913077156530 & 17.568 & 2.623 & - & -60.78 & -62 & M \\
\hline NGC6440_16 & 267.2288277646500 & -20.3981523854640 & 18.226 & 2.522 & - & 187.35 & - & \\
\hline NGC6440_17 & 267.2282071898930 & -20.4036506064310 & 16.477 & 3.202 & - & 121.48 & - & \\
\hline NGC6440_18 & 267.1830234769110 & -20.4099430892500 & 17.703 & 2.515 & - & 82.84 & - & \\
\hline NGC6440_19 & 267.2175680421177 & -20.4169636952160 & 18.247 & 2.349 & - & 63.82 & - & \\
\hline NGC6453_1 & 267.7109046327830 & -34.5432736160450 & 17.199 & 1.696 & - & 10.50 & 8 & \\
\hline
\end{tabular}


Table B.1 - continued.

\begin{tabular}{|c|c|c|c|c|c|c|c|c|}
\hline Star ID & $\begin{array}{l}\text { RA (J2000) } \\
(\operatorname{deg})\end{array}$ & $\begin{array}{l}\text { DEC (J2000) } \\
(\mathrm{deg})\end{array}$ & $\begin{array}{c}\mathrm{V} \\
(\mathrm{mag})\end{array}$ & $\begin{array}{c}\text { V-I } \\
(\mathrm{mag})\end{array}$ & $\begin{array}{c}\text { B-V } \\
(\mathrm{mag})\end{array}$ & $\begin{array}{r}\text { Vhelio } \\
(\mathrm{km} / \mathrm{s})\end{array}$ & $\begin{array}{r}\text { Vhelio-CaT } \\
(\mathrm{km} / \mathrm{s})\end{array}$ & members \\
\hline NGC6453_2 & 267.7099404707681 & -34.5472647329590 & 16.528 & 1.682 & - & -189.04 & -186 & \\
\hline NGC6453_3 & 267.7117060844460 & -34.5547399421890 & 17.277 & 1.797 & - & 83.37 & 96 & \\
\hline NGC6453_4 & 267.7292470188960 & -34.5605918640070 & 17.585 & 1.606 & - & -107.16 & -115 & \\
\hline NGC6453_5 & 267.7102053888260 & -34.5691401085550 & 17.429 & 1.770 & - & -32.00 & -9 & \\
\hline NGC6453_6 & 267.7058201621750 & -34.5732638316630 & 16.916 & 1.789 & - & -188.36 & -184 & \\
\hline NGC6453_7 & 267.7289160763439 & -34.5823533377810 & 17.387 & 1.724 & - & -66.95 & -51 & \\
\hline NGC6453_8 & 267.7226546849880 & -34.5879325333010 & 16.314 & 1.772 & - & -90.08 & -95 & \\
\hline NGC6453_10 & 267.7124990715680 & -34.5983430405210 & 15.536 & 1.895 & - & -158.83 & -118 & M \\
\hline NGC6453_11 & 267.7231285105429 & -34.6042698746130 & 16.405 & 1.851 & - & -140.70 & -115 & M \\
\hline NGC6453_13 & 267.7068837766470 & -34.6157585913080 & 17.533 & 1.790 & - & 36.24 & 66 & \\
\hline NGC6453_14 & 267.6983936335840 & -34.6228403407740 & 17.334 & 1.662 & - & -165.87 & -144 & \\
\hline NGC6453_15 & 267.7245315396250 & -34.6329408571700 & 16.787 & 1.662 & - & -160.70 & -137 & M \\
\hline NGC6453_16 & 267.7355219144729 & -34.6352324389380 & 17.151 & 1.777 & - & -189.33 & -153 & \\
\hline NGC6453_17 & 267.7228026610230 & -34.6422184403970 & 17.543 & 1.628 & - & -36.75 & 1 & \\
\hline NGC6453_18 & 267.7081228730470 & -34.6483015798160 & 17.352 & 1.717 & - & -133.40 & -91 & \\
\hline NGC6538_2 & 271.1891346933031 & -7.5368165863050 & 17.781 & 2.381 & - & -15.60 & 9 & \\
\hline NGC6538_3 & 271.2225768215739 & -7.5417614936001 & 18.400 & 2.342 & - & -9.51 & 8 & \\
\hline NGC6538_4 & 271.2115914147460 & -7.5466212743910 & 16.401 & 2.872 & - & 51.55 & 57 & M \\
\hline NGC6538_5 & 271.2152706610500 & -7.5529669666963 & 16.797 & 2.547 & - & 10.82 & 28 & M \\
\hline NGC6538_6 & 271.2172879051260 & -7.5588502786937 & 18.358 & 2.309 & - & 71.93 & 106 & \\
\hline NGC6538_7 & 271.2209245927010 & -7.5663514469969 & 17.934 & 2.521 & - & 51.64 & 70 & \\
\hline NGC6538_8 & 271.2207360141699 & -7.5730120587253 & 17.613 & 2.422 & - & 4.51 & 38 & M \\
\hline NGC6538_9 & 271.2084174690189 & -7.5788454320773 & 18.434 & 2.271 & - & 32.01 & 28 & M \\
\hline NGC6538_10 & 271.1990876725411 & -7.5857819095202 & 17.873 & 2.442 & - & 48.05 & 31 & M \\
\hline NGC6538_11 & 271.2128916424860 & -7.5903446252700 & 16.261 & 3.137 & - & 88.66 & 54 & \\
\hline NGC6538_13 & 271.2003494122119 & -7.6051381264440 & 16.110 & 2.981 & - & 39.20 & 32 & \\
\hline NGC6538_14 & 271.1988435054970 & -7.6100848355027 & 16.554 & 2.613 & - & 23.50 & 31 & M \\
\hline NGC6538_15 & 271.1793873281880 & -7.6175695473128 & 17.061 & 2.428 & - & 47.08 & 31 & \\
\hline NGC6538_17 & 271.2098010791651 & -7.6296450723905 & 18.250 & 2.281 & - & -14.98 & -6 & \\
\hline NGC6538_18 & 271.1994704411969 & -7.6369369734792 & 18.316 & 2.320 & - & 36.64 & 31 & M \\
\hline Pal7_1 & 272.7129181253690 & -7.1538632982032 & 17.357 & 2.592 & - & 169.18 & 162 & M \\
\hline Pal7_3 & 272.6941865319670 & -7.1655922878238 & 17.052 & 2.559 & - & 155.32 & 161 & M \\
\hline Pal7_4 & 272.7038149516796 & -7.1737082694418 & 15.969 & 3.115 & - & 179.41 & 180 & M \\
\hline Pal7_5 & 272.6553330306050 & -7.1729570563556 & 17.146 & 2.675 & - & 168.15 & - & \\
\hline Pal7_7 & 272.6814999578997 & -7.1895031554166 & 16.472 & 2.922 & - & 123.67 & 160 & M \\
\hline Pal7_8 & 272.6940609349913 & -7.1960174655907 & 17.303 & 2.626 & - & 162.24 & 172 & M \\
\hline Pal7_9 & 272.6856585188370 & -7.2029350900190 & 16.323 & 3.030 & - & 159.29 & 153 & M \\
\hline Pal7_10 & 272.6907524082347 & -7.2083277352292 & 16.186 & 2.833 & - & 162.91 & 165 & M \\
\hline Pal7_11 & 272.6889448185320 & -7.2141697502301 & 16.844 & 2.560 & - & 158.08 & 160 & M \\
\hline Pal7_12 & 272.6739324450837 & -7.2210562124949 & 17.387 & 2.542 & - & 153.47 & 169 & M \\
\hline Pal7_13 & 272.6947842420930 & -7.2270852702464 & 17.204 & 2.479 & - & -4.88 & - & \\
\hline Pal7_14 & 272.6732928068980 & -7.2332635490277 & 17.142 & 2.563 & - & -48.90 & - & \\
\hline Pal7_15 & 272.6350120593880 & -7.2349830905768 & 17.186 & 2.594 & - & 139.93 & 173 & M \\
\hline
\end{tabular}


Table B.1 - continued.

\begin{tabular}{|c|c|c|c|c|c|c|c|c|}
\hline Star ID & $\begin{array}{l}\text { RA (J2000) } \\
(\mathrm{deg})\end{array}$ & $\begin{array}{l}\text { DEC (J2000) } \\
(\mathrm{deg})\end{array}$ & $\begin{array}{c}\mathrm{V} \\
(\mathrm{mag})\end{array}$ & $\begin{array}{c}\mathrm{V}-\mathrm{I} \\
(\mathrm{mag})\end{array}$ & $\begin{array}{l}\mathrm{B}-\mathrm{V} \\
(\mathrm{mag})\end{array}$ & $\begin{array}{r}\mathrm{V}_{\text {helio }} \\
(\mathrm{km} / \mathrm{s})\end{array}$ & $\begin{array}{r}\text { Vhelio-CaT } \\
(\mathrm{km} / \mathrm{s})\end{array}$ & members \\
\hline Pal7_16 & 272.6481250359010 & -7.2447529133249 & 16.850 & 2.574 & - & 36.18 & 156 & \\
\hline Pal7_17 & 272.6667908626877 & -7.2499074682881 & 17.100 & 2.498 & - & 141.10 & 180 & M \\
\hline Pal7_18 & 272.6644089239120 & -7.2579028580247 & 16.091 & 2.816 & - & 157.52 & 162 & M \\
\hline Pal7_19 & 272.6662718121410 & -7.2637347001366 & 17.182 & 2.511 & - & 104.85 & - & \\
\hline NGC6569_1 & 273.4336292334870 & -31.7704262455560 & 17.092 & 1.649 & - & -39.46 & -40 & M \\
\hline NGC6569_2 & 273.4455484196830 & -31.7765360025320 & 15.317 & 2.032 & - & 71.91 & - & \\
\hline NGC6569_3 & 273.4264817161510 & -31.7834977305820 & 16.853 & 1.769 & - & -97.91 & - & \\
\hline NGC6569_4 & 273.4091675473910 & -31.7897212799030 & 17.040 & 1.775 & - & -56.15 & -54 & M \\
\hline NGC6569_5 & 273.3803188915610 & -31.7961359918000 & 16.084 & 1.690 & - & -157.26 & - & \\
\hline NGC6569_6 & 273.4318560737670 & -31.8015552547670 & 17.281 & 1.536 & - & 87.49 & - & \\
\hline NGC6569_7 & 273.4181355474257 & -31.8088466519520 & 17.069 & 1.657 & - & 27.40 & - & \\
\hline NGC6569_8 & 273.4162067086087 & -31.8157065176360 & 17.282 & 1.618 & - & -62.73 & - & M \\
\hline NGC6569_9 & 273.4101047591937 & -31.8185969759490 & 16.990 & 1.586 & - & -41.93 & -45 & M \\
\hline NGC6569_10 & 273.4226581650420 & -31.8270607419080 & 16.352 & 1.747 & - & -45.95 & -42 & M \\
\hline NGC6569_11 & 273.4008204511300 & -31.8316386498970 & 16.765 & 1.651 & - & -52.06 & -39 & \\
\hline NGC6569_12 & 273.4080675327830 & -31.8415956101290 & 17.147 & 1.588 & - & -55.60 & -55 & M \\
\hline NGC6569_13 & 273.4228352776827 & -31.8457683686530 & 16.760 & 1.798 & - & -54.59 & -52 & M \\
\hline NGC6569_14 & 273.4091489443977 & -31.8518340957680 & 17.424 & 1.682 & - & -46.50 & - & \\
\hline NGC6569_15 & 273.4246883581039 & -31.8585619278720 & 17.326 & 1.656 & - & -31.28 & - & \\
\hline NGC6569_16 & 273.4040223818470 & -31.8642141148940 & 16.822 & 1.669 & - & -114.62 & - & \\
\hline NGC6569_17 & 273.4264176766977 & -31.8704671125370 & 17.199 & 1.709 & - & -32.93 & - & \\
\hline NGC6569_19 & 273.3876649349320 & -31.8844286986320 & 17.394 & 1.644 & - & -4.56 & - & \\
\hline NGC6749_1 & 286.2847567371839 & 1.9596903589911 & 17.585 & 2.754 & - & -43.71 & -70 & \\
\hline NGC6749_2 & 286.2868656096230 & 1.9525164460737 & 18.576 & 2.564 & - & 33.15 & 64 & \\
\hline NGC6749_3 & 286.3284566482919 & 1.9445190618570 & 18.593 & 2.452 & - & -72.70 & -52 & \\
\hline NGC6749_4 & 286.3376510500939 & 1.9399435480177 & 17.455 & 2.657 & - & -47.40 & -37 & \\
\hline NGC6749_5 & 286.3067566745090 & 1.9354624935870 & 17.176 & 2.970 & - & -78.84 & -62 & \\
\hline NGC6749_6 & 286.3152627412300 & 1.9288996628548 & 17.325 & 2.821 & - & -85.30 & -44 & \\
\hline NGC6749_7 & 286.3096862766980 & 1.9192341679555 & 17.031 & 2.924 & - & -84.10 & -64 & \\
\hline NGC6749_8 & 286.3217847655430 & 1.9126298767745 & 17.949 & 2.716 & - & -72.06 & -58 & M \\
\hline NGC6749_9 & 286.3199408737951 & 1.9074423842532 & 16.898 & 2.950 & - & -85.06 & -63 & \\
\hline NGC6749_10 & 286.3322975895430 & 1.9038472343615 & 16.993 & 2.858 & - & -54.09 & -30 & M \\
\hline NGC6749_11 & 286.3260013003970 & 1.8982261632430 & 17.073 & 2.860 & - & -69.88 & -48 & M \\
\hline NGC6749_13 & 286.3332319080549 & 1.8832013430046 & 17.504 & 2.738 & - & 2.75 & 34 & \\
\hline NGC6749_14 & 286.3491620208251 & 1.8790442360165 & 17.856 & 2.717 & - & -31.05 & -35 & \\
\hline NGC6749_15 & 286.3258746626020 & 1.8689929106830 & 17.353 & 2.853 & - & -67.39 & -53 & M \\
\hline NGC6749_16 & 286.3078692410110 & 1.8652342887235 & 18.171 & 2.701 & - & -76.61 & -49 & \\
\hline NGC6749_17 & 286.3067004905620 & 1.8590099439023 & 18.230 & 2.588 & - & 0.52 & 21 & \\
\hline NGC6749_18 & 286.3123536019180 & 1.8515819277174 & 17.544 & 2.844 & - & 43.34 & 53 & \\
\hline Pal10_1 & 289.5125606154301 & 18.6299558446680 & 19.242 & 2.904 & - & 65.92 & 38 & \\
\hline Pal10_2 & 289.4646838783200 & 18.6277908909462 & 19.017 & 2.947 & - & -22.78 & -1 & M \\
\hline Pal10_3 & 289.4688827393061 & 18.6215883550814 & 19.378 & 2.944 & - & 18.76 & 20 & \\
\hline Pal10_4 & 289.4899243412699 & 18.6123372877962 & 18.869 & 3.045 & - & -49.04 & -35 & M \\
\hline
\end{tabular}


Table B.1 - continued.

\begin{tabular}{|c|c|c|c|c|c|c|c|c|}
\hline Star ID & $\begin{array}{l}\text { RA (J2000) } \\
(\operatorname{deg})\end{array}$ & $\begin{array}{l}\text { DEC (J2000) } \\
(\mathrm{deg})\end{array}$ & $\begin{array}{c}\mathrm{V} \\
(\mathrm{mag})\end{array}$ & $\begin{array}{c}\text { V-I } \\
(\mathrm{mag})\end{array}$ & $\begin{array}{c}\text { B-V } \\
\text { (mag) }\end{array}$ & $\begin{array}{r}\text { Vhelio } \\
(\mathrm{km} / \mathrm{s})\end{array}$ & $\begin{array}{r}\text { Vhelio-CaT } \\
(\mathrm{km} / \mathrm{s})\end{array}$ & members \\
\hline Pal10_6 & 289.4656372047450 & 18.6046132108929 & 18.147 & 3.368 & - & -112.84 & -101 & \\
\hline Pal10_7 & 289.4652506135770 & 18.5959932118453 & 18.449 & 3.072 & - & -27.55 & -43 & \\
\hline Pal10_8 & 289.5027113535670 & 18.5872513699130 & 18.901 & 3.143 & - & -46.90 & -42 & M \\
\hline Pal10_9 & 289.5280987147370 & 18.5773513020153 & 18.924 & 3.085 & - & -65.87 & -69 & $\mathrm{M}$ \\
\hline Pal10_10 & 289.4984921319600 & 18.5759838819809 & 18.537 & 3.167 & - & -41.28 & -35 & M \\
\hline Pal10_11 & 289.5162605322620 & 18.5685585953761 & 18.165 & 3.354 & - & -35.28 & -34 & $\mathrm{M}$ \\
\hline Pal10_13 & 289.5236933727690 & 18.5551426686763 & 18.546 & 3.291 & - & -11.91 & -15 & M \\
\hline Pal10_16 & 289.5093507683760 & 18.5361496794223 & 18.655 & 2.994 & - & -48.26 & -18 & $\mathrm{M}$ \\
\hline Pal10_17 & 289.5264177399050 & 18.5275771598552 & 18.554 & 3.104 & - & -17.88 & -25 & M \\
\hline Pal11_2 & 296.2918886563560 & -7.9607733525394 & 17.783 & 1.313 & - & -75.74 & -77 & $\mathrm{M}$ \\
\hline Pal11_3 & 296.2983701502389 & -7.9679129619890 & 17.991 & 1.374 & - & -105.55 & -94 & \\
\hline Pal11_6 & 296.3121063991420 & -7.9855545899986 & 17.931 & 1.327 & - & -107.49 & -66 & M \\
\hline Pal11_7 & 296.3266258731740 & -7.9901956699317 & 17.953 & 1.347 & - & -76.90 & -67 & M \\
\hline Pal11_8 & 296.3100194350170 & -7.9998724816952 & 16.791 & 1.482 & - & -91.10 & -59 & $\mathrm{M}$ \\
\hline Pal11_9 & 296.3095590678440 & -8.0041382789580 & 16.292 & 1.572 & - & -64.31 & -57 & M \\
\hline Pal11_10 & 296.3031036009810 & -8.0127983094640 & 18.018 & 1.255 & - & -72.92 & -60 & $\mathrm{M}$ \\
\hline Pal11_11 & 296.3336414063500 & -8.0177424751638 & 17.018 & 1.526 & - & -80.89 & -59 & $\mathrm{M}$ \\
\hline Pal11_12 & 296.3194836366321 & -8.0224404692151 & 17.345 & 1.426 & - & -99.30 & -63 & $\mathrm{M}$ \\
\hline Pal11_13 & 296.3052561711690 & -8.0296541122148 & 18.129 & 1.248 & - & -83.91 & -60 & M \\
\hline Pal11_16 & 296.3055388234640 & -8.0477687172836 & 18.132 & 1.263 & - & -49.68 & -49 & \\
\hline Pal11_17 & 296.2948864246590 & -8.0569353684763 & 16.173 & 1.561 & - & -58.82 & -54 & M \\
\hline M75_2 & 301.5722924809030 & -21.8724239925710 & 15.351 & 1.584 & - & -180.58 & -168 & $\mathrm{M}$ \\
\hline M75_3 & 301.5332302439650 & -21.8792783822790 & 16.052 & 1.399 & - & -268.32 & -195 & \\
\hline M75_4 & 301.5112863868840 & -21.8838393125550 & 17.305 & 1.161 & - & -209.35 & -201 & M \\
\hline M75_5 & 301.5398310455530 & -21.8901698871600 & 16.911 & 1.222 & - & -211.96 & -196 & $\mathrm{M}$ \\
\hline M75_6 & 301.5363529935929 & -21.8994513202970 & 16.667 & 1.223 & - & -170.35 & -193 & M \\
\hline M75_7 & 301.5284174072768 & -21.9043265871400 & 15.075 & 1.691 & - & -159.03 & -191 & M \\
\hline M75_8 & 301.5367067586659 & -21.9096006038980 & 15.446 & 1.494 & - & -242.29 & -187 & \\
\hline M75_9 & 301.5170655412350 & -21.9142047369940 & 15.189 & 1.677 & - & -208.00 & -212 & M \\
\hline M75_10 & 301.5041416139131 & -21.9220494603590 & 15.723 & 1.448 & - & -168.25 & -192 & $\mathrm{M}$ \\
\hline M75_11 & 301.5300056045850 & -21.9276243871480 & 15.446 & 1.543 & - & -209.20 & -185 & M \\
\hline M75_13 & 301.5065335843889 & -21.9420631700740 & 16.847 & 1.147 & - & -194.14 & -195 & M \\
\hline M75_14 & 301.5168102614069 & -21.9491974411900 & 16.919 & 1.150 & - & -194.05 & -195 & $\mathrm{M}$ \\
\hline NGC7006_9 & 315.3682175549997 & 16.1917858619990 & 17.000 & 1.195 & - & -406.56 & -377 & \\
\hline NGC7006_18 & 315.3795161685969 & 16.1968831361999 & 18.478 & 1.067 & - & -375.70 & -379 & M \\
\hline NGC7006_19 & 315.3593718729330 & 16.1997473878717 & 17.738 & 1.104 & - & -283.61 & -379 & \\
\hline NGC7006_21 & 315.3686452062430 & 16.2046868611790 & 19.389 & 0.940 & - & -433.24 & -387 & M \\
\hline NGC7006_22 & 315.3765269702437 & 16.2072140496116 & 19.165 & 0.963 & - & -359.48 & -369 & \\
\hline NGC7006_23 & 315.3705323766769 & 16.2116463895666 & 16.947 & 1.200 & - & -389.45 & -386 & $\mathrm{M}$ \\
\hline NGC7006_24 & 315.3786288060080 & 16.2195341119763 & 17.252 & 1.189 & - & -380.73 & -379 & M \\
\hline NGC7006_25 & 315.3822326435830 & 16.2264922925027 & 18.094 & 0.991 & - & -378.19 & -385 & $\mathrm{M}$ \\
\hline NGC7006_26 & 315.3388329980339 & 16.2098296634600 & 17.809 & 1.045 & - & -477.01 & -370 & \\
\hline NGC7006_27 & 315.3393898303030 & 16.2235240225451 & 18.997 & 0.931 & - & -84.08 & - & \\
\hline
\end{tabular}


Table B.1 - continued.

\begin{tabular}{|c|c|c|c|c|c|c|c|c|}
\hline Star ID & $\begin{array}{l}\text { RA (J2000) } \\
(\mathrm{deg})\end{array}$ & $\begin{array}{l}\text { DEC (J2000) } \\
(\mathrm{deg})\end{array}$ & $\begin{array}{c}\mathrm{V} \\
(\mathrm{mag})\end{array}$ & $\begin{array}{c}\mathrm{V}-\mathrm{I} \\
(\mathrm{mag})\end{array}$ & $\begin{array}{c}\mathrm{B}-\mathrm{V} \\
(\mathrm{mag})\end{array}$ & $\begin{array}{r}\text { Vhelio } \\
(\mathrm{km} / \mathrm{s})\end{array}$ & $\begin{array}{r}\text { Vhelio-CaT } \\
(\mathrm{km} / \mathrm{s})\end{array}$ & members \\
\hline NGC7006_28 & 315.3876249350990 & 16.2337482232222 & 18.878 & 1.055 & - & -69.38 & - & \\
\hline NGC7006_29 & 315.3737767394420 & 16.1656969965715 & 18.654 & 1.007 & - & -93.62 & - & \\
\hline NGC7006_31 & 315.3696240893173 & 16.1583516837794 & 17.635 & 1.158 & - & -34.91 & 一 & \\
\hline NGC7006_32 & 315.3863374169697 & 16.1547441200559 & 18.302 & 1.065 & - & -379.42 & - & \\
\hline
\end{tabular}

${ }^{a}$ Due to low number of observed stars it was hard to define the membership of M 107. See discussion in Chapter 4

Table B.2 - List of 49 well known stars selected from ELODIE spectral library. Literature parameters are average from PASTEL catalogue. Results from this work are using MILES library. See details in Section 3.4 .2 .2

\begin{tabular}{|c|c|c|c|c|c|c|c|c|c|c|c|}
\hline ELODIE & Star & $\mathrm{RR}_{\text {tot }}$ & $\mathrm{N}$ & $\mathrm{S}_{\mathrm{lim}}$ & $\mathrm{T}_{\text {eff }}(\mathrm{K})$ & $\begin{array}{c}\log (g) \\
\text { (literature) }\end{array}$ & {$[\mathrm{Fe} / \mathrm{H}]$} & $\mathrm{T}_{\text {eff }}(\mathrm{K})$ & $\begin{array}{l}\log g \\
\quad \text { (this }\end{array}$ & $\begin{array}{l}{[\mathrm{Fe} / \mathrm{H}]} \\
\text { work) }\end{array}$ & {$[\mathrm{Mg} / \mathrm{Fe}]$} \\
\hline 1 & HD000245 & 0.107 & 2 & 1.042 & $5490 \pm 153$ & $3.48 \pm 0.15$ & $-0.77 \pm 0.08$ & $5378 \pm 60$ & $3.67 \pm 0.06$ & $-0.84 \pm 0.20$ & $0.34 \pm 0.08$ \\
\hline 9 & HD002796 & 0.062 & 1 & 1.000 & $4931 \pm 60$ & $1.45 \pm 0.34$ & $-2.32 \pm 0.11$ & $4945 \pm 133$ & $1.36 \pm 0.08$ & $-2.31 \pm 0.11$ & $0.37 \pm 0.08$ \\
\hline 19 & HD004395 & 0.042 & 5 & 1.116 & $5487 \pm 38$ & $3.33 \pm 0.05$ & $-0.35 \pm 0.04$ & $5330 \pm 149$ & $3.24 \pm 0.16$ & $-0.34 \pm 0.11$ & $0.18 \pm 0.07$ \\
\hline 31 & HD006833 & 0.092 & 1 & 1.000 & $4426 \pm 95$ & $1.28 \pm 0.32$ & $-0.91 \pm 0.14$ & $4380 \pm 217$ & $1.25 \pm 0.64$ & $-0.99 \pm 0.29$ & $0.30 \pm 0.11$ \\
\hline 32 & HD006920 & 0.035 & 8 & 1.319 & $5886 \pm 111$ & $3.60 \pm 0.28$ & $-0.10 \pm 0.09$ & $5854 \pm 100$ & $3.60 \pm 0.05$ & $-0.10 \pm 0.14$ & $0.10 \pm 0.09$ \\
\hline 33 & HD008724 & 0.018 & 2 & 1.001 & $4586 \pm 84$ & $1.39 \pm 0.26$ & $-1.73 \pm 0.13$ & $4626 \pm 6$ & $1.40 \pm 0.06$ & $-1.75 \pm 0.00$ & $0.37 \pm 0.08$ \\
\hline 47 & HD013530 & 0.013 & 6 & 1.036 & $4772 \pm 106$ & $2.60 \pm 0.39$ & $-0.54 \pm 0.11$ & $4769 \pm 87$ & $2.63 \pm 0.21$ & $-0.54 \pm 0.15$ & $0.37 \pm 0.08$ \\
\hline 66 & HD015596 & 0.047 & 9 & 1.211 & $4808 \pm 59$ & $2.66 \pm 0.32$ & $-0.65 \pm 0.06$ & $4760 \pm 58$ & $2.54 \pm 0.14$ & $-0.66 \pm 0.07$ & $0.40 \pm 0.06$ \\
\hline 67 & HD015596 & 0.037 & 10 & 1.298 & $4808 \pm 59$ & $2.66 \pm 0.32$ & $-0.65 \pm 0.06$ & $4751 \pm 52$ & $2.57 \pm 0.12$ & $-0.64 \pm 0.06$ & $0.37 \pm 0.07$ \\
\hline 68 & HD015596 & 0.047 & 9 & 1.248 & $4808 \pm 59$ & $2.66 \pm 0.32$ & $-0.65 \pm 0.06$ & $4759 \pm 56$ & $2.54 \pm 0.13$ & $-0.66 \pm 0.06$ & $0.40 \pm 0.06$ \\
\hline 69 & HD016458 & 0.118 & 2 & 1.115 & $4593 \pm 123$ & $1.84 \pm 0.26$ & $-0.35 \pm 0.05$ & $4992 \pm 513$ & $1.97 \pm 0.95$ & $-0.34 \pm 0.25$ & $0.33 \pm 0.23$ \\
\hline 88 & HD020512 & 0.086 & 3 & 1.118 & $5212 \pm 63$ & $3.65 \pm 0.14$ & $-0.22 \pm 0.19$ & $5074 \pm 38$ & $3.35 \pm 0.14$ & $-0.22 \pm 0.20$ & $0.12 \pm 0.03$ \\
\hline 89 & HD020512 & 0.094 & 3 & 1.101 & $5212 \pm 63$ & $3.65 \pm 0.14$ & $-0.22 \pm 0.19$ & $5074 \pm 39$ & $3.35 \pm 0.15$ & $-0.21 \pm 0.21$ & $0.12 \pm 0.03$ \\
\hline 117 & HD026297 & 0.133 & 11 & 1.263 & $4445 \pm 140$ & $1.02 \pm 0.28$ & $-1.74 \pm 0.15$ & $4460 \pm 73$ & $1.15 \pm 0.19$ & $-1.66 \pm 0.08$ & $0.48 \pm 0.04$ \\
\hline 151 & HD035369 & 0.032 & 6 & 1.062 & $4885 \pm 112$ & $2.57 \pm 0.27$ & $-0.21 \pm 0.08$ & $4900 \pm 45$ & $2.65 \pm 0.08$ & $-0.21 \pm 0.05$ & $0.06 \pm 0.02$ \\
\hline 227 & HD045282 & 0.023 & 1 & 1.000 & $5264 \pm 86$ & $3.19 \pm 0.16$ & $-1.43 \pm 0.12$ & $5348 \pm 110$ & $3.24 \pm 0.29$ & $-1.44 \pm 0.05$ & $0.22 \pm 0.07$ \\
\hline 228 & HD045282 & 0.065 & 3 & 1.042 & $5264 \pm 86$ & $3.19 \pm 0.16$ & $-1.43 \pm 0.12$ & $5268 \pm 48$ & $3.14 \pm 0.23$ & $-1.52 \pm 0.05$ & $0.36 \pm 0.06$ \\
\hline 253 & HD046480 & 0.016 & 15 & 1.218 & $4785 \pm 26$ & $2.63 \pm 0.12$ & $-0.49 \pm 0.01$ & $4791 \pm 55$ & $2.65 \pm 0.13$ & $-0.50 \pm 0.08$ & $0.31 \pm 0.05$ \\
\hline 254 & HD046480 & 0.016 & 15 & 1.214 & $4785 \pm 26$ & $2.63 \pm 0.12$ & $-0.49 \pm 0.01$ & $4791 \pm 55$ & $2.65 \pm 0.13$ & $-0.50 \pm 0.08$ & $0.31 \pm 0.05$ \\
\hline 314 & HD063791 & 0.040 & 4 & 1.028 & $4715 \pm 78$ & $1.75 \pm 0.08$ & $-1.68 \pm 0.08$ & $4868 \pm 275$ & $1.78 \pm 0.47$ & $-1.66 \pm 0.38$ & $0.44 \pm 0.12$ \\
\hline 384 & HD087140 & 0.033 & 2 & 1.038 & $5129 \pm 103$ & $2.66 \pm 0.25$ & $-1.80 \pm 0.13$ & $5090 \pm 5$ & $2.58 \pm 0.10$ & $-1.82 \pm 0.06$ & $0.42 \pm 0.01$ \\
\hline 425 & HD108317 & 0.039 & 2 & 1.034 & $5259 \pm 111$ & $2.68 \pm 0.25$ & $-2.27 \pm 0.05$ & $5117 \pm 40$ & $2.70 \pm 0.15$ & $-2.33 \pm 0.11$ & $0.45 \pm 0.08$ \\
\hline 452 & HD 117876 & 0.075 & 3 & 1.091 & $4747 \pm 128$ & $2.27 \pm 0.03$ & $-0.48 \pm 0.02$ & $4806 \pm 18$ & $2.25 \pm 0.15$ & $-0.44 \pm 0.03$ & $0.42 \pm 0.10$ \\
\hline 454 & HD 122956 & 0.023 & 3 & 1.018 & $4633 \pm 78$ & $1.46 \pm 0.18$ & $-1.72 \pm 0.11$ & $4646 \pm 16$ & $1.43 \pm 0.04$ & $-1.73 \pm 0.02$ & $0.42 \pm 0.07$ \\
\hline 459 & HD 124897 & 0.136 & 5 & 1.023 & $4302 \pm 115$ & $1.66 \pm 0.31$ & $-0.52 \pm 0.11$ & $4346 \pm 98$ & $1.87 \pm 0.46$ & $-0.55 \pm 0.24$ & $0.29 \pm 0.15$ \\
\hline 470 & HD 135722 & 0.011 & 3 & 1.016 & $4795 \pm 76$ & $2.60 \pm 0.41$ & $-0.40 \pm 0.10$ & $4846 \pm 8$ & $2.60 \pm 0.03$ & $-0.40 \pm 0.03$ & $0.14 \pm 0.04$ \\
\hline 473 & HD137759 & 0.142 & 2 & 1.014 & $4549 \pm 118$ & $2.88 \pm 0.21$ & $0.13 \pm 0.11$ & $4558 \pm 64$ & $2.54 \pm 0.07$ & $0.14 \pm 0.02$ & $-0.03 \pm 0.11$ \\
\hline 509 & HD159181 & 0.180 & 4 & 1.215 & $5234 \pm 158$ & $1.56 \pm 0.19$ & $0.15 \pm 0.12$ & $5235 \pm 48$ & $1.82 \pm 0.31$ & $0.14 \pm 0.24$ & $0.04 \pm 0.19$ \\
\hline 566 & HD166161 & 0.059 & 2 & 1.040 & $5210 \pm 167$ & $2.25 \pm 0.42$ & $-1.22 \pm 0.13$ & $5071 \pm 122$ & $2.16 \pm 0.11$ & $-1.18 \pm 0.05$ & $0.31 \pm 0.06$ \\
\hline 568 & HD166208 & 0.161 & 1 & 1.000 & $5037 \pm 56$ & $2.71 \pm 0.08$ & $0.07 \pm 0.11$ & $4919 \pm 98$ & $2.52 \pm 0.05$ & $0.08 \pm 0.04$ & $0.17 \pm 0.01$ \\
\hline 652 & HD175305 & 0.082 & 1 & 1.000 & $5053 \pm 140$ & $2.49 \pm 0.26$ & $-1.43 \pm 0.07$ & $4899 \pm 17$ & $2.30 \pm 0.03$ & $-1.43 \pm 0.05$ & $0.27 \pm 0.06$ \\
\hline 701 & HD 187111 & 0.125 & 4 & 1.149 & $4299 \pm 75$ & $0.74 \pm 0.30$ & $-1.78 \pm 0.18$ & $4343 \pm 77$ & $0.79 \pm 0.21$ & $-1.59 \pm 0.09$ & $0.47 \pm 0.04$ \\
\hline 763 & HD198149 & 0.078 & 2 & 1.015 & $4956 \pm 177$ & $3.35 \pm 0.22$ & $-0.12 \pm 0.18$ & $5027 \pm 10$ & $3.12 \pm 0.06$ & $-0.12 \pm 0.06$ & $0.12 \pm 0.01$ \\
\hline 791 & HD204543 & 0.026 & 1 & 1.000 & $4667 \pm 68$ & $1.30 \pm 0.23$ & $-1.80 \pm 0.10$ & $4617 \pm 43$ & $1.31 \pm 0.08$ & $-1.76 \pm 0.10$ & $0.24 \pm 0.07$ \\
\hline 792 & HD204613 & 0.101 & 2 & 1.208 & $5742 \pm 135$ & $3.72 \pm 0.19$ & $-0.51 \pm 0.16$ & $5614 \pm 111$ & $3.45 \pm 0.09$ & $-0.48 \pm 0.08$ & $0.21 \pm 0.15$ \\
\hline 803 & HD207130 & 0.093 & 3 & 1.011 & $4760 \pm 53$ & $2.63 \pm 0.15$ & $0.01 \pm 0.11$ & $4727 \pm 16$ & $2.40 \pm 0.12$ & $0.01 \pm 0.03$ & $0.07 \pm 0.01$ \\
\hline 825 & HD216143 & 0.045 & 2 & 1.019 & $4495 \pm 82$ & $1.12 \pm 0.38$ & $-2.20 \pm 0.06$ & $4480 \pm 8$ & $1.15 \pm 0.06$ & $-2.12 \pm 0.01$ & $0.39 \pm 0.05$ \\
\hline 826 & HD216174 & 0.066 & 2 & 1.145 & $4413 \pm 23$ & $2.11 \pm 0.36$ & $-0.55 \pm 0.02$ & $4381 \pm 9$ & $2.21 \pm 0.02$ & $-0.53 \pm 0.00$ & $0.33 \pm 0.04$ \\
\hline 836 & HD218857 & 0.054 & 8 & 1.098 & $5119 \pm 44$ & $2.50 \pm 0.37$ & $-1.91 \pm 0.09$ & $5067 \pm 89$ & $2.37 \pm 0.31$ & $-1.93 \pm 0.15$ & $0.41 \pm 0.04$ \\
\hline 848 & HD221345 & 0.019 & 12 & 1.224 & $4635 \pm 108$ & $2.49 \pm 0.32$ & $-0.30 \pm 0.07$ & $4666 \pm 45$ & $2.50 \pm 0.09$ & $-0.30 \pm 0.04$ & $0.18 \pm 0.07$ \\
\hline 849 & HD221377 & 0.182 & 3 & 1.150 & $6176 \pm 188$ & $3.61 \pm 0.17$ & $-0.88 \pm 0.17$ & $6027 \pm 57$ & $3.24 \pm 0.15$ & $-1.01 \pm 0.15$ & $0.57 \pm 0.12$ \\
\hline
\end{tabular}


Table B.2 - continued.

\begin{tabular}{|c|c|c|c|c|c|c|c|c|c|c|c|}
\hline ELODIE & Star & $\mathrm{RR}_{\text {tot }}$ & $\mathrm{N}$ & $\mathrm{S}_{\mathrm{lim}}$ & $\mathrm{T}_{\text {eff }}(\mathrm{K})$ & $\log (g)$ & {$[\mathrm{Fe} / \mathrm{H}]$} & $\mathrm{T}_{\text {eff }}(\mathrm{K})$ & $\log g$ & {$[\mathrm{Fe} / \mathrm{H}]$} & {$[\mathrm{Mg} / \mathrm{Fe}]$} \\
\hline 871 & HD232078 & 0.101 & 1 & 1.000 & $3939 \pm 175$ & $0.31 \pm 0.34$ & $-1.58 \pm 0.15$ & $3983 \pm 186$ & $0.30 \pm 0.53$ & $-1.73 \pm 0.76$ & $0.27 \pm 0.15$ \\
\hline 878 & $\mathrm{BD}+233130$ & 0.033 & 1 & 1.000 & $5119 \pm 140$ & $2.39 \pm 0.38$ & $-2.62 \pm 0.19$ & $5039 \pm 20$ & $2.42 \pm 0.16$ & $-2.55 \pm 0.01$ & $0.60 \pm 0.04$ \\
\hline 883 & $\mathrm{BD}+302611$ & 0.139 & 2 & 1.013 & $4292 \pm 100$ & $0.96 \pm 0.37$ & $-1.41 \pm 0.19$ & $4421 \pm 274$ & $0.83 \pm 0.72$ & $-1.43 \pm 0.05$ & $0.46 \pm 0.13$ \\
\hline 927 & HD000245 & 0.110 & 2 & 1.032 & $5490 \pm 153$ & $3.48 \pm 0.15$ & $-0.77 \pm 0.08$ & $5377 \pm 59$ & $3.67 \pm 0.06$ & $-0.84 \pm 0.19$ & $0.34 \pm 0.08$ \\
\hline 941 & HD003546 & 0.035 & 15 & 1.412 & $4906 \pm 168$ & $2.45 \pm 0.43$ & $-0.64 \pm 0.12$ & $4868 \pm 71$ & $2.51 \pm 0.19$ & $-0.65 \pm 0.09$ & $0.27 \pm 0.03$ \\
\hline 1395 & HD105546 & 0.047 & 2 & 1.018 & $5234 \pm 79$ & $2.38 \pm 0.14$ & $-1.39 \pm 0.15$ & $5387 \pm 190$ & $2.30 \pm 0.14$ & $-1.37 \pm 0.23$ & $0.54 \pm 0.06$ \\
\hline 1452 & HD122563 & 0.043 & 1 & 1.000 & $4565 \pm 131$ & $1.17 \pm 0.24$ & $-2.62 \pm 0.14$ & $4566 \pm 440$ & $1.12 \pm 1.31$ & $-2.63 \pm 0.37$ & $0.60 \pm 0.22$ \\
\hline 1483 & HD136512 & 0.098 & 2 & 1.060 & $4719 \pm 66$ & $2.72 \pm 0.04$ & $-0.33 \pm 0.16$ & $4747 \pm 46$ & $2.62 \pm 0.25$ & $-0.30 \pm 0.00$ & $0.08 \pm 0.03$ \\
\hline 1484 & HD136512 & 0.110 & 4 & 1.072 & $4719 \pm 66$ & $2.72 \pm 0.04$ & $-0.33 \pm 0.16$ & $4754 \pm 29$ & $2.53 \pm 0.17$ & $-0.30 \pm 0.08$ & $0.14 \pm 0.11$ \\
\hline 1485 & HD 136512 & 0.111 & 4 & 1.072 & $4719 \pm 66$ & $2.72 \pm 0.04$ & $-0.33 \pm 0.16$ & $4754 \pm 29$ & $2.53 \pm 0.17$ & $-0.30 \pm 0.08$ & $0.14 \pm 0.11$ \\
\hline 1486 & HD136512 & 0.070 & 14 & 1.149 & $4719 \pm 66$ & $2.72 \pm 0.04$ & $-0.33 \pm 0.16$ & $4824 \pm 25$ & $2.54 \pm 0.08$ & $-0.33 \pm 0.05$ & $0.21 \pm 0.06$ \\
\hline 1487 & HD136512 & 0.080 & 13 & 1.144 & $4719 \pm 66$ & $2.72 \pm 0.04$ & $-0.33 \pm 0.16$ & $4821 \pm 27$ & $2.52 \pm 0.08$ & $-0.32 \pm 0.06$ & $0.21 \pm 0.06$ \\
\hline 1576 & HD162211 & 0.058 & 4 & 1.210 & $4568 \pm 74$ & $2.74 \pm 0.11$ & $0.04 \pm 0.06$ & $4581 \pm 72$ & $2.59 \pm 0.17$ & $0.04 \pm 0.02$ & $0.04 \pm 0.07$ \\
\hline 1811 & HD188326 & 0.172 & 3 & 1.092 & $5272 \pm 40$ & $3.80 \pm 0.01$ & $-0.18 \pm 0.00$ & $5074 \pm 39$ & $3.34 \pm 0.15$ & $-0.20 \pm 0.21$ & $0.12 \pm 0.03$ \\
\hline 1812 & HD188326 & 0.161 & 3 & 1.084 & $5272 \pm 40$ & $3.80 \pm 0.01$ & $-0.18 \pm 0.00$ & $5074 \pm 41$ & $3.34 \pm 0.16$ & $-0.20 \pm 0.21$ & $0.12 \pm 0.03$ \\
\hline 1876 & HD212943 & 0.081 & 4 & 1.085 & $4625 \pm 67$ & $2.79 \pm 0.05$ & $-0.29 \pm 0.09$ & $4656 \pm 35$ & $2.61 \pm 0.10$ & $-0.30 \pm 0.04$ & $0.17 \pm 0.07$ \\
\hline 1893 & HD216219 & 0.093 & 1 & 1.000 & $5628 \pm 106$ & $3.12 \pm 0.22$ & $-0.41 \pm 0.10$ & $5727 \pm 152$ & $3.36 \pm 0.50$ & $-0.39 \pm 0.21$ & $0.06 \pm 0.19$ \\
\hline 1916 & HD219449 & 0.070 & 16 & 1.259 & $4647 \pm 75$ & $2.56 \pm 0.26$ & $-0.03 \pm 0.07$ & $4626 \pm 35$ & $2.39 \pm 0.07$ & $-0.03 \pm 0.03$ & $0.03 \pm 0.03$ \\
\hline
\end{tabular}


Table B.3 - Atmospheric parameters for all stars analysed in the six clusters: $\mathrm{T}_{\text {eff }}, \log (g),[\mathrm{Fe} / \mathrm{H}],[\mathrm{Mg} / \mathrm{Fe}]$ and $[\alpha / \mathrm{Fe}]$. Membership identification is copied from Table B.1 to guide the reader.

\begin{tabular}{|c|c|c|c|c|c|c|c|c|c|c|c|c|}
\hline NGC ID & $\mathrm{T}_{e f f}^{(a)}(\mathrm{K})$ & $\mathrm{T}_{e f f}^{(b)}(\mathrm{K})$ & $\mathrm{T}_{e f f}^{(a v g)}(\mathrm{K})$ & $\log (g)^{(a)}$ & $\log (g)^{(b)}$ & $\log (g)^{(a v g)}$ & {$[\mathrm{Fe} / \mathrm{H}]^{(a)}$} & {$[\mathrm{Fe} / \mathrm{H}]^{(b)}$} & {$[\mathrm{Fe} / \mathrm{H}]^{(a v g)}$} & {$[\mathrm{Mg} / \mathrm{Fe}]^{(a)}$} & {$[\alpha / \mathrm{Fe}]^{(b)}$} & members \\
\hline $6528 \_2$ & $3969 \pm 80$ & $4074 \pm 114$ & $4004 \pm 66$ & $1.54 \pm 0.18$ & $1.9 \pm 0.4$ & $1.60 \pm 0.17$ & $0.02 \pm 0.16$ & $-0.50 \pm 0.00$ & $-0.24 \pm 0.16$ & $-0.04 \pm 0.18$ & $0.20 \pm 0.14$ & \\
\hline 6528_3 & $3640 \pm 100$ & $3525 \pm 75$ & $3566 \pm 60$ & $0.70 \pm 0.00$ & $2.9 \pm 0.8$ & $0.96 \pm 0.28$ & $-0.10 \pm 0.07$ & $-0.70 \pm 0.25$ & $-0.14 \pm 0.06$ & $0.23 \pm 0.00$ & $0.32 \pm 0.07$ & \\
\hline 6528_4 & $3900 \pm 79$ & $3623 \pm 125$ & $3821 \pm 67$ & $1.38 \pm 0.19$ & $0.8 \pm 0.7$ & $1.34 \pm 0.18$ & $-0.07 \pm 0.20$ & $-1.35 \pm 0.23$ & $-0.62 \pm 0.15$ & $0.06 \pm 0.20$ & $0.26 \pm 0.11$ & \\
\hline $6528 \_5$ & $4027 \pm 79$ & $4198 \pm 151$ & $4064 \pm 70$ & $1.54 \pm 0.34$ & $2.0 \pm 0.4$ & $1.74 \pm 0.26$ & $-0.31 \pm 0.22$ & $-0.85 \pm 0.23$ & $-0.57 \pm 0.16$ & $0.12 \pm 0.18$ & $0.31 \pm 0.10$ & \\
\hline 6528_6 & $4344 \pm 215$ & $4625 \pm 125$ & $4554 \pm 108$ & $2.1 \pm 0.5$ & $2.8 \pm 0.23$ & $2.71 \pm 0.21$ & $0.04 \pm 0.17$ & $-0.21 \pm 0.30$ & $-0.02 \pm 0.15$ & $0.03 \pm 0.12$ & $0.18 \pm 0.12$ & \\
\hline $6528 \_7$ & $3930 \pm 58$ & $4124 \pm 125$ & $3964 \pm 53$ & $1.51 \pm 0.01$ & $2.05 \pm 0.35$ & $1.51 \pm 0.01$ & $0.10 \pm 0.13$ & $-0.25 \pm 0.25$ & $0.02 \pm 0.12$ & $0.16 \pm 0.06$ & $0.30 \pm 0.08$ & \\
\hline $6528 \_8$ & $4159 \pm 162$ & $4225 \pm 175$ & $4189 \pm 119$ & $1.7 \pm 0.4$ & $2.0 \pm 0.5$ & $1.88 \pm 0.32$ & $-0.08 \pm 0.20$ & $-0.75 \pm 0.25$ & $-0.34 \pm 0.16$ & $0.02 \pm 0.19$ & $0.28 \pm 0.09$ & M \\
\hline 6528_9 & $4244 \pm 170$ & $4400 \pm 122$ & $4347 \pm 99$ & $1.9 \pm 0.4$ & $2.6 \pm 0.4$ & $2.28 \pm 0.27$ & $-0.06 \pm 0.19$ & $-0.70 \pm 0.24$ & $-0.31 \pm 0.15$ & $0.03 \pm 0.17$ & $0.27 \pm 0.09$ & $\mathrm{M}$ \\
\hline 6528_10 & $4557 \pm 236$ & $4925 \pm 115$ & $4855 \pm 103$ & $2.4 \pm 0.5$ & $3.30 \pm 0.24$ & $3.14 \pm 0.22$ & $-0.10 \pm 0.25$ & $0.10 \pm 0.10$ & $0.07 \pm 0.09$ & $0.06 \pm 0.17$ & $0.19 \pm 0.11$ & $\mathrm{M}$ \\
\hline 6528_11 & $3911 \pm 70$ & $3800 \pm 187$ & $3897 \pm 66$ & $1.43 \pm 0.18$ & $1.3 \pm 0.7$ & $1.42 \pm 0.17$ & $-0.04 \pm 0.21$ & $-1.15 \pm 0.32$ & $-0.37 \pm 0.18$ & $0.06 \pm 0.17$ & $0.29 \pm 0.11$ & $\mathrm{M}$ \\
\hline 6528_13 & $4853 \pm 198$ & $4876 \pm 167$ & $4866 \pm 128$ & $2.5 \pm 0.4$ & $2.6 \pm 0.5$ & $2.53 \pm 0.32$ & $-0.44 \pm 0.22$ & $-0.80 \pm 0.25$ & $-0.60 \pm 0.17$ & $0.24 \pm 0.20$ & $0.22 \pm 0.11$ & \\
\hline 6528_14 & $4239 \pm 172$ & $4397 \pm 164$ & $4322 \pm 119$ & $1.9 \pm 0.5$ & $2.2 \pm 0.4$ & $2.07 \pm 0.30$ & $-0.13 \pm 0.21$ & $-0.71 \pm 0.33$ & $-0.30 \pm 0.18$ & $0.03 \pm 0.11$ & $0.28 \pm 0.12$ & \\
\hline 6528_15 & $4083 \pm 176$ & $4325 \pm 114$ & $4253 \pm 96$ & $1.61 \pm 0.19$ & $2.30 \pm 0.25$ & $1.86 \pm 0.15$ & $0.05 \pm 0.16$ & $-0.35 \pm 0.23$ & $-0.08 \pm 0.13$ & $0.05 \pm 0.11$ & $0.17 \pm 0.13$ & \\
\hline 6528_16 & $3938 \pm 40$ & $4075 \pm 114$ & $3953 \pm 38$ & $1.48 \pm 0.12$ & $1.95 \pm 0.35$ & $1.53 \pm 0.11$ & $-0.04 \pm 0.15$ & $-0.80 \pm 0.24$ & $-0.25 \pm 0.13$ & $0.05 \pm 0.10$ & $0.29 \pm 0.09$ & \\
\hline 6528_17 & $4500 \pm 147$ & $4700 \pm 186$ & $4577 \pm 115$ & $2.35 \pm 0.27$ & $2.70 \pm 0.33$ & $2.49 \pm 0.21$ & $-0.37 \pm 0.13$ & $-0.6 \pm 0.4$ & $-0.39 \pm 0.12$ & $0.17 \pm 0.16$ & $0.31 \pm 0.10$ & \\
\hline 6528_18 & $4709 \pm 194$ & $4676 \pm 195$ & $4692 \pm 138$ & $2.5 \pm 0.4$ & $2.5 \pm 0.5$ & $2.52 \pm 0.29$ & $-0.22 \pm 0.25$ & $-0.6 \pm 0.4$ & $-0.35 \pm 0.21$ & $0.12 \pm 0.18$ & $0.16 \pm 0.12$ & \\
\hline 6528_19 & $4496 \pm 94$ & $4701 \pm 99$ & $4593 \pm 68$ & $2.53 \pm 0.30$ & $2.90 \pm 0.20$ & $2.79 \pm 0.17$ & $0.13 \pm 0.09$ & $-0.02 \pm 0.25$ & $0.11 \pm 0.08$ & $0.04 \pm 0.08$ & $0.17 \pm 0.13$ & \\
\hline 6553_1 & $4141 \pm 107$ & $4425 \pm 195$ & $4207 \pm 94$ & $1.58 \pm 0.14$ & $2.2 \pm 0.5$ & $1.62 \pm 0.14$ & $-0.08 \pm 0.09$ & $-0.40 \pm 0.20$ & $-0.14 \pm 0.08$ & $0.08 \pm 0.06$ & $0.32 \pm 0.07$ & M \\
\hline 6553_3 & $4384 \pm 155$ & $4475 \pm 174$ & $4424 \pm 116$ & $1.9 \pm 0.4$ & $2.2 \pm 0.5$ & $1.98 \pm 0.31$ & $-0.06 \pm 0.08$ & $-0.40 \pm 0.20$ & $-0.10 \pm 0.07$ & $0.12 \pm 0.12$ & $0.29 \pm 0.09$ & M \\
\hline 6553_4 & $3837 \pm 100$ & $3574 \pm 114$ & $3723 \pm 75$ & $1.17 \pm 0.18$ & $1.1 \pm 0.7$ & $1.17 \pm 0.17$ & $0.10 \pm 0.17$ & $-1.35 \pm 0.23$ & $-0.41 \pm 0.14$ & $0.09 \pm 0.07$ & $0.34 \pm 0.07$ & M \\
\hline $6553 \_5$ & $3970 \pm 71$ & $4076 \pm 160$ & $3987 \pm 65$ & $1.65 \pm 0.10$ & $1.6 \pm 0.6$ & $1.65 \pm 0.10$ & $0.06 \pm 0.13$ & $-0.75 \pm 0.33$ & $-0.04 \pm 0.12$ & $0.05 \pm 0.11$ & $0.34 \pm 0.07$ & M \\
\hline 6553_6 & $4312 \pm 135$ & $4400 \pm 122$ & $4360 \pm 91$ & $1.9 \pm 0.4$ & $1.9 \pm 0.4$ & $1.90 \pm 0.28$ & $0.004 \pm 0.09$ & $-0.50 \pm 0.00$ & $-0.13 \pm 0.08$ & $-0.01 \pm 0.17$ & $0.26 \pm 0.11$ & M \\
\hline 6553_7 & $3730 \pm 60$ & $3550 \pm 100$ & $3682 \pm 51$ & $0.90 \pm 0.13$ & $1.2 \pm 0.9$ & $0.91 \pm 0.13$ & $0.27 \pm 0.18$ & $-0.95 \pm 0.27$ & $-0.10 \pm 0.15$ & $0.08 \pm 0.10$ & $0.28 \pm 0.10$ & M \\
\hline 6553_8 & $3640 \pm 0$ & $3500 \pm 0$ & $3528 \pm 45$ & $0.70 \pm 0.00$ & $2.8 \pm 0.8$ & $0.98 \pm 0.28$ & $-0.10 \pm 0.06$ & $-0.70 \pm 0.24$ & $-0.14 \pm 0.06$ & $0.23 \pm 0.10$ & $0.32 \pm 0.07$ & \\
\hline 6553_9 & $4259 \pm 15$ & $4399 \pm 165$ & $4260 \pm 15$ & $1.47 \pm 0.08$ & $2.1 \pm 0.5$ & $1.49 \pm 0.08$ & $-0.13 \pm 0.00$ & $-0.45 \pm 0.15$ & $-0.13 \pm 0.01$ & $0.11 \pm 0.10$ & $0.28 \pm 0.10$ & M \\
\hline 6553_10 & $4388 \pm 155$ & $4525 \pm 174$ & $4449 \pm 116$ & $1.9 \pm 0.4$ & $2.3 \pm 0.5$ & $2.02 \pm 0.31$ & $-0.06 \pm 0.08$ & $-0.35 \pm 0.23$ & $-0.09 \pm 0.07$ & $0.13 \pm 0.12$ & $0.29 \pm 0.09$ & $\mathrm{M}$ \\
\hline
\end{tabular}


Table B.3 - continued.

\begin{tabular}{|c|c|c|c|c|c|c|c|c|c|c|c|c|}
\hline NGC ID & $\mathrm{T}_{e f f}^{(a)}(\mathrm{K})$ & $\mathrm{T}_{e f f}^{(b)}(\mathrm{K})$ & $\mathrm{T}_{\text {eff }}^{(\text {avg) }}(\mathrm{K})$ & $\log (g)^{(a)}$ & $\log (g)^{(b)}$ & $\log (g)^{(a v g)}$ & {$[\mathrm{Fe} / \mathrm{H}]^{(a)}$} & {$[\mathrm{Fe} / \mathrm{H}]^{(b)}$} & {$[\mathrm{Fe} / \mathrm{H}]^{(a v g)}$} & {$[\mathrm{Mg} / \mathrm{Fe}]^{(a)}$} & {$[\alpha / \mathrm{Fe}]^{(b)}$} & members \\
\hline 6553_11 & $3941 \pm 44$ & $3801 \pm 218$ & $3936 \pm 43$ & $1.47 \pm 0.19$ & $1.0 \pm 0.8$ & $1.45 \pm 0.19$ & $0.01 \pm 0.14$ & $-0.9 \pm 0.4$ & $-0.07 \pm 0.13$ & $0.07 \pm 0.09$ & $0.27 \pm 0.09$ & M \\
\hline 6553_13 & $4219 \pm 57$ & $4350 \pm 122$ & $4242 \pm 52$ & $1.63 \pm 0.26$ & $2.0 \pm 0.4$ & $1.74 \pm 0.22$ & $-0.01 \pm 0.13$ & $-0.50 \pm 0.00$ & $-0.22 \pm 0.10$ & $0.02 \pm 0.10$ & $0.25 \pm 0.10$ & M \\
\hline 6553_14 & $3889 \pm 64$ & $3896 \pm 301$ & $3889 \pm 63$ & $1.38 \pm 0.16$ & $1.3 \pm 1.0$ & $1.38 \pm 0.16$ & $-0.01 \pm 0.17$ & $-0.95 \pm 0.42$ & $-0.15 \pm 0.16$ & $0.004 \pm 0.20$ & $0.33 \pm 0.10$ & M \\
\hline 6553_15 & $3640 \pm 0$ & $3500 \pm 0$ & $3528 \pm 45$ & $0.70 \pm 0.00$ & $3.1 \pm 0.8$ & $1.01 \pm 0.28$ & $-0.10 \pm 0.06$ & $-0.60 \pm 0.20$ & $-0.15 \pm 0.06$ & $0.23 \pm 0.10$ & $0.32 \pm 0.07$ & \\
\hline 6553_16 & $3932 \pm 29$ & $4101 \pm 123$ & $3941 \pm 28$ & $1.54 \pm 0.13$ & $1.9 \pm 0.4$ & $1.58 \pm 0.12$ & $-0.20 \pm 0.05$ & $-0.70 \pm 0.24$ & $-0.22 \pm 0.05$ & $0.05 \pm 0.09$ & $0.33 \pm 0.06$ & M \\
\hline 6553_17 & $3640 \pm 100$ & $3548 \pm 99$ & $3594 \pm 70$ & $0.70 \pm 0.00$ & $2.4 \pm 0.7$ & $1.15 \pm 0.34$ & $-0.05 \pm 0.05$ & $-0.90 \pm 0.20$ & $-0.10 \pm 0.05$ & $0.23 \pm 0.10$ & $0.35 \pm 0.07$ & \\
\hline 6553_18 & $3640 \pm 100$ & $3500 \pm 0.0$ & $3528 \pm 45$ & $0.70 \pm 0.00$ & $3.4 \pm 0.5$ & $1.78 \pm 0.31$ & $-0.10 \pm 0.07$ & $0.20 \pm 0.17$ & $-0.06 \pm 0.06$ & $0.23 \pm 0.10$ & $0.33 \pm 0.06$ & \\
\hline 6553_19 & $4259 \pm 15$ & $4176 \pm 114$ & $4258 \pm 15$ & $1.47 \pm 0.08$ & $1.75 \pm 0.33$ & $1.49 \pm 0.08$ & $-0.13 \pm 0.00$ & $-0.65 \pm 0.23$ & $-0.13 \pm 0.01$ & $0.11 \pm 0.10$ & $0.27 \pm 0.09$ & M \\
\hline M71_2 & $4743 \pm 210$ & $4850 \pm 122$ & $4823 \pm 106$ & $2.5 \pm 0.5$ & $2.95 \pm 0.27$ & $2.85 \pm 0.24$ & $-0.48 \pm 0.24$ & $-0.85 \pm 0.23$ & $-0.67 \pm 0.17$ & $0.25 \pm 0.20$ & $0.28 \pm 0.13$ & M \\
\hline M71_4 & $4305 \pm 77$ & $4449 \pm 187$ & $4326 \pm 71$ & $1.8 \pm 0.5$ & $2.2 \pm 0.6$ & $1.99 \pm 0.36$ & $-0.56 \pm 0.24$ & $-0.85 \pm 0.23$ & $-0.71 \pm 0.17$ & $0.32 \pm 0.18$ & $0.28 \pm 0.10$ & M \\
\hline M71_5 & $4722 \pm 223$ & $4899 \pm 198$ & $4821 \pm 148$ & $2.4 \pm 0.6$ & $3.10 \pm 0.30$ & $2.97 \pm 0.27$ & $-0.51 \pm 0.26$ & $-0.85 \pm 0.32$ & $-0.64 \pm 0.20$ & $0.27 \pm 0.20$ & $0.29 \pm 0.09$ & M \\
\hline M71_6 & $4364 \pm 94$ & $4523 \pm 174$ & $4400 \pm 83$ & $1.9 \pm 0.4$ & $2.2 \pm 0.6$ & $2.02 \pm 0.32$ & $-0.55 \pm 0.22$ & $-0.95 \pm 0.35$ & $-0.66 \pm 0.19$ & $0.26 \pm 0.16$ & $0.30 \pm 0.08$ & M \\
\hline M71_7 & $3991 \pm 99$ & $4026 \pm 207$ & $3997 \pm 89$ & $1.5 \pm 0.4$ & $1.7 \pm 0.7$ & $1.53 \pm 0.35$ & $-0.32 \pm 0.21$ & $-1.25 \pm 0.33$ & $-0.58 \pm 0.17$ & $0.15 \pm 0.18$ & $0.34 \pm 0.07$ & M \\
\hline M71_8 & $4409 \pm 132$ & $4850 \pm 122$ & $4646 \pm 90$ & $2.1 \pm 0.5$ & $3.15 \pm 0.23$ & $2.95 \pm 0.21$ & $0.16 \pm 0.13$ & $0.26 \pm 0.21$ & $0.18 \pm 0.11$ & $0.01 \pm 0.14$ & $0.13 \pm 0.10$ & \\
\hline M71_9 & $4303 \pm 102$ & $4350 \pm 166$ & $4316 \pm 87$ & $1.9 \pm 0.5$ & $2.0 \pm 0.5$ & $1.97 \pm 0.33$ & $-0.54 \pm 0.23$ & $-1.05 \pm 0.27$ & $-0.76 \pm 0.17$ & $0.27 \pm 0.21$ & $0.28 \pm 0.10$ & M \\
\hline M71_10 & $3861 \pm 77$ & $3549 \pm 99$ & $3743 \pm 61$ & $1.23 \pm 0.21$ & $0.5 \pm 0.6$ & $1.16 \pm 0.20$ & $-0.06 \pm 0.26$ & $-1.70 \pm 0.24$ & $-0.96 \pm 0.18$ & $0.11 \pm 0.07$ & $0.29 \pm 0.14$ & M \\
\hline M71_13 & $4685 \pm 209$ & $4850 \pm 122$ & $4808 \pm 106$ & $2.4 \pm 0.5$ & $2.95 \pm 0.27$ & $2.82 \pm 0.24$ & $-0.44 \pm 0.26$ & $-0.80 \pm 0.25$ & $-0.63 \pm 0.18$ & $0.23 \pm 0.20$ & $0.25 \pm 0.10$ & M \\
\hline M71_14 & $4901 \pm 212$ & $5175 \pm 114$ & $5113 \pm 101$ & $2.7 \pm 0.5$ & $3.15 \pm 0.32$ & $3.01 \pm 0.27$ & $-0.44 \pm 0.25$ & $-0.65 \pm 0.23$ & $-0.55 \pm 0.17$ & $0.25 \pm 0.20$ & $0.27 \pm 0.11$ & M \\
\hline M71_15 & $4840 \pm 259$ & $5101 \pm 165$ & $5025 \pm 139$ & $2.5 \pm 0.6$ & $3.10 \pm 0.30$ & $2.98 \pm 0.27$ & $-0.50 \pm 0.30$ & $-0.55 \pm 0.15$ & $-0.54 \pm 0.13$ & $0.26 \pm 0.19$ & $0.27 \pm 0.11$ & M \\
\hline M71_16 & $4694 \pm 214$ & $4974 \pm 175$ & $4862 \pm 135$ & $2.5 \pm 0.4$ & $2.95 \pm 0.35$ & $2.76 \pm 0.27$ & $-0.13 \pm 0.25$ & $-0.11 \pm 0.27$ & $-0.12 \pm 0.18$ & $0.08 \pm 0.18$ & $0.20 \pm 0.13$ & \\
\hline 6558_3 & $4758 \pm 228$ & $4926 \pm 160$ & $4871 \pm 131$ & $2.5 \pm 0.5$ & $3.10 \pm 0.30$ & $2.94 \pm 0.25$ & $-0.29 \pm 0.26$ & $-0.70 \pm 0.24$ & $-0.51 \pm 0.18$ & $0.15 \pm 0.20$ & $0.30 \pm 0.10$ & \\
\hline $6558 \_4$ & $4678 \pm 172$ & $4625 \pm 167$ & $4651 \pm 120$ & $2.55 \pm 0.33$ & $3.0 \pm 0.4$ & $2.75 \pm 0.26$ & $-0.21 \pm 0.19$ & $-1.30 \pm 0.25$ & $-0.61 \pm 0.15$ & $0.09 \pm 0.14$ & $0.29 \pm 0.09$ & \\
\hline $6558 \_5$ & $4921 \pm 445$ & $4900 \pm 200$ & $4903 \pm 182$ & $2.0 \pm 0.6$ & $2.00 \pm 0.32$ & $2.00 \pm 0.29$ & $-1.2 \pm 0.6$ & $-1.80 \pm 0.24$ & $-1.72 \pm 0.22$ & $0.37 \pm 0.19$ & $0.21 \pm 0.11$ & \\
\hline 6558_6 & $4738 \pm 329$ & $4951 \pm 186$ & $4899 \pm 162$ & $2.1 \pm 0.8$ & $2.8 \pm 0.4$ & $2.68 \pm 0.39$ & $-0.9 \pm 0.4$ & $-1.20 \pm 0.24$ & $-1.11 \pm 0.20$ & $0.22 \pm 0.07$ & $0.26 \pm 0.13$ & M \\
\hline $6558 \_7$ & $4928 \pm 317$ & $4926 \pm 114$ & $4926 \pm 108$ & $2.5 \pm 0.7$ & $2.70 \pm 0.25$ & $2.68 \pm 0.24$ & $-0.64 \pm 0.32$ & $-1.15 \pm 0.23$ & $-0.98 \pm 0.19$ & $0.26 \pm 0.13$ & $0.26 \pm 0.13$ & M \\
\hline 6558_8 & $3640 \pm 100$ & $3524 \pm 74$ & $3565 \pm 59$ & $0.70 \pm 0.5$ & $2.8 \pm 0.9$ & $1.05 \pm 0.36$ & $0.00 \pm 0.07$ & $-0.95 \pm 0.15$ & $-0.16 \pm 0.06$ & $0.23 \pm 0.00$ & $0.31 \pm 0.08$ & M \\
\hline 6558_9 & $4663 \pm 336$ & $5076 \pm 195$ & $4972 \pm 168$ & $1.8 \pm 0.9$ & $2.9 \pm 0.5$ & $2.68 \pm 0.46$ & $-1.2 \pm 0.4$ & $-1.10 \pm 0.20$ & $-1.11 \pm 0.18$ & $0.41 \pm 0.16$ & $0.21 \pm 0.11$ & M \\
\hline 6558_10 & $4398 \pm 157$ & $4799 \pm 100$ & $4684 \pm 84$ & $2.1 \pm 0.5$ & $3.15 \pm 0.23$ & $2.95 \pm 0.21$ & $0.10 \pm 0.13$ & $0.16 \pm 0.19$ & $0.12 \pm 0.11$ & $0.02 \pm 0.13$ & $0.12 \pm 0.12$ & \\
\hline
\end{tabular}


Table B.3 - continued.

\begin{tabular}{|c|c|c|c|c|c|c|c|c|c|c|c|c|}
\hline NGC ID & $\mathrm{T}_{e f f}^{(a)}(\mathrm{K})$ & $\mathrm{T}_{e f f}^{(b)}(\mathrm{K})$ & $\mathrm{T}_{e f f}^{(a v g)}(\mathrm{K})$ & $\log (g)^{(a)}$ & $\log (g)^{(b)}$ & $\log (g)^{(a v g)}$ & {$[\mathrm{Fe} / \mathrm{H}]^{(a)}$} & {$[\mathrm{Fe} / \mathrm{H}]^{(b)}$} & {$[\mathrm{Fe} / \mathrm{H}]^{(a v g)}$} & {$[\mathrm{Mg} / \mathrm{Fe}]^{(a)}$} & {$[\alpha / \mathrm{Fe}]^{(b)}$} & members \\
\hline 6558_11 & $4887 \pm 413$ & $5325 \pm 114$ & $5294 \pm 110$ & $2.2 \pm 0.9$ & $3.30 \pm 0.25$ & $3.22 \pm 0.24$ & $-1.1 \pm 0.5$ & $-1.00 \pm 0.00$ & $-1.00 \pm 0.05$ & $0.37 \pm 0.19$ & $0.19 \pm 0.14$ & M \\
\hline 6558_12 & $4516 \pm 177$ & $4548 \pm 245$ & $4527 \pm 143$ & $2.3 \pm 0.5$ & $2.2 \pm 0.6$ & $2.26 \pm 0.37$ & $-0.18 \pm 0.27$ & $-0.58 \pm 0.34$ & $-0.33 \pm 0.21$ & $0.10 \pm 0.18$ & $0.25 \pm 0.10$ & \\
\hline 6558_13 & $4683 \pm 94$ & $5076 \pm 115$ & $4840 \pm 73$ & $2.49 \pm 0.14$ & $3.25 \pm 0.25$ & $2.67 \pm 0.12$ & $-0.22 \pm 0.09$ & $-0.25 \pm 0.25$ & $-0.22 \pm 0.08$ & $0.09 \pm 0.15$ & $0.31 \pm 0.08$ & \\
\hline 6558_14 & $4643 \pm 454$ & $4675 \pm 115$ & $4673 \pm 111$ & $3.0 \pm 1.0$ & $4.4 \pm 0.5$ & $4.12 \pm 0.44$ & $0.30 \pm 0.13$ & $-0.25 \pm 0.25$ & $0.18 \pm 0.12$ & $0.18 \pm 0.07$ & $0.12 \pm 0.07$ & \\
\hline 6558_15 & $4885 \pm 409$ & $5500 \pm 0$ & $5491 \pm 50$ & $1.9 \pm 0.8$ & $3.0 \pm 0.4$ & $2.79 \pm 0.35$ & $-1.40 \pm 0.25$ & $-1.00 \pm 0.00$ & $-1.02 \pm 0.05$ & $0.44 \pm 0.15$ & $0.20 \pm 0.13$ & \\
\hline 6558_16 & $4690 \pm 186$ & $4825 \pm 195$ & $4754 \pm 135$ & $2.50 \pm 0.35$ & $2.9 \pm 0.5$ & $2.64 \pm 0.28$ & $-0.20 \pm 0.22$ & $-0.50 \pm 0.32$ & $-0.29 \pm 0.18$ & $0.10 \pm 0.16$ & $0.23 \pm 0.13$ & \\
\hline 6558_17 & $4829 \pm 192$ & $4800 \pm 187$ & $4814 \pm 134$ & $2.7 \pm 0.6$ & $3.30 \pm 0.33$ & $3.14 \pm 0.28$ & $-0.56 \pm 0.26$ & $-1.4 \pm 0.4$ & $-0.84 \pm 0.21$ & $0.33 \pm 0.18$ & $0.36 \pm 0.05$ & \\
\hline 6558_18 & $4888 \pm 241$ & $5151 \pm 122$ & $5097 \pm 109$ & $2.6 \pm 0.4$ & $3.15 \pm 0.23$ & $3.04 \pm 0.20$ & $-0.36 \pm 0.26$ & $-0.50 \pm 0.00$ & $-0.50 \pm 0.05$ & $0.20 \pm 0.22$ & $0.26 \pm 0.11$ & \\
\hline 6558_19 & $4912 \pm 435$ & $5175 \pm 160$ & $5144 \pm 150$ & $2.1 \pm 0.9$ & $3.20 \pm 0.25$ & $3.12 \pm 0.24$ & $-1.5 \pm 0.4$ & $-1.45 \pm 0.15$ & $-1.46 \pm 0.14$ & $0.46 \pm 0.15$ & $0.22 \pm 0.12$ & \\
\hline 6426_1 & $4849 \pm 25$ & $5026 \pm 175$ & $4853 \pm 25$ & $3.45 \pm 0.04$ & $4.5 \pm 0.4$ & $3.46 \pm 0.04$ & $0.09 \pm 0.14$ & $-0.75 \pm 0.25$ & $-0.11 \pm 0.12$ & $-0.02 \pm 0.01$ & $0.34 \pm 0.07$ & \\
\hline 6426_2 & $4643 \pm 51$ & $5075 \pm 115$ & $4714 \pm 47$ & $3.05 \pm 0.21$ & $4.7 \pm 0.4$ & $3.41 \pm 0.19$ & $0.30 \pm 0.05$ & $-0.11 \pm 0.27$ & $0.28 \pm 0.05$ & $0.18 \pm 0.10$ & $0.14 \pm 0.09$ & \\
\hline 6426_3 & $4250 \pm 163$ & $4625 \pm 125$ & $4486 \pm 99$ & $3.50 \pm 0.34$ & $3.95 \pm 0.35$ & $3.72 \pm 0.24$ & $0.10 \pm 0.06$ & $-0.60 \pm 0.20$ & $0.05 \pm 0.05$ & $-0.02 \pm 0.10$ & $0.17 \pm 0.12$ & \\
\hline 6426_4 & $4881 \pm 350$ & $4900 \pm 122$ & $4898 \pm 116$ & $2.0 \pm 0.8$ & $1.9 \pm 0.4$ & $1.92 \pm 0.39$ & $-1.93 \pm 0.35$ & $-2.5 \pm 0.1$ & $-2.46 \pm 0.10$ & $0.37 \pm 0.12$ & $0.28 \pm 0.10$ & M \\
\hline 6426_7 & $5008 \pm 435$ & $5250 \pm 10$ & $5250 \pm 10$ & $2.1 \pm 0.8$ & $2.1 \pm 0.4$ & $2.11 \pm 0.34$ & $-1.9 \pm 0.4$ & $-2.5 \pm 0.1$ & $-2.47 \pm 0.10$ & $0.41 \pm 0.17$ & $0.23 \pm 0.13$ & M \\
\hline 6426_9 & $5071 \pm 374$ & $5250 \pm 10$ & $5250 \pm 10$ & $2.3 \pm 0.9$ & $2.30 \pm 0.33$ & $2.30 \pm 0.31$ & $-2.1 \pm 0.4$ & $-2.5 \pm 0.1$ & $-2.47 \pm 0.10$ & $0.38 \pm 0.19$ & $0.20 \pm 0.14$ & M \\
\hline 6426_10 & $4487 \pm 70$ & $4951 \pm 149$ & $4571 \pm 63$ & $1.15 \pm 0.12$ & $2.1 \pm 0.4$ & $1.22 \pm 0.12$ & $-2.06 \pm 0.14$ & $-2.05 \pm 0.15$ & $-2.05 \pm 0.10$ & $0.39 \pm 0.11$ & $0.21 \pm 0.14$ & M \\
\hline 6426_11 & $4967 \pm 156$ & $5475 \pm 207$ & $5150 \pm 125$ & $3.1 \pm 0.4$ & $4.65 \pm 0.32$ & $4.13 \pm 0.26$ & $-0.44 \pm 0.32$ & $-0.70 \pm 0.24$ & $-0.61 \pm 0.19$ & $0.25 \pm 0.16$ & $0.30 \pm 0.08$ & \\
\hline 6426_13 & $5015 \pm 331$ & $5000 \pm 10$ & $5000 \pm 10$ & $2.2 \pm 0.8$ & $2.0 \pm 0.4$ & $2.08 \pm 0.37$ & $-2.0 \pm 0.4$ & $-2.5 \pm 0.1$ & $-2.47 \pm 0.10$ & $0.37 \pm 0.19$ & $0.25 \pm 0.10$ & M \\
\hline 6426_18 & $4824 \pm 52$ & $4875 \pm 125$ & $4831 \pm 48$ & $3.24 \pm 0.23$ & $3.5 \pm 0.4$ & $3.31 \pm 0.20$ & $0.01 \pm 0.07$ & $-0.5 \pm 0.1$ & $-0.15 \pm 0.06$ & $0.05 \pm 0.07$ & $0.24 \pm 0.12$ & \\
\hline Ter8_1 & $4885 \pm 314$ & $5250 \pm 0$ & $5067 \pm 314$ & $2.0 \pm 0.7$ & $2.30 \pm 0.33$ & $2.24 \pm 0.30$ & $-1.82 \pm 0.34$ & $-2.00 \pm 0.00$ & $-1.97 \pm 0.14$ & $0.40 \pm 0.13$ & $0.20 \pm 0.14$ & M \\
\hline Ter8_4 & $4565 \pm 193$ & $4299 \pm 99$ & $4354 \pm 88$ & $1.3 \pm 0.5$ & $0.4 \pm 0.4$ & $0.67 \pm 0.31$ & $-1.78 \pm 0.23$ & $-2.50 \pm 0.00$ & $-2.28 \pm 0.13$ & $0.40 \pm 0.14$ & $0.20 \pm 0.14$ & M \\
\hline Ter8_5 & $4952 \pm 326$ & $5000 \pm 0$ & $4976 \pm 326$ & $2.1 \pm 0.8$ & $1.9 \pm 0.4$ & $1.98 \pm 0.37$ & $-1.9 \pm 0.4$ & $-2.50 \pm 0.00$ & $-2.42 \pm 0.14$ & $0.40 \pm 0.17$ & $0.25 \pm 0.10$ & M \\
\hline Ter8_6 & $4956 \pm 337$ & $5250 \pm 0$ & $4978 \pm 337$ & $2.1 \pm 0.8$ & $2.6 \pm 0.4$ & $2.50 \pm 0.39$ & $-1.9 \pm 0.4$ & $-2.00 \pm 0.00$ & $-1.98 \pm 0.14$ & $0.40 \pm 0.17$ & $0.15 \pm 0.13$ & M \\
\hline Ter8_8 & $4947 \pm 333$ & $5175 \pm 114$ & $5151 \pm 108$ & $2.1 \pm 0.8$ & $2.7 \pm 0.4$ & $2.56 \pm 0.36$ & $-1.9 \pm 0.4$ & $-2.15 \pm 0.23$ & $-2.06 \pm 0.19$ & $0.40 \pm 0.18$ & $0.19 \pm 0.15$ & M \\
\hline Ter8_9 & $4694 \pm 293$ & $4549 \pm 99$ & $4564 \pm 94$ & $1.6 \pm 0.5$ & $0.9 \pm 0.4$ & $1.12 \pm 0.30$ & $-1.80 \pm 0.33$ & $-2.40 \pm 0.20$ & $-2.24 \pm 0.17$ & $0.42 \pm 0.13$ & $0.19 \pm 0.14$ & M \\
\hline Ter8_10 & $4849 \pm 195$ & $4825 \pm 114$ & $4831 \pm 99$ & $3.5 \pm 0.5$ & $4.30 \pm 0.25$ & $4.13 \pm 0.22$ & $0.09 \pm 0.09$ & $-0.65 \pm 0.23$ & $-0.01 \pm 0.08$ & $-0.02 \pm 0.03$ & $0.23 \pm 0.13$ & \\
\hline Ter8_11 & $4655 \pm 247$ & $4951 \pm 100$ & $4909 \pm 92$ & $1.5 \pm 0.5$ & $2.05 \pm 0.35$ & $1.84 \pm 0.28$ & $-1.90 \pm 0.25$ & $-2.00 \pm 0.00$ & $-1.97 \pm 0.13$ & $0.45 \pm 0.12$ & $0.16 \pm 0.14$ & M \\
\hline Ter8_13 & $4967 \pm 367$ & $5250 \pm 0$ & $5108 \pm 367$ & $2.1 \pm 0.8$ & $2.4 \pm 0.4$ & $2.34 \pm 0.34$ & $-1.8 \pm 0.4$ & $-2.00 \pm 0.00$ & $-1.98 \pm 0.14$ & $0.41 \pm 0.17$ & $0.22 \pm 0.12$ & M \\
\hline
\end{tabular}


Table B.3 - continued.

\begin{tabular}{|c|c|c|c|c|c|c|c|c|c|c|c|c|}
\hline NGC ID & $\mathrm{T}_{e f f}^{(a)}(\mathrm{K})$ & $\mathrm{T}_{e f f}^{(b)}(\mathrm{K})$ & $\mathrm{T}_{e f f}^{(a v g)}(\mathrm{K})$ & $\log (g)^{(a)}$ & $\log (g)^{(b)}$ & $\log (g)^{(a v g)}$ & {$[\mathrm{Fe} / \mathrm{H}]^{(a)}$} & {$[\mathrm{Fe} / \mathrm{H}]^{(b)}$} & {$[\mathrm{Fe} / \mathrm{H}]^{(a v g)}$} & {$[\mathrm{Mg} / \mathrm{Fe}]^{(a)}$} & {$[\alpha / \mathrm{Fe}]^{(b)}$} & members \\
\hline Ter8_14 & $4756 \pm 142$ & $4324 \pm 114$ & $4493 \pm 89$ & $1.8 \pm 0.4$ & $0.4 \pm 0.4$ & $1.16 \pm 0.28$ & $-1.68 \pm 0.11$ & $-2.50 \pm 0.00$ & $-1.98 \pm 0.09$ & $0.43 \pm 0.14$ & $0.19 \pm 0.14$ & M \\
\hline Ter8_15 & $4984 \pm 344$ & $5225 \pm 75$ & $5214 \pm 73$ & $2.1 \pm 0.8$ & $2.20 \pm 0.25$ & $2.19 \pm 0.24$ & $-1.8 \pm 0.4$ & $-2.00 \pm 0.00$ & $-1.97 \pm 0.14$ & $0.39 \pm 0.17$ & $0.19 \pm 0.15$ & M \\
\hline Ter8_16 & $4871 \pm 333$ & $5050 \pm 100$ & $5035 \pm 96$ & $1.9 \pm 0.6$ & $2.20 \pm 0.33$ & $2.14 \pm 0.29$ & $-1.75 \pm 0.34$ & $-2.00 \pm 0.00$ & $-1.96 \pm 0.14$ & $0.42 \pm 0.12$ & $0.21 \pm 0.14$ & M \\
\hline Ter8_18 & $4722 \pm 195$ & $4700 \pm 245$ & $4713 \pm 153$ & $1.7 \pm 0.5$ & $1.6 \pm 0.5$ & $1.68 \pm 0.38$ & $-1.74 \pm 0.30$ & $-2.30 \pm 0.24$ & $-2.08 \pm 0.19$ & $0.37 \pm 0.13$ & $0.27 \pm 0.09$ & M \\
\hline HP1_8 & $4682 \pm 264$ & $4375 \pm 125$ & $4431 \pm 113$ & $2.3 \pm 0.6$ & $1.60 \pm 0.37$ & $1.79 \pm 0.31$ & $-0.4 \pm 0.4$ & $-1.25 \pm 0.25$ & $-1.01 \pm 0.21$ & $0.17 \pm 0.23$ & $0.28 \pm 0.12$ & M \\
\hline HP1_10 & $4467 \pm 176$ & $4575 \pm 159$ & $4526 \pm 118$ & $1.7 \pm 0.7$ & $1.95 \pm 0.35$ & $1.90 \pm 0.31$ & $-0.8 \pm 0.4$ & $-1.30 \pm 0.25$ & $-1.16 \pm 0.21$ & $0.22 \pm 0.25$ & $0.27 \pm 0.09$ & M \\
\hline HP1_11 & $4353 \pm 185$ & $4125 \pm 230$ & $4263 \pm 144$ & $1.7 \pm 0.6$ & $0.85 \pm 0.59$ & $1.27 \pm 0.42$ & $-0.67 \pm 0.31$ & $-1.65 \pm 0.32$ & $-1.14 \pm 0.22$ & $0.29 \pm 0.23$ & $0.28 \pm 0.13$ & M \\
\hline HP1_12 & $4823 \pm 340$ & $4448 \pm 149$ & $4508 \pm 136$ & $2.4 \pm 0.8$ & $1.75 \pm 0.33$ & $1.84 \pm 0.31$ & $-0.6 \pm 0.4$ & $-1.65 \pm 0.23$ & $-1.39 \pm 0.20$ & $0.28 \pm 0.21$ & $0.28 \pm 0.10$ & M \\
\hline HP1_13 & $4232 \pm 49$ & $4026 \pm 174$ & $4217 \pm 47$ & $1.37 \pm 0.33$ & $0.60 \pm 0.62$ & $1.20 \pm 0.29$ & $-0.79 \pm 0.21$ & $-1.45 \pm 0.35$ & $-0.96 \pm 0.18$ & $0.48 \pm 0.15$ & $0.30 \pm 0.10$ & M \\
\hline HP1_14 & $4517 \pm 348$ & $4175 \pm 114$ & $4208 \pm 108$ & $1.6 \pm 0.9$ & $0.40 \pm 0.44$ & $0.63 \pm 0.40$ & $-1.0 \pm 0.5$ & $-2.00 \pm 0.1$ & $-1.96 \pm 0.10$ & $0.34 \pm 0.18$ & $0.27 \pm 0.09$ & \\
\hline HP1_15 & $4868 \pm 451$ & $4600 \pm 200$ & $4644 \pm 183$ & $2.0 \pm 0.9$ & $1.40 \pm 0.73$ & $1.64 \pm 0.57$ & $-1.3 \pm 0.5$ & $-1.90 \pm 0.20$ & $-1.82 \pm 0.19$ & $0.41 \pm 0.16$ & $0.11 \pm 0.09$ & \\
\hline HP1_19 & $4823 \pm 367$ & $4927 \pm 297$ & $4886 \pm 231$ & $2.0 \pm 0.9$ & $2.55 \pm 0.61$ & $2.38 \pm 0.50$ & $-1.2 \pm 0.5$ & $-1.50 \pm 0.39$ & $-1.39 \pm 0.31$ & $0.40 \pm 0.15$ & $0.31 \pm 0.10$ & \\
\hline HP1_20 & $4681 \pm 267$ & $4325 \pm 115$ & $4381 \pm 106$ & $2.4 \pm 0.6$ & $1.85 \pm 0.23$ & $1.92 \pm 0.21$ & $-0.4 \pm 0.4$ & $-1.35 \pm 0.23$ & $-1.11 \pm 0.20$ & $0.19 \pm 0.21$ & $0.21 \pm 0.11$ & \\
\hline HP1_21 & $4607 \pm 241$ & $4800 \pm 187$ & $4727 \pm 148$ & $2.4 \pm 0.5$ & $2.70 \pm 0.40$ & $2.58 \pm 0.31$ & $-0.14 \pm 0.27$ & $-0.26 \pm 0.30$ & $-0.19 \pm 0.20$ & $0.08 \pm 0.19$ & $0.33 \pm 0.06$ & \\
\hline HP1_22 & $4758 \pm 323$ & $4498 \pm 192$ & $4566 \pm 165$ & $2.2 \pm 0.8$ & $1.85 \pm 0.45$ & $1.93 \pm 0.39$ & $-0.8 \pm 0.4$ & $-1.55 \pm 0.27$ & $-1.32 \pm 0.22$ & $0.35 \pm 0.17$ & $0.28 \pm 0.10$ & M \\
\hline HP1_23 & $4420 \pm 200$ & $4377 \pm 125$ & $4389 \pm 106$ & $1.9 \pm 0.6$ & $1.55 \pm 0.35$ & $1.64 \pm 0.30$ & $-0.62 \pm 0.33$ & $-1.25 \pm 0.25$ & $-1.02 \pm 0.20$ & $0.27 \pm 0.22$ & $0.29 \pm 0.09$ & M \\
\hline HP1_24 & $4561 \pm 301$ & $4099 \pm 122$ & $4164 \pm 113$ & $1.9 \pm 0.7$ & $0.79 \pm 0.60$ & $1.26 \pm 0.46$ & $-0.71 \pm 0.31$ & $-1.80 \pm 0.24$ & $-1.39 \pm 0.19$ & $0.36 \pm 0.18$ & $0.25 \pm 0.10$ & M \\
\hline HP1_31 & $4379 \pm 186$ & $4750 \pm 100$ & $4667 \pm 88$ & $2.0 \pm 0.4$ & $2.40 \pm 0.20$ & $2.32 \pm 0.18$ & $0.04 \pm 0.19$ & $0.08 \pm 0.10$ & $0.07 \pm 0.09$ & $0.02 \pm 0.16$ & $0.24 \pm 0.14$ & \\
\hline HP1_32 & $4836 \pm 388$ & $4850 \pm 166$ & $4848 \pm 153$ & $2.1 \pm 0.9$ & $3.00 \pm 0.45$ & $2.82 \pm 0.40$ & $-1.2 \pm 0.5$ & $-1.75 \pm 0.25$ & $-1.64 \pm 0.22$ & $0.41 \pm 0.16$ & $0.31 \pm 0.08$ & \\
\hline HP1_33 & $4799 \pm 262$ & $5199 \pm 244$ & $5013 \pm 179$ & $2.5 \pm 0.5$ & $3.25 \pm 0.33$ & $3.02 \pm 0.28$ & $-0.37 \pm 0.33$ & $-0.40 \pm 0.30$ & $-0.39 \pm 0.22$ & $0.19 \pm 0.22$ & $0.29 \pm 0.10$ & \\
\hline HP1_34 & $4373 \pm 92$ & $4224 \pm 231$ & $4353 \pm 85$ & $1.9 \pm 0.4$ & $1.16 \pm 0.70$ & $1.72 \pm 0.35$ & $-0.53 \pm 0.24$ & $-1.10 \pm 0.37$ & $-0.70 \pm 0.20$ & $0.28 \pm 0.20$ & $0.28 \pm 0.14$ & \\
\hline HP1_35 & $4716 \pm 245$ & $4675 \pm 114$ & $4682 \pm 103$ & $2.4 \pm 0.5$ & $2.15 \pm 0.32$ & $2.22 \pm 0.27$ & $-0.27 \pm 0.33$ & $-0.65 \pm 0.23$ & $-0.53 \pm 0.19$ & $0.15 \pm 0.22$ & $0.26 \pm 0.13$ & \\
\hline HP1_36 & $4397 \pm 153$ & $4675 \pm 114$ & $4576 \pm 91$ & $2.1 \pm 0.4$ & $2.35 \pm 0.23$ & $2.29 \pm 0.20$ & $0.08 \pm 0.14$ & $0.08 \pm 0.10$ & $0.08 \pm 0.08$ & $0.01 \pm 0.14$ & $0.18 \pm 0.12$ & \\
\hline HP1_37 & $4844 \pm 327$ & $4875 \pm 125$ & $4871 \pm 117$ & $2.5 \pm 0.6$ & $2.65 \pm 0.39$ & $2.61 \pm 0.33$ & $-0.4 \pm 0.4$ & $-0.75 \pm 0.25$ & $-0.65 \pm 0.21$ & $0.20 \pm 0.23$ & $0.24 \pm 0.14$ & \\
\hline HP1_38 & $4875 \pm 308$ & $5251 \pm 224$ & $5121 \pm 181$ & $2.5 \pm 0.6$ & $3.05 \pm 0.15$ & $3.02 \pm 0.15$ & $-0.37 \pm 0.33$ & $-0.30 \pm 0.25$ & $-0.33 \pm 0.20$ & $0.18 \pm 0.22$ & $0.26 \pm 0.11$ & \\
\hline HP1_40 & $4490 \pm 239$ & $4499 \pm 250$ & $4494 \pm 173$ & $2.2 \pm 0.5$ & $2.10 \pm 0.62$ & $2.16 \pm 0.39$ & $-0.08 \pm 0.26$ & $-0.53 \pm 0.44$ & $-0.20 \pm 0.22$ & $0.06 \pm 0.17$ & $0.23 \pm 0.11$ & \\
\hline HP1_41 & $4356 \pm 211$ & $4649 \pm 123$ & $4575 \pm 106$ & $2.1 \pm 0.5$ & $2.85 \pm 0.32$ & $2.63 \pm 0.27$ & $0.05 \pm 0.16$ & $-0.12 \pm 0.32$ & $0.02 \pm 0.14$ & $0.02 \pm 0.12$ & $0.17 \pm 0.13$ & \\
\hline HP1_42 & $4418 \pm 125$ & $4723 \pm 175$ & $4521 \pm 102$ & $2.2 \pm 0.5$ & $3.10 \pm 0.44$ & $2.71 \pm 0.33$ & $0.15 \pm 0.13$ & $0.20 \pm 0.17$ & $0.17 \pm 0.10$ & $0.03 \pm 0.15$ & $0.12 \pm 0.07$ & \\
\hline
\end{tabular}


Table B.3 - continued.

\begin{tabular}{|c|c|c|c|c|c|c|c|c|c|c|c|c|}
\hline NGC ID & $\mathrm{T}_{e f f}^{(a)}(\mathrm{K})$ & $\mathrm{T}_{e f f}^{(b)}(\mathrm{K})$ & $\mathrm{T}_{e f f}^{(a v g)}(\mathrm{K})$ & $\log (g)^{(a)}$ & $\log (g)^{(b)}$ & $\log (g)^{(a v g)}$ & {$[\mathrm{Fe} / \mathrm{H}]^{(a)}$} & {$[\mathrm{Fe} / \mathrm{H}]^{(b)}$} & {$[\mathrm{Fe} / \mathrm{H}]^{(a v g)}$} & {$[\mathrm{Mg} / \mathrm{Fe}]^{(a)}$} & {$[\alpha / \mathrm{Fe}]^{(b)}$} & members \\
\hline HP1_43 & $4402 \pm 157$ & $4524 \pm 207$ & $4447 \pm 125$ & $2.2 \pm 0.5$ & $2.40 \pm 0.62$ & $2.28 \pm 0.39$ & $0.04 \pm 0.13$ & $-0.28 \pm 0.27$ & $-0.02 \pm 0.12$ & $0.04 \pm 0.14$ & $0.27 \pm 0.10$ & \\
\hline HP1_44 & $4449 \pm 224$ & $4775 \pm 175$ & $4651 \pm 138$ & $2.2 \pm 0.5$ & $3.65 \pm 0.39$ & $3.10 \pm 0.31$ & $-0.09 \pm 0.26$ & $-0.20 \pm 0.24$ & $-0.15 \pm 0.18$ & $0.06 \pm 0.15$ & $0.12 \pm 0.10$ & \\
\hline HP1_45 & $4386 \pm 140$ & $4479 \pm 209$ & $4415 \pm 116$ & $2.2 \pm 0.4$ & $2.36 \pm 0.55$ & $2.26 \pm 0.32$ & $0.13 \pm 0.13$ & $-0.13 \pm 0.30$ & $0.09 \pm 0.12$ & $0.02 \pm 0.15$ & $0.12 \pm 0.10$ & \\
\hline Djorg2_1 & $3946 \pm 59$ & $4250 \pm 193$ & $3972 \pm 56$ & $1.51 \pm 0.19$ & $2.4 \pm 0.6$ & $1.59 \pm 0.18$ & $0.02 \pm 0.18$ & $-0.35 \pm 0.23$ & $-0.12 \pm 0.14$ & $-0.01 \pm 0.23$ & $0.18 \pm 0.10$ & \\
\hline Djorg2_3 & $4498 \pm 110$ & $4750 \pm 224$ & $4547 \pm 99$ & $2.2 \pm 0.4$ & $2.6 \pm 0.5$ & $2.35 \pm 0.30$ & $0.03 \pm 0.15$ & $-0.12 \pm 0.32$ & $0.01 \pm 0.14$ & $0.03 \pm 0.17$ & $0.21 \pm 0.13$ & \\
\hline Djorg2_4 & $4116 \pm 165$ & $4149 \pm 123$ & $4137 \pm 98$ & $1.6 \pm 0.4$ & $1.9 \pm 0.4$ & $1.78 \pm 0.26$ & $-0.09 \pm 0.18$ & $-0.70 \pm 0.25$ & $-0.30 \pm 0.15$ & $0.03 \pm 0.18$ & $0.22 \pm 0.12$ & \\
\hline Djorg2_5 & $4384 \pm 137$ & $4400 \pm 165$ & $4390 \pm 105$ & $2.1 \pm 0.4$ & $2.1 \pm 0.4$ & $2.11 \pm 0.28$ & $-0.11 \pm 0.20$ & $-0.70 \pm 0.33$ & $-0.27 \pm 0.17$ & $0.09 \pm 0.17$ & $0.33 \pm 0.08$ & \\
\hline Djorg2_6 & $4556 \pm 195$ & $4524 \pm 236$ & $4543 \pm 150$ & $2.3 \pm 0.5$ & $2.0 \pm 0.5$ & $2.19 \pm 0.33$ & $-0.16 \pm 0.27$ & $-0.60 \pm 0.30$ & $-0.35 \pm 0.20$ & $0.08 \pm 0.18$ & $0.28 \pm 0.11$ & \\
\hline Djorg2_7 & $4577 \pm 199$ & $4475 \pm 134$ & $4507 \pm 111$ & $2.4 \pm 0.5$ & $1.8 \pm 0.4$ & $2.04 \pm 0.32$ & $-0.15 \pm 0.27$ & $-1.55 \pm 0.27$ & $-0.85 \pm 0.19$ & $0.10 \pm 0.20$ & $0.27 \pm 0.09$ & M \\
\hline Djorg2_8 & $4559 \pm 195$ & $4600 \pm 122$ & $4588 \pm 104$ & $2.3 \pm 0.6$ & $1.75 \pm 0.25$ & $1.83 \pm 0.23$ & $-0.3 \pm 0.4$ & $-1.30 \pm 0.24$ & $-1.00 \pm 0.20$ & $0.17 \pm 0.23$ & $0.29 \pm 0.09$ & \\
\hline Djorg2_9 & $4515 \pm 170$ & $4376 \pm 166$ & $4444 \pm 119$ & $2.1 \pm 0.5$ & $1.6 \pm 0.4$ & $1.85 \pm 0.31$ & $-0.53 \pm 0.19$ & $-1.25 \pm 0.33$ & $-0.71 \pm 0.16$ & $0.26 \pm 0.21$ & $0.27 \pm 0.11$ & M \\
\hline Djorg2_10 & $4250 \pm 33$ & $4127 \pm 200$ & $4247 \pm 33$ & $1.4 \pm 0.4$ & $1.0 \pm 0.7$ & $1.28 \pm 0.32$ & $-0.77 \pm 0.23$ & $-1.29 \pm 0.33$ & $-0.94 \pm 0.19$ & $0.46 \pm 0.17$ & $0.27 \pm 0.13$ & M \\
\hline Djorg2_11 & $4190 \pm 153$ & $4351 \pm 123$ & $4288 \pm 96$ & $1.7 \pm 0.4$ & $1.95 \pm 0.35$ & $1.85 \pm 0.26$ & $-0.22 \pm 0.25$ & $-0.70 \pm 0.24$ & $-0.47 \pm 0.17$ & $0.09 \pm 0.19$ & $0.32 \pm 0.07$ & \\
\hline Djorg2_13 & $4549 \pm 191$ & $4625 \pm 167$ & $4592 \pm 126$ & $2.3 \pm 0.5$ & $2.4 \pm 0.6$ & $2.32 \pm 0.37$ & $-0.29 \pm 0.28$ & $-0.70 \pm 0.24$ & $-0.53 \pm 0.18$ & $0.15 \pm 0.20$ & $0.29 \pm 0.09$ & \\
\hline Djorg2_14 & $4256 \pm 218$ & $4350 \pm 122$ & $4328 \pm 107$ & $2.0 \pm 0.5$ & $2.30 \pm 0.25$ & $2.23 \pm 0.22$ & $0.17 \pm 0.17$ & $-0.30 \pm 0.25$ & $0.02 \pm 0.14$ & $0.00 \pm 0.13$ & $0.15 \pm 0.14$ & \\
\hline Djorg2_15 & $4745 \pm 240$ & $4700 \pm 218$ & $4721 \pm 161$ & $2.4 \pm 0.5$ & $2.2 \pm 0.6$ & $2.35 \pm 0.40$ & $-0.42 \pm 0.29$ & $-0.85 \pm 0.23$ & $-0.68 \pm 0.18$ & $0.21 \pm 0.20$ & $0.28 \pm 0.07$ & M \\
\hline Djorg2_16 & $4230 \pm 180$ & $4251 \pm 158$ & $4242 \pm 119$ & $1.8 \pm 0.4$ & $1.9 \pm 0.5$ & $1.89 \pm 0.33$ & $-0.15 \pm 0.21$ & $-1.00 \pm 0.32$ & $-0.41 \pm 0.18$ & $0.08 \pm 0.16$ & $0.34 \pm 0.07$ & \\
\hline Djorg2_17 & $4763 \pm 371$ & $4624 \pm 125$ & $4638 \pm 118$ & $2.2 \pm 0.9$ & $1.8 \pm 0.4$ & $1.90 \pm 0.36$ & $-0.9 \pm 0.5$ & $-1.50 \pm 0.10$ & $-1.48 \pm 0.10$ & $0.34 \pm 0.18$ & $0.26 \pm 0.14$ & \\
\hline M22a_1 & $4551 \pm 56$ & $4926 \pm 114$ & $4624 \pm 50$ & $2.39 \pm 0.19$ & $2.90 \pm 0.20$ & $2.63 \pm 0.14$ & $-0.02 \pm 0.09$ & $-0.07 \pm 0.29$ & $-0.02 \pm 0.09$ & $0.05 \pm 0.09$ & $0.27 \pm 0.11$ & \\
\hline M22a_2 & $4395 \pm 124$ & $4725 \pm 175$ & $4505 \pm 101$ & $1.0 \pm 0.4$ & $2.10 \pm 0.44$ & $1.50 \pm 0.30$ & $-1.64 \pm 0.25$ & $-1.90 \pm 0.20$ & $-1.80 \pm 0.16$ & $0.47 \pm 0.08$ & $0.20 \pm 0.08$ & \\
\hline M22a_3 & $4491 \pm 136$ & $4798 \pm 147$ & $4633 \pm 100$ & $1.18 \pm 0.18$ & $1.85 \pm 0.32$ & $1.34 \pm 0.16$ & $-1.87 \pm 0.20$ & $-1.95 \pm 0.15$ & $-1.92 \pm 0.12$ & $0.45 \pm 0.11$ & $0.18 \pm 0.12$ & M \\
\hline M22a_4 & $4430 \pm 32$ & $4849 \pm 122$ & $4457 \pm 31$ & $1.03 \pm 0.01$ & $1.85 \pm 0.32$ & $1.03 \pm 0.01$ & $-2.10 \pm 0.1$ & $-2.00 \pm 0.1$ & $-2.05 \pm 0.07$ & $0.51 \pm 0.01$ & $0.21 \pm 0.14$ & M \\
\hline M22a_5 & $4892 \pm 339$ & $5200 \pm 100$ & $5175 \pm 96$ & $1.9 \pm 0.6$ & $2.70 \pm 0.25$ & $2.58 \pm 0.23$ & $-1.58 \pm 0.32$ & $-1.60 \pm 0.20$ & $-1.59 \pm 0.17$ & $0.41 \pm 0.14$ & $0.26 \pm 0.13$ & M \\
\hline M22a_6 & $4739 \pm 414$ & $5100 \pm 122$ & $5071 \pm 117$ & $1.4 \pm 0.4$ & $2.40 \pm 0.30$ & $2.04 \pm 0.24$ & $-1.78 \pm 0.34$ & $-2.00 \pm 0.1$ & $-1.98 \pm 0.10$ & $0.48 \pm 0.12$ & $0.15 \pm 0.12$ & M \\
\hline M22a_7 & $4668 \pm 283$ & $4824 \pm 159$ & $4787 \pm 139$ & $1.5 \pm 0.5$ & $1.95 \pm 0.35$ & $1.80 \pm 0.29$ & $-1.71 \pm 0.27$ & $-1.95 \pm 0.15$ & $-1.89 \pm 0.13$ & $0.46 \pm 0.10$ & $0.23 \pm 0.13$ & \\
\hline M22a_8 & $4659 \pm 299$ & $4950 \pm 100$ & $4921 \pm 95$ & $1.5 \pm 0.5$ & $2.25 \pm 0.33$ & $2.02 \pm 0.28$ & $-1.57 \pm 0.31$ & $-1.60 \pm 0.20$ & $-1.59 \pm 0.17$ & $0.45 \pm 0.14$ & $0.22 \pm 0.13$ & M \\
\hline M22a_9 & $4967 \pm 335$ & $5150 \pm 166$ & $5114 \pm 149$ & $2.1 \pm 0.7$ & $2.75 \pm 0.51$ & $2.52 \pm 0.41$ & $-1.62 \pm 0.31$ & $-1.95 \pm 0.15$ & $-1.89 \pm 0.14$ & $0.42 \pm 0.13$ & $0.23 \pm 0.09$ & M \\
\hline M22a_10 & $4773 \pm 277$ & $4848 \pm 198$ & $4823 \pm 161$ & $1.7 \pm 0.5$ & $1.95 \pm 0.35$ & $1.87 \pm 0.29$ & $-1.59 \pm 0.34$ & $-1.90 \pm 0.20$ & $-1.82 \pm 0.17$ & $0.43 \pm 0.15$ & $0.21 \pm 0.11$ & M \\
\hline
\end{tabular}


Table B.3 - continued.

\begin{tabular}{|c|c|c|c|c|c|c|c|c|c|c|c|c|}
\hline NGC ID & $\mathrm{T}_{e f f}^{(a)}(\mathrm{K})$ & $\mathrm{T}_{e f f}^{(b)}(\mathrm{K})$ & $\mathrm{T}_{\text {eff }}^{(a v g)}(\mathrm{K})$ & $\log (g)^{(a)}$ & $\log (g)^{(b)}$ & $\log (g)^{(a v g)}$ & {$[\mathrm{Fe} / \mathrm{H}]^{(a)}$} & {$[\mathrm{Fe} / \mathrm{H}]^{(b)}$} & {$[\mathrm{Fe} / \mathrm{H}]^{(a v g)}$} & {$[\mathrm{Mg} / \mathrm{Fe}]^{(a)}$} & {$[\alpha / \mathrm{Fe}]^{(b)}$} & members \\
\hline M22a_11 & $5023 \pm 383$ & $5174 \pm 160$ & $5152 \pm 148$ & $2.3 \pm 0.8$ & $3.15 \pm 0.32$ & $3.03 \pm 0.30$ & $-1.6 \pm 0.4$ & $-1.95 \pm 0.15$ & $-1.91 \pm 0.14$ & $0.40 \pm 0.18$ & $0.27 \pm 0.13$ & M \\
\hline M22a_13 & $5126 \pm 427$ & $5550 \pm 100$ & $5528 \pm 97$ & $2.3 \pm 0.8$ & $4.00 \pm 0.32$ & $3.77 \pm 0.30$ & $-1.7 \pm 0.5$ & $-1.90 \pm 0.20$ & $-1.87 \pm 0.19$ & $0.42 \pm 0.17$ & $0.17 \pm 0.16$ & M \\
\hline M22a_14 & $5091 \pm 269$ & $5325 \pm 115$ & $5289 \pm 106$ & $2.4 \pm 0.8$ & $2.65 \pm 0.45$ & $2.59 \pm 0.39$ & $-2.0 \pm 0.4$ & $-2.35 \pm 0.23$ & $-2.26 \pm 0.20$ & $0.37 \pm 0.20$ & $0.22 \pm 0.12$ & M \\
\hline M22a_15 & $4939 \pm 325$ & $5200 \pm 187$ & $5135 \pm 162$ & $2.1 \pm 0.7$ & $2.80 \pm 0.46$ & $2.59 \pm 0.38$ & $-1.64 \pm 0.29$ & $-1.90 \pm 0.20$ & $-1.82 \pm 0.16$ & $0.42 \pm 0.12$ & $0.31 \pm 0.09$ & \\
\hline M22a_16 & $4955 \pm 324$ & $5175 \pm 195$ & $5117 \pm 167$ & $2.1 \pm 0.7$ & $2.60 \pm 0.54$ & $2.41 \pm 0.43$ & $-1.61 \pm 0.31$ & $-1.85 \pm 0.23$ & $-1.76 \pm 0.18$ & $0.42 \pm 0.13$ & $0.18 \pm 0.12$ & M \\
\hline M22a_17 & $4934 \pm 341$ & $5250 \pm 100$ & $5225 \pm 96$ & $2.1 \pm 0.8$ & $2.95 \pm 0.42$ & $2.77 \pm 0.37$ & $-1.75 \pm 0.33$ & $-2.00 \pm 0.1$ & $-1.98 \pm 0.10$ & $0.41 \pm 0.13$ & $0.20 \pm 0.13$ & \\
\hline M22a_18 & $4981 \pm 336$ & $5250 \pm 100$ & $5228 \pm 96$ & $2.2 \pm 0.8$ & $2.75 \pm 0.51$ & $2.59 \pm 0.43$ & $-1.73 \pm 0.34$ & $-2.00 \pm 0.1$ & $-1.98 \pm 0.10$ & $0.41 \pm 0.13$ & $0.25 \pm 0.10$ & M \\
\hline M22a_19 & $4958 \pm 339$ & $5250 \pm 100$ & $5227 \pm 96$ & $2.1 \pm 0.8$ & $2.60 \pm 0.37$ & $2.51 \pm 0.34$ & $-1.8 \pm 0.4$ & $-2.00 \pm 0.1$ & $-1.99 \pm 0.10$ & $0.40 \pm 0.16$ & $0.24 \pm 0.14$ & M \\
\hline M22b_2 & $4927 \pm 322$ & $5075 \pm 114$ & $5059 \pm 107$ & $2.0 \pm 0.6$ & $2.20 \pm 0.46$ & $2.13 \pm 0.37$ & $-1.60 \pm 0.32$ & $-1.95 \pm 0.15$ & $-1.89 \pm 0.14$ & $0.40 \pm 0.13$ & $0.24 \pm 0.14$ & M \\
\hline M22b_3 & $4516 \pm 263$ & $4975 \pm 75$ & $4940 \pm 72$ & $1.2 \pm 0.5$ & $2.50 \pm 0.39$ & $2.01 \pm 0.31$ & $-1.56 \pm 0.26$ & $-1.55 \pm 0.15$ & $-1.55 \pm 0.13$ & $0.48 \pm 0.11$ & $0.26 \pm 0.13$ & M \\
\hline M22b_4 & $4767 \pm 396$ & $5201 \pm 99$ & $5175 \pm 96$ & $1.5 \pm 0.5$ & $2.20 \pm 0.25$ & $2.06 \pm 0.22$ & $-1.7 \pm 0.4$ & $-2.00 \pm 0.1$ & $-1.98 \pm 0.10$ & $0.41 \pm 0.16$ & $0.20 \pm 0.13$ & M \\
\hline M22b_5 & $4926 \pm 332$ & $5075 \pm 115$ & $5059 \pm 109$ & $2.0 \pm 0.6$ & $2.25 \pm 0.25$ & $2.21 \pm 0.23$ & $-1.65 \pm 0.30$ & $-2.00 \pm 0.1$ & $-1.97 \pm 0.09$ & $0.40 \pm 0.13$ & $0.25 \pm 0.10$ & M \\
\hline M22b_6 & $4958 \pm 341$ & $5100 \pm 229$ & $5056 \pm 190$ & $2.1 \pm 0.8$ & $2.05 \pm 0.42$ & $2.06 \pm 0.37$ & $-1.8 \pm 0.4$ & $-2.15 \pm 0.23$ & $-2.06 \pm 0.20$ & $0.38 \pm 0.19$ & $0.23 \pm 0.13$ & M \\
\hline M22b_7 & $4790 \pm 350$ & $5375 \pm 125$ & $5309 \pm 118$ & $1.7 \pm 0.4$ & $2.80 \pm 0.33$ & $2.35 \pm 0.25$ & $-1.5 \pm 0.4$ & $-1.25 \pm 0.25$ & $-1.32 \pm 0.21$ & $0.46 \pm 0.13$ & $0.26 \pm 0.13$ & M \\
\hline M22b_8 & $4710 \pm 287$ & $4925 \pm 114$ & $4896 \pm 106$ & $1.5 \pm 0.5$ & $2.00 \pm 0.45$ & $1.78 \pm 0.33$ & $-1.7 \pm 0.4$ & $-2.00 \pm 0.1$ & $-1.98 \pm 0.10$ & $0.40 \pm 0.13$ & $0.25 \pm 0.10$ & M \\
\hline M22b_9 & $4921 \pm 217$ & $5275 \pm 208$ & $5105 \pm 150$ & $3.35 \pm 0.19$ & $3.85 \pm 0.23$ & $3.55 \pm 0.15$ & $-0.00 \pm 0.10$ & $-0.30 \pm 0.24$ & $-0.05 \pm 0.09$ & $0.09 \pm 0.11$ & $0.17 \pm 0.11$ & \\
\hline M22b_10 & $4694 \pm 416$ & $4721 \pm 208$ & $4716 \pm 186$ & $1.4 \pm 0.6$ & $1.89 \pm 0.37$ & $1.76 \pm 0.31$ & $-1.6 \pm 0.4$ & $-1.85 \pm 0.23$ & $-1.79 \pm 0.20$ & $0.46 \pm 0.14$ & $0.23 \pm 0.13$ & M \\
\hline M22b_11 & $4915 \pm 560$ & $4724 \pm 208$ & $4747 \pm 195$ & $1.6 \pm 0.6$ & $1.75 \pm 0.25$ & $1.73 \pm 0.23$ & $-1.7 \pm 0.4$ & $-2.35 \pm 0.23$ & $-2.19 \pm 0.20$ & $0.55 \pm 0.03$ & $0.27 \pm 0.13$ & M \\
\hline M22b_12 & $5089 \pm 432$ & $5250 \pm 100$ & $5242 \pm 97$ & $2.3 \pm 0.8$ & $2.40 \pm 0.37$ & $2.38 \pm 0.34$ & $-1.7 \pm 0.4$ & $-2.00 \pm 0.1$ & $-1.98 \pm 0.10$ & $0.41 \pm 0.18$ & $0.22 \pm 0.12$ & M \\
\hline M22b_13 & $5024 \pm 463$ & $5200 \pm 100$ & $5192 \pm 98$ & $2.2 \pm 0.9$ & $3.10 \pm 0.37$ & $2.97 \pm 0.34$ & $-1.7 \pm 0.5$ & $-2.00 \pm 0.1$ & $-1.99 \pm 0.10$ & $0.43 \pm 0.17$ & $0.25 \pm 0.10$ & M \\
\hline M22b_15 & $4785 \pm 347$ & $5125 \pm 168$ & $5060 \pm 151$ & $1.7 \pm 0.5$ & $2.90 \pm 0.44$ & $2.38 \pm 0.33$ & $-1.7 \pm 0.4$ & $-2.05 \pm 0.15$ & $-2.01 \pm 0.14$ & $0.43 \pm 0.15$ & $0.24 \pm 0.12$ & M \\
\hline M22b_16 & $4768 \pm 271$ & $4799 \pm 148$ & $4792 \pm 130$ & $1.7 \pm 0.5$ & $1.85 \pm 0.32$ & $1.81 \pm 0.27$ & $-1.6 \pm 0.4$ & $-1.95 \pm 0.15$ & $-1.91 \pm 0.14$ & $0.38 \pm 0.13$ & $0.19 \pm 0.14$ & M \\
\hline M22b_17 & $4791 \pm 353$ & $5250 \pm 194$ & $5144 \pm 170$ & $1.7 \pm 0.5$ & $2.70 \pm 0.51$ & $2.19 \pm 0.36$ & $-1.7 \pm 0.4$ & $-1.70 \pm 0.24$ & $-1.70 \pm 0.21$ & $0.41 \pm 0.17$ & $0.23 \pm 0.13$ & M \\
\hline M22b_18 & $4799 \pm 409$ & $5250 \pm 100$ & $5225 \pm 97$ & $1.6 \pm 0.6$ & $2.90 \pm 0.37$ & $2.54 \pm 0.31$ & $-1.86 \pm 0.34$ & $-2.00 \pm 0.1$ & $-1.99 \pm 0.10$ & $0.48 \pm 0.11$ & $0.16 \pm 0.14$ & M \\
\hline M22b_19 & $4804 \pm 267$ & $5250 \pm 100$ & $5195 \pm 94$ & $1.7 \pm 0.5$ & $2.90 \pm 0.37$ & $2.48 \pm 0.30$ & $-1.62 \pm 0.33$ & $-1.50 \pm 0.1$ & $-1.51 \pm 0.10$ & $0.44 \pm 0.13$ & $0.19 \pm 0.14$ & M \\
\hline M22c_1 & $5089 \pm 407$ & $5500 \pm 100$ & $5477 \pm 97$ & $2.3 \pm 0.9$ & $3.00 \pm 0.39$ & $2.89 \pm 0.36$ & $-1.9 \pm 0.4$ & $-2.00 \pm 0.1$ & $-1.99 \pm 0.10$ & $0.41 \pm 0.17$ & $0.14 \pm 0.13$ & M \\
\hline M22c_2 & $4919 \pm 297$ & $5075 \pm 114$ & $5055 \pm 106$ & $2.1 \pm 0.7$ & $2.50 \pm 0.39$ & $2.41 \pm 0.34$ & $-1.67 \pm 0.25$ & $-1.90 \pm 0.20$ & $-1.81 \pm 0.16$ & $0.41 \pm 0.13$ & $0.20 \pm 0.12$ & \\
\hline M22c_3 & $5027 \pm 313$ & $5300 \pm 100$ & $5275 \pm 95$ & $2.3 \pm 0.7$ & $2.65 \pm 0.39$ & $2.57 \pm 0.34$ & $-1.67 \pm 0.29$ & $-1.90 \pm 0.20$ & $-1.83 \pm 0.16$ & $0.42 \pm 0.13$ & $0.25 \pm 0.10$ & M \\
\hline
\end{tabular}


Table B.3 - continued.

\begin{tabular}{|c|c|c|c|c|c|c|c|c|c|c|c|c|}
\hline NGC ID & $\mathrm{T}_{e f f}^{(a)}(\mathrm{K})$ & $\mathrm{T}_{e f f}^{(b)}(\mathrm{K})$ & $\mathrm{T}_{e f f}^{(a v g)}(\mathrm{K})$ & $\log (g)^{(a)}$ & $\log (g)^{(b)}$ & $\log (g)^{(a v g)}$ & {$[\mathrm{Fe} / \mathrm{H}]^{(a)}$} & {$[\mathrm{Fe} / \mathrm{H}]^{(b)}$} & {$[\mathrm{Fe} / \mathrm{H}]^{(a v g)}$} & {$[\mathrm{Mg} / \mathrm{Fe}]^{(a)}$} & {$[\alpha / \mathrm{Fe}]^{(b)}$} & members \\
\hline M22c_4 & $4943 \pm 325$ & $5175 \pm 195$ & $5114 \pm 167$ & $2.1 \pm 0.7$ & $2.35 \pm 0.59$ & $2.25 \pm 0.45$ & $-1.64 \pm 0.29$ & $-1.80 \pm 0.24$ & $-1.73 \pm 0.18$ & $0.41 \pm 0.12$ & $0.27 \pm 0.13$ & M \\
\hline M22c_5 & $4987 \pm 312$ & $5250 \pm 100$ & $5225 \pm 95$ & $2.2 \pm 0.7$ & $2.70 \pm 0.33$ & $2.61 \pm 0.30$ & $-1.70 \pm 0.25$ & $-2.00 \pm 0.1$ & $-1.96 \pm 0.09$ & $0.43 \pm 0.12$ & $0.20 \pm 0.14$ & \\
\hline M22c_6 & $5197 \pm 243$ & $5425 \pm 115$ & $5383 \pm 104$ & $2.7 \pm 0.7$ & $3.30 \pm 0.46$ & $3.12 \pm 0.38$ & $-1.7 \pm 0.4$ & $-2.00 \pm 0.1$ & $-1.98 \pm 0.10$ & $0.37 \pm 0.22$ & $0.27 \pm 0.12$ & M \\
\hline M22c_7 & $4937 \pm 317$ & $5150 \pm 122$ & $5123 \pm 114$ & $2.0 \pm 0.5$ & $2.65 \pm 0.32$ & $2.46 \pm 0.27$ & $-1.55 \pm 0.31$ & $-1.70 \pm 0.24$ & $-1.64 \pm 0.19$ & $0.41 \pm 0.14$ & $0.25 \pm 0.13$ & $\mathrm{M}$ \\
\hline M22c_8 & $4943 \pm 330$ & $5100 \pm 122$ & $5081 \pm 114$ & $2.1 \pm 0.7$ & $2.35 \pm 0.23$ & $2.33 \pm 0.22$ & $-1.7 \pm 0.4$ & $-2.00 \pm 0.1$ & $-1.98 \pm 0.10$ & $0.42 \pm 0.13$ & $0.18 \pm 0.13$ & $\mathrm{M}$ \\
\hline M22c_9 & $4923 \pm 324$ & $5000 \pm 100$ & $4993 \pm 96$ & $2.0 \pm 0.6$ & $2.30 \pm 0.33$ & $2.23 \pm 0.29$ & $-1.60 \pm 0.32$ & $-2.00 \pm 0.1$ & $-1.96 \pm 0.10$ & $0.40 \pm 0.13$ & $0.19 \pm 0.14$ & $\mathrm{M}$ \\
\hline M22c_10 & $4964 \pm 342$ & $5100 \pm 123$ & $5084 \pm 116$ & $2.1 \pm 0.8$ & $2.25 \pm 0.25$ & $2.24 \pm 0.24$ & $-1.7 \pm 0.4$ & $-2.00 \pm 0.1$ & $-1.98 \pm 0.10$ & $0.40 \pm 0.13$ & $0.18 \pm 0.14$ & M \\
\hline M22c_11 & $4812 \pm 351$ & $5225 \pm 75$ & $5207 \pm 73$ & $1.7 \pm 0.5$ & $3.20 \pm 0.33$ & $2.74 \pm 0.28$ & $-1.72 \pm 0.33$ & $-2.00 \pm 0.1$ & $-1.98 \pm 0.10$ & $0.43 \pm 0.14$ & $0.21 \pm 0.13$ & M \\
\hline M22c_12 & $4820 \pm 170$ & $5100 \pm 122$ & $5005 \pm 99$ & $2.7 \pm 0.4$ & $3.35 \pm 0.23$ & $3.19 \pm 0.20$ & $-0.19 \pm 0.18$ & $-0.30 \pm 0.24$ & $-0.23 \pm 0.14$ & $0.10 \pm 0.15$ & $0.21 \pm 0.11$ & \\
\hline M22c_13 & $4929 \pm 324$ & $5125 \pm 167$ & $5084 \pm 148$ & $2.0 \pm 0.6$ & $2.55 \pm 0.42$ & $2.37 \pm 0.34$ & $-1.59 \pm 0.32$ & $-1.80 \pm 0.25$ & $-1.72 \pm 0.20$ & $0.42 \pm 0.13$ & $0.27 \pm 0.12$ & M \\
\hline M22c_14 & $4793 \pm 515$ & $5075 \pm 160$ & $5050 \pm 153$ & $1.4 \pm 0.6$ & $2.30 \pm 0.46$ & $1.97 \pm 0.37$ & $-1.8 \pm 0.4$ & $-2.05 \pm 0.15$ & $-2.02 \pm 0.14$ & $0.53 \pm 0.04$ & $0.18 \pm 0.14$ & $\mathrm{M}$ \\
\hline M22c_15 & $4920 \pm 325$ & $5300 \pm 217$ & $5183 \pm 180$ & $2.0 \pm 0.6$ & $2.85 \pm 0.59$ & $2.43 \pm 0.42$ & $-1.62 \pm 0.30$ & $-1.65 \pm 0.23$ & $-1.64 \pm 0.18$ & $0.41 \pm 0.12$ & $0.20 \pm 0.11$ & M \\
\hline M22c_16 & $5006 \pm 349$ & $5425 \pm 114$ & $5385 \pm 108$ & $2.2 \pm 0.8$ & $3.00 \pm 0.50$ & $2.78 \pm 0.42$ & $-1.60 \pm 0.31$ & $-1.65 \pm 0.23$ & $-1.63 \pm 0.18$ & $0.43 \pm 0.13$ & $0.24 \pm 0.14$ & M \\
\hline M22c_17 & $4946 \pm 323$ & $5350 \pm 122$ & $5300 \pm 114$ & $2.1 \pm 0.7$ & $3.10 \pm 0.37$ & $2.88 \pm 0.33$ & $-1.64 \pm 0.29$ & $-1.80 \pm 0.25$ & $-1.73 \pm 0.19$ & $0.41 \pm 0.12$ & $0.26 \pm 0.11$ & M \\
\hline M22c_18 & $4888 \pm 330$ & $5050 \pm 100$ & $5036 \pm 96$ & $2.0 \pm 0.7$ & $2.20 \pm 0.33$ & $2.16 \pm 0.30$ & $-1.71 \pm 0.29$ & $-2.00 \pm 0.1$ & $-1.97 \pm 0.09$ & $0.41 \pm 0.13$ & $0.20 \pm 0.14$ & $\mathrm{M}$ \\
\hline 5824_1 & $4760 \pm 188$ & $4825 \pm 115$ & $4807 \pm 98$ & $2.8 \pm 0.4$ & $3.34 \pm 0.32$ & $3.13 \pm 0.25$ & $-0.22 \pm 0.15$ & $-0.65 \pm 0.23$ & $-0.35 \pm 0.13$ & $0.12 \pm 0.13$ & $0.26 \pm 0.09$ & \\
\hline 5824_2 & $4401 \pm 162$ & $4725 \pm 74$ & $4669 \pm 67$ & $1.01 \pm 0.21$ & $1.45 \pm 0.35$ & $1.13 \pm 0.18$ & $-1.99 \pm 0.17$ & $-2.00 \pm 0.1$ & $-2.00 \pm 0.09$ & $0.45 \pm 0.14$ & $0.13 \pm 0.13$ & M \\
\hline 5824_3 & $4471 \pm 195$ & $4600 \pm 122$ & $4564 \pm 103$ & $1.11 \pm 0.23$ & $1.55 \pm 0.35$ & $1.24 \pm 0.19$ & $-2.15 \pm 0.1$ & $-2.30 \pm 0.24$ & $-2.17 \pm 0.09$ & $0.40 \pm 0.14$ & $0.24 \pm 0.09$ & M \\
\hline $5824 \_4$ & $4438 \pm 226$ & $4401 \pm 122$ & $4409 \pm 107$ & $1.1 \pm 0.8$ & $1.00 \pm 0.59$ & $1.04 \pm 0.47$ & $-1.59 \pm 0.21$ & $-2.10 \pm 0.20$ & $-1.86 \pm 0.14$ & $0.52 \pm 0.11$ & $0.30 \pm 0.08$ & M \\
\hline $5824 \_5$ & $4975 \pm 343$ & $5250 \pm 100$ & $5228 \pm 96$ & $2.1 \pm 0.8$ & $2.60 \pm 0.49$ & $2.46 \pm 0.42$ & $-1.8 \pm 0.4$ & $-2.00 \pm 0.1$ & $-1.99 \pm 0.10$ & $0.40 \pm 0.17$ & $0.26 \pm 0.09$ & M \\
\hline 5824_6 & $4851 \pm 324$ & $5000 \pm 100$ & $4987 \pm 96$ & $1.9 \pm 0.7$ & $2.00 \pm 0.39$ & $1.98 \pm 0.34$ & $-1.75 \pm 0.28$ & $-2.00 \pm 0.1$ & $-1.97 \pm 0.09$ & $0.40 \pm 0.12$ & $0.17 \pm 0.13$ & M \\
\hline $5824 \_7$ & $4614 \pm 324$ & $4701 \pm 99$ & $4694 \pm 95$ & $1.4 \pm 0.6$ & $1.65 \pm 0.45$ & $1.56 \pm 0.36$ & $-1.64 \pm 0.34$ & $-2.00 \pm 0.1$ & $-1.97 \pm 0.10$ & $0.43 \pm 0.15$ & $0.23 \pm 0.14$ & M \\
\hline $5824 \_8$ & $4538 \pm 161$ & $5000 \pm 100$ & $4871 \pm 85$ & $1.3 \pm 0.4$ & $2.10 \pm 0.37$ & $1.73 \pm 0.27$ & $-2.02 \pm 0.18$ & $-2.00 \pm 0.1$ & $-2.00 \pm 0.09$ & $0.48 \pm 0.12$ & $0.25 \pm 0.14$ & \\
\hline 5824_9 & $4565 \pm 334$ & $4850 \pm 122$ & $4816 \pm 115$ & $1.2 \pm 0.4$ & $1.50 \pm 0.39$ & $1.35 \pm 0.28$ & $-1.7 \pm 0.4$ & $-1.80 \pm 0.24$ & $-1.77 \pm 0.21$ & $0.47 \pm 0.11$ & $0.20 \pm 0.13$ & M \\
\hline 5824_10 & $4400 \pm 212$ & $4475 \pm 74$ & $4467 \pm 70$ & $1.1 \pm 0.6$ & $1.30 \pm 0.46$ & $1.23 \pm 0.37$ & $-1.79 \pm 0.32$ & $-2.05 \pm 0.15$ & $-2.00 \pm 0.14$ & $0.52 \pm 0.11$ & $0.27 \pm 0.12$ & M \\
\hline 5824_11 & $4893 \pm 334$ & $5025 \pm 75$ & $5019 \pm 73$ & $2.0 \pm 0.6$ & $2.05 \pm 0.41$ & $2.03 \pm 0.34$ & $-1.67 \pm 0.29$ & $-2.00 \pm 0.1$ & $-1.96 \pm 0.09$ & $0.42 \pm 0.13$ & $0.26 \pm 0.11$ & \\
\hline 5824_13 & $4618 \pm 240$ & $4926 \pm 114$ & $4869 \pm 103$ & $1.3 \pm 0.4$ & $2.01 \pm 0.45$ & $1.61 \pm 0.30$ & $-1.89 \pm 0.24$ & $-2.00 \pm 0.1$ & $-1.98 \pm 0.09$ & $0.46 \pm 0.11$ & $0.25 \pm 0.10$ & M \\
\hline 5824_14 & $4539 \pm 73$ & $5025 \pm 75$ & $4775 \pm 52$ & $1.20 \pm 0.10$ & $2.10 \pm 0.37$ & $1.26 \pm 0.10$ & $-1.97 \pm 0.19$ & $-2.00 \pm 0.1$ & $-1.99 \pm 0.09$ & $0.33 \pm 0.08$ & $0.20 \pm 0.14$ & M \\
\hline
\end{tabular}


Table B.3 - continued.

\begin{tabular}{|c|c|c|c|c|c|c|c|c|c|c|c|c|}
\hline NGC ID & $\mathrm{T}_{\text {eff }}^{(a)}(\mathrm{K})$ & $\mathrm{T}_{e f f}^{(b)}(\mathrm{K})$ & $\mathrm{T}_{e f f}^{(a v g)}(\mathrm{K})$ & $\log (g)^{(a)}$ & $\log (g)^{(b)}$ & $\log (g)^{(a v g)}$ & {$[\mathrm{Fe} / \mathrm{H}]^{(a)}$} & {$[\mathrm{Fe} / \mathrm{H}]^{(b)}$} & {$[\mathrm{Fe} / \mathrm{H}]^{(a v g)}$} & {$[\mathrm{Mg} / \mathrm{Fe}]^{(a)}$} & {$[\alpha / \mathrm{Fe}]^{(b)}$} & members \\
\hline 5824_15 & $4891 \pm 343$ & $5000 \pm 100$ & $4991 \pm 96$ & $2.0 \pm 0.7$ & $2.00 \pm 0.39$ & $2.00 \pm 0.34$ & $-1.70 \pm 0.30$ & $-2.00 \pm 0.1$ & $-1.97 \pm 0.09$ & $0.41 \pm 0.13$ & $0.22 \pm 0.12$ & $\mathrm{M}$ \\
\hline 5824_16 & $4932 \pm 348$ & $5250 \pm 100$ & $5226 \pm 96$ & $2.0 \pm 0.7$ & $2.15 \pm 0.32$ & $2.12 \pm 0.29$ & $-1.8 \pm 0.4$ & $-2.00 \pm 0.1$ & $-1.99 \pm 0.10$ & $0.44 \pm 0.12$ & $0.20 \pm 0.14$ & M \\
\hline 5824_17 & $4488 \pm 80$ & $4899 \pm 123$ & $4610 \pm 67$ & $1.12 \pm 0.12$ & $1.95 \pm 0.42$ & $1.18 \pm 0.12$ & $-2.03 \pm 0.16$ & $-2.00 \pm 0.1$ & $-2.01 \pm 0.08$ & $0.41 \pm 0.11$ & $0.28 \pm 0.12$ & $\mathrm{M}$ \\
\hline 5824_18 & $4531 \pm 184$ & $4701 \pm 99$ & $4663 \pm 87$ & $1.27 \pm 0.35$ & $1.60 \pm 0.37$ & $1.43 \pm 0.25$ & $-1.93 \pm 0.20$ & $-2.10 \pm 0.20$ & $-2.01 \pm 0.14$ & $0.48 \pm 0.11$ & $0.21 \pm 0.14$ & M \\
\hline 5824_19 & $4480 \pm 184$ & $4578 \pm 224$ & $4519 \pm 142$ & $1.20 \pm 0.26$ & $1.36 \pm 0.71$ & $1.22 \pm 0.24$ & $-1.85 \pm 0.19$ & $-2.15 \pm 0.23$ & $-1.97 \pm 0.15$ & $0.47 \pm 0.12$ & $0.27 \pm 0.11$ & M \\
\hline 47Tuc_502 & $3640 \pm 100$ & $3627 \pm 125$ & $3635 \pm 78$ & $0.70 \pm 0.2$ & $3.5 \pm 0.5$ & $1.08 \pm 0.19$ & $-0.10 \pm 0.07$ & $-0.04 \pm 0.25$ & $-0.10 \pm 0.07$ & $0.23 \pm 0.1$ & $0.37 \pm 0.05$ & \\
\hline 47Tuc_509 & $3983 \pm 84$ & $3773 \pm 284$ & $3966 \pm 81$ & $1.47 \pm 0.30$ & $1.1 \pm 0.9$ & $1.43 \pm 0.28$ & $-0.31 \pm 0.20$ & $-1.60 \pm 0.44$ & $-0.53 \pm 0.18$ & $0.12 \pm 0.18$ & $0.32 \pm 0.10$ & M \\
\hline 47Tuc_514 & $4651 \pm 217$ & $4823 \pm 196$ & $4746 \pm 145$ & $2.3 \pm 0.5$ & $2.9 \pm 0.5$ & $2.61 \pm 0.36$ & $-0.47 \pm 0.24$ & $-0.75 \pm 0.25$ & $-0.60 \pm 0.17$ & $0.25 \pm 0.20$ & $0.28 \pm 0.10$ & M \\
\hline 47Tuc_517 & $4688 \pm 224$ & $4728 \pm 175$ & $4713 \pm 138$ & $2.4 \pm 0.5$ & $2.6 \pm 0.4$ & $2.51 \pm 0.33$ & $-0.47 \pm 0.25$ & $-0.90 \pm 0.20$ & $-0.73 \pm 0.16$ & $0.26 \pm 0.20$ & $0.30 \pm 0.08$ & M \\
\hline 47Tuc_519 & $4263 \pm 154$ & $4277 \pm 134$ & $4271 \pm 101$ & $1.8 \pm 0.4$ & $1.96 \pm 0.35$ & $1.89 \pm 0.26$ & $-0.46 \pm 0.22$ & $-1.05 \pm 0.15$ & $-0.86 \pm 0.12$ & $0.21 \pm 0.21$ & $0.23 \pm 0.13$ & M \\
\hline 47Tuc_525 & $4272 \pm 152$ & $4277 \pm 134$ & $4275 \pm 101$ & $1.8 \pm 0.4$ & $1.95 \pm 0.35$ & $1.88 \pm 0.26$ & $-0.47 \pm 0.22$ & $-1.10 \pm 0.20$ & $-0.81 \pm 0.15$ & $0.22 \pm 0.20$ & $0.27 \pm 0.11$ & M \\
\hline 47Tuc_533 & $4278 \pm 107$ & $4300 \pm 147$ & $4286 \pm 86$ & $1.8 \pm 0.4$ & $1.8 \pm 0.4$ & $1.82 \pm 0.28$ & $-0.51 \pm 0.23$ & $-0.94 \pm 0.27$ & $-0.69 \pm 0.18$ & $0.29 \pm 0.20$ & $0.29 \pm 0.09$ & M \\
\hline 47Tuc_534 & $4879 \pm 363$ & $5125 \pm 167$ & $5082 \pm 152$ & $2.3 \pm 0.8$ & $2.8 \pm 0.4$ & $2.70 \pm 0.36$ & $-0.7 \pm 0.4$ & $-0.80 \pm 0.25$ & $-0.77 \pm 0.21$ & $0.29 \pm 0.15$ & $0.19 \pm 0.12$ & M \\
\hline 47Tuc_535 & $4582 \pm 175$ & $4596 \pm 121$ & $4591 \pm 100$ & $2.3 \pm 0.4$ & $2.3 \pm 0.4$ & $2.32 \pm 0.30$ & $-0.46 \pm 0.19$ & $-0.81 \pm 0.24$ & $-0.59 \pm 0.15$ & $0.25 \pm 0.21$ & $0.23 \pm 0.11$ & M \\
\hline 47Tuc_539 & $4488 \pm 180$ & $4628 \pm 125$ & $4582 \pm 103$ & $2.1 \pm 0.5$ & $2.49 \pm 0.31$ & $2.38 \pm 0.26$ & $-0.46 \pm 0.21$ & $-0.74 \pm 0.25$ & $-0.58 \pm 0.16$ & $0.25 \pm 0.21$ & $0.25 \pm 0.08$ & M \\
\hline 47Tuc_551 & $4949 \pm 309$ & $5148 \pm 123$ & $5121 \pm 114$ & $2.5 \pm 0.6$ & $2.8 \pm 0.4$ & $2.71 \pm 0.33$ & $-0.61 \pm 0.29$ & $-0.70 \pm 0.25$ & $-0.66 \pm 0.19$ & $0.30 \pm 0.13$ & $0.20 \pm 0.14$ & M \\
\hline 47Tuc_553 & $4530 \pm 187$ & $4623 \pm 125$ & $4594 \pm 104$ & $2.2 \pm 0.5$ & $2.42 \pm 0.35$ & $2.35 \pm 0.29$ & $-0.47 \pm 0.18$ & $-0.75 \pm 0.25$ & $-0.57 \pm 0.15$ & $0.24 \pm 0.20$ & $0.22 \pm 0.10$ & M \\
\hline 47Tuc_554 & $3909 \pm 34$ & $3750 \pm 156$ & $3902 \pm 33$ & $1.43 \pm 0.17$ & $0.7 \pm 0.5$ & $1.35 \pm 0.16$ & $-0.28 \pm 0.19$ & $-1.40 \pm 0.20$ & $-0.81 \pm 0.14$ & $0.12 \pm 0.20$ & $0.26 \pm 0.11$ & M \\
\hline 47Tuc_559 & $4780 \pm 282$ & $4749 \pm 193$ & $4759 \pm 159$ & $2.5 \pm 0.6$ & $2.85 \pm 0.32$ & $2.77 \pm 0.28$ & $-0.52 \pm 0.32$ & $-1.05 \pm 0.35$ & $-0.76 \pm 0.24$ & $0.27 \pm 0.20$ & $0.32 \pm 0.10$ & M \\
\hline 47Tuc_571 & $4972 \pm 439$ & $5277 \pm 260$ & $5198 \pm 224$ & $2.4 \pm 0.9$ & $2.8 \pm 0.5$ & $2.72 \pm 0.41$ & $-0.7 \pm 0.5$ & $-0.80 \pm 0.33$ & $-0.77 \pm 0.28$ & $0.25 \pm 0.17$ & $0.26 \pm 0.11$ & M \\
\hline 47Tuc_581 & $4868 \pm 346$ & $5071 \pm 113$ & $5051 \pm 107$ & $2.2 \pm 0.8$ & $2.44 \pm 0.34$ & $2.40 \pm 0.31$ & $-0.8 \pm 0.4$ & $-0.91 \pm 0.19$ & $-0.89 \pm 0.17$ & $0.36 \pm 0.13$ & $0.22 \pm 0.15$ & M \\
\hline 2808_1 & $4676 \pm 331$ & $4925 \pm 115$ & $4898 \pm 108$ & $1.8 \pm 0.9$ & $1.65 \pm 0.23$ & $1.66 \pm 0.22$ & $-1.0 \pm 0.5$ & $-1.15 \pm 0.23$ & $-1.12 \pm 0.21$ & $0.32 \pm 0.17$ & $0.16 \pm 0.12$ & M \\
\hline $2808 \_2$ & $4736 \pm 167$ & $5124 \pm 125$ & $4985 \pm 100$ & $2.52 \pm 0.30$ & $3.25 \pm 0.25$ & $2.95 \pm 0.19$ & $-0.30 \pm 0.22$ & $-0.25 \pm 0.25$ & $-0.28 \pm 0.17$ & $0.16 \pm 0.19$ & $0.21 \pm 0.09$ & \\
\hline 2808_3 & $4812 \pm 360$ & $4825 \pm 115$ & $4824 \pm 109$ & $2.2 \pm 0.9$ & $2.50 \pm 0.39$ & $2.45 \pm 0.36$ & $-0.9 \pm 0.5$ & $-1.35 \pm 0.23$ & $-1.27 \pm 0.21$ & $0.34 \pm 0.17$ & $0.24 \pm 0.12$ & M \\
\hline $2808 \_4$ & $4949 \pm 314$ & $4800 \pm 245$ & $4856 \pm 193$ & $2.5 \pm 0.7$ & $2.00 \pm 0.45$ & $2.15 \pm 0.38$ & $-0.57 \pm 0.30$ & $-1.20 \pm 0.24$ & $-0.95 \pm 0.19$ & $0.24 \pm 0.13$ & $0.24 \pm 0.14$ & M \\
\hline $2808 \_5$ & $4801 \pm 399$ & $5276 \pm 208$ & $5175 \pm 184$ & $1.9 \pm 0.8$ & $2.65 \pm 0.45$ & $2.47 \pm 0.39$ & $-1.4 \pm 0.4$ & $-1.20 \pm 0.24$ & $-1.25 \pm 0.21$ & $0.42 \pm 0.16$ & $0.19 \pm 0.14$ & M \\
\hline $2808 \_6$ & $4910 \pm 354$ & $4750 \pm 158$ & $4777 \pm 144$ & $2.5 \pm 0.9$ & $2.20 \pm 0.56$ & $2.28 \pm 0.48$ & $-0.7 \pm 0.5$ & $-1.40 \pm 0.20$ & $-1.30 \pm 0.19$ & $0.32 \pm 0.20$ & $0.26 \pm 0.09$ & M \\
\hline 2808_7 & $4903 \pm 401$ & $4799 \pm 149$ & $4812 \pm 140$ & $2.3 \pm 0.9$ & $1.85 \pm 0.32$ & $1.90 \pm 0.30$ & $-0.8 \pm 0.5$ & $-1.45 \pm 0.15$ & $-1.40 \pm 0.14$ & $0.31 \pm 0.20$ & $0.19 \pm 0.14$ & $\mathrm{M}$ \\
\hline
\end{tabular}


Table B.3 - continued.

\begin{tabular}{|c|c|c|c|c|c|c|c|c|c|c|c|c|}
\hline NGC ID & $\mathrm{T}_{e f f}^{(a)}(\mathrm{K})$ & $\mathrm{T}_{e f f}^{(b)}(\mathrm{K})$ & $\mathrm{T}_{e f f}^{(a v g)}(\mathrm{K})$ & $\log (g)^{(a)}$ & $\log (g)^{(b)}$ & $\log (g)^{(a v g)}$ & {$[\mathrm{Fe} / \mathrm{H}]^{(a)}$} & {$[\mathrm{Fe} / \mathrm{H}]^{(b)}$} & {$[\mathrm{Fe} / \mathrm{H}]^{(a v g)}$} & {$[\mathrm{Mg} / \mathrm{Fe}]^{(a)}$} & {$[\alpha / \mathrm{Fe}]^{(b)}$} & members \\
\hline 2808_8 & $4786 \pm 413$ & $4651 \pm 122$ & $4662 \pm 117$ & $2.3 \pm 0.8$ & $1.40 \pm 0.49$ & $1.65 \pm 0.42$ & $-0.51 \pm 0.34$ & $-1.20 \pm 0.24$ & $-0.97 \pm 0.20$ & $0.12 \pm 0.06$ & $0.21 \pm 0.14$ & M \\
\hline 2808_9 & $4687 \pm 362$ & $4925 \pm 114$ & $4904 \pm 109$ & $1.7 \pm 0.8$ & $2.00 \pm 0.45$ & $1.93 \pm 0.39$ & $-1.4 \pm 0.4$ & $-1.50 \pm 0.1$ & $-1.49 \pm 0.10$ & $0.42 \pm 0.14$ & $0.22 \pm 0.12$ & \\
\hline 2808_10 & $4072 \pm 114$ & $3925 \pm 296$ & $4053 \pm 106$ & $1.4 \pm 0.4$ & $0.90 \pm 0.73$ & $1.28 \pm 0.35$ & $-0.47 \pm 0.22$ & $-1.50 \pm 0.39$ & $-0.72 \pm 0.19$ & $0.19 \pm 0.24$ & $0.32 \pm 0.07$ & M \\
\hline 2808_11 & $4662 \pm 415$ & $4900 \pm 122$ & $4881 \pm 117$ & $1.9 \pm 0.9$ & $2.05 \pm 0.35$ & $2.03 \pm 0.33$ & $-0.8 \pm 0.5$ & $-1.00 \pm 0.1$ & $-0.99 \pm 0.10$ & $0.30 \pm 0.17$ & $0.20 \pm 0.12$ & \\
\hline 2808_13 & $4961 \pm 279$ & $4773 \pm 236$ & $4851 \pm 180$ & $2.7 \pm 0.6$ & $1.90 \pm 0.37$ & $2.12 \pm 0.31$ & $-0.52 \pm 0.31$ & $-1.25 \pm 0.25$ & $-0.96 \pm 0.19$ & $0.24 \pm 0.17$ & $0.25 \pm 0.13$ & M \\
\hline 2808_14 & $4968 \pm 300$ & $5049 \pm 99$ & $5041 \pm 94$ & $2.5 \pm 0.6$ & $2.40 \pm 0.37$ & $2.43 \pm 0.31$ & $-0.57 \pm 0.31$ & $-0.90 \pm 0.20$ & $-0.80 \pm 0.17$ & $0.25 \pm 0.13$ & $0.19 \pm 0.14$ & M \\
\hline 2808_15 & $4638 \pm 430$ & $5075 \pm 225$ & $4981 \pm 199$ & $1.5 \pm 0.8$ & $2.00 \pm 0.39$ & $1.90 \pm 0.35$ & $-1.2 \pm 0.5$ & $-1.15 \pm 0.23$ & $-1.16 \pm 0.21$ & $0.38 \pm 0.18$ & $0.22 \pm 0.14$ & M \\
\hline 2808_16 & $4328 \pm 48$ & $4526 \pm 175$ & $4342 \pm 46$ & $1.20 \pm 0.05$ & $1.90 \pm 0.44$ & $1.21 \pm 0.05$ & $-0.72 \pm 0.24$ & $-1.10 \pm 0.20$ & $-0.94 \pm 0.15$ & $0.15 \pm 0.14$ & $0.22 \pm 0.11$ & M \\
\hline 2808_17 & $4832 \pm 264$ & $4625 \pm 125$ & $4663 \pm 113$ & $2.5 \pm 0.7$ & $2.05 \pm 0.27$ & $2.11 \pm 0.25$ & $-0.51 \pm 0.29$ & $-1.25 \pm 0.25$ & $-0.93 \pm 0.19$ & $0.19 \pm 0.15$ & $0.25 \pm 0.10$ & M \\
\hline 2808_18 & $4433 \pm 74$ & $4576 \pm 159$ & $4458 \pm 67$ & $2.36 \pm 0.23$ & $2.55 \pm 0.47$ & $2.40 \pm 0.21$ & $-0.45 \pm 0.08$ & $-0.85 \pm 0.32$ & $-0.47 \pm 0.08$ & $0.16 \pm 0.15$ & $0.27 \pm 0.11$ & \\
\hline 2808_19 & $4874 \pm 359$ & $5175 \pm 114$ & $5147 \pm 109$ & $2.3 \pm 0.9$ & $3.15 \pm 0.32$ & $3.05 \pm 0.30$ & $-1.0 \pm 0.5$ & $-1.05 \pm 0.15$ & $-1.05 \pm 0.14$ & $0.34 \pm 0.19$ & $0.18 \pm 0.14$ & M \\
\hline 3201_1 & $4819 \pm 392$ & $5225 \pm 75$ & $5211 \pm 73$ & $1.9 \pm 0.9$ & $3.20 \pm 0.25$ & $3.11 \pm 0.24$ & $-1.60 \pm 0.32$ & $-1.50 \pm 0.1$ & $-1.51 \pm 0.10$ & $0.43 \pm 0.13$ & $0.19 \pm 0.15$ & \\
\hline $3201 \_2$ & $4757 \pm 322$ & $4975 \pm 175$ & $4925 \pm 154$ & $1.7 \pm 0.7$ & $2.45 \pm 0.35$ & $2.30 \pm 0.31$ & $-1.56 \pm 0.32$ & $-1.65 \pm 0.23$ & $-1.62 \pm 0.19$ & $0.43 \pm 0.14$ & $0.22 \pm 0.12$ & M \\
\hline 3201_5 & $4775 \pm 369$ & $5250 \pm 100$ & $5218 \pm 97$ & $1.8 \pm 0.8$ & $2.60 \pm 0.37$ & $2.46 \pm 0.34$ & $-1.57 \pm 0.32$ & $-1.50 \pm 0.1$ & $-1.51 \pm 0.10$ & $0.43 \pm 0.14$ & $0.24 \pm 0.14$ & M \\
\hline 3201_6 & $4509 \pm 274$ & $5076 \pm 160$ & $4932 \pm 138$ & $1.2 \pm 0.6$ & $2.55 \pm 0.41$ & $2.12 \pm 0.34$ & $-1.48 \pm 0.34$ & $-1.30 \pm 0.25$ & $-1.36 \pm 0.20$ & $0.46 \pm 0.12$ & $0.21 \pm 0.13$ & \\
\hline $3201 \_7$ & $4891 \pm 346$ & $5225 \pm 75$ & $5210 \pm 73$ & $2.0 \pm 0.7$ & $2.60 \pm 0.37$ & $2.47 \pm 0.33$ & $-1.56 \pm 0.31$ & $-1.55 \pm 0.15$ & $-1.55 \pm 0.14$ & $0.43 \pm 0.14$ & $0.27 \pm 0.11$ & M \\
\hline 3201_8 & $4408 \pm 178$ & $4800 \pm 100$ & $4706 \pm 87$ & $1.0 \pm 0.5$ & $2.00 \pm 0.39$ & $1.62 \pm 0.31$ & $-1.52 \pm 0.20$ & $-1.50 \pm 0.1$ & $-1.50 \pm 0.09$ & $0.45 \pm 0.14$ & $0.25 \pm 0.14$ & M \\
\hline 3201_9 & $4774 \pm 347$ & $5025 \pm 75$ & $5014 \pm 73$ & $1.7 \pm 0.7$ & $2.45 \pm 0.35$ & $2.30 \pm 0.31$ & $-1.54 \pm 0.30$ & $-1.50 \pm 0.1$ & $-1.50 \pm 0.09$ & $0.45 \pm 0.14$ & $0.19 \pm 0.13$ & M \\
\hline 3201_10 & $4752 \pm 340$ & $5049 \pm 99$ & $5026 \pm 95$ & $1.7 \pm 0.8$ & $2.40 \pm 0.37$ & $2.28 \pm 0.34$ & $-1.50 \pm 0.27$ & $-1.40 \pm 0.20$ & $-1.44 \pm 0.16$ & $0.46 \pm 0.15$ & $0.18 \pm 0.14$ & M \\
\hline 3201_11 & $4997 \pm 287$ & $5250 \pm 111$ & $5217 \pm 104$ & $2.2 \pm 0.7$ & $3.00 \pm 0.39$ & $2.81 \pm 0.34$ & $-1.47 \pm 0.27$ & $-1.55 \pm 0.15$ & $-1.53 \pm 0.13$ & $0.42 \pm 0.16$ & $0.21 \pm 0.14$ & M \\
\hline 3201_13 & $4928 \pm 465$ & $5075 \pm 195$ & $5053 \pm 180$ & $2.1 \pm 0.9$ & $2.65 \pm 0.39$ & $2.56 \pm 0.36$ & $-1.52 \pm 0.35$ & $-1.60 \pm 0.20$ & $-1.58 \pm 0.17$ & $0.45 \pm 0.15$ & $0.23 \pm 0.13$ & M \\
\hline 3201_14 & $4851 \pm 353$ & $5050 \pm 100$ & $5035 \pm 96$ & $1.9 \pm 0.8$ & $2.45 \pm 0.35$ & $2.36 \pm 0.32$ & $-1.51 \pm 0.28$ & $-1.50 \pm 0.1$ & $-1.50 \pm 0.09$ & $0.44 \pm 0.15$ & $0.15 \pm 0.10$ & M \\
\hline 3201_15 & $4925 \pm 385$ & $5250 \pm 100$ & $5229 \pm 97$ & $2.1 \pm 0.8$ & $3.00 \pm 0.39$ & $2.83 \pm 0.35$ & $-1.53 \pm 0.31$ & $-1.50 \pm 0.1$ & $-1.50 \pm 0.10$ & $0.44 \pm 0.14$ & $0.19 \pm 0.14$ & M \\
\hline 3201_16 & $4600 \pm 367$ & $4750 \pm 250$ & $4702 \pm 207$ & $1.7 \pm 1.0$ & $2.05 \pm 0.47$ & $1.99 \pm 0.43$ & $-1.0 \pm 0.5$ & $-1.25 \pm 0.25$ & $-1.20 \pm 0.22$ & $0.31 \pm 0.16$ & $0.18 \pm 0.15$ & M \\
\hline 3201_17 & $4893 \pm 349$ & $5101 \pm 229$ & $5038 \pm 191$ & $2.0 \pm 0.8$ & $2.40 \pm 0.54$ & $2.27 \pm 0.45$ & $-1.54 \pm 0.30$ & $-1.65 \pm 0.23$ & $-1.61 \pm 0.18$ & $0.43 \pm 0.15$ & $0.27 \pm 0.11$ & M \\
\hline 3201_18 & $4813 \pm 380$ & $5175 \pm 160$ & $5121 \pm 147$ & $1.9 \pm 0.8$ & $2.75 \pm 0.40$ & $2.58 \pm 0.36$ & $-1.59 \pm 0.32$ & $-1.60 \pm 0.20$ & $-1.60 \pm 0.17$ & $0.43 \pm 0.14$ & $0.25 \pm 0.12$ & M \\
\hline M68_49 & $5111 \pm 355$ & $5500 \pm 100$ & $5471 \pm 96$ & $2.3 \pm 0.7$ & $3.05 \pm 0.42$ & $2.85 \pm 0.36$ & $-1.8 \pm 0.5$ & $-2.00 \pm 0.1$ & $-1.99 \pm 0.10$ & $0.39 \pm 0.19$ & $0.25 \pm 0.10$ & M \\
\hline M68_30 & $4907 \pm 300$ & $5025 \pm 175$ & $4995 \pm 151$ & $1.9 \pm 0.7$ & $2.65 \pm 0.39$ & $2.47 \pm 0.34$ & $-1.9 \pm 0.4$ & $-2.35 \pm 0.23$ & $-2.24 \pm 0.20$ & $0.39 \pm 0.11$ & $0.12 \pm 0.13$ & M \\
\hline
\end{tabular}


Table B.3 - continued.

\begin{tabular}{|c|c|c|c|c|c|c|c|c|c|c|c|c|}
\hline NGC ID & $\mathrm{T}_{e f f}^{(a)}(\mathrm{K})$ & $\mathrm{T}_{e f f}^{(b)}(\mathrm{K})$ & $\mathrm{T}_{e f f}^{(a v g)}(\mathrm{K})$ & $\log (g)^{(a)}$ & $\log (g)^{(b)}$ & $\log (g)^{(\operatorname{avg})}$ & {$[\mathrm{Fe} / \mathrm{H}]^{(a)}$} & {$[\mathrm{Fe} / \mathrm{H}]^{(b)}$} & {$[\mathrm{Fe} / \mathrm{H}]^{(a v g)}$} & {$[\mathrm{Mg} / \mathrm{Fe}]^{(a)}$} & {$[\alpha / \mathrm{Fe}]^{(b)}$} & members \\
\hline M68_74 & $5041 \pm 354$ & $5049 \pm 99$ & $5049 \pm 96$ & $2.2 \pm 0.8$ & $2.45 \pm 0.61$ & $2.36 \pm 0.49$ & $-2.0 \pm 0.4$ & $-2.40 \pm 0.20$ & $-2.32 \pm 0.18$ & $0.39 \pm 0.18$ & $0.20 \pm 0.14$ & M \\
\hline M68_II47 & $4990 \pm 434$ & $4825 \pm 115$ & $4836 \pm 111$ & $2.0 \pm 0.7$ & $2.10 \pm 0.37$ & $2.08 \pm 0.33$ & $-1.8 \pm 0.4$ & $-2.50 \pm 0.1$ & $-2.46 \pm 0.10$ & $0.41 \pm 0.18$ & $0.22 \pm 0.14$ & M \\
\hline M68_119 & $4908 \pm 326$ & $4775 \pm 75$ & $4782 \pm 73$ & $2.0 \pm 0.7$ & $2.35 \pm 0.32$ & $2.29 \pm 0.29$ & $-1.74 \pm 0.34$ & $-2.45 \pm 0.15$ & $-2.33 \pm 0.14$ & $0.36 \pm 0.13$ & $0.21 \pm 0.13$ & M \\
\hline M68_144 & $5219 \pm 385$ & $5775 \pm 75$ & $5755 \pm 74$ & $2.5 \pm 0.8$ & $3.60 \pm 0.54$ & $3.26 \pm 0.45$ & $-2.0 \pm 0.4$ & $-2.50 \pm 0.1$ & $-2.47 \pm 0.10$ & $0.37 \pm 0.20$ & $0.10 \pm 0.10$ & \\
\hline M68_185 & $4862 \pm 15$ & $5100 \pm 122$ & $4866 \pm 15$ & $3.4 \pm 0.2$ & $4.45 \pm 0.35$ & $3.66 \pm 0.17$ & $0.02 \pm 0.09$ & $-0.65 \pm 0.23$ & $-0.07 \pm 0.08$ & $0.04 \pm 0.03$ & $0.21 \pm 0.09$ & \\
\hline M68_239 & $4910 \pm 300$ & $5226 \pm 74$ & $5208 \pm 72$ & $1.9 \pm 0.7$ & $2.21 \pm 0.25$ & $2.17 \pm 0.24$ & $-1.9 \pm 0.4$ & $-2.00 \pm 0.1$ & $-1.99 \pm 0.10$ & $0.39 \pm 0.11$ & $0.19 \pm 0.15$ & M \\
\hline M68_II72 & $5096 \pm 393$ & $5075 \pm 160$ & $5078 \pm 148$ & $2.2 \pm 0.8$ & $2.55 \pm 0.61$ & $2.42 \pm 0.49$ & $-1.9 \pm 0.4$ & $-2.40 \pm 0.20$ & $-2.30 \pm 0.18$ & $0.38 \pm 0.18$ & $0.12 \pm 0.12$ & M \\
\hline M68_II79 & $4862 \pm 16$ & $5274 \pm 175$ & $4865 \pm 16$ & $3.4 \pm 0.2$ & $4.25 \pm 0.25$ & $3.73 \pm 0.16$ & $0.02 \pm 0.09$ & $-0.18 \pm 0.27$ & $0.00 \pm 0.09$ & $0.04 \pm 0.03$ & $0.09 \pm 0.08$ & \\
\hline M5_II45 & $4692 \pm 330$ & $4874 \pm 202$ & $4825 \pm 172$ & $1.7 \pm 0.8$ & $2.40 \pm 0.54$ & $2.18 \pm 0.45$ & $-1.48 \pm 0.27$ & $-1.65 \pm 0.23$ & $-1.58 \pm 0.18$ & $0.43 \pm 0.15$ & $0.27 \pm 0.11$ & M \\
\hline M5_II50 & $4705 \pm 304$ & $4645 \pm 227$ & $4667 \pm 182$ & $2.0 \pm 0.8$ & $1.99 \pm 0.39$ & $1.99 \pm 0.35$ & $-0.9 \pm 0.4$ & $-1.35 \pm 0.23$ & $-1.24 \pm 0.20$ & $0.29 \pm 0.13$ & $0.21 \pm 0.11$ & M \\
\hline M5_II51 & $4727 \pm 329$ & $4600 \pm 122$ & $4615 \pm 115$ & $1.9 \pm 0.9$ & $1.85 \pm 0.23$ & $1.85 \pm 0.22$ & $-1.1 \pm 0.4$ & $-1.80 \pm 0.24$ & $-1.61 \pm 0.21$ & $0.42 \pm 0.16$ & $0.26 \pm 0.11$ & M \\
\hline M5_II80 & $4560 \pm 273$ & $4773 \pm 73$ & $4759 \pm 70$ & $1.5 \pm 0.8$ & $2.30 \pm 0.39$ & $2.15 \pm 0.35$ & $-1.2 \pm 0.4$ & $-1.45 \pm 0.15$ & $-1.42 \pm 0.14$ & $0.38 \pm 0.15$ & $0.21 \pm 0.13$ & M \\
\hline M5_I2 & $4810 \pm 356$ & $4748 \pm 250$ & $4768 \pm 205$ & $2.1 \pm 0.8$ & $1.95 \pm 0.42$ & $1.98 \pm 0.37$ & $-0.8 \pm 0.4$ & $-1.25 \pm 0.25$ & $-1.12 \pm 0.21$ & $0.32 \pm 0.14$ & $0.25 \pm 0.12$ & M \\
\hline M5_I50 & $4739 \pm 377$ & $4848 \pm 122$ & $4838 \pm 116$ & $2.0 \pm 1.0$ & $2.55 \pm 0.35$ & $2.49 \pm 0.33$ & $-1.0 \pm 0.5$ & $-1.30 \pm 0.24$ & $-1.24 \pm 0.22$ & $0.38 \pm 0.16$ & $0.20 \pm 0.11$ & M \\
\hline M5_I61 & $4618 \pm 340$ & $4597 \pm 164$ & $4601 \pm 148$ & $2.0 \pm 0.9$ & $1.99 \pm 0.38$ & $1.99 \pm 0.35$ & $-0.9 \pm 0.4$ & $-1.45 \pm 0.15$ & $-1.38 \pm 0.14$ & $0.29 \pm 0.18$ & $0.27 \pm 0.13$ & M \\
\hline M5_I68 & $4484 \pm 226$ & $4224 \pm 174$ & $4321 \pm 138$ & $2.0 \pm 0.6$ & $1.60 \pm 0.43$ & $1.74 \pm 0.35$ & $-0.59 \pm 0.32$ & $-1.55 \pm 0.35$ & $-1.03 \pm 0.24$ & $0.28 \pm 0.19$ & $0.27 \pm 0.09$ & M \\
\hline M5_I71 & $4702 \pm 412$ & $4927 \pm 113$ & $4911 \pm 109$ & $1.9 \pm 0.9$ & $2.06 \pm 0.41$ & $2.03 \pm 0.37$ & $-0.9 \pm 0.5$ & $-1.00 \pm 0.1$ & $-1.00 \pm 0.10$ & $0.34 \pm 0.17$ & $0.25 \pm 0.13$ & M \\
\hline M4_1 & $4764 \pm 371$ & $4974 \pm 174$ & $4936 \pm 158$ & $2.1 \pm 0.9$ & $2.90 \pm 0.43$ & $2.75 \pm 0.388$ & $-0.9 \pm 0.4$ & $-1.15 \pm 0.23$ & $-1.09 \pm 0.20$ & $0.38 \pm 0.16$ & $0.26 \pm 0.13$ & M \\
\hline M4_3 & $4816 \pm 367$ & $5200 \pm 150$ & $5145 \pm 139$ & $2.1 \pm 0.9$ & $3.25 \pm 0.25$ & $3.17 \pm 0.241$ & $-1.2 \pm 0.4$ & $-1.15 \pm 0.23$ & $-1.16 \pm 0.20$ & $0.40 \pm 0.17$ & $0.28 \pm 0.10$ & M \\
\hline M4_4 & $4946 \pm 364$ & $5151 \pm 122$ & $5130 \pm 116$ & $2.6 \pm 0.9$ & $3.20 \pm 0.25$ & $3.16 \pm 0.241$ & $-0.7 \pm 0.5$ & $-1.00 \pm 0.1$ & $-0.99 \pm 0.10$ & $0.32 \pm 0.21$ & $0.21 \pm 0.11$ & M \\
\hline M4_5 & $4883 \pm 348$ & $4999 \pm 157$ & $4979 \pm 143$ & $2.5 \pm 0.8$ & $2.75 \pm 0.25$ & $2.73 \pm 0.239$ & $-0.7 \pm 0.4$ & $-1.00 \pm 0.31$ & $-0.89 \pm 0.25$ & $0.33 \pm 0.17$ & $0.30 \pm 0.10$ & M \\
\hline M4_6 & $4504 \pm 163$ & $4701 \pm 268$ & $4557 \pm 139$ & $2.0 \pm 0.7$ & $2.50 \pm 0.59$ & $2.29 \pm 0.451$ & $-0.62 \pm 0.19$ & $-1.00 \pm 0.39$ & $-0.69 \pm 0.17$ & $0.17 \pm 0.14$ & $0.28 \pm 0.07$ & \\
\hline M4_8 & $4781 \pm 378$ & $5025 \pm 174$ & $4982 \pm 158$ & $2.2 \pm 0.9$ & $2.80 \pm 0.24$ & $2.76 \pm 0.232$ & $-0.8 \pm 0.5$ & $-0.95 \pm 0.35$ & $-0.90 \pm 0.29$ & $0.34 \pm 0.17$ & $0.30 \pm 0.10$ & \\
\hline M4_9 & $4896 \pm 359$ & $4925 \pm 195$ & $4919 \pm 172$ & $2.5 \pm 0.9$ & $2.65 \pm 0.39$ & $2.63 \pm 0.358$ & $-0.8 \pm 0.5$ & $-1.25 \pm 0.25$ & $-1.16 \pm 0.22$ & $0.33 \pm 0.20$ & $0.27 \pm 0.09$ & \\
\hline M4_10 & $4792 \pm 280$ & $4951 \pm 186$ & $4902 \pm 155$ & $2.3 \pm 0.7$ & $2.95 \pm 0.35$ & $2.82 \pm 0.313$ & $-0.84 \pm 0.31$ & $-1.20 \pm 0.24$ & $-1.07 \pm 0.19$ & $0.36 \pm 0.17$ & $0.25 \pm 0.13$ & M \\
\hline M4_11 & $4875 \pm 336$ & $4999 \pm 157$ & $4977 \pm 142$ & $2.5 \pm 0.8$ & $2.80 \pm 0.24$ & $2.78 \pm 0.230$ & $-0.8 \pm 0.4$ & $-1.00 \pm 0.31$ & $-0.92 \pm 0.25$ & $0.34 \pm 0.17$ & $0.26 \pm 0.13$ & M \\
\hline M4_13 & $4798 \pm 387$ & $5125 \pm 125$ & $5094 \pm 119$ & $2.2 \pm 1.0$ & $3.15 \pm 0.23$ & $3.10 \pm 0.224$ & $-1.0 \pm 0.5$ & $-1.00 \pm 0.1$ & $-1.00 \pm 0.10$ & $0.38 \pm 0.17$ & $0.24 \pm 0.14$ & M \\
\hline M4_16 & $5038 \pm 282$ & $4850 \pm 299$ & $4950 \pm 205$ & $2.9 \pm 0.5$ & $2.35 \pm 0.63$ & $2.69 \pm 0.392$ & $-0.49 \pm 0.31$ & $-1.20 \pm 0.33$ & $-0.82 \pm 0.23$ & $0.27 \pm 0.24$ & $0.31 \pm 0.10$ & \\
\hline
\end{tabular}


Table B.3 - continued.

\begin{tabular}{|c|c|c|c|c|c|c|c|c|c|c|c|c|}
\hline NGC ID & $\mathrm{T}_{\text {eff }}^{(a)}(\mathrm{K})$ & $\mathrm{T}_{\text {eff }}^{(b)}(\mathrm{K})$ & $\mathrm{T}_{e f f}^{(a v g)}(\mathrm{K})$ & $\log (g)^{(a)}$ & $\log (g)^{(b)}$ & $\log (g)^{(a v g)}$ & {$[\mathrm{Fe} / \mathrm{H}]^{(a)}$} & {$[\mathrm{Fe} / \mathrm{H}]^{(b)}$} & {$[\mathrm{Fe} / \mathrm{H}]^{(a v g)}$} & {$[\mathrm{Mg} / \mathrm{Fe}]^{(a)}$} & {$[\alpha / \mathrm{Fe}]^{(b)}$} & members \\
\hline M4_17 & $4980 \pm 260$ & $4728 \pm 208$ & $4827 \pm 163$ & $3.0 \pm 0.4$ & $2.26 \pm 0.60$ & $2.77 \pm 0.333$ & $-0.52 \pm 0.30$ & $-1.35 \pm 0.23$ & $-1.04 \pm 0.18$ & $0.28 \pm 0.22$ & $0.29 \pm 0.09$ & $\mathrm{M}$ \\
\hline M4_18 & $5030 \pm 44$ & $5072 \pm 336$ & $5031 \pm 44$ & $2.99 \pm 0.09$ & $2.35 \pm 0.59$ & $2.98 \pm 0.089$ & $-0.49 \pm 0.1$ & $-1.10 \pm 0.44$ & $-0.52 \pm 0.10$ & $0.22 \pm 0.05$ & $0.31 \pm 0.08$ & \\
\hline M4_19 & $6330 \pm 241$ & $5976 \pm 175$ & $6098 \pm 142$ & $3.63 \pm 0.007$ & $3.85 \pm 0.32$ & $3.63 \pm 0.007$ & $0.14 \pm 0.08$ & $-0.35 \pm 0.23$ & $0.09 \pm 0.08$ & $0.09 \pm 0.007$ & $0.09 \pm 0.10$ & \\
\hline M107_F & $4262 \pm 123$ & $4201 \pm 185$ & $4243 \pm 102$ & $1.8 \pm 0.4$ & $1.30 \pm 0.45$ & $1.58 \pm 0.30$ & $-0.45 \pm 0.24$ & $-1.10 \pm 0.37$ & $-0.64 \pm 0.20$ & $0.24 \pm 0.20$ & $0.29 \pm 0.09$ & \\
\hline M107_62 & $4497 \pm 178$ & $4525 \pm 208$ & $4509 \pm 135$ & $2.1 \pm 0.4$ & $2.55 \pm 0.42$ & $2.31 \pm 0.29$ & $-0.56 \pm 0.21$ & $-0.95 \pm 0.42$ & $-0.64 \pm 0.19$ & $0.33 \pm 0.20$ & $0.24 \pm 0.11$ & \\
\hline M107_100 & $4801 \pm 206$ & $4951 \pm 242$ & $4864 \pm 157$ & $2.4 \pm 0.5$ & $2.90 \pm 0.49$ & $2.66 \pm 0.35$ & $-0.40 \pm 0.23$ & $-0.50 \pm 0.31$ & $-0.44 \pm 0.18$ & $0.23 \pm 0.22$ & $0.12 \pm 0.13$ & \\
\hline M107_I & $4779 \pm 210$ & $4798 \pm 245$ & $4787 \pm 160$ & $2.5 \pm 0.4$ & $2.65 \pm 0.67$ & $2.54 \pm 0.34$ & $-0.31 \pm 0.29$ & $-0.70 \pm 0.25$ & $-0.53 \pm 0.19$ & $0.17 \pm 0.22$ & $0.19 \pm 0.13$ & \\
\hline M107_C & $4845 \pm 269$ & $4799 \pm 99$ & $4804 \pm 93$ & $2.4 \pm 0.6$ & $2.30 \pm 0.25$ & $2.31 \pm 0.23$ & $-0.55 \pm 0.27$ & $-1.00 \pm 0.1$ & $-0.95 \pm 0.09$ & $0.28 \pm 0.21$ & $0.20 \pm 0.14$ & $\mathrm{M}^{(c)}$ \\
\hline M10_1 & $4944 \pm 316$ & $5200 \pm 218$ & $5118 \pm 179$ & $2.0 \pm 0.6$ & $2.80 \pm 0.56$ & $2.43 \pm 0.41$ & $-1.59 \pm 0.28$ & $-1.75 \pm 0.25$ & $-1.68 \pm 0.19$ & $0.41 \pm 0.13$ & $0.19 \pm 0.11$ & $\mathrm{M}$ \\
\hline M10_2 & $4788 \pm 291$ & $5126 \pm 167$ & $5043 \pm 145$ & $1.7 \pm 0.5$ & $2.10 \pm 0.30$ & $1.99 \pm 0.26$ & $-1.59 \pm 0.31$ & $-1.55 \pm 0.15$ & $-1.56 \pm 0.14$ & $0.45 \pm 0.13$ & $0.23 \pm 0.13$ & $\mathrm{M}$ \\
\hline M10_3 & $4949 \pm 296$ & $5300 \pm 187$ & $5200 \pm 158$ & $2.0 \pm 0.6$ & $3.05 \pm 0.35$ & $2.78 \pm 0.30$ & $-1.55 \pm 0.29$ & $-1.60 \pm 0.20$ & $-1.58 \pm 0.16$ & $0.42 \pm 0.14$ & $0.28 \pm 0.13$ & M \\
\hline M10_4 & $4807 \pm 306$ & $5101 \pm 123$ & $5060 \pm 114$ & $1.8 \pm 0.6$ & $2.60 \pm 0.37$ & $2.38 \pm 0.31$ & $-1.66 \pm 0.29$ & $-1.80 \pm 0.25$ & $-1.74 \pm 0.19$ & $0.40 \pm 0.12$ & $0.26 \pm 0.13$ & M \\
\hline M10_5 & $4776 \pm 285$ & $5176 \pm 114$ & $5121 \pm 106$ & $1.7 \pm 0.5$ & $2.20 \pm 0.25$ & $2.10 \pm 0.22$ & $-1.62 \pm 0.32$ & $-1.50 \pm 0.1$ & $-1.51 \pm 0.10$ & $0.44 \pm 0.12$ & $0.19 \pm 0.14$ & M \\
\hline M10_6 & $4686 \pm 300$ & $4950 \pm 187$ & $4876 \pm 159$ & $1.5 \pm 0.6$ & $2.10 \pm 0.30$ & $1.98 \pm 0.27$ & $-1.62 \pm 0.31$ & $-1.70 \pm 0.24$ & $-1.67 \pm 0.19$ & $0.46 \pm 0.13$ & $0.25 \pm 0.10$ & $\mathrm{M}$ \\
\hline M10_7 & $4605 \pm 313$ & $5000 \pm 100$ & $4963 \pm 95$ & $1.3 \pm 0.5$ & $2.11 \pm 0.37$ & $1.82 \pm 0.30$ & $-1.52 \pm 0.25$ & $-1.50 \pm 0.1$ & $-1.50 \pm 0.09$ & $0.48 \pm 0.12$ & $0.25 \pm 0.14$ & M \\
\hline M10_8 & $4906 \pm 341$ & $5151 \pm 122$ & $5123 \pm 115$ & $2.0 \pm 0.7$ & $3.00 \pm 0.32$ & $2.83 \pm 0.29$ & $-1.61 \pm 0.30$ & $-1.70 \pm 0.24$ & $-1.66 \pm 0.19$ & $0.43 \pm 0.13$ & $0.23 \pm 0.16$ & M \\
\hline M10_9 & $4361 \pm 125$ & $4750 \pm 100$ & $4598 \pm 78$ & $0.90 \pm 0.31$ & $2.05 \pm 0.41$ & $1.32 \pm 0.25$ & $-1.53 \pm 0.21$ & $-1.50 \pm 0.1$ & $-1.51 \pm 0.09$ & $0.42 \pm 0.12$ & $0.22 \pm 0.13$ & $\mathrm{M}$ \\
\hline M10_10 & $4589 \pm 307$ & $5000 \pm 100$ & $4961 \pm 95$ & $1.3 \pm 0.5$ & $2.20 \pm 0.33$ & $1.93 \pm 0.28$ & $-1.57 \pm 0.28$ & $-1.50 \pm 0.1$ & $-1.51 \pm 0.09$ & $0.48 \pm 0.11$ & $0.21 \pm 0.15$ & M \\
\hline M10_11 & $4947 \pm 323$ & $5251 \pm 223$ & $5152 \pm 184$ & $2.1 \pm 0.7$ & $3.00 \pm 0.59$ & $2.63 \pm 0.45$ & $-1.62 \pm 0.30$ & $-1.70 \pm 0.24$ & $-1.67 \pm 0.19$ & $0.43 \pm 0.13$ & $0.14 \pm 0.09$ & M \\
\hline M10_13 & $4948 \pm 324$ & $5425 \pm 114$ & $5372 \pm 108$ & $2.1 \pm 0.7$ & $3.05 \pm 0.35$ & $2.86 \pm 0.31$ & $-1.64 \pm 0.29$ & $-1.60 \pm 0.20$ & $-1.61 \pm 0.16$ & $0.42 \pm 0.12$ & $0.18 \pm 0.12$ & M \\
\hline M10_15 & $4949 \pm 336$ & $5375 \pm 125$ & $5323 \pm 117$ & $2.1 \pm 0.7$ & $3.05 \pm 0.35$ & $2.86 \pm 0.31$ & $-1.69 \pm 0.30$ & $-1.65 \pm 0.23$ & $-1.66 \pm 0.18$ & $0.41 \pm 0.12$ & $0.23 \pm 0.16$ & M \\
\hline M10_16 & $4853 \pm 326$ & $5201 \pm 148$ & $5142 \pm 135$ & $1.8 \pm 0.5$ & $2.25 \pm 0.40$ & $2.07 \pm 0.31$ & $-1.59 \pm 0.34$ & $-1.55 \pm 0.15$ & $-1.56 \pm 0.14$ & $0.42 \pm 0.14$ & $0.23 \pm 0.13$ & \\
\hline M10_17 & $4970 \pm 338$ & $5425 \pm 115$ & $5378 \pm 109$ & $2.1 \pm 0.7$ & $3.20 \pm 0.25$ & $3.08 \pm 0.24$ & $-1.60 \pm 0.31$ & $-1.50 \pm 0.1$ & $-1.51 \pm 0.10$ & $0.43 \pm 0.13$ & $0.20 \pm 0.14$ & \\
\hline 6397_1 & $5006 \pm 354$ & $5250 \pm 100$ & $5232 \pm 96$ & $2.2 \pm 0.9$ & $2.60 \pm 0.49$ & $2.51 \pm 0.43$ & $-2.0 \pm 0.4$ & $-2.50 \pm 0.1$ & $-2.47 \pm 0.10$ & $0.40 \pm 0.19$ & $0.27 \pm 0.09$ & $\mathrm{M}$ \\
\hline 6397_2 & $5191 \pm 409$ & $5350 \pm 122$ & $5337 \pm 117$ & $2.5 \pm 0.9$ & $2.85 \pm 0.81$ & $2.69 \pm 0.60$ & $-2.1 \pm 0.4$ & $-2.50 \pm 0.1$ & $-2.48 \pm 0.10$ & $0.41 \pm 0.24$ & $0.05 \pm 0.07$ & \\
\hline 6397_4 & $5071 \pm 253$ & $5050 \pm 100$ & $5053 \pm 93$ & $2.3 \pm 0.7$ & $2.25 \pm 0.51$ & $2.27 \pm 0.41$ & $-2.0 \pm 0.4$ & $-2.40 \pm 0.20$ & $-2.32 \pm 0.18$ & $0.36 \pm 0.20$ & $0.21 \pm 0.13$ & $\mathrm{M}$ \\
\hline 6397_5 & $5137 \pm 330$ & $5500 \pm 100$ & $5469 \pm 96$ & $2.4 \pm 0.8$ & $3.40 \pm 0.37$ & $3.22 \pm 0.34$ & $-1.9 \pm 0.4$ & $-2.00 \pm 0.1$ & $-1.99 \pm 0.10$ & $0.41 \pm 0.18$ & $0.19 \pm 0.14$ & M \\
\hline
\end{tabular}


Table B.3 - continued.

\begin{tabular}{|c|c|c|c|c|c|c|c|c|c|c|c|c|}
\hline NGC ID & $\mathrm{T}_{e f f}^{(a)}(\mathrm{K})$ & $\mathrm{T}_{e f f}^{(b)}(\mathrm{K})$ & $\mathrm{T}_{e f f}^{(a v g)}(\mathrm{K})$ & $\log (g)^{(a)}$ & $\log (g)^{(b)}$ & $\log (g)^{(a v g)}$ & {$[\mathrm{Fe} / \mathrm{H}]^{(a)}$} & {$[\mathrm{Fe} / \mathrm{H}]^{(b)}$} & {$[\mathrm{Fe} / \mathrm{H}]^{(a v g)}$} & {$[\mathrm{Mg} / \mathrm{Fe}]^{(a)}$} & {$[\alpha / \mathrm{Fe}]^{(b)}$} & members \\
\hline 6397_6 & $5036 \pm 402$ & $5350 \pm 122$ & $5323 \pm 117$ & $2.2 \pm 0.8$ & $2.80 \pm 0.71$ & $2.54 \pm 0.53$ & $-1.9 \pm 0.4$ & $-2.30 \pm 0.24$ & $-2.19 \pm 0.21$ & $0.40 \pm 0.17$ & $0.18 \pm 0.12$ & M \\
\hline $6397 \_7$ & $5043 \pm 312$ & $5425 \pm 115$ & $5379 \pm 108$ & $2.2 \pm 0.8$ & $3.15 \pm 0.32$ & $3.02 \pm 0.30$ & $-1.9 \pm 0.4$ & $-2.00 \pm 0.1$ & $-1.99 \pm 0.10$ & $0.41 \pm 0.18$ & $0.25 \pm 0.12$ & M \\
\hline $6397 \_8$ & $4963 \pm 324$ & $5075 \pm 114$ & $5062 \pm 108$ & $2.1 \pm 0.8$ & $2.45 \pm 0.42$ & $2.37 \pm 0.37$ & $-2.0 \pm 0.4$ & $-2.35 \pm 0.23$ & $-2.26 \pm 0.20$ & $0.39 \pm 0.18$ & $0.20 \pm 0.13$ & M \\
\hline 6397_9 & $5115 \pm 284$ & $5250 \pm 100$ & $5235 \pm 94$ & $2.4 \pm 0.8$ & $2.50 \pm 0.39$ & $2.48 \pm 0.35$ & $-2.1 \pm 0.4$ & $-2.50 \pm 0.1$ & $-2.48 \pm 0.10$ & $0.35 \pm 0.26$ & $0.25 \pm 0.13$ & M \\
\hline 6397_10 & $4992 \pm 317$ & $5125 \pm 125$ & $5107 \pm 116$ & $2.2 \pm 0.8$ & $2.40 \pm 0.66$ & $2.32 \pm 0.51$ & $-2.0 \pm 0.4$ & $-2.30 \pm 0.25$ & $-2.22 \pm 0.21$ & $0.39 \pm 0.18$ & $0.24 \pm 0.12$ & M \\
\hline 6397_11 & $4906 \pm 332$ & $5250 \pm 100$ & $5221 \pm 96$ & $2.1 \pm 0.8$ & $2.60 \pm 0.37$ & $2.51 \pm 0.34$ & $-1.81 \pm 0.34$ & $-2.00 \pm 0.1$ & $-1.98 \pm 0.10$ & $0.43 \pm 0.13$ & $0.22 \pm 0.12$ & M \\
\hline 6397_13 & $5006 \pm 307$ & $5050 \pm 99$ & $5046 \pm 95$ & $2.3 \pm 0.7$ & $2.15 \pm 0.55$ & $2.21 \pm 0.43$ & $-1.8 \pm 0.4$ & $-2.40 \pm 0.20$ & $-2.28 \pm 0.18$ & $0.37 \pm 0.12$ & $0.22 \pm 0.12$ & M \\
\hline 6397_14 & $5105 \pm 278$ & $5225 \pm 74$ & $5217 \pm 72$ & $2.4 \pm 0.8$ & $2.85 \pm 0.45$ & $2.74 \pm 0.39$ & $-1.8 \pm 0.4$ & $-2.05 \pm 0.15$ & $-2.02 \pm 0.14$ & $0.38 \pm 0.20$ & $0.17 \pm 0.15$ & M \\
\hline 6397_14b & $4957 \pm 311$ & $5154 \pm 197$ & $5097 \pm 167$ & $2.2 \pm 0.7$ & $2.30 \pm 0.45$ & $2.27 \pm 0.38$ & $-1.70 \pm 0.26$ & $-2.10 \pm 0.20$ & $-1.95 \pm 0.16$ & $0.40 \pm 0.12$ & $0.30 \pm 0.10$ & M \\
\hline 6397_15 & $5042 \pm 363$ & $5450 \pm 100$ & $5421 \pm 96$ & $2.2 \pm 0.8$ & $3.15 \pm 0.45$ & $2.92 \pm 0.39$ & $-2.0 \pm 0.4$ & $-2.10 \pm 0.20$ & $-2.08 \pm 0.18$ & $0.40 \pm 0.17$ & $0.22 \pm 0.14$ & M \\
\hline 6397_16 & $4929 \pm 329$ & $5226 \pm 74$ & $5211 \pm 72$ & $2.1 \pm 0.8$ & $2.20 \pm 0.25$ & $2.19 \pm 0.24$ & $-1.75 \pm 0.27$ & $-2.00 \pm 0.1$ & $-1.97 \pm 0.09$ & $0.42 \pm 0.12$ & $0.18 \pm 0.14$ & M \\
\hline 6397_16b & $4797 \pm 306$ & $5000 \pm 100$ & $4980 \pm 95$ & $1.8 \pm 0.6$ & $2.00 \pm 0.39$ & $1.94 \pm 0.33$ & $-1.79 \pm 0.26$ & $-2.00 \pm 0.1$ & $-1.97 \pm 0.09$ & $0.42 \pm 0.12$ & $0.17 \pm 0.13$ & M \\
\hline 6397_19 & $5124 \pm 306$ & $5300 \pm 100$ & $5283 \pm 95$ & $2.4 \pm 0.8$ & $2.95 \pm 0.42$ & $2.83 \pm 0.37$ & $-2.0 \pm 0.4$ & $-2.40 \pm 0.20$ & $-2.32 \pm 0.18$ & $0.39 \pm 0.19$ & $0.20 \pm 0.13$ & M \\
\hline 6397_319 & $4849 \pm 93$ & $5125 \pm 125$ & $4947 \pm 75$ & $3.45 \pm 0.11$ & $4.35 \pm 0.23$ & $3.62 \pm 0.10$ & $0.09 \pm 0.06$ & $-0.06 \pm 0.23$ & $0.08 \pm 0.06$ & $-0.02 \pm 0.04$ & $0.14 \pm 0.09$ & \\
\hline 6397_344 & $3640 \pm 60$ & $3525 \pm 74$ & $3595 \pm 47$ & $0.70 \pm 0.13$ & $2.39 \pm 0.83$ & $0.74 \pm 0.13$ & $0.00 \pm 0.18$ & $-1.10 \pm 0.30$ & $-0.29 \pm 0.15$ & $0.23 \pm 0.10$ & $0.30 \pm 0.10$ & \\
\hline 6397_361 & $4617 \pm 198$ & $5000 \pm 100$ & $4922 \pm 89$ & $1.4 \pm 0.5$ & $2.05 \pm 0.41$ & $1.79 \pm 0.32$ & $-1.91 \pm 0.22$ & $-2.00 \pm 0.1$ & $-1.98 \pm 0.09$ & $0.44 \pm 0.13$ & $0.26 \pm 0.11$ & M \\
\hline 6397_365 & $5200 \pm 98$ & $4922 \pm 224$ & $5156 \pm 90$ & $2.63 \pm 0.13$ & $2.24 \pm 0.56$ & $2.61 \pm 0.13$ & $-1.60 \pm 0.09$ & $-2.35 \pm 0.23$ & $-1.70 \pm 0.08$ & $0.40 \pm 0.05$ & $0.25 \pm 0.10$ & M \\
\hline 6441_1 & $4514 \pm 148$ & $4600 \pm 165$ & $4552 \pm 110$ & $2.30 \pm 0.40$ & $2.50 \pm 0.50$ & $2.38 \pm 0.31$ & $0.06 \pm 0.09$ & $-0.33 \pm 0.26$ & $0.02 \pm 0.09$ & $0.02 \pm 0.13$ & $0.16 \pm 0.12$ & \\
\hline 6441_2 & $4314 \pm 137$ & $4424 \pm 225$ & $4344 \pm 117$ & $2.00 \pm 0.40$ & $2.25 \pm 0.56$ & $2.08 \pm 0.33$ & $0.08 \pm 0.16$ & $-0.29 \pm 0.32$ & $0.01 \pm 0.14$ & $0.00 \pm 0.14$ & $0.28 \pm 0.13$ & \\
\hline 6441_3 & $4507 \pm 157$ & $4601 \pm 166$ & $4551 \pm 114$ & $2.20 \pm 0.40$ & $2.50 \pm 0.45$ & $2.33 \pm 0.30$ & $-0.30 \pm 0.27$ & $-0.75 \pm 0.25$ & $-0.54 \pm 0.18$ & $0.16 \pm 0.19$ & $0.27 \pm 0.11$ & M \\
\hline 6441_4 & $4608 \pm 171$ & $4800 \pm 186$ & $4696 \pm 126$ & $2.50 \pm 0.40$ & $2.90 \pm 0.20$ & $2.82 \pm 0.18$ & $-0.12 \pm 0.20$ & $-0.40 \pm 0.37$ & $-0.18 \pm 0.18$ & $0.08 \pm 0.14$ & $0.23 \pm 0.13$ & M \\
\hline $6441 \_5$ & $4839 \pm 188$ & $4949 \pm 186$ & $4895 \pm 132$ & $2.60 \pm 0.40$ & $2.75 \pm 0.33$ & $2.69 \pm 0.25$ & $-0.29 \pm 0.29$ & $-0.60 \pm 0.37$ & $-0.41 \pm 0.23$ & $0.16 \pm 0.22$ & $0.24 \pm 0.14$ & M \\
\hline 6441_6 & $3894 \pm 74$ & $3643 \pm 198$ & $3863 \pm 69$ & $1.44 \pm 0.15$ & $0.67 \pm 0.68$ & $1.40 \pm 0.15$ & $-0.07 \pm 0.20$ & $-1.26 \pm 0.33$ & $-0.39 \pm 0.17$ & $0.09 \pm 0.20$ & $0.23 \pm 0.13$ & M \\
\hline 6441_7 & $4376 \pm 193$ & $4625 \pm 125$ & $4552 \pm 105$ & $2.10 \pm 0.50$ & $2.90 \pm 0.20$ & $2.79 \pm 0.19$ & $0.01 \pm 0.18$ & $-0.21 \pm 0.30$ & $-0.05 \pm 0.15$ & $0.03 \pm 0.14$ & $0.19 \pm 0.11$ & \\
\hline $6441 \_8$ & $4500 \pm 163$ & $4475 \pm 175$ & $4488 \pm 119$ & $2.20 \pm 0.40$ & $1.80 \pm 0.40$ & $2.00 \pm 0.28$ & $-0.08 \pm 0.24$ & $-0.65 \pm 0.23$ & $-0.38 \pm 0.17$ & $0.06 \pm 0.18$ & $0.26 \pm 0.10$ & M \\
\hline 6441_9 & $4571 \pm 193$ & $4725 \pm 175$ & $4655 \pm 130$ & $2.20 \pm 0.40$ & $1.90 \pm 0.43$ & $2.06 \pm 0.29$ & $-0.22 \pm 0.23$ & $-0.65 \pm 0.23$ & $-0.43 \pm 0.16$ & $0.08 \pm 0.15$ & $0.30 \pm 0.08$ & M \\
\hline 6441_10 & $4520 \pm 177$ & $4726 \pm 260$ & $4585 \pm 146$ & $2.30 \pm 0.40$ & $2.65 \pm 0.59$ & $2.41 \pm 0.33$ & $-0.23 \pm 0.27$ & $-0.45 \pm 0.35$ & $-0.31 \pm 0.21$ & $0.13 \pm 0.18$ & $0.27 \pm 0.09$ & M \\
\hline 6441_11 & $4369 \pm 179$ & $4750 \pm 158$ & $4584 \pm 118$ & $2.10 \pm 0.50$ & $2.50 \pm 0.32$ & $2.38 \pm 0.27$ & $0.27 \pm 0.09$ & $0.18 \pm 0.18$ & $0.25 \pm 0.08$ & $0.05 \pm 0.07$ & $0.21 \pm 0.11$ & \\
\hline
\end{tabular}


Table B.3 - continued.

\begin{tabular}{|c|c|c|c|c|c|c|c|c|c|c|c|c|}
\hline NGC ID & $\mathrm{T}_{e f f}^{(a)}(\mathrm{K})$ & $\mathrm{T}_{e f f}^{(b)}(\mathrm{K})$ & $\mathrm{T}_{e f f}^{(a v g)}(\mathrm{K})$ & $\log (g)^{(a)}$ & $\log (g)^{(b)}$ & $\log (g)^{(a v g)}$ & {$[\mathrm{Fe} / \mathrm{H}]^{(a)}$} & {$[\mathrm{Fe} / \mathrm{H}]^{(b)}$} & {$[\mathrm{Fe} / \mathrm{H}]^{(a v g)}$} & {$[\mathrm{Mg} / \mathrm{Fe}]^{(a)}$} & {$[\alpha / \mathrm{Fe}]^{(b)}$} & members \\
\hline 6441_13 & $4804 \pm 282$ & $4801 \pm 187$ & $4802 \pm 156$ & $2.50 \pm 0.60$ & $2.50 \pm 0.55$ & $2.50 \pm 0.41$ & $-0.40 \pm 0.40$ & $-0.80 \pm 0.25$ & $-0.69 \pm 0.21$ & $0.19 \pm 0.21$ & $0.23 \pm 0.13$ & M \\
\hline 6441_14 & $4222 \pm 162$ & $4475 \pm 174$ & $4339 \pm 119$ & $1.90 \pm 0.40$ & $2.65 \pm 0.39$ & $2.28 \pm 0.28$ & $-0.09 \pm 0.21$ & $-0.55 \pm 0.35$ & $-0.21 \pm 0.18$ & $0.01 \pm 0.14$ & $0.31 \pm 0.07$ & \\
\hline 6441_15 & $4075 \pm 147$ & $4324 \pm 114$ & $4230 \pm 90$ & $1.60 \pm 0.13$ & $2.40 \pm 0.37$ & $1.69 \pm 0.12$ & $0.01 \pm 0.17$ & $-0.35 \pm 0.23$ & $-0.12 \pm 0.14$ & $0.05 \pm 0.12$ & $0.20 \pm 0.11$ & \\
\hline 6441_16 & $4544 \pm 229$ & $4650 \pm 166$ & $4614 \pm 134$ & $2.40 \pm 0.50$ & $2.60 \pm 0.37$ & $2.53 \pm 0.30$ & $-0.19 \pm 0.27$ & $-0.70 \pm 0.33$ & $-0.39 \pm 0.21$ & $0.11 \pm 0.18$ & $0.30 \pm 0.10$ & \\
\hline 6441_17 & $4413 \pm 137$ & $4824 \pm 114$ & $4656 \pm 88$ & $2.10 \pm 0.40$ & $3.15 \pm 0.23$ & $2.89 \pm 0.20$ & $0.11 \pm 0.13$ & $0.21 \pm 0.21$ & $0.14 \pm 0.11$ & $0.03 \pm 0.15$ & $0.14 \pm 0.11$ & \\
\hline 6441_18 & $4267 \pm 150$ & $4400 \pm 122$ & $4347 \pm 95$ & $1.87 \pm 0.28$ & $2.45 \pm 0.42$ & $2.05 \pm 0.23$ & $0.13 \pm 0.18$ & $-0.20 \pm 0.24$ & $0.01 \pm 0.14$ & $-0.00 \pm 0.10$ & $0.13 \pm 0.11$ & \\
\hline 6441_19 & $4355 \pm 141$ & $4750 \pm 193$ & $4492 \pm 114$ & $1.90 \pm 0.50$ & $2.60 \pm 0.44$ & $2.29 \pm 0.33$ & $0.15 \pm 0.17$ & $0.15 \pm 0.27$ & $0.15 \pm 0.14$ & $0.02 \pm 0.17$ & $0.18 \pm 0.09$ & \\
\hline 6752_2 & $4904 \pm 382$ & $5101 \pm 123$ & $5082 \pm 117$ & $1.80 \pm 0.50$ & $2.26 \pm 0.33$ & $2.12 \pm 0.28$ & $-1.50 \pm 0.31$ & $-1.50 \pm 0.10$ & $-1.50 \pm 0.10$ & $0.45 \pm 0.15$ & $0.18 \pm 0.13$ & M \\
\hline 6752_4 & $4811 \pm 329$ & $5000 \pm 223$ & $4941 \pm 185$ & $1.80 \pm 0.60$ & $2.70 \pm 0.51$ & $2.32 \pm 0.39$ & $-1.58 \pm 0.31$ & $-1.70 \pm 0.24$ & $-1.66 \pm 0.19$ & $0.44 \pm 0.15$ & $0.25 \pm 0.10$ & M \\
\hline 6752_10 & $4960 \pm 433$ & $4952 \pm 245$ & $4954 \pm 213$ & $2.10 \pm 0.70$ & $2.25 \pm 0.64$ & $2.18 \pm 0.47$ & $-1.50 \pm 0.40$ & $-1.80 \pm 0.25$ & $-1.72 \pm 0.21$ & $0.44 \pm 0.15$ & $0.23 \pm 0.13$ & M \\
\hline 6752_11 & $4916 \pm 316$ & $5076 \pm 195$ & $5032 \pm 166$ & $1.80 \pm 0.40$ & $1.65 \pm 0.67$ & $1.76 \pm 0.34$ & $-1.57 \pm 0.30$ & $-1.75 \pm 0.25$ & $-1.68 \pm 0.19$ & $0.46 \pm 0.12$ & $0.20 \pm 0.11$ & M \\
\hline 6752_12 & $5487 \pm 383$ & $5499 \pm 224$ & $5496 \pm 193$ & $2.60 \pm 0.40$ & $1.89 \pm 0.74$ & $2.44 \pm 0.35$ & $-1.39 \pm 0.22$ & $-1.70 \pm 0.25$ & $-1.53 \pm 0.17$ & $0.54 \pm 0.11$ & $0.21 \pm 0.13$ & M \\
\hline 6752_16 & $3730 \pm 63$ & $3713 \pm 338$ & $3729 \pm 62$ & $0.90 \pm 0.14$ & $2.76 \pm 1.42$ & $0.92 \pm 0.14$ & $0.27 \pm 0.19$ & $-2.03 \pm 0.47$ & $-0.05 \pm 0.18$ & $0.08 \pm 0.10$ & $0.35 \pm 0.07$ & \\
\hline M15_2 & $5014 \pm 240$ & $5050 \pm 100$ & $5045 \pm 92$ & $2.20 \pm 0.60$ & $2.35 \pm 0.32$ & $2.32 \pm 0.28$ & $-2.20 \pm 0.40$ & $-2.50 \pm 0.10$ & $-2.48 \pm 0.10$ & $0.45 \pm 0.12$ & $0.18 \pm 0.15$ & M \\
\hline M15_3 & $4909 \pm 337$ & $4950 \pm 187$ & $4940 \pm 164$ & $2.00 \pm 0.80$ & $2.00 \pm 0.45$ & $2.00 \pm 0.39$ & $-1.90 \pm 0.35$ & $-2.40 \pm 0.20$ & $-2.28 \pm 0.17$ & $0.38 \pm 0.19$ & $0.25 \pm 0.14$ & M \\
\hline M15_4 & $4977 \pm 325$ & $5175 \pm 115$ & $5153 \pm 108$ & $2.10 \pm 0.80$ & $2.00 \pm 0.39$ & $2.02 \pm 0.35$ & $-2.00 \pm 0.40$ & $-2.50 \pm 0.10$ & $-2.47 \pm 0.10$ & $0.39 \pm 0.18$ & $0.20 \pm 0.13$ & M \\
\hline M15_5 & $4978 \pm 405$ & $5075 \pm 115$ & $5068 \pm 110$ & $2.10 \pm 0.80$ & $2.20 \pm 0.33$ & $2.19 \pm 0.31$ & $-1.80 \pm 0.40$ & $-2.50 \pm 0.10$ & $-2.46 \pm 0.10$ & $0.41 \pm 0.19$ & $0.27 \pm 0.09$ & M \\
\hline M15_6 & $4909 \pm 305$ & $5000 \pm 100$ & $4991 \pm 95$ & $1.90 \pm 0.70$ & $1.90 \pm 0.37$ & $1.90 \pm 0.33$ & $-2.07 \pm 0.35$ & $-2.50 \pm 0.10$ & $-2.47 \pm 0.10$ & $0.39 \pm 0.19$ & $0.21 \pm 0.13$ & M \\
\hline M15_7 & $4456 \pm 37$ & $4475 \pm 75$ & $4460 \pm 33$ & $1.11 \pm 0.10$ & $0.85 \pm 0.45$ & $1.10 \pm 0.10$ & $-2.12 \pm 0.02$ & $-2.50 \pm 0.10$ & $-2.13 \pm 0.02$ & $0.43 \pm 0.09$ & $0.26 \pm 0.13$ & M \\
\hline M15_8 & $4771 \pm 340$ & $4750 \pm 100$ & $4752 \pm 96$ & $1.70 \pm 0.70$ & $1.45 \pm 0.41$ & $1.51 \pm 0.35$ & $-2.00 \pm 0.40$ & $-2.50 \pm 0.10$ & $-2.47 \pm 0.10$ & $0.42 \pm 0.10$ & $0.23 \pm 0.13$ & M \\
\hline M15_9 & $4771 \pm 339$ & $4750 \pm 100$ & $4752 \pm 96$ & $1.70 \pm 0.70$ & $1.55 \pm 0.41$ & $1.59 \pm 0.35$ & $-2.00 \pm 0.40$ & $-2.50 \pm 0.10$ & $-2.47 \pm 0.10$ & $0.42 \pm 0.10$ & $0.16 \pm 0.12$ & M \\
\hline M15_10 & $4903 \pm 352$ & $5150 \pm 122$ & $5123 \pm 116$ & $2.00 \pm 0.80$ & $2.50 \pm 0.32$ & $2.43 \pm 0.30$ & $-1.80 \pm 0.40$ & $-2.20 \pm 0.24$ & $-2.09 \pm 0.21$ & $0.41 \pm 0.12$ & $0.25 \pm 0.10$ & M \\
\hline M15_12 & $4975 \pm 311$ & $5000 \pm 100$ & $4998 \pm 95$ & $2.10 \pm 0.70$ & $2.10 \pm 0.37$ & $2.10 \pm 0.33$ & $-2.10 \pm 0.40$ & $-2.50 \pm 0.10$ & $-2.48 \pm 0.10$ & $0.38 \pm 0.19$ & $0.19 \pm 0.13$ & M \\
\hline M15_13 & $5061 \pm 257$ & $5050 \pm 100$ & $5051 \pm 93$ & $2.30 \pm 0.60$ & $2.10 \pm 0.44$ & $2.17 \pm 0.35$ & $-2.00 \pm 0.40$ & $-2.50 \pm 0.10$ & $-2.47 \pm 0.10$ & $0.34 \pm 0.21$ & $0.28 \pm 0.10$ & M \\
\hline M15_14 & $5078 \pm 326$ & $5050 \pm 100$ & $5052 \pm 96$ & $2.30 \pm 0.80$ & $2.20 \pm 0.56$ & $2.23 \pm 0.46$ & $-1.80 \pm 0.40$ & $-2.40 \pm 0.20$ & $-2.28 \pm 0.18$ & $0.38 \pm 0.20$ & $0.24 \pm 0.12$ & M \\
\hline M15_15 & $4943 \pm 280$ & $5000 \pm 111$ & $4992 \pm 104$ & $2.00 \pm 0.60$ & $2.10 \pm 0.44$ & $2.07 \pm 0.35$ & $-1.90 \pm 0.40$ & $-2.45 \pm 0.15$ & $-2.38 \pm 0.14$ & $0.39 \pm 0.10$ & $0.25 \pm 0.10$ & M \\
\hline M15_17 & $4856 \pm 344$ & $4950 \pm 187$ & $4928 \pm 164$ & $2.00 \pm 0.80$ & $2.00 \pm 0.45$ & $2.00 \pm 0.39$ & $-1.86 \pm 0.34$ & $-2.40 \pm 0.20$ & $-2.26 \pm 0.17$ & $0.42 \pm 0.12$ & $0.27 \pm 0.09$ & M \\
\hline M15_18 & $5152 \pm 323$ & $5250 \pm 100$ & $5241 \pm 96$ & $2.50 \pm 0.80$ & $2.15 \pm 0.32$ & $2.20 \pm 0.30$ & $-2.10 \pm 0.40$ & $-2.50 \pm 0.10$ & $-2.48 \pm 0.10$ & $0.37 \pm 0.20$ & $0.19 \pm 0.14$ & M \\
\hline
\end{tabular}


Table B.3 - continued.

\begin{tabular}{|c|c|c|c|c|c|c|c|c|c|c|c|c|}
\hline NGC ID & $\mathrm{T}_{e f f}^{(a)}(\mathrm{K})$ & $\mathrm{T}_{e f f}^{(b)}(\mathrm{K})$ & $\mathrm{T}_{e f f}^{(a v g)}(\mathrm{K})$ & $\log (g)^{(a)}$ & $\log (g)^{(b)}$ & $\log (g)^{(\operatorname{avg})}$ & {$[\mathrm{Fe} / \mathrm{H}]^{(a)}$} & {$[\mathrm{Fe} / \mathrm{H}]^{(b)}$} & {$[\mathrm{Fe} / \mathrm{H}]^{(a v g)}$} & {$[\mathrm{Mg} / \mathrm{Fe}]^{(a)}$} & {$[\alpha / \mathrm{Fe}]^{(b)}$} & members \\
\hline 2298_11 & $4766 \pm 339$ & $4750 \pm 100$ & $4751 \pm 96$ & $1.70 \pm 0.60$ & $1.90 \pm 0.37$ & $1.84 \pm 0.31$ & $-1.64 \pm 0.34$ & $-2.00 \pm 0.10$ & $-1.97 \pm 0.10$ & $0.43 \pm 0.15$ & $0.19 \pm 0.14$ & M \\
\hline 2298_14 & $4694 \pm 329$ & $4775 \pm 74$ & $4771 \pm 73$ & $1.60 \pm 0.60$ & $1.70 \pm 0.33$ & $1.68 \pm 0.29$ & $-1.63 \pm 0.32$ & $-1.95 \pm 0.15$ & $-1.89 \pm 0.14$ & $0.45 \pm 0.13$ & $0.20 \pm 0.14$ & M \\
\hline 2298_16 & $5006 \pm 336$ & $5250 \pm 100$ & $5230 \pm 96$ & $2.20 \pm 0.80$ & $2.30 \pm 0.33$ & $2.29 \pm 0.31$ & $-1.80 \pm 0.40$ & $-2.00 \pm 0.10$ & $-1.99 \pm 0.10$ & $0.40 \pm 0.18$ & $0.19 \pm 0.14$ & M \\
\hline 2298_17 & $4589 \pm 327$ & $4799 \pm 188$ & $4747 \pm 163$ & $1.30 \pm 0.60$ & $2.05 \pm 0.52$ & $1.73 \pm 0.39$ & $-1.68 \pm 0.35$ & $-1.80 \pm 0.24$ & $-1.76 \pm 0.20$ & $0.47 \pm 0.12$ & $0.23 \pm 0.13$ & M \\
\hline 2298_18 & $4894 \pm 344$ & $5000 \pm 100$ & $4992 \pm 96$ & $2.00 \pm 0.70$ & $1.95 \pm 0.41$ & $1.96 \pm 0.35$ & $-1.70 \pm 0.40$ & $-2.00 \pm 0.10$ & $-1.98 \pm 0.10$ & $0.42 \pm 0.14$ & $0.14 \pm 0.11$ & M \\
\hline 4372_20 & $4882 \pm 356$ & $4625 \pm 125$ & $4653 \pm 118$ & $2.30 \pm 1.10$ & $1.80 \pm 0.71$ & $1.95 \pm 0.60$ & $-1.70 \pm 0.40$ & $-2.25 \pm 0.25$ & $-2.10 \pm 0.21$ & $0.39 \pm 0.14$ & $0.14 \pm 0.13$ & M \\
\hline 4372_14 & $4731 \pm 192$ & $4675 \pm 224$ & $4707 \pm 146$ & $1.80 \pm 0.50$ & $2.30 \pm 0.51$ & $2.05 \pm 0.36$ & $-1.70 \pm 0.40$ & $-2.05 \pm 0.27$ & $-1.94 \pm 0.22$ & $0.40 \pm 0.16$ & $0.15 \pm 0.10$ & M \\
\hline 4372_13 & $4392 \pm 179$ & $4423 \pm 116$ & $4414 \pm 97$ & $1.00 \pm 0.50$ & $1.05 \pm 0.27$ & $1.04 \pm 0.24$ & $-1.82 \pm 0.27$ & $-2.15 \pm 0.23$ & $-2.01 \pm 0.18$ & $0.48 \pm 0.12$ & $0.24 \pm 0.12$ & M \\
\hline 4372_95 & $4984 \pm 371$ & $4750 \pm 100$ & $4766 \pm 97$ & $2.00 \pm 0.90$ & $1.84 \pm 0.32$ & $1.86 \pm 0.30$ & $-2.00 \pm 0.40$ & $-2.50 \pm 0.10$ & $-2.47 \pm 0.10$ & $0.43 \pm 0.09$ & $0.17 \pm 0.15$ & M \\
\hline 4372_91 & $4890 \pm 336$ & $4925 \pm 114$ & $4922 \pm 108$ & $2.10 \pm 0.80$ & $2.05 \pm 0.35$ & $2.06 \pm 0.32$ & $-1.82 \pm 0.34$ & $-2.15 \pm 0.23$ & $-2.05 \pm 0.19$ & $0.43 \pm 0.12$ & $0.27 \pm 0.11$ & M \\
\hline 4372_89 & $4828 \pm 333$ & $4977 \pm 236$ & $4927 \pm 193$ & $1.80 \pm 0.70$ & $2.20 \pm 0.40$ & $2.10 \pm 0.35$ & $-1.90 \pm 0.40$ & $-2.30 \pm 0.25$ & $-2.19 \pm 0.21$ & $0.41 \pm 0.10$ & $0.29 \pm 0.09$ & M \\
\hline $4372 \_85$ & $5235 \pm 360$ & $5575 \pm 115$ & $5544 \pm 109$ & $2.50 \pm 0.80$ & $3.55 \pm 0.52$ & $3.24 \pm 0.44$ & $-2.34 \pm 0.25$ & $-2.50 \pm 0.10$ & $-2.48 \pm 0.09$ & $0.35 \pm 0.26$ & $0.10 \pm 0.10$ & \\
\hline 4372_74 & $4677 \pm 217$ & $3524 \pm 73$ & $3642 \pm 70$ & $1.70 \pm 0.60$ & $0.10 \pm 0.20$ & $0.26 \pm 0.19$ & $-1.70 \pm 0.40$ & $-2.35 \pm 0.23$ & $-2.19 \pm 0.20$ & $0.31 \pm 0.09$ & $0.14 \pm 0.13$ & M \\
\hline 4372_76 & $4679 \pm 288$ & $4850 \pm 229$ & $4784 \pm 179$ & $1.50 \pm 0.50$ & $2.00 \pm 0.39$ & $1.81 \pm 0.31$ & $-1.90 \pm 0.25$ & $-2.15 \pm 0.23$ & $-2.04 \pm 0.17$ & $0.42 \pm 0.12$ & $0.23 \pm 0.11$ & M \\
\hline $4372 \_77$ & $4993 \pm 365$ & $5024 \pm 74$ & $5023 \pm 73$ & $2.20 \pm 0.80$ & $3.20 \pm 0.33$ & $3.05 \pm 0.31$ & $-1.90 \pm 0.40$ & $-2.50 \pm 0.10$ & $-2.46 \pm 0.10$ & $0.39 \pm 0.17$ & $0.24 \pm 0.13$ & \\
\hline Rup106_2 & $4830 \pm 151$ & $4921 \pm 116$ & $4887 \pm 92$ & $3.10 \pm 0.50$ & $3.54 \pm 0.42$ & $3.36 \pm 0.32$ & $-0.14 \pm 0.14$ & $-0.50 \pm 0.10$ & $-0.38 \pm 0.08$ & $0.12 \pm 0.11$ & $0.26 \pm 0.11$ & \\
\hline Rup106_3 & $4780 \pm 373$ & $5000 \pm 250$ & $4932 \pm 208$ & $1.70 \pm 0.60$ & $1.95 \pm 0.35$ & $1.89 \pm 0.30$ & $-1.60 \pm 0.40$ & $-1.75 \pm 0.25$ & $-1.71 \pm 0.21$ & $0.43 \pm 0.16$ & $0.10 \pm 0.08$ & M \\
\hline Rup106_4 & $4341 \pm 121$ & $4750 \pm 100$ & $4584 \pm 77$ & $0.86 \pm 0.32$ & $1.85 \pm 0.39$ & $1.26 \pm 0.25$ & $-1.56 \pm 0.22$ & $-1.50 \pm 0.10$ & $-1.51 \pm 0.09$ & $0.42 \pm 0.13$ & $0.16 \pm 0.14$ & \\
\hline Rup106_5 & $5013 \pm 266$ & $5350 \pm 123$ & $5291 \pm 111$ & $2.90 \pm 0.50$ & $3.60 \pm 0.37$ & $3.35 \pm 0.30$ & $-0.47 \pm 0.27$ & $-0.50 \pm 0.10$ & $-0.50 \pm 0.09$ & $0.26 \pm 0.20$ & $0.25 \pm 0.14$ & \\
\hline Rup106_6 & $4673 \pm 461$ & $4800 \pm 245$ & $4772 \pm 216$ & $1.50 \pm 0.80$ & $1.95 \pm 0.41$ & $1.86 \pm 0.36$ & $-1.60 \pm 0.50$ & $-1.70 \pm 0.24$ & $-1.68 \pm 0.22$ & $0.40 \pm 0.20$ & $0.14 \pm 0.11$ & M \\
\hline Rup106_7 & $4713 \pm 159$ & $4949 \pm 150$ & $4838 \pm 109$ & $2.80 \pm 0.50$ & $3.25 \pm 0.33$ & $3.11 \pm 0.28$ & $-0.01 \pm 0.09$ & $-0.15 \pm 0.23$ & $-0.03 \pm 0.08$ & $0.06 \pm 0.07$ & $0.25 \pm 0.10$ & \\
\hline Rup106_8 & $4864 \pm 198$ & $5028 \pm 302$ & $4913 \pm 166$ & $2.80 \pm 0.50$ & $2.81 \pm 0.45$ & $2.81 \pm 0.33$ & $-0.52 \pm 0.28$ & $-0.90 \pm 0.37$ & $-0.66 \pm 0.22$ & $0.27 \pm 0.19$ & $0.30 \pm 0.10$ & \\
\hline Rup106_9 & $4638 \pm 286$ & $4899 \pm 229$ & $4797 \pm 179$ & $1.50 \pm 0.60$ & $1.95 \pm 0.41$ & $1.81 \pm 0.34$ & $-1.70 \pm 0.40$ & $-1.85 \pm 0.23$ & $-1.81 \pm 0.20$ & $0.43 \pm 0.19$ & $0.14 \pm 0.13$ & M \\
\hline Rup106_10 & $4917 \pm 310$ & $5152 \pm 122$ & $5120 \pm 114$ & $1.90 \pm 0.50$ & $2.40 \pm 0.38$ & $2.22 \pm 0.30$ & $-1.54 \pm 0.34$ & $-1.70 \pm 0.24$ & $-1.65 \pm 0.20$ & $0.43 \pm 0.15$ & $0.09 \pm 0.07$ & M \\
\hline Rup106_11 & $4417 \pm 193$ & $4950 \pm 100$ & $4838 \pm 89$ & $1.00 \pm 0.60$ & $2.10 \pm 0.30$ & $1.88 \pm 0.27$ & $-1.61 \pm 0.20$ & $-1.50 \pm 0.10$ & $-1.52 \pm 0.09$ & $0.51 \pm 0.09$ & $0.14 \pm 0.13$ & M \\
\hline Rup106_12 & $4856 \pm 391$ & $5225 \pm 75$ & $5212 \pm 73$ & $1.80 \pm 0.60$ & $2.20 \pm 0.25$ & $2.14 \pm 0.23$ & $-1.60 \pm 0.40$ & $-1.50 \pm 0.10$ & $-1.51 \pm 0.10$ & $0.45 \pm 0.15$ & $0.19 \pm 0.15$ & $\mathrm{M}$ \\
\hline Rup106_14 & $4904 \pm 335$ & $5250 \pm 100$ & $5222 \pm 96$ & $1.90 \pm 0.60$ & $2.55 \pm 0.47$ & $2.30 \pm 0.37$ & $-1.58 \pm 0.32$ & $-1.50 \pm 0.10$ & $-1.51 \pm 0.10$ & $0.41 \pm 0.14$ & $0.12 \pm 0.10$ & M \\
\hline
\end{tabular}


Table B.3 - continued.

\begin{tabular}{|c|c|c|c|c|c|c|c|c|c|c|c|c|}
\hline NGC ID & $\mathrm{T}_{e f f}^{(a)}(\mathrm{K})$ & $\mathrm{T}_{e f f}^{(b)}(\mathrm{K})$ & $\mathrm{T}_{e f f}^{(a v g)}(\mathrm{K})$ & $\log (g)^{(a)}$ & $\log (g)^{(b)}$ & $\log (g)^{(a v g)}$ & {$[\mathrm{Fe} / \mathrm{H}]^{(a)}$} & {$[\mathrm{Fe} / \mathrm{H}]^{(b)}$} & {$[\mathrm{Fe} / \mathrm{H}]^{(a v g)}$} & {$[\mathrm{Mg} / \mathrm{Fe}]^{(a)}$} & {$[\alpha / \mathrm{Fe}]^{(b)}$} & members \\
\hline Rup106_16 & $5050 \pm 6$ & $5226 \pm 207$ & $5050 \pm 6$ & $3.49 \pm 0.32$ & $4.55 \pm 0.35$ & $3.97 \pm 0.24$ & $-0.10 \pm 0.04$ & $-0.60 \pm 0.20$ & $-0.12 \pm 0.04$ & $0.24 \pm 0.07$ & $0.12 \pm 0.11$ & \multirow{3}{*}{$\mathrm{M}$} \\
\hline Rup106_17 & $4803 \pm 448$ & $5100 \pm 122$ & $5079 \pm 118$ & $1.70 \pm 0.70$ & $2.20 \pm 0.40$ & $2.08 \pm 0.35$ & $-1.50 \pm 0.50$ & $-1.50 \pm 0.10$ & $-1.50 \pm 0.10$ & $0.45 \pm 0.17$ & $0.11 \pm 0.09$ & \\
\hline Rup106_18 & $4761 \pm 355$ & $5350 \pm 165$ & $5246 \pm 150$ & $1.90 \pm 0.90$ & $3.35 \pm 0.39$ & $3.12 \pm 0.36$ & $-1.30 \pm 0.40$ & $-1.05 \pm 0.15$ & $-1.08 \pm 0.14$ & $0.46 \pm 0.14$ & $0.22 \pm 0.10$ & \\
\hline 5634_6 & $4815 \pm 349$ & $4899 \pm 229$ & $4874 \pm 191$ & $1.80 \pm 0.60$ & $2.00 \pm 0.39$ & $1.94 \pm 0.33$ & $-1.61 \pm 0.33$ & $-1.85 \pm 0.23$ & $-1.77 \pm 0.19$ & $0.43 \pm 0.14$ & $0.19 \pm 0.11$ & M \\
\hline $5634 \_7$ & $4867 \pm 353$ & $5000 \pm 112$ & $4988 \pm 106$ & $1.90 \pm 0.70$ & $2.25 \pm 0.40$ & $2.16 \pm 0.35$ & $-1.70 \pm 0.40$ & $-1.95 \pm 0.15$ & $-1.92 \pm 0.14$ & $0.42 \pm 0.14$ & $0.19 \pm 0.14$ & M \\
\hline $5634 \_8$ & $4902 \pm 344$ & $5175 \pm 160$ & $5127 \pm 145$ & $2.00 \pm 0.70$ & $2.15 \pm 0.32$ & $2.12 \pm 0.29$ & $-1.61 \pm 0.33$ & $-1.60 \pm 0.20$ & $-1.60 \pm 0.17$ & $0.41 \pm 0.14$ & $0.25 \pm 0.14$ & M \\
\hline 5634_9 & $4921 \pm 334$ & $5100 \pm 122$ & $5079 \pm 115$ & $2.00 \pm 0.70$ & $2.55 \pm 0.42$ & $2.40 \pm 0.36$ & $-1.61 \pm 0.31$ & $-1.80 \pm 0.24$ & $-1.73 \pm 0.19$ & $0.42 \pm 0.13$ & $0.22 \pm 0.13$ & M \\
\hline 5634_11 & $4389 \pm 177$ & $4676 \pm 114$ & $4591 \pm 96$ & $1.00 \pm 0.50$ & $1.50 \pm 0.39$ & $1.31 \pm 0.31$ & $-1.55 \pm 0.20$ & $-1.65 \pm 0.23$ & $-1.59 \pm 0.15$ & $0.45 \pm 0.14$ & $0.24 \pm 0.12$ & M \\
\hline 5634_13 & $4981 \pm 345$ & $5200 \pm 187$ & $5150 \pm 164$ & $2.10 \pm 0.80$ & $2.70 \pm 0.46$ & $2.55 \pm 0.40$ & $-1.80 \pm 0.40$ & $-1.90 \pm 0.20$ & $-1.88 \pm 0.18$ & $0.40 \pm 0.17$ & $0.10 \pm 0.12$ & M \\
\hline 5634_14 & $4767 \pm 350$ & $4775 \pm 74$ & $4774 \pm 73$ & $1.70 \pm 0.60$ & $1.65 \pm 0.39$ & $1.66 \pm 0.33$ & $-1.60 \pm 0.33$ & $-1.95 \pm 0.15$ & $-1.89 \pm 0.14$ & $0.45 \pm 0.14$ & $0.24 \pm 0.11$ & M \\
\hline 5634_15 & $4338 \pm 119$ & $4599 \pm 201$ & $4406 \pm 102$ & $0.85 \pm 0.32$ & $1.45 \pm 0.52$ & $1.01 \pm 0.27$ & $-1.56 \pm 0.22$ & $-1.60 \pm 0.20$ & $-1.58 \pm 0.15$ & $0.42 \pm 0.13$ & $0.20 \pm 0.12$ & M \\
\hline 5634_17 & $4769 \pm 56$ & $5250 \pm 193$ & $4806 \pm 54$ & $2.98 \pm 0.33$ & $4.65 \pm 0.39$ & $3.68 \pm 0.25$ & $-0.01 \pm 0.07$ & $-0.25 \pm 0.25$ & $-0.03 \pm 0.07$ & $0.16 \pm 0.13$ & $0.10 \pm 0.08$ & \\
\hline 5694_3 & $4849 \pm 56$ & $4874 \pm 167$ & $4852 \pm 53$ & $3.45 \pm 0.33$ & $4.35 \pm 0.32$ & $3.91 \pm 0.23$ & $0.09 \pm 0.07$ & $-0.60 \pm 0.20$ & $0.01 \pm 0.07$ & $-0.02 \pm 0.13$ & $0.26 \pm 0.11$ & \\
\hline $5694 \_5$ & $4877 \pm 340$ & $4950 \pm 100$ & $4944 \pm 96$ & $1.80 \pm 0.60$ & $1.55 \pm 0.42$ & $1.63 \pm 0.34$ & $-1.69 \pm 0.33$ & $-2.00 \pm 0.10$ & $-1.97 \pm 0.10$ & $0.42 \pm 0.12$ & $0.14 \pm 0.11$ & M \\
\hline 5694_6 & $4972 \pm 360$ & $5175 \pm 114$ & $5157 \pm 109$ & $2.10 \pm 0.70$ & $2.10 \pm 0.30$ & $2.10 \pm 0.28$ & $-1.80 \pm 0.40$ & $-2.00 \pm 0.10$ & $-1.99 \pm 0.10$ & $0.40 \pm 0.17$ & $0.13 \pm 0.11$ & $\mathrm{M}$ \\
\hline $5694 \_7$ & $4970 \pm 388$ & $4925 \pm 114$ & $4929 \pm 110$ & $2.10 \pm 0.80$ & $1.80 \pm 0.60$ & $1.91 \pm 0.48$ & $-1.80 \pm 0.40$ & $-2.15 \pm 0.23$ & $-2.06 \pm 0.20$ & $0.40 \pm 0.18$ & $0.18 \pm 0.12$ & M \\
\hline 5694_8 & $4981 \pm 330$ & $5225 \pm 75$ & $5213 \pm 73$ & $2.10 \pm 0.80$ & $2.20 \pm 0.25$ & $2.19 \pm 0.24$ & $-1.90 \pm 0.40$ & $-2.00 \pm 0.10$ & $-1.99 \pm 0.10$ & $0.40 \pm 0.17$ & $0.19 \pm 0.15$ & M \\
\hline 5694_9 & $4664 \pm 320$ & $4750 \pm 100$ & $4742 \pm 95$ & $1.50 \pm 0.60$ & $1.45 \pm 0.42$ & $1.47 \pm 0.34$ & $-1.71 \pm 0.32$ & $-2.00 \pm 0.10$ & $-1.97 \pm 0.10$ & $0.45 \pm 0.12$ & $0.23 \pm 0.13$ & M \\
\hline 5694_10 & $5012 \pm 430$ & $5175 \pm 114$ & $5164 \pm 111$ & $2.10 \pm 0.80$ & $2.05 \pm 0.35$ & $2.06 \pm 0.32$ & $-1.80 \pm 0.40$ & $-2.00 \pm 0.10$ & $-1.99 \pm 0.10$ & $0.41 \pm 0.17$ & $0.10 \pm 0.10$ & \\
\hline 5694_11 & $4935 \pm 331$ & $5175 \pm 115$ & $5149 \pm 108$ & $2.00 \pm 0.70$ & $2.25 \pm 0.25$ & $2.22 \pm 0.24$ & $-1.80 \pm 0.40$ & $-2.00 \pm 0.10$ & $-1.99 \pm 0.10$ & $0.39 \pm 0.17$ & $0.15 \pm 0.14$ & M \\
\hline 5694_13 & $4643 \pm 82$ & $4874 \pm 125$ & $4712 \pm 69$ & $3.00 \pm 0.40$ & $4.30 \pm 0.25$ & $3.93 \pm 0.21$ & $0.30 \pm 0.19$ & $0.05 \pm 0.20$ & $0.18 \pm 0.14$ & $0.18 \pm 0.07$ & $0.10 \pm 0.08$ & \\
\hline 5694_15 & $4925 \pm 329$ & $5200 \pm 100$ & $5177 \pm 96$ & $2.00 \pm 0.70$ & $2.20 \pm 0.25$ & $2.18 \pm 0.24$ & $-1.80 \pm 0.35$ & $-2.00 \pm 0.10$ & $-1.98 \pm 0.10$ & $0.41 \pm 0.13$ & $0.16 \pm 0.14$ & M \\
\hline 5694_17 & $4821 \pm 323$ & $4875 \pm 125$ & $4868 \pm 117$ & $1.80 \pm 0.60$ & $2.05 \pm 0.35$ & $1.99 \pm 0.30$ & $-1.68 \pm 0.28$ & $-2.00 \pm 0.10$ & $-1.96 \pm 0.09$ & $0.41 \pm 0.11$ & $0.20 \pm 0.13$ & M \\
\hline 5897_138 & $5078 \pm 420$ & $5050 \pm 245$ & $5057 \pm 212$ & $2.20 \pm 0.80$ & $2.05 \pm 0.42$ & $2.08 \pm 0.37$ & $-1.60 \pm 0.40$ & $-2.20 \pm 0.24$ & $-2.04 \pm 0.21$ & $0.44 \pm 0.15$ & $0.26 \pm 0.13$ & $\mathrm{M}$ \\
\hline 5897_142 & $4720 \pm 380$ & $4400 \pm 164$ & $4450 \pm 151$ & $1.60 \pm 0.70$ & $1.20 \pm 0.46$ & $1.32 \pm 0.38$ & $-1.60 \pm 0.40$ & $-2.25 \pm 0.25$ & $-2.07 \pm 0.21$ & $0.43 \pm 0.16$ & $0.28 \pm 0.13$ & M \\
\hline 5897_160 & $4587 \pm 335$ & $4426 \pm 114$ & $4442 \pm 108$ & $1.30 \pm 0.60$ & $1.50 \pm 0.39$ & $1.44 \pm 0.33$ & $-1.70 \pm 0.40$ & $-2.15 \pm 0.23$ & $-2.04 \pm 0.20$ & $0.44 \pm 0.14$ & $0.20 \pm 0.14$ & M \\
\hline 5897_255 & $4301 \pm 42$ & $4250 \pm 100$ & $4293 \pm 39$ & $0.86 \pm 0.31$ & $0.66 \pm 0.32$ & $0.76 \pm 0.22$ & $-1.54 \pm 0.22$ & $-2.00 \pm 0.10$ & $-1.92 \pm 0.09$ & $0.38 \pm 0.13$ & $0.23 \pm 0.15$ & M \\
\hline
\end{tabular}


Table B.3 - continued.

\begin{tabular}{|c|c|c|c|c|c|c|c|c|c|c|c|c|}
\hline NGC ID & $\mathrm{T}_{e f f}^{(a)}(\mathrm{K})$ & $\mathrm{T}_{e f f}^{(b)}(\mathrm{K})$ & $\mathrm{T}_{e f f}^{(a v g)}(\mathrm{K})$ & $\log (g)^{(a)}$ & $\log (g)^{(b)}$ & $\log (g)^{(a v g)}$ & {$[\mathrm{Fe} / \mathrm{H}]^{(a)}$} & {$[\mathrm{Fe} / \mathrm{H}]^{(b)}$} & {$[\mathrm{Fe} / \mathrm{H}]^{(a v g)}$} & {$[\mathrm{Mg} / \mathrm{Fe}]^{(a)}$} & {$[\alpha / \mathrm{Fe}]^{(b)}$} & members \\
\hline 5897_302 & $4583 \pm 218$ & $4750 \pm 100$ & $4721 \pm 91$ & $1.40 \pm 0.50$ & $1.60 \pm 0.49$ & $1.50 \pm 0.35$ & $-1.87 \pm 0.31$ & $-2.00 \pm 0.10$ & $-1.99 \pm 0.10$ & $0.42 \pm 0.14$ & $0.24 \pm 0.11$ & M \\
\hline 5897_306 & $4971 \pm 473$ & $4750 \pm 100$ & $4759 \pm 98$ & $2.00 \pm 0.80$ & $1.90 \pm 0.37$ & $1.92 \pm 0.34$ & $-1.50 \pm 0.40$ & $-2.00 \pm 0.10$ & $-1.97 \pm 0.10$ & $0.43 \pm 0.17$ & $0.19 \pm 0.14$ & M \\
\hline 5897_316 & $4590 \pm 310$ & $4675 \pm 115$ & $4665 \pm 108$ & $1.30 \pm 0.60$ & $1.80 \pm 0.56$ & $1.57 \pm 0.41$ & $-1.71 \pm 0.34$ & $-2.00 \pm 0.10$ & $-1.98 \pm 0.10$ & $0.46 \pm 0.13$ & $0.24 \pm 0.09$ & M \\
\hline 5897_318 & $4930 \pm 420$ & $4799 \pm 148$ & $4813 \pm 140$ & $2.00 \pm 0.70$ & $2.00 \pm 0.39$ & $2.00 \pm 0.34$ & $-1.50 \pm 0.40$ & $-1.95 \pm 0.15$ & $-1.89 \pm 0.14$ & $0.43 \pm 0.15$ & $0.22 \pm 0.13$ & M \\
\hline 5927_37 & $4432 \pm 120$ & $4547 \pm 98$ & $4501 \pm 76$ & $2.10 \pm 0.40$ & $2.09 \pm 0.37$ & $2.09 \pm 0.27$ & $-0.10 \pm 0.21$ & $-0.41 \pm 0.20$ & $-0.26 \pm 0.14$ & $0.08 \pm 0.16$ & $0.20 \pm 0.14$ & M \\
\hline 5927_65 & $3927 \pm 20$ & $3997 \pm 159$ & $3928 \pm 20$ & $1.49 \pm 0.08$ & $1.54 \pm 0.65$ & $1.49 \pm 0.08$ & $-0.18 \pm 0.03$ & $-1.01 \pm 0.32$ & $-0.19 \pm 0.03$ & $0.01 \pm 0.09$ & $0.33 \pm 0.08$ & M \\
\hline 5927_133 & $4018 \pm 117$ & $4025 \pm 174$ & $4020 \pm 97$ & $1.51 \pm 0.32$ & $1.60 \pm 0.62$ & $1.53 \pm 0.28$ & $-0.20 \pm 0.20$ & $-1.00 \pm 0.31$ & $-0.44 \pm 0.17$ & $0.08 \pm 0.16$ & $0.29 \pm 0.09$ & M \\
\hline $5927 \_372$ & $3669 \pm 42$ & $3524 \pm 73$ & $3633 \pm 36$ & $0.76 \pm 0.09$ & $2.35 \pm 0.63$ & $0.79 \pm 0.09$ & $0.05 \pm 0.15$ & $-0.95 \pm 0.15$ & $-0.45 \pm 0.11$ & $0.18 \pm 0.07$ & $0.29 \pm 0.09$ & M \\
\hline 5927_335 & $3886 \pm 117$ & $3924 \pm 160$ & $3899 \pm 94$ & $1.31 \pm 0.19$ & $2.25 \pm 0.60$ & $1.40 \pm 0.18$ & $0.05 \pm 0.19$ & $-0.65 \pm 0.32$ & $-0.13 \pm 0.16$ & $0.05 \pm 0.22$ & $0.29 \pm 0.11$ & M \\
\hline 5927_190 & $3938 \pm 29$ & $3843 \pm 278$ & $3937 \pm 29$ & $1.49 \pm 0.07$ & $1.08 \pm 0.94$ & $1.49 \pm 0.07$ & $-0.16 \pm 0.07$ & $-1.16 \pm 0.39$ & $-0.19 \pm 0.07$ & $0.03 \pm 0.09$ & $0.31 \pm 0.10$ & M \\
\hline 5946_1 & $4285 \pm 163$ & $4450 \pm 186$ & $4356 \pm 123$ & $1.90 \pm 0.40$ & $2.40 \pm 0.37$ & $2.17 \pm 0.27$ & $0.09 \pm 0.20$ & $-0.16 \pm 0.29$ & $0.01 \pm 0.16$ & $0.03 \pm 0.13$ & $0.19 \pm 0.13$ & \\
\hline 5946_2 & $4796 \pm 229$ & $5049 \pm 312$ & $4885 \pm 185$ & $2.50 \pm 0.50$ & $2.80 \pm 0.51$ & $2.65 \pm 0.36$ & $-0.26 \pm 0.31$ & $-0.55 \pm 0.41$ & $-0.36 \pm 0.25$ & $0.16 \pm 0.22$ & $0.22 \pm 0.12$ & \\
\hline 5946_3 & $4414 \pm 175$ & $4701 \pm 99$ & $4631 \pm 86$ & $2.20 \pm 0.50$ & $2.90 \pm 0.20$ & $2.80 \pm 0.19$ & $0.05 \pm 0.13$ & $-0.04 \pm 0.24$ & $0.03 \pm 0.11$ & $0.02 \pm 0.14$ & $0.18 \pm 0.12$ & \\
\hline 5946_4 & $4960 \pm 487$ & $4975 \pm 208$ & $4973 \pm 191$ & $2.10 \pm 0.90$ & $1.45 \pm 0.52$ & $1.61 \pm 0.45$ & $-1.60 \pm 0.40$ & $-1.85 \pm 0.23$ & $-1.79 \pm 0.20$ & $0.42 \pm 0.18$ & $0.18 \pm 0.11$ & \\
\hline $5946 \_5$ & $4725 \pm 418$ & $4701 \pm 100$ & $4702 \pm 97$ & $1.80 \pm 0.90$ & $1.60 \pm 0.37$ & $1.63 \pm 0.34$ & $-1.40 \pm 0.50$ & $-1.60 \pm 0.20$ & $-1.57 \pm 0.19$ & $0.40 \pm 0.16$ & $0.21 \pm 0.14$ & M \\
\hline $5946 \_6$ & $4866 \pm 451$ & $4950 \pm 100$ & $4946 \pm 98$ & $1.90 \pm 0.80$ & $2.00 \pm 0.45$ & $1.98 \pm 0.39$ & $-1.60 \pm 0.40$ & $-2.00 \pm 0.10$ & $-1.98 \pm 0.10$ & $0.41 \pm 0.18$ & $0.25 \pm 0.10$ & \\
\hline $5946 \_7$ & $4619 \pm 325$ & $4876 \pm 201$ & $4805 \pm 171$ & $1.50 \pm 0.60$ & $1.95 \pm 0.42$ & $1.80 \pm 0.34$ & $-1.52 \pm 0.28$ & $-1.60 \pm 0.20$ & $-1.57 \pm 0.16$ & $0.45 \pm 0.15$ & $0.29 \pm 0.10$ & M \\
\hline $5946 \_8$ & $4743 \pm 418$ & $4675 \pm 114$ & $4680 \pm 110$ & $1.90 \pm 0.90$ & $1.60 \pm 0.37$ & $1.64 \pm 0.34$ & $-1.30 \pm 0.50$ & $-1.65 \pm 0.23$ & $-1.59 \pm 0.21$ & $0.40 \pm 0.17$ & $0.24 \pm 0.12$ & $\mathrm{M}$ \\
\hline 5946_9 & $4748 \pm 353$ & $5000 \pm 100$ & $4981 \pm 96$ & $1.70 \pm 0.70$ & $2.10 \pm 0.37$ & $2.01 \pm 0.33$ & $-1.59 \pm 0.33$ & $-1.50 \pm 0.10$ & $-1.51 \pm 0.10$ & $0.44 \pm 0.14$ & $0.19 \pm 0.13$ & M \\
\hline 5946_10 & $4941 \pm 529$ & $4675 \pm 160$ & $4698 \pm 153$ & $2.00 \pm 0.90$ & $1.55 \pm 0.61$ & $1.69 \pm 0.50$ & $-1.50 \pm 0.50$ & $-2.10 \pm 0.20$ & $-2.02 \pm 0.19$ & $0.42 \pm 0.18$ & $0.24 \pm 0.14$ & \\
\hline 5946_11 & $4919 \pm 474$ & $4825 \pm 115$ & $4830 \pm 111$ & $2.00 \pm 0.90$ & $1.90 \pm 0.37$ & $1.91 \pm 0.34$ & $-1.57 \pm 0.34$ & $-2.00 \pm 0.10$ & $-1.97 \pm 0.10$ & $0.45 \pm 0.15$ & $0.25 \pm 0.10$ & \\
\hline 5946_14 & $4794 \pm 430$ & $4725 \pm 75$ & $4727 \pm 74$ & $1.80 \pm 0.90$ & $1.45 \pm 0.42$ & $1.51 \pm 0.38$ & $-1.60 \pm 0.40$ & $-2.00 \pm 0.10$ & $-1.98 \pm 0.10$ & $0.44 \pm 0.15$ & $0.25 \pm 0.10$ & \\
\hline 5946_15 & $4693 \pm 353$ & $4900 \pm 200$ & $4850 \pm 174$ & $1.60 \pm 0.70$ & $1.75 \pm 0.34$ & $1.72 \pm 0.31$ & $-1.50 \pm 0.40$ & $-1.60 \pm 0.20$ & $-1.58 \pm 0.18$ & $0.41 \pm 0.15$ & $0.12 \pm 0.12$ & M \\
\hline 5946_16 & $4878 \pm 468$ & $4875 \pm 125$ & $4875 \pm 121$ & $1.90 \pm 0.90$ & $0.50 \pm 0.39$ & $0.72 \pm 0.36$ & $-1.60 \pm 0.40$ & $-2.00 \pm 0.10$ & $-1.98 \pm 0.10$ & $0.42 \pm 0.18$ & $0.12 \pm 0.10$ & \\
\hline 5946_19 & $4594 \pm 210$ & $4899 \pm 165$ & $4782 \pm 130$ & $2.22 \pm 0.33$ & $2.65 \pm 0.39$ & $2.40 \pm 0.25$ & $-0.21 \pm 0.23$ & $-0.23 \pm 0.28$ & $-0.22 \pm 0.18$ & $0.09 \pm 0.15$ & $0.28 \pm 0.12$ & \\
\hline BH176_2 & $4664 \pm 241$ & $4975 \pm 175$ & $4868 \pm 142$ & $2.40 \pm 0.50$ & $2.85 \pm 0.23$ & $2.77 \pm 0.21$ & $-0.17 \pm 0.29$ & $-0.06 \pm 0.32$ & $-0.12 \pm 0.21$ & $0.10 \pm 0.20$ & $0.25 \pm 0.13$ & M \\
\hline BH176_3 & $4551 \pm 247$ & $5025 \pm 208$ & $4829 \pm 159$ & $2.30 \pm 0.50$ & $2.90 \pm 0.44$ & $2.64 \pm 0.33$ & $-0.09 \pm 0.26$ & $0.26 \pm 0.21$ & $0.12 \pm 0.16$ & $0.06 \pm 0.17$ & $0.28 \pm 0.09$ & \\
\hline
\end{tabular}


Table B.3 - continued.

\begin{tabular}{|c|c|c|c|c|c|c|c|c|c|c|c|c|}
\hline NGC ID & $\mathrm{T}_{e f f}^{(a)}(\mathrm{K})$ & $\mathrm{T}_{e f f}^{(b)}(\mathrm{K})$ & $\mathrm{T}_{e f f}^{(a v g)}(\mathrm{K})$ & $\log (g)^{(a)}$ & $\log (g)^{(b)}$ & $\log (g)^{(a v g)}$ & {$[\mathrm{Fe} / \mathrm{H}]^{(a)}$} & {$[\mathrm{Fe} / \mathrm{H}]^{(b)}$} & {$[\mathrm{Fe} / \mathrm{H}]^{(a v g)}$} & {$[\mathrm{Mg} / \mathrm{Fe}]^{(a)}$} & {$[\alpha / \mathrm{Fe}]^{(b)}$} & members \\
\hline BH176_4 & $3988 \pm 108$ & $3600 \pm 122$ & $3818 \pm 81$ & $1.50 \pm 0.40$ & $2.35 \pm 0.23$ & $2.14 \pm 0.20$ & $-0.16 \pm 0.23$ & $-2.00 \pm 0.10$ & $-1.71 \pm 0.09$ & $0.07 \pm 0.18$ & $0.19 \pm 0.11$ & \\
\hline BH176_6 & $4099 \pm 199$ & $4275 \pm 208$ & $4183 \pm 144$ & $1.70 \pm 0.40$ & $2.15 \pm 0.45$ & $1.90 \pm 0.30$ & $-0.04 \pm 0.21$ & $-0.28 \pm 0.27$ & $-0.13 \pm 0.17$ & $0.05 \pm 0.16$ & $0.18 \pm 0.11$ & M \\
\hline BH176_7 & $5319 \pm 414$ & $6175 \pm 115$ & $6114 \pm 110$ & $3.00 \pm 0.60$ & $4.50 \pm 0.32$ & $4.17 \pm 0.28$ & $-0.25 \pm 0.32$ & $0.04 \pm 0.08$ & $0.02 \pm 0.08$ & $0.14 \pm 0.15$ & $0.07 \pm 0.06$ & M \\
\hline BH176_8 & $3973 \pm 119$ & $3575 \pm 114$ & $3766 \pm 83$ & $1.45 \pm 0.30$ & $1.75 \pm 0.90$ & $1.48 \pm 0.28$ & $-0.12 \pm 0.23$ & $-1.85 \pm 0.23$ & $-0.98 \pm 0.16$ & $0.08 \pm 0.17$ & $0.30 \pm 0.09$ & \\
\hline BH176_9 & $4244 \pm 274$ & $4525 \pm 175$ & $4444 \pm 147$ & $1.80 \pm 0.60$ & $2.35 \pm 0.55$ & $2.10 \pm 0.41$ & $-0.02 \pm 0.19$ & $-0.04 \pm 0.25$ & $-0.03 \pm 0.15$ & $0.03 \pm 0.15$ & $0.21 \pm 0.09$ & M \\
\hline BH176_10 & $3954 \pm 78$ & $4000 \pm 157$ & $3963 \pm 70$ & $1.47 \pm 0.22$ & $1.50 \pm 0.55$ & $1.47 \pm 0.20$ & $-0.03 \pm 0.18$ & $-0.50 \pm 0.31$ & $-0.15 \pm 0.16$ & $0.05 \pm 0.19$ & $0.22 \pm 0.12$ & M \\
\hline BH176_11 & $3951 \pm 103$ & $3950 \pm 149$ & $3951 \pm 85$ & $1.45 \pm 0.22$ & $1.40 \pm 0.62$ & $1.44 \pm 0.21$ & $-0.04 \pm 0.18$ & $-0.60 \pm 0.30$ & $-0.19 \pm 0.15$ & $0.06 \pm 0.20$ & $0.26 \pm 0.11$ & $\mathrm{M}$ \\
\hline BH176_13 & $3640 \pm 100$ & $3500 \pm 100$ & $3570 \pm 71$ & $0.70 \pm 0.20$ & $3.00 \pm 0.71$ & $0.87 \pm 0.19$ & $-0.10 \pm 0.06$ & $-0.00 \pm 0.26$ & $-0.09 \pm 0.06$ & $0.23 \pm 0.10$ & $0.34 \pm 0.07$ & M \\
\hline BH176_14 & $4146 \pm 218$ & $4400 \pm 122$ & $4339 \pm 107$ & $1.70 \pm 0.50$ & $2.40 \pm 0.20$ & $2.30 \pm 0.19$ & $-0.05 \pm 0.20$ & $-0.16 \pm 0.29$ & $-0.09 \pm 0.16$ & $0.06 \pm 0.15$ & $0.15 \pm 0.12$ & M \\
\hline BH176_15 & $4240 \pm 270$ & $4350 \pm 123$ & $4331 \pm 112$ & $1.80 \pm 0.60$ & $2.30 \pm 0.24$ & $2.23 \pm 0.22$ & $-0.07 \pm 0.23$ & $-0.30 \pm 0.25$ & $-0.18 \pm 0.17$ & $0.05 \pm 0.17$ & $0.15 \pm 0.14$ & M \\
\hline BH176_16 & $4210 \pm 255$ & $4425 \pm 114$ & $4389 \pm 104$ & $1.80 \pm 0.60$ & $2.35 \pm 0.23$ & $2.28 \pm 0.21$ & $-0.08 \pm 0.25$ & $-0.07 \pm 0.29$ & $-0.08 \pm 0.19$ & $0.06 \pm 0.18$ & $0.26 \pm 0.11$ & M \\
\hline BH176_17 & $3810 \pm 12$ & $3500 \pm 100$ & $3806 \pm 12$ & $1.10 \pm 0.21$ & $2.20 \pm 0.56$ & $1.24 \pm 0.20$ & $0.00 \pm 0.23$ & $-1.90 \pm 0.20$ & $-1.08 \pm 0.15$ & $0.04 \pm 0.12$ & $0.26 \pm 0.09$ & \\
\hline BH176_18 & $4101 \pm 213$ & $4274 \pm 208$ & $4190 \pm 149$ & $1.60 \pm 0.50$ & $2.05 \pm 0.42$ & $1.86 \pm 0.32$ & $-0.06 \pm 0.22$ & $-0.11 \pm 0.27$ & $-0.08 \pm 0.17$ & $0.06 \pm 0.17$ & $0.18 \pm 0.10$ & M \\
\hline Lynga7_1 & $4447 \pm 143$ & $4425 \pm 195$ & $4439 \pm 115$ & $2.20 \pm 0.40$ & $2.30 \pm 0.46$ & $2.24 \pm 0.30$ & $-0.43 \pm 0.17$ & $-1.15 \pm 0.39$ & $-0.54 \pm 0.16$ & $0.20 \pm 0.16$ & $0.27 \pm 0.11$ & \\
\hline Lynga7_2 & $4130 \pm 68$ & $4202 \pm 186$ & $4139 \pm 64$ & $1.85 \pm 0.23$ & $1.75 \pm 0.46$ & $1.83 \pm 0.21$ & $-0.27 \pm 0.04$ & $-1.10 \pm 0.37$ & $-0.28 \pm 0.04$ & $0.10 \pm 0.02$ & $0.32 \pm 0.07$ & \\
\hline Lynga7_3 & $4271 \pm 174$ & $4348 \pm 122$ & $4322 \pm 100$ & $1.85 \pm 0.35$ & $2.00 \pm 0.32$ & $1.93 \pm 0.24$ & $-0.02 \pm 0.14$ & $-0.80 \pm 0.24$ & $-0.22 \pm 0.12$ & $0.03 \pm 0.16$ & $0.29 \pm 0.09$ & \\
\hline Lynga7_4 & $4459 \pm 156$ & $4450 \pm 187$ & $4455 \pm 120$ & $2.20 \pm 0.40$ & $2.40 \pm 0.49$ & $2.28 \pm 0.31$ & $-0.47 \pm 0.21$ & $-1.10 \pm 0.37$ & $-0.62 \pm 0.18$ & $0.24 \pm 0.19$ & $0.28 \pm 0.10$ & M \\
\hline Lynga7_5 & $3640 \pm 100$ & $3523 \pm 73$ & $3564 \pm 59$ & $0.70 \pm 0.20$ & $3.09 \pm 0.77$ & $0.85 \pm 0.19$ & $-0.10 \pm 0.06$ & $-0.56 \pm 0.27$ & $-0.12 \pm 0.06$ & $0.23 \pm 0.10$ & $0.34 \pm 0.08$ & \\
\hline Lynga7_6 & $4706 \pm 170$ & $4950 \pm 149$ & $4844 \pm 112$ & $2.50 \pm 0.40$ & $3.25 \pm 0.33$ & $2.95 \pm 0.25$ & $-0.39 \pm 0.22$ & $-0.65 \pm 0.23$ & $-0.51 \pm 0.16$ & $0.21 \pm 0.20$ & $0.29 \pm 0.10$ & \\
\hline Lynga7_8 & $4361 \pm 110$ & $4349 \pm 122$ & $4356 \pm 82$ & $1.90 \pm 0.40$ & $1.89 \pm 0.30$ & $1.89 \pm 0.24$ & $-0.38 \pm 0.28$ & $-0.85 \pm 0.23$ & $-0.66 \pm 0.18$ & $0.21 \pm 0.20$ & $0.25 \pm 0.10$ & M \\
\hline Lynga7_9 & $4153 \pm 117$ & $4324 \pm 114$ & $4241 \pm 82$ & $1.58 \pm 0.14$ & $2.10 \pm 0.37$ & $1.65 \pm 0.13$ & $0.01 \pm 0.11$ & $-0.35 \pm 0.23$ & $-0.06 \pm 0.10$ & $-0.01 \pm 0.16$ & $0.25 \pm 0.13$ & \\
\hline Lynga7_10 & $4483 \pm 152$ & $4600 \pm 122$ & $4554 \pm 95$ & $2.20 \pm 0.40$ & $2.55 \pm 0.35$ & $2.40 \pm 0.26$ & $-0.37 \pm 0.22$ & $-0.80 \pm 0.24$ & $-0.57 \pm 0.16$ & $0.19 \pm 0.19$ & $0.27 \pm 0.11$ & M \\
\hline Lynga7_11 & $4186 \pm 150$ & $4349 \pm 122$ & $4284 \pm 95$ & $1.67 \pm 0.23$ & $2.05 \pm 0.35$ & $1.78 \pm 0.19$ & $-0.06 \pm 0.13$ & $-0.80 \pm 0.24$ & $-0.23 \pm 0.11$ & $0.01 \pm 0.16$ & $0.28 \pm 0.10$ & \\
\hline Lynga7_14 & $4711 \pm 236$ & $4899 \pm 165$ & $4837 \pm 135$ & $2.50 \pm 0.50$ & $2.95 \pm 0.27$ & $2.85 \pm 0.24$ & $-0.29 \pm 0.30$ & $-0.75 \pm 0.25$ & $-0.56 \pm 0.19$ & $0.16 \pm 0.21$ & $0.28 \pm 0.12$ & \\
\hline Lynga7_15 & $3885 \pm 67$ & $3575 \pm 115$ & $3806 \pm 58$ & $1.28 \pm 0.20$ & $0.75 \pm 0.64$ & $1.23 \pm 0.19$ & $-0.20 \pm 0.29$ & $-1.65 \pm 0.23$ & $-1.09 \pm 0.18$ & $0.15 \pm 0.18$ & $0.29 \pm 0.10$ & \\
\hline Lynga7_16 & $4434 \pm 156$ & $4874 \pm 125$ & $4702 \pm 98$ & $2.20 \pm 0.50$ & $3.20 \pm 0.24$ & $3.01 \pm 0.22$ & $0.09 \pm 0.12$ & $0.23 \pm 0.20$ & $0.13 \pm 0.10$ & $0.01 \pm 0.14$ & $0.09 \pm 0.07$ & \\
\hline Pal14_3 & $4657 \pm 367$ & $4900 \pm 122$ & $4876 \pm 116$ & $1.70 \pm 0.90$ & $1.75 \pm 0.51$ & $1.74 \pm 0.44$ & $-1.10 \pm 0.50$ & $-1.20 \pm 0.24$ & $-1.18 \pm 0.22$ & $0.36 \pm 0.15$ & $0.22 \pm 0.14$ & M \\
\hline Pal14_4 & $4654 \pm 381$ & $4850 \pm 122$ & $4832 \pm 117$ & $1.80 \pm 1.00$ & $2.60 \pm 0.37$ & $2.50 \pm 0.35$ & $-1.10 \pm 0.40$ & $-1.30 \pm 0.24$ & $-1.25 \pm 0.21$ & $0.40 \pm 0.14$ & $0.21 \pm 0.11$ & $\mathrm{M}$ \\
\hline
\end{tabular}


Table B.3 - continued.

\begin{tabular}{|c|c|c|c|c|c|c|c|c|c|c|c|c|}
\hline NGC ID & $\mathrm{T}_{e f f}^{(a)}(\mathrm{K})$ & $\mathrm{T}_{e f f}^{(b)}(\mathrm{K})$ & $\mathrm{T}_{\text {eff }}^{(a v g)}(\mathrm{K})$ & $\log (g)^{(a)}$ & $\log (g)^{(b)}$ & $\log (g)^{(a v g)}$ & {$[\mathrm{Fe} / \mathrm{H}]^{(a)}$} & {$[\mathrm{Fe} / \mathrm{H}]^{(b)}$} & {$[\mathrm{Fe} / \mathrm{H}]^{(a v g)}$} & {$[\mathrm{Mg} / \mathrm{Fe}]^{(a)}$} & {$[\alpha / \mathrm{Fe}]^{(b)}$} & members \\
\hline Pal14_5 & $4815 \pm 422$ & $5025 \pm 175$ & $4994 \pm 162$ & $1.90 \pm 0.90$ & $2.35 \pm 0.55$ & $2.23 \pm 0.47$ & $-1.30 \pm 0.50$ & $-1.35 \pm 0.23$ & $-1.34 \pm 0.21$ & $0.42 \pm 0.17$ & $0.26 \pm 0.11$ & M \\
\hline Pal14_7 & $4420 \pm 324$ & $4650 \pm 122$ & $4622 \pm 115$ & $1.50 \pm 0.70$ & $2.15 \pm 0.32$ & $2.04 \pm 0.29$ & $-0.90 \pm 0.40$ & $-1.20 \pm 0.24$ & $-1.12 \pm 0.21$ & $0.30 \pm 0.17$ & $0.28 \pm 0.10$ & M \\
\hline Pal14_8 & $4805 \pm 380$ & $4950 \pm 245$ & $4907 \pm 206$ & $2.00 \pm 0.90$ & $2.05 \pm 0.42$ & $2.04 \pm 0.38$ & $-1.30 \pm 0.50$ & $-1.30 \pm 0.24$ & $-1.30 \pm 0.22$ & $0.41 \pm 0.16$ & $0.22 \pm 0.10$ & M \\
\hline Pal14_10 & $4849 \pm 420$ & $4551 \pm 150$ & $4585 \pm 141$ & $3.45 \pm 0.04$ & $4.25 \pm 0.46$ & $3.46 \pm 0.04$ & $0.09 \pm 0.01$ & $-1.35 \pm 0.23$ & $0.089 \pm 0.007$ & $-0.02 \pm 0.10$ & $0.34 \pm 0.07$ & \\
\hline Pal14_11 & $4296 \pm 13$ & $4599 \pm 166$ & $4298 \pm 13$ & $1.05 \pm 0.10$ & $2.05 \pm 0.35$ & $1.13 \pm 0.10$ & $-0.90 \pm 0.40$ & $-1.25 \pm 0.25$ & $-1.15 \pm 0.21$ & $0.13 \pm 0.12$ & $0.24 \pm 0.12$ & M \\
\hline 6284_1 & $4696 \pm 201$ & $4750 \pm 158$ & $4729 \pm 124$ & $2.50 \pm 0.40$ & $2.65 \pm 0.39$ & $2.58 \pm 0.28$ & $-0.16 \pm 0.24$ & $-0.50 \pm 0.32$ & $-0.282 \pm 0.19$ & $0.09 \pm 0.17$ & $0.25 \pm 0.08$ & \\
\hline $6284 \_2$ & $4488 \pm 182$ & $4447 \pm 216$ & $4471 \pm 139$ & $2.10 \pm 0.50$ & $1.80 \pm 0.45$ & $1.93 \pm 0.33$ & $-0.51 \pm 0.20$ & $-1.15 \pm 0.32$ & $-0.690 \pm 0.17$ & $0.22 \pm 0.18$ & $0.27 \pm 0.11$ & \\
\hline 6284_3 & $4919 \pm 124$ & $5398 \pm 165$ & $5092 \pm 99$ & $2.80 \pm 0.40$ & $3.65 \pm 0.23$ & $3.44 \pm 0.20$ & $0.01 \pm 0.10$ & $0.23 \pm 0.19$ & $0.055 \pm 0.09$ & $0.04 \pm 0.10$ & $0.08 \pm 0.07$ & \\
\hline 6284_4 & $4903 \pm 189$ & $5151 \pm 122$ & $5078 \pm 103$ & $2.60 \pm 0.40$ & $3.20 \pm 0.25$ & $3.03 \pm 0.21$ & $-0.47 \pm 0.23$ & $-0.50 \pm 0.10$ & $-0.495 \pm 0.09$ & $0.25 \pm 0.19$ & $0.21 \pm 0.11$ & \\
\hline $6284 \_5$ & $4676 \pm 188$ & $4898 \pm 123$ & $4832 \pm 103$ & $2.60 \pm 0.40$ & $3.15 \pm 0.23$ & $3.01 \pm 0.20$ & $-0.24 \pm 0.19$ & $-0.35 \pm 0.23$ & $-0.285 \pm 0.15$ & $0.12 \pm 0.15$ & $0.26 \pm 0.13$ & \\
\hline $6284 \_6$ & $4727 \pm 181$ & $4750 \pm 100$ & $4745 \pm 88$ & $2.30 \pm 0.40$ & $2.05 \pm 0.41$ & $2.18 \pm 0.29$ & $-0.49 \pm 0.24$ & $-1.00 \pm 0.10$ & $-0.925 \pm 0.09$ & $0.29 \pm 0.24$ & $0.25 \pm 0.10$ & \\
\hline $6284 \_7$ & $4935 \pm 319$ & $4801 \pm 245$ & $4851 \pm 194$ & $2.50 \pm 0.70$ & $1.95 \pm 0.42$ & $2.10 \pm 0.36$ & $-0.61 \pm 0.34$ & $-1.20 \pm 0.24$ & $-1.004 \pm 0.20$ & $0.24 \pm 0.12$ & $0.25 \pm 0.10$ & M \\
\hline $6284 \_8$ & $4602 \pm 167$ & $4625 \pm 125$ & $4616 \pm 100$ & $2.41 \pm 0.29$ & $2.10 \pm 0.30$ & $2.26 \pm 0.21$ & $-0.33 \pm 0.16$ & $-0.75 \pm 0.25$ & $-0.452 \pm 0.13$ & $0.14 \pm 0.17$ & $0.28 \pm 0.12$ & \\
\hline 6284_9 & $4469 \pm 184$ & $4975 \pm 208$ & $4692 \pm 138$ & $1.30 \pm 0.50$ & $2.10 \pm 0.44$ & $1.75 \pm 0.33$ & $-1.40 \pm 0.40$ & $-1.35 \pm 0.23$ & $-1.362 \pm 0.20$ & $0.42 \pm 0.11$ & $0.30 \pm 0.10$ & M \\
\hline 6284_10 & $4668 \pm 276$ & $4875 \pm 125$ & $4840 \pm 114$ & $1.80 \pm 0.80$ & $2.55 \pm 0.35$ & $2.43 \pm 0.32$ & $-1.10 \pm 0.40$ & $-1.25 \pm 0.25$ & $-1.208 \pm 0.21$ & $0.36 \pm 0.17$ & $0.31 \pm 0.09$ & M \\
\hline 6284_11 & $4824 \pm 175$ & $4800 \pm 187$ & $4813 \pm 128$ & $2.60 \pm 0.40$ & $2.60 \pm 0.44$ & $2.60 \pm 0.30$ & $-0.48 \pm 0.21$ & $-0.90 \pm 0.37$ & $-0.582 \pm 0.18$ & $0.28 \pm 0.20$ & $0.28 \pm 0.10$ & \\
\hline 6284_13 & $4793 \pm 275$ & $4825 \pm 225$ & $4812 \pm 174$ & $2.20 \pm 0.70$ & $2.20 \pm 0.56$ & $2.20 \pm 0.44$ & $-0.80 \pm 0.40$ & $-1.20 \pm 0.24$ & $-1.094 \pm 0.21$ & $0.28 \pm 0.14$ & $0.28 \pm 0.10$ & M \\
\hline 6284_14 & $4821 \pm 369$ & $5199 \pm 100$ & $5173 \pm 97$ & $2.20 \pm 0.90$ & $3.05 \pm 0.35$ & $2.94 \pm 0.33$ & $-0.90 \pm 0.50$ & $-1.00 \pm 0.10$ & $-0.996 \pm 0.10$ & $0.34 \pm 0.17$ & $0.22 \pm 0.12$ & M \\
\hline 6284_15 & $4933 \pm 421$ & $4950 \pm 218$ & $4946 \pm 194$ & $2.40 \pm 0.90$ & $2.60 \pm 0.37$ & $2.57 \pm 0.34$ & $-0.80 \pm 0.50$ & $-1.30 \pm 0.24$ & $-1.206 \pm 0.22$ & $0.33 \pm 0.20$ & $0.25 \pm 0.10$ & M \\
\hline 6284_16 & $4165 \pm 140$ & $4473 \pm 208$ & $4261 \pm 116$ & $1.70 \pm 0.40$ & $2.60 \pm 0.66$ & $1.94 \pm 0.34$ & $-0.40 \pm 0.20$ & $-0.85 \pm 0.23$ & $-0.594 \pm 0.15$ & $0.16 \pm 0.18$ & $0.34 \pm 0.07$ & \\
\hline 6284_17 & $4633 \pm 140$ & $5000 \pm 157$ & $4795 \pm 105$ & $2.47 \pm 0.34$ & $3.00 \pm 0.31$ & $2.76 \pm 0.23$ & $-0.13 \pm 0.18$ & $-0.02 \pm 0.25$ & $-0.092 \pm 0.15$ & $0.06 \pm 0.12$ & $0.23 \pm 0.13$ & \\
\hline 6284_18 & $4948 \pm 259$ & $4875 \pm 202$ & $4903 \pm 159$ & $2.70 \pm 0.60$ & $2.50 \pm 0.55$ & $2.59 \pm 0.41$ & $-0.57 \pm 0.30$ & $-1.15 \pm 0.23$ & $-0.935 \pm 0.18$ & $0.29 \pm 0.20$ & $0.28 \pm 0.10$ & M \\
\hline 6316_1 & $4137 \pm 161$ & $4375 \pm 230$ & $4215 \pm 132$ & $1.76 \pm 0.28$ & $2.10 \pm 0.58$ & $1.82 \pm 0.25$ & $-0.07 \pm 0.19$ & $-0.41 \pm 0.34$ & $-0.15 \pm 0.17$ & $0.02 \pm 0.13$ & $0.19 \pm 0.11$ & \\
\hline 6316_2 & $4274 \pm 159$ & $4373 \pm 125$ & $4335 \pm 98$ & $1.90 \pm 0.50$ & $2.10 \pm 0.37$ & $2.03 \pm 0.30$ & $-0.32 \pm 0.26$ & $-0.80 \pm 0.24$ & $-0.58 \pm 0.18$ & $0.16 \pm 0.19$ & $0.31 \pm 0.10$ & \\
\hline 6316_3 & $4817 \pm 224$ & $4901 \pm 229$ & $4858 \pm 160$ & $2.50 \pm 0.60$ & $2.90 \pm 0.44$ & $2.76 \pm 0.35$ & $-0.51 \pm 0.24$ & $-0.80 \pm 0.33$ & $-0.61 \pm 0.19$ & $0.28 \pm 0.19$ & $0.28 \pm 0.12$ & \\
\hline 6316_4 & $4586 \pm 190$ & $4675 \pm 114$ & $4652 \pm 98$ & $2.20 \pm 0.40$ & $2.65 \pm 0.32$ & $2.47 \pm 0.25$ & $-0.21 \pm 0.21$ & $-0.65 \pm 0.23$ & $-0.41 \pm 0.16$ & $0.11 \pm 0.16$ & $0.27 \pm 0.09$ & \\
\hline 6316_5 & $4233 \pm 128$ & $4376 \pm 125$ & $4306 \pm 89$ & $1.78 \pm 0.24$ & $2.15 \pm 0.32$ & $1.91 \pm 0.19$ & $0.12 \pm 0.18$ & $-0.25 \pm 0.25$ & $-0.01 \pm 0.15$ & $-0.00 \pm 0.11$ & $0.20 \pm 0.13$ & \\
\hline 6316_6 & $4698 \pm 192$ & $4801 \pm 218$ & $4743 \pm 144$ & $2.50 \pm 0.50$ & $2.85 \pm 0.59$ & $2.65 \pm 0.38$ & $-0.41 \pm 0.24$ & $-0.75 \pm 0.25$ & $-0.57 \pm 0.17$ & $0.22 \pm 0.20$ & $0.30 \pm 0.08$ & \\
\hline
\end{tabular}


Table B.3 - continued.

\begin{tabular}{|c|c|c|c|c|c|c|c|c|c|c|c|c|}
\hline NGC ID & $\mathrm{T}_{e f f}^{(a)}(\mathrm{K})$ & $\mathrm{T}_{e f f}^{(b)}(\mathrm{K})$ & $\mathrm{T}_{e f f}^{(a v g)}(\mathrm{K})$ & $\log (g)^{(a)}$ & $\log (g)^{(b)}$ & $\log (g)^{(a v g)}$ & {$[\mathrm{Fe} / \mathrm{H}]^{(a)}$} & {$[\mathrm{Fe} / \mathrm{H}]^{(b)}$} & {$[\mathrm{Fe} / \mathrm{H}]^{(a v g)}$} & {$[\mathrm{Mg} / \mathrm{Fe}]^{(a)}$} & {$[\alpha / \mathrm{Fe}]^{(b)}$} & members \\
\hline 6316_7 & $4529 \pm 195$ & $4474 \pm 208$ & $4503 \pm 142$ & $2.30 \pm 0.50$ & $1.90 \pm 0.44$ & $2.07 \pm 0.33$ & $-0.19 \pm 0.28$ & $-0.80 \pm 0.24$ & $-0.54 \pm 0.18$ & $0.11 \pm 0.18$ & $0.27 \pm 0.13$ & M \\
\hline $6316 \_8$ & $4634 \pm 185$ & $4699 \pm 218$ & $4661 \pm 141$ & $2.40 \pm 0.40$ & $2.35 \pm 0.63$ & $2.39 \pm 0.34$ & $-0.43 \pm 0.22$ & $-0.80 \pm 0.24$ & $-0.60 \pm 0.16$ & $0.21 \pm 0.20$ & $0.30 \pm 0.09$ & \\
\hline 6316_9 & $3980 \pm 119$ & $3924 \pm 194$ & $3965 \pm 101$ & $1.40 \pm 0.40$ & $1.00 \pm 0.74$ & $1.31 \pm 0.35$ & $-0.35 \pm 0.14$ & $-1.20 \pm 0.33$ & $-0.48 \pm 0.13$ & $0.17 \pm 0.21$ & $0.30 \pm 0.10$ & M \\
\hline 6316_10 & $4388 \pm 143$ & $4399 \pm 165$ & $4393 \pm 108$ & $2.00 \pm 0.40$ & $2.09 \pm 0.44$ & $2.04 \pm 0.30$ & $-0.35 \pm 0.27$ & $-0.85 \pm 0.23$ & $-0.64 \pm 0.18$ & $0.20 \pm 0.19$ & $0.32 \pm 0.07$ & M \\
\hline 6316_11 & $4393 \pm 116$ & $4527 \pm 175$ & $4434 \pm 97$ & $2.00 \pm 0.40$ & $2.20 \pm 0.40$ & $2.10 \pm 0.28$ & $-0.50 \pm 0.21$ & $-0.85 \pm 0.23$ & $-0.66 \pm 0.16$ & $0.23 \pm 0.18$ & $0.28 \pm 0.07$ & M \\
\hline 6316_14 & $4157 \pm 162$ & $4201 \pm 186$ & $4176 \pm 122$ & $1.60 \pm 0.40$ & $1.75 \pm 0.46$ & $1.66 \pm 0.30$ & $-0.35 \pm 0.23$ & $-1.10 \pm 0.37$ & $-0.56 \pm 0.20$ & $0.11 \pm 0.17$ & $0.32 \pm 0.07$ & M \\
\hline 6316_15 & $4285 \pm 200$ & $4500 \pm 100$ & $4457 \pm 89$ & $1.90 \pm 0.50$ & $2.30 \pm 0.24$ & $2.23 \pm 0.22$ & $0.14 \pm 0.18$ & $0.10 \pm 0.10$ & $0.11 \pm 0.09$ & $0.03 \pm 0.12$ & $0.16 \pm 0.10$ & \\
\hline 6316_17 & $4147 \pm 156$ & $4223 \pm 175$ & $4181 \pm 117$ & $1.70 \pm 0.25$ & $1.90 \pm 0.37$ & $1.76 \pm 0.21$ & $0.01 \pm 0.18$ & $-0.70 \pm 0.25$ & $-0.24 \pm 0.15$ & $0.01 \pm 0.12$ & $0.31 \pm 0.10$ & M \\
\hline 6316_18 & $4515 \pm 136$ & $4649 \pm 123$ & $4589 \pm 91$ & $2.29 \pm 0.35$ & $2.25 \pm 0.51$ & $2.28 \pm 0.29$ & $-0.23 \pm 0.26$ & $-0.70 \pm 0.25$ & $-0.47 \pm 0.18$ & $0.09 \pm 0.16$ & $0.30 \pm 0.08$ & M \\
\hline 6316_19 & $4413 \pm 133$ & $4874 \pm 125$ & $4658 \pm 91$ & $2.10 \pm 0.50$ & $2.95 \pm 0.35$ & $2.67 \pm 0.29$ & $0.13 \pm 0.14$ & $0.35 \pm 0.15$ & $0.23 \pm 0.10$ & $0.02 \pm 0.16$ & $0.17 \pm 0.09$ & \\
\hline 6356_1 & $4698 \pm 214$ & $4849 \pm 255$ & $4761 \pm 164$ & $2.30 \pm 0.50$ & $2.40 \pm 0.62$ & $2.34 \pm 0.39$ & $-0.43 \pm 0.24$ & $-0.65 \pm 0.32$ & $-0.51 \pm 0.19$ & $0.19 \pm 0.21$ & $0.30 \pm 0.08$ & M \\
\hline 6356_2 & $4533 \pm 93$ & $4648 \pm 199$ & $4554 \pm 84$ & $2.42 \pm 0.32$ & $2.65 \pm 0.55$ & $2.48 \pm 0.28$ & $0.01 \pm 0.10$ & $-0.30 \pm 0.24$ & $-0.04 \pm 0.09$ & $0.08 \pm 0.12$ & $0.24 \pm 0.09$ & \\
\hline 6356_3 & $4822 \pm 395$ & $5274 \pm 175$ & $5200 \pm 160$ & $2.20 \pm 1.00$ & $3.35 \pm 0.32$ & $3.24 \pm 0.30$ & $-1.20 \pm 0.50$ & $-1.10 \pm 0.20$ & $-1.11 \pm 0.19$ & $0.43 \pm 0.16$ & $0.31 \pm 0.07$ & \\
\hline 6356_4 & $3959 \pm 87$ & $3926 \pm 114$ & $3947 \pm 69$ & $1.47 \pm 0.32$ & $1.40 \pm 0.62$ & $1.46 \pm 0.28$ & $-0.22 \pm 0.21$ & $-1.15 \pm 0.23$ & $-0.64 \pm 0.16$ & $0.13 \pm 0.16$ & $0.25 \pm 0.10$ & \\
\hline 6356_5 & $4379 \pm 97$ & $4549 \pm 187$ & $4415 \pm 86$ & $2.00 \pm 0.40$ & $2.40 \pm 0.54$ & $2.14 \pm 0.32$ & $-0.50 \pm 0.20$ & $-0.80 \pm 0.24$ & $-0.62 \pm 0.15$ & $0.25 \pm 0.20$ & $0.32 \pm 0.09$ & M \\
\hline 6356_6 & $4724 \pm 195$ & $4827 \pm 194$ & $4776 \pm 138$ & $2.40 \pm 0.50$ & $2.46 \pm 0.52$ & $2.43 \pm 0.36$ & $-0.47 \pm 0.23$ & $-0.75 \pm 0.25$ & $-0.60 \pm 0.17$ & $0.25 \pm 0.20$ & $0.29 \pm 0.09$ & M \\
\hline 6356_7 & $4344 \pm 179$ & $4347 \pm 122$ & $4346 \pm 101$ & $2.00 \pm 0.40$ & $1.99 \pm 0.32$ & $1.99 \pm 0.25$ & $-0.29 \pm 0.24$ & $-0.85 \pm 0.23$ & $-0.58 \pm 0.17$ & $0.16 \pm 0.18$ & $0.29 \pm 0.09$ & M \\
\hline 6356_8 & $4001 \pm 101$ & $4075 \pm 115$ & $4033 \pm 76$ & $1.50 \pm 0.40$ & $1.90 \pm 0.38$ & $1.71 \pm 0.28$ & $-0.30 \pm 0.18$ & $-1.25 \pm 0.25$ & $-0.62 \pm 0.15$ & $0.11 \pm 0.17$ & $0.32 \pm 0.07$ & M \\
\hline 6356_9 & $4369 \pm 91$ & $4496 \pm 194$ & $4392 \pm 82$ & $2.00 \pm 0.40$ & $2.19 \pm 0.56$ & $2.06 \pm 0.33$ & $-0.51 \pm 0.20$ & $-0.75 \pm 0.25$ & $-0.60 \pm 0.16$ & $0.27 \pm 0.20$ & $0.32 \pm 0.10$ & M \\
\hline 6356_10 & $4814 \pm 192$ & $4829 \pm 224$ & $4820 \pm 146$ & $2.50 \pm 0.40$ & $2.01 \pm 0.39$ & $2.25 \pm 0.28$ & $-0.24 \pm 0.30$ & $-0.70 \pm 0.24$ & $-0.52 \pm 0.19$ & $0.12 \pm 0.23$ & $0.29 \pm 0.10$ & M \\
\hline 6356_11 & $4322 \pm 125$ & $4476 \pm 208$ & $4363 \pm 107$ & $2.10 \pm 0.40$ & $2.40 \pm 0.49$ & $2.22 \pm 0.31$ & $0.06 \pm 0.11$ & $-0.29 \pm 0.32$ & $0.02 \pm 0.10$ & $-0.02 \pm 0.16$ & $0.26 \pm 0.10$ & \\
\hline 6356_13 & $4182 \pm 186$ & $4255 \pm 194$ & $4217 \pm 134$ & $1.65 \pm 0.26$ & $1.96 \pm 0.52$ & $1.71 \pm 0.23$ & $-0.06 \pm 0.19$ & $-0.94 \pm 0.35$ & $-0.26 \pm 0.17$ & $0.03 \pm 0.12$ & $0.31 \pm 0.08$ & M \\
\hline 6356_14 & $4232 \pm 182$ & $4327 \pm 159$ & $4286 \pm 120$ & $1.90 \pm 0.40$ & $1.96 \pm 0.47$ & $1.93 \pm 0.30$ & $-0.11 \pm 0.20$ & $-0.85 \pm 0.32$ & $-0.32 \pm 0.17$ & $0.03 \pm 0.13$ & $0.30 \pm 0.10$ & M \\
\hline 6356_15 & $3922 \pm 48$ & $3950 \pm 186$ & $3924 \pm 46$ & $1.54 \pm 0.04$ & $1.50 \pm 0.71$ & $1.54 \pm 0.04$ & $0.17 \pm 0.14$ & $-0.58 \pm 0.41$ & $0.09 \pm 0.13$ & $0.17 \pm 0.05$ & $0.34 \pm 0.07$ & \\
\hline 6356_16 & $4711 \pm 183$ & $4801 \pm 187$ & $4755 \pm 131$ & $2.40 \pm 0.50$ & $2.41 \pm 0.54$ & $2.40 \pm 0.37$ & $-0.49 \pm 0.22$ & $-0.80 \pm 0.25$ & $-0.63 \pm 0.17$ & $0.25 \pm 0.20$ & $0.31 \pm 0.10$ & M \\
\hline 6356_17 & $3861 \pm 96$ & $3549 \pm 99$ & $3710 \pm 69$ & $1.24 \pm 0.13$ & $0.90 \pm 0.62$ & $1.23 \pm 0.13$ & $0.07 \pm 0.17$ & $-1.40 \pm 0.20$ & $-0.55 \pm 0.13$ & $0.09 \pm 0.07$ & $0.30 \pm 0.08$ & M \\
\hline 6356_18 & $4419 \pm 205$ & $4349 \pm 165$ & $4377 \pm 128$ & $2.20 \pm 0.50$ & $2.05 \pm 0.47$ & $2.12 \pm 0.34$ & $-0.26 \pm 0.29$ & $-1.00 \pm 0.22$ & $-0.73 \pm 0.18$ & $0.14 \pm 0.18$ & $0.28 \pm 0.10$ & M \\
\hline 6356_19 & $4350 \pm 80$ & $4499 \pm 194$ & $4372 \pm 74$ & $2.00 \pm 0.40$ & $2.10 \pm 0.49$ & $2.04 \pm 0.31$ & $-0.52 \pm 0.20$ & $-0.70 \pm 0.25$ & $-0.59 \pm 0.16$ & $0.24 \pm 0.17$ & $0.29 \pm 0.09$ & M \\
\hline
\end{tabular}


Table B.3 - continued.

\begin{tabular}{|c|c|c|c|c|c|c|c|c|c|c|c|c|}
\hline NGC ID & $\mathrm{T}_{e f f}^{(a)}(\mathrm{K})$ & $\mathrm{T}_{e f f}^{(b)}(\mathrm{K})$ & $\mathrm{T}_{e f f}^{(a v g)}(\mathrm{K})$ & $\log (g)^{(a)}$ & $\log (g)^{(b)}$ & $\log (g)^{(a v g)}$ & {$[\mathrm{Fe} / \mathrm{H}]^{(a)}$} & {$[\mathrm{Fe} / \mathrm{H}]^{(b)}$} & {$[\mathrm{Fe} / \mathrm{H}]^{(a v g)}$} & {$[\mathrm{Mg} / \mathrm{Fe}]^{(a)}$} & {$[\alpha / \mathrm{Fe}]^{(b)}$} & members \\
\hline 6355_1 & $4128 \pm 122$ & $4401 \pm 122$ & $4264 \pm 86$ & $1.71 \pm 0.20$ & $2.05 \pm 0.35$ & $1.79 \pm 0.17$ & $0.05 \pm 0.20$ & $-0.20 \pm 0.24$ & $-0.05 \pm 0.15$ & $0.06 \pm 0.07$ & $0.28 \pm 0.10$ & \\
\hline $6355 \_2$ & $4538 \pm 128$ & $4601 \pm 229$ & $4553 \pm 112$ & $2.40 \pm 0.40$ & $2.50 \pm 0.55$ & $2.43 \pm 0.32$ & $-0.10 \pm 0.21$ & $-0.70 \pm 0.33$ & $-0.27 \pm 0.18$ & $0.07 \pm 0.14$ & $0.28 \pm 0.10$ & \\
\hline 6355_3 & $4638 \pm 208$ & $4700 \pm 292$ & $4659 \pm 169$ & $2.30 \pm 0.50$ & $2.60 \pm 0.73$ & $2.40 \pm 0.41$ & $-0.34 \pm 0.32$ & $-0.90 \pm 0.37$ & $-0.58 \pm 0.24$ & $0.19 \pm 0.22$ & $0.33 \pm 0.06$ & \\
\hline 6355_4 & $4404 \pm 142$ & $4800 \pm 100$ & $4669 \pm 82$ & $2.00 \pm 0.40$ & $2.75 \pm 0.25$ & $2.54 \pm 0.21$ & $0.12 \pm 0.14$ & $0.18 \pm 0.18$ & $0.14 \pm 0.11$ & $0.03 \pm 0.16$ & $0.16 \pm 0.11$ & \\
\hline $6355 \_5$ & $4292 \pm 139$ & $4449 \pm 187$ & $4348 \pm 112$ & $1.80 \pm 0.40$ & $2.40 \pm 0.58$ & $1.99 \pm 0.33$ & $-0.50 \pm 0.21$ & $-1.00 \pm 0.32$ & $-0.65 \pm 0.18$ & $0.25 \pm 0.20$ & $0.33 \pm 0.06$ & \\
\hline 6355_6 & $4490 \pm 159$ & $4600 \pm 123$ & $4559 \pm 97$ & $2.20 \pm 0.40$ & $2.60 \pm 0.37$ & $2.42 \pm 0.27$ & $-0.44 \pm 0.20$ & $-0.80 \pm 0.25$ & $-0.58 \pm 0.16$ & $0.22 \pm 0.18$ & $0.25 \pm 0.10$ & \\
\hline $6355 \_7$ & $4884 \pm 459$ & $5125 \pm 168$ & $5097 \pm 158$ & $2.00 \pm 0.90$ & $2.60 \pm 0.37$ & $2.51 \pm 0.34$ & $-1.50 \pm 0.40$ & $-1.35 \pm 0.23$ & $-1.39 \pm 0.20$ & $0.44 \pm 0.16$ & $0.28 \pm 0.10$ & $\mathrm{M}$ \\
\hline $6355 \_8$ & $4304 \pm 7$ & $4672 \pm 224$ & $4304 \pm 7$ & $1.04 \pm 0.10$ & $1.94 \pm 0.42$ & $1.09 \pm 0.10$ & $-1.37 \pm 0.10$ & $-1.35 \pm 0.23$ & $-1.37 \pm 0.09$ & $0.26 \pm 0.01$ & $0.21 \pm 0.15$ & $\mathrm{M}$ \\
\hline 6355_9 & $4422 \pm 143$ & $4725 \pm 75$ & $4660 \pm 66$ & $1.13 \pm 0.29$ & $1.70 \pm 0.33$ & $1.38 \pm 0.22$ & $-1.45 \pm 0.27$ & $-1.55 \pm 0.15$ & $-1.53 \pm 0.13$ & $0.41 \pm 0.11$ & $0.21 \pm 0.15$ & M \\
\hline 6355_10 & $4705 \pm 420$ & $4950 \pm 187$ & $4910 \pm 171$ & $1.40 \pm 0.60$ & $1.95 \pm 0.35$ & $1.81 \pm 0.30$ & $-1.60 \pm 0.40$ & $-1.70 \pm 0.24$ & $-1.67 \pm 0.21$ & $0.46 \pm 0.14$ & $0.32 \pm 0.09$ & M \\
\hline 6355_11 & $4649 \pm 388$ & $4475 \pm 174$ & $4504 \pm 159$ & $1.90 \pm 0.90$ & $1.40 \pm 0.54$ & $1.53 \pm 0.46$ & $-1.00 \pm 0.50$ & $-1.65 \pm 0.23$ & $-1.54 \pm 0.21$ & $0.36 \pm 0.16$ & $0.25 \pm 0.14$ & M \\
\hline 6355_13 & $4685 \pm 380$ & $4725 \pm 134$ & $4720 \pm 127$ & $1.70 \pm 0.90$ & $1.80 \pm 0.33$ & $1.79 \pm 0.31$ & $-1.40 \pm 0.50$ & $-1.60 \pm 0.20$ & $-1.57 \pm 0.19$ & $0.41 \pm 0.14$ & $0.28 \pm 0.10$ & M \\
\hline 6355_14 & $4795 \pm 158$ & $4902 \pm 122$ & $4862 \pm 97$ & $2.60 \pm 0.40$ & $3.10 \pm 0.30$ & $2.92 \pm 0.24$ & $-0.41 \pm 0.23$ & $-0.70 \pm 0.24$ & $-0.55 \pm 0.17$ & $0.25 \pm 0.19$ & $0.25 \pm 0.10$ & \\
\hline 6355_15 & $4913 \pm 262$ & $5250 \pm 274$ & $5074 \pm 189$ & $2.70 \pm 0.50$ & $3.40 \pm 0.37$ & $3.15 \pm 0.30$ & $-0.36 \pm 0.31$ & $-0.85 \pm 0.32$ & $-0.60 \pm 0.22$ & $0.20 \pm 0.22$ & $0.35 \pm 0.07$ & \\
\hline 6355_17 & $4707 \pm 322$ & $4925 \pm 195$ & $4867 \pm 167$ & $1.80 \pm 0.80$ & $3.00 \pm 0.32$ & $2.83 \pm 0.30$ & $-1.30 \pm 0.40$ & $-1.75 \pm 0.25$ & $-1.62 \pm 0.21$ & $0.41 \pm 0.15$ & $0.29 \pm 0.09$ & \\
\hline 6355_18 & $4739 \pm 232$ & $4724 \pm 175$ & $4729 \pm 140$ & $2.50 \pm 0.50$ & $2.20 \pm 0.56$ & $2.37 \pm 0.37$ & $-0.28 \pm 0.29$ & $-0.90 \pm 0.20$ & $-0.70 \pm 0.16$ & $0.16 \pm 0.21$ & $0.29 \pm 0.09$ & \\
\hline 6352_1 & $4736 \pm 208$ & $5074 \pm 194$ & $4917 \pm 142$ & $2.60 \pm 0.50$ & $3.20 \pm 0.33$ & $3.02 \pm 0.28$ & $-0.17 \pm 0.25$ & $-0.45 \pm 0.27$ & $-0.30 \pm 0.18$ & $0.12 \pm 0.17$ & $0.31 \pm 0.10$ & M \\
\hline $6352 \_2$ & $4723 \pm 190$ & $5126 \pm 229$ & $4887 \pm 146$ & $2.60 \pm 0.40$ & $3.10 \pm 0.43$ & $2.83 \pm 0.29$ & $-0.15 \pm 0.19$ & $-0.20 \pm 0.33$ & $-0.16 \pm 0.16$ & $0.09 \pm 0.14$ & $0.27 \pm 0.09$ & \\
\hline $6352 \_5$ & $4384 \pm 205$ & $4425 \pm 196$ & $4405 \pm 142$ & $2.10 \pm 0.50$ & $2.20 \pm 0.51$ & $2.15 \pm 0.36$ & $-0.13 \pm 0.25$ & $-0.85 \pm 0.23$ & $-0.52 \pm 0.17$ & $0.09 \pm 0.19$ & $0.32 \pm 0.07$ & M \\
\hline 6352_6 & $4766 \pm 227$ & $4899 \pm 199$ & $4841 \pm 150$ & $2.50 \pm 0.60$ & $3.15 \pm 0.32$ & $3.01 \pm 0.28$ & $-0.48 \pm 0.25$ & $-0.90 \pm 0.30$ & $-0.65 \pm 0.19$ & $0.26 \pm 0.20$ & $0.29 \pm 0.09$ & M \\
\hline $6352 \_8$ & $4149 \pm 104$ & $4347 \pm 122$ & $4232 \pm 79$ & $1.60 \pm 0.50$ & $2.05 \pm 0.35$ & $1.90 \pm 0.29$ & $-0.48 \pm 0.27$ & $-0.86 \pm 0.23$ & $-0.70 \pm 0.18$ & $0.18 \pm 0.18$ & $0.30 \pm 0.10$ & M \\
\hline 6352_9 & $4810 \pm 163$ & $5002 \pm 192$ & $4890 \pm 124$ & $2.60 \pm 0.40$ & $3.05 \pm 0.35$ & $2.85 \pm 0.26$ & $-0.42 \pm 0.24$ & $-0.65 \pm 0.23$ & $-0.54 \pm 0.17$ & $0.23 \pm 0.20$ & $0.25 \pm 0.13$ & M \\
\hline 6352_10 & $4391 \pm 111$ & $4625 \pm 167$ & $4463 \pm 92$ & $2.00 \pm 0.50$ & $2.65 \pm 0.45$ & $2.36 \pm 0.33$ & $-0.51 \pm 0.21$ & $-0.80 \pm 0.25$ & $-0.63 \pm 0.16$ & $0.25 \pm 0.20$ & $0.27 \pm 0.11$ & M \\
\hline 6352_11 & $3921 \pm 48$ & $3699 \pm 217$ & $3911 \pm 47$ & $1.35 \pm 0.24$ & $0.90 \pm 0.73$ & $1.31 \pm 0.23$ & $-0.34 \pm 0.18$ & $-1.50 \pm 0.39$ & $-0.54 \pm 0.16$ & $0.15 \pm 0.19$ & $0.32 \pm 0.07$ & M \\
\hline 6352_13 & $4629 \pm 178$ & $4775 \pm 209$ & $4691 \pm 135$ & $2.44 \pm 0.35$ & $2.85 \pm 0.60$ & $2.54 \pm 0.30$ & $-0.39 \pm 0.18$ & $-0.65 \pm 0.23$ & $-0.49 \pm 0.14$ & $0.19 \pm 0.18$ & $0.27 \pm 0.09$ & M \\
\hline 6352_14 & $4550 \pm 264$ & $4225 \pm 175$ & $4324 \pm 146$ & $2.20 \pm 0.60$ & $2.80 \pm 0.51$ & $2.55 \pm 0.39$ & $-0.50 \pm 0.31$ & $-2.15 \pm 0.32$ & $-1.30 \pm 0.22$ & $0.28 \pm 0.20$ & $0.34 \pm 0.07$ & \\
\hline 6352_15 & $4694 \pm 202$ & $4875 \pm 167$ & $4801 \pm 129$ & $2.40 \pm 0.50$ & $3.10 \pm 0.30$ & $2.91 \pm 0.26$ & $-0.43 \pm 0.25$ & $-0.75 \pm 0.33$ & $-0.55 \pm 0.20$ & $0.23 \pm 0.20$ & $0.28 \pm 0.10$ & M \\
\hline 6352_16 & $4364 \pm 98$ & $4625 \pm 125$ & $4464 \pm 77$ & $1.90 \pm 0.50$ & $2.25 \pm 0.40$ & $2.11 \pm 0.31$ & $-0.52 \pm 0.24$ & $-0.75 \pm 0.25$ & $-0.63 \pm 0.17$ & $0.27 \pm 0.21$ & $0.34 \pm 0.07$ & M \\
\hline
\end{tabular}


Table B.3 - continued.

\begin{tabular}{|c|c|c|c|c|c|c|c|c|c|c|c|c|}
\hline NGC ID & $\mathrm{T}_{e f f}^{(a)}(\mathrm{K})$ & $\mathrm{T}_{e f f}^{(b)}(\mathrm{K})$ & $\mathrm{T}_{e f f}^{(a v g)}(\mathrm{K})$ & $\log (g)^{(a)}$ & $\log (g)^{(b)}$ & $\log (g)^{(a v g)}$ & {$[\mathrm{Fe} / \mathrm{H}]^{(a)}$} & {$[\mathrm{Fe} / \mathrm{H}]^{(b)}$} & {$[\mathrm{Fe} / \mathrm{H}]^{(a v g)}$} & {$[\mathrm{Mg} / \mathrm{Fe}]^{(a)}$} & {$[\alpha / \mathrm{Fe}]^{(b)}$} & members \\
\hline 6352_17 & $3984 \pm 15$ & $4351 \pm 123$ & $3989 \pm 15$ & $1.13 \pm 0.18$ & $2.20 \pm 0.33$ & $1.38 \pm 0.16$ & $-0.44 \pm 0.10$ & $-0.80 \pm 0.25$ & $-0.49 \pm 0.09$ & $0.03 \pm 0.09$ & $0.26 \pm 0.11$ & M \\
\hline 6352_18 & $4390 \pm 106$ & $4676 \pm 114$ & $4522 \pm 78$ & $2.00 \pm 0.40$ & $2.00 \pm 0.39$ & $2.00 \pm 0.28$ & $-0.44 \pm 0.25$ & $-0.65 \pm 0.23$ & $-0.55 \pm 0.17$ & $0.21 \pm 0.20$ & $0.32 \pm 0.07$ & M \\
\hline 6366_1 & $4849 \pm 293$ & $5000 \pm 158$ & $4966 \pm 139$ & $2.50 \pm 0.60$ & $3.25 \pm 0.25$ & $3.14 \pm .23$ & $-0.48 \pm 0.33$ & $-0.85 \pm 0.23$ & $-0.73 \pm 0.19$ & $0.24 \pm 0.21$ & $0.27 \pm 0.09$ & M \\
\hline 6366_2 & $4976 \pm 355$ & $5250 \pm 193$ & $5187 \pm 170$ & $2.60 \pm 0.70$ & $3.15 \pm 0.23$ & $3.10 \pm .22$ & $-0.60 \pm 0.40$ & $-0.65 \pm 0.23$ & $-0.64 \pm 0.20$ & $0.26 \pm 0.20$ & $0.23 \pm 0.13$ & M \\
\hline 6366_3 & $4876 \pm 279$ & $5150 \pm 166$ & $5079 \pm 142$ & $2.60 \pm 0.60$ & $3.30 \pm 0.33$ & $3.14 \pm .29$ & $-0.40 \pm 0.32$ & $-0.60 \pm 0.20$ & $-0.54 \pm 0.17$ & $0.21 \pm 0.21$ & $0.32 \pm 0.07$ & M \\
\hline 6366_4 & $4874 \pm 256$ & $4950 \pm 187$ & $4923 \pm 151$ & $2.70 \pm 0.50$ & $3.20 \pm 0.25$ & $3.10 \pm .22$ & $-0.40 \pm 0.27$ & $-0.80 \pm 0.24$ & $-0.62 \pm 0.18$ & $0.23 \pm 0.21$ & $0.29 \pm 0.09$ & M \\
\hline 6366_5 & $4601 \pm 171$ & $4622 \pm 125$ & $4615 \pm 101$ & $2.39 \pm 0.32$ & $2.10 \pm 0.37$ & $2.27 \pm .24$ & $-0.31 \pm 0.20$ & $-0.76 \pm 0.25$ & $-0.49 \pm 0.16$ & $0.14 \pm 0.17$ & $0.27 \pm 0.11$ & M \\
\hline 6366_6 & $5079 \pm 365$ & $5276 \pm 208$ & $5228 \pm 181$ & $2.70 \pm 0.70$ & $2.65 \pm 0.45$ & $2.66 \pm .38$ & $-0.45 \pm 0.32$ & $-0.70 \pm 0.24$ & $-0.61 \pm 0.19$ & $0.22 \pm 0.18$ & $0.19 \pm 0.14$ & \\
\hline 6366_7 & $4882 \pm 319$ & $4750 \pm 250$ & $4800 \pm 197$ & $2.60 \pm 0.50$ & $2.85 \pm 0.45$ & $2.74 \pm .33$ & $-0.35 \pm 0.28$ & $-1.10 \pm 0.37$ & $-0.62 \pm 0.22$ & $0.19 \pm 0.21$ & $0.31 \pm 0.10$ & M \\
\hline 6366_8 & $4860 \pm 293$ & $5049 \pm 187$ & $4995 \pm 158$ & $2.60 \pm 0.70$ & $3.30 \pm 0.33$ & $3.17 \pm .30$ & $-0.50 \pm 0.34$ & $-0.95 \pm 0.35$ & $-0.72 \pm 0.24$ & $0.26 \pm 0.21$ & $0.34 \pm 0.07$ & M \\
\hline 6366_9 & $4526 \pm 153$ & $4624 \pm 200$ & $4562 \pm 122$ & $2.30 \pm 0.30$ & $2.59 \pm 0.58$ & $2.36 \pm .27$ & $-0.40 \pm 0.16$ & $-0.80 \pm 0.33$ & $-0.48 \pm 0.14$ & $0.21 \pm 0.10$ & $0.26 \pm 0.11$ & M \\
\hline 6366_10 & $4864 \pm 325$ & $5175 \pm 160$ & $5114 \pm 143$ & $2.50 \pm 0.70$ & $3.30 \pm 0.25$ & $3.21 \pm .24$ & $-0.40 \pm 0.40$ & $-0.70 \pm 0.24$ & $-0.62 \pm 0.21$ & $0.22 \pm 0.21$ & $0.32 \pm 0.07$ & M \\
\hline 6366_11 & $4748 \pm 223$ & $4776 \pm 175$ & $4765 \pm 137$ & $2.50 \pm 0.50$ & $2.80 \pm 0.24$ & $2.74 \pm .22$ & $-0.41 \pm 0.27$ & $-0.95 \pm 0.35$ & $-0.61 \pm 0.21$ & $0.22 \pm 0.19$ & $0.29 \pm 0.09$ & $\mathrm{M}$ \\
\hline 6366_13 & $4838 \pm 237$ & $4750 \pm 193$ & $4785 \pm 150$ & $2.60 \pm 0.50$ & $2.95 \pm 0.35$ & $2.83 \pm .29$ & $-0.41 \pm 0.26$ & $-1.10 \pm 0.30$ & $-0.71 \pm 0.20$ & $0.22 \pm 0.21$ & $0.26 \pm 0.13$ & M \\
\hline 6366_14 & $4819 \pm 350$ & $4725 \pm 208$ & $4750 \pm 179$ & $2.40 \pm 0.80$ & $3.25 \pm 0.46$ & $3.04 \pm .40$ & $-0.70 \pm 0.40$ & $-1.75 \pm 0.25$ & $-1.46 \pm 0.21$ & $0.32 \pm 0.19$ & $0.33 \pm 0.08$ & \\
\hline 6366_15 & $4630 \pm 223$ & $4600 \pm 279$ & $4618 \pm 174$ & $2.30 \pm 0.50$ & $2.30 \pm 0.64$ & $2.30 \pm .39$ & $-0.37 \pm 0.28$ & $-1.05 \pm 0.35$ & $-0.64 \pm 0.22$ & $0.21 \pm 0.21$ & $0.32 \pm 0.07$ & M \\
\hline 6366_16 & $4843 \pm 252$ & $4974 \pm 175$ & $4932 \pm 144$ & $2.60 \pm 0.60$ & $3.20 \pm 0.24$ & $3.12 \pm .22$ & $-0.43 \pm 0.30$ & $-0.75 \pm 0.25$ & $-0.62 \pm 0.19$ & $0.22 \pm 0.21$ & $0.30 \pm 0.10$ & M \\
\hline 6366_17 & $4768 \pm 201$ & $4976 \pm 261$ & $4846 \pm 159$ & $2.60 \pm 0.40$ & $2.90 \pm 0.49$ & $2.72 \pm .31$ & $-0.24 \pm 0.25$ & $-0.45 \pm 0.35$ & $-0.31 \pm 0.20$ & $0.14 \pm 0.16$ & $0.29 \pm 0.09$ & \\
\hline 6366_18 & $4376 \pm 115$ & $4474 \pm 175$ & $4406 \pm 96$ & $2.00 \pm 0.50$ & $2.25 \pm 0.51$ & $2.12 \pm .36$ & $-0.55 \pm 0.23$ & $-1.05 \pm 0.35$ & $-0.70 \pm 0.19$ & $0.28 \pm 0.22$ & $0.31 \pm 0.07$ & M \\
\hline 6401_1 & $4512 \pm 219$ & $4625 \pm 167$ & $4583 \pm 133$ & $2.30 \pm 0.50$ & $2.75 \pm 0.40$ & $2.57 \pm 0.31$ & $-0.08 \pm 0.24$ & $-0.80 \pm 0.25$ & $-0.43 \pm 0.17$ & $0.07 \pm 0.17$ & $0.27 \pm 0.11$ & \\
\hline 6401_2 & $4610 \pm 169$ & $4701 \pm 186$ & $4651 \pm 125$ & $2.50 \pm 0.40$ & $2.90 \pm 0.37$ & $2.72 \pm 0.27$ & $-0.08 \pm 0.20$ & $-0.70 \pm 0.24$ & $-0.33 \pm 0.15$ & $0.04 \pm 0.14$ & $0.24 \pm 0.14$ & \\
\hline 6401_3 & $4932 \pm 330$ & $4726 \pm 74$ & $4736 \pm 72$ & $2.20 \pm 0.70$ & $1.70 \pm 0.33$ & $1.79 \pm 0.30$ & $-0.80 \pm 0.40$ & $-1.55 \pm 0.15$ & $-1.46 \pm 0.14$ & $0.38 \pm 0.16$ & $0.20 \pm 0.14$ & M \\
\hline 6401_4 & $3989 \pm 80$ & $4098 \pm 122$ & $4022 \pm 67$ & $1.67 \pm 0.11$ & $1.94 \pm 0.35$ & $1.69 \pm 0.10$ & $0.11 \pm 0.09$ & $-0.50 \pm 0.10$ & $-0.16 \pm 0.07$ & $0.08 \pm 0.09$ & $0.27 \pm 0.11$ & \\
\hline 6401_5 & $4551 \pm 124$ & $4950 \pm 100$ & $4792 \pm 78$ & $2.30 \pm 0.40$ & $3.20 \pm 0.24$ & $2.96 \pm 0.21$ & $0.03 \pm 0.13$ & $0.15 \pm 0.15$ & $0.08 \pm 0.10$ & $0.03 \pm 0.12$ & $0.11 \pm 0.09$ & \\
\hline 6401_6 & $4625 \pm 237$ & $5072 \pm 336$ & $4773 \pm 194$ & $2.20 \pm 0.40$ & $2.39 \pm 0.62$ & $2.26 \pm 0.34$ & $-0.35 \pm 0.30$ & $-0.60 \pm 0.44$ & $-0.43 \pm 0.25$ & $0.21 \pm 0.25$ & $0.30 \pm 0.08$ & \\
\hline $6401 \_7$ & $4841 \pm 310$ & $4975 \pm 261$ & $4919 \pm 200$ & $2.40 \pm 0.80$ & $2.60 \pm 0.58$ & $2.53 \pm 0.47$ & $-0.60 \pm 0.40$ & $-0.95 \pm 0.35$ & $-0.80 \pm 0.26$ & $0.32 \pm 0.19$ & $0.29 \pm 0.09$ & M \\
\hline $6401 \_8$ & $4759 \pm 232$ & $4675 \pm 159$ & $4702 \pm 131$ & $2.40 \pm 0.60$ & $2.20 \pm 0.40$ & $2.26 \pm 0.33$ & $-0.55 \pm 0.26$ & $-1.15 \pm 0.32$ & $-0.79 \pm 0.20$ & $0.29 \pm 0.19$ & $0.29 \pm 0.09$ & M \\
\hline 6401_9 & $4856 \pm 219$ & $4700 \pm 100$ & $4727 \pm 91$ & $2.40 \pm 0.50$ & $1.70 \pm 0.24$ & $1.83 \pm 0.22$ & $-0.46 \pm 0.30$ & $-1.10 \pm 0.20$ & $-0.90 \pm 0.17$ & $0.21 \pm 0.20$ & $0.25 \pm 0.10$ & M \\
\hline
\end{tabular}


Table B.3 - continued.

\begin{tabular}{|c|c|c|c|c|c|c|c|c|c|c|c|c|}
\hline NGC ID & $\mathrm{T}_{\text {eff }}^{(a)}(\mathrm{K})$ & $\mathrm{T}_{e f f}^{(b)}(\mathrm{K})$ & $\mathrm{T}_{\text {eff }}^{(a v g)}(\mathrm{K})$ & $\log (g)^{(a)}$ & $\log (g)^{(b)}$ & $\log (g)^{(a v g)}$ & {$[\mathrm{Fe} / \mathrm{H}]^{(a)}$} & {$[\mathrm{Fe} / \mathrm{H}]^{(b)}$} & {$[\mathrm{Fe} / \mathrm{H}]^{(a v g)}$} & {$[\mathrm{Mg} / \mathrm{Fe}]^{(a)}$} & {$[\alpha / \mathrm{Fe}]^{(b)}$} & members \\
\hline 6401_10 & $4259 \pm 159$ & $4349 \pm 122$ & $4316 \pm 97$ & $1.83 \pm 0.32$ & $2.24 \pm 0.40$ & $1.99 \pm 0.25$ & $-0.01 \pm 0.14$ & $-0.80 \pm 0.24$ & $-0.21 \pm 0.12$ & $-0.02 \pm 0.17$ & $0.33 \pm 0.08$ & \multirow{9}{*}{$\begin{array}{l}\mathrm{M} \\
\mathrm{M}\end{array}$} \\
\hline 6401_11 & $4392 \pm 159$ & $4475 \pm 208$ & $4423 \pm 126$ & $2.10 \pm 0.40$ & $2.35 \pm 0.55$ & $2.19 \pm 0.32$ & $0.13 \pm 0.14$ & $-0.11 \pm 0.35$ & $0.10 \pm 0.13$ & $0.05 \pm 0.16$ & $0.09 \pm 0.08$ & \\
\hline 6401_12 & $4520 \pm 258$ & $4025 \pm 208$ & $4220 \pm 162$ & $1.90 \pm 0.60$ & $0.05 \pm 0.15$ & $0.16 \pm 0.15$ & $-0.60 \pm 0.40$ & $-2.20 \pm 0.24$ & $-1.78 \pm 0.21$ & $0.32 \pm 0.24$ & $0.14 \pm 0.13$ & \\
\hline 6401_13 & $4858 \pm 426$ & $5025 \pm 175$ & $5001 \pm 162$ & $2.00 \pm 0.90$ & $2.30 \pm 0.60$ & $2.21 \pm 0.50$ & $-1.20 \pm 0.50$ & $-1.35 \pm 0.23$ & $-1.32 \pm 0.21$ & $0.39 \pm 0.17$ & $0.29 \pm 0.09$ & \\
\hline 6401_14 & $4869 \pm 200$ & $4599 \pm 199$ & $4733 \pm 141$ & $2.50 \pm 0.50$ & $2.00 \pm 0.45$ & $2.22 \pm 0.33$ & $-0.46 \pm 0.29$ & $-1.40 \pm 0.20$ & $-1.10 \pm 0.16$ & $0.25 \pm 0.22$ & $0.23 \pm 0.13$ & \\
\hline 6401_15 & $4475 \pm 200$ & $4451 \pm 186$ & $4462 \pm 136$ & $2.30 \pm 0.50$ & $2.25 \pm 0.51$ & $2.28 \pm 0.36$ & $-0.09 \pm 0.25$ & $-1.10 \pm 0.37$ & $-0.41 \pm 0.21$ & $0.07 \pm 0.16$ & $0.33 \pm 0.06$ & \\
\hline 6401_16 & $4546 \pm 166$ & $4749 \pm 159$ & $4652 \pm 115$ & $2.30 \pm 0.40$ & $2.60 \pm 0.44$ & $2.44 \pm 0.30$ & $-0.27 \pm 0.26$ & $-0.50 \pm 0.32$ & $-0.36 \pm 0.20$ & $0.14 \pm 0.19$ & $0.32 \pm 0.07$ & \\
\hline 6401_17 & $4554 \pm 181$ & $4600 \pm 278$ & $4568 \pm 152$ & $2.40 \pm 0.50$ & $2.45 \pm 0.65$ & $2.42 \pm 0.40$ & $-0.17 \pm 0.26$ & $-0.65 \pm 0.39$ & $-0.32 \pm 0.22$ & $0.10 \pm 0.17$ & $0.30 \pm 0.10$ & \\
\hline 6401_18 & $4339 \pm 230$ & $4275 \pm 132$ & $4291 \pm 115$ & $2.00 \pm 0.60$ & $1.85 \pm 0.32$ & $1.88 \pm 0.28$ & $-0.09 \pm 0.25$ & $-1.00 \pm 0.22$ & $-0.60 \pm 0.17$ & $0.06 \pm 0.17$ & $0.26 \pm 0.11$ & \\
\hline Pal6_1 & $4240 \pm 163$ & $4325 \pm 159$ & $4283 \pm 114$ & $1.90 \pm 0.50$ & $1.95 \pm 0.41$ & $1.93 \pm 0.32$ & $-0.24 \pm 0.27$ & $-0.85 \pm 0.32$ & $-0.49 \pm 0.21$ & $0.10 \pm 0.19$ & $0.31 \pm 0.09$ & \multirow{10}{*}{ M } \\
\hline Pal6_2 & $4828 \pm 301$ & $5150 \pm 278$ & $5002 \pm 204$ & $2.40 \pm 0.70$ & $3.35 \pm 0.55$ & $2.99 \pm 0.43$ & $-0.50 \pm 0.40$ & $-0.75 \pm 0.34$ & $-0.64 \pm 0.26$ & $0.25 \pm 0.22$ & $0.34 \pm 0.07$ & \\
\hline Pal6_3 & $4900 \pm 155$ & $5249 \pm 158$ & $5071 \pm 111$ & $3.23 \pm 0.35$ & $4.25 \pm 0.25$ & $3.91 \pm 0.20$ & $-0.03 \pm 0.09$ & $-0.25 \pm 0.25$ & $-0.05 \pm 0.08$ & $0.07 \pm 0.08$ & $0.11 \pm 0.08$ & \\
\hline Pal6_4 & $4383 \pm 177$ & $4475 \pm 208$ & $4422 \pm 135$ & $1.90 \pm 0.40$ & $1.95 \pm 0.42$ & $1.92 \pm 0.29$ & $0.09 \pm 0.18$ & $-0.14 \pm 0.30$ & $0.03 \pm 0.15$ & $0.09 \pm 0.12$ & $0.16 \pm 0.11$ & \\
\hline Pal6_5 & $4561 \pm 199$ & $4475 \pm 208$ & $4520 \pm 144$ & $2.10 \pm 0.40$ & $1.90 \pm 0.44$ & $2.01 \pm 0.30$ & $-0.17 \pm 0.21$ & $-0.85 \pm 0.23$ & $-0.48 \pm 0.16$ & $0.12 \pm 0.14$ & $0.24 \pm 0.12$ & \\
\hline Pal6_6 & $4037 \pm 146$ & $3875 \pm 230$ & $3991 \pm 123$ & $1.57 \pm 0.31$ & $1.55 \pm 0.61$ & $1.57 \pm 0.28$ & $-0.12 \pm 0.21$ & $-1.30 \pm 0.40$ & $-0.37 \pm 0.19$ & $0.06 \pm 0.16$ & $0.26 \pm 0.09$ & \\
\hline Pal6_7 & $4042 \pm 144$ & $4150 \pm 166$ & $4088 \pm 109$ & $1.50 \pm 0.40$ & $1.75 \pm 0.51$ & $1.60 \pm 0.31$ & $-0.10 \pm 0.20$ & $-0.55 \pm 0.27$ & $-0.26 \pm 0.16$ & $0.05 \pm 0.17$ & $0.27 \pm 0.09$ & \\
\hline Pal6_8 & $4372 \pm 211$ & $4498 \pm 224$ & $4431 \pm 154$ & $2.10 \pm 0.50$ & $2.10 \pm 0.58$ & $2.10 \pm 0.38$ & $0.04 \pm 0.15$ & $-0.16 \pm 0.29$ & $-0.00 \pm 0.13$ & $0.02 \pm 0.12$ & $0.23 \pm 0.13$ & \\
\hline Pal6_9 & $4273 \pm 259$ & $3850 \pm 199$ & $4007 \pm 158$ & $1.90 \pm 0.60$ & $0.40 \pm 0.54$ & $1.07 \pm 0.40$ & $-0.08 \pm 0.25$ & $-1.55 \pm 0.27$ & $-0.76 \pm 0.18$ & $0.07 \pm 0.17$ & $0.27 \pm 0.15$ & \\
\hline Pal6_10 & $3989 \pm 124$ & $4500 \pm 100$ & $4299 \pm 78$ & $1.67 \pm 0.01$ & $2.30 \pm 0.24$ & $1.67 \pm 0.01$ & $0.11 \pm 0.02$ & $0.10 \pm 0.10$ & $0.11 \pm 0.02$ & $0.08 \pm 0.21$ & $0.30 \pm 0.10$ & \\
\hline Pal6_11 & $4377 \pm 149$ & $3899 \pm 200$ & $4206 \pm 119$ & $2.00 \pm 0.50$ & $0.55 \pm 0.52$ & $1.30 \pm 0.36$ & $-0.45 \pm 0.23$ & $-1.70 \pm 0.40$ & $-0.76 \pm 0.20$ & $0.22 \pm 0.20$ & $0.23 \pm 0.13$ & \multirow{7}{*}{$\begin{array}{l}\mathrm{M} \\
\mathrm{M} \\
\mathrm{M}\end{array}$} \\
\hline Pal6_13 & $4691 \pm 269$ & $4150 \pm 255$ & $4406 \pm 185$ & $2.40 \pm 0.70$ & $1.10 \pm 0.73$ & $1.78 \pm 0.51$ & $-0.40 \pm 0.40$ & $-1.85 \pm 0.39$ & $-1.14 \pm 0.28$ & $0.21 \pm 0.21$ & $0.32 \pm 0.10$ & \\
\hline Pal6_14 & $4469 \pm 226$ & $3925 \pm 160$ & $4106 \pm 130$ & $2.20 \pm 0.60$ & $0.30 \pm 0.46$ & $1.00 \pm 0.37$ & $-0.17 \pm 0.32$ & $-1.65 \pm 0.32$ & $-0.91 \pm 0.23$ & $0.09 \pm 0.20$ & $0.27 \pm 0.09$ & \\
\hline Pal6_15 & $4407 \pm 152$ & $4350 \pm 123$ & $4373 \pm 95$ & $1.70 \pm 0.40$ & $1.95 \pm 0.42$ & $1.82 \pm 0.29$ & $-0.13 \pm 0.10$ & $-0.70 \pm 0.24$ & $-0.21 \pm 0.09$ & $0.14 \pm 0.10$ & $0.33 \pm 0.06$ & \\
\hline Pal6_16 & $4339 \pm 228$ & $4275 \pm 134$ & $4291 \pm 115$ & $2.10 \pm 0.60$ & $1.70 \pm 0.46$ & $1.85 \pm 0.37$ & $0.02 \pm 0.18$ & $-0.95 \pm 0.27$ & $-0.28 \pm 0.15$ & $0.03 \pm 0.14$ & $0.27 \pm 0.11$ & \\
\hline Pal6_17 & $4352 \pm 247$ & $4150 \pm 165$ & $4212 \pm 137$ & $2.00 \pm 0.60$ & $1.30 \pm 0.56$ & $1.63 \pm 0.41$ & $-0.10 \pm 0.28$ & $-1.20 \pm 0.33$ & $-0.56 \pm 0.21$ & $0.07 \pm 0.18$ & $0.32 \pm 0.07$ & \\
\hline Pal6_18 & $4353 \pm 259$ & $4051 \pm 187$ & $4154 \pm 152$ & $2.00 \pm 0.60$ & $1.00 \pm 0.71$ & $1.58 \pm 0.46$ & $-0.08 \pm 0.26$ & $-1.40 \pm 0.37$ & $-0.52 \pm 0.21$ & $0.06 \pm 0.17$ & $0.33 \pm 0.06$ & \\
\hline 6440_1 & $4023 \pm 118$ & $4249 \pm 222$ & $4073 \pm 104$ & $1.56 \pm 0.16$ & $2.05 \pm 0.56$ & $1.60 \pm 0.15$ & $-0.02 \pm 0.15$ & $-0.53 \pm 0.38$ & $-0.09 \pm 0.14$ & $0.04 \pm 0.10$ & $0.13 \pm 0.12$ & \\
\hline 6440_3 & $4733 \pm 136$ & $5250 \pm 156$ & $4956 \pm 103$ & $2.51 \pm 0.25$ & $3.25 \pm 0.25$ & $2.88 \pm 0.18$ & $-0.20 \pm 0.19$ & $-0.01 \pm 0.18$ & $-0.10 \pm 0.13$ & $0.11 \pm 0.17$ & $0.22 \pm 0.12$ & \\
\hline
\end{tabular}


Table B.3 - continued.

\begin{tabular}{|c|c|c|c|c|c|c|c|c|c|c|c|c|}
\hline NGC ID & $\mathrm{T}_{e f f}^{(a)}(\mathrm{K})$ & $\mathrm{T}_{e f f}^{(b)}(\mathrm{K})$ & $\mathrm{T}_{e f f}^{(a v g)}(\mathrm{K})$ & $\log (g)^{(a)}$ & $\log (g)^{(b)}$ & $\log (g)^{(a v g)}$ & {$[\mathrm{Fe} / \mathrm{H}]^{(a)}$} & {$[\mathrm{Fe} / \mathrm{H}]^{(b)}$} & {$[\mathrm{Fe} / \mathrm{H}]^{(a v g)}$} & {$[\mathrm{Mg} / \mathrm{Fe}]^{(a)}$} & {$[\alpha / \mathrm{Fe}]^{(b)}$} & members \\
\hline 6440_4 & $4899 \pm 232$ & $5151 \pm 165$ & $5066 \pm 135$ & $2.60 \pm 0.50$ & $3.15 \pm 0.45$ & $2.90 \pm 0.33$ & $-0.46 \pm 0.24$ & $-0.55 \pm 0.15$ & $-0.52 \pm 0.13$ & $0.25 \pm 0.19$ & $0.22 \pm 0.12$ & \\
\hline $6440 \_5$ & $3946 \pm 27$ & $4074 \pm 114$ & $3953 \pm 26$ & $1.53 \pm 0.13$ & $1.90 \pm 0.30$ & $1.59 \pm 0.12$ & $-0.06 \pm 0.22$ & $-0.85 \pm 0.23$ & $-0.44 \pm 0.16$ & $0.06 \pm 0.08$ & $0.27 \pm 0.11$ & \\
\hline 6440_6 & $4270 \pm 142$ & $4351 \pm 123$ & $4316 \pm 93$ & $1.82 \pm 0.27$ & $2.15 \pm 0.32$ & $1.96 \pm 0.21$ & $0.11 \pm 0.18$ & $-0.30 \pm 0.25$ & $-0.03 \pm 0.15$ & $0.02 \pm 0.10$ & $0.22 \pm 0.15$ & M \\
\hline $6440 \_7$ & $4399 \pm 149$ & $4375 \pm 125$ & $4385 \pm 96$ & $2.10 \pm 0.40$ & $2.10 \pm 0.37$ & $2.10 \pm 0.27$ & $0.06 \pm 0.16$ & $-0.75 \pm 0.25$ & $-0.17 \pm 0.13$ & $0.04 \pm 0.15$ & $0.28 \pm 0.12$ & M \\
\hline 6440_8 & $3907 \pm 77$ & $3621 \pm 166$ & $3857 \pm 70$ & $1.40 \pm 0.18$ & $0.78 \pm 0.71$ & $1.36 \pm 0.17$ & $-0.04 \pm 0.18$ & $-1.26 \pm 0.33$ & $-0.32 \pm 0.16$ & $0.07 \pm 0.17$ & $0.26 \pm 0.11$ & M \\
\hline 6440_10 & $4267 \pm 206$ & $4374 \pm 167$ & $4332 \pm 130$ & $1.80 \pm 0.40$ & $2.25 \pm 0.40$ & $2.02 \pm 0.28$ & $0.05 \pm 0.19$ & $-0.75 \pm 0.33$ & $-0.15 \pm 0.16$ & $0.07 \pm 0.13$ & $0.33 \pm 0.08$ & M \\
\hline 6440_11 & $3684 \pm 45$ & $3500 \pm 100$ & $3653 \pm 41$ & $0.80 \pm 0.10$ & $2.75 \pm 0.75$ & $0.83 \pm 0.10$ & $0.13 \pm 0.13$ & $-1.15 \pm 0.23$ & $-0.18 \pm 0.11$ & $0.16 \pm 0.07$ & $0.31 \pm 0.08$ & \\
\hline 6440_13 & $4498 \pm 110$ & $4524 \pm 236$ & $4503 \pm 100$ & $2.04 \pm 0.32$ & $2.00 \pm 0.50$ & $2.03 \pm 0.27$ & $-0.24 \pm 0.26$ & $-0.65 \pm 0.32$ & $-0.40 \pm 0.20$ & $0.15 \pm 0.18$ & $0.33 \pm 0.08$ & M \\
\hline 6440_14 & $3916 \pm 113$ & $3600 \pm 122$ & $3771 \pm 83$ & $1.36 \pm 0.04$ & $1.19 \pm 0.64$ & $1.36 \pm 0.04$ & $0.08 \pm 0.22$ & $-1.30 \pm 0.24$ & $-0.55 \pm 0.16$ & $0.11 \pm 0.09$ & $0.32 \pm 0.06$ & M \\
\hline 6440_15 & $4459 \pm 139$ & $4352 \pm 123$ & $4399 \pm 92$ & $1.72 \pm 0.27$ & $1.96 \pm 0.42$ & $1.79 \pm 0.23$ & $-0.13 \pm 0.10$ & $-0.70 \pm 0.24$ & $-0.21 \pm 0.09$ & $0.20 \pm 0.08$ & $0.33 \pm 0.06$ & M \\
\hline 6440_16 & $3989 \pm 124$ & $4401 \pm 199$ & $4104 \pm 105$ & $1.67 \pm 0.01$ & $2.30 \pm 0.40$ & $1.67 \pm 0.01$ & $0.11 \pm 0.02$ & $-0.02 \pm 0.25$ & $0.11 \pm 0.02$ & $0.08 \pm 0.21$ & $0.18 \pm 0.14$ & \\
\hline 6440_17 & $3685 \pm 45$ & $3524 \pm 74$ & $3641 \pm 38$ & $0.80 \pm 0.10$ & $2.41 \pm 0.83$ & $0.82 \pm 0.10$ & $0.13 \pm 0.13$ & $-1.00 \pm 0.22$ & $-0.16 \pm 0.11$ & $0.16 \pm 0.07$ & $0.30 \pm 0.10$ & \\
\hline 6440_18 & $3976 \pm 84$ & $4275 \pm 134$ & $4060 \pm 71$ & $1.54 \pm 0.17$ & $2.35 \pm 0.45$ & $1.64 \pm 0.16$ & $0.03 \pm 0.17$ & $-0.45 \pm 0.27$ & $-0.11 \pm 0.14$ & $-0.03 \pm 0.17$ & $0.23 \pm 0.13$ & \\
\hline 6440_19 & $4268 \pm 150$ & $4351 \pm 123$ & $4318 \pm 95$ & $1.86 \pm 0.29$ & $2.30 \pm 0.25$ & $2.11 \pm 0.19$ & $0.16 \pm 0.18$ & $-0.30 \pm 0.25$ & $0.00 \pm 0.15$ & $0.00 \pm 0.11$ & $0.16 \pm 0.14$ & \\
\hline 6453_1 & $4777 \pm 183$ & $5026 \pm 234$ & $4872 \pm 144$ & $2.50 \pm 0.40$ & $2.76 \pm 0.46$ & $2.61 \pm 0.30$ & $-0.31 \pm 0.23$ & $-0.40 \pm 0.37$ & $-0.34 \pm 0.20$ & $0.15 \pm 0.19$ & $0.29 \pm 0.12$ & \\
\hline 6453_2 & $4620 \pm 194$ & $4751 \pm 157$ & $4699 \pm 122$ & $2.50 \pm 0.50$ & $3.15 \pm 0.39$ & $2.90 \pm 0.31$ & $-0.22 \pm 0.25$ & $-0.75 \pm 0.25$ & $-0.48 \pm 0.18$ & $0.12 \pm 0.17$ & $0.29 \pm 0.09$ & \\
\hline 6453_3 & $4805 \pm 212$ & $4826 \pm 115$ & $4821 \pm 101$ & $2.50 \pm 0.50$ & $2.85 \pm 0.23$ & $2.79 \pm 0.21$ & $-0.46 \pm 0.25$ & $-0.85 \pm 0.23$ & $-0.67 \pm 0.17$ & $0.26 \pm 0.19$ & $0.25 \pm 0.10$ & \\
\hline 6453_4 & $4434 \pm 143$ & $4824 \pm 114$ & $4672 \pm 89$ & $2.20 \pm 0.50$ & $3.15 \pm 0.23$ & $2.98 \pm 0.21$ & $0.11 \pm 0.15$ & $0.12 \pm 0.10$ & $0.12 \pm 0.08$ & $0.04 \pm 0.14$ & $0.20 \pm 0.12$ & \\
\hline 6453_5 & $4888 \pm 252$ & $5000 \pm 224$ & $4951 \pm 167$ & $2.50 \pm 0.60$ & $2.75 \pm 0.46$ & $2.66 \pm 0.37$ & $-0.47 \pm 0.30$ & $-0.70 \pm 0.24$ & $-0.61 \pm 0.19$ & $0.25 \pm 0.19$ & $0.24 \pm 0.08$ & \\
\hline 6453_6 & $4744 \pm 188$ & $4900 \pm 166$ & $4832 \pm 124$ & $2.50 \pm 0.40$ & $2.65 \pm 0.32$ & $2.59 \pm 0.25$ & $-0.07 \pm 0.22$ & $-0.23 \pm 0.28$ & $-0.13 \pm 0.17$ & $0.03 \pm 0.12$ & $0.26 \pm 0.10$ & \\
\hline $6453 \_7$ & $4542 \pm 28$ & $4700 \pm 218$ & $4545 \pm 28$ & $2.19 \pm 0.27$ & $2.30 \pm 0.56$ & $2.21 \pm 0.24$ & $-0.09 \pm 0.08$ & $-0.33 \pm 0.26$ & $-0.11 \pm 0.08$ & $0.13 \pm 0.11$ & $0.25 \pm 0.08$ & \\
\hline 6453_8 & $4859 \pm 186$ & $4774 \pm 236$ & $4826 \pm 146$ & $2.20 \pm 0.50$ & $1.95 \pm 0.42$ & $2.05 \pm 0.32$ & $-0.73 \pm 0.15$ & $-1.70 \pm 0.25$ & $-0.99 \pm 0.13$ & $0.38 \pm 0.13$ & $0.26 \pm 0.09$ & \\
\hline 6453_10 & $4396 \pm 176$ & $4650 \pm 166$ & $4531 \pm 121$ & $1.10 \pm 0.50$ & $1.15 \pm 0.50$ & $1.12 \pm 0.35$ & $-1.51 \pm 0.21$ & $-1.65 \pm 0.23$ & $-1.57 \pm 0.16$ & $0.44 \pm 0.14$ & $0.17 \pm 0.09$ & M \\
\hline 6453_11 & $4826 \pm 379$ & $4726 \pm 74$ & $4730 \pm 72$ & $2.10 \pm 0.90$ & $1.70 \pm 0.33$ & $1.75 \pm 0.31$ & $-1.00 \pm 0.60$ & $-1.55 \pm 0.15$ & $-1.52 \pm 0.15$ & $0.36 \pm 0.18$ & $0.22 \pm 0.15$ & M \\
\hline 6453_13 & $4371 \pm 158$ & $4451 \pm 149$ & $4413 \pm 108$ & $2.10 \pm 0.40$ & $2.15 \pm 0.39$ & $2.13 \pm 0.28$ & $0.11 \pm 0.14$ & $-0.13 \pm 0.25$ & $0.05 \pm 0.12$ & $0.03 \pm 0.15$ & $0.13 \pm 0.09$ & \\
\hline 6453_14 & $4915 \pm 272$ & $5077 \pm 160$ & $5036 \pm 138$ & $2.50 \pm 0.60$ & $2.56 \pm 0.41$ & $2.54 \pm 0.34$ & $-0.48 \pm 0.32$ & $-0.80 \pm 0.25$ & $-0.68 \pm 0.20$ & $0.23 \pm 0.20$ & $0.26 \pm 0.13$ & \\
\hline 6453_15 & $4893 \pm 469$ & $4950 \pm 149$ & $4945 \pm 142$ & $2.00 \pm 0.90$ & $1.95 \pm 0.42$ & $1.96 \pm 0.38$ & $-1.40 \pm 0.50$ & $-1.55 \pm 0.15$ & $-1.54 \pm 0.14$ & $0.43 \pm 0.16$ & $0.12 \pm 0.10$ & M \\
\hline 6453_16 & $4295 \pm 220$ & $4500 \pm 100$ & $4465 \pm 91$ & $2.00 \pm 0.50$ & $2.15 \pm 0.23$ & $2.12 \pm 0.21$ & $0.23 \pm 0.12$ & $0.04 \pm 0.08$ & $0.10 \pm 0.07$ & $0.02 \pm 0.14$ & $0.18 \pm 0.12$ & \\
\hline
\end{tabular}


Table B.3 - continued.

\begin{tabular}{|c|c|c|c|c|c|c|c|c|c|c|c|c|}
\hline NGC ID & $\mathrm{T}_{e f f}^{(a)}(\mathrm{K})$ & $\mathrm{T}_{e f f}^{(b)}(\mathrm{K})$ & $\mathrm{T}_{\text {eff }}^{(\text {avg) }}(\mathrm{K})$ & $\log (g)^{(a)}$ & $\log (g)^{(b)}$ & $\log (g)^{(a v g)}$ & {$[\mathrm{Fe} / \mathrm{H}]^{(a)}$} & {$[\mathrm{Fe} / \mathrm{H}]^{(b)}$} & {$[\mathrm{Fe} / \mathrm{H}]^{(a v g)}$} & {$[\mathrm{Mg} / \mathrm{Fe}]^{(a)}$} & {$[\alpha / \mathrm{Fe}]^{(b)}$} & members \\
\hline 6453_17 & $4655 \pm 206$ & $4824 \pm 160$ & $4761 \pm 126$ & $2.50 \pm 0.50$ & $2.85 \pm 0.39$ & $2.72 \pm 0.31$ & $-0.15 \pm 0.25$ & $-0.40 \pm 0.30$ & $-0.25 \pm 0.19$ & $0.10 \pm 0.15$ & $0.31 \pm 0.10$ & \\
\hline 6453_18 & $4459 \pm 144$ & $4775 \pm 135$ & $4628 \pm 98$ & $2.20 \pm 0.50$ & $2.80 \pm 0.24$ & $2.69 \pm 0.22$ & $0.10 \pm 0.13$ & $0.07 \pm 0.27$ & $0.09 \pm 0.12$ & $0.03 \pm 0.15$ & $0.14 \pm 0.08$ & \\
\hline $6538 \_2$ & $4214 \pm 169$ & $4374 \pm 125$ & $4318 \pm 100$ & $1.90 \pm 0.40$ & $2.49 \pm 0.39$ & $2.20 \pm 0.28$ & $-0.15 \pm 0.22$ & $-0.75 \pm 0.25$ & $-0.41 \pm 0.17$ & $0.06 \pm 0.18$ & $0.28 \pm 0.07$ & \\
\hline 6538_3 & $4540 \pm 180$ & $4576 \pm 159$ & $4560 \pm 119$ & $2.30 \pm 0.50$ & $2.55 \pm 0.41$ & $2.45 \pm 0.32$ & $-0.28 \pm 0.26$ & $-0.85 \pm 0.32$ & $-0.51 \pm 0.20$ & $0.15 \pm 0.18$ & $0.26 \pm 0.10$ & \\
\hline 6538_4 & $3890 \pm 115$ & $3576 \pm 115$ & $3733 \pm 81$ & $1.29 \pm 0.18$ & $0.80 \pm 0.60$ & $1.25 \pm 0.17$ & $0.03 \pm 0.18$ & $-1.35 \pm 0.23$ & $-0.49 \pm 0.14$ & $0.05 \pm 0.19$ & $0.26 \pm 0.09$ & M \\
\hline $6538 \_5$ & $4416 \pm 161$ & $4347 \pm 122$ & $4372 \pm 97$ & $1.90 \pm 0.40$ & $1.99 \pm 0.32$ & $1.95 \pm 0.25$ & $-0.21 \pm 0.25$ & $-0.81 \pm 0.24$ & $-0.52 \pm 0.17$ & $0.17 \pm 0.14$ & $0.29 \pm 0.09$ & M \\
\hline 6538_6 & $4798 \pm 208$ & $4900 \pm 165$ & $4860 \pm 129$ & $2.60 \pm 0.40$ & $2.75 \pm 0.40$ & $2.67 \pm 0.28$ & $-0.25 \pm 0.29$ & $-0.75 \pm 0.25$ & $-0.54 \pm 0.19$ & $0.14 \pm 0.21$ & $0.25 \pm 0.14$ & \\
\hline 6538_7 & $4283 \pm 174$ & $4201 \pm 100$ & $4221 \pm 86$ & $1.90 \pm 0.40$ & $1.90 \pm 0.37$ & $1.90 \pm 0.27$ & $0.12 \pm 0.19$ & $-0.60 \pm 0.20$ & $-0.22 \pm 0.14$ & $0.04 \pm 0.11$ & $0.22 \pm 0.12$ & \\
\hline $6538 \_8$ & $4499 \pm 109$ & $4501 \pm 193$ & $4499 \pm 95$ & $2.04 \pm 0.32$ & $2.30 \pm 0.60$ & $2.10 \pm 0.28$ & $-0.24 \pm 0.25$ & $-0.85 \pm 0.23$ & $-0.57 \pm 0.17$ & $0.15 \pm 0.18$ & $0.30 \pm 0.08$ & M \\
\hline 6538_9 & $4777 \pm 262$ & $4701 \pm 218$ & $4732 \pm 168$ & $2.50 \pm 0.50$ & $2.30 \pm 0.56$ & $2.41 \pm 0.37$ & $-0.41 \pm 0.31$ & $-0.85 \pm 0.23$ & $-0.69 \pm 0.18$ & $0.21 \pm 0.21$ & $0.30 \pm 0.08$ & M \\
\hline 6538_10 & $4505 \pm 177$ & $4600 \pm 165$ & $4556 \pm 121$ & $2.20 \pm 0.50$ & $2.45 \pm 0.47$ & $2.33 \pm 0.34$ & $-0.41 \pm 0.24$ & $-0.75 \pm 0.25$ & $-0.57 \pm 0.17$ & $0.21 \pm 0.21$ & $0.28 \pm 0.07$ & M \\
\hline 6538_11 & $3888 \pm 117$ & $3569 \pm 112$ & $3721 \pm 81$ & $1.28 \pm 0.18$ & $0.61 \pm 0.59$ & $1.22 \pm 0.17$ & $0.03 \pm 0.18$ & $-1.36 \pm 0.22$ & $-0.53 \pm 0.14$ & $0.05 \pm 0.19$ & $0.25 \pm 0.10$ & \\
\hline 6538_13 & $3892 \pm 100$ & $3549 \pm 99$ & $3720 \pm 70$ & $1.20 \pm 0.20$ & $0.89 \pm 0.63$ & $1.17 \pm 0.19$ & $-0.03 \pm 0.40$ & $-1.55 \pm 0.27$ & $-1.07 \pm 0.22$ & $0.13 \pm 0.08$ & $0.29 \pm 0.10$ & \\
\hline 6538_14 & $4145 \pm 157$ & $4200 \pm 187$ & $4168 \pm 120$ & $1.60 \pm 0.40$ & $1.80 \pm 0.56$ & $1.67 \pm 0.33$ & $-0.25 \pm 0.20$ & $-1.10 \pm 0.37$ & $-0.44 \pm 0.18$ & $0.08 \pm 0.17$ & $0.33 \pm 0.06$ & M \\
\hline 6538_15 & $4702 \pm 271$ & $4700 \pm 187$ & $4701 \pm 154$ & $2.30 \pm 0.60$ & $2.65 \pm 0.32$ & $2.57 \pm 0.28$ & $-0.40 \pm 0.40$ & $-1.10 \pm 0.37$ & $-0.78 \pm 0.27$ & $0.20 \pm 0.22$ & $0.32 \pm 0.07$ & \\
\hline 6538_17 & $4456 \pm 127$ & $4775 \pm 75$ & $4693 \pm 64$ & $2.40 \pm 0.40$ & $3.30 \pm 0.24$ & $3.06 \pm 0.21$ & $0.30 \pm 0.07$ & $0.20 \pm 0.17$ & $0.29 \pm 0.06$ & $0.06 \pm 0.08$ & $0.13 \pm 0.10$ & \\
\hline 6538_18 & $4661 \pm 233$ & $4999 \pm 193$ & $4861 \pm 149$ & $2.40 \pm 0.50$ & $3.40 \pm 0.37$ & $3.05 \pm 0.30$ & $-0.47 \pm 0.27$ & $-0.65 \pm 0.23$ & $-0.57 \pm 0.18$ & $0.26 \pm 0.20$ & $0.32 \pm 0.07$ & M \\
\hline Pal7_1 & $4286 \pm 149$ & $4250 \pm 156$ & $4269 \pm 108$ & $1.90 \pm 0.50$ & $1.85 \pm 0.32$ & $1.86 \pm 0.27$ & $-0.38 \pm 0.26$ & $-1.05 \pm 0.27$ & $-0.70 \pm 0.19$ & $0.20 \pm 0.21$ & $0.28 \pm 0.10$ & M \\
\hline Pal7_3 & $4452 \pm 206$ & $4447 \pm 187$ & $4449 \pm 138$ & $2.20 \pm 0.50$ & $1.99 \pm 0.50$ & $2.10 \pm 0.35$ & $-0.06 \pm 0.24$ & $-0.75 \pm 0.25$ & $-0.39 \pm 0.17$ & $0.05 \pm 0.17$ & $0.28 \pm 0.14$ & M \\
\hline Pal7_4 & $3900 \pm 102$ & $3549 \pm 100$ & $3720 \pm 71$ & $1.26 \pm 0.17$ & $1.14 \pm 0.63$ & $1.25 \pm 0.16$ & $0.07 \pm 0.17$ & $-1.40 \pm 0.20$ & $-0.55 \pm 0.13$ & $0.02 \pm 0.20$ & $0.35 \pm 0.07$ & M \\
\hline Pal7_5 & $4322 \pm 153$ & $4250 \pm 193$ & $4294 \pm 120$ & $1.90 \pm 0.50$ & $2.55 \pm 0.65$ & $2.14 \pm 0.40$ & $-0.50 \pm 0.20$ & $-1.85 \pm 0.39$ & $-0.78 \pm 0.18$ & $0.25 \pm 0.19$ & $0.36 \pm 0.05$ & \\
\hline Pal7_7 & $3994 \pm 98$ & $3625 \pm 168$ & $3900 \pm 85$ & $1.50 \pm 0.40$ & $1.10 \pm 0.62$ & $1.38 \pm 0.34$ & $-0.27 \pm 0.19$ & $-1.85 \pm 0.23$ & $-0.91 \pm 0.15$ & $0.11 \pm 0.17$ & $0.32 \pm 0.09$ & M \\
\hline Pal7_8 & $4346 \pm 216$ & $4348 \pm 122$ & $4347 \pm 106$ & $2.10 \pm 0.50$ & $2.05 \pm 0.35$ & $2.07 \pm 0.29$ & $-0.00 \pm 0.19$ & $-0.80 \pm 0.24$ & $-0.31 \pm 0.15$ & $0.03 \pm 0.14$ & $0.28 \pm 0.10$ & M \\
\hline Pal7_9 & $3932 \pm 43$ & $3674 \pm 195$ & $3920 \pm 42$ & $1.42 \pm 0.21$ & $0.89 \pm 0.74$ & $1.38 \pm 0.20$ & $-0.20 \pm 0.23$ & $-1.45 \pm 0.27$ & $-0.73 \pm 0.18$ & $0.12 \pm 0.17$ & $0.30 \pm 0.12$ & M \\
\hline Pal7_10 & $3942 \pm 46$ & $3776 \pm 175$ & $3931 \pm 44$ & $1.50 \pm 0.15$ & $1.15 \pm 0.71$ & $1.49 \pm 0.15$ & $-0.06 \pm 0.16$ & $-1.35 \pm 0.23$ & $-0.48 \pm 0.13$ & $0.06 \pm 0.12$ & $0.30 \pm 0.10$ & M \\
\hline Pal7_11 & $4296 \pm 194$ & $4374 \pm 125$ & $4351 \pm 105$ & $2.00 \pm 0.50$ & $2.05 \pm 0.35$ & $2.03 \pm 0.29$ & $0.00 \pm 0.20$ & $-0.75 \pm 0.25$ & $-0.29 \pm 0.16$ & $0.03 \pm 0.15$ & $0.29 \pm 0.09$ & M \\
\hline Pal7_12 & $4428 \pm 197$ & $4350 \pm 123$ & $4372 \pm 104$ & $2.20 \pm 0.50$ & $2.00 \pm 0.32$ & $2.06 \pm 0.27$ & $-0.20 \pm 0.28$ & $-0.80 \pm 0.25$ & $-0.53 \pm 0.19$ & $0.11 \pm 0.18$ & $0.27 \pm 0.09$ & M \\
\hline Pal7_13 & $4522 \pm 247$ & $4575 \pm 275$ & $4546 \pm 184$ & $1.90 \pm 0.50$ & $2.35 \pm 0.64$ & $2.07 \pm 0.39$ & $-0.50 \pm 0.40$ & $-1.20 \pm 0.40$ & $-0.85 \pm 0.28$ & $0.30 \pm 0.26$ & $0.32 \pm 0.07$ & \\
\hline
\end{tabular}


Table B.3 - continued.

\begin{tabular}{|c|c|c|c|c|c|c|c|c|c|c|c|c|}
\hline NGC ID & $\mathrm{T}_{e f f}^{(a)}(\mathrm{K})$ & $\mathrm{T}_{e f f}^{(b)}(\mathrm{K})$ & $\mathrm{T}_{e f f}^{(a v g)}(\mathrm{K})$ & $\log (g)^{(a)}$ & $\log (g)^{(b)}$ & $\log (g)^{(a v g)}$ & {$[\mathrm{Fe} / \mathrm{H}]^{(a)}$} & {$[\mathrm{Fe} / \mathrm{H}]^{(b)}$} & {$[\mathrm{Fe} / \mathrm{H}]^{(a v g)}$} & {$[\mathrm{Mg} / \mathrm{Fe}]^{(a)}$} & {$[\alpha / \mathrm{Fe}]^{(b)}$} & members \\
\hline Pal7_14 & $4241 \pm 176$ & $4325 \pm 115$ & $4300 \pm 96$ & $1.90 \pm 0.40$ & $2.30 \pm 0.40$ & $2.10 \pm 0.28$ & $-0.13 \pm 0.23$ & $-0.85 \pm 0.23$ & $-0.49 \pm 0.16$ & $0.06 \pm 0.18$ & $0.30 \pm 0.08$ & \\
\hline Pal7_15 & $4256 \pm 168$ & $4525 \pm 207$ & $4363 \pm 131$ & $1.90 \pm 0.40$ & $2.60 \pm 0.62$ & $2.11 \pm 0.34$ & $-0.27 \pm 0.28$ & $-0.55 \pm 0.35$ & $-0.38 \pm 0.22$ & $0.14 \pm 0.20$ & $0.32 \pm 0.07$ & M \\
\hline Pal7_16 & $4273 \pm 169$ & $4423 \pm 195$ & $4338 \pm 128$ & $1.90 \pm 0.50$ & $2.35 \pm 0.67$ & $2.06 \pm 0.40$ & $-0.24 \pm 0.26$ & $-0.75 \pm 0.25$ & $-0.50 \pm 0.18$ & $0.12 \pm 0.17$ & $0.30 \pm 0.10$ & \\
\hline Pal7_17 & $4364 \pm 108$ & $4399 \pm 199$ & $4372 \pm 95$ & $2.00 \pm 0.40$ & $2.20 \pm 0.51$ & $2.08 \pm 0.31$ & $-0.39 \pm 0.28$ & $-0.90 \pm 0.20$ & $-0.73 \pm 0.16$ & $0.22 \pm 0.20$ & $0.29 \pm 0.09$ & M \\
\hline Pal7_18 & $3961 \pm 96$ & $3827 \pm 160$ & $3925 \pm 82$ & $1.36 \pm 0.26$ & $1.26 \pm 0.64$ & $1.35 \pm 0.24$ & $-0.26 \pm 0.27$ & $-1.30 \pm 0.25$ & $-0.82 \pm 0.18$ & $0.18 \pm 0.21$ & $0.30 \pm 0.08$ & M \\
\hline Pal7_19 & $4550 \pm 196$ & $4475 \pm 175$ & $4508 \pm 131$ & $2.30 \pm 0.50$ & $2.65 \pm 0.50$ & $2.47 \pm 0.35$ & $-0.29 \pm 0.26$ & $-1.10 \pm 0.30$ & $-0.64 \pm 0.20$ & $0.16 \pm 0.18$ & $0.29 \pm 0.09$ & \\
\hline 6569_1 & $4764 \pm 207$ & $4828 \pm 337$ & $4781 \pm 176$ & $2.30 \pm 0.60$ & $2.40 \pm 0.62$ & $2.35 \pm 0.43$ & $-0.60 \pm 0.18$ & $-1.10 \pm 0.44$ & $-0.67 \pm 0.17$ & $0.38 \pm 0.22$ & $0.30 \pm 0.08$ & M \\
\hline $6569 \_2$ & $4057 \pm 100$ & $4103 \pm 251$ & $4063 \pm 93$ & $1.50 \pm 0.40$ & $1.76 \pm 0.71$ & $1.56 \pm 0.35$ & $-0.22 \pm 0.32$ & $-1.14 \pm 0.39$ & $-0.59 \pm 0.25$ & $0.10 \pm 0.27$ & $0.34 \pm 0.07$ & \\
\hline 6569_3 & $4398 \pm 99$ & $4625 \pm 125$ & $4486 \pm 78$ & $2.10 \pm 0.40$ & $2.65 \pm 0.32$ & $2.44 \pm 0.25$ & $-0.46 \pm 0.18$ & $-0.75 \pm 0.25$ & $-0.56 \pm 0.15$ & $0.21 \pm 0.20$ & $0.27 \pm 0.11$ & \\
\hline 6569_4 & $4809 \pm 243$ & $5000 \pm 224$ & $4912 \pm 165$ & $2.40 \pm 0.60$ & $2.85 \pm 0.45$ & $2.69 \pm 0.36$ & $-0.56 \pm 0.26$ & $-0.70 \pm 0.25$ & $-0.63 \pm 0.18$ & $0.29 \pm 0.19$ & $0.29 \pm 0.07$ & $\mathrm{M}$ \\
\hline $6569 \_5$ & $4349 \pm 117$ & $4576 \pm 195$ & $4409 \pm 100$ & $2.10 \pm 0.40$ & $2.65 \pm 0.39$ & $2.38 \pm 0.28$ & $0.11 \pm 0.14$ & $-0.19 \pm 0.32$ & $0.06 \pm 0.13$ & $-0.00 \pm 0.15$ & $0.15 \pm 0.10$ & \\
\hline 6569_6 & $4778 \pm 170$ & $4950 \pm 187$ & $4856 \pm 126$ & $2.50 \pm 0.50$ & $3.20 \pm 0.24$ & $3.07 \pm 0.22$ & $-0.50 \pm 0.24$ & $-0.80 \pm 0.25$ & $-0.64 \pm 0.17$ & $0.30 \pm 0.19$ & $0.29 \pm 0.09$ & \\
\hline 6569_7 & $4507 \pm 95$ & $4799 \pm 148$ & $4592 \pm 80$ & $2.30 \pm 0.40$ & $3.05 \pm 0.27$ & $2.82 \pm 0.22$ & $0.10 \pm 0.11$ & $0.10 \pm 0.24$ & $0.10 \pm 0.10$ & $0.11 \pm 0.11$ & $0.14 \pm 0.09$ & \\
\hline 6569_8 & $4891 \pm 248$ & $4875 \pm 321$ & $4885 \pm 196$ & $2.60 \pm 0.60$ & $2.50 \pm 0.55$ & $2.55 \pm 0.41$ & $-0.51 \pm 0.28$ & $-1.05 \pm 0.42$ & $-0.68 \pm 0.23$ & $0.27 \pm 0.20$ & $0.31 \pm 0.08$ & $\mathrm{M}$ \\
\hline 6569_9 & $4808 \pm 238$ & $4851 \pm 123$ & $4842 \pm 109$ & $2.50 \pm 0.60$ & $2.55 \pm 0.41$ & $2.53 \pm 0.34$ & $-0.48 \pm 0.27$ & $-0.80 \pm 0.25$ & $-0.65 \pm 0.18$ & $0.24 \pm 0.19$ & $0.25 \pm 0.10$ & M \\
\hline 6569_10 & $4642 \pm 175$ & $4497 \pm 222$ & $4586 \pm 137$ & $2.40 \pm 0.40$ & $1.89 \pm 0.49$ & $2.20 \pm 0.31$ & $-0.42 \pm 0.21$ & $-1.10 \pm 0.30$ & $-0.64 \pm 0.17$ & $0.22 \pm 0.22$ & $0.31 \pm 0.10$ & M \\
\hline 6569_11 & $4809 \pm 358$ & $5025 \pm 74$ & $5016 \pm 73$ & $2.20 \pm 0.90$ & $2.25 \pm 0.40$ & $2.24 \pm 0.37$ & $-0.90 \pm 0.50$ & $-0.95 \pm 0.15$ & $-0.95 \pm 0.14$ & $0.31 \pm 0.17$ & $0.23 \pm 0.14$ & \\
\hline 6569_12 & $4876 \pm 275$ & $5050 \pm 187$ & $4995 \pm 155$ & $2.60 \pm 0.60$ & $3.05 \pm 0.35$ & $2.94 \pm 0.30$ & $-0.49 \pm 0.31$ & $-0.70 \pm 0.24$ & $-0.62 \pm 0.19$ & $0.26 \pm 0.20$ & $0.24 \pm 0.09$ & M \\
\hline 6569_13 & $4692 \pm 180$ & $4774 \pm 175$ & $4734 \pm 126$ & $2.00 \pm 0.40$ & $2.50 \pm 0.45$ & $2.22 \pm 0.30$ & $-0.61 \pm 0.24$ & $-0.95 \pm 0.35$ & $-0.72 \pm 0.20$ & $0.44 \pm 0.18$ & $0.30 \pm 0.08$ & M \\
\hline 6569_14 & $4560 \pm 161$ & $4825 \pm 195$ & $4667 \pm 124$ & $2.40 \pm 0.40$ & $2.95 \pm 0.52$ & $2.60 \pm 0.32$ & $-0.06 \pm 0.19$ & $-0.06 \pm 0.23$ & $-0.06 \pm 0.15$ & $0.07 \pm 0.13$ & $0.21 \pm 0.11$ & \\
\hline 6569_15 & $4531 \pm 31$ & $4975 \pm 134$ & $4554 \pm 30$ & $2.17 \pm 0.23$ & $2.95 \pm 0.27$ & $2.50 \pm 0.18$ & $-0.10 \pm 0.07$ & $0.00 \pm 0.26$ & $-0.09 \pm 0.07$ & $0.12 \pm 0.11$ & $0.27 \pm 0.11$ & \\
\hline 6569_16 & $4789 \pm 165$ & $4974 \pm 174$ & $4876 \pm 120$ & $2.50 \pm 0.40$ & $2.75 \pm 0.25$ & $2.68 \pm 0.21$ & $-0.37 \pm 0.22$ & $-0.55 \pm 0.35$ & $-0.42 \pm 0.19$ & $0.19 \pm 0.21$ & $0.28 \pm 0.10$ & \\
\hline 6569_17 & $4565 \pm 85$ & $4824 \pm 114$ & $4657 \pm 68$ & $2.70 \pm 0.40$ & $3.15 \pm 0.23$ & $3.04 \pm 0.20$ & $0.27 \pm 0.03$ & $0.12 \pm 0.10$ & $0.26 \pm 0.03$ & $0.13 \pm 0.06$ & $0.17 \pm 0.11$ & \\
\hline 6569_19 & $4755 \pm 195$ & $4926 \pm 159$ & $4857 \pm 123$ & $2.50 \pm 0.50$ & $3.10 \pm 0.30$ & $2.94 \pm 0.26$ & $-0.42 \pm 0.24$ & $-0.70 \pm 0.24$ & $-0.56 \pm 0.17$ & $0.22 \pm 0.20$ & $0.28 \pm 0.10$ & \\
\hline 6749_1 & $4765 \pm 364$ & $4575 \pm 195$ & $4618 \pm 172$ & $1.70 \pm 0.70$ & $1.40 \pm 0.49$ & $1.50 \pm 0.40$ & $-1.64 \pm 0.32$ & $-2.25 \pm 0.25$ & $-2.02 \pm 0.20$ & $0.43 \pm 0.13$ & $0.11 \pm 0.09$ & \\
\hline 6749_2 & $4650 \pm 215$ & $5175 \pm 115$ & $5059 \pm 101$ & $2.40 \pm 0.50$ & $3.45 \pm 0.35$ & $3.10 \pm 0.29$ & $-0.06 \pm 0.21$ & $0.08 \pm 0.10$ & $0.05 \pm 0.09$ & $0.03 \pm 0.14$ & $0.13 \pm 0.11$ & \\
\hline 6749_3 & $4666 \pm 411$ & $4425 \pm 115$ & $4442 \pm 110$ & $1.50 \pm 0.70$ & $0.10 \pm 0.20$ & $0.21 \pm 0.19$ & $-1.60 \pm 0.60$ & $-2.50 \pm 0.10$ & $-2.48 \pm 0.10$ & $0.45 \pm 0.14$ & $0.17 \pm 0.13$ & \\
\hline 6749_4 & $4483 \pm 288$ & $4250 \pm 100$ & $4275 \pm 94$ & $1.60 \pm 0.70$ & $0.25 \pm 0.25$ & $0.40 \pm 0.24$ & $-1.00 \pm 0.60$ & $-2.45 \pm 0.15$ & $-2.36 \pm 0.15$ & $0.38 \pm 0.19$ & $0.16 \pm 0.14$ & \\
\hline
\end{tabular}


Table B.3 - continued.

\begin{tabular}{|c|c|c|c|c|c|c|c|c|c|c|c|c|}
\hline NGC ID & $\mathrm{T}_{e f f}^{(a)}(\mathrm{K})$ & $\mathrm{T}_{e f f}^{(b)}(\mathrm{K})$ & $\mathrm{T}_{e f f}^{(a v g)}(\mathrm{K})$ & $\log (g)^{(a)}$ & $\log (g)^{(b)}$ & $\log (g)^{(a v g)}$ & {$[\mathrm{Fe} / \mathrm{H}]^{(a)}$} & {$[\mathrm{Fe} / \mathrm{H}]^{(b)}$} & {$[\mathrm{Fe} / \mathrm{H}]^{(a v g)}$} & {$[\mathrm{Mg} / \mathrm{Fe}]^{(a)}$} & {$[\alpha / \mathrm{Fe}]^{(b)}$} & members \\
\hline 6749_5 & $4346 \pm 171$ & $4151 \pm 165$ & $4245 \pm 119$ & $0.90 \pm 0.40$ & $0.30 \pm 0.33$ & $0.54 \pm 0.25$ & $-1.56 \pm 0.34$ & $-2.15 \pm 0.23$ & $-1.96 \pm 0.19$ & $0.45 \pm 0.09$ & $0.15 \pm 0.16$ & \\
\hline 6749_6 & $4152 \pm 54$ & $4124 \pm 202$ & $4150 \pm 52$ & $0.73 \pm 0.02$ & $0.05 \pm 0.15$ & $0.72 \pm 0.02$ & $-1.65 \pm 0.28$ & $-2.35 \pm 0.23$ & $-2.07 \pm 0.18$ & $0.45 \pm 0.01$ & $0.20 \pm 0.13$ & \\
\hline 6749_7 & $4346 \pm 172$ & $4100 \pm 229$ & $4257 \pm 138$ & $0.90 \pm 0.40$ & $0.10 \pm 0.20$ & $0.26 \pm 0.18$ & $-1.56 \pm 0.34$ & $-2.15 \pm 0.23$ & $-1.96 \pm 0.19$ & $0.45 \pm 0.09$ & $0.16 \pm 0.12$ & \\
\hline $6749 \_8$ & $4646 \pm 233$ & $4100 \pm 200$ & $4332 \pm 152$ & $2.00 \pm 0.40$ & $0.05 \pm 0.15$ & $0.29 \pm 0.14$ & $-0.61 \pm 0.32$ & $-2.10 \pm 0.20$ & $-1.68 \pm 0.17$ & $0.33 \pm 0.21$ & $0.15 \pm 0.12$ & M \\
\hline 6749_9 & $4596 \pm 270$ & $4049 \pm 188$ & $4227 \pm 154$ & $2.00 \pm 0.50$ & $0.05 \pm 0.15$ & $0.21 \pm 0.14$ & $-0.70 \pm 0.40$ & $-2.15 \pm 0.23$ & $-1.79 \pm 0.20$ & $0.34 \pm 0.19$ & $0.20 \pm 0.13$ & \\
\hline 6749_10 & $4624 \pm 224$ & $4025 \pm 208$ & $4302 \pm 152$ & $2.10 \pm 0.40$ & $0.10 \pm 0.20$ & $0.50 \pm 0.18$ & $-0.52 \pm 0.34$ & $-2.15 \pm 0.23$ & $-1.64 \pm 0.19$ & $0.30 \pm 0.21$ & $0.19 \pm 0.11$ & M \\
\hline 6749_11 & $4737 \pm 183$ & $4126 \pm 201$ & $4460 \pm 135$ & $2.12 \pm 0.32$ & $0.20 \pm 0.24$ & $0.89 \pm 0.19$ & $-0.60 \pm 0.32$ & $-2.15 \pm 0.23$ & $-1.62 \pm 0.19$ & $0.32 \pm 0.21$ & $0.17 \pm 0.15$ & M \\
\hline 6749_13 & $4228 \pm 48$ & $4375 \pm 125$ & $4247 \pm 45$ & $1.53 \pm 0.15$ & $1.95 \pm 0.35$ & $1.60 \pm 0.14$ & $-0.03 \pm 0.10$ & $-0.25 \pm 0.25$ & $-0.06 \pm 0.09$ & $-0.05 \pm 0.18$ & $0.24 \pm 0.10$ & \\
\hline 6749_14 & $4535 \pm 8$ & $4250 \pm 100$ & $4533 \pm 8$ & $2.24 \pm 0.10$ & $0.40 \pm 0.43$ & $2.15 \pm 0.10$ & $-0.13 \pm 0.10$ & $-2.50 \pm 0.10$ & $-1.31 \pm 0.07$ & $0.01 \pm 0.02$ & $0.17 \pm 0.15$ & \\
\hline 6749_15 & $4732 \pm 168$ & $4101 \pm 200$ & $4470 \pm 129$ & $1.99 \pm 0.30$ & $0.20 \pm 0.33$ & $1.18 \pm 0.22$ & $-0.74 \pm 0.23$ & $-2.20 \pm 0.24$ & $-1.44 \pm 0.17$ & $0.40 \pm 0.17$ & $0.17 \pm 0.15$ & M \\
\hline 6749_16 & $4623 \pm 315$ & $4300 \pm 100$ & $4329 \pm 95$ & $1.50 \pm 0.70$ & $0.20 \pm 0.24$ & $0.34 \pm 0.23$ & $-1.72 \pm 0.33$ & $-2.50 \pm 0.10$ & $-2.43 \pm 0.10$ & $0.45 \pm 0.14$ & $0.21 \pm 0.14$ & \\
\hline 6749_17 & $4526 \pm 149$ & $4776 \pm 207$ & $4611 \pm 121$ & $2.30 \pm 0.40$ & $2.45 \pm 0.57$ & $2.35 \pm 0.33$ & $-0.22 \pm 0.25$ & $-0.30 \pm 0.24$ & $-0.26 \pm 0.17$ & $0.11 \pm 0.20$ & $0.25 \pm 0.10$ & \\
\hline 6749_18 & $4341 \pm 162$ & $4449 \pm 187$ & $4387 \pm 122$ & $2.00 \pm 0.40$ & $1.95 \pm 0.42$ & $1.98 \pm 0.29$ & $0.01 \pm 0.13$ & $-0.40 \pm 0.20$ & $-0.11 \pm 0.11$ & $0.00 \pm 0.13$ & $0.27 \pm 0.12$ & \\
\hline Pal10_1 & $4187 \pm 194$ & $4475 \pm 208$ & $4321 \pm 142$ & $1.70 \pm 0.40$ & $2.45 \pm 0.52$ & $1.98 \pm 0.32$ & $0.07 \pm 0.16$ & $-0.11 \pm 0.35$ & $0.04 \pm 0.15$ & $0.01 \pm 0.15$ & $0.10 \pm 0.09$ & \\
\hline Pal10_2 & $4219 \pm 228$ & $4400 \pm 166$ & $4337 \pm 134$ & $1.90 \pm 0.50$ & $2.15 \pm 0.45$ & $2.04 \pm 0.33$ & $-0.00 \pm 0.19$ & $-0.23 \pm 0.28$ & $-0.08 \pm 0.16$ & $0.05 \pm 0.14$ & $0.17 \pm 0.12$ & M \\
\hline Pal10_3 & $4490 \pm 258$ & $4550 \pm 150$ & $4535 \pm 130$ & $2.20 \pm 0.50$ & $2.80 \pm 0.33$ & $2.62 \pm 0.28$ & $-0.15 \pm 0.27$ & $-0.90 \pm 0.30$ & $-0.49 \pm 0.20$ & $0.08 \pm 0.18$ & $0.24 \pm 0.12$ & \\
\hline Pal10_4 & $4250 \pm 207$ & $4225 \pm 175$ & $4235 \pm 134$ & $1.80 \pm 0.50$ & $1.85 \pm 0.39$ & $1.83 \pm 0.31$ & $-0.09 \pm 0.27$ & $-0.65 \pm 0.23$ & $-0.41 \pm 0.18$ & $0.07 \pm 0.17$ & $0.21 \pm 0.11$ & M \\
\hline Pal10_6 & $4048 \pm 125$ & $3976 \pm 284$ & $4036 \pm 114$ & $1.56 \pm 0.32$ & $1.30 \pm 0.84$ & $1.53 \pm 0.30$ & $-0.20 \pm 0.20$ & $-1.45 \pm 0.41$ & $-0.44 \pm 0.18$ & $0.09 \pm 0.14$ & $0.30 \pm 0.08$ & \\
\hline Pal10_7 & $4326 \pm 246$ & $4226 \pm 208$ & $4267 \pm 159$ & $2.00 \pm 0.50$ & $2.15 \pm 0.59$ & $2.06 \pm 0.38$ & $-0.17 \pm 0.28$ & $-1.40 \pm 0.30$ & $-0.74 \pm 0.20$ & $0.10 \pm 0.19$ & $0.33 \pm 0.08$ & \\
\hline Pal10_8 & $4212 \pm 190$ & $4100 \pm 165$ & $4148 \pm 125$ & $1.80 \pm 0.50$ & $1.50 \pm 0.54$ & $1.66 \pm 0.37$ & $-0.01 \pm 0.15$ & $-0.80 \pm 0.33$ & $-0.14 \pm 0.14$ & $0.05 \pm 0.13$ & $0.27 \pm 0.13$ & M \\
\hline Pal10_9 & $4369 \pm 165$ & $4475 \pm 134$ & $4433 \pm 104$ & $1.79 \pm 0.33$ & $2.05 \pm 0.52$ & $1.86 \pm 0.28$ & $-0.07 \pm 0.13$ & $-0.50 \pm 0.22$ & $-0.18 \pm 0.11$ & $0.08 \pm 0.14$ & $0.33 \pm 0.08$ & M \\
\hline Pal10_10 & $4147 \pm 99$ & $4050 \pm 150$ & $4118 \pm 83$ & $1.59 \pm 0.13$ & $1.35 \pm 0.55$ & $1.58 \pm 0.13$ & $-0.06 \pm 0.10$ & $-0.90 \pm 0.30$ & $-0.14 \pm 0.09$ & $0.03 \pm 0.13$ & $0.30 \pm 0.10$ & M \\
\hline Pal10_11 & $4362 \pm 150$ & $4425 \pm 115$ & $4402 \pm 91$ & $1.56 \pm 0.15$ & $1.90 \pm 0.44$ & $1.60 \pm 0.14$ & $-0.13 \pm 0.10$ & $-0.50 \pm 0.10$ & $-0.32 \pm 0.07$ & $0.17 \pm 0.09$ & $0.30 \pm 0.08$ & M \\
\hline Pal10_13 & $4134 \pm 108$ & $4177 \pm 114$ & $4154 \pm 78$ & $1.58 \pm 0.14$ & $1.70 \pm 0.25$ & $1.61 \pm 0.12$ & $-0.07 \pm 0.10$ & $-0.65 \pm 0.23$ & $-0.16 \pm 0.09$ & $0.08 \pm 0.06$ & $0.25 \pm 0.10$ & M \\
\hline Pal10_16 & $4427 \pm 144$ & $4299 \pm 100$ & $4341 \pm 82$ & $1.80 \pm 0.40$ & $1.85 \pm 0.32$ & $1.83 \pm 0.25$ & $-0.07 \pm 0.10$ & $-0.50 \pm 0.10$ & $-0.29 \pm 0.07$ & $0.08 \pm 0.16$ & $0.24 \pm 0.14$ & M \\
\hline Pal10_17 & $4214 \pm 68$ & $4275 \pm 134$ & $4227 \pm 61$ & $1.48 \pm 0.07$ & $1.85 \pm 0.32$ & $1.50 \pm 0.07$ & $-0.13 \pm 0.10$ & $-0.55 \pm 0.15$ & $-0.26 \pm 0.08$ & $0.12 \pm 0.01$ & $0.28 \pm 0.12$ & M \\
\hline Pal11_2 & $4508 \pm 174$ & $4800 \pm 185$ & $4645 \pm 127$ & $2.30 \pm 0.40$ & $3.00 \pm 0.31$ & $2.74 \pm 0.25$ & $-0.38 \pm 0.22$ & $-0.50 \pm 0.31$ & $-0.42 \pm 0.18$ & $0.18 \pm 0.18$ & $0.26 \pm 0.13$ & M \\
\hline Pal11_3 & $3980 \pm 47$ & $4624 \pm 125$ & $4060 \pm 44$ & $1.73 \pm 0.19$ & $4.45 \pm 0.42$ & $2.19 \pm 0.17$ & $-0.27 \pm 0.09$ & $-0.55 \pm 0.15$ & $-0.34 \pm 0.08$ & $0.11 \pm 0.13$ & $0.26 \pm 0.13$ & \\
\hline
\end{tabular}


Table B.3 - continued.

\begin{tabular}{|c|c|c|c|c|c|c|c|c|c|c|c|c|}
\hline NGC ID & $\mathrm{T}_{e f f}^{(a)}(\mathrm{K})$ & $\mathrm{T}_{e f f}^{(b)}(\mathrm{K})$ & $\mathrm{T}_{e f f}^{(a v g)}(\mathrm{K})$ & $\log (g)^{(a)}$ & $\log (g)^{(b)}$ & $\log (g)^{(a v g)}$ & {$[\mathrm{Fe} / \mathrm{H}]^{(a)}$} & {$[\mathrm{Fe} / \mathrm{H}]^{(b)}$} & {$[\mathrm{Fe} / \mathrm{H}]^{(a v g)}$} & {$[\mathrm{Mg} / \mathrm{Fe}]^{(a)}$} & {$[\alpha / \mathrm{Fe}]^{(b)}$} & members \\
\hline Pal11_6 & $4568 \pm 179$ & $4725 \pm 206$ & $4636 \pm 135$ & $2.40 \pm 0.40$ & $2.85 \pm 0.45$ & $2.60 \pm 0.30$ & $-0.34 \pm 0.17$ & $-0.65 \pm 0.32$ & $-0.41 \pm 0.15$ & $0.17 \pm 0.15$ & $0.31 \pm 0.10$ & M \\
\hline Pal11_7 & $4624 \pm 196$ & $4675 \pm 159$ & $4655 \pm 124$ & $2.50 \pm 0.50$ & $2.65 \pm 0.32$ & $2.61 \pm 0.27$ & $-0.22 \pm 0.23$ & $-0.65 \pm 0.32$ & $-0.37 \pm 0.19$ & $0.12 \pm 0.17$ & $0.30 \pm 0.10$ & M \\
\hline Pal11_8 & $4361 \pm 114$ & $4397 \pm 164$ & $4373 \pm 94$ & $2.02 \pm 0.32$ & $2.14 \pm 0.45$ & $2.06 \pm 0.26$ & $-0.30 \pm 0.19$ & $-0.71 \pm 0.33$ & $-0.40 \pm 0.16$ & $0.22 \pm 0.19$ & $0.31 \pm 0.10$ & M \\
\hline Pal11_9 & $4265 \pm 160$ & $4350 \pm 122$ & $4318 \pm 97$ & $1.91 \pm 0.32$ & $2.05 \pm 0.35$ & $1.97 \pm 0.24$ & $-0.20 \pm 0.12$ & $-0.80 \pm 0.24$ & $-0.32 \pm 0.11$ & $0.05 \pm 0.12$ & $0.27 \pm 0.11$ & M \\
\hline Pal11_10 & $4661 \pm 187$ & $4802 \pm 186$ & $4732 \pm 132$ & $2.50 \pm 0.40$ & $3.06 \pm 0.47$ & $2.74 \pm 0.30$ & $-0.34 \pm 0.20$ & $-0.60 \pm 0.20$ & $-0.47 \pm 0.14$ & $0.19 \pm 0.18$ & $0.24 \pm 0.14$ & M \\
\hline Pal11_11 & $4358 \pm 149$ & $4399 \pm 165$ & $4377 \pm 111$ & $2.10 \pm 0.28$ & $2.10 \pm 0.43$ & $2.10 \pm 0.23$ & $-0.08 \pm 0.13$ & $-0.70 \pm 0.33$ & $-0.16 \pm 0.12$ & $0.08 \pm 0.14$ & $0.32 \pm 0.07$ & M \\
\hline Pal11_12 & $4463 \pm 154$ & $4450 \pm 187$ & $4458 \pm 119$ & $2.30 \pm 0.40$ & $2.30 \pm 0.46$ & $2.30 \pm 0.30$ & $-0.07 \pm 0.21$ & $-0.75 \pm 0.25$ & $-0.35 \pm 0.16$ & $0.07 \pm 0.15$ & $0.34 \pm 0.07$ & $\mathrm{M}$ \\
\hline Pal11_13 & $4645 \pm 193$ & $4898 \pm 123$ & $4825 \pm 104$ & $2.50 \pm 0.40$ & $3.15 \pm 0.23$ & $2.99 \pm 0.20$ & $-0.26 \pm 0.21$ & $-0.35 \pm 0.23$ & $-0.30 \pm 0.16$ & $0.14 \pm 0.16$ & $0.25 \pm 0.13$ & M \\
\hline Pal11_16 & $4643 \pm 201$ & $4875 \pm 125$ & $4810 \pm 106$ & $3.00 \pm 0.60$ & $4.35 \pm 0.39$ & $3.95 \pm 0.33$ & $0.30 \pm 0.04$ & $0.10 \pm 0.10$ & $0.27 \pm 0.04$ & $0.18 \pm 0.12$ & $0.10 \pm 0.08$ & \\
\hline Pal11_17 & $4118 \pm 118$ & $4301 \pm 101$ & $4224 \pm 77$ & $1.70 \pm 0.40$ & $1.95 \pm 0.35$ & $1.84 \pm 0.26$ & $-0.19 \pm 0.20$ & $-0.60 \pm 0.20$ & $-0.39 \pm 0.14$ & $0.07 \pm 0.14$ & $0.30 \pm 0.08$ & M \\
\hline M75_2 & $4378 \pm 116$ & $4377 \pm 125$ & $4377 \pm 85$ & $1.90 \pm 0.50$ & $1.65 \pm 0.23$ & $1.69 \pm 0.21$ & $-0.49 \pm 0.25$ & $-1.00 \pm 0.10$ & $-0.93 \pm 0.09$ & $0.20 \pm 0.18$ & $0.23 \pm 0.13$ & M \\
\hline M75_3 & $4743 \pm 238$ & $4799 \pm 99$ & $4791 \pm 92$ & $2.30 \pm 0.60$ & $2.40 \pm 0.37$ & $2.37 \pm 0.31$ & $-0.50 \pm 0.22$ & $-0.90 \pm 0.20$ & $-0.72 \pm 0.15$ & $0.19 \pm 0.16$ & $0.21 \pm 0.13$ & \\
\hline M75_4 & $4811 \pm 392$ & $5050 \pm 100$ & $5035 \pm 97$ & $2.20 \pm 0.90$ & $2.30 \pm 0.33$ & $2.29 \pm 0.31$ & $-0.80 \pm 0.50$ & $-0.95 \pm 0.15$ & $-0.94 \pm 0.14$ & $0.32 \pm 0.17$ & $0.19 \pm 0.15$ & M \\
\hline M75_5 & $4878 \pm 328$ & $5076 \pm 115$ & $5054 \pm 108$ & $2.40 \pm 0.80$ & $2.80 \pm 0.25$ & $2.76 \pm 0.24$ & $-0.70 \pm 0.40$ & $-0.85 \pm 0.23$ & $-0.81 \pm 0.20$ & $0.26 \pm 0.14$ & $0.18 \pm 0.12$ & M \\
\hline M75_6 & $4532 \pm 278$ & $5050 \pm 245$ & $4824 \pm 184$ & $1.30 \pm 0.60$ & $1.95 \pm 0.42$ & $1.74 \pm 0.34$ & $-1.40 \pm 0.40$ & $-1.20 \pm 0.24$ & $-1.25 \pm 0.21$ & $0.45 \pm 0.13$ & $0.17 \pm 0.11$ & M \\
\hline M75_7 & $4224 \pm 61$ & $4126 \pm 200$ & $4216 \pm 58$ & $1.40 \pm 0.40$ & $1.10 \pm 0.70$ & $1.33 \pm 0.35$ & $-0.66 \pm 0.31$ & $-1.30 \pm 0.33$ & $-0.96 \pm 0.23$ & $0.40 \pm 0.20$ & $0.24 \pm 0.14$ & M \\
\hline M75_8 & $4539 \pm 182$ & $4425 \pm 114$ & $4458 \pm 97$ & $2.20 \pm 0.50$ & $1.75 \pm 0.25$ & $1.84 \pm 0.22$ & $-0.46 \pm 0.19$ & $-1.15 \pm 0.23$ & $-0.74 \pm 0.15$ & $0.17 \pm 0.18$ & $0.21 \pm 0.11$ & \\
\hline M75_9 & $4289 \pm 90$ & $4250 \pm 192$ & $4282 \pm 82$ & $1.50 \pm 0.40$ & $1.45 \pm 0.72$ & $1.49 \pm 0.35$ & $-0.69 \pm 0.26$ & $-1.15 \pm 0.23$ & $-0.95 \pm 0.17$ & $0.40 \pm 0.18$ & $0.26 \pm 0.13$ & M \\
\hline M75_10 & $4805 \pm 359$ & $4500 \pm 194$ & $4569 \pm 170$ & $2.30 \pm 0.90$ & $2.40 \pm 0.49$ & $2.38 \pm 0.43$ & $-0.70 \pm 0.50$ & $-1.95 \pm 0.35$ & $-1.54 \pm 0.29$ & $0.31 \pm 0.19$ & $0.31 \pm 0.07$ & M \\
\hline M75_11 & $4406 \pm 125$ & $4426 \pm 159$ & $4414 \pm 98$ & $2.00 \pm 0.50$ & $1.80 \pm 0.46$ & $1.89 \pm 0.34$ & $-0.48 \pm 0.20$ & $-1.00 \pm 0.22$ & $-0.72 \pm 0.15$ & $0.19 \pm 0.19$ & $0.21 \pm 0.13$ & M \\
\hline M75_13 & $4863 \pm 408$ & $5100 \pm 229$ & $5044 \pm 200$ & $2.00 \pm 0.90$ & $2.40 \pm 0.62$ & $2.27 \pm 0.51$ & $-1.50 \pm 0.40$ & $-1.65 \pm 0.23$ & $-1.61 \pm 0.20$ & $0.43 \pm 0.15$ & $0.25 \pm 0.13$ & M \\
\hline M75_14 & $4685 \pm 387$ & $5301 \pm 187$ & $5184 \pm 168$ & $1.60 \pm 0.70$ & $2.35 \pm 0.32$ & $2.22 \pm 0.29$ & $-1.40 \pm 0.40$ & $-1.10 \pm 0.20$ & $-1.16 \pm 0.18$ & $0.44 \pm 0.14$ & $0.11 \pm 0.09$ & M \\
\hline 7006_9 & $5195 \pm 430$ & $6125 \pm 125$ & $6053 \pm 120$ & $2.40 \pm 0.80$ & $4.20 \pm 0.46$ & $3.75 \pm 0.40$ & $-2.00 \pm 0.50$ & $-2.50 \pm 0.10$ & $-2.48 \pm 0.10$ & $0.40 \pm 0.19$ & $0.06 \pm 0.07$ & \\
\hline 7006_18 & $4901 \pm 461$ & $4900 \pm 122$ & $4900 \pm 118$ & $2.10 \pm 0.90$ & $2.55 \pm 0.27$ & $2.51 \pm 0.26$ & $-1.40 \pm 0.50$ & $-1.70 \pm 0.24$ & $-1.64 \pm 0.22$ & $0.40 \pm 0.17$ & $0.19 \pm 0.11$ & M \\
\hline 7006_19 & $5013 \pm 393$ & $5000 \pm 100$ & $5001 \pm 97$ & $2.20 \pm 0.80$ & $0.80 \pm 0.81$ & $1.51 \pm 0.57$ & $-1.70 \pm 0.50$ & $-2.50 \pm 0.10$ & $-2.47 \pm 0.10$ & $0.42 \pm 0.18$ & $0.20 \pm 0.14$ & \\
\hline 7006_21 & $5029 \pm 452$ & $5375 \pm 125$ & $5350 \pm 120$ & $2.20 \pm 0.90$ & $3.30 \pm 0.24$ & $3.23 \pm 0.23$ & $-1.70 \pm 0.50$ & $-1.75 \pm 0.25$ & $-1.74 \pm 0.22$ & $0.42 \pm 0.17$ & $0.28 \pm 0.12$ & M \\
\hline 7006_22 & $5020 \pm 465$ & $5400 \pm 122$ & $5375 \pm 118$ & $2.20 \pm 0.90$ & $3.40 \pm 0.37$ & $3.23 \pm 0.34$ & $-1.70 \pm 0.50$ & $-2.50 \pm 0.10$ & $-2.47 \pm 0.10$ & $0.42 \pm 0.17$ & $0.07 \pm 0.06$ & \\
\hline 7006_23 & $4807 \pm 402$ & $4924 \pm 225$ & $4896 \pm 196$ & $1.90 \pm 0.90$ & $1.90 \pm 0.37$ & $1.90 \pm 0.34$ & $-1.60 \pm 0.40$ & $-1.80 \pm 0.24$ & $-1.75 \pm 0.21$ & $0.44 \pm 0.15$ & $0.28 \pm 0.10$ & M \\
\hline
\end{tabular}


Table B.3 - continued.

\begin{tabular}{|c|c|c|c|c|c|c|c|c|c|c|c|c|}
\hline NGC ID & $\mathrm{T}_{\text {eff }}^{(a)}(\mathrm{K})$ & $\mathrm{T}_{\text {eff }}^{(b)}(\mathrm{K})$ & $\mathrm{T}_{e f f}^{(a v g)}(\mathrm{K})$ & $\log (g)^{(a)}$ & $\log (g)^{(b)}$ & $\log (g)^{(a v g)}$ & {$[\mathrm{Fe} / \mathrm{H}]^{(a)}$} & {$[\mathrm{Fe} / \mathrm{H}]^{(b)}$} & {$[\mathrm{Fe} / \mathrm{H}]^{(a v g)}$} & {$[\mathrm{Mg} / \mathrm{Fe}]^{(a)}$} & {$[\alpha / \mathrm{Fe}]^{(b)}$} & members \\
\hline 7006_24 & $4623 \pm 340$ & $4800 \pm 218$ & $4748 \pm 184$ & $1.60 \pm 0.80$ & $1.90 \pm 0.44$ & $1.83 \pm 0.39$ & $-1.43 \pm 0.35$ & $-1.65 \pm 0.23$ & $-1.58 \pm 0.19$ & $0.43 \pm 0.14$ & $0.28 \pm 0.10$ & M \\
\hline $7006 \_25$ & $4947 \pm 446$ & $5050 \pm 187$ & $5035 \pm 172$ & $2.10 \pm 0.90$ & $1.85 \pm 0.32$ & $1.88 \pm 0.30$ & $-1.60 \pm 0.40$ & $-1.80 \pm 0.24$ & $-1.75 \pm 0.21$ & $0.42 \pm 0.17$ & $0.18 \pm 0.12$ & M \\
\hline 7006_26 & $4960 \pm 464$ & $5200 \pm 100$ & $5189 \pm 98$ & $2.10 \pm 0.90$ & $2.90 \pm 0.44$ & $2.75 \pm 0.40$ & $-1.70 \pm 0.50$ & $-2.45 \pm 0.15$ & $-2.39 \pm 0.14$ & $0.42 \pm 0.17$ & $0.08 \pm 0.10$ & \\
\hline 7006_27 & $4957 \pm 376$ & $4800 \pm 150$ & $4822 \pm 139$ & $2.20 \pm 0.90$ & $4.45 \pm 0.61$ & $3.74 \pm 0.50$ & $-1.70 \pm 0.50$ & $-2.50 \pm 0.10$ & $-2.47 \pm 0.10$ & $0.42 \pm 0.16$ & $0.34 \pm 0.07$ & \\
\hline 7006_28 & $5020 \pm 366$ & $4625 \pm 168$ & $4693 \pm 152$ & $2.30 \pm 0.90$ & $4.55 \pm 0.52$ & $3.99 \pm 0.45$ & $-1.60 \pm 0.60$ & $-2.50 \pm 0.10$ & $-2.48 \pm 0.10$ & $0.42 \pm 0.17$ & $0.36 \pm 0.05$ & \\
\hline 7006_29 & $5060 \pm 475$ & $5750 \pm 158$ & $5682 \pm 150$ & $2.60 \pm 1.00$ & $4.80 \pm 0.24$ & $4.68 \pm 0.23$ & $-0.90 \pm 0.50$ & $-1.00 \pm 0.10$ & $-1.00 \pm 0.10$ & $0.37 \pm 0.24$ & $0.25 \pm 0.14$ & \\
\hline 7006_31 & $3980 \pm 188$ & $4600 \pm 123$ & $4415 \pm 103$ & $1.70 \pm 1.20$ & $4.35 \pm 0.55$ & $3.89 \pm 0.50$ & $-0.27 \pm 0.26$ & $-0.85 \pm 0.23$ & $-0.60 \pm 0.17$ & $0.11 \pm 0.09$ & $0.30 \pm 0.08$ & \\
\hline 7006_32 & $5064 \pm 433$ & $5625 \pm 125$ & $5582 \pm 120$ & $2.20 \pm 0.80$ & $3.65 \pm 0.45$ & $3.30 \pm 0.39$ & $-1.90 \pm 0.40$ & $-2.50 \pm 0.10$ & $-2.46 \pm 0.10$ & $0.41 \pm 0.18$ & $0.06 \pm 0.07$ & \\
\hline
\end{tabular}

${ }^{a}$ MILES library ${ }^{b}$ COELHO library ${ }^{c}$ Due to low number of observed stars it was hard to define the membership of M 107. See discussion in Chapter 4 
Appendix C

\section{FORS2 pipeline - wavelength calibration for low resolution spectra of GRIS1400V}

\section{C.1 Default pipeline reduction}

I used the esorex and the monolithic pipelines for the fors2, using the respectives calibration files, as follows:

$>$ esorex fors_calib cal.sof

$>$ esorex fors_science sci.sof

where the file cal.sof was:

../../BIAS/FORS2.2012-05-21T12_21_30.622.fits BIAS

... . .BAIAS/FORS2.2012-05-21T12_22_04.555.fits BIAS

... . ./BIAS/FORS2.2012-05-21T12_22_38.548.fits BIAS

../../BIAS/FORS2.2012-05-21T12_23_12.561.fits BIAS

../../BIAS/FORS2.2012-05-21T12_23_46.563.fits BIAS

...FORS2.2012-05-22T11_34_08.770.fits SCREEN_FLAT_MOS

../FORS2.2012-05-22T11_35_38.858.fits SCREEN_FLAT_MOS

../FORS2.2012-05-22T11_37_09.905.fits SCREEN_FLAT_MOS

...FORS2.2012-05-22T11_38_40.843.fits SCREEN_FLAT_MOS

.. /FORS2.2012-05-22T11_40_11.880.fits SCREEN_FLAT_MOS

...FORS2.2012-05-22T11_42_40.562.fits LAMP_MOS

. ./ . /Mg-CAL/M.FORS2 . 2012-05-31T13_47_50.389.fits MASTER_LINECAT

.././Mg-CAL/M.FORS2.2012-05-31T13_45_57.523.fits GRISM_TABLE 
and the file sci.sof was:

FORS2.2012-05-22T03_20_50.977.fits SCIENCE_MOS

master_bias.fits MASTER_BIAS

master_norm_flat_mos.fits MASTER_NORM_FLAT_MOS

disp_coeff mos.fits DISP_COEFF_MOS

curv_coeff_mos.fits CURV_COEFF_MOS

slit_location_mos.fits SLIT_LOCATION_MOS

. . . .Mg-CAL/M.FORS2.2012-05-31T13_45_57.523.fits GRISM_TABLE

So the results can be seen in the Figure C.1.

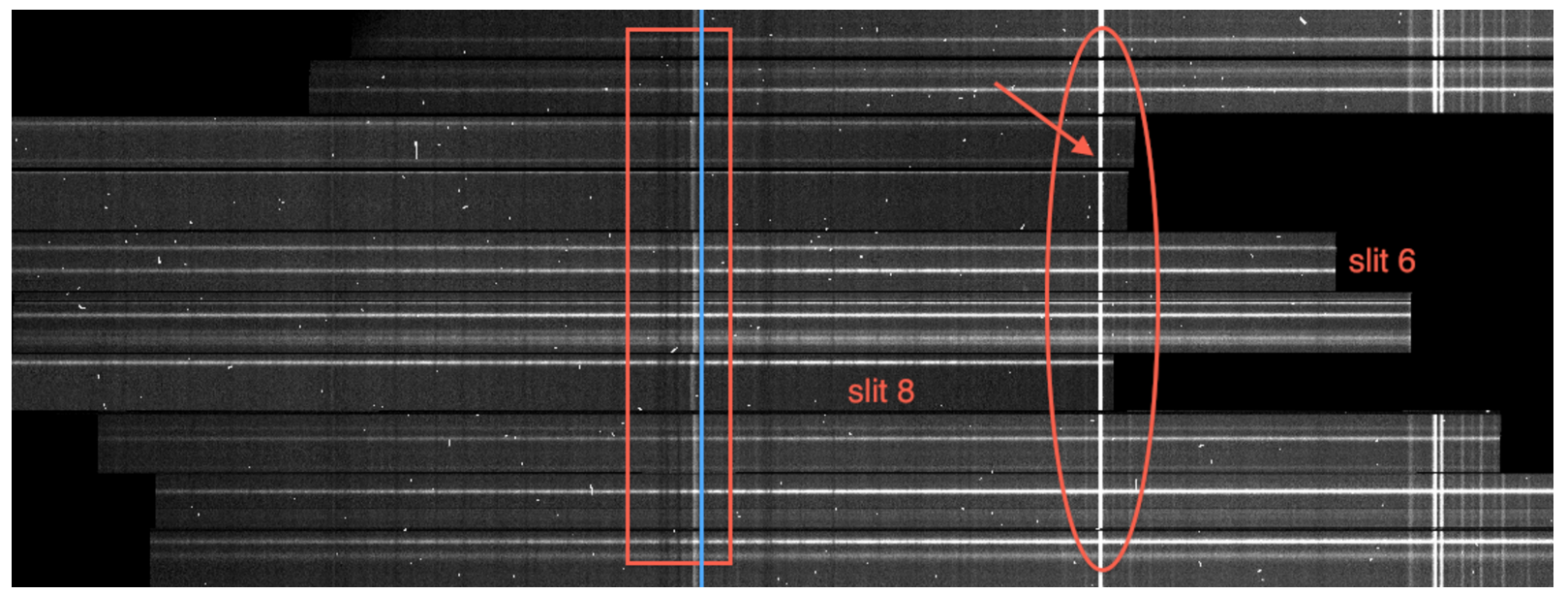

Figure C.1: Output (mapped_all_sci_mos.fits) of FORS2 pipeline using default parameters. The unique skyline used by the pipeline is indicated by the arrow. The blue line is just a vertical reference.

As you can see, there are too few skylines, and the strongest one (highlighted by the oval in the Figure C.1) is the $5577 \AA$, which is the unique line considered by the pipeline for these low resolution spectra. You can check it in the page 109 of the FORS pipeline manual, issue 4.3, copied here in the Figure C.2. This table present ONLY ONE line, in the middle/red part of the grism $1400 \mathrm{~V}$ spectra, which is not enough. So I found out a way to include my own line list, even with weak lines, and the results are present in the next Section. 


\begin{tabular}{|c|c|c|c|}
\hline Wavelength & Low resolution & Wavelength & Low resolution \\
\hline $\begin{array}{l}5577.338 \\
5889.953 \\
5895.923 \\
5915.301 \\
5932.862 \\
5953.420 \\
6257.961 \\
6287.434 \\
6300.304 \\
6306.869 \\
6363.780 \\
6498.729 \\
6533.044 \\
6553.617 \\
6841.945 \\
6863.955 \\
6870.994 \\
6889.288 \\
6900.833 \\
6912.623 \\
6923.220 \\
6939.521 \\
6969.930 \\
7003.858 \\
7244.907 \\
7276.405 \\
7284.439 \\
7316.282\end{array}$ & 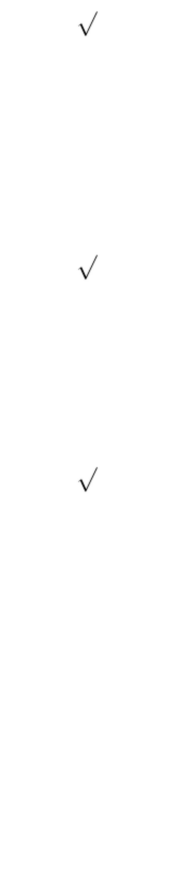 & $\begin{array}{l}7329.148 \\
7340.885 \\
7358.659 \\
7571.746 \\
7750.640 \\
7759.996 \\
7794.112 \\
7808.467 \\
7821.503 \\
7841.266 \\
7913.708 \\
7949.204 \\
7964.650 \\
7993.332 \\
8014.059 \\
8310.719 \\
8344.602 \\
8382.392 \\
8399.170 \\
8415.231 \\
8430.174 \\
8452.250 \\
8493.389 \\
8791.186 \\
8827.096 \\
8885.850 \\
8903.114 \\
8943.395 \\
8988.366\end{array}$ & 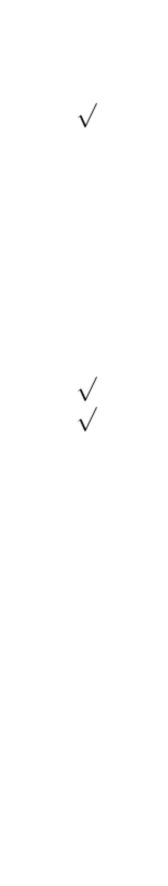 \\
\hline
\end{tabular}

Table 9.9.1: Default sky lines wavelengths used by the recipe fors_science. The marked lines are those used on data from low resolution grisms, i.e., all grisms with $300 \mathrm{gr} / \mathrm{mm}$ or less.

Figure C.2: Default list of skylines from manual.

\section{C.2 New list of sky lines for GRIS1400V}

I selected more skylines in the spectral region (Figure C.3) based on the UVES sky emission spectrum 1 and also a visual inspection of possible lines to be used. I included also a weak line more in the blue, in order to correct the entire dispersion. I also changed the parameter skyalign in order to fit a polynomial, as described in the manual, page 114, section 9.9.3. And then, my steps were:

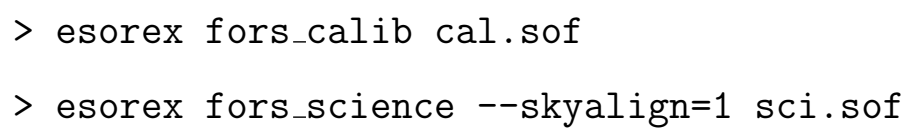

where the file cal.sof was the same as above, and the file sci.sof had one more line, indicating the new line list (and then not using the default list automatically):

\footnotetext{
${ }^{1}$ http://www.eso.org/observing/dfo/quality/UVES/pipeline/sky_spectrum.html
} 


Table Browser for 1: shortlist-fors.fits
\begin{tabular}{|c|c|c|}
\hline \multicolumn{1}{|c|}{ WLEN } & COMMENT \\
\hline 1 & 4861.3 & 18.3 \\
2 & 5197.9 & 252.8 \\
3 & 5200.3 & 146.7 \\
4 & 5577.3 & 7787.2 \\
5 & 5888.2 & 158.4 \\
6 & 5890. & 796.8 \\
7 & 5895.9 & 487. \\
\hline
\end{tabular}

Figure C.3: New list of skylines with the right column being a relative intensity for reference.

FORS2.2012-05-22T03_20_50.977.fits SCIENCE_MOS

master_bias.fits MASTER_BIAS

master_norm_flat_mos.fits MASTER_NORM_FLAT_MOS

disp_coeff_mos.fits DISP_COEFF_MOS

curv_coeff_mos.fits CURV_COEFF_MOS

slit_location_mos.fits SLIT_LOCATION_MOS

.././CAL/shortlist-fors.fits MASTER_SKYLINECAT

.././Mg-CAL/M.FORS2.2012-05-31T13_45_57.523.fits GRISM_TABLE

And now, with more skylines, and using a polynomial fitting (instead of the default median assuming that the displacement is equal for the whole wavelength range) for the dispersion, it was possible to correct the dispersion even in the blue, as shown by the Figure C.4.

\section{C.3 Comparisons bewteen the 2 procedures}

The Figure C.5 shows the comparison of the extracted spectra from one of the corrected slits (slit8 as an example), highlighted in the Figures C.1 and C.4. It is easy to notice that the new approach changes significantly the spectra.

In order to have a reference spectrum, I plot in the Figure C.6 the same of the Figure C.5. but for a slit where the dispersion does not significantly change between one procedure and another.

And finally, I use the slit 6 (of the new procedure) as a reference to overplot the 


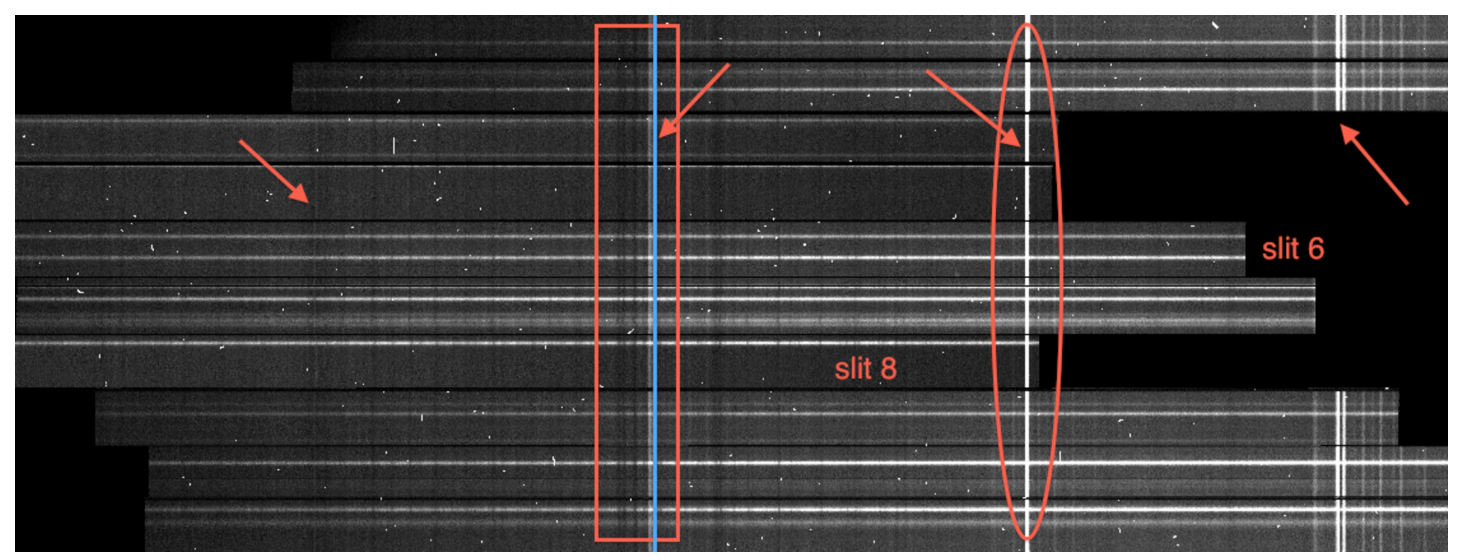

Figure C.4: Output (mapped_all_sci_mos.fits) of FORS2 pipeline using the new approach. The skylines used by the pipeline are indicated by the arrows. The blue line is just a vertical reference.

spectra of slit 8 from the 2 procedures. You can see in the Figure C.7 that the new approach successful corrects the dispersion in wavelength for low resolution spectra of FORS2/GRIS1400V. 

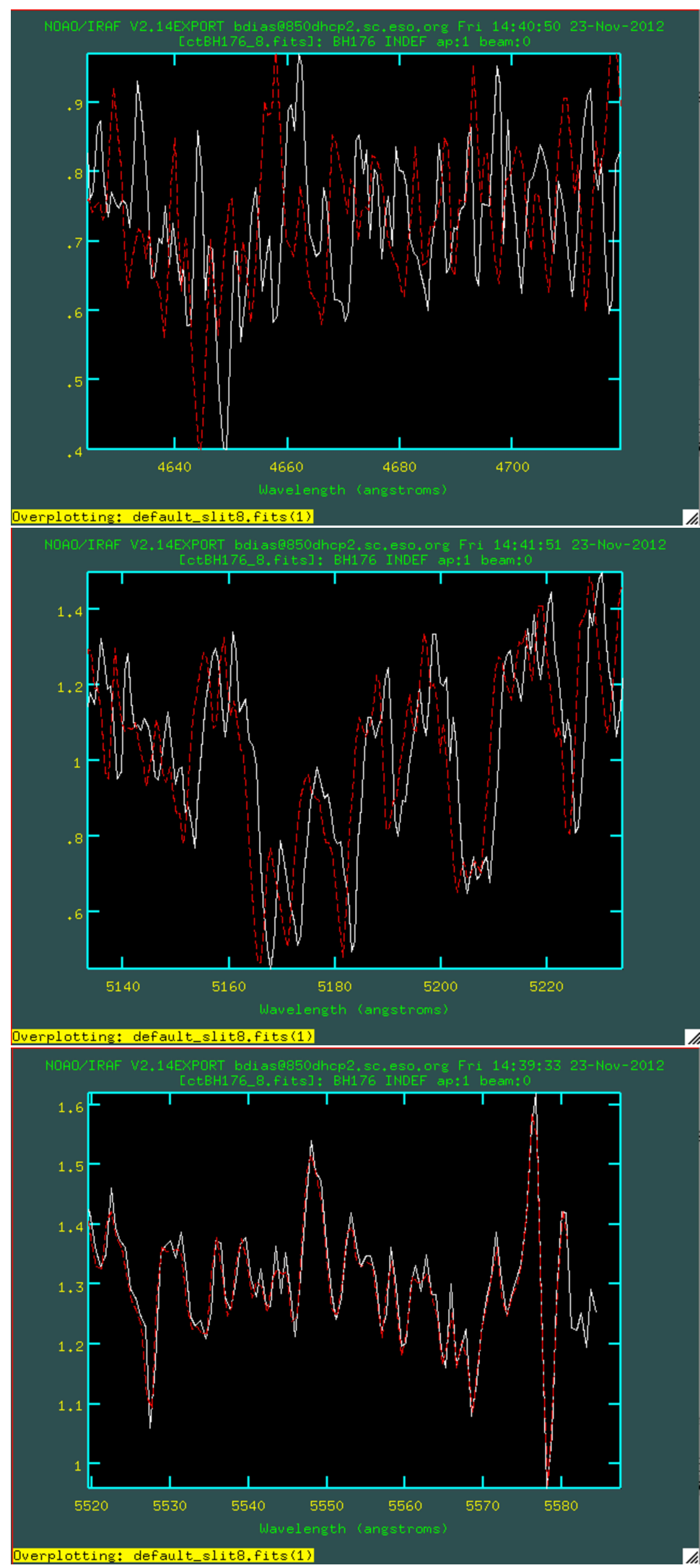

Figure C.5: Comparison between the corrected spectrum of the slit \#8 (solid white line) with the original one using the default paramenters (dashed red line) for 3 regions, in particular the central region is the $\mathrm{Mg}$ triplet, very important for this work. 

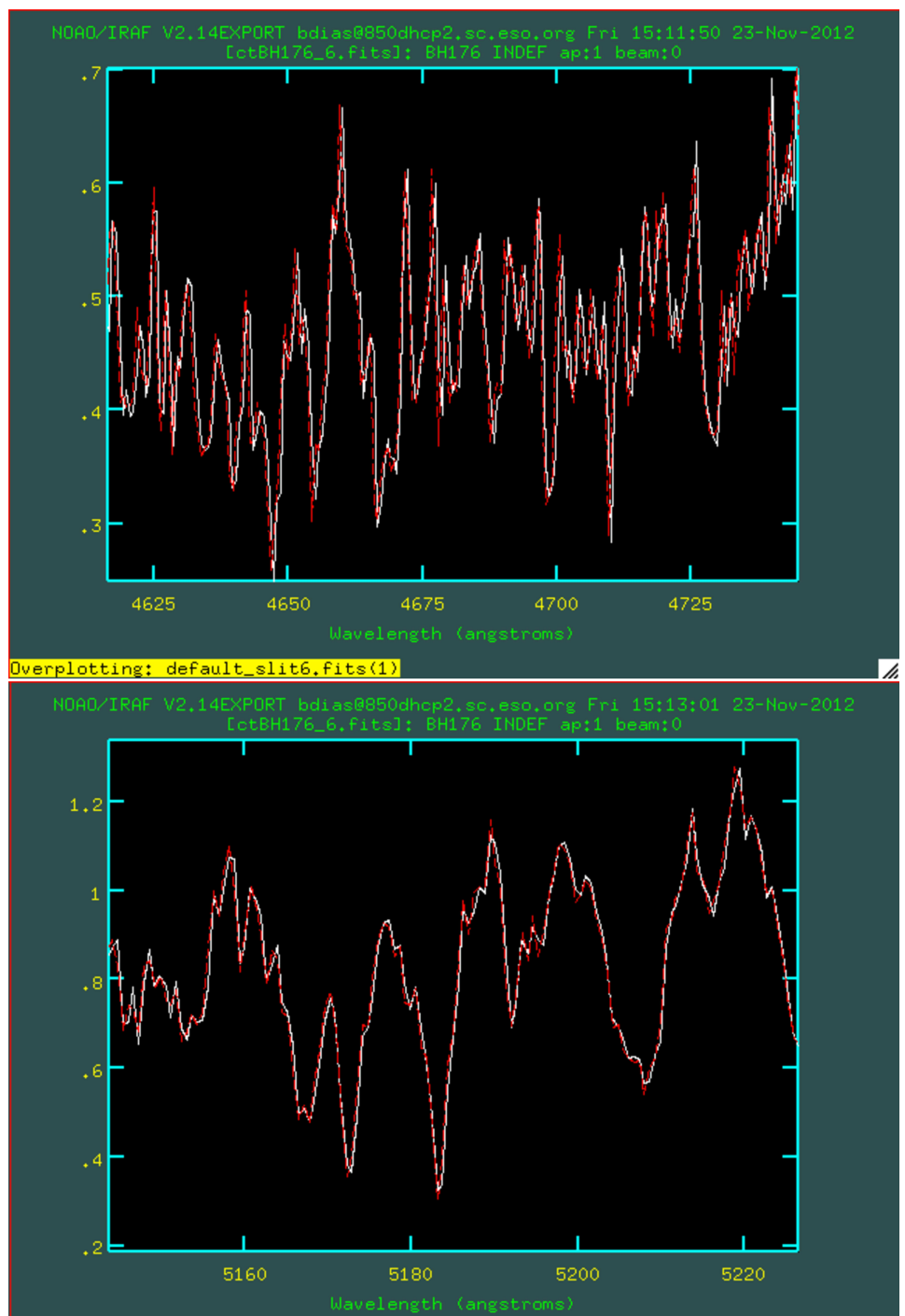

Overplotting: default_slit6.fits(1)

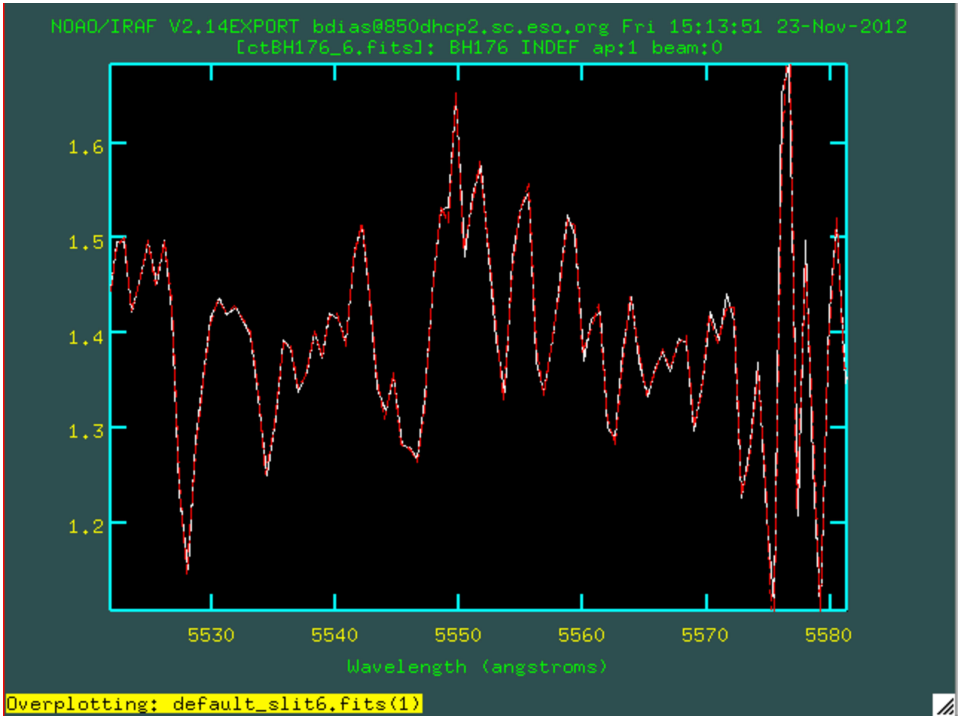

Figure C.6: Same of Figure C.5, but for the slit \#6. 

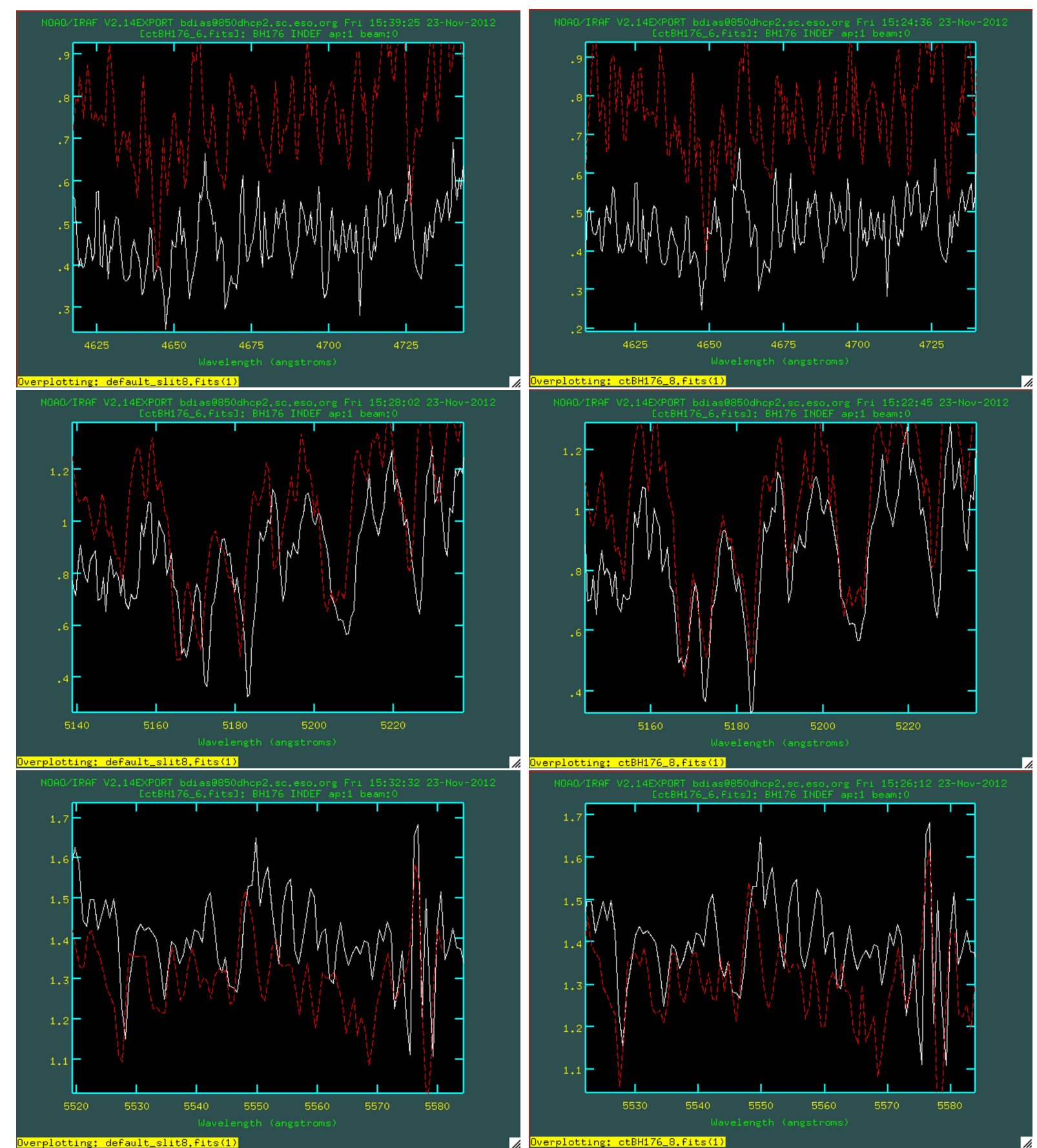

Figure C.7: Comparison between the default procedure (plots on the left) with the new procedure (plots on the right). The solid white line is the slit6, and the dashed red one is the slit8. 


\section{Appendix D}

\section{Papers in the period 2010-2014}

We present here the abstract of all papers published and one submitted during the $\mathrm{PhD}$ as first author or co-author in order of publication date.

\section{New Galactic star clusters discovered in the VVV survey}

J. Borissova ${ }^{1}$, C. Bonatto ${ }^{2}$, R. Kurtev ${ }^{1}$, J. R. A. Clarke ${ }^{1}$, F. Peñaloza ${ }^{1}$, S. E. Sale ${ }^{1,5}$, D. Minniti ${ }^{5,18}$, J. Alonso-García ${ }^{5}$, E. Artigau ${ }^{3}$, R. Barbá ${ }^{16}$, E. Bica ${ }^{2}$, G. L. Baume ${ }^{4}$, M. Catelan ${ }^{5}$, A. N. Chenè ${ }^{1,6}$, B. Dias ${ }^{7}$, S. L. Folkes ${ }^{1}$, D. Froebrich ${ }^{8}$,

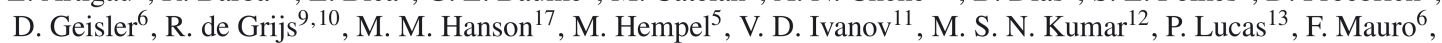
C. Moni Bidin ${ }^{6}$, M. Rejkuba ${ }^{15}$, R. K. Saito ${ }^{5}$, M. Tamura ${ }^{14}$, and I. Toledo ${ }^{5}$

(Affiliations can be found after the references)

Received 6 February 2011 / Accepted 3 June 2011

\section{ABSTRACT}

Context. VISTA Variables in the Vía Láctea (VVV) is one of the six ESO Public Surveys operating on the new 4-m Visible and Infrared Survey Telescope for Astronomy (VISTA). VVV is scanning the Milky Way bulge and an adjacent section of the disk, where star formation activity is high. One of the principal goals of the VVV Survey is to find new star clusters of different ages.

Aims. In order to trace the early epochs of star cluster formation we concentrated our search in the directions to those of known star formation regions, masers, radio, and infrared sources.

Methods. The disk area covered by VVV was visually inspected using the pipeline processed and calibrated $K_{\mathrm{S}}$-band tile images for stellar overdensities. Subsequently, we examined the composite $J H K_{\mathrm{S}}$ and $Z J K_{\mathrm{S}}$ color images of each candidate. PSF photometry of $15 \times 15$ arcmin fields centered on the candidates was then performed on the Cambridge Astronomy Survey Unit reduced images. After statistical field-star decontamination, color-magnitude and color-color diagrams were constructed and analyzed.

Results. We report the discovery of 96 new infrared open clusters and stellar groups. Most of the new cluster candidates are faint and compact (with small angular sizes), highly reddened, and younger than $5 \mathrm{Myr}$. For relatively well populated cluster candidates we derived their fundamental parameters such as reddening, distance, and age by fitting the solar-metallicity Padova isochrones to the color-magnitude diagrams.

Key words. open clusters and associations: general - stars: early-type - infrared: general

Figure D.1: Abstract of Borissova et al. (2011). 


\section{VVV DR1: The first data release of the Milky Way bulge and southern plane from the near-infrared ESO public survey VISTA variables in the Vía Láctea ${ }^{\star}$}

R. K. Saito ${ }^{1}$, M. Hempel ${ }^{1}$, D. Minniti1 ${ }^{1,2,3}$, P. W. Lucas $^{4}$, M. Rejkuba ${ }^{5}$, I. Toledo ${ }^{6}$, O. A. Gonzalez ${ }^{5}$, J. Alonso-García ${ }^{1}$, M. J. Irwin ${ }^{7}$, E. Gonzalez-Solares ${ }^{7}$, S. T. Hodgkin ${ }^{7}$, J. R. Lewis ${ }^{7}$, N. Cross ${ }^{8}$, V. D. Ivanov ${ }^{9}$, E. Kerins ${ }^{10}$,

J. P. Emerson ${ }^{11}$, M. Soto ${ }^{12}$, E. B. Amôres ${ }^{13,14}$, S. Gurovich ${ }^{15}$, I. Dékány ${ }^{1}$, R. Angeloni ${ }^{1}$, J. C. Beamin ${ }^{1}$, M. Catelan ${ }^{1}$,

N. Padilla 1,16 , M. Zoccali 1,17 , P. Pietrukowicz ${ }^{18}$, C. Moni Bidin ${ }^{19}$, F. Mauro ${ }^{19}$, D. Geisler ${ }^{19}$, S. L. Folkes ${ }^{20}$,

S. E. Sale ${ }^{1,20}$, J. Borissova ${ }^{20}$, R. Kurtev ${ }^{20}$, A. V. Ahumada ${ }^{9,21,22}$, M. V. Alonso ${ }^{15,21}$, A. Adamson 23 , J. I. Arias' ${ }^{12}$,

R. M. Bandyopadhyay ${ }^{24}$, R. H. Barbá12,25, B. Barbuy ${ }^{26}$, G. L. Baume 27, L. R. Bedin ${ }^{28}$, A. Bellini ${ }^{29}$, R. Benjamin $^{30}$,

E. Bica ${ }^{31}$, C. Bonatto ${ }^{31}$, L. Bronfman ${ }^{32}$, G. Carraro ${ }^{9}$, A. N. Chenè ${ }^{19,20}$, J. J. Clariá21 ${ }^{2}$, J. R. A. Clarke ${ }^{20}$, C. Contreras ${ }^{4}$, A. Corvillón ${ }^{1}$, R. de Grijs ${ }^{33,34}$, B. Dias ${ }^{26}$, J. E. Drew ${ }^{4}$, C. Fariña ${ }^{27}$, C. Feinstein ${ }^{27}$, E. Fernández-Lajús ${ }^{27}$,

R. C. Gamen ${ }^{27}$, W. Gieren ${ }^{19}$, B. Goldman ${ }^{35}$, C. González-Fernández ${ }^{36}$, R. J. J. Grand ${ }^{37}$, G. Gunthardt ${ }^{21}$,

N. C. Hambly ${ }^{8}$, M. M. Hanson ${ }^{38}$, K. G. Hełminiak ${ }^{1}$, M. G. Hoare ${ }^{39}$, L. Huckvale ${ }^{10}$, A. Jordán ${ }^{1}$, K. Kinemuchi $^{40}$,

A. Longmore ${ }^{41}$, M. López-Corredoira ${ }^{42,43}$, T. Maccarone ${ }^{44}$, D. Majaess ${ }^{45}$, E. L. Martín ${ }^{46}$, N. Masetti ${ }^{47}$,

R. E. Mennickent ${ }^{19}$, I. F. Mirabel ${ }^{48,49}$, L. Monaco ${ }^{9}$, L. Morelli ${ }^{29}$, V. Motta ${ }^{20}$, T. Palma ${ }^{21}$, M. C. Parisi ${ }^{21}$, Q. Parker ${ }^{50,51}$, F. Peñaloza ${ }^{20}$, G. Pietrzyński ${ }^{18,19}$, G. Pignata ${ }^{52}$, B. Popescu ${ }^{38}$, M. A. Read ${ }^{8}$, A. Rojas ${ }^{1}$, A. Roman-Lopes ${ }^{12}$,

M. T. Ruiz ${ }^{32}$, I. Saviane ${ }^{9}$, M. R. Schreiber ${ }^{20}$, A. C. Schröder ${ }^{53,54}$, S. Sharma ${ }^{20,55}$, M. D. Smith ${ }^{56}$, L. Sodré Jr ${ }^{26}$, J. Stead ${ }^{39}$, A. W. Stephens ${ }^{57}$, M. Tamura ${ }^{58}$, C. Tappert ${ }^{20}$, M. A. Thompson ${ }^{4}$, E. Valenti ${ }^{5}$, L. Vanzi ${ }^{16,59}$, N. A. Walton ${ }^{7}$, W. Weidmann ${ }^{21}$, and A. Zijlstra ${ }^{10}$

(Affiliations can be found after the references)

Received 5 November 2011 / Accepted 22 November 2011

\section{ABSTRACT}

Context. The ESO public survey VISTA variables in the Vía Láctea (VVV) started in 2010. VVV targets 562 sq. deg in the Galactic bulge and an adjacent plane region and is expected to run for about five years.

Aims. We describe the progress of the survey observations in the first observing season, the observing strategy, and quality of the data obtained. Methods. The observations are carried out on the 4-m VISTA telescope in the $Z Y J H K_{\mathrm{s}}$ filters. In addition to the multi-band imaging the variability monitoring campaign in the $K_{\mathrm{s}}$ filter has started. Data reduction is carried out using the pipeline at the Cambridge Astronomical Survey Unit. The photometric and astrometric calibration is performed via the numerous 2MASS sources observed in each pointing.

Results. The first data release contains the aperture photometry and astrometric catalogues for 348 individual pointings in the $Z Y J H K_{\mathrm{s}}$ filters taken in the 2010 observing season. The typical image quality is $\sim 0^{\prime} 9-1{ }^{\prime \prime} 0$. The stringent photometric and image quality requirements of the survey are satisfied in $100 \%$ of the $J H K_{\mathrm{s}}$ images in the disk area and $90 \%$ of the $J H K_{\mathrm{s}}$ images in the bulge area. The completeness in the $Z$ and $Y$ images is $84 \%$ in the disk, and $40 \%$ in the bulge. The first season catalogues contain $1.28 \times 10^{8}$ stellar sources in the bulge and $1.68 \times 10^{8}$ in the disk area detected in at least one of the photometric bands. The combined, multi-band catalogues contain more than $1.63 \times 10^{8}$ stellar sources. About $10 \%$ of these are double detections because of overlapping adjacent pointings. These overlapping multiple detections are used to characterise the quality of the data. The images in the $J H K_{\mathrm{s}}$ bands extend typically $\sim 4$ mag deeper than 2MASS. The magnitude limit and photometric quality depend strongly on crowding in the inner Galactic regions. The astrometry for $K_{\mathrm{s}}=15-18$ mag has rms $\sim 35-175$ mas.

Conclusions. The VVV Survey data products offer a unique dataset to map the stellar populations in the Galactic bulge and the adjacent plane and provide an exciting new tool for the study of the structure, content, and star-formation history of our Galaxy, as well as for investigations of the newly discovered star clusters, star-forming regions in the disk, high proper motion stars, asteroids, planetary nebulae, and other interesting objects.

Key words. Galaxy: bulge - Galaxy: disk - Galaxy: stellar content - stars: abundances - infrared: stars - surveys

Figure D.2: Abstract of Saito et al. (2012). 


\section{Astronomy Astrophysics}

\section{Milky Way demographics with the VVV survey ${ }^{\star} \star \star \star$}

\section{The 84-million star colour-magnitude diagram of the Galactic bulge}

R. K. Saito ${ }^{1,2,3}$, D. Minniti ${ }^{1,3,4}$, B. Dias ${ }^{5}$, M. Hempel ${ }^{1,3}$, M. Rejkuba ${ }^{6}$, J. Alonso-García ${ }^{1,3}$, B. Barbuy ${ }^{5}$, M. Catelan ${ }^{1,3}$, J. P. Emerson ${ }^{7}$, O. A. Gonzalez ${ }^{6}$, P. W. Lucas ${ }^{8}$, and M. Zoccali1 ${ }^{1,3}$

1 Departamento de Astronomía y Astrofísica, Pontificia Universidad Católica de Chile, Vicuña Mackenna 4860, Casilla 306, Santiago 22, Chile

e-mail: rsaito@astro.puc.cl

Departamento de Física y Astronomía, Facultad de Ciencias, Universidad de Valparaíso, Ave. Gran Bretaña 1111, Playa Ancha, Casilla 5030, Valparaíso, Chile

3 The Milky Way Millennium Nucleus, Av. Vicuña Mackenna 4860, 782-0436 Macul, Santiago, Chile

4 Vatican Observatory, Vatican City State V-00120, Italy

5 Universidade de São Paulo, IAG, Rua do Matão 1226, Cidade Universitária, 05508-900 São Paulo, Brazil

${ }^{6}$ European Southern Observatory, Karl-Schwarzschild-Strasse 2, 85748 Garching b. München, Germany

7 Astronomy Unit, School of Physics and Astronomy, Queen Mary University of London, Mile End Road, London, E1 4NS, UK

8 Centre for Astrophysics Research, University of Hertfordshire, College Lane, Hatfield AL10 9AB, UK

Received 20 April 2012 / Accepted 20 June 2012

\section{ABSTRACT}

Context. The Milky Way (MW) bulge is a fundamental Galactic component for understanding the formation and evolution of galaxies, in particular our own. The ESO Public Survey VISTA Variables in the Vía Láctea is a deep near-IR survey mapping the Galactic bulge and southern plane. Particularly for the bulge area, VVV is covering 315 $\mathrm{deg}^{2}$. Data taken during 2010 and 2011 covered the entire bulge area in the $J H K_{\mathrm{s}}$ bands.

Aims. We used VVV data for the whole bulge area as a single and homogeneous data set to build for the first time a single colour-magnitude diagram (CMD) for the entire Galactic bulge.

Methods. Photometric data in the $J H K_{\mathrm{s}}$ bands were combined to produce a single and huge data set containing 173150467 sources in the three bands, for the $\sim 315 \mathrm{deg}^{2}$ covered by VVV in the bulge. Selecting only the data points flagged as stellar, the total number of sources is 84095284 .

Results. We built the largest colour-magnitude diagrams published up to date, containing 173.1+ million sources for all data points, and more than 84.0 million sources accounting for the stellar sources only. The CMD has a complex shape, mostly owing to the complexity of the stellar population and the effects of extinction and reddening towards the Galactic centre. The red clump (RC) giants are seen double in magnitude at $b \sim-8^{\circ}-10^{\circ}$, while in the inner part $\left(b \sim-3^{\circ}\right)$ they appear to be spreading in colour, or even splitting into a secondary peak. Stellar population models show the predominance of main-sequence and giant stars. The analysis of the outermost bulge area reveals a well-defined sequence of late $\mathrm{K}$ and $\mathrm{M}$ dwarfs, seen at $\left(J-K_{\mathrm{s}}\right) \sim 0.7-0.9 \mathrm{mag}$ and $K_{\mathrm{s}} \gtrsim 14 \mathrm{mag}$. Conclusions. The interpretation of the CMD yields important information about the MW bulge, showing the fingerprint of its structure and content. We report a well-defined red dwarf sequence in the outermost bulge, which is important for the planetary transit searches of VVV. The double RC in magnitude seen in the outer bulge is the signature of the X-shaped MW bulge, while the spreading of the $\mathrm{RC}$ in colour, and even its splitting into a secondary peak, are caused by reddening effects. The region around the Galactic centre is harder to interpret because it is strongly affected by reddening and extinction.

Key words. Galaxy: bulge - Galaxy: center - Galaxy: structure - Galaxy: stellar content - surveys

Figure D.3: Abstract of Saito et al. (2012). 


\title{
Self-consistent physical parameters for five intermediate-age SMC stellar clusters from CMD modelling ${ }^{\star}$
}

\author{
B. Dias ${ }^{1,2}$, L. O. Kerber ${ }^{1,3}$, B. Barbuy ${ }^{1}$, B. Santiago ${ }^{4}$, S. Ortolani $^{5}$, and E. Balbinot ${ }^{4}$ \\ 1 Instituto de Astronomia, Geofísica e Ciências Atmosféricas, Universidade de São Paulo, Rua do Matão 1226, Cidade Universitária, \\ 05508-900 São Paulo, SP, Brazil \\ e-mail: bdias@astro.iag.usp.br \\ 2 European Southern Observatory, Alonso de Cordova 3107, Santiago, Chile \\ 3 LATO-DCET-UESC, Rodovia Ilhéus-Itabuna km 16, Ilhéus, Bahia 45662-000, Brazil \\ 4 Universidade Federal do Rio Grande do Sul, IF, CP 15051, 91501-970, RS, Porto Alegre, Brazil \\ 5 Dipartimento di Fisica e Astronomia Galileo Galilei, University of Padova, vicolo dell'Osservatorio 3, 35122 Padova, Italy \\ Received 16 June 2013 / Accepted 15 November 2013
}

\section{ABSTRACT}

Context. Stellar clusters in the Small Magellanic Cloud (SMC) are useful probes for studying the chemical and dynamical evolution of this neighbouring dwarf galaxy, enabling inspection of a large period covering over $10 \mathrm{Gyr}$.

Aims. The main goals of this work are the derivation of age, metallicity, distance modulus, reddening, core radius, and central density profiles for six sample clusters, in order to place them in the context of the Small Cloud evolution. The studied clusters are AM 3 , HW 1, HW 34, HW 40, Lindsay 2, and Lindsay 3; HW 1, HW 34, and Lindsay 2 are studied for the first time.

Methods. Optical colour-magnitude diagrams ( $V, B-V$ CMDs) and radial density profiles were built from images obtained with the $4.1 \mathrm{~m}$ Southern Astrophysical Research (SOAR) telescope, reaching $V \sim 23$. The determination of structural parameters were carried out by applying King profile fitting. The other parameters were derived in a self-consistent way by means of isochrone fitting, which uses likelihood statistics to identify the synthetic CMDs that best reproduce the observed ones. Membership probabilities were determined comparing the cluster and control field CMDs. Completeness and photometric uncertainties were obtained by performing artificial star tests.

Results. The results confirm that these clusters (except HW 34, identified as a field fluctuation) are intermediate-age clusters, with ages between 1.2 Gyr (Lindsay 3) and $~ 5.0 \mathrm{Gyr}$ (HW 1). In particular HW 1, Lindsay 2 and Lindsay 3 are located in a region that we called West Halo, where studies of ages and metallicity gradients are still lacking. Moreover, Lindsay 2 was identified as a moderately metal-poor cluster with $[\mathrm{Fe} / \mathrm{H}]=-1.4 \pm 0.2 \mathrm{dex}$, lower than expected from the age-metallicity relation by Pagel \& Tautvaisiene (1998). We also found distances varying from $\sim 53 \mathrm{kpc}$ to $66 \mathrm{kpc}$, compatible with the large depth of the SMC.

Key words. galaxies: star clusters: general - Magellanic Clouds - Hertzsprung-Russell and C-M diagrams

Figure D.4: Abstract of Dias et al. (2014). 


\title{
Deriving metallicities from calcium triplet spectroscopy

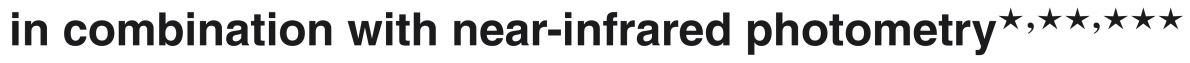

\author{
F. Mauro ${ }^{1}$, C. Moni Bidin ${ }^{1,2}$, D. Geisler ${ }^{1}$, I. Saviane ${ }^{3}$, G. S. Da Costa ${ }^{4}$, A. C. Gormaz-Matamala ${ }^{1}$, S. Vasquez ${ }^{3,5}$, \\ A.-N. Chené ${ }^{1,6,7}$, R. Cohen ${ }^{1}$, and B. Dias ${ }^{3,8}$ \\ ${ }^{1}$ Departamento de Astronomía, Universidad de Concepción, Casilla 160-C, Concepción, Chile \\ e-mail: skz5k2@gmail.com \\ 2 Instituto de Astronomía, Universidad Católica del Norte, Av. Angamos 0610, Casilla 1280, Antofagasta, Chile \\ 3 European Southern Observatory, Ave. Alonso de Cordova 3107, Casilla 19, 19001 Santiago, Chile \\ ${ }^{4}$ Research School of Astronomy \& Astrophyiscs, Australian National University, Canberra ACT 0200, Australia \\ 5 Instituto de Astrofísica, Facultad de Fisíca, Pontificia Universidad Católica de Chile, Av. Vicuña MacKenna 4860, Santiago, Chile \\ 6 Gemini Observatory, Hawaii, USA \\ 7 Departamento de Fisíca y Astronomía, Universidad de Valparaíso, Av. Gran Bretaña 1111, Casilla 5030, Playa Ancha, Chile \\ 8 Instituto de Astronomia, Geofísica e Ciências Atmosféricas, Universidade de São Paulo, rua do Matão 1226, Cidade Universitária, \\ 05508-900 São Paulo, Brazil
}

Received 28 October 2013 / Accepted 10 January 2014

\section{ABSTRACT}

Context. When they are established with sufficient precision, the ages, metallicities and kinematics of Galactic globular clusters (GGCs) can shed much light on the dynamical and chemical evolution of the Galactic halo and bulge. While the most fundamental way of determining GC abundances is by means of high-resolution spectroscopy, in practice this method is limited to only the brighter stars in the nearest and less reddened objects. This restriction has, over the years, led to the development of a large number of techniques that measure the overall abundance indirectly from parameters that correlate with overall metallicity. One of the most efficient methods is measuring the equivalent width (EW) of the calcium II triplet (CaT) at $\lambda \approx 8500 \AA$ in red giants, which are corrected for the luminosity and temperature effects using the $V$ magnitude differences from the horizontal branch (HB).

Aims. We establish a similar method in the near-infrared (NIR), by combining the power of the differential magnitudes technique with the advantages of NIR photometry to minimize differential reddening effects.

Methods. We used the $K_{\mathrm{s}}$ magnitude difference between the star and the reddest part of the HB (RHB) or of the red clump (RC) to generate reduced equivalent widths ( $\mathrm{rEW}$ ) from previously presented datasets. Then we calibrated these rEW against three previously reported different metallicity scales; one of which we corrected using high-resolution spectroscopic metallicities.

Results. We calculated the calibration relations for the two datasets and the three metallicity scales and found that they are approximately equivalent, with almost negligible differences. We compared our NIR calibrations with the corresponding optical ones, and found them to be equivalent, which shows that the luminosity-corrected rEW using the $K_{\mathrm{s}}$ magnitude is compatible with the one obtained from the $V$ magnitude. We then used the metallicities obtained from the calibration to investigate the internal metallicity distributions of the GCs.

Conclusions. We have established that the $([\mathrm{Fe} / \mathrm{H}]: \mathrm{rEW})$ relation is independent of the magnitude used for the luminosity correction and find that the calibration relations change only slightly for different metallicity scales. The CaT technique using NIR photometry is thus a powerful tool to derive metallicities. In particular, it can be used to study the internal metallicity spread of a GC. We confirm the presence of at least two metallicity populations in NGC 6656 and find that several other GCs present peculiar metallicity distributions.

Key words. stars: abundances - globular clusters: general - stars: kinematics and dynamics

Figure D.5: Abstract of Mauro et al. (2014). 


\title{
FORS2/VLT survey of Milky Way globular clusters
}

\section{Description of the method for derivation of metal abundances in the optical and application to NGC 6528, NGC 6553, M 71, NGC 6558, NGC 6426 and Terzan 8 *}

\author{
B. Dias ${ }^{1,2}$, B. Barbuy ${ }^{1}$, I. Saviane ${ }^{2}$, E. V. Held ${ }^{3}$, G. S. Da Costa ${ }^{4}$, S. Ortolani ${ }^{3,5}$, S. Vasquez ${ }^{2,6}$, M. Gullieuszik ${ }^{3}$, and D.
} $\mathrm{Katz}^{7}$

\footnotetext{
1 Universidade de São Paulo, Dept. de Astronomia, Rua do Matão 1226, São Paulo 05508-090, Brazil e-mail: bdias@astro.iag.usp.br

2 European Southern Observatory, Alonso de Cordova 3107, Santiago, Chile

3 INAF, Osservatorio Astronomico di Padova, Vicolo dell'Osservatorio 5, 35122 Padova, Italy

${ }^{4}$ Research School of Astronomy \& Astrophysics, Australian National University, Mount Stromlo Observatory, via Cotter Road, Weston Creek, ACT 2611, Australia

5 Università di Padova, Dipartimento di Astronomia, Vicolo dell'Osservatorio 2, 35122 Padova, Italy

6 Instituto de Astrofisica, Facultad de Fisica, Pontificia Universidad Catolica de Chile, Casilla 306, Santiago 22, Chile

7 GEPI, Observatoire de Paris, CNRS, Université Paris Diderot, 5 Place Jules Janssen 92190 Meudon, France

Received: ; accepted:
}

\section{ABSTRACT}

Context. We have observed almost $1 / 3$ of the globular clusters in the Milky Way, targeting distant and/or highly reddened objects, besides a few reference clusters. A large sample of red giant stars was observed with FORS2@VLT/ESO at R 2000. The method for derivation of stellar parameters is presented with application to six reference clusters.

Aims. We aim at deriving the stellar parameters effective temperature, gravity, metallicity and alpha-element enhancement, as well as radial velocity, for membership confirmation of individual stars in each cluster. We analyse the spectra collected for the reference globular clusters NGC 6528 ([Fe/H] -0.1), NGC $6553([\mathrm{Fe} / \mathrm{H}] \sim-0.2)$, M $71([\mathrm{Fe} / \mathrm{H}] \sim-0.8)$, NGC $6558([\mathrm{Fe} / \mathrm{H}] \sim-1.0)$, NGC 6426 $([\mathrm{Fe} / \mathrm{H}] \sim-2.1)$ and Terzan $8([\mathrm{Fe} / \mathrm{H}] \sim-2.2)$. They cover the full range of globular cluster metallicities, and are located in the bulge, disc and halo.

Methods. Full spectrum fitting techniques are applied, by comparing each target spectrum with a stellar library in the optical region at 4560-5860 $\AA$. We employed the library of observed spectra MILES, and the synthetic library by Coelho et al. (2005). Validation of the method is achieved through recovery of the known atmospheric parameters for 49 well-studied stars that cover a wide range in the parameter space.

Results. We identified 4 member stars in NGC 6528, 13 in NGC 6553, 10 in M 71, 5 in NGC 6558, 5 in NGC 6426 and 12 in Terzan 8 Radial velocities, effective temperatures, gravities, metallicities and alpha-element enhancements were derived. We derived $\left\langle v_{\text {helio }}\right\rangle$ $=-242 \pm 11 \mathrm{~km} / \mathrm{s},[\mathrm{Fe} / \mathrm{H}]=-2.39 \pm 0.04,[\mathrm{Mg} / \mathrm{Fe}]=0.38 \pm 0.06$ for NGC 6426 from spectroscopy for the first time.

Conclusions. The method proved to be reliable for red giant stars observed with resolution $\mathrm{R} \sim 2000$, yielding results compatible with high-resolution spectroscopy. The derived $\alpha$-element abundances show $[\alpha / \mathrm{Fe}]$ vs. $[\mathrm{Fe} / \mathrm{H}]$ consistent with that of field stars at the same metallicities.

Key words. Stars: abundances - Stars: kinematics and dynamics - Stars: Population II - Galaxy: globular clusters - Galaxy: globular clusters: individual: NGC 6528, NGC 6553, M 71, NGC 6558, NGC 6426, Terzan 8 - Galaxy: stellar content

Figure D.6: Abstract of Dias et al. (2014b), submitted to A\&A and accepted after revision. 